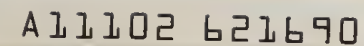

NATL INST OF STANDARDS \& TECH R.I.C.

(1)

A11102621690

Smlth, Bradford M/Inltlal graphics excha

1986 V 19 C.1 NBS-P

9

\title{
Initial Graphics Exchange Specification (IGES), Version 3.0
}

Brad Smith

Joan Wellington

U.S. DEPARTMENT OF COMMERCE

National Bureau of Standards

National Engineering Laboratory

Center for Manufacturing Engineering

Automated Production Technology Division

Gaithersburg, MD 20899

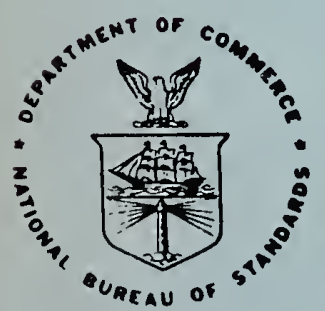

U.S. DEPARTMENT OF COMMERCE

NATIONAL BUREAU OF STANDARDS 


\section{ACKNOWLEDGEMENT}

Version 3.0 of this IGES Document is the joint effort of a large number of people who comprise the IGES Organization. Individually, these experts have shown tremendous personal commitment. Collectively, they have demonstrated a spirit of cooperation that is unique in consensus standards development. Each company has ample reason to be proud of the contributions of the IGES member they sponsor.

While individual contributions are too numerous to mention, I wish to recognize the efforts of my Secretary, Mrs. Mary Marello, for the word processing of this Document. Her accuracy and attention to detail are second only to her dedication in making IGES the useful tool it is. My personal thanks to Mary for an outstanding job.

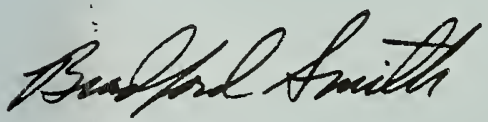

Bradford Smith

Chairman, IGES 


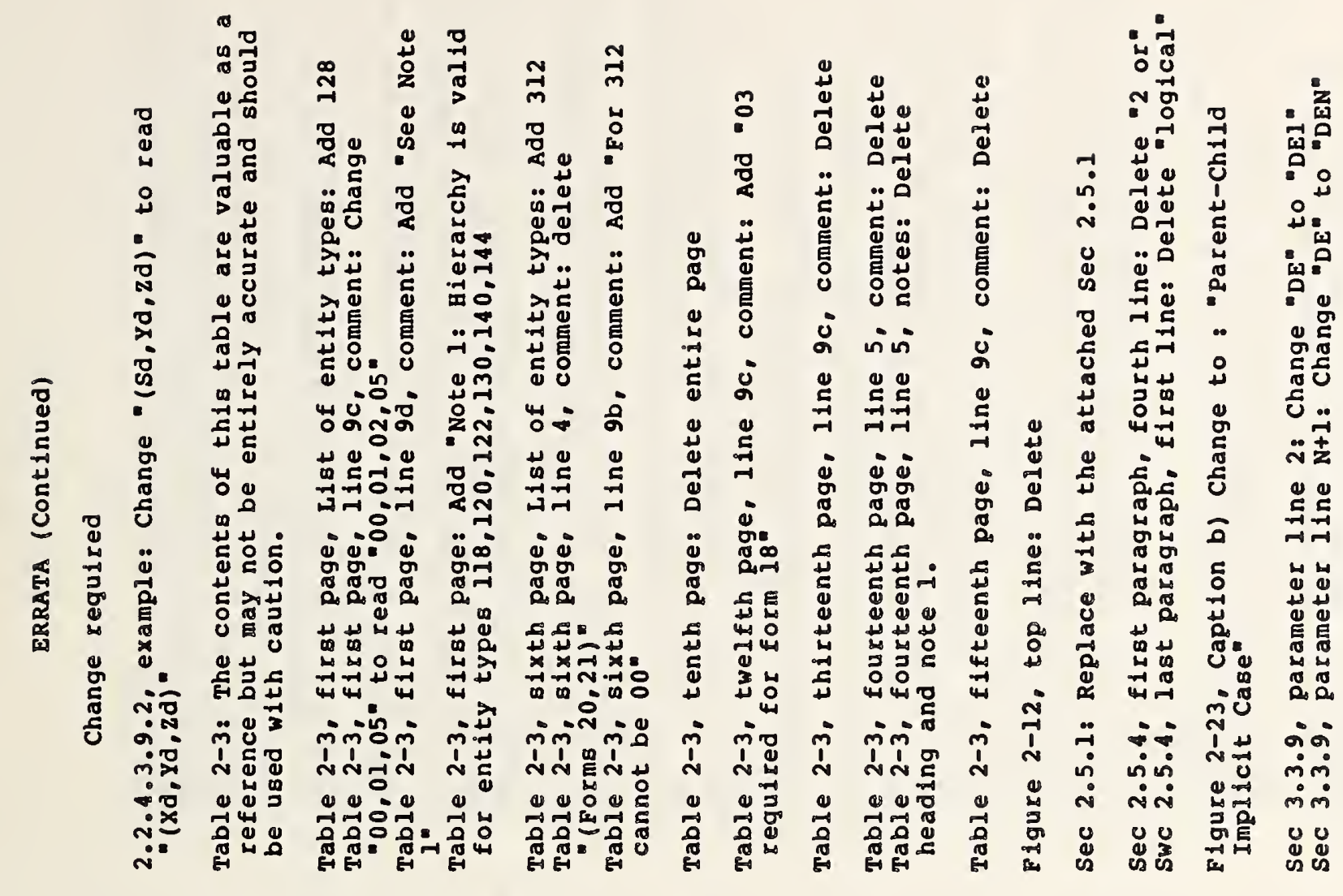

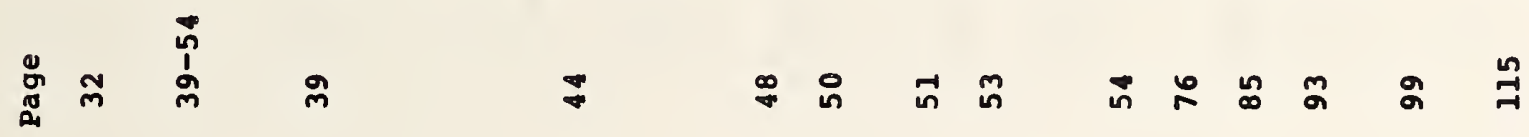

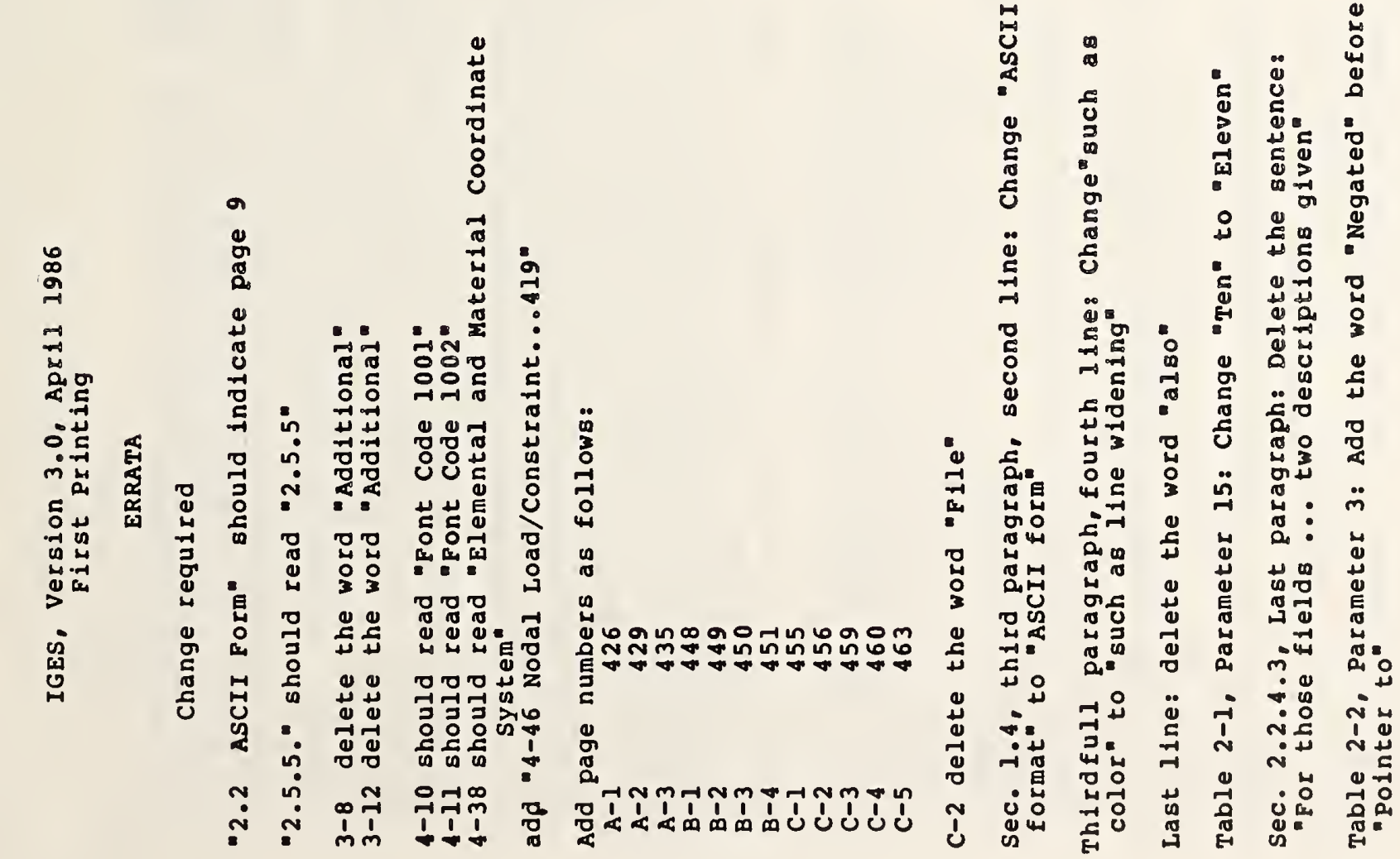

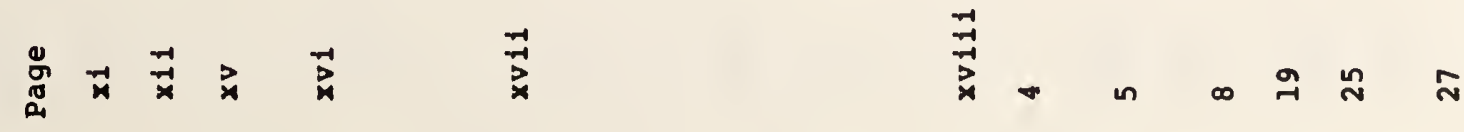




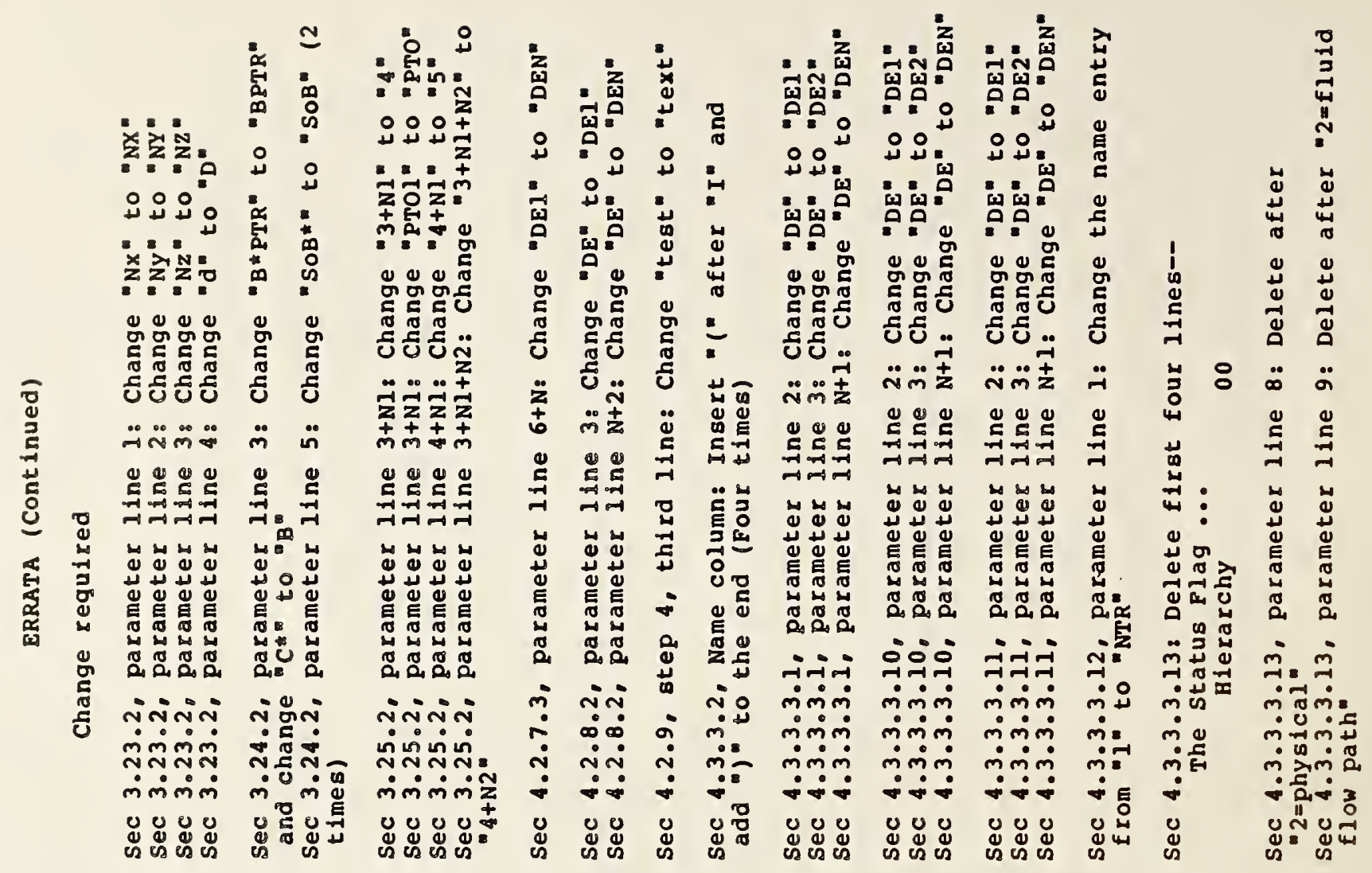

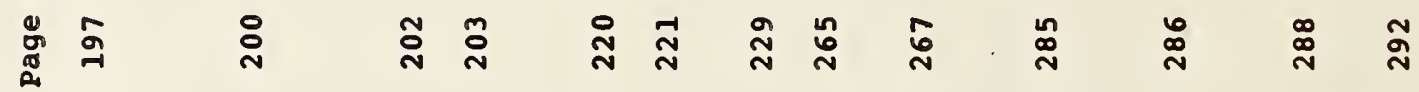

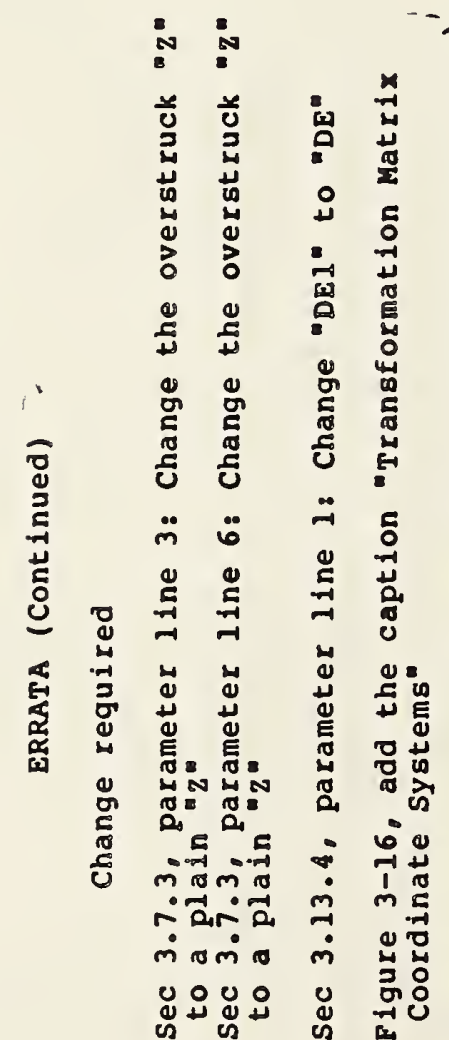

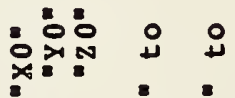

요워

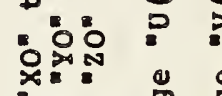
अ० รั. สู่อ๊ป

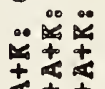

$\ddot{0} \ddot{0}$

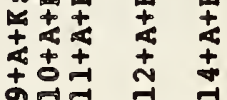

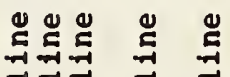

प

苟

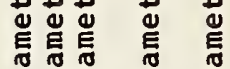

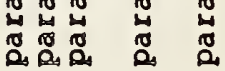

กิง

둥

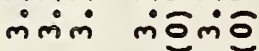

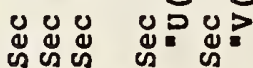

空. -

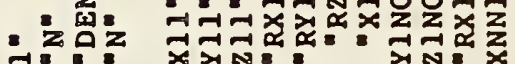

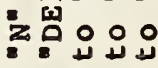

요열

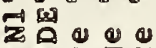

a

๒ูํำ

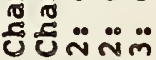

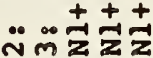

藏骂号

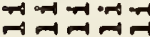

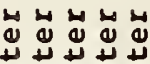

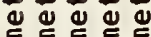

言望苗

$\rightarrow \pi \begin{gathered}\pi \\ \pi\end{gathered}$

a, कos as a

$+\infty+\infty$

สีสี่สีสี

mंxंmini

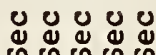

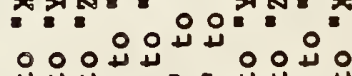

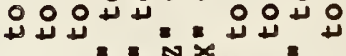

xis

ข

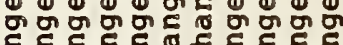

ช

ชับठठ

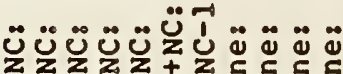

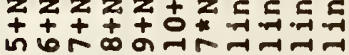

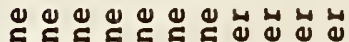

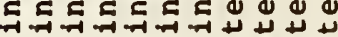

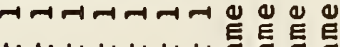
菏

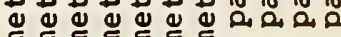

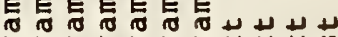
山4 ล

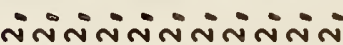

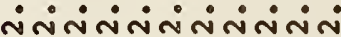

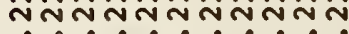

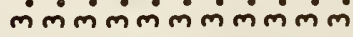

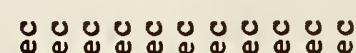
๗

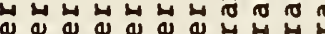

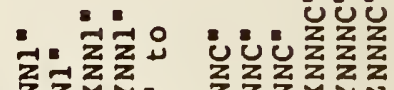

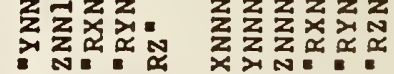

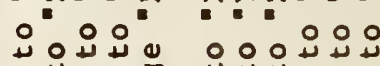
$\downarrow x x^{\circ}$ N舟西

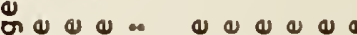

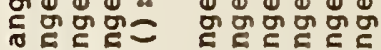

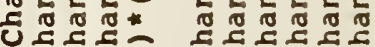

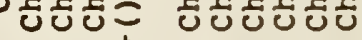
$\ddot{j} \ddot{\omega} \ddot{\omega} \ddot{u}$

E

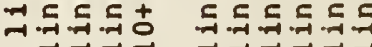
નનનન નનનનન

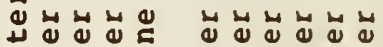
岒

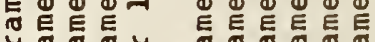
ถั 山م

$\vec{x} \vec{x} \vec{x} \vec{x} \ddot{x} \vec{x}$

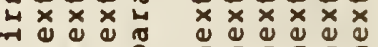

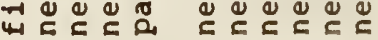
NiñNin niñniñ

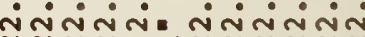
งกำกงกง

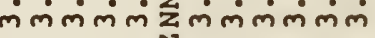
U

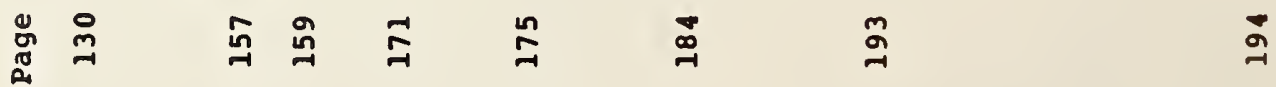




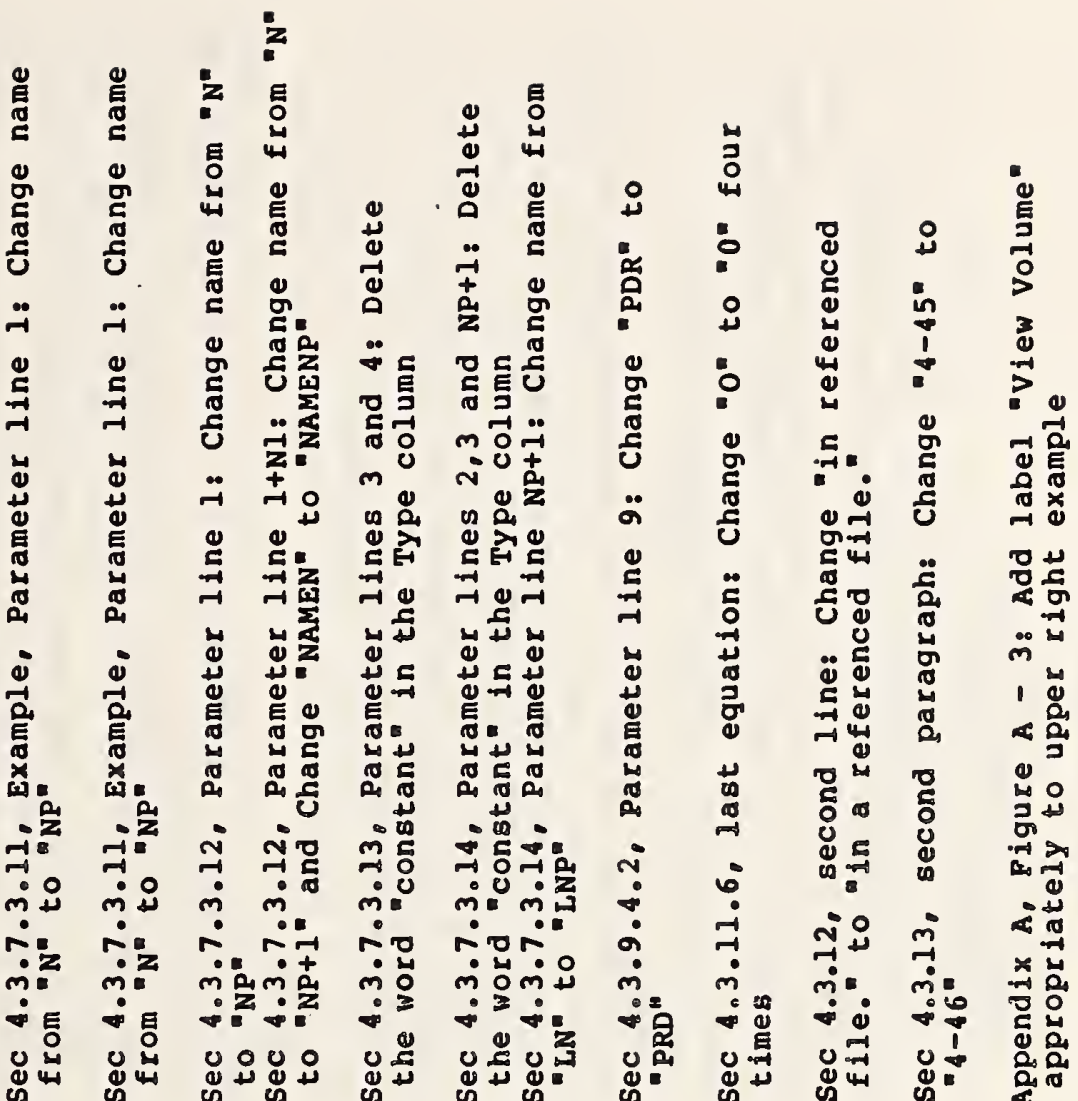

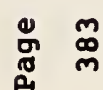

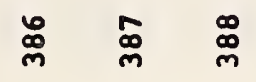

命

$\stackrel{\circ}{\Rightarrow} \nRightarrow \stackrel{\text { ॥ }}{\sharp}$

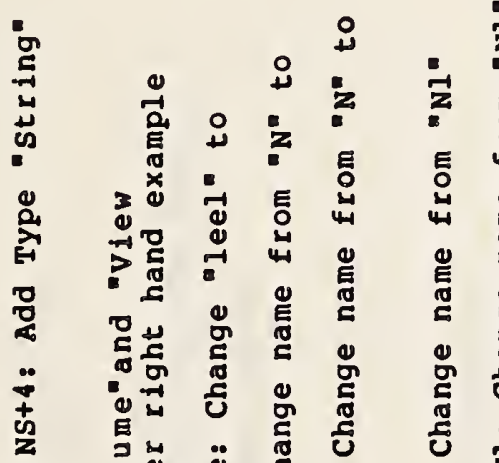

ปิ

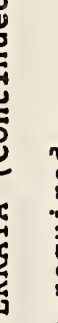

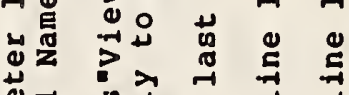

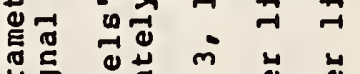

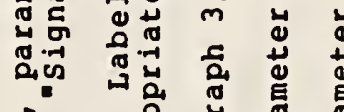

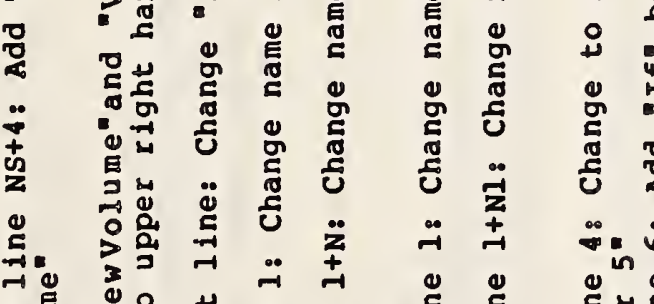

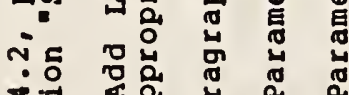

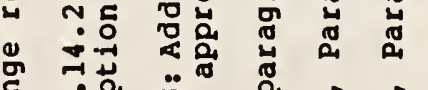

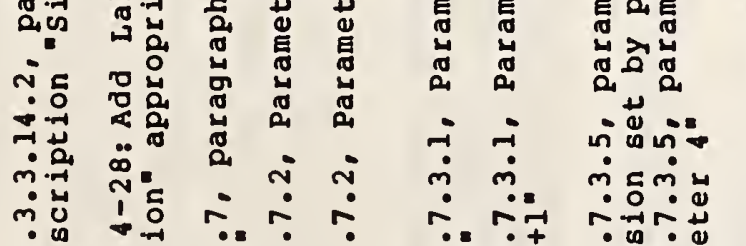

$\dot{m}$ o

$\div$ 过

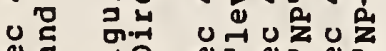

जा का

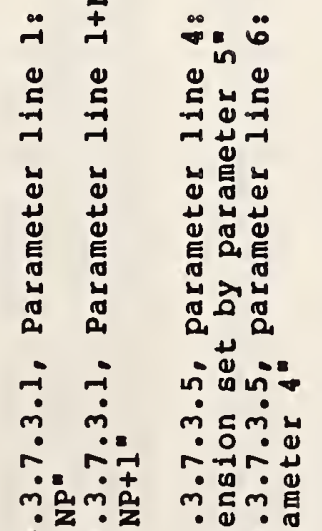

00000 离政

为然
ह

)

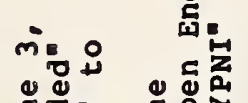

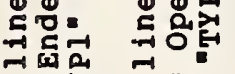

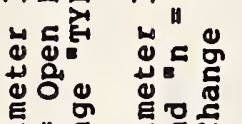

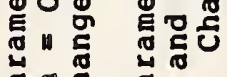

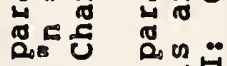

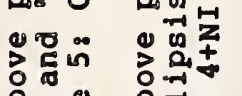

舟邑

तح

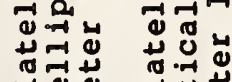

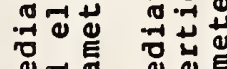

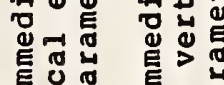

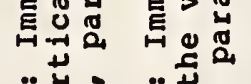

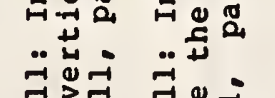

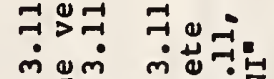

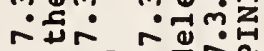

ㅁ

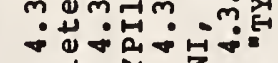

ن요

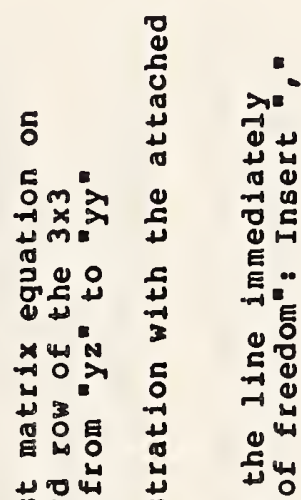

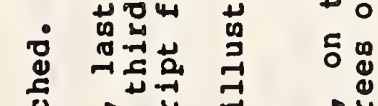

iे ठै

1 50

政

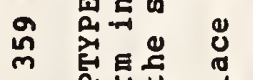

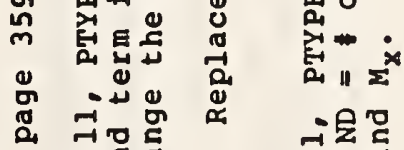

o

워

$\dot{m}^{\infty} \ddot{x}+$

กิ:

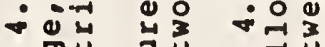

ن

范 


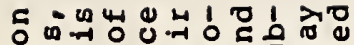
年

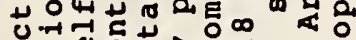

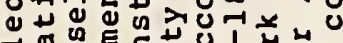

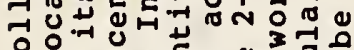
ช 0 중 เ

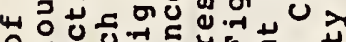

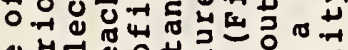

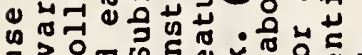

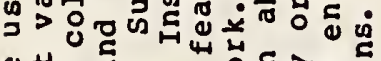

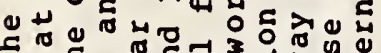
4 ๑ 舟

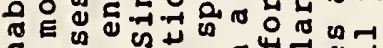
ธ。 ○ 0 व ह o ชี

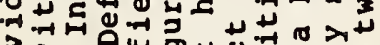
3 3 .

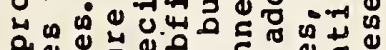
0. ธ

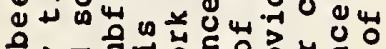

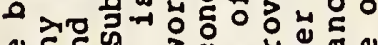

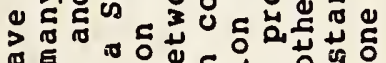
舟 कo ต מ.

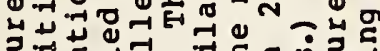

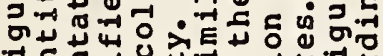

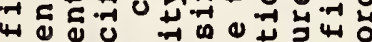
1 0 o

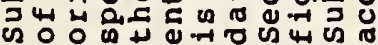

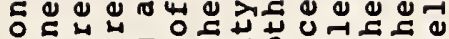
-

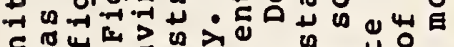
出

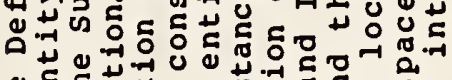
म ว ข ชิ $\rightarrow$ O

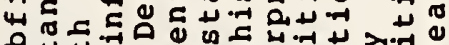

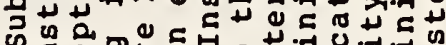

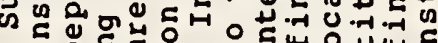
ه 0 4 崩 ने का व

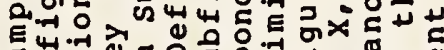

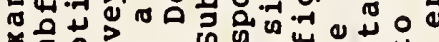

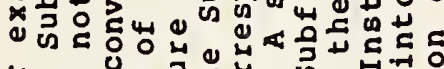
山ै ए 든 ० ป.

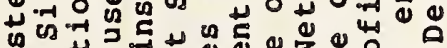
为

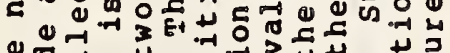

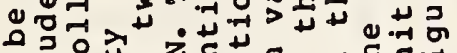

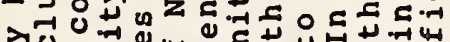

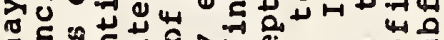
E

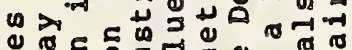
ข

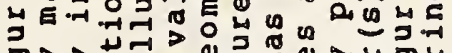

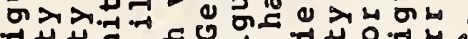
फ

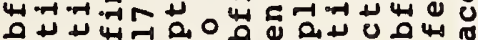

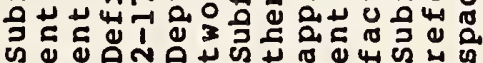

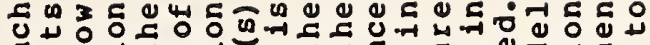

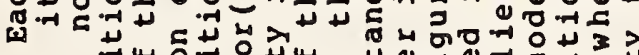

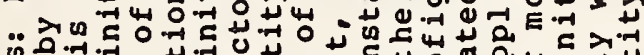

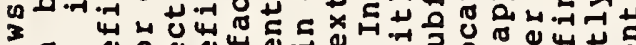

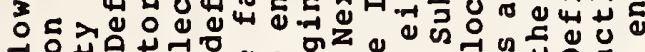

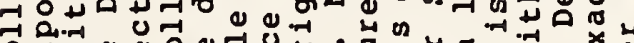
운 ס

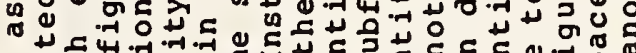

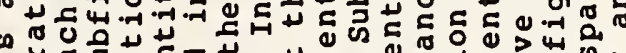
ન 品 \&

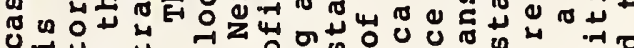

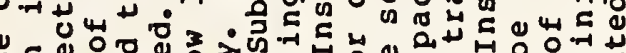

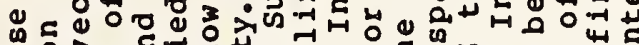

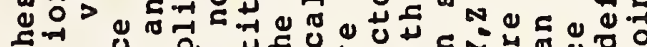
山⿷

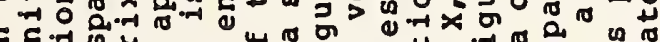
न لـ

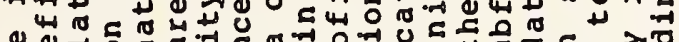
ญั

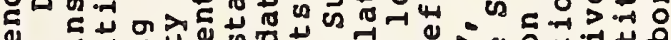

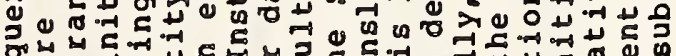
Oे

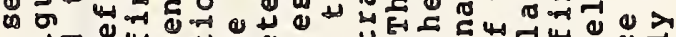
वाँ

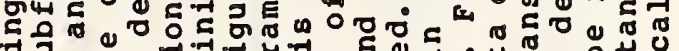

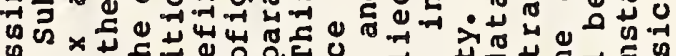
ص ठ도

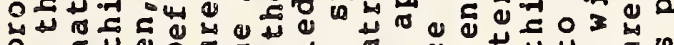

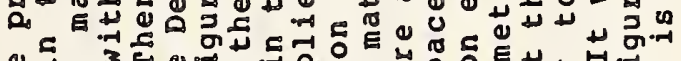

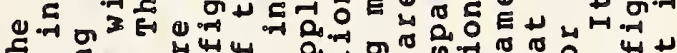

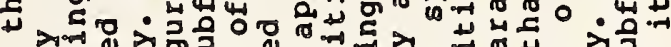
-

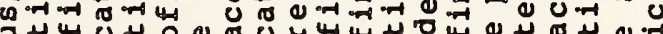

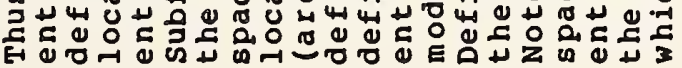

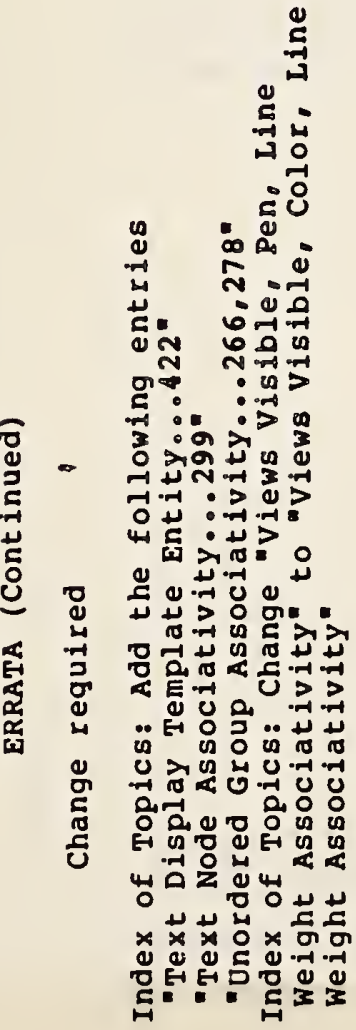




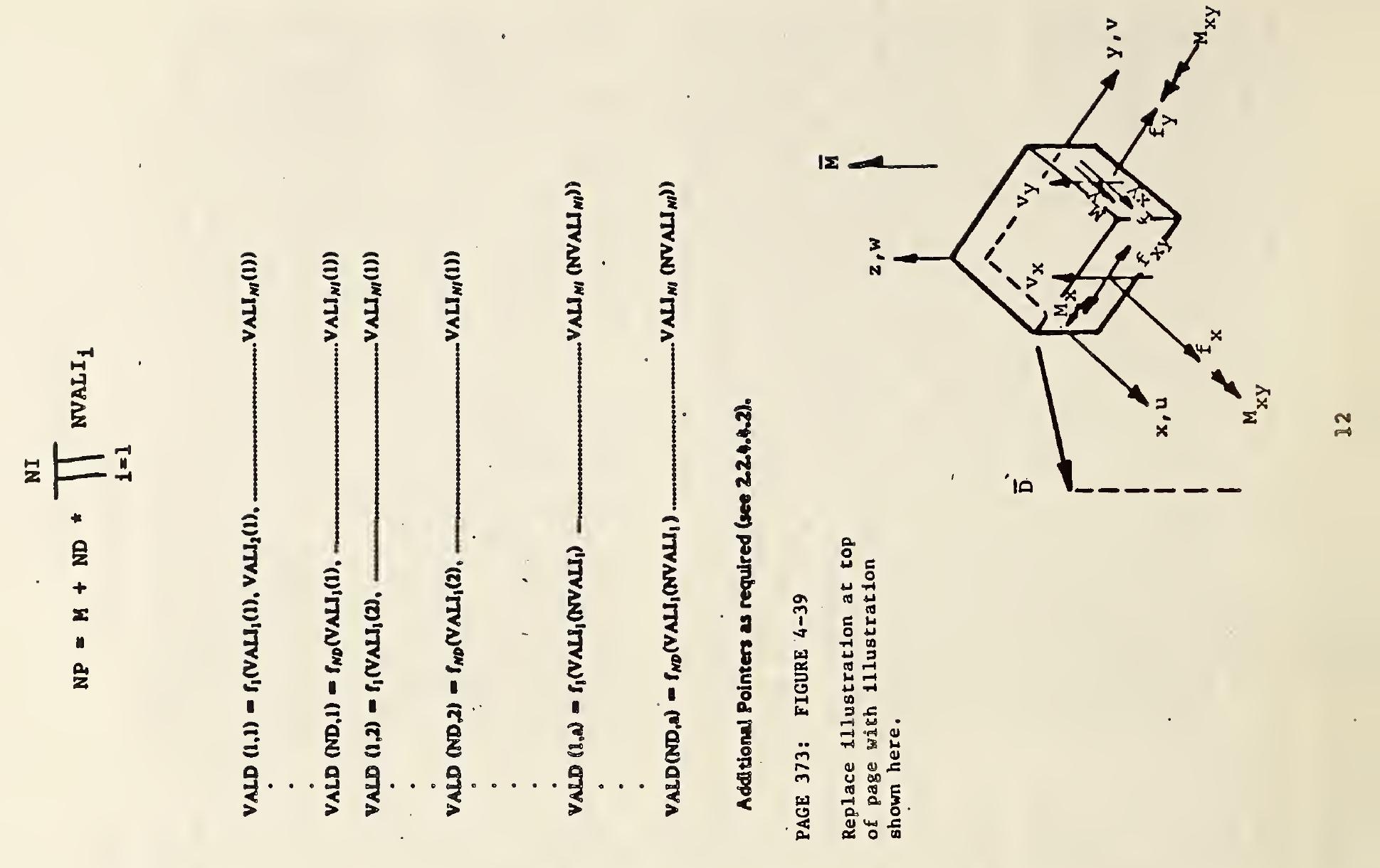

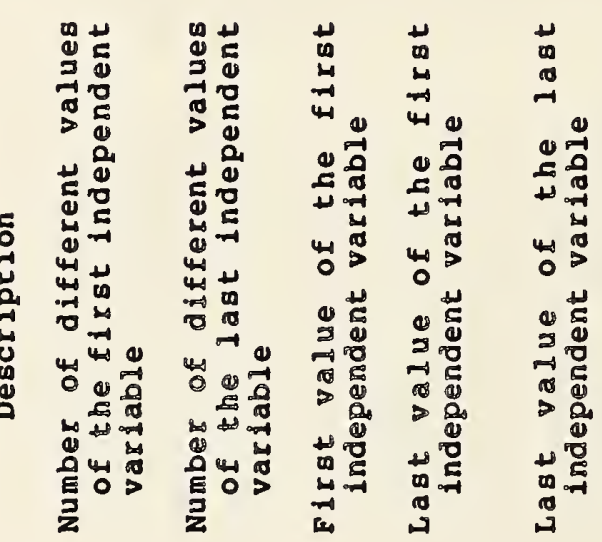

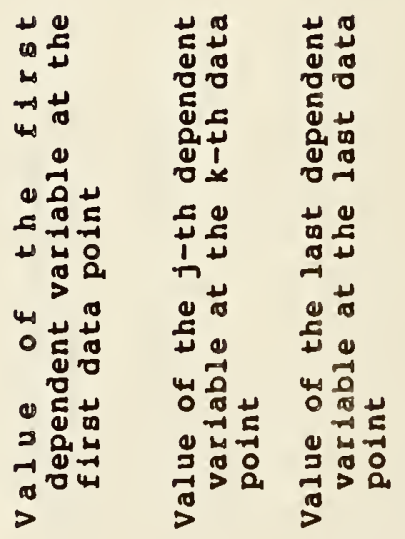

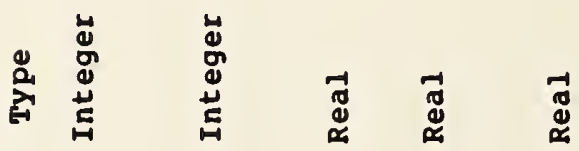

蛋

$\cong$

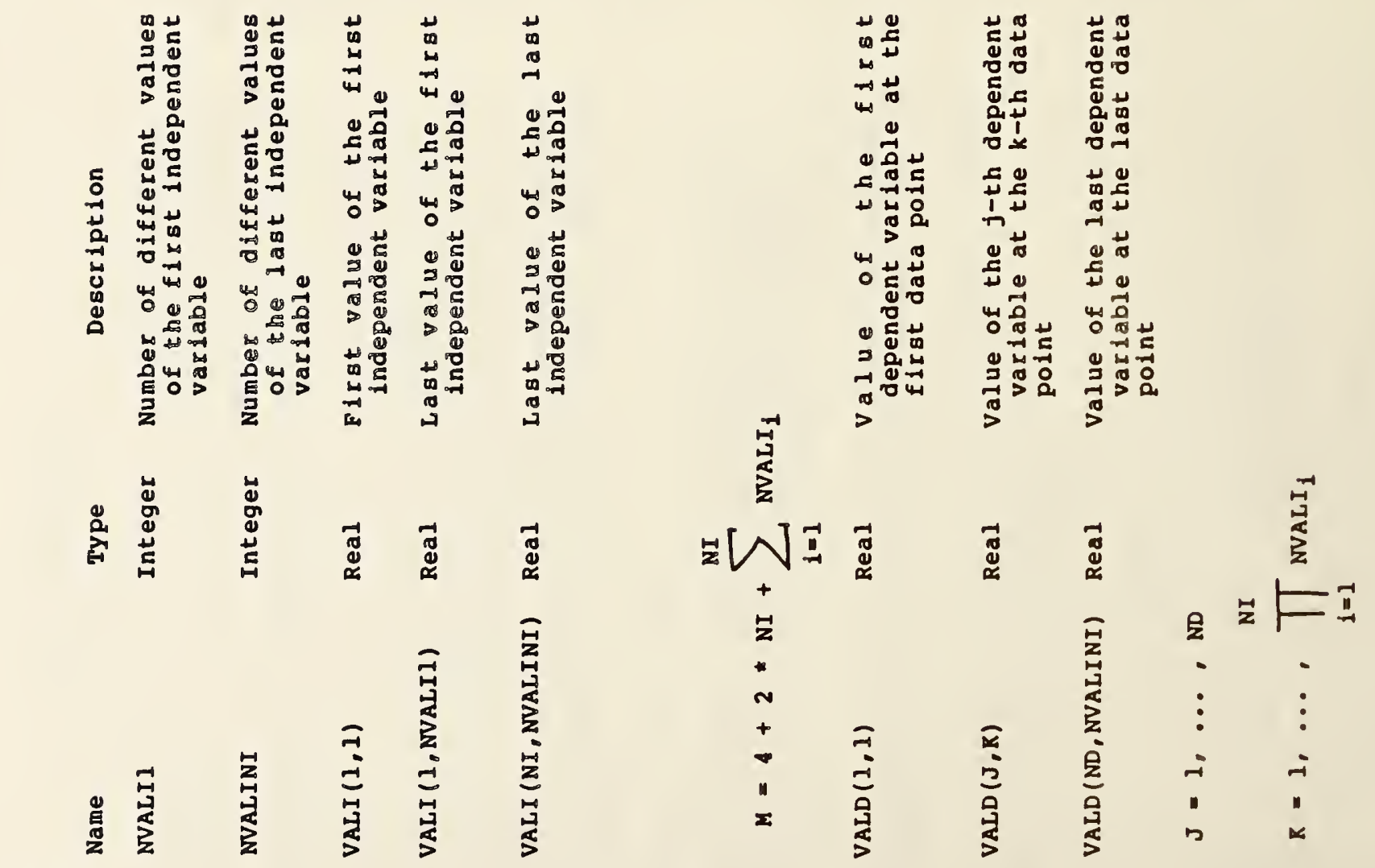

㟲

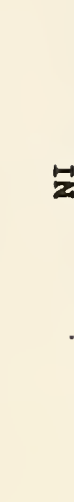

年 


\section{INITIAL GRAPHICS EXCHANGE SPECIFICATION (IGES), VERSION 3.0}

Brad Smith

Joan Wellington

U.S. DEPARTMENT OF COMMERCE

National Bureau of Standards

National Engineering Laboratory

Center for Manufacturing Engineering

Automated Production Technology Division

Gaithersburg, MD 20899

April 1986

U.S. DEPARTMENT OF COMMERCE, Malcolm Baldrige, Secretary NATIONAL BUREAU OF STANDARDS, Ernest Ambler, Diroctor 

Special recognition is accorded to the following officers of the IGES Organization who have contributed heavily to both the technical content and the editorial review of this document.

$\begin{array}{lll}\text { Jeff } & \text { Altemueller } & \text { McDonnell Douglas AIS Co. } \\ \text { Raymond E. } & \text { Barker } & \text { Caterpillar Tractor Co. } \\ \text { William G. } & \text { Beazley } & \text { W. G. Beazley \& Assoc. } \\ \text { Kalman } & \text { Brauner } & \text { The Boeing Commercial Airplane Co. } \\ \text { William C. } & \text { Burkett } & \text { McDonnell Aircraft Co. } \\ \text { Noel } & \text { Christensen } & \text { Allied Bendix Aerospace } \\ \text { Edward } & \text { Clapp } & \text { IBM Corporation } \\ \text { Robert } & \text { Colsher } & \text { IGES Data Analysis Corp. } \\ \text { Spencer } & \text { DePauw } & \text { Caterpillar Tractor Co. } \\ \text { Jim } & \text { Fleming } & \text { Cummins Engine Co. Inc. } \\ \text { William B. } & \text { Gruttke } & \text { McDonnell Douglas AIS Co. } \\ \text { Dennette A. } & \text { Harrod Jr } & \text { Applicon Corporation } \\ \text { Robert } & \text { Ivey } & \text { Westinghouse Electric Corp. } \\ \text { J.C. } & \text { Kelly } & \text { Sandia National Laboratories } \\ \text { Philip } & \text { Kennicott } & \text { General Electric Co. } \\ \text { Linda } & \text { Martino } & \text { IBM Corporation } \\ \text { Larry } & \text { O'Connell } & \text { Sandia National Laboratories } \\ \text { Curtis H. } & \text { Parks } & \text { General Dynamics Corp } \\ \text { Kent } & \text { Reed } & \text { National Bureau of Standards } \\ \text { Patrick W. } & \text { Rourke } & \text { Newport News Shipbuilding } \\ \text { Douglas } & \text { Schenck } & \text { McDonnell Douglas } \\ \text { Fred } & \text { Stahl } & \text { IBM Corporation } \\ & & \end{array}$





\begin{tabular}{|c|c|}
\hline Joseph & Aebischer \\
\hline Jeff & Al temLlel l er \\
\hline Eil1 & Anderson \\
\hline Fiobert $E$. & Anderson \\
\hline Colin & Ashley \\
\hline Faul & Atallah \\
\hline $\begin{array}{l}\text { Dave } \\
\text { Fiaymond E. }\end{array}$ & $\begin{array}{l}\text { Barteer } \\
\text { Barl:er }\end{array}$ \\
\hline William $G$. & Bearley \\
\hline Fionald & Eiel cher- \\
\hline F'eter & E巨חjamin \\
\hline Ted & Вегепуi \\
\hline Dieter $W$. & Eergman \\
\hline Kevin & Bl actuel 1 \\
\hline Donald L. & El ant: \\
\hline Sапdra & Bowen \\
\hline Jemes $A$ : & Er adford \\
\hline Norman & Brainard \\
\hline Kalman & Er ELInEr \\
\hline Fiaymond $A$. & Er.engs \\
\hline DEvid & Eriggs \\
\hline Stan & Briggs \\
\hline Fonald & Eryant \\
\hline Willi am C. & Eutr-l:ett \\
\hline Fichard & Butler \\
\hline Bradley $K$. & $\operatorname{col} 11$ \\
\hline Gerald $E_{\text {: }}$ & Campbel1 \\
\hline James & Carterry \\
\hline Lt Fotert & Carringer \\
\hline Dr. Nien-Hua & Etrao \\
\hline Ken & Christensen \\
\hline $\begin{array}{l}\text { NoEl } \\
\text { Al }\end{array}$ & $\begin{array}{l}\text { Christensen } \\
\text { Cinque }\end{array}$ \\
\hline Edward & El $\mathrm{APp}$ \\
\hline Alan $L$. & Clart: \\
\hline Margaret & Cline \\
\hline Fotsert & Clines \\
\hline $\begin{array}{l}\text { Fichard } \\
\text { Charles E. }\end{array}$ & $\begin{array}{l}\text { Cochr an } \\
\text { cockrell }\end{array}$ \\
\hline $\begin{array}{l}\text { Fobert } \\
\text { Fichard }\end{array}$ & $\begin{array}{l}\text { Colsher } \\
\text { Costabile }\end{array}$ \\
\hline $\begin{array}{l}\text { Gary } A . \\
\text { Fobert } M .\end{array}$ & $\begin{array}{l}\text { Cox } \\
\text { Eutry }\end{array}$ \\
\hline Farlis & Dantiers \\
\hline Fiaul $H_{\text {. }}$ & Davis \\
\hline $\begin{array}{l}\text { Anthory James } \\
\text { Marl: }\end{array}$ & $\begin{array}{l}\text { Day } \\
\text { Demeranvil1e }\end{array}$ \\
\hline Spencer & DeFaúw \\
\hline Karen & Deutsch \\
\hline Fichard N. & DiFronaD \\
\hline Fielph & Disa \\
\hline George & Donnel 1 an \\
\hline
\end{tabular}

Martin Marietta Data Systems

MeDonnel 1 Douglas AIS Co

General Dynamics Corp.

NAVAIF EngineEring Support office

FAFEC Ltd

Electronic Data Systems Corp:

Hercules Aerospace

Caterpillar Tractor Co.

W. G. Beazley \& Assoc.

MrDonnel 1 Douglas Automation Co.

Lockheed Missiles And Space Co.

Deere and Compary

$I \cdot F \quad C$

Lawrence Livermore Nationjl Laboratory

Sperry Information Systems

Electranic Data Systems

Allied Bendix Aerospace

Electronic Data Systems

The Boeing Commercial Airplane Co.

Naval Ship Fi $D$ D Center

The Bueing Commercial Airplare Co.

Naval Air Fieworl: Facility

Xeros Corporation

MeDorriell Aircraft Co.

Computervision Corp.

F'DA Engineering

Computervision Corp.

NAUFAC

US Air Force ICAM Office

A $T$ 足 $T$ BEII Latoratories

Ford Motar Comfany

Allied Eendix Aerospace

M. Fosenblatt is Som

IEM CorF.

Ford Mator Co.

Lackheed Geargia

Martin Marietta Aerospace

Fratt o ufictiey

NASA Larigley Fiesestch Center

IGES Data Aralysis Corp.

Xerox Corporation

LTY Aerospace and Defense Company

TFiW Electrorics Defense

TFiW Electrorics

Fratt \& whitrey UTC

Sikorst:y Aircraft UTC

Sperry Defense Froducts Group

Caterpillar Tractor Co.

CADAM InC

Metagraphics Iric.

Fratt \& Whitney

Honeywell Electra-Optics Div. 
Fion

Alan E.

Marc $W^{\circ}$

Colin F.

Fiobert $J$.

Fichard

Ni.ct:

Jim

Henry $H_{\text {. }}$

Danny

Feter

Lel and $H_{4}$

Will i am Fi。

Fichard

Fioger

walter $F$.

Wi. 11 i alí

Albert. J

Mitchel. 1

Burtor

Fiainer

Scatt $A$.

William $H_{\text {" }}$

Willi am E.

John

C. Scott

Dennette $A_{1}$

Michael

Don

Edward $C$.

Willi am $A_{\text {. }}$

Fichard

Fiobert

Dwight L.

Scott $E_{\text {. }}$

Antriony

Esfan

Thomas

J. [.

Debbie

Fhilip

Stephen $T$.

Gabor

Faren L.

Sudhir

Stanley J.

M. J.

Harry

Jotin

Debor ah

Lanise M.

Kaiman

Ertuce
Dowrier

Dragoo

Durnin

Ear 1

Easterday

Eppes Jr

Fl:i aras

Fleming

Forig

Forthiman

Fossel inari

Frayseth

Freeman

Futir

Gale

Geisinger

Gerrein

Gibbors

Gilbert

Gischrier

Gl atz

Gordon

Grey

Grutti:e

Hardt

Harris

Harrod Jr

Hastings

Hemmelgarn

Hong

Hussang

Isler

Ivey

Jaeger

Jorgensori

Joyce

Karnvar

reane

Hel Iy

Kengott

rennj.cott

kertis

Kiss

Fontry

Kshirsagar

fulta

rutt:us

Ladd

Lamoureux

LaFiey

Leach

Lee

Lepisto
Hughes Aircraft Co. EDSG

McDorinel 1 Douglas MIS Co

Lockheed-Georgi. a Company

Automation Technology Froducts

Martin Marietta Energy Systems

us Army Missile Command

Tel:tronis Inc.

Cummine Engine Co. Inc: .

F'DA Engineering

Bruning CAD

Douglas Aircraft

Eechtel Fietroleum

Al. 1 ied Eendix Aerospace

The Eoeing Commercial Airplane Co.

D. Appleton Co.

Construction Specifications Institute

General Electric Co.

Westinghouse Electric Corp.

Grumman Aerospace Corp.

General Dynamics - Electric Boat: Div.

University of Karlsruhe

NASA Goddard Space Flight Center

Martin Marietta Energy Systems

McDorinell Douglas AIS C.o.

Dana Corp.

Hugties Aircraft Co

Applicon

Control Data Corporation

Interrationa]. TechneGroup Inc

Electronic Data Systems

Horeywell Inc. Avionics Division

Sandia National Laboratories

Westingticuse E]ectric Corp.

Los Alamos National Laboratory

Contro]. Data Corporation

Naval Facilities Engineering Command

AT 品 T Bell Laboratories

The Boeing Commercial Airplane Co.

Sandia National. Laboratories

AutoTrol Technology Corp.

General Electric Co.

Naval Weapons Center

Bel1 Communications Fessearch

Electronic Data Systems Corporation

SDFiC

General Dynamics

Hughes Aircraft Co:

DuForit

IIT Fiesearch Institute

Westingtiouse Electric Corp.

US Military Academy

Naval Facilities Engineering Comand

Department of Defense 


\begin{tabular}{|c|c|c|}
\hline Olga & Lichten & IEM CorF: \\
\hline Michael & LiEwald & Hugties Aircaft Co. \\
\hline Vincent & $\operatorname{Lin}$ & Automation Technology Froducts \\
\hline Fiobert & Lipman & Naval Ship $F$ \& D Center \\
\hline Lincoln & LittlE & Cummins Engine Co. Inc. \\
\hline Julie & Long & Electronic Data Systems \\
\hline Bi 11 & Loye & Interconnics \\
\hline $\begin{array}{l}\text { Fiotert } \\
\text { Spencer }\end{array}$ & $\begin{array}{l}\text { Maclatchie } \\
\text { Magleby }\end{array}$ & $\begin{array}{l}\text { FlanFrint Company } \\
\text { General Dynamics Corp. }\end{array}$ \\
\hline Eil1 & Marl: 5 & Hugties Aircraft Co \\
\hline Linda & Martino & IEM CORP. \\
\hline Steven & Mastrangelo & Micro Contral Systems Inc. \\
\hline Fialph J. & Mayer & Adra Systems, Inc: \\
\hline Faphael & MCBain & General Dynamics Corp. \\
\hline Marie & MCFar 1 and & Hughes Aircraft Co. \\
\hline Stanley & MCMil1Eח & Gerber Systems Technolagy Inc. \\
\hline H. W. Guy & Meyer & Wisconsin Dept of Transportation \\
\hline Thurter J. & Moffett & Northirup \\
\hline Charles $E_{\text {. }}$ & Morril 1 & IEM Corp. \\
\hline$F \cdot G$. & Marrison Jr & MCDonnel 1 Aircraft Co. \\
\hline $\begin{array}{l}\text { Jaseph A. } \\
\text { Fuger }\end{array}$ & $\begin{array}{l}\text { Mudd } \\
\text { Mage }\end{array}$ & Bechtel Fietroleum Inc. \\
\hline Faul A. & Nel 5 on & Hugties Aircraft Ca. \\
\hline David & Norling & Boeing Computer Services \\
\hline Fred J. & Norton & Lawrence Livermore National Laboratory \\
\hline Frof. Horst & Nowacki & Technical University Berlin \\
\hline Larry & Q'ConineI 1 & Sandia National Laboratories \\
\hline W. Fiob & Daties & Los Alamos National Laboratory \\
\hline Jeffrey & otten & General Electric Co \\
\hline Conחie & Fañica & General Motars BOC Hats \\
\hline Geoffrey H. & Fartier & Intergraph Corp. \\
\hline Curtis $H$. & Fiarl:s & General Dyriamies Corp. \\
\hline 61 al!s & F'asemanா & Volk:swageriwerg AG \\
\hline Al an & F'Eltzman & Software Consultant \\
\hline StEVE & F'eters & Fierce, Goodwin, Alexander \\
\hline Freda & Fhelps & Lawrence Livermore National Laboratory \\
\hline उ०ח & Fittman & Hot: Computer Services Corp: \\
\hline Vladimir & Foctiop & AutoDest: Inc. \\
\hline Tim & Frotiast:a & Allied Bendix Aerospace \\
\hline Llayd & Furves & NASA Goddard Space Flight Center \\
\hline rent & FiEed & National Bureaul of Standards \\
\hline Gaylen Fi. & Firiaudot & National Eureau of Standards \\
\hline Fhillip & Fiosol & Martin Marietta Data Systems \\
\hline Fitrict: W. & Fiount:e & Newport News Shiptuilding \\
\hline Walter J. & Fiygie1 & Ford Motor Co. \\
\hline riandy & Schmid & Hughes Aircraft Co EDSG \\
\hline Dirt: & Sctiroeter & Martin Marietta Orlando Aerospace \\
\hline Arrigld & Stak & Grumman Data Systems \\
\hline Jerry & Stiaver & Gerber Systems Technology \\
\hline Chia Hui & Stith & SDFC \\
\hline Marie & Skutch & Westinghouse Electric Corp. \\
\hline Er adford & Smiti & National Eureau of Standards \\
\hline Keith M. & Smith & Summa Technologies Inc. \\
\hline
\end{tabular}




\begin{tabular}{|c|c|}
\hline Jj. & Snyder" \\
\hline $\begin{array}{l}\text { Michaed V. } \\
\text { Fred }\end{array}$ & $\begin{array}{l}\text { Spiricisa } \\
\text { Stahl. }\end{array}$ \\
\hline $\begin{array}{l}\text { Jeffrey } \\
\text { John }\end{array}$ & $\begin{array}{l}\text { Star } \\
\text { Stedman }\end{array}$ \\
\hline $\begin{array}{l}\text { Edward } T . \\
\text { Char Ies } L \text {. } \\
\text { Fandy } \\
\text { Julia }\end{array}$ & $\begin{array}{l}\text { Sticl: } 1 \text { E } \\
\text { Stoddard } \\
\text { Terada } \\
\text { Terry }\end{array}$ \\
\hline David & Thei 1 en \\
\hline Marl: A. & Triie]. \\
\hline Alistair & Thompson \\
\hline$C{ } \mathrm{~B}_{\text {" }} " \mathrm{Bi} 11 "$ & Thompsor \\
\hline FaL!I & Thompson \\
\hline Vince E. & Thompson \\
\hline Dietmer & Tr-ippner \\
\hline Janes & Tule ent:o \\
\hline Jaities $A_{1 .}$ & Turnier \\
\hline Fobert In & Ventura \\
\hline EEr $1 F$ & WEaVEr \\
\hline Donald & Wechsler \\
\hline Jerry $A$. & WEISS \\
\hline Uwe & Weissf $1 \square g$ \\
\hline Fiotert & White \\
\hline George & Whitehurst \\
\hline Wi 11 i em & Whitemari \\
\hline Gary & Whitt \\
\hline Feter & Wil $100 \pi$ \\
\hline Fiobert M. & Wil $150 \pi$ \\
\hline Fichard C. & WInfrey \\
\hline Edward Fin & Witteriberg \\
\hline Deborati $A$. & Wright \\
\hline Sheree & Yang \\
\hline Siu Fun & $Y u$ \\
\hline John & Zimmerman \\
\hline
\end{tabular}

Martun Marietta Energy Systens Computervision Corp.

I BW Corp.

University of Califormia

Mentor Graphics Corp.

Martin Marietta Energy Systems

Fratt \& Whitriey

CADAM InC.

Martin Marietta Energy Systems

Allied Eeridix Aerospace

McDorne]. 1 Douglas futomation Co.

Carribridge Interactive Systems Ltd.

Southern Company Servj.ces Inc

Control Data

Eurris : McDonnel]. Engineering Co EMW

MeDermott, Inc.

University of Michigan

Martin Marietta Aerospace

Ballistics Fiesearch Laboratory

The MITFE Corporation

McDornel 1 Aircraft. Co.

IEM Germany Dept 2081

Corriputervision Corp.

NASA Langley Fiesearch Center

Enterprise Systems Design Inc.

Inter"graph Corp.

General Electric. Co

Martin Marietta Energy Systems

Digital Equipment Corp.

Ford Matar Ca.

United Technologies-Sitorsty Aircraft

Ford Aarospace communj.cations Co Hewlett-Fackard Co.

Allied Bendix Aerospace 


\section{FOREWORD}

Version 3.0 of the Initial Graphics Exchange Specification greatly improves upon earlier publications of IGES Version 1.0 in 1980 , ANSI Y $14.26 \mathrm{M}$ in 1981 , and IGES Version 2.0 in 1983 by extending and refining the specification in many areas. The refinements, which improve on the clarity and precision of the specification, are in many cases the result of considerable implementation experience gained with IGES across a large number of systems. The many technical extensions in this document reflect a desire to expand the specification's capability to communicate a wider range of product data developed and used by computer aided design and manufacturing systems. They are the result of two and one half years of technical work and were approved by IGES committee members in February 1985.

Despite the many changes to the earlier work, IGES Version 3.0 remains for all practical purposes upwards compatible with Version 2.0. This means that a translator fully conforming to Version 3.0 can correctly interpret IGES files written in accordance with prior versions of IGES. Minor technical changes do exist which do violate this objective or which would have violated this objective had any earlier translator been written to put out some of the more complex entity structures of Version 2.0. One example of a nonupwards compatible change is in the specification of connect points where the earlier approach was shown to be logically incorrect. Other changes introduced into Version 3.0 make obsolete the older entities, but it is believed that these older entities had never been implemented.

Considerable clarifications have been made in the 3.0 document in areas of the View and Drawing entities, in the Global parameters including default values, in the Units flag, in the Transformation Matrix pointer, in the Leader entity and in the Parametric Spline Curve and Surface entities.

New entity capability has been added in the geometry area for Offset Curves, Offset Surfaces and Curves on a Parametric Surface. A Trimmed Surface entity allows the definition of a surface boundary.

In the annotation area new capability exists for representing a larger range of annotation style of the base value and the tolerance limits. Additionally, a more compact definition for crosshatching is given.

Version 3.0 introduces greatly enhanced capability for user defined MACRO's essential for standard part libraries. By adding Labels, Branching and Calling arguments to the previous MACRO capability, the Version 3.0 MACRO is able to represent parametrized IGES constructs that are user defined. Coupled with another new extension for External File Reference, users will be able to implement large standard libraries of symbols or components.

Finally, Version 3.0 introduces a means of reducing IGES file size to one third of its previous size. Called the Compressed ASCII Format, the optional technique addresses storage size and telecommunications costs. Utility programs are included in an Appendix for two way conversion to the Compressed IGES physical file format. 
FOREWORD

List of Figures

xV

List of Tables

xviii

1 General

1.1 Purpose

1.2 Field of Application

1.3 Concepts of Product Definition

1.4 Concepts of the File Structure

1.5 Concepts of the Information Structures for Wire-Frame Model Descriptions

1.5.1 Property Entity

1.5.2 Associativity Entities

1.5.3 View Entity

1.5.4 Drawing Entity

1.5.5 Transformation Matrix Entity

1.5.6 MACRO Entities

1.5.7 Implementor Defined Entities

2.2 ASCII Form

2.2.1 Sequence Numbers

2.2.2 Constants

2.2.3 Rules for Forming and Interpreting Free Formatted Data

2.2.4 File Structure

$\begin{array}{lll}2.3 & \text { Compressed ASCII Form } & 61\end{array}$

2.3.1 File Structure $\quad 61$

2.4 Binary Form $\quad 63$

2.4.1 Constants 63

2.4.2 File Structure 69

2.5 Specific File Structures $\quad 85$

$\begin{array}{lll}2.5 .1 & \text { Subfigures } & 85\end{array}$

2.5.2 Connectivity 88 
2.5.3 MACROs 90

2.5.4 External Reference Linkage 90

2.5.5. Drawings and Views 93

2.5.6 Finite Element Modeling 95

2.5.7 Multiple Transformation Entities 98

3 Geometry 103

$\begin{array}{lll}3.1 & \text { General } & 103\end{array}$

3.1.1 Coordinate Systems 103

$\begin{array}{ll}3.1 .2 & \text { Directionality } \\ 3.1 .3 & 106\end{array}$

3.1.3 Geometric Entities 107

$\begin{array}{ll}3.2 \text { Circular Arc Entity } & 108\end{array}$

3.3 Composite Curve Entity 111

3.4 Conic Arc Entity 116

3.5 Copious Data Entity 122

$\begin{array}{ll}3.6 & \text { Plane Entity } \\ 3.7 & 126\end{array}$

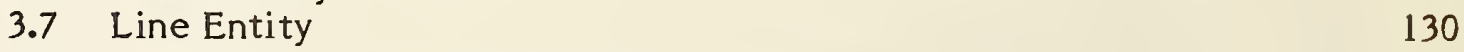

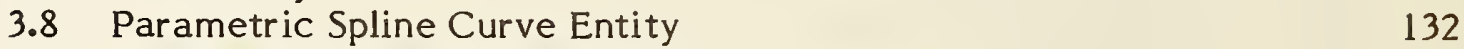

3.9 Parametric Spline Surface Entity 138

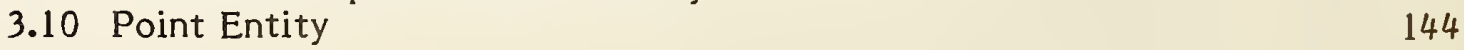

3.11 Ruled Surface Entity 146

3.12 Surface of Revolution Entity 151

3.13 Tabulated Cylinder Entity 155

3.14 Transformation Matrix Entity 158

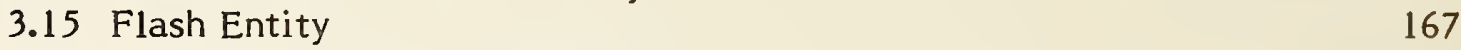

3.16 Rational B-Spline Curve Entity 169

3.17 Rational B-Spline Surface Entity 172

3.18 Offset Curve Entity 176

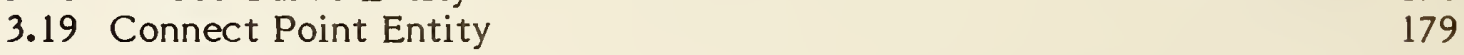

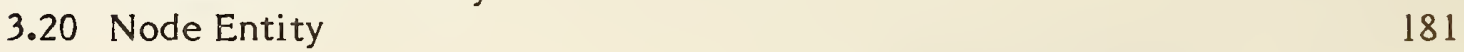

3.21 Finite Element Entity 184

3.22 Nodal Displacement and Rotation Entity 192

3.23 Offset Surface Entity 195

3.24 Curve On A Parametric Surface Entity 198

3.25 Trimmed (Parametric) Surface Entity 201

4 Non-Geometry 205

4.1 General 205

4.2 Annotation Entities 206

4.2.1 Entity Type/Type Number 206

$\begin{array}{ll}\text { 4.2.2 Construction } & 206\end{array}$

$\begin{array}{ll}4.2 .3 & \text { Definition Space } \\ 4.2 .4 & 207\end{array}$

4.2.4 Angular Dimension Entity 209

4.2.5 Centerline Entity 213

4.2.6 Diameter Dimension Entity 215

$\begin{array}{ll}\text { 4.2.7 Flag Note Entity } & 217\end{array}$

4.2.8 General Label Entity 221 
4.2.9 General Note Entity 223

4.2.10 Leader (Arrow) Entity 238

4.2.11 Linear Dimension Entity 243

4.2.12 Ordinate Dimension Entity 245

4.2.13 Point Dimension Entity 247

4.2.14 Radius Dimension Entity 249

4.2.15 Section Entity 251

4.2.16 General Symbol Entity 254

4.2.17 Sectioned Area Entity 256

4.2.18 Witness Line Entity 259

4.3 Structure Entities 261

4.3.1 Entity Type/Type Number 261

4.3.2 Associativity Definition Entity 262

4.3.3 Associativity Instance Entity 264

4.3.4 Drawing Entity 303

4.3.5 Line Font Definition Entity 308

$\begin{array}{ll}\text { 4.3.6 MACRO Capability } & 314\end{array}$

4.3.6.1 General 314

$\begin{array}{ll}\text { 4.3.6.2 MACRO Syntax } & 315\end{array}$

4.3.6.3 MACRO Definition Entity 321

4.3.6.4 MACRO Instance Entity 334

4.3.6.5 Examples of MACRO Usage 335

4.3.7 Property Entity 344

4.3.8 Subfigure Definitions 394

4.3.8.1 Subfigure Definition Entity 394

4.3.8.2 Network Subfigure Definition Entity 395

4.3.9 Subfigure Instances 397

4.3.9.1 Singular Subfigure Instance Entity 397

4.3.9.2 Rectangular Array Subfigure Instance Entity 397

4.3.9.3 Circular Array Subfigure Instance Entity 401

4.3.9.4 Network Subfigure Instance Entity 403

4.3.10 Text Font Definition Entity 405

4.3.11 View Entity 411

4.3.12 External Reference Entity 417

4.3.13 Nodal Load/Constraint Entity 419

4.3.14 Color Definition Entity 421

4.3.15 Text Display Template Entity 422

APPENDIX A: PART FILE EXAMPLES 425

APPENDIX B: ELECTRICAL/ELECTRONIC PRODUCT REPRESENTATION 439

APPENDIX C: $\quad$ PLANT FLOW SHEET REPRESENTATION 453 
APPENDIX D: $\quad$ SPLINE REPRESENTATIONS 469

$\begin{array}{lll}\text { APPENDIX E: } & \text { CONIC ARCS } & 479\end{array}$

APPENDIX F: $\quad$ COLOR SPACE VARIATIONS 481

APPENDIX G: COMPRESSED ASCII FORMAT _ 483

$\begin{array}{ll}\text { REFERENCES } & 497\end{array}$

$\begin{array}{ll}\text { GLOSSARY } & 499\end{array}$

$\begin{array}{ll}\text { INDEX } & 515\end{array}$ 
Figure No.

1-1 Categories of Product Definition 3

2-1 Start Section 17

2-2 Directory Entry (DE) Section 26

2-3 Parameter Data Section $\quad 57$

2-4 Terminate Section 59

2-5 Compressed ASCII Form $\quad 62$

2-6 Format of Control Byte $\quad 65$

2-7 Integer Primitive Format $\quad 66$

2-8 Real Number Primitive Format $\quad 68$

2-9 String Constant Primitive Format $\quad 70$

2-10 Binary General File Structure $\quad 71$

2-11 Format of Binary Information Section $\quad 73$

2-12 Format of Start Section $\quad 76$

2-13 Global Section Format $\quad 77$

2-14 Format of DE Subrecord $\quad 79$

2-15 Format of Parameter Section $\quad 81$

2-16 Format of Terminate Section $\quad 83$

2-17 Subfigure Structures $\quad 86$

2-18 General Connectivity Pointer Diagràm $\quad 87$

2-19 MACRO Definition/Instance Structure $\quad 91$

2-20 External Linkages 94

2-21 Finite Element Modeling File Structure 96

2-22 Finite Element Modeling Logical Structure 97

2-23 Multiple Transformation Cases 99

3-1 Examples of the Circular Arc Entity 110

3-2 Example of the Composite Curve Entity 112

3-3 Examples of the Conic Arc Entity 118

3-4 Examples of the Plane Entity 127

3-5 Single Parent Associativity As Used With a

3-6 Examples of the Line Entity 131

3-7 Example of the Parametric Spline Curve Entity 134

3-8 Additional Examples of the Parametric Spline Curve Entity 135

3-9 Example of the Parametric Spline Surface Entity 140

3-10 Examples of the Point Entity 145

3-11 Example of the Ruled Surface Entity 148

3-12 Additional Examples of the Ruled Surface Entity 149

3-13 Examples of the Surface of Revolution Entity 153

3-14 Surface of Revolution Start and Terminating Angles 154

3-15 Example of the Tabulated Cylinder Entity 156

3-16 Transformation Matrix Coordinate Systems 159

3-17 Displacement Components 163

3-18 Flash Entities $\quad 168$

3-19 Node Definition in Each Coordinate System 183

3-20 Finite Element Topology Set 186

3-21 Offset Surface in 3-D Euclidean Space 196 
4-1 Construction of ZT Depth of Annotation Entities

4-2 Angular Dimension: Construction of Arcs in the Associated Leaders

4-3 Examples of the Angular Dimension Entity 211

4-4 Examples of the Centerline Entity

Examples of the Diameter Dimension Entity

216

4-6

4-7

Flag Note

217

Examples of the Flag Note Entity

219

4-8

Examples of the General Label Entity

222

4-9

Examples of the General Note Entity

224

4-10 Font 1001

Font 1002

226

4-12 Character Set and Octal Code for Font Code Zero

General Note Text Construction

General Note Example of Text Operations

Structure of Leaders Internal to a Dimension

Arrowhead Definitions

Drawing Entity Example 1

4-31 Construction of Line Fonts 
A-1 Electrical Part Example

A-2 Mechanical Part Example

A-3 Drawing and View Example

B-1 General Pointer and Entity Model

B-2 Sample Schematic

B-3 Entity Relations Chart for Sample Schematic (Figure 2)

B-4 Schematic/Physical Diagram for Sample Schematic (Figure 2)

C-1 Plant Flow Sheet Example

C-2 Exploded View of Flow Sheet

C-3 Network Subfigure Entities

C-4 Example Entities

C-5 Flow Sheet Pointer Structure 
Table No.

2-1 Parameters in the Global Section

2-2 Directory Entry Field Description

2-3 Entity DE Field Requirements

2-4 Physical Parent/Child Relationships

3-1 Finite Element Topology

C-1 Entities in PI\&D Test Case

457

C-2 Plant Flowsheet Example File

465 


\subsection{Purpose}

This document establishes information structures to be used for the digital representation and communication of product definition data. Use of the specification established herein permits the compatible exchange of product definition data used by various CAD/CAM (Computer Aided Design and Computer Aided Manufacturing) systems.

\subsection{Field of Application}

This Specification defines a file structure format, a language format, and the representation of geometric, topological, and non-geometric product definition data in these formats. Product definition data represented in these formats will be exchanged via a variety of physical media. The specific features and protocols for the communications media are the subject of other standards. The methodology for representing product definition data in this Specification is extensible and independent of the modeling methods used.

Section 2 defines the communications file structure and format. It explains the function of each of the sections of a file. The geometry data representation in Section 3 deals with two- and three-dimensional edge-vertex models and with simple surface representations. Section 4 specifies non-geometric representations, including common drafting practices, data organization methods, and data definition methods.

In Sections 3 and 4, the product is described in terms of geometric and nongeometric information, with non-geometric information being divided into annotation, definition, and organization. The geometry category consists of elements such as points, curves, and surfaces that model the product. The annotation category consists of those elements which are used to clarify or enhance the geometry, including dimensions, drafting notation, and text. The definition 
category provides the ability to define specific properties or characteristics of individual or collections of data entities. The structure category identifies groupings of elements from geometric, annotation, or property data which are to be evaluated and manipulated as single items.

\subsection{Concepts of Product Definition}

This Specification is concerned with the data required to describe and communicate the essential engineering characteristics of physical objects as manufactured products. Such products are described in terms of their physical shape, their dimensions, and information which further describes or explains the product. The processes which generate or utilize the product definition data typically include design, engineering analysis, production planning, fabrication, material handling, assembly, inspection, marketing, and field service.

The requirements for a common data communication format for product definition can be understood in terms of today's CAD/CAM environment. Traditionally, engineering drawings and associated documentation are used to communicate product definition data. Commercial interactive graphics systems, originally developed as aids to producing these two-dimensional drawings, are rapidly developing sophisticated three-dimensional edge-vertex modeling capability. In parallel, extensive research work is being conducted in advanced geometric modeling techniques (e.g., parametric representations and solid primitives) and in CAM applications utilizing product definition data in manufacturing (e.g., NC machining and computer-controlled coordinate measurement). The result is rapid growth of CAD/CAM applications which should be able to exchange product definition data, but which usually employ incompatible data representations and formats. In addressing this compatibility problem, this Specification is concerned with needs and capabilities of current and advanced methods of CAD/CAM product definition development.

Product definition data may be categorized by their principal roles in defining a product. An example of such a categorization is presented in Figure 1-1. This Specification specifies communication formats (information structures) for subsets of the product definition. 
Product Identification

Product Structure

o

DESIGN/ANALYSIS

Idealized Models

- BASIC SHAPE

Geometric

Topological

- AUGMENTING PHYSICAL CHARACTERISTICS

Dimensions and Tolerances

Intrinsic Properties

- PROCESSING INFORMATION

- PRESENTATIONAL INFORMATION

FIGURE 1-1 CATEGORIES OF PRODUCT DEFINITION 
A format to allow the exchange of a product definition between CAD/CAM systems must, as a minimum, support the communication of geometric data, annotation, and organization of the data. The file format defined by this Specification treats the product definition as a file of entities, each entity being represented in an application-independent format, to and from which the native representation of a specific CAD/CAM system can be mapped. The entity representations provided in this Specification include forms common to the CAD/CAM systems currently available and forms which support the system technologies currently emerging.

The fundamental unit of information in the file is the entity. Entities are categorized as geometric and non-geometric. Geometric entities represent the definition of the physical shape and include points, curves, surfaces, and relations which are collections of similarly structured entities. Non-geometric entities typically serve to enrich the model by providing a viewing perspective in which a planar drawing may be composed and by providing annotation and dimensioning appropriate to the drawing. Non-geometric entities further serve to provide specific attributes or characteristics for individual or groups of entities and to provide definitions and instances for groupings of entities. The definitions of the:ie groupings may reside in another IGES file. Typical non-geometric entities for drawing definition, annotation, and dimensioning are the view, drawing, general note, witness line, and leader. Typical non-geometric entities for attributes and groupings are the property and the associativity entities.

A file consists of five or six sections: Flag (in the case of the binary or compressed ASCII format), Start, Global, Directory Entry, Parameter Data, and Terminate. A file may include any number of entities of any type as required to represent the product definition. Each entity occurrence consists of a directory entry and a parameter data entry. The directory entry provides an index and includes descriptive attributes about the data, while the parameter data provides the specific entity definition. The directory data are organized in fixed fields and are consistent for all entities to provide simple access to frequently used descriptive data. The parameter data are entity specific and are variable in length and 
format. The directory data and parameter data for all entities in the file are organized into separate sections, with pointers providing bi-directional links between the directory entry and parameter data for each entity. The Specification provides for groupings whose definitions will be found in a file other than the one in which they are used.

Each entity defined by the file structure of Section 2 has a specific assigned entity type number. While not all are assigned at this time, entity numbers 0001 through 0599 and 0700 through 5000 are allocated for specific assignment. Entity type numbers 0600 through 0699 and 10000 through 99999 are for implementor-defined (i.e. MACRO) entities. For user defined entities see section 1.5.7. The Index of Topics includes an alphabetical listing of entity types.

Some entity types include a form number as an attribute. The form number serves to fur ther define or classify an entity within its specific type.

The entity set includes a provision for associativities and properties. The associativity provides a mechanism to establish relationships among entities, and to define the meaning of the relationship. The property allows specific characteristics, such as color, to be assigned to an entity or collection of entities. Each entity format includes a structure for an arbitrary number of pointers to associativities and properties. The file structure provides for both standard associativities and properties to be included in the Specification, and unique definitions which will be defined by the user.

\subsection{Concepts of the Information Structures for Wire-Frame Model Descriptions}

The wire-frame model refers to the entity set defined by Sections 3 and 4, and comprises an entity-based product definition file. The entity types, as described in 1.4, are categorized as geometric and non-geometric. In general, the geometric entities are defined independently of one another (surfaces are an exception). Features have been provided to define and compose relationships among entities to enhance the model. The non-geometric entities include structures in which an entity may be defined by a collection of other entities and structures which are independent. 
Several entity types which are used to provide relations or definitions are essential to the file structure methodology of this Specification and are described below.

1.5.1 Property Entity. The PROPERTY entity allows non-geometric numeric or textual information to be related to any entity. Any entity occurrence may reference one or more property entity occurrences as required.

Property entities themselves may exist independently of other entities. In this case the property is defined to be a property of the level indicated in the level field of the directory entry (DE) of the property. This allows for a general property to apply to all entities of a given level or for the assignment of an applications function to a level. Because the level field in a DE is also allowed to point to a property of levels, properties may be applied to multiple levels.

1.5.2 Associativity Entities. The Associativity Entities are designed for use when several entities must be logically related to one another. Two types of entities are involved here: ASSOCIATIVITY DEFINITION and ASSOCIATIVITY INSTANCE. The associativity definition entity is used to specify the structure of the logical relationship, and the associativity instance entity is used to specify the information involved in a particular occurrence of the relationship.

Some associativities are defined as part of this Specification. These intrinsic definitions include GROUP and VIEWS VISIBLE associativities, and are defined in Section 4.3.

1.5.3 View Entity. A drawing or equivalent human-readable representation of the geometric model of a product is a two-dimensional projection of a selected subset of the model, together with non-geometric information such as text. The VIEW entity and VIEWS VISIBLE forms of associativity control such representations. These provide information for orientation, clipping, line removal, and other characteristics associated with individual views rather than with the model itself.

1.5.4 Drawing Entity. The DRAWING entity allows a set of views to be identified and arranged for human presentation. Note that the view and drawing entities contain only the rules and parameters for extracting drawings from the geometric model. The actual product definition is not duplicated in various views, eliminating risk of conflicting or ambiguous information. 
1.5.5 Transformation Matrix Entity. The TRANSFORMATION MATRIX entity allows translation and rotation to be applied as needed, to any entity in the construction of the model and to the development of views and drawings of the model.

1.5.6 MACRO Entities. This Specification includes a MACRO DEFINITION entity for defining new entity types which may then be used in the defining file in the same manner as the intrinsically defined entities. A language for defining these new entity types is specified in 4.3.6.

1.5.7 Implementor Defined Entities. This specification allows implementors to include entities in their files that are not defined in this document but which have specific user meanings. This feature supports the objective of the Specification to act as an archiving format where the receiving system is the same as the sending system. In this way, the implementor is able to archive those data forms which may be unique to a particular system.

From time-to-time, files with such implementor-defined entities are used with applications which attempt to edit the file. In this situation, processing problems can arise because, without an entity definition, the editor cannot know which parameter values are pointers that have to be updated, and which are simply data values that should not be updated.

To avoid this problem, implementors should use MACRO definitions and instances of MACRO entities with entity type numbers in the range of 5001 to 9999 inclusively. (See Section 4.3.6 for information on how to use the MACRO capabilities of the specification.) This means that for each different implementordefined entity type, there will be a MACRO Definition Entity (Type 306). In order to accomplish the desired result, all that needs to be present in the parameter data for these MACRO definitions is the first MACRO statement which defines the parameter list, and an ENDM statement to terminate the definition. 


\subsection{Appendices}

As an aid to the implementor/user, a series of appendices is included with this Specification. Appendix A gives three part file examples. Appendix B describes an electrical-electronic product representation, and Appendix C, a plant flowsheet representation. Appendix D provides explanation of spline representation and approaches for conversion techniques. Appendix $E$ discusses the numerical stability of conic arcs. Appendix F provides mappings between color spaces. A set of FORTRAN utilities is documented in Appendix $G$ to convert the ASCII Form of physical file structure to and from the Compressed ASCII Form. In addition, a List of References, a Glossary and an Index of Topics are also included. 
Two different formats are defined to represent IGES data. These formats are ASCII (ANSI68, ANSI74, ANS177), and Binary. The binary form uses a byte oriented (bit string) structure which may be especially suited to the transmission of large files.

The constants, free format rules, file organization, and information structure are discussed in terms of displayed or printed ASCII form. Following this discussion, the binary form is defined together with necessary changes in constants and file structure.

The ASCII form has two format types: a fixed (80 character) line length format, and a compressed format. In the fixed line length ASCII format, the entire file is partitioned into 80 character units beginning with the first character in the file. These units are called lines. The term "column" refers to the character position in a line. The file is divided into sections. The section identification character shall occupy column 73 of each line. Columns 74 through 80 are specified for the section sequence number of each line. For the compressed ASCII format, the DIRECTORY ENTRY and PARAMETER DATA sections are excepted from the above two rules, and will be defined in a later section. The remaining columns are assigned to fields as defined in the file section description. The term "record" refers to the set of parameters for one entity within one file section. A record consists of one or more lines.

\section{Sequence Numbers}

A sequence number is a string of from one to seven digits and is the means of indexing lines within the various sections of the data file. The sequence numbers for each section begin with $1(0000001)$ and continue sequentially without interruption to the value corresponding to the number of lines in the section. A sequence number may have either leading zeros or leading blanks and is right-justified in its field in the line (columns 74-80). 
The sequence number is preceded in the line by a single letter code in column 73 identifying the section.in which the line resides:

$\begin{array}{lc}\text { Section } & \text { Letter Code } \\ \text { Flag (optional) } & \text { B or C } \\ \text { Start } & \text { S } \\ \text { Global } & \text { G } \\ \text { Directory Entry } & \text { D } \\ \text { Parameter Data } & \text { P } \\ \text { Terminate } & \text { T }\end{array}$

Letter codes "B" and "C" are used to signify binary (see section 2.4.2.1) and compressed ASCII (see section 2.3.1) information, respectively.

\subsubsection{Constants}

This specification defines five types of constants: integer (or fixed point), real (or floating point), string, pointer, and language statement. Regardless of whether the constants appear in a fixed or free format in the data file, cer tain rules apply to their formation and interpretation:

- Blanks are only significant in string and language statement constants. A numeric field of all blanks is considered to denote the default value for that field. No blanks are allowed between the beginning of a numeric constant (i.e., its sign, first numeric digit, or decimal point) and the end of that constant (i.e., the last character position allocated in fixed format or the delimiter character in free format). Leading blanks in the parameters containing numeric constants are ignored. Blanks between the end of any constant and the delimiter following the constant are not allowed.

- Numeric constants cannot contain embedded commas.

- The absolute magnitude of an integer constant may not exceed the value $2 * *(N-1)-1$, where $N$ is the number of bits used to represent the integer value (Global parameter 7 ). 
Similarly, the absolute magnitude and precision of a real constant may not exceed that indicated by Global parameters 8-9 (for single precision) and 10-11 (for double precision).

- Only string and language statement constants may cross field/line boundaries. When such a constant does cross a boundary, it is considered to extend to the last usable column on the current line and then to continue with the first column on the succeeding line. The last usable column on lines in the Parameter Data section is column 64; on lines in all other sections it is column 72. A string constant may not be broken before the Hollerith delimiter $(H)$.

- A numeric constant may be either signed or unsigned. If signed, the leading plus or minus determines the sense of the constant. If unsigned, the sense is assumed to be non-negative.

\subsubsection{Integer Constants}

An integer constant (sometimes called a fixed point constant) is always an exact representation of an integer value. It may assume a positive, negative or zero value. It may assume only an integral value.

The form of an integer constant is an optional sign followed by a non-empty string of digits. The digit string is interpreted as a decimal number.

The following are examples of valid integer constants (assuming the value of Global parameter 7 is 32 ) :

$\begin{array}{rrrr}1 & 150 & 2147483647 & +3451 \\ 0 & -10 & -2147483647 & \end{array}$




\subsubsection{Real Constants}

A real constant (sometimes called a floating point constant) is a processor approximation of the value of a real number. It may assume a positive, negative, or zero value. A real constant may be either a basic real constant, a basic real constant followed by an exponent, or an integer constant followed by an exponent.

A real constant may be of either single or double precision. The distinction is in the precision of the processor's representation of the real number which is specified in Global parameters 8 through 11. A double precision constant may be either a basic real constant followed by a double precision exponent, or an integer constant followed by a double precision exponent.

The form of a basic real constant is, in order, an optional sign, an integer part, a decimal point, and a fractional part. Both the integer part and the fractional part are strings of digits; either of these parts may be omitted but not both. A basic real constant is interpreted as a decimal number.

The form of a real exponent is the letter $E$ followed by an optionally signed integer constant. A real exponent denotes a decimal power of ten by which the preceding constant is multiplied.

The form of a double precision exponent is the letter $D$ followed by an optionally signed integer constant. A double precision exponent denotes a decimal power of ten by which the preceding constant is multiplied.

The following are examples of valid real constants:

$\begin{array}{llll}256.091 & 0 . & -0.58 & +4.21 \\ 1.36 \mathrm{E} 1 & -13 \mathrm{E}-02 & 0.1 \mathrm{E}-3 & 1 . \mathrm{E}+4 \\ 145.98763 \mathrm{D} 4 & -2145.980001 \mathrm{D}-5 & 0.123456789 \mathrm{D}+09 & -.43 \mathrm{E} 2\end{array}$




\subsubsection{String Constants}

String constants are represented in the Hollerith form as specified in Appendix $\mathrm{C}$ of the current FORTRAN standard (ANSI78). A string constant is an arbitrary sequence of ASCII characters. Blanks, parameter delimiters, and record delimiters are treated simply as characters within the string. There is no limit on the length of a string constant.

The form of a string constant is a non-zero, unsigned integer constant (character count), followed by the letter $\mathrm{H}$, followed by a string of characters consisting of the number of contiguous characters specified by the character count.

The following are examples of valid string constants:

$3 \mathrm{H} 123$

8H0.457E03
1OHABC.,;ABCD

12H HELLO THERE

\subsubsection{Pointer Constants}

A pointer constant is a non-empty string of from one to seven digits. Pointer constants are used to identify a line, in either the same or a different section of the data file. The magnitude of the pointer constant corresponds to the sequence number of the referenced line, and the referenced file section is determined by the context of the reference. Pointer constants are unsigned except where they are alternative parameters in a field. Pointer constants whose magnitude requires fewer than seven digits may use leading zeros or leading blanks in fixed format fields.

\subsubsection{Language Statement Constants}

The language statement constant is an arbitrary character string made up of alphanumeric, punctuation, and blank characters from the ASCII character set. The language statement constant is not preceded by the 
character count and the Hollerith delimiter $\mathrm{H}$ as is the string constant. Section 4.3.6 defines the syntax of the language statement constant as used for the MACRO entity. The length of the language statement constant is determined by means of the Parameter Data line count in the Directory Entry record for the entity (see Directory Entry parameter 14).

\subsubsection{Rules for Forming and Interpreting Free Formatted Data}

The data in several sections of a file may be entered in free format. The free format will apply to a range of columns of a line in the section and to the same range of columns of successive lines as needed. This free format feature allows the specification of parameters in the prescribed order without restricting the placement of the parameter to a particular location on a line. When free format is permitted, the following rules apply (in addition to those in section 2.2.2):

- The parameter delimiter (Global parameter 1 - defaulting to a comma) is used to separate parameters.

- The record delimiter (Global parameter 2 - defaulting to a semicolon) is used to terminate the record (i.e., to terminate a list of parameters).

- When two parameter delimiters, or a parameter and record delimiter, appear adjacent to each other, or are separated by only blanks, the parameter is considered not to have been specified in the file and should be given its default value. Unless specifically noted, the default value for a numeric parameter is zero, and the default value for a string parameter is null. It is the responsibility of the preprocessor to ensure that these default values are reasonable for the particular parameter in question.

- When a record delimiter appears before the list of parameters is complete, all remaining parameters should be given their default values (see above for a discussion of assigning default values). 
- The end of the data portion of the physical line (i.e., column 72 in the Global Section, and column 64 in the Parameter Data Section) is not to be construed to act as either a parameter delimiter or a record delimiter.

- The parameter delimiter and record delimiter characters do not maintain their special significance when included within a string constant.

- A numeric constant, including its trailing delimiter, cannot extend across a line boundary. 


\subsubsection{File Structure}

The file contains six subsections which must appear in order as follows:
a. Flag Section (optional)
b. Start Section
c. Global Section
d. Directory Entry Section
e. Parameter Data Section
f. Terminate Section.

The Flag Section of the file is used, when present, to indicate that the file is in the Binary Form (2.4.2.1) or in the Compressed ASCII Form (2.3.1).

2.2.4.1 Start Section. The start section of the file is designed to provide a manreadable prolog to the file. There must be at least one start record, and all records in the section must have the letter $S$ in column 73 and a sequence number in column 74 through 80 (See 2.2.1). The information in columns 1 through 72 is not formatted in any special way except that the ASCII character set must be used. An example of a start section is shown in Figure $2-1$. 


\section{START SECTION}

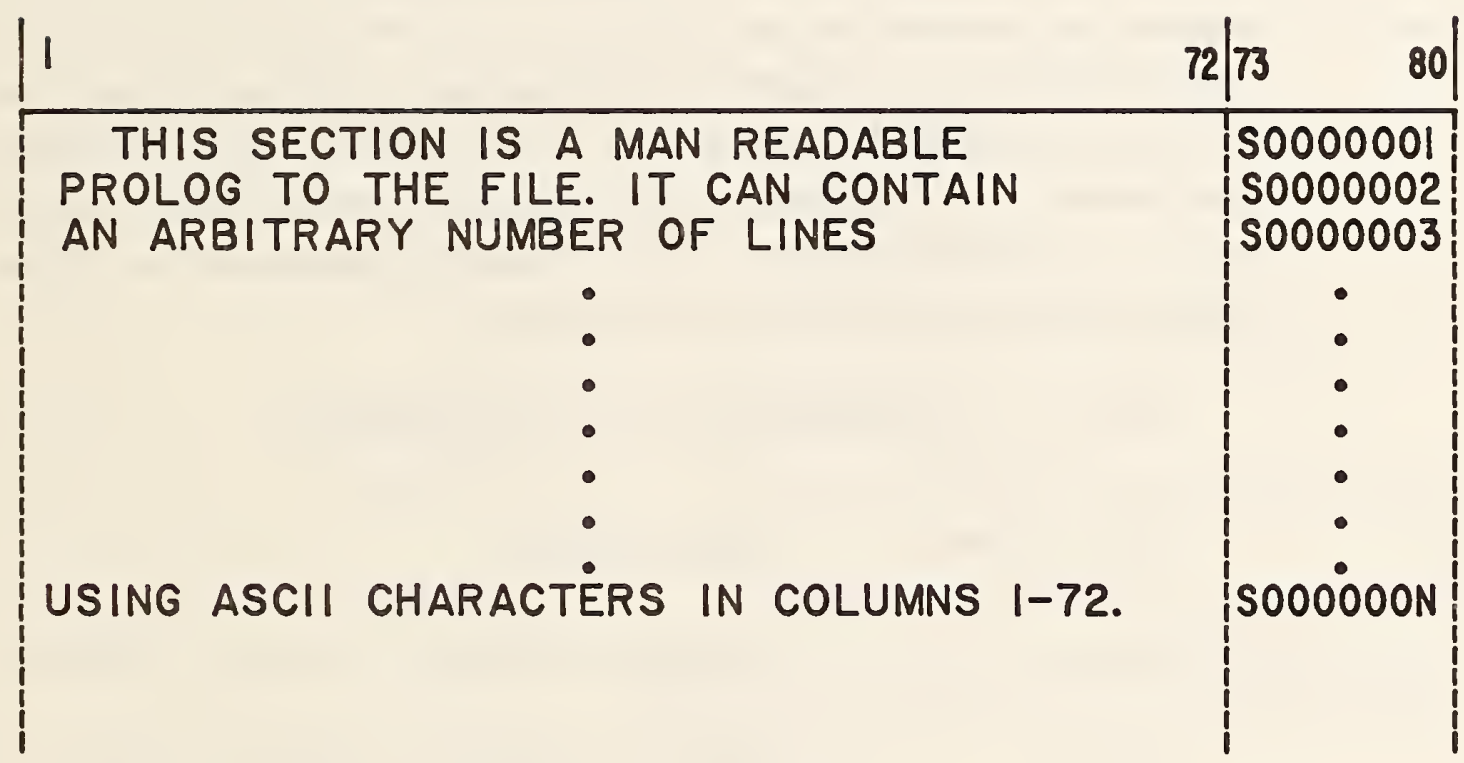

FIG. 2-I START SECTION 


\subsubsection{Global Section}

The global section of the file contains the information describing the preprocessor and information needed by the post-processor to handle the file. All records in the global section shall contain the letter $G$ in column 73 and a sequence number (See 2.2.1). The first two global parameters are used to define the parameter delimiter and record delimiter characters if necessary. The default characters are "cornma" and "semicolon" respectively.

The parameters for the global section are input in free format as described in 2.2.3. As implied in 2.2.3, the global parameters will end with the record delimiter. If the global section specifies new delimiter characters, they take over immediately and are used in the global section as well as the rest of the file. This is possible, because the comma and semicolon delimiter specifications are the first two global parameters. The parameters in the global section are described in Table 2-1 and the paragraphs that follow. Unless explicitly stated, no defaults are provided.

\section{TABLE 2- 1 PARAMETERS IN THE GLOBAL SECTION}

INDEX

1

2

3

4

5

6
FIELD TYPE

String

String

String

String

String

String

\section{DESCRIPTION}

Parameter delimiter character (default is comma)

Record delimiter character (default is semicolon)

Product identification from sending system

File name

System ID

Preprocessor version 
Integer

Integer

Integer

Integer

Integer

String

Real

Integer

String

Integer

Real

String
Number of bits for integer representation

Maximum power of ten representable in a single precision floating point number on the sending system

Number of significant digits in a single precision floating point number on the sending system

Maximum power of ten representable in a double precision floating point number on the sending system

Number of significant digits in a double precision floating point number on the sending system

Product identification for the receiving system

Model space scale (example: .125 indicates a ratio of 1 unit model space to 8 units real world)

Unit flag

Units. Ten values of the unit flag have been defined. (See 2.2.4.2.15).

Maximum number of line weight gradations (132768). Refer to the directory entry parameter 12 (See 2.2.4.3) for use of this parameter.

Size of maximum line width in units. Refer to the directory entry parameter 12 (See 2.2.4.3) for use of this parameter.

Date \& time of file generation 13HYYMMDD.HHNNSS where: $Y Y$ is year (last 2 digits) MM is month (01-12)

DD is day (01-31)

$\mathrm{HH}$ is hour (00-23)

NN is minute (00-59)

$\mathrm{SS}$ is second (00-59) 
DATA FORM - ASCII - Global Section

19

20

21

22

23

24
Real

Real

String

String

Integer

Integer
Minimum user-intended resolution or granularity of the model expressed in units defined by parameter 15 (example .0001 )

Approximate maximum coordinate value occurring in the model expressed in units defined by parameter 15. (Example: 1000.0 means for all coordinates $|X|,|Y|,|Z| \leqslant 1000$.)

Name of author

Organization

Integer value corresponding to the version of IGES used to create this file. (See 2.2.4.2.23 for Correspondence Table.)

Drafting standard in compliance to which the data encoded in this file was generated. (See 2.2.4.2.24) 
2.2.4.2.1 Parameter Delimiter Character. This parameter indicates which character is used to separate parameter values in the Global and Parameter Data sections. Each occurrence of this character denotes the end of the current parameter and the start of the next parameter. Two exceptions exist: (1) string constants in which the delimiter character may be part of the string; (2) language statements in which the delimiter character may be a part of the language syntax. The default value is a comma. See 2.2.3.

2.2.4.2.2 Record Delimiter. This parameter indicates which character is used to denote the end of a list of parameters in the Global section and each Parameter Data section entry. Each occurrence of this character denotes the end of the current parameter list. Two exceptions exist: (1) string constants in which the delimiter character may be part of the string; (2) language statements in which the delimiter character may be a part of the language syntax. The default value is a semicolon. See 2.2.3.

2.2.4.2.3 Product Identification From Sender. This is the name of the identifier which is used by the sender to reference this product.

2.2.4.2.4 File Name. This is the name of the IGES file.

2.2.4.2.5 System ID. This parameter is an identification code which should uniquely identify the system which generated this file. It includes both the name of the system and the version of software on that system.

2.2.4.2.6 Preprocessor Version. This parameter identifies the version of the preprocessor software which created this file.

2.2.4.2.7 Number of Bits for Integer Representation. This parameter indicates how many bits are present in the integer representation of the sending system. This parameter sets limits on the range of values for integer parameters in the file.

2.2.4.2.8 Single Precision Magnitude. This parameter indicates the maximum power of ten which may be represented as a single precision floating point number on the sending system. 
2.2.4.2.9 Single Precision Significance. This parameter indicates the number of decimal digits of significance which can be accurately represented in the single precision floating point representation on the sending system.

2.2.4.2.10 Double Precision Magnitude. This parameter indicates the maximum power of ten which may be represented as a double precision floating point number on the sending system.

2.2.4.2.11 Double Precision Significance. This parameter indicates the number of decimal digits of significance which can be accurately represented in the double precision floating point representation on the sending system.

Example: For an IEEE 754-1985 (IEEE85) floating point representation with 32 bits, the magnitude and significance parameters have the values 38 and 6, respectively; for a representation with 64 bits, the values are 308 and 15, respectively.

2.2.4.2.12 Product Identification for the Receiver. This is the name or identifier which is intended to be used by the receiver to reference this product.

2.2.4.2.13 Model Space Scale. The ratio of model space to real world space.

2.2.4.2.14 Unit Flag. An integer value denoting the measuring system used in the file. The values in the file are assumed to be:

$$
\begin{aligned}
& \text { Unit flag = } 1 \text { (Inches) } \\
& =2 \text { (Millimeters) } \\
& =3 \text { (See Parameter } 15 \text { for name of units) } \\
& =4 \text { (Feet) } \\
& =5 \text { (Miles) } \\
& =6 \text { (Meters) } \\
& =7 \text { (Kilometers) } \\
& =8 \text { (Mils, i.e. } 0.001 \text { inch) } \\
& =9 \text { (Microns) } \\
& =10 \text { (Centimeters) } \\
& =11 \text { (Microinches) }
\end{aligned}
$$

This is the controlling definition of units. A value of '3' should only be used when it is intended to transfer data to a system using the same units, in 
which case parameter 15 must be used to provide additional information as to those units.

2.2.4.2.15 Units. A string constant naming the model units in the system, e.g.:

$\begin{array}{ll}\text { 2HIN or 4HINCH (model units are inches) } \\ \text { 2HMM } & \text { (model units are millimeters) } \\ \text { 2HFT } & \text { (model units are feet) } \\ \text { 2HMI } & \text { (model units are miles) } \\ \text { 1HM } & \text { (model units are meters) } \\ \text { 2HKM } & \text { (model units are kilometers) } \\ \text { 3HMIL } & \text { (model units are mils) } \\ \text { 2HUM } & \text { (model units are microns) } \\ \text { 2HCM } & \text { (model units are centimeters) } \\ \text { 3HUIN } & \text { (model units are microinches) }\end{array}$

When the unit flag is given a value of 3, the string constant naming the desired unit should conform to MIL-STD-12D, (DOD12D) or ANSI/IEEE 260, (IEEE260).

2.2.4.2.16 Maximum Number of Line Weight Gradations. This is the number of equal subdivisions of line thickness.

2.2.4.2.17 Size of Maximum Line Width in Units. This is the actual width of the thickest line possible in the (scaled) file.

2.2.4.2.18 Date and Time of File Generation. This is a time stamp of when the file was created.

2.2.4.2.19 Minimum User-Intended Resolution. This parameter indicates the smallest distance in model space units that the system should consider as discernable. Coordinate locations in the file which are less than this distance apart should be considered to be coincident.

2.2.4.2.20 Approximate Maximum Coordinate Value. This is the upper bound on the values of all coordinate data actually occurring in this model. The absolute magnitude of each of the coordinates in this model is less than or equal to this value. 
2.2.4.2.21 Name of Author. The name of the person responsible for the generation of the data contained in this file.

2.2.4.2.22 Organization. The organization or group with whom the author is associated.

2.2.4.2.23 Version Number. Each version of IGES is assigned a unique integer value corresponding to that version. This field contains the integer value corresponding to the version of IGES used to create this file. The correspondences are specified in the table below.

\begin{tabular}{|c|c|}
\hline VALUE & IGES VERSION \\
\hline 1 & 1.0 \\
\hline 2 & ANSI Y $14.26 \mathrm{M}-1981$ \\
\hline 3 & 2.0 \\
\hline 4 & 3.0 \\
\hline
\end{tabular}

If no valid integer value is entered in this field, the default value of 3 (corresponding to IGES Version 2.0) should be assumed.

2.2.4.2.24 Drafting standard code. The drafting standard according to which the data in this file was generated.

0 None - No standard specifiéd

1 ISO - International Organization for Standardization

2 AFNOR - French Association for Standardization

3 ANSI - American National Standards Institute

4 BSI - British Standards Institute

5 CSA - Canadian Standards Association

6 DIN - German Institute for Standar dization

7 JIS - Japanese Institute for Standardization 


\subsubsection{Directory Entry Section}

The directory entry section has one directory entry for each entity in the file. The directory entry for each entity is fixed in size and contains twenty fields of eight characters. each spread across two consecutive eighty character lines. Data are right justified in each field. With the exception of the fields numbered $10,16,17,18$, and 20 , entries in all fields in this section will be either integer constants or pointer constants. In this section, the word "number" is sometimes used in place of the phrase "integer constant."

The purposes of the directory entry section are to provide an index for the file and to contain attribute information for each entity. The order of the directory entries within the directory entry section is arbitrary with the exception that a definition entity must precede all of its instances.

Within the directory entry section, a field consisting wholly of blanks is to be considered to have not been specified and should be given a default value where possible. Default values are not allowed in fields $1,2,10,11,14$, and 20. The actual values to be assigned as defaults will vary depending on the entity type. This rule does not apply to compressed ASCII.

Some of the fields in the directory entry can contain either an attribute value directly, or a pointer to a set of such values. In these fields, a positive value indicates an integer constant, while for a negative value, the absolute value should be taken and the result interpreted as a pointer constant.

Since zero is not a valid pointer constant value, a zero in a field requiring a pointer is not permitted unless a specific interpretation has been allowed for in this Specification.

Table 2-2 and the following paragraphs describe each directory entry field. For those fields accommodating either an attribute value or a poit .er, there are two descriptions given. Figure 2-2 gives an abbreviated listing of the fields making up the directory entry for each entity. 
DATA FORM - ASCII - $\begin{aligned} \text { Directory Entry } \\ \text { Section }\end{aligned}$

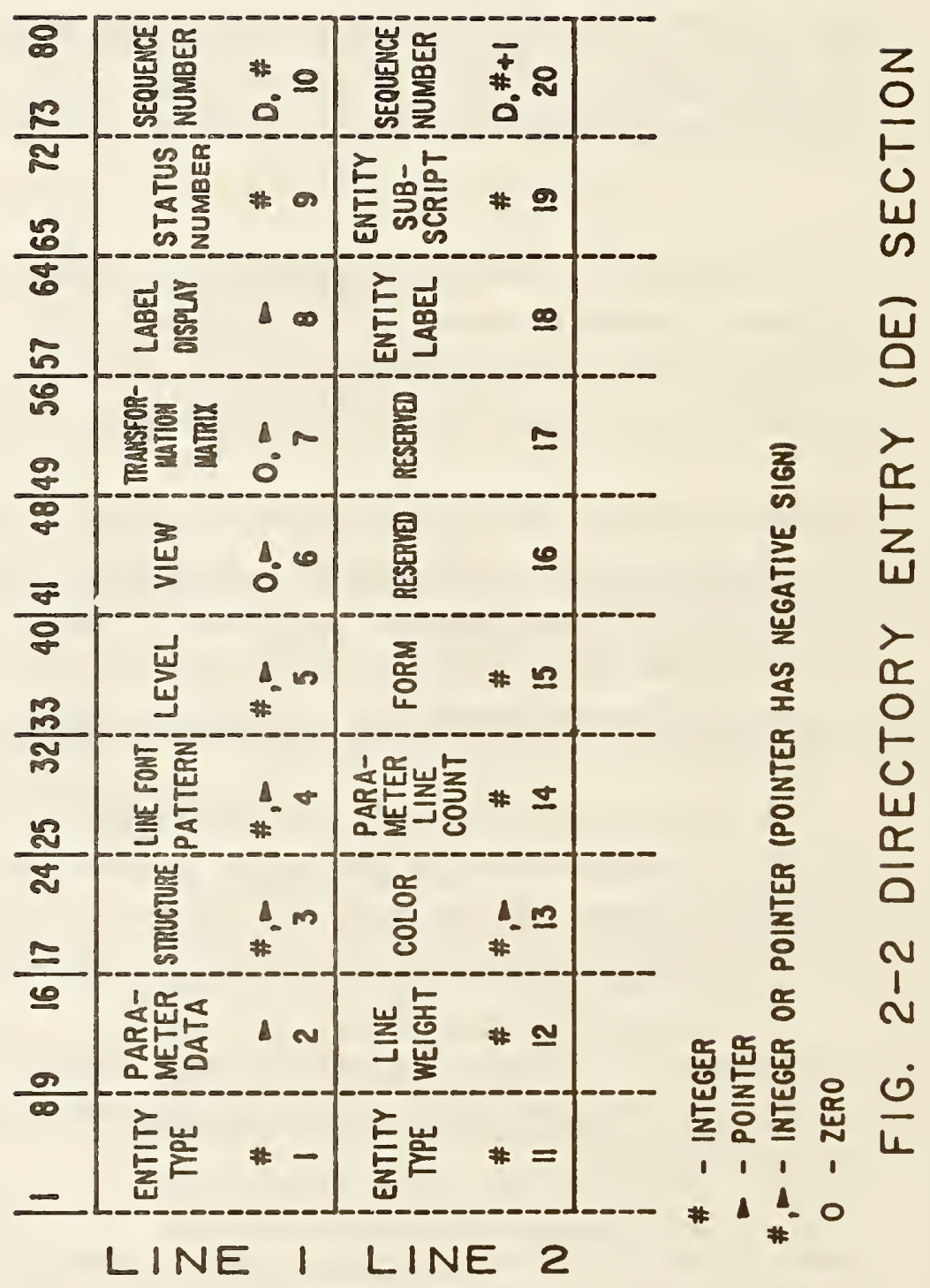


TABLE 2-2 DIRECTORY ENTRY FIELD DESCRIPTION

NO. FIELD NAME

1 Entity Type Number

- 2 Parameter

Data

3 Structure

$4 \quad$ Line Font Pattern

5 Level

6 View

7 Transformation Matrix

Pointer to the directory entry of a transformation matrix (type number 124) used in defining this entity; zero (default) implies the identity transformation matrix and zero translation vector will be used. 
8 Label Display Associativity

9 Status Number

10 Section Code and Sequence Number

11 Entity Type Number
Pointer to the directory entry of a label display associativity (Entity 402 Form 5).

Provides four two-digit status values which are entered from left to right in the status number field in the order given below.

1-2 Blank Status

00 Visible

01 Blanked

3-4 Subordinate Entity Switch

00 Independent

01 Physically Dependent

02 Logically Dependent

03 Both (01) and (02)

5-6 Entity Use Flag

00 Geometry

01 Annotation

02 Definition

03 Other

04 Logical/Positional

05 2D Parametric

7-8 Hierarchy

00 Global top down

01 Global defer

02 Use hierarchy property

Physical count of this line from the beginning of the directory entry section, preceded by the letter $\mathrm{D}$ (odd number).

(Same as Field 1.) 
12 Line Weight Number System display thickness; given as a gradation value in the range of 0 to the maximum (parameter 16 of the global section).

13 Color Number

14 Parameter Line Count Number

15 Form Number

\section{6-17 Reserved for} future use

18 Entity Label

19 Entity Subscript Number

20 Section Code and Sequence Number
Color number or pointer to the directory entry of a color definition entity.

Number of lines in the parameter data record for this entity.

Certain entities have different interpretations. These interpretations are uniquely identified by a form number. Possible form numbers are listed within each entity description.

Up to eight alphanumeric characters (right justified).

1 to 8 digit unsigned number associated with the label.

Same meaning as field 10

(even number). 
2.2.4.3.1 Entity Type Number. The integer number indicating the type of entity.

2.2.4.3.2 Parameter Data. This is the sequence number of the first parameter data record for this entity. The letter $\mathrm{P}$ is not included.

2.2.4.3.3 Structure. Non-negative integer values are permitted in this field, but should be disregarded. (In versions prior to Version 3.0, non-negative integers were used in this field to designate version numbers.) For a negative value, the absolute value of this field is interpreted as a pointer to the structure definition entity which specifies the schema for this entity type number.

2.2.4.3.4 Line Font Pattern. This indicates a display pattern to be used to display a geometric entity. A positive value indicates that the receiving system's corresponding version of the solid, dashed, phantom and centerline fonts should be used. A negative value indicates that the absolute value should be interpreted as a pointer to a line font definition entity (type 304) which provides the information specifying the display pattern.

$$
\begin{array}{ll}
1=\text { Solid } & 3=\text { Phantom } \\
2=\text { Dashed } & 4=\text { Centerline }
\end{array}
$$

2.2.4.3.5 Level. This value specifies a graphic display level or levels to be associated with this entity. A positive value indicates the graphic level this entity exists on. A negative value indicates the absolute value is a pointer to a property entity (type 406, form 1) which contains a list of levels to be associated with the entity. This feature allows an entity to exist on multiple graphic levels.

2.2.4.3.6 View. Three options exist. This value is either a pointer to the directory entry of a view entity (type 410), a pointer to an associativity instance (type 402 , form 3 or 4 , views visible), or the integer zero (default). The first option applies when the entity is visible in a single view. The second option applies when the entity is visible in more than one view. (Form 4 applies when the display characteristics of the entity are view dependent.) The third option applies when the entity is displayed with the same characteristics in all views. 
2.2.4.3.7 Transformation Matrix. This value is either a pointer to the directory entry of a Transformation Matrix entity (type 124) or the integer zero (default). Zero implies the identity rotation matrix and zero translation vector will be used. The Transformation Matrix entity provides Form Numbers according to the form of the transformation matrix. See Section 3.14.

2.2.4.3.8 Label Display Associativity. This is a pointer to the directory entry of a label display associativity (type 402, form 5) which defines how the entity's label and subscript are to be displayed in different views.

2.2.4.3.9 Status Number. This value contains four pieces of information which are concatenated into a single integer number. The four two-digit values are concatenated from left to right in the order given below.

2.2.4.3.9.1 Blank Status. This value defines whether the entity is meant to be visible on the output device of the receiving system. A value of 00 implies the entity is to be displayed and a value of 01 implies the entity is not to be displayed.

2.2.4.3.9.2 Subordinate Entity Switch. This value indicates whether or not the entity is referenced by other entities in the file; and, if so, what type of relationship exists. An entity can be independent, physically dependent, logically dependent, or physically and logically dependent. The values are defined as follows:

00: Independent. The entity is not referenced (i.e., pointed to) by any other entities in the file. It can exist alone in the native database.

01: Physically Dependent. This entity (the child) is referenced by another entity (the parent) in the file. The child cannot exist unless the parent exists. The matrix pointed to by the entity (as a child) must be applied to the entity's definition in order to determine its location in the parent's definition space.

Entity $A$ is subordinate to entity $B$ if, and only if, the parameter data entry of entity B contains a pointer to entity A. This implies that entities are NOT subordinate to the view (or views visible associativity) entity that defines the view within which the entity is displayed. 
The structure formed by a parent entity and its physically subordinate components is indivisible and may therefore be considered to form a single entity.

The following are examples of physically subordinate entities:

- A leader line entity pointed to by a subordinate entity.

- A line entity pointed to by a plane entity.

- A circular arc entity pointed to by a composite curve entity.

- A composite curve entity pointed to by a subfigure definition (note that the subfigure definition would NOT point to the constituent entities of the composite curve).

Consider the following example:

- Entity $A$ is physically subordinate to entity $B$.

- Entity A points to a transformation matrix Ml.

- Transformation matrix M1 points to a transformation matrix M2.

- Entity $B$ is subordinate to a subfigure definition entity $C$.

- Entity $B$ points to a transformation matrix M3.

- Entity $\mathrm{C}$ is instanced in a subfigure instance $\mathrm{D}$.

- The parameter data of entity D specifies its scale factor as Sd and position as ( $\mathrm{d} d, Y d, Z d)$.

- Entity D points to a transformation matrix M4.

- Entity $D$ points to a view entity $E$.

- The view scale factor defined in the parameter data of entity $E$ is Se.

- Entity $E$ occurs within a drawing $F$ at drawing coordinates ( Fx, Fy).

o Entity E points to a transformation matrix M5.

In order to obtain the drawing space coordinates of entity A, the following operations are performed:

1. The coordinates of entity A are transformed by MI.

2. The coordinates resulting from the preceeding step are transformed by $\mathrm{M} 2$.

3. The coordinates resulting from the preceeding step are transformed by $\mathrm{M} 3$. 
4. The coordinates resulting from the preceeding step are scaled by Sd.

5. The coordinates resulting from the preceeding step are transformed by $\mathrm{M} 4$.

6. The coordinates resulting from the preceeding step are translated by the vector $(X d, Y d, Z d)$. The cuordinates resulting from this step are the model space coordinates of entity $A$.

7. The coordinates resulting from the preceeding step are transformed by $\mathrm{M} 5$.

8. The coordinates resulting from the preceeding step are scaled by the scale factor Se.

9. The coordinates resulting from the preceeding step are translated by the vector $(F x, F y)$.

02: Logically Dependent. This entity (the child) can exist alone in the native database, but is referenced by some sort of logical grouping entity, or entities (the parents), such as associativities. The matrix pointed to by the parent entity has no effect on the location of the child.

An example of a logically subordinate entity is that of a line entity pointed to by a group associativity entity.

03: Both Physically and Logically Dependent. This entity is physically dependent upon another ertity in the file and is subject to the physical dependency rules described above. This entity is also referenced by one or more logical grouping entities, and is also subject to the logical dependency rules described above. Additionally, an entity cannot be physically and logically dependent upon the same parent entity.

The case of an entity being both logically and physically subordinate refers to the fact that the transformation matrix pointed to by a parent entity is not applied to its logically subordinate children.

An example of a logically and physically subordinate entity is that of a line entity occuring in a subfigure definition and pointed to by a group associativity entity. 


\section{DATA FORM - ASCII - Directory Entry Section}

2.2.4.3.9.3 Entity Use Flag。 This value indicates the intent of the entity. It classifies the entity as intending to serve in the following manners:

00 Geometry. This is the default value. The entity is used to define the geometry of the structure of the product.

01 Annotation. The entity is used to add annotation or description to the file. This includes geometric entities used to form annotation or description.

02 Definition. The entity is used in definition structures of the file. It is not intended to be valid outside of the other entities which reference the definition structure. An example is the entities in a subfigure definition which are intended to be valid in the subfigure instances that reference the subfigure definition. This class includes all entities in the 300 entity type number range.

03 Other. The entity is being used for other purposes such as defining structural features in the file. This category corresponds roughly to the 400 range, but there are exceptions. For example, a subfigure instance (408) could define geometry, thus having an entity use $\mathrm{flag}=0$ or it could define a drawing format, thus having an entity use flag $=01$. An associativity instance would ordinarily have the value 03 . Exceptions include associativities concerned with display where it would have the value 01 . The view and drawing entities have value 01 (annotation). Transformation depends on its use: If used only for annotation (e.g., defining a view) the value is 01 ; if used for defining geometry or for defining geometry and annotation, value is 00 . 
04 Logical/Positional. The entity is used as a logical and/or positional reference by other entities. This usage does not prevent the entity from referencing other entities or having its own attributes. Some entities which may be instanced in this way are nodes, connect points, and points when their primary use is as a reference.

05 2D Parametric. The entity takes its values in two dimensional $X Y$ parameter space considered as a subset of three dimensional $X Y Z$ space by ignoring the $Z$ coordinate. The transformation matrix from definition space to parameter space must be 2-dimensional (i.e., in entity 124, section 3.14.8, $\mathrm{T}_{3}=\mathrm{R}_{13}=\mathrm{R}_{31}=\mathrm{R}_{32}=\mathrm{R}_{23}=0.0$ and $\mathrm{R}_{33}=1.0$ ). In addition, the coordinates do not have units of length (i.e., the model space scale and units conversion do not apply). This is intended for use in defining curves on surfaces.

2.2.4.3.9.4 Hierarchy. This value indicates the relationship between entities in a hierarchical structure and is used to determine which entity's directory entry attributes should be applied. It applies to line font, view, entity level, blank status, line weight, and color number. Three values are provided:

00: All the above directory entry attributes will apply to entities physically subordinate to this entity.

01: None of the above directory entry attributes of this entity will apply to physically subordinate entities. The physically subordinate entities will use their own directory entity attributes.

02: Individual setting of each of above direct entry attributes are allowed. A hierarchy property entity form 10 (see 4.3.7.3.10) specifies whether 00 or 01 is applied for each directory entry attribute to physically subordinate entities. 
Example: If an entity $A$ has 00 in its DE status digits 7 and 8, all entities subordinate to $A$ will have the attributes assigned to $\mathrm{A}$. Consequently, the attributes assigned to all entities subordinate to $A$ are ignored.

If an entity $A$ has 01 in its DE status digits 7 and 8, the entities immediately subordinate to $A$ will retain their own status. Consequently, the attributes assigned to $\mathrm{A}$ are ignored.

2.2.4.3.10 Sequence Number. A number which specifies the position of the DE line in the directory entry section. The sequence number of the first DE line for any entity is always odd and the sequence number of the second line is always even.

\subsection{Entity Type Number. This is the same as Field 1.}

2.2.4.3.12 Line Weight Number. This value denotes the thickness (or width) with which an entity should be displayed. A specific series of possible thicknesses are specified by global parameters 16 and 17. The largest thickness possible is that specified in global parameter 17 and is denoted by setting this value equal to the value in global parameter 16. The smallest thickness possible is equal to the result of dividing global parameter 17 by global parameter 16 and is denoted by setting this value equal to 1 . Thicknesses between the smallest and largest thickness are available in increments equal to the smallest possible thickness and are denoted by setting this value equal to the integer number of (adjacent) increments required.

Thus, display thickness is:

Line Weight Number * (Global parameter 17/Global parameter 16)

A value of 0 indicates that the default line weight display of the receiving system is to be used. 
2.2.4.3.13 Color Number. Field 13 is a color number and is used for specifying color when the precise shade is unimportant or is a pointer to a more precise color definition. It is up to the receiving system to generate colors which approximately fit the following description.

Color No.

$\begin{array}{ll}0 & \text { No color assigned } \\ 1 & \text { Black } \\ 2 & \text { Red } \\ 3 & \text { Green } \\ 4 & \text { Blue } \\ 5 & \text { Yellow } \\ 6 & \text { Magenta } \\ 7 & \text { Cyan } \\ 8 & \text { White }\end{array}$

If the color number is negative, its absolute value is a pointer to the directory entry of a color definition entity (type 314 ).

2.2.4.3.14 Parameter Line Count Number. This is the number of lines in the parameter data section which contain the parameter data record for this entity.

2.2.4.3.15 Form Number. This value indicates an individual interpretation of the entity to be used when processing the parameter data for this entity. Some entity types allow multiple interpretations of their parameter data. This parameter along with the entity type number uniquely identify the interpretation of the parameter data.

2.2.4.3.16 Reserved Field. This field is reserved for future use and should be left blank.

\subsection{Reserved Field. Same as Field 16.}

2.2.4.3.18 Entity Label. This is the application-specified alphanumeric identifier or name for this entity. It is used in conjunction with the entity subscript number (Field 19) to provide the application-specified alphanumeric identifier for the entity. 
$\begin{aligned} \text { DATA FORM - ASCII - } & \text { Directory Entry } \\ & \text { Section }\end{aligned}$

2.2.4.3.19 Entity Subscript Number. This is a numeric qualifier for the entity label (Field 18).

2.2.4.3.20 Sequence Number. Same as 2.2.4.3.10

2.2.4.3.21 Table 2-3 summarizes DE Field Requirements for mechanical product entities.

Each DE field is placed in one of three following categories.

Ignore

The field has no meaning for this entity. The preprocessor should set the field to 0 or blank. The postprocessor should ignore the field altogether.

Valid: May be defaulted to

0 or blank is a valid setting for this field. If the field is blank, the postprocessor will interpret it a 0 or blank as specified. Other valid settings must be set explicitly by the preprocessor.

Valid: Non-zero required

A non-zero value must be set by the preprocessor. Zero or blank are not valid settings for this field, therefore no default is allowed. 
TABLE 2-3 ENTITY DE FIELD REQUIREMENTS

Entity Type(s): 100, 104, 106 (Forms 1-3, 11-13, 63) 110, 112, 116, 126, 114, 108, 118, 120, 122, 130, 140, 142,144

\begin{tabular}{|c|c|c|c|c|}
\hline ATTRIBUTE & IGNORE & $\begin{array}{l}\text { VALID: } \\
\text { May be } \\
\text { defaulted to }\end{array}$ & \begin{tabular}{|l|} 
VALID: \\
Non-Zero \\
Required \\
\end{tabular} & COMMENT \\
\hline 1. TYPE & & & $\mathrm{X}$ & \\
\hline 2. PD ptr. & & & $\mathrm{X}$ & \\
\hline 3. Structure & $\mathrm{X}$ & & & \\
\hline 4. Line Font & & & $\mathrm{X}$ & $\begin{array}{l}\text { Ignored for 116, } 106 \\
\text { (Forms 1,2, 3) }\end{array}$ \\
\hline 5. Level & & 0 & & \\
\hline 6. View ptr. & & 0 & & $\begin{array}{l}0 \text { means display in all } \\
\text { views }\end{array}$ \\
\hline 7. Matrix ptr. & & 0 & & \\
\hline 8. Label ptr. & & 0 & & \\
\hline 9a. Blank S & & 00 & & \\
\hline 9b. Subord Sw & & 00 & & \\
\hline 9c. Entity use & & 00 & & $\begin{array}{l}00,01,05 \text { are the only } \\
\text { valid values. } 01 \text { implies } \\
\text { the entity is used as } \\
\text { annotation rather than } \\
\text { defining geometry. }\end{array}$ \\
\hline 9d. Hierarchy & $\mathrm{X}$ & & & \\
\hline 10. Seq. num. & & & $\mathrm{X}$ & \\
\hline 11. TYPE & & & $\mathrm{X}$ & \\
\hline 12. Line wt. & & 0 & & $\begin{array}{l}\text { A value of } 0 \text { means to use } \\
\text { the receiving system's } \\
\text { default line weight. }\end{array}$ \\
\hline 13. Color & & 0 & & $\begin{array}{l}\text { A value of } 0 \text { means any } \\
\text { color may be used. }\end{array}$ \\
\hline 14. PD Count & & & $\mathrm{X}$ & \\
\hline 15. Form & & 0 & & Non-zero required for 106 \\
\hline 16. Reserved & $\mathrm{X}$ & & & \\
\hline 17. Reserved & $\mathrm{X}$ & & & \\
\hline 18. Label & & Blank & & \\
\hline 19. Subscript & & 0 & & \\
\hline 20. Seq. num. & & & $\mathrm{X}$ & \\
\hline
\end{tabular}


Entity Type(s): 102

\begin{tabular}{|l|l|l|l|l|}
\hline ATTRIBUTE & IGNORE & $\begin{array}{l}\text { VALID: } \\
\text { May be } \\
\text { defaulted to }\end{array}$ & $\begin{array}{l}\text { VALID: } \\
\text { Non-Zero } \\
\text { Required }\end{array}$ & COMMENT \\
\hline 1. TYPE & & & $\mathrm{X}$ & \\
\hline 2. PD ptr. & & & $\mathrm{X}$ & \\
\hline 3. Structure & $\mathrm{X}$ & & $\mathrm{X}$ & \\
\hline 4. Line Font & & & Note 1 \\
\hline 5. Level & & 0 & Note 1 \\
\hline 6. View ptr. & & 0 & & Note 1 \\
\hline 7. Matrix ptr. & & 0 & & \\
\hline 8. Label ptr. & & 0 & & \\
\hline 9a. Blank S & & 00 & & Note 1 \\
\hline 9b. Subord Sw & & 00 & & \\
\hline 9c. Entity use & & 00 & & \\
\hline 9d. Hierarchy & & 00 & & \\
\hline 10. Seq. num. & & & $\mathrm{X}$ & \\
\hline 11. TYPE & & & $\mathrm{X}$ & \\
\hline 12. Line wt. & & 0 & & Note 1 \\
\hline 13. Color & & 0 & $\mathrm{X}$ & \\
\hline 14. PD Count & & & $\mathrm{X}$ & \\
\hline 15. Form & & 0 & & \\
\hline 16. Reserved & $\mathrm{X}$ & & & \\
\hline 17. Reserved & $\mathrm{X}$ & & & \\
\hline 18. Label & & $\mathrm{Blank}$ & & \\
\hline 19. Subscript & & 0 & & \\
\hline 20. Seq. num. & & & & \\
\hline
\end{tabular}

\section{Notes:}

1. This field is ignored if the hierarchy value for field is 01 . 
Entity Type(s): 124 (Forms 0,1)

\begin{tabular}{|l|l|l|l|l|}
\hline ATTRIBUTE & IGNORE & $\begin{array}{l}\text { VALID: } \\
\text { May be } \\
\text { defaulted to }\end{array}$ & $\begin{array}{l}\text { VALID: } \\
\text { Non-Zero } \\
\text { Required }\end{array}$ & COMMENT \\
\hline 1. TYPE & & & $\mathrm{X}$ & \\
\hline 2. PD ptr. & & & $\mathrm{X}$ & \\
\hline 3. Structure & $\mathrm{X}$ & & & \\
\hline 4. Line Font & $\mathrm{X}$ & & & \\
\hline 5. Level & $\mathrm{X}$ & & & \\
\hline 6. View ptr. & $\mathrm{X}$ & & & \\
\hline 7. Matrix ptr. & & 0 & & \\
\hline 8. Label ptr. & $\mathrm{X}$ & & & \\
\hline 9a. Blank S & $\mathrm{X}$ & & & \\
\hline 9b. Subord Sw & $\mathrm{X}$ & & & \\
\hline 9c. Entity use & $\mathrm{X}$ & & & \\
\hline 9d. Hierarchy & $\mathrm{X}$ & & & \\
\hline 10. Seq. num. & & & $\mathrm{X}$ & \\
\hline 11. TYPE & & & $\mathrm{X}$ & \\
\hline 12. Line wt. & $\mathrm{X}$ & & & \\
\hline 13. Color & $\mathrm{X}$ & & & \\
\hline 14. PD Count & & & & \\
\hline 15. Form & & 0 & & \\
\hline 16. Reserved & $\mathrm{X}$ & & & \\
\hline 17. Reserved & $\mathrm{X}$ & & & \\
\hline 18. Label & & $\mathrm{Blank}$ & & \\
\hline 19. Subscript & & 0 & & \\
\hline 20. Seq. num. & & & & \\
\hline
\end{tabular}


DATA FORM - ASCII - $\begin{aligned} \text { Directory Entry } \\ \text { Section }\end{aligned}$

Entity Type(s): 124 (Forms 10,11,12)

\begin{tabular}{|c|c|c|c|c|}
\hline ATTRIBUTE & IGNORE & $\begin{array}{l}\text { VALID: } \\
\text { May be } \\
\text { defaulted to }\end{array}$ & $\begin{array}{l}\text { VALID: } \\
\text { Non-Zero } \\
\text { Required }\end{array}$ & COMMENT \\
\hline 1. TYPE & & & $\mathrm{X}$ & \\
\hline 2. PD ptr. & & & $\mathrm{X}$ & \\
\hline 3. Structure & $\mathrm{X}$ & & & \\
\hline 4. Line Font & $\mathrm{X}$ & & & \\
\hline 5. Level & $\mathrm{X}$ & & & \\
\hline 6. View ptr. & $\mathrm{X}$ & & & \\
\hline 7. Matrix ptr. & & 0 & & \\
\hline 8. Label ptr. & $\mathrm{X}$ & & & \\
\hline 9a. Blank S & & 00 & & $\begin{array}{l}\text { If not blanked then } \\
\text { display coordinate system } \\
\text { in color indicated in DE } \\
\text { field } 13\end{array}$ \\
\hline 9b. Subord Sw & & 00 & & $\begin{array}{l}01 \text { is used to stack } \\
\text { coordinate systems, } 02 \text { is } \\
\text { used to group coordinate } \\
\text { systems }\end{array}$ \\
\hline 9c. Entity use & $\mathrm{X}$ & & & \\
\hline 9d. Hierarchy & $\mathrm{X}$ & & & \\
\hline 10. Seq. num. & & & $\mathrm{X}$ & \\
\hline 11. TYPE & & & $\mathrm{X}$ & \\
\hline 12. Line wt. & $\mathrm{X}$ & & & \\
\hline 13. Color & & 0 & & \\
\hline 14. PD Count & & & $\mathrm{X}$ & \\
\hline 15. Form & & 0 & & \\
\hline 16. Reserved & $\mathrm{X}$ & & & \\
\hline 17. Reserved & $\mathrm{X}$ & & & \\
\hline 18. Label & & Blank & & \\
\hline 19. Subscript & & 0 & & \\
\hline 20. Seq. num. & & & $\mathrm{X}$ & \\
\hline
\end{tabular}


Entity Type(s): 200 Series except 212 and 214

\begin{tabular}{|l|l|l|l|l|}
\hline ATTRIBUTE & IGNORE & $\begin{array}{l}\text { VALID: } \\
\text { May be } \\
\text { defaulted to }\end{array}$ & $\begin{array}{l}\text { VALID: } \\
\text { Non-Zero } \\
\text { Required }\end{array}$ & COMMENT \\
\hline 1. TYPE & & & $\mathrm{X}$ & \\
\hline 2. PD ptr. & & & $\mathrm{X}$ & \\
\hline 3. Structure & $\mathrm{X}$ & & & \\
\hline 4. Line Font & & & $\mathrm{X}$ & \\
\hline 5. Level & & 0 & & \\
\hline 6. View ptr. & & 0 & & \\
\hline 7. Matrix ptr. & & 0 & & \\
\hline 8. Label ptr. & & 0 & & \\
\hline 9a. Blank S & & 00 & & \\
\hline 9b. Subord Sw & & 00 & & \\
\hline 9c. Entity use & & & $\mathrm{X}$ & Must be 01 \\
\hline 9d. Hierarchy & & 00 & & \\
\hline 10. Seq. num. & & & $\mathrm{X}$ & \\
\hline 11. TYPE & & & $\mathrm{X}$ & \\
\hline 12. Line wt. & & 0 & & \\
\hline 13. Color & & 0 & & \\
\hline 14. PD Count & & & $\mathrm{X}$ & \\
\hline 15. Form & & 0 & & \\
\hline 16. Reserved & $\mathrm{X}$ & & & \\
\hline 17. Reserved & $\mathrm{X}$ & & & \\
\hline 18. Label & & $\mathrm{Blank}$ & & \\
\hline 19. Subscript & & 0 & & \\
\hline 20. Seq. num. & & & & \\
\hline
\end{tabular}


Entity Type(s): 212. 214 and 106 (Forms 20, 21, 31-40)

\begin{tabular}{|l|l|l|l|l|}
\hline ATTRIBUTE & IGNORE & $\begin{array}{l}\text { VALID: } \\
\text { May be } \\
\text { defaulted to }\end{array}$ & $\begin{array}{l}\text { VALID: } \\
\text { Non-Zero } \\
\text { Required }\end{array}$ & COMMENT \\
\hline 1. TYPE & & & $\mathrm{X}$ & \\
\hline 2. PD ptr. & & & $\mathrm{X}$ & \\
\hline 3. Structure & $\mathrm{X}$ & & & \\
\hline 4. Line Font & & & $\mathrm{X}$ & $\begin{array}{l}\text { Ignored for 106 (Forms 20, } \\
\text { 21) }\end{array}$ \\
\hline 5. Level & & 0 & & \\
\hline 6. View ptr. & & 0 & & \\
\hline 7. Matrix ptr. & & 0 & & \\
\hline 8. Label ptr. & & 0 & & \\
\hline 9a. Blank S & & 00 & & \\
\hline 9b. Subord Sw & & 00 & & \\
\hline 9c. Entity use & & & $\mathrm{X}$ & Must be 01 \\
\hline 9d. Hierarchy & $\mathrm{X}$ & & & \\
\hline 10. Seq. num. & & & $\mathrm{X}$ & \\
\hline 11. TYPE & & & $\mathrm{X}$ & \\
\hline 12. Line wt. & & 0 & & \\
\hline 13. Color & & 0 & & \\
\hline 14. PD Count & & & & \\
\hline 15. Form & & 0 & & \\
\hline 16. Reserved & $\mathrm{X}$ & & $\mathrm{X}$ & \\
\hline 17. Reserved & $\mathrm{X}$ & & & \\
\hline 18. Label & & $\mathrm{Blank}$ & & \\
\hline 19. Subscript & & 0 & & \\
\hline 20. Seq. num. & & & & \\
\hline
\end{tabular}


Entity Type(s): 302, 306, 310

\begin{tabular}{|l|l|l|l|l|}
\hline ATTRIBUTE & IGNORE & $\begin{array}{l}\text { VALID: } \\
\text { May be } \\
\text { defaulted to }\end{array}$ & $\begin{array}{l}\text { VALID: } \\
\text { Non-Zero } \\
\text { Required }\end{array}$ & COMMENT \\
\hline 1. TYPE & & & $\mathrm{X}$ & \\
\hline 2. PD ptr. & & & $\mathrm{X}$ & \\
\hline 3. Structure & $\mathrm{X}$ & & & \\
\hline 4. Line Font & $\mathrm{X}$ & & & \\
\hline 5. Level & $\mathrm{X}$ & & & \\
\hline 6. View ptr. & $\mathrm{X}$ & & & \\
\hline 7. Matrix ptr. & $\mathrm{X}$ & & & \\
\hline 8. Label ptr. & $\mathrm{X}$ & & & \\
\hline 9a. Blank S & $\mathrm{X}$ & & & \\
\hline 9b. Subord Sw & & 00 & & Must be 00 \\
\hline 9c. Entity use & & & $\mathrm{X}$ & Must be 02 \\
\hline 9d. Hierarchy & $\mathrm{X}$ & & & \\
\hline 10. Seq. num. & & & $\mathrm{X}$ & \\
\hline 11. TYPE & & & $\mathrm{X}$ & \\
\hline 12. Line wt. & $\mathrm{X}$ & & & \\
\hline 13. Color & $\mathrm{X}$ & & & \\
\hline 14. PD Count & & & $\mathrm{X}$ & \\
\hline 15. Form & & 0 & & \\
\hline 16. Reserved & $\mathrm{X}$ & & $\mathrm{X}$ & \\
\hline 17. Reserved & $\mathrm{X}$ & & & \\
\hline 18. Label & & $\mathrm{Blank}$ & & \\
\hline 19. Subscript & & 0 & & \\
\hline 20. Seq. num. & & & & \\
\hline
\end{tabular}


DATA FORM - ASCII - $\begin{aligned} \text { Directory Entry } \\ \text { Section }\end{aligned}$

Entity Type(s): 304

\begin{tabular}{|l|l|l|l|l|}
\hline ATTRIBUTE & IGNORE & $\begin{array}{l}\text { VALID: } \\
\text { May be } \\
\text { defaulted to }\end{array}$ & $\begin{array}{l}\text { VALID: } \\
\text { Non-Zero } \\
\text { Required }\end{array}$ & COMMENT \\
\hline 1. TYPE & & & $\mathrm{X}$ & \\
\hline 2. PD ptr. & & & $\mathrm{X}$ & \\
\hline 3. Structure & $\mathrm{X}$ & & & \\
\hline 4. Line Font & & & $\mathrm{X}$ & \\
\hline 5. Level & $\mathrm{X}$ & & & \\
\hline 6. View ptr. & $\mathrm{X}$ & & & \\
\hline 7. Matrix ptr. & & 0 & & \\
\hline 8. Label ptr. & $\mathrm{X}$ & & & \\
\hline 9a. Blank S & $\mathrm{X}$ & & & \\
\hline 9b. Subord Sw & & 00 & & Must be 00 \\
\hline 9c. Entity use & & & $\mathrm{X}$ & Must be 02 \\
\hline 9d. Hierarchy & $\mathrm{X}$ & & & \\
\hline 10. Seq. num. & & & $\mathrm{X}$ & \\
\hline 11. TYPE & & & $\mathrm{X}$ & \\
\hline 12. Line wt. & $\mathrm{X}$ & & & \\
\hline 13. Color & $\mathrm{X}$ & & & \\
\hline 14. PD Count & & & $\mathrm{X}$ & \\
\hline 15. Form & & & $\mathrm{X}$ & \\
\hline 16. Reserved & $\mathrm{X}$ & & & \\
\hline 17. Reserved & $\mathrm{X}$ & & & \\
\hline 18. Label & & $\mathrm{Blank}$ & & \\
\hline 19. Subscript & & 0 & & \\
\hline 20. Seq. num. & & & & \\
\hline
\end{tabular}


Entity Type(s): 308,320 Subfigure definition

\begin{tabular}{|l|l|l|l|l|}
\hline ATTRIBUTE & IGNORE & $\begin{array}{l}\text { VALID: } \\
\text { May be } \\
\text { defaulted to }\end{array}$ & $\begin{array}{l}\text { VALID: } \\
\text { Non-Zero } \\
\text { Required }\end{array}$ & COMMENT \\
\hline 1. TYPE & & & $\mathrm{X}$ & \\
\hline 2. PD ptr. & & & $\mathrm{X}$ & \\
\hline 3. Structure & $\mathrm{X}$ & & $\mathrm{X}$ & \\
\hline 4. Line. Font & & & Note 1 \\
\hline 5. Level & $\mathrm{X}$ & & Note 1 \\
\hline 6. View ptr. & $\mathrm{X}$ & & & \\
\hline 7. Matrix ptr. & & 0 & & \\
\hline 8. Label ptr. & & 0 & & \\
\hline 9a. Blank S & $\mathrm{X}$ & & & \\
\hline 9b. Subord Sw & & 00 & $\mathrm{X}$ & Must be 02 \\
\hline 9c. Entity use & & & & \\
\hline 9d. Hierarchy & & 00 & $\mathrm{X}$ & \\
\hline 10. Seq. num. & & & $\mathrm{X}$ & \\
\hline 11. TYPE & & & & Note 1 \\
\hline 12. Line wt. & & 0 & & Note 1 \\
\hline 13. Color & & 0 & $\mathrm{X}$ & \\
\hline 14. PD Count & & & & \\
\hline 15. Form & & 0 & & \\
\hline 16. Reserved & $\mathrm{X}$ & & & \\
\hline 17. Reserved & $\mathrm{X}$ & & $\mathrm{X}$ & \\
\hline 18. Label & & 0 & & \\
\hline 19. Subscript & & & & \\
\hline 20. Seq. num. & & & & \\
\hline
\end{tabular}

\section{Notes:}

1. This field is ignored if the hierarchy value for the field is 01. 
Entity Type(s): 312

\begin{tabular}{|l|l|l|l|l|}
\hline ATTRIBUTE & IGNORE & $\begin{array}{l}\text { VALID: } \\
\text { May be } \\
\text { defaulted to }\end{array}$ & $\begin{array}{l}\text { VALID: } \\
\text { Non-Zero } \\
\text { Required }\end{array}$ & COMMENT \\
\hline 1. TYPE & & & $\mathrm{X}$ & \\
\hline 2. PD ptr. & & & $\mathrm{X}$ & \\
\hline 3. Structure & $\mathrm{X}$ & & & \\
\hline 4. Line Font & $\mathrm{X}$ & & & \\
\hline 5. Level & $\mathrm{X}$ & & & \\
\hline 6. View ptr. & $\mathrm{X}$ & & & \\
\hline 7. Matrix ptr. & $\mathrm{X}$ & & & \\
\hline 8. Label ptr. & $\mathrm{X}$ & & & \\
\hline 9a. Blank S & $\mathrm{X}$ & & & \\
\hline 9b. Subord Sw & & & $\mathrm{X}$ & Must be 02 \\
\hline 9c. Entity use & & & $\mathrm{X}$ & Must be 02 \\
\hline 9d. Hierarchy & & & $\mathrm{X}$ & Must be 01 \\
\hline 10. Seq. num. & & & $\mathrm{X}$ & \\
\hline 11. TYPE & & & $\mathrm{X}$ & \\
\hline 12. Line wt. & $\mathrm{X}$ & & & \\
\hline 13. Color & $\mathrm{X}$ & & & \\
\hline 14. PD Count & & & $\mathrm{X}$ & \\
\hline 15. Form & & 0 & & \\
\hline 16. Reserved & $\mathrm{X}$ & & & \\
\hline 17. Reserved & $\mathrm{X}$ & & & \\
\hline 18. Label & & $\mathrm{Blank}$ & & \\
\hline 19. Subscript & & 0 & & \\
\hline 20. Seq. num. & & & & \\
\hline
\end{tabular}


Entity Type(s): 314

\begin{tabular}{|l|l|l|l|l|}
\hline ATTRIBUTE & IGNORE & $\begin{array}{l}\text { VALID: } \\
\text { May be } \\
\text { defaulted to }\end{array}$ & $\begin{array}{l}\text { VALID: } \\
\text { Non-Zero } \\
\text { Required }\end{array}$ & COMMENT \\
\hline 1. TYPE & & & $\mathrm{X}$ & \\
\hline 2. PD ptr. & & & $\mathrm{X}$ & \\
\hline 3. Structure & $\mathrm{X}$ & & & \\
\hline 4. Line Font & $\mathrm{X}$ & & & \\
\hline 5. Level & $\mathrm{X}$ & & & \\
\hline 6. View ptr. & $\mathrm{X}$ & & & \\
\hline 7. Matrix ptr. & $\mathrm{X}$ & & & \\
\hline 8. Label ptr. & $\mathrm{X}$ & & & \\
\hline 9a. Blank S & $\mathrm{X}$ & & & \\
\hline 9b. Subord Sw & & 00 & & Must be 00 \\
\hline 9c. Entity use & & & $\mathrm{X}$ & Must be 02 \\
\hline 9d. Hierarchy & $\mathrm{X}$ & & & \\
\hline 10. Seq. num. & & & $\mathrm{X}$ & \\
\hline 11. TYPE & & & $\mathrm{X}$ & \\
\hline 12. Line wt. & $\mathrm{X}$ & & & \\
\hline 13. Color & & 0 & $\mathrm{X}$ & \\
\hline 14. PD Count & & & & \\
\hline 15. Form & & 0 & & \\
\hline 16. Reserved & $\mathrm{X}$ & & & \\
\hline 17. Reserved & $\mathrm{X}$ & & & \\
\hline 18. Label & & $\mathrm{Blank}$ & & \\
\hline 19. Subscript & & 0 & & \\
\hline 20. Seq. num. & & & & \\
\hline
\end{tabular}


Entity Type(s): 402

\begin{tabular}{|l|l|l|l|l|}
\hline ATTRIBUTE & IGNORE & $\begin{array}{l}\text { VALID: } \\
\text { May be } \\
\text { defaulted to }\end{array}$ & $\begin{array}{l}\text { VALID: } \\
\text { Non-Zero } \\
\text { Required }\end{array}$ & COMMENT \\
\hline 1. TYPE & & & $\mathrm{X}$ & \\
\hline 2. PD ptr. & & & $\mathrm{X}$ & \\
\hline 3. Structure & & & $\mathrm{X}$ & $\begin{array}{l}\text { Ignored for furm numbers } \\
\text { to 5000 }\end{array}$ \\
\hline 4. Line Font & $\mathrm{X}$ & & & \\
\hline 5. Level & $\mathrm{X}$ & & & \\
\hline 6. View ptr. & $\mathrm{X}$ & & & \\
\hline 7. Matrix ptr. & $\mathrm{X}$ & & & \\
\hline 8. Label ptr. & $\mathrm{X}$ & & & \\
\hline 9a. Blank S & $\mathrm{X}$ & & & \\
\hline 9b. Subord Sw & & 00 & & 00 required for Forms 3 \& \\
\hline 9c. Entity use & $\mathrm{X}$ & & & \\
\hline 9d. Hierarchy & $\mathrm{X}$ & & & \\
\hline 10. Seq. num. & & & $\mathrm{X}$ & \\
\hline 11. TYPE & & & $\mathrm{X}$ & \\
\hline 12. Line wt. & $\mathrm{X}$ & & & \\
\hline 13. Color & $\mathrm{X}$ & & & \\
\hline 14. PD Count & & & $\mathrm{X}$ & \\
\hline 15. Form & & & & \\
\hline 16. Reserved & $\mathrm{X}$ & & $\mathrm{X}$ & \\
\hline 17. Reserved & $\mathrm{X}$ & & & \\
\hline 18. Label & & $\mathrm{Blank}$ & & \\
\hline 19. Subscript & & 0 & & \\
\hline 20. Seq. num. & & & & \\
\hline
\end{tabular}


Entity Type(s): 404

\begin{tabular}{|l|l|l|l|l|}
\hline ATTRIBUTE & IGNORE & $\begin{array}{l}\text { VALID: } \\
\text { May be } \\
\text { defaulted to }\end{array}$ & $\begin{array}{l}\text { VALID: } \\
\text { Non-Zero } \\
\text { Required }\end{array}$ & COMMENT \\
\hline 1. TYPE & & & $\mathrm{X}$ & \\
\hline 2. PD ptr. & & & $\mathrm{X}$ & \\
\hline 3. Structure & $\mathrm{X}$ & & & \\
\hline 4. Line Font & $\mathrm{X}$ & & & \\
\hline 5. Level & $\mathrm{X}$ & & & \\
\hline 6. View ptr. & $\mathrm{X}$ & & & \\
\hline 7. Matrix ptr. & $\mathrm{X}$ & & & \\
\hline 8. Label ptr. & $\mathrm{X}$ & & & \\
\hline 9a. Blank S & $\mathrm{X}$ & & & \\
\hline 9b. Subord Sw & & 00 & & Must be independent \\
\hline 9c. Entity use & & & $\mathrm{X}$ & Must be 03 \\
\hline 9d. Hierarchy & $\mathrm{X}$ & & & \\
\hline 10. Seq. num. & & & $\mathrm{X}$ & \\
\hline 11. TYPE & & & $\mathrm{X}$ & \\
\hline 12. Line wt. & $\mathrm{X}$ & & & \\
\hline 13. Color & $\mathrm{X}$ & & & \\
\hline 14. PD Count & & & $\mathrm{X}$ & \\
\hline 15. Form & & 0 & & \\
\hline 16. Reserved & $\mathrm{X}$ & & & \\
\hline 17. Reserved & $\mathrm{X}$ & & & \\
\hline 18. Label & & $\mathrm{Blank}$ & & \\
\hline 19. Subscript & & 0 & & \\
\hline 20. Seq. num. & & & & \\
\hline
\end{tabular}


Entity Type(s): 406

\begin{tabular}{|l|l|l|l|l|}
\hline ATTRIBUTE & IGNORE & $\begin{array}{l}\text { VALID: } \\
\text { May be } \\
\text { defaulted to }\end{array}$ & $\begin{array}{l}\text { VALID: } \\
\text { Non-Zero } \\
\text { Required }\end{array}$ & COMMENT \\
\hline 1. TYPE & & & $\mathrm{X}$ & \\
\hline 2. PD ptr. & & & $\mathrm{X}$ & \\
\hline 3. Structure & $\mathrm{X}$ & & & \\
\hline 4. Line Font & $\mathrm{X}$ & & & \\
\hline 5. Level & & 0 & & \\
\hline 6. View ptr. & $\mathrm{X}$ & & & \\
\hline 7. Matrix ptr. & $\mathrm{X}$ & & & \\
\hline 8. Label ptr. & $\mathrm{X}$ & & & \\
\hline 9a. Blank S & $\mathrm{X}$ & & & \\
\hline 9b. Subord Sw & & 00 & & See Note 1 \\
\hline 9c. Entity use & $\mathrm{X}$ & & & \\
\hline 9d. Hierarchy & $\mathrm{X}$ & & & \\
\hline 10. Seq. num. & & & $\mathrm{X}$ & \\
\hline 11. TYPE & & & $\mathrm{X}$ & \\
\hline 12. Line wt. & $\mathrm{X}$ & & & \\
\hline 13. Color & $\mathrm{X}$ & & & \\
\hline 14. PD Count & & & $\mathrm{X}$ & \\
\hline 15. Form & & & $\mathrm{X}$ & \\
\hline 16. Reserved & $\mathrm{X}$ & & & \\
\hline 17. Reserved & $\mathrm{X}$ & & & \\
\hline 18. Label & & $\mathrm{Blank}$ & & \\
\hline 19. Subscript & & 0 & & \\
\hline 20. Seq. num. & & & & \\
\hline
\end{tabular}

Notes:

1. The setting for this field should be 02. The specification currently states a setting of 00 implies the property should be applied to all entities occurring at the same level. 
Entity Type(s): 408, 412, 414, 420

\begin{tabular}{|l|l|l|l|l|}
\hline ATTRIBUTE & IGNORE & $\begin{array}{l}\text { VALID: } \\
\text { May be } \\
\text { defaulted to }\end{array}$ & $\begin{array}{l}\text { VALID: } \\
\text { Non-Zero } \\
\text { Required }\end{array}$ & COMMENT \\
\hline 1. TYPE & & & $\mathrm{X}$ & \\
\hline 2. PD ptr. & & & $\mathrm{X}$ & \\
\hline 3. Structure & $\mathrm{X}$ & & & \\
\hline 4. Line Font & $\mathrm{X}$ & & & \\
\hline 5. Level & & 0 & & See Note 1 \\
\hline 6. View ptr. & & 0 & & \\
\hline 7. Matrix ptr. & & 0 & & \\
\hline 8. Label ptr. & & 0 & & \\
\hline 9a. Blank S & & 00 & & \\
\hline 9b. Subord Sw & & 00 & & \\
\hline 9c. Entity use & & 00 & & \\
\hline 9d. Hierarchy & $\mathrm{X}$ & & & \\
\hline 10. Seq. num. & & & $\mathrm{X}$ & \\
\hline 11. TYPE & & & $\mathrm{X}$ & \\
\hline 12. Line wt. & $\mathrm{X}$ & & & \\
\hline 13. Color & $\mathrm{X}$ & & & \\
\hline 14. PD Count & & & $\mathrm{X}$ & \\
\hline 15. Form & & 0 & & \\
\hline 16. Reserved & $\mathrm{X}$ & & & \\
\hline 17. Reserved & $\mathrm{X}$ & & & \\
\hline 18. Label & & $\mathrm{Blank}$ & & \\
\hline 19. Subscript & & 0 & & \\
\hline 20. Seq. num. & & & & \\
\hline
\end{tabular}

Notes:

1. The specification currently states entities within a subfigure are displayed at the same level as the instance. 
DATA FORM - ASCII - Directory Entry

Section

Entity Type(s): 410

\begin{tabular}{|l|l|l|l|l|}
\hline ATTRIBUTE & IGNORE & $\begin{array}{l}\text { VALID: } \\
\text { May be } \\
\text { defaulted to }\end{array}$ & $\begin{array}{l}\text { VALID: } \\
\text { Non-Zero } \\
\text { Required }\end{array}$ & COMMENT \\
\hline 1. TYPE & & & $\mathrm{X}$ & \\
\hline 2. PD ptr. & & & $\mathrm{X}$ & \\
\hline 3. Structure & $\mathrm{X}$ & & & \\
\hline 4. Line Font & $\mathrm{X}$ & & & \\
\hline 5. Level & $\mathrm{X}$ & & & \\
\hline 6. View ptr. & $\mathrm{X}$ & & & \\
\hline 7. Matrix ptr. & & 0 & & \\
\hline 8. Label ptr. & $\mathrm{X}$ & & & \\
\hline 9a. Blank S & $\mathrm{X}$ & & & \\
\hline 9b. Subord Sw & & 0 & & $\begin{array}{l}\text { Dependent if 404 points to } \\
\text { it. Independent if not. }\end{array}$ \\
\hline 9c. Entity use & & & $\mathrm{X}$ & Must be 03 \\
\hline 9d. Hierarchy & $\mathrm{X}$ & & & \\
\hline 10. Seq. num. & & & $\mathrm{X}$ & \\
\hline 11. TYPE & & & $\mathrm{X}$ & \\
\hline 12. Line wt. & $\mathrm{X}$ & & & \\
\hline 13. Color & $\mathrm{X}$ & & $\mathrm{X}$ & \\
\hline 14. PD Count & & & & \\
\hline 15. Form & & 0 & & \\
\hline 16. Reserved & $\mathrm{X}$ & & & \\
\hline 17. Reserved & $\mathrm{X}$ & & & \\
\hline 18. Label & & $\mathrm{Blank}$ & & \\
\hline 19. Subscript & & 0 & & \\
\hline 20. Seq. num. & & & & \\
\hline
\end{tabular}




\subsubsection{Parameter Data Section}

The parameter data section of the file contains the parameter data associated with each entity. The following information is true for all parameter data.

2.2.4.4.1 Parameter data are placed in free format (see 2.2.3) with the first field always containing the entity type number. Therefore, the entity type number and a parameter delimiter (default is comma) precede parameter one of each entity. The free format part of a parameter line ends in column 64 . Column 65 shall contain a blank. Columns 66 through 72 on all parameter lines contain the sequence number of the first line in the directory entry of the entity for which parameter data is being presented. Column 73 of all lines in the parameter section shall contain the letter $\mathrm{P}$ and columns 74 through 80 shall contain the sequence number (See 2.2.1).

2.2.4.4.2 Two groups of parameters are defined at the end of the specified parameters for each entity. The first group of parameters may contain pointers to Associativity Instances, General Notes, and/or Text Template entities. The second group of parameters may contain pointers to one or more properties. The pointers to associativity instances are "back pointers" in that they point back from a member of an associativity instance to the associativity instance. These pointers may be required by the associativity definition.

If the given entity references associated text, a pointer to the General Note may be included in the first group of pointers. If so, the indicated General Note specifies the string constant and the indicated display parameters.

If instead, the given entity contains a string constant to be displayed, a pointer to a Text Template entity may be included in the first group of pointers. The Text Template entities provide display parameters for text supplied by the entity which points to the template. 
Either group of parameters, or both, may be empty or may be defaulted to no pointers.

When present, the pointers comprising these parameters are added after all the other specified (or defaulted) parameters, but ahead of the record delimiter as follows:

Let $\mathrm{NV}=$ last parameter number

Index Name $\quad \underline{\text { Type }}$ Description

$\begin{array}{llll}* & \cdot & - & \cdot \\ N V+1 & N A & \text { Integer } & \text { Number of pointers to } \\ & & \text { Associativity Instances/Text } \\ & & \text { Entities }\end{array}$

NV+2 DE Pointer

$\begin{array}{cccl}\cdot & \cdot & \cdot & \text { Associativity } \\ \mathrm{NV}+\mathrm{NA}+1 & \cdot & \cdot & \text { Entity List } \\ \mathrm{NV}+\mathrm{NA}+2 & \mathrm{NP} & \text { Integer } & \begin{array}{l}\text { Number of } \\ \text { Properties }\end{array} \\ \mathrm{NV}+\mathrm{NA}+3 & \mathrm{DE} & \text { Pointer } & \\ \cdot & \cdot & \cdot & \\ \mathrm{NV}+\mathrm{NA}+\mathrm{NP}+2 & \cdot & \cdot & \end{array}$

2.2.4.4.3 Any desired comment may be added after the record delimiter. Note that additional lines may be used for this purpose by keeping the directory entry pointer in columns 65-72 constant and include them in the count of parameter lines for the entity (DE field 14). Figure 2-3 shows a parameter data section. 
PARAMETER DATA (PD) SECTION

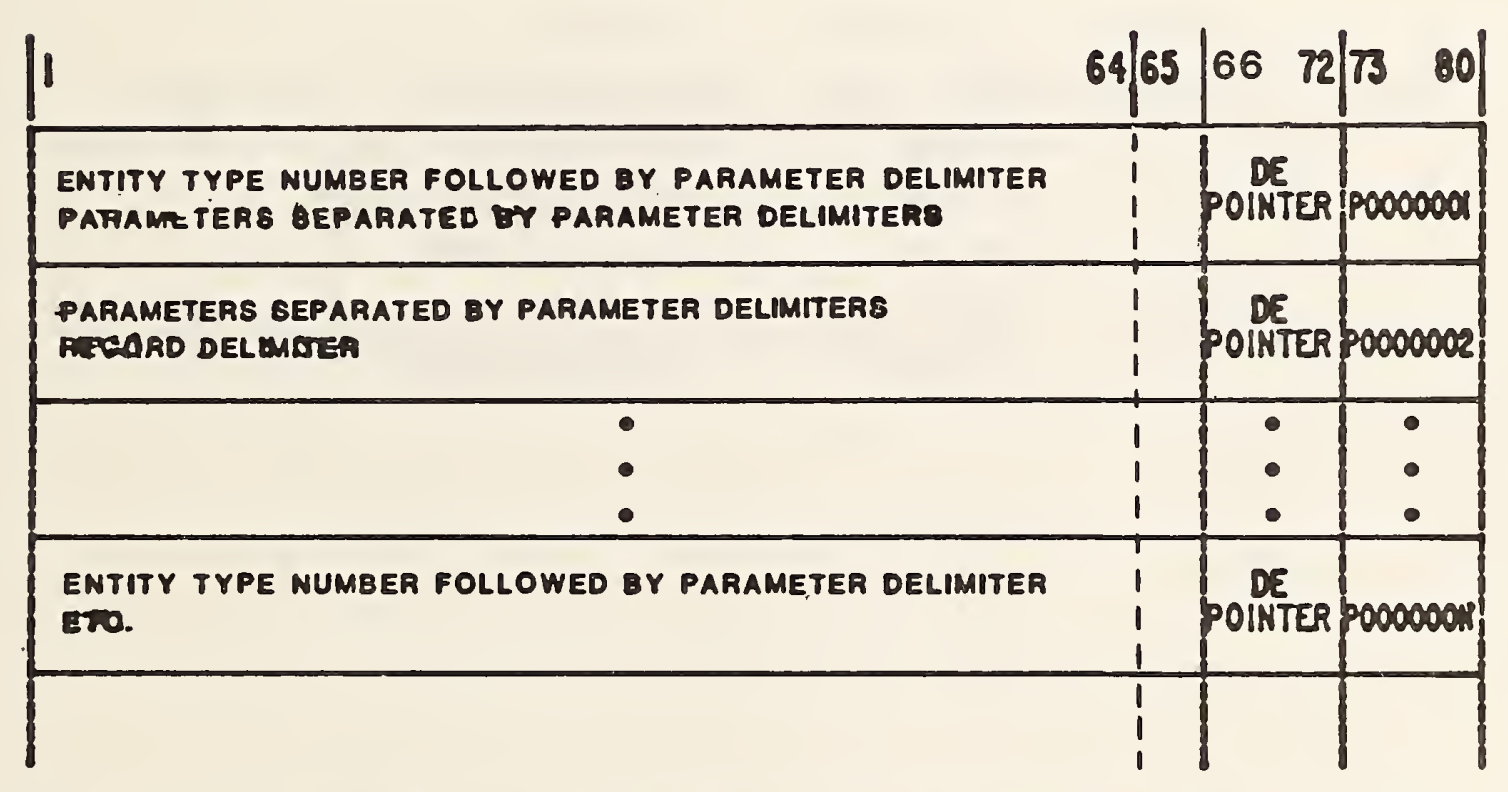

DE POINTER: THE SEQUENCE NUMBER OF THE FIRST DIRECTORY EUTRY LINE FOR THIS ENTITY

FIG. 2-3 PARAMETER DATA SECTION 
DATA FORM - ASCII - Terminate Section

\subsubsection{Terminate Section}

There is only one line in the terminate section of the file. It is divided into ten fields of eight columns each. The terminate section must be the last line of the file. It has a "T" in column 73 and columns 74 through 80 contain the sequence number with a value of one (1).

The fields on the terminate line contain the character representing the section type and the last sequence number used in each of the previous sections. The fields are defined below and shown in Figure 2-4.

$\begin{array}{lll}\text { FIELD } & \text { COLUMNS } & \text { SECTION } \\ 1 & 1-8 & \text { Start Section } \\ 2 & 9-16 & \text { Global Section } \\ 3 & 17-24 & \text { Directory Entry Section } \\ 4 & 25-32 & \text { Parameter Section } \\ 5-9 & 33-72 & \text { (not used) } \\ 10 & 73-80 & \text { Terminate Section }\end{array}$




\section{TERMINATE SECTION}

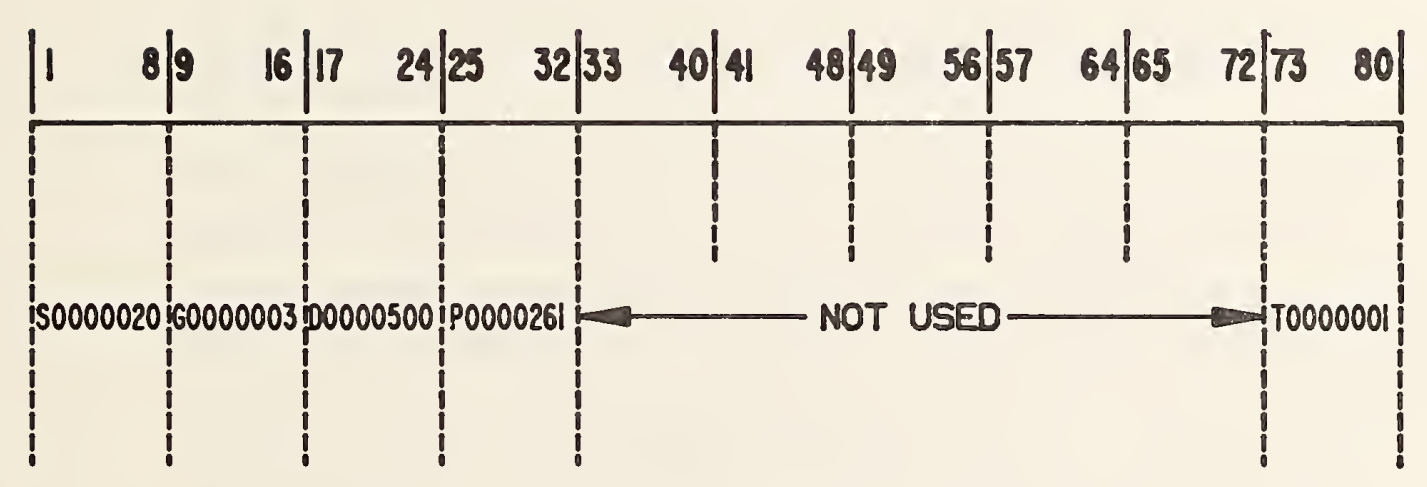

FIG. 2-4 TERMINATE SECTION 

The format described here is intended to serve as an alternative to the ASCII Form when the size of a file is a problem. The Compressed ASCII Form is intended to be simply converted to and from the regular ASCII Form. As an example of such a conversion, the National Bureau of Standards has software available to convert from ASCII to Compressed ASCII and from Compressed ASCII to regular ASCII (See Appendix G).

\subsubsection{File Structure}

A single line shall precede the START section with the character "C" in character position 73 to identify the file as compressed ASCII. The START, GLOBAL and TERMINATE sections remain the same as the ASCII Form, while the DE and PD sections are combined into a single DATA section. The PD portion of the DATA section is written in free format in a manner similar to the present PD section. Each line is of variable length and is to terminate before character position 65, thus assuring that character position 65 , if it existed (i.e., if the line is read into a fixed 80 character buffer) would always contain the blank character. The lines corresponding to a single entity begin with one or more lines giving the data presently contained in the DE record.

The "neo-DE" records begin with the letter "D", followed, without intervening blanks, by an integer equal to the present sequence number of the first DE record. See Figure 2-5. The D〈sequence number $\rangle$ group of characters is followed by one or more DE field specifiers. The DE field specifier consists of the symbol "(a" (commercial at) followed by an integer giving the $D E$ field being specified. The @ field number group is followed by a character sequence consisting of the symbol "-" (underline), followed by the value of the field.

All DE field numbers remain the same as with the present DE record. Fields 2, 10, 11, and 20 are not specified, as they are either redundant or meaningless in the Compressed ASCII form. When several DE 
fields are being specified, additional lines can be used. The sequence of field specifiers must be broken only between complete specifiers, thus assuring that new records will begin with the symbol "@".

Field values will be specified only when they change. Thus, a field retains its value from entity to entity unless it is changed, and only the first entity in a file is assured of containing a complete set of fields and values. No separation mark is used between @ 〈field number $\geq$ 〈value groups but the DE field specifier record(s) end with a record delimiter (default ";").

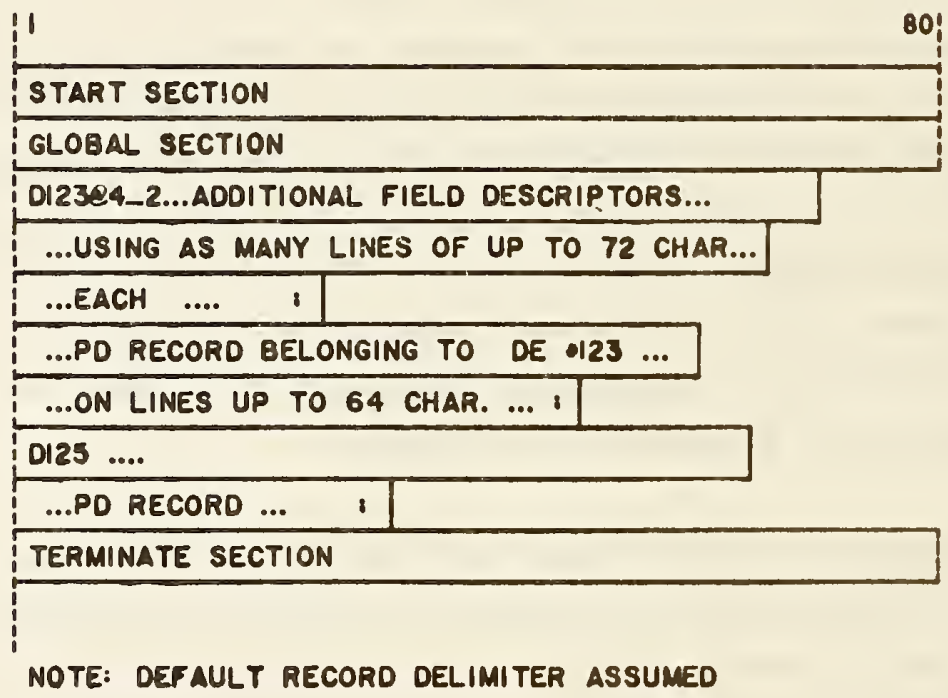

FIGURE 2-5 COMPRESSED ASCII FORM

The PD records remain the same as in the ASCII Form with the exception that they terminate at the end of parameter data (i.e., before character position 65). 
The format defined in Section 2.2, referred to as the ASCII Form, has 80 character fixed length record lines. This section describes a bit stream binary representation of data which may be used as an alternative format to the ASCII Form.

This data is transportable by user selected communication protocols with the data treated as "transparent" or bit stream data. All entity parameterizations and data organization are otherwise identical to the ASCII Form.

2.4.1 Constants. The following constants need to be represented in the Binary Form:
- integer numbers
- real numbers
- string constants
- pointers
- language constants 
A control byte will precede each value or set of values of the same type, unless otherwise specified. The control byte will specify the format of the following value or set of values, the quantity of subsequent values with that format, and whether values other than the initial value following the control byte are present. If the control byte indicates that values subsequent to the initial value of the set are absent, all subsequent values, up to the quantity indicated are assumed to have the same value as the initial value following the control byte.

The repetition portion of the control byte is unsigned and biased by 1 so that the true quantity of numbers to which the repetition field applies is one more than the unsigned value of the field.

The format of the control byte is as shown in Figure 2-6.

\subsubsection{Integer Numbers.}

The structure of an integer number shall be a sign bit followed by a two's complement integer of length $\mathrm{i}-1$ as shown in Figure 2-7.

Two lengths, $i$, of integer data can be selected by the system which generates the file.

The length of single precision data is $i_{s}$ and the length of double precision data is $i_{d}$, defined in section 2.4.2.1.

\subsubsection{Real Numbers.}

The structure of a real number shall be a sign bit followed by a biased exponent value of $\mathrm{NX}$ bits which is a power of 2 and a binary fraction of NF bits. (NX and NF are defined in section 2.4.2.1) The value of the number is the sign applied to the fractional part multiplied by two raised to the power specified by the exponent part. The sign field consists of one bit. A sign of 0 indicates a positive number and a sign of 1 indicates a negative number. The exponent field consists of $\mathrm{NX}$ bits and is interpreted as an unsigned integer, BX, of ten referred to as the biased exponent. The value of the exponent is its unbiased value $X$ which is obtained by deducting the bias $B=2 * *(N X-1)$. 


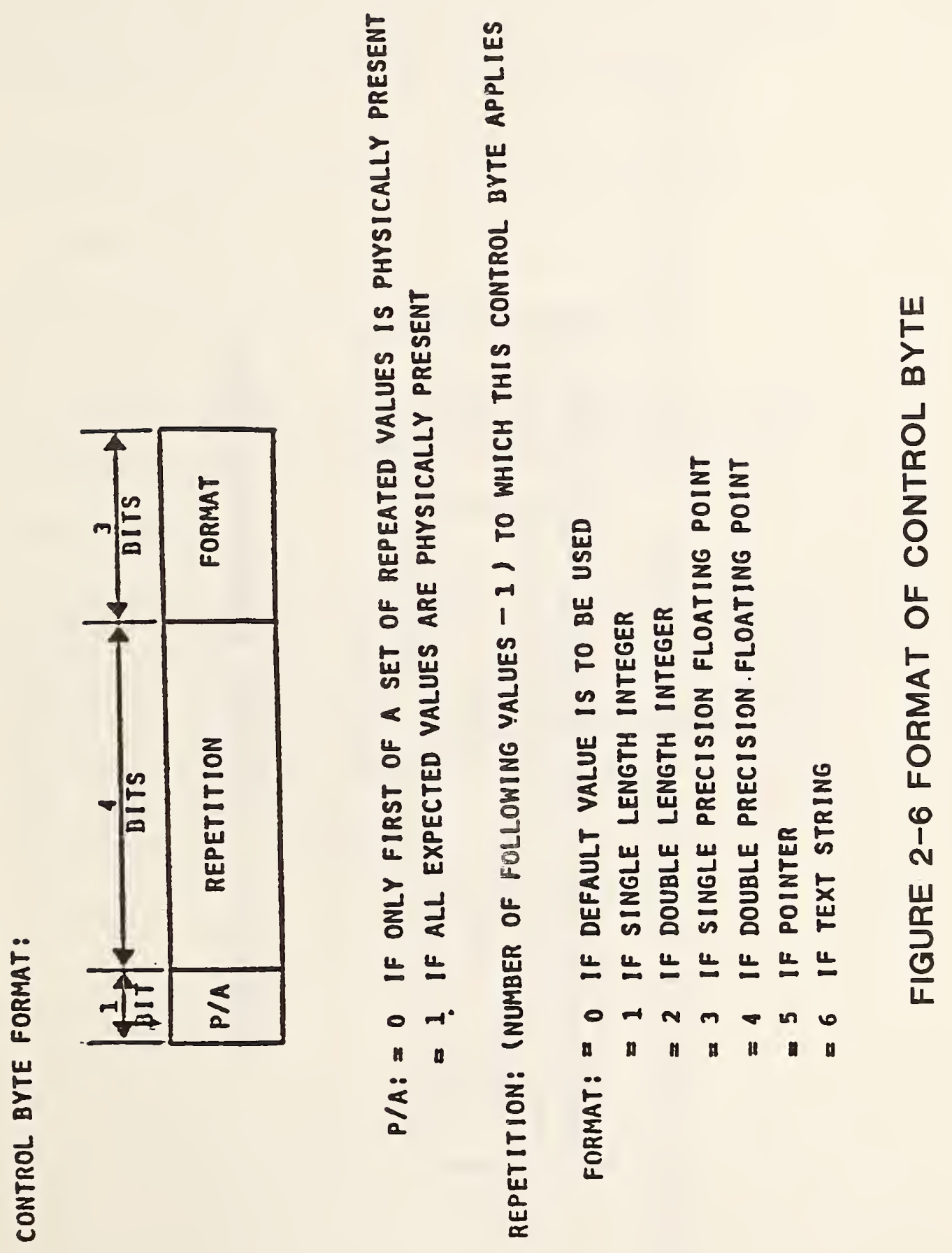




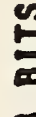

曲

님

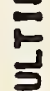

2

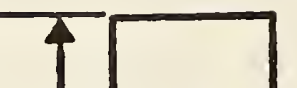

$6 \quad 1$

2

$\frac{1}{2}$

?

$\frac{E}{E}$

를

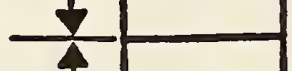

씰

두

ㄴ $\quad$ IE

는

$\frac{1}{2}$

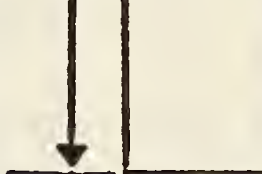

놀

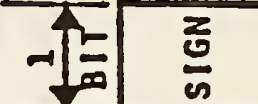

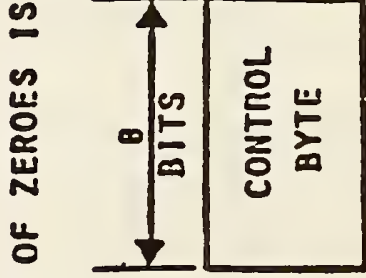

8

필

N

$\frac{W}{\frac{U}{\square}}$ 
The fraction field consists of NF bits interpreted as the low order bits of a normalized $(\mathrm{NF}+1)$-bit fraction part, $\mathrm{F}$. The fraction lies between 0.5 (inclusive) and 1.0 (exclusive). Since the most significant bit of a normalized fraction is always 1 it is not explicitly represented.

Numbers with a non-zero biased exponent have a value given by:

$$
(-1)^{\operatorname{SIGN}_{* 2}(B X-B)} * F
$$

The structure of a real number is shown in Figure 2-8.

Two lengths of real data can be selected by specifying the length of each exponent (NX) and the length of each fractional portion (NF).

\subsubsection{String Constants.}

Following the control byte will be a character count with a length of $i_{s}$, defined in section 2.4.2.1. Where the character count exceeds the capability of an $i_{s}$ length integer, the string is broken up into substrings. In order to indicate that another substring follows the current string, a negative character count is used. The number of characters in the substring is the absolute value of the character count. A positive character count indicates the last substring. 


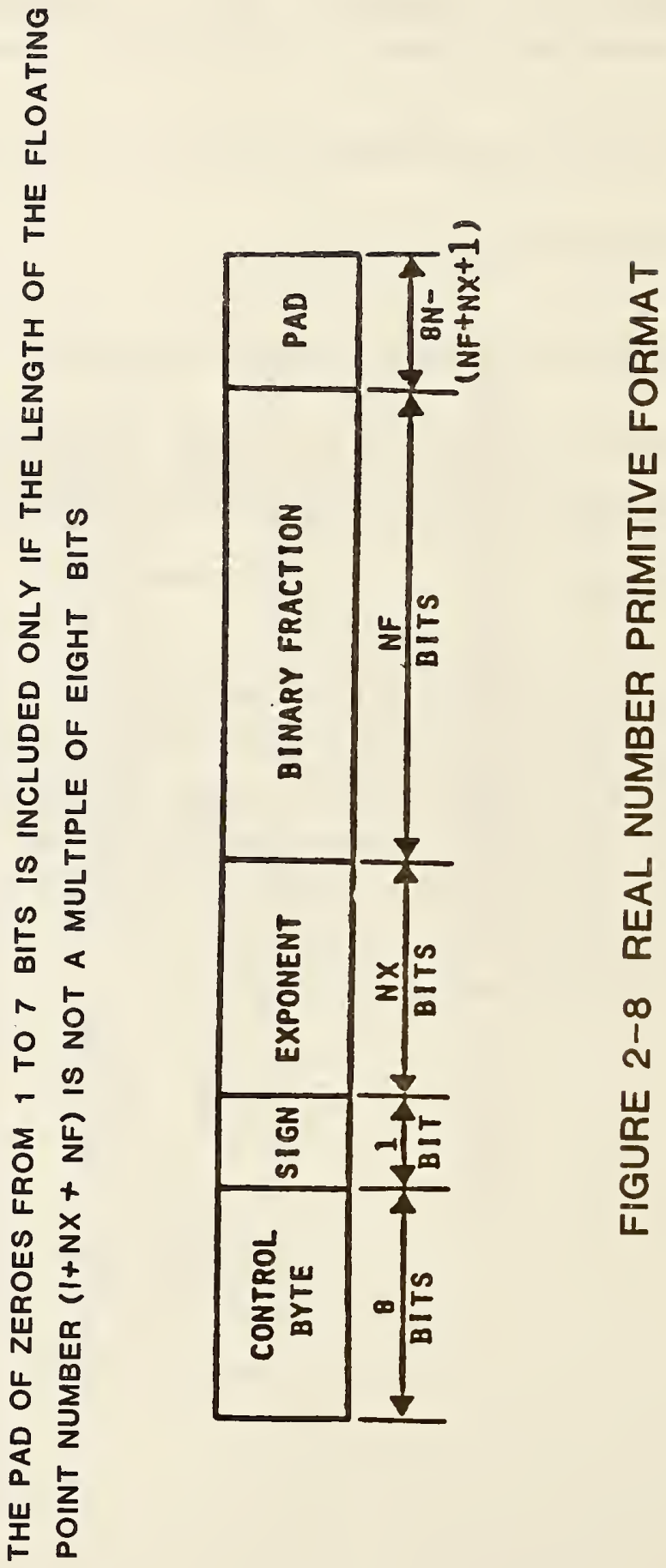


The structure of the string constant is shown in Figure 2-9.

\subsubsection{Pointers.}

The structure of a pointer shall be a 32 bit integer. The pointer shall contain the relative byte position of the entity byte count of the DE or PD entity to which it is pointing. A pointer to the first DE entity will have a value of 1 . A pointer to the second $D E$ entity will have a value equal to the number of bytes of the first DE entity plus one. A pointer to the first $P D$ entity will have a value of 1 . Pointers with values of zero or negative are not actual pointers but may have a default meaning depending upon the context. For example, a defining matrix value of zero would imply that the identity rotation matrix and zero translation vector are used. This case might also be handled by using the control byte, instead, to indicate a default value.

2.4.1.5 Language Primitives. Language primitives are the string constants of the MACRO definition entity which, in the ASCII Form, are not preceded by $\mathrm{nH}$ and are terminated with a record delimiter. In Binary Form the format of language primitives will be identical to string constants. Each language primitive (MACRO statement) will be an individual string constant.

2.4.2 File Structure. The general file structure is as shown in Figure 2-10 and is comprised of the following six sections:

$\begin{array}{ll}\text { - } & \text { Binary information section } \\ \text { - } & \text { Start section } \\ \text { o } & \text { Global section } \\ \text { - } & \text { Directory entry section } \\ \text { - } & \text { Parameter Data section } \\ \text { - } & \text { Terminate section }\end{array}$

Following each section is zero, one or many 8-bit null padding characters. These characters do not belong to the section and have no meaning. They are provided to assist the creator of a file with 


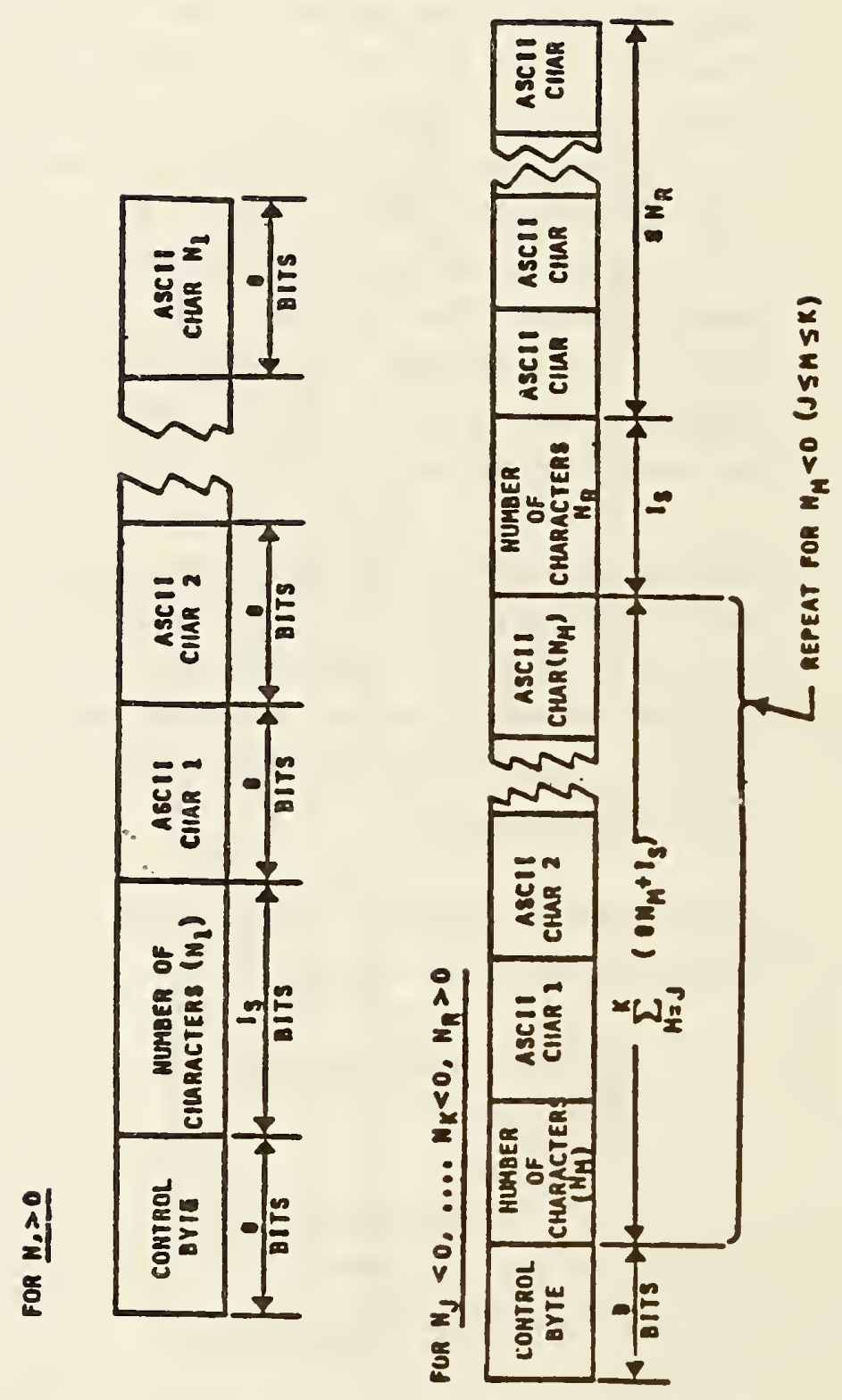

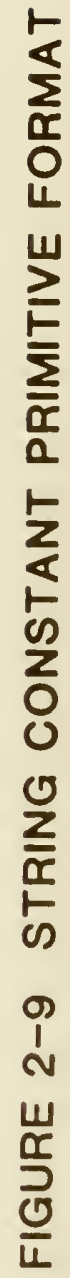




\begin{tabular}{|c|}
\hline BINARY INFORMATION SECTION \\
\hline START SECTION \\
\hline GLOBAL SECTION \\
DIRECTORY ENTRY SECTION \\
PARAMETER SECTION : \\
\hline TERMINATE SECTION
\end{tabular}

FIGURE 2-10 BINARY GENERAL FILE STRUCTURE 
physical system limitations such as word or sector boundaries.

Following the terminate section of the file shall be zero, one, or many null padding characters followed by an 8-bit end of information designator, the ASCII letter E. Any information following the letter E shall be ignored.

2.4.2.1 Binary Information Section. The format of the binary information section is as shown in Figure 2-11. It is comprised of the following data items, all of which are integers unless otherwise specified.

- Binary information section identifier consisting of the ASCII letter $B$.

- Binary information section byte count. This byte count excludes the 5 bytes required for the section identifier and section byte count. This byte count also excludes any null padding characters. The value of this byte count will be 75 .

- Length $\mathrm{i}_{s}$ of single length integer primitives.

- Length $i_{d}$ of double length integer primitives.

- Length $\mathrm{NX}_{\mathrm{S}}$ of exponent of single precision real primitives.

- Length $\mathrm{NF}_{s}$ of binary fraction of single precision real primitives.

- Length $\mathrm{NX} \mathrm{d}_{d}$ of exponent of double precision real primitives.

- Length $\mathrm{NF}_{\mathrm{d}}$ of binary fraction of double precision real primitives.

- ASCII letter B.

- Binary information section displacement. This is the byte count of the total length of the binary information section including all null padding characters. This length is the actual length from the initial B of the binary information section up to but not including the $S$ of the start section.

- ASCII letter S.

- Start section displacement. This is the byte count of the total length of the start section including all control bytes and null padding characters. This length is the actual length from the initial $S$ of the start section up to but not including the $G$ of the 


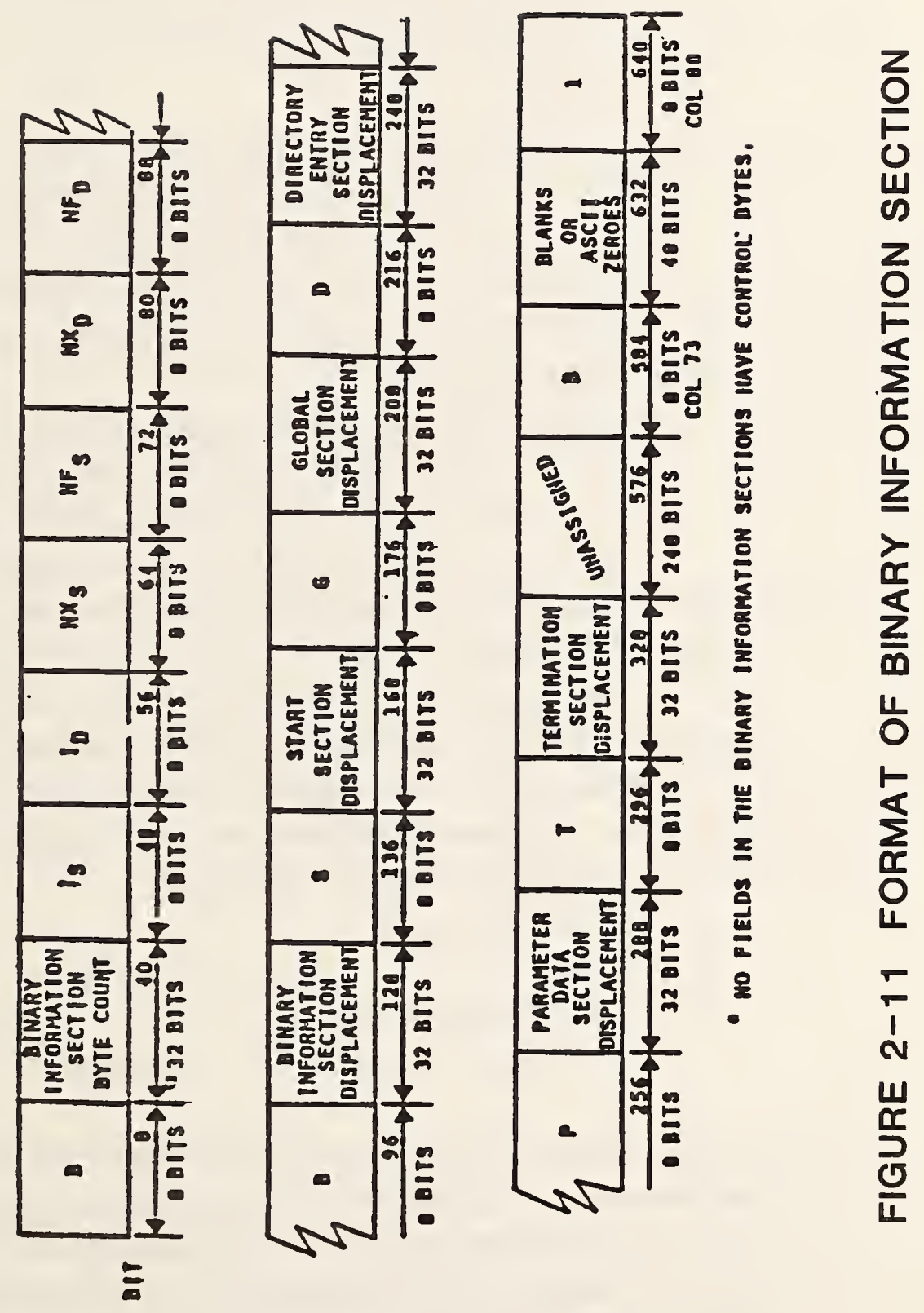


global section.

0 ASCII letter G.

- Global section displacement. This is the byte count of the total length of the global section including all control bytes and null padding characters. This length is the actual length from the initial $G$ of the global section up to but not including the $D$ of the directory entry section.

- ASCII letter D.

- Directory entry section displacement. This is the byte count of the total length of the descriptive entity section including all control bytes and null padding characters. The length is the actual length from the initial $D$ of the directory entry section up to but not including the $\mathrm{P}$ of the parameter data section.

0 ASCII letter P.

- Parameter data section displacement. This is the byte count of the total length of the parameter data section including all control bytes and null padding characters. This length is the actual length from the initial $P$ of the parameter data section up to but not including the $\mathrm{T}$ of the terminate section.

- ASCII letter T.

- Terminate section displacement. This is the byte count of the total length of the terminate section including all null padding characters. This length is the actual length from the initial $T$ of the terminate section up to but not including the letter $\mathrm{E}$ of the end of information designator.

- 31 unassigned bytes.

- ASCII letter B.

- 6 ASCII blanks or zeroes.

- ASCII character 1 .

No control bytes are applied to this section. Thus the characters in the equivalent of columns 73 through 80 of the binary information section are similar in format to the section identification of the ASCII Form and can be used to determine if a file is ASCII or binary. If the file contains an $\mathrm{S}$ in column 73 of its first 80 bytes, it is ASCII (or C if compressed ASCII). If it contains a $B$, it is binary. 
2.4.2.2 Start Section. The format of the start section is as shown in Figure 2-12. It is comprised of the following data items:

- A start section identifier consisting of the ASCII letter S

- Byte count for the start section. The byte count excludes the 5 bytes required for the start section identifier and section byte count. This byte count also excludes any null padding characters.

- One or more language or text primitives which are logically equivalent to columns 1 through 72 of the ASCII Form. There is no required physical correspondence between the ASCII Form and language/text primitives. One language/text primitive may contain the equivalent of several complete or partial ASCII records. Carriage return characters may be embedded in the language/text primitives. Control bytes only apply to the language and text primitives. No control bytes precede the section identifier and byte count.

2.4.2.3 Global Section. The format of the global section is as shown in Figure 213. The global section is comprised of the following data items:
- Global section identifier consisting of the ASCII letter G
- Global section byte count. This byte count excludes the 5 bytes required for the global section identifier and the section byte count. This byte count also excludes any null padding charac- ters.
- 24 global parameters.

Control bytes apply only to the 24 global parameters.

The global parameters have the same sequence and meaning as the ASCII Form global parameters with the exception that global parameters 1 (parameter delimiter character), 2 (record delimiter), 7 (number of bits for integer representation), 8 (single precision magnitude), 9 (single precision significance), 10 (double precision magnitude), and 11 (double precision significance) shall be ignored in Binary Form. The binary information section shall supersede these global parameters. 


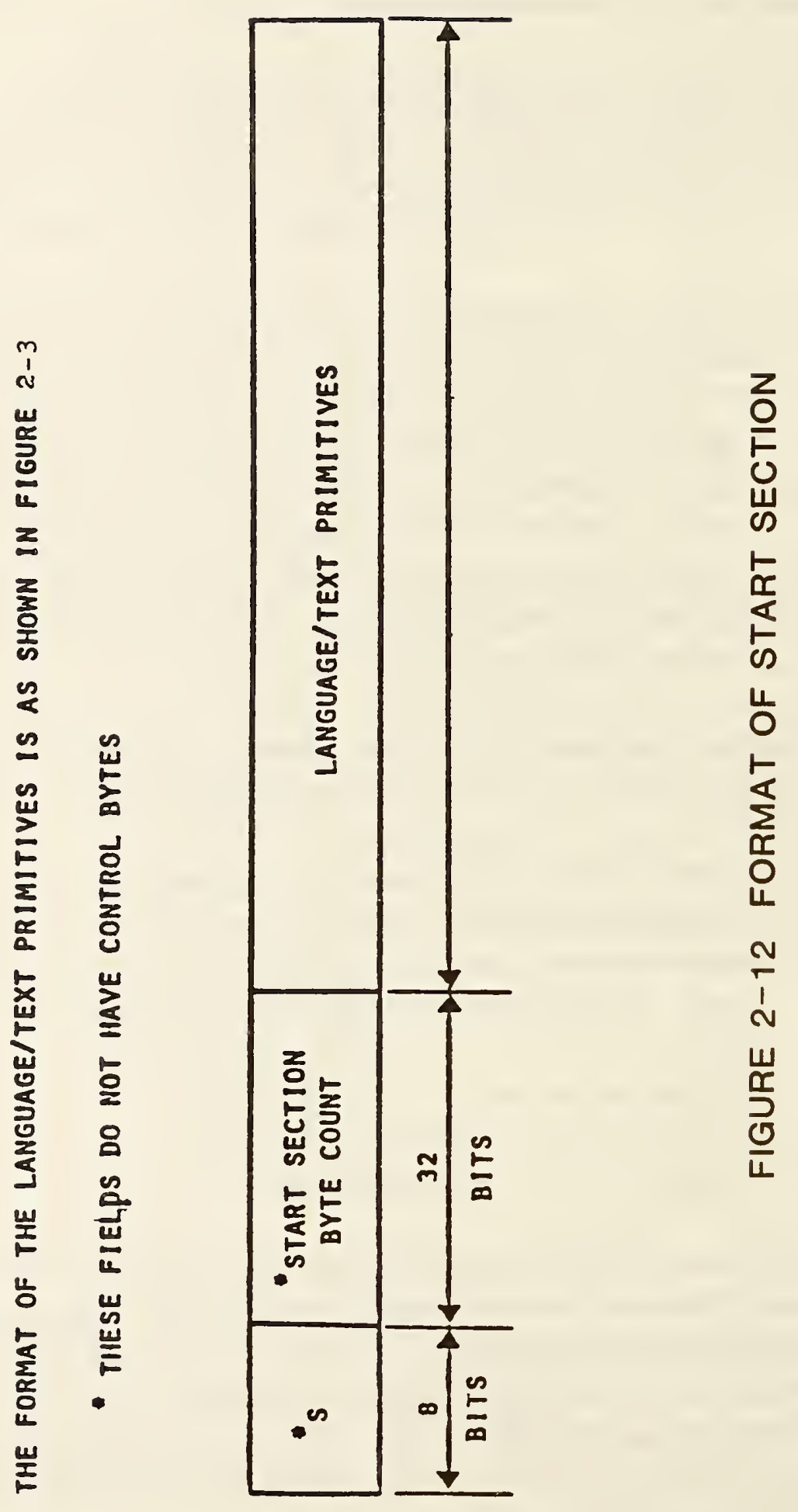




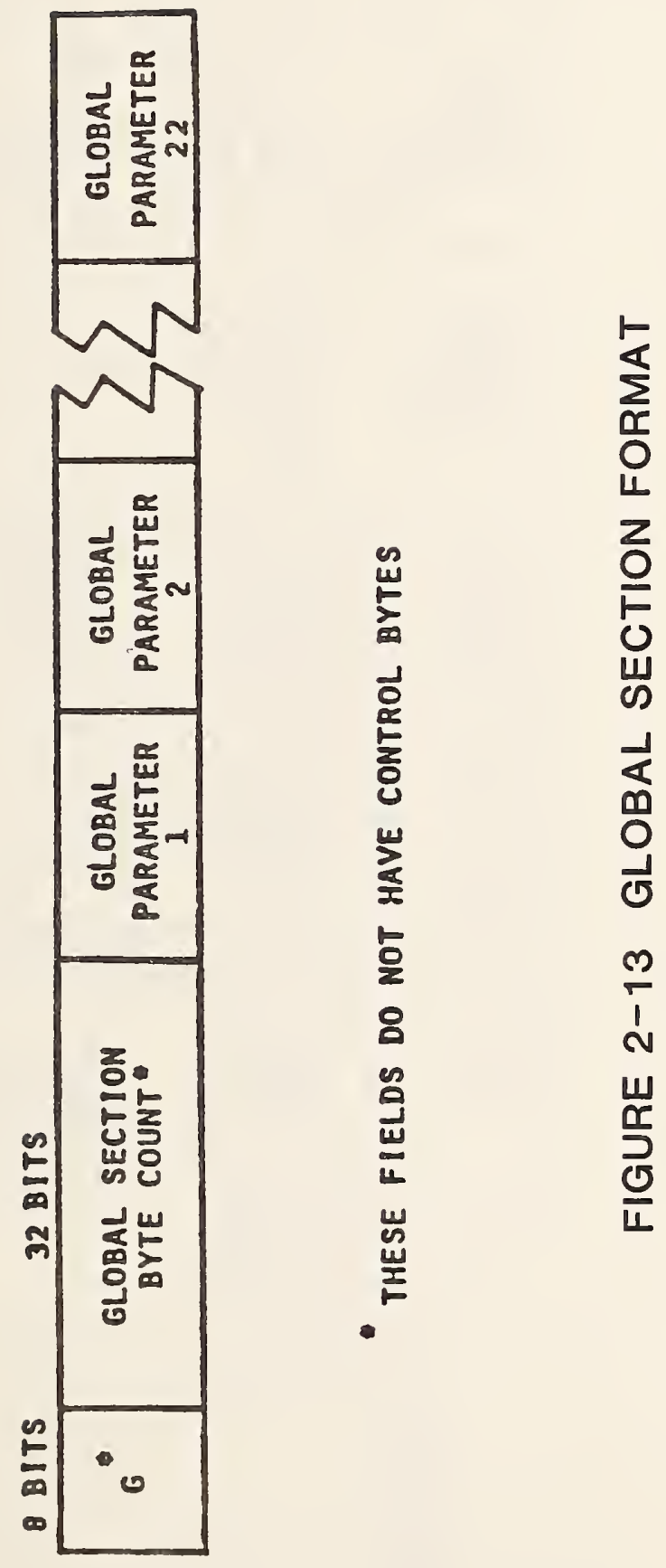


2.4.2.4 Directory Entry Section. The format of the directory entry section is as shown in Figure 2-14. The directory entry section is comprised of the following data items:

- Directory entry section identifier consisting of the ASCII letter D

- Directory entry section byte count. This byte count excludes the 5 bytes required for the section identifier and section byte count. This byte count also excludes any null padding characters.

o For each directory entry, the following 17 data fields are present:

- entity byte count, which is the length in bytes, including control bytes, of the subsequent 16 data fields.

- entity type

- parameter data pointer

- structure

- line font number or pointer

- level number or pointer

- view pointer

- $\quad$ transformation matrix pointer

- label display associativity pointer

- $\quad$ status number

- line weight

- color number or pointer

- form number

- $\quad$ reserved field 1

- $\quad$ reserved field 2

- entity label

- $\quad$ entity subscript 


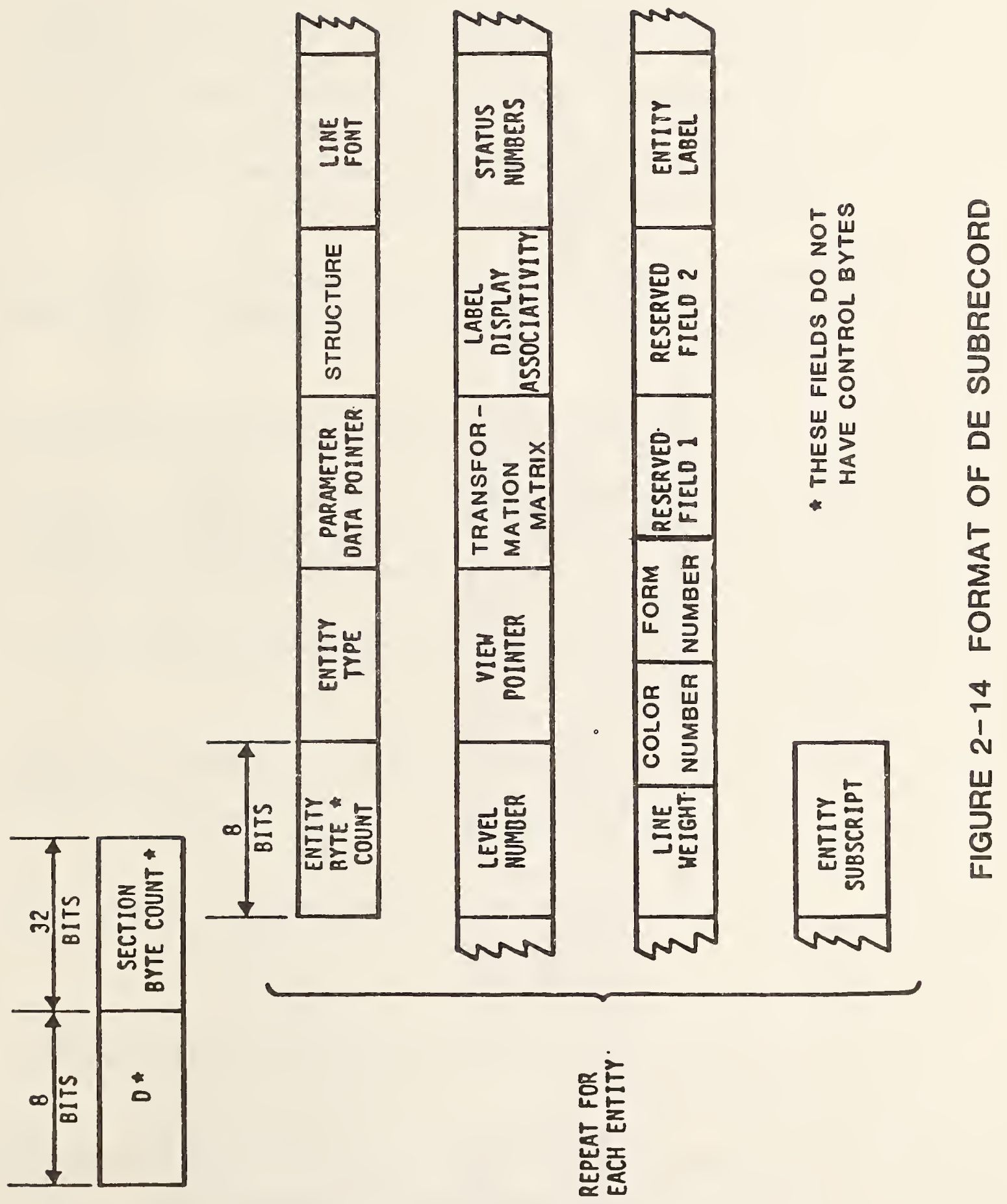


Control bytes apply only to the last 16 data fields.

The directory entry data fields, except for the entity byte count, are identical to and have the same sequence as fields in the ASCII Form. Within a single file, the length of DE record for each entity (in bytes) shall be consistent. If in the future additional fields are required, it is preferable to increase the number of fields for each directory entry and add any new fields subsequent to existing fields.

2.4.2.5 Parameter Section. The format of the parameter data section is as shown in Figure 2-15. The parameter data section is comprised of the following data iterns:

- Parameter data section identifier consisting of the ASCII letter $\mathrm{P}$

- Parameter data section byte count. This byte count excludes the 5 bytes required for the section identifier and section byte count. This byte count also excludes any null padding characters.

- For each parameter data entity, the following data fields are required:

- entity byte count, which is composed of the lengths, including control bytes, of all subsequent data fields for this entity.

- entity type

- directory entry pointer (relative to directory entry section)

- parameter data

Control bytes apply only to the entity type, directory entry pointer and parameter data fields.

The parameter data entity fields, except for the entity byte count, are identical to and have the same sequence as the the ASCII Form. 

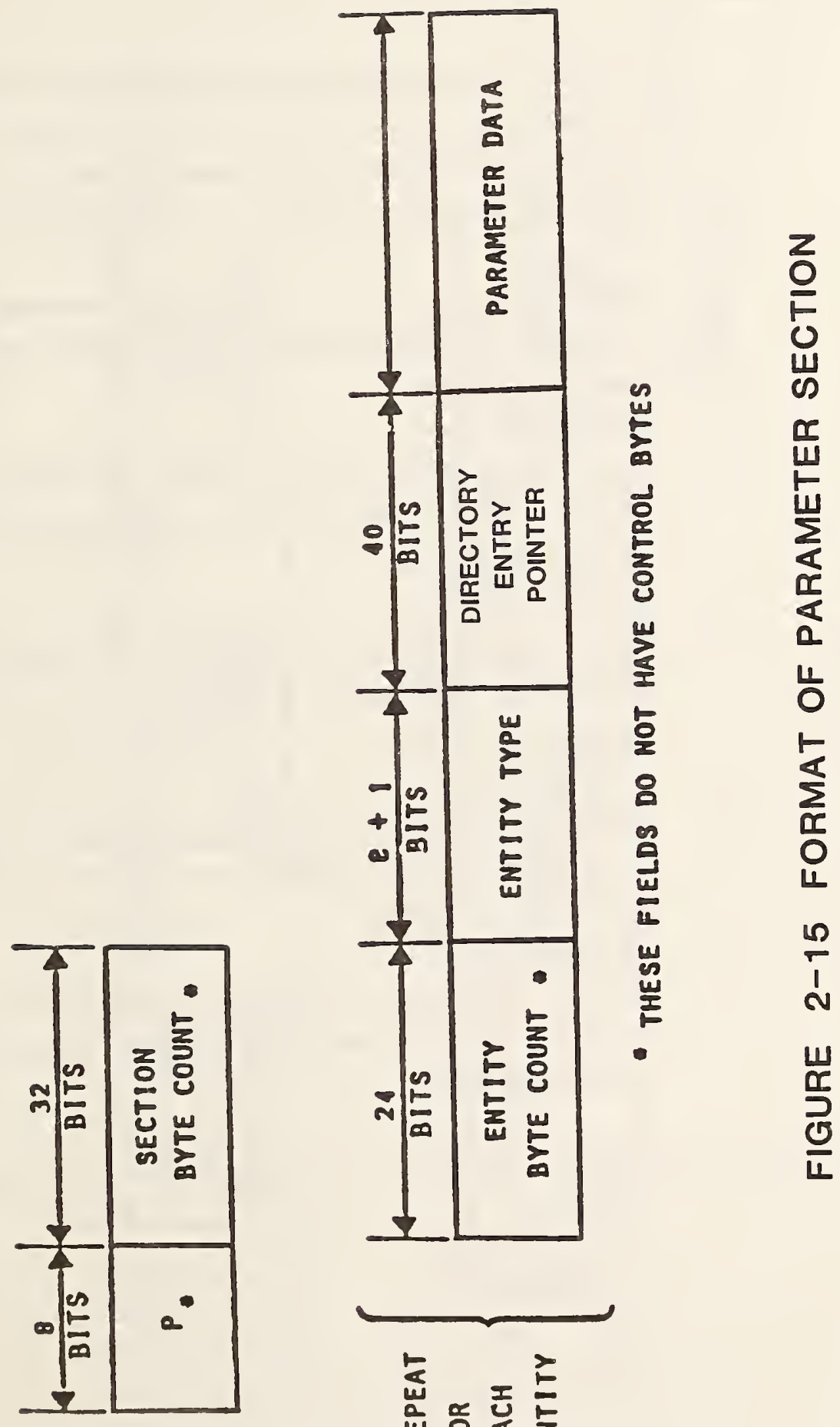

茪里 
2.4.2.6 Terminate Section. The format of the terminate section is as shown in Figure 2-16. The terminate section is comprised of the following data items:

- Terminate section identifier consisting of the ASCII letter T

- Terminate section byte count. This byte count excludes the 5 bytes required for the section identifier and section by te count. This byte count also excludes any null padding characters.

- ASCII letter B

- Binary identification section byte count, including the section identifier, and section byte count, but excluding any null padding characters.

- ASCII letter S

- Start section byte count, including the section identifier, section byte count, and all control bytes but excluding any null padding characters.

- ASCII letter G

- Global section byte count, including the section identifier, section byte count, and all control bytes but excluding any null padding characters.

- ASCII letter D

- Directory entry section byte count, including the section identifier, section byte count, and all control bytes but excluding any null padding characters.

- ASCII letter $P$

- Parameter data section byte count, including the section identifier, section byte count, and all control bytes but excluding any null padding characters.

The terminate section has no control bytes applied to any of its data. 


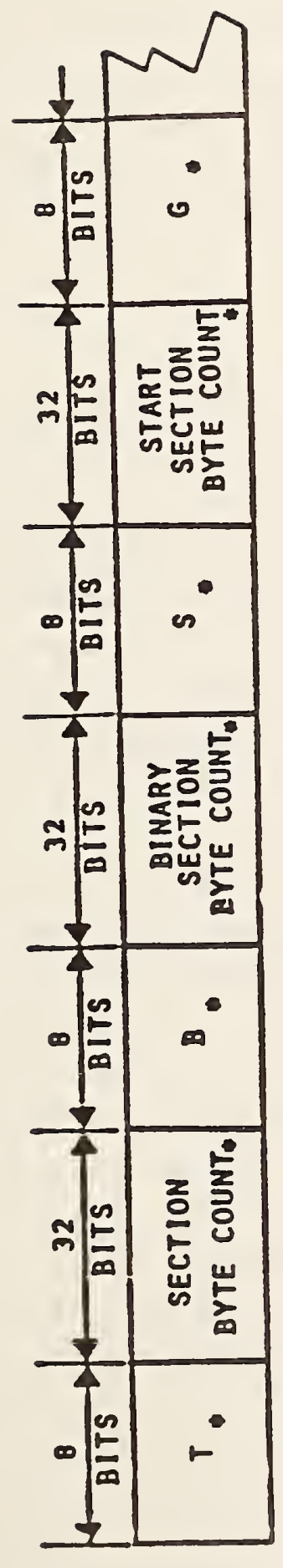

$\sum$

울

8

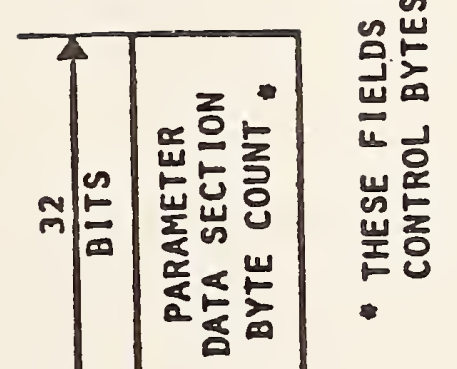

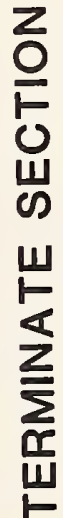

$\frac{1}{0}$

$\frac{1}{0}$

$\frac{0}{1}$

$\frac{4}{\frac{5}{4}}$ 
THIS PAGE LEFT BLANK 
Individual entities of the file structure are described in Sections 3 and 4 of this document. Complete files of these entities must be structured uniformly to convey specialized information between applications. Some of the ways in which those entities tie together to form relationships are described in this Section.

\subsubsection{Subfigures}

The concept of subfigures is supported by defining a collection of entities once and then instancing that collection multiple times in the model at various locations, orientations, and scales. The entity use flag defines how the entity will be used in the model. The structure consists of a Subfigure Definition Entity (type 308) which points to a collection of entities (see Figure 2-17). One or more subfigure instance entities may point to the subfigure definition entity and specify an $X, Y, Z$ location and scale factor. Rotation of the instance is specified by having the subfigure instance point to a transformation matrix entity.

Two types of subfigure definitions and four types of subfigure instances are defined. The Subfigure Definition Entity can be instanced: (1) individually by the Singular Subfigure Instance Entity, (2) as a rectangular grid of instances by the Rectangular Array Subfigure Instance Entity or, (3) as a circularly located set of instances by the Circular Array Subfigure Instance Entity. Items which have locations specified for logical connections (represented by one or more Connect Point Entities) in their subfigure definitions, and thus instances, or which require independent scale factors in the $X, Y$, and $Z$ axes shall be defined by a Network Subfigure Definition Entity and (4) instanced by the Network Subfigure Instance Entity (see Section 2.5.2 and Figure 218). 
DATA FORM - Specific File

structures

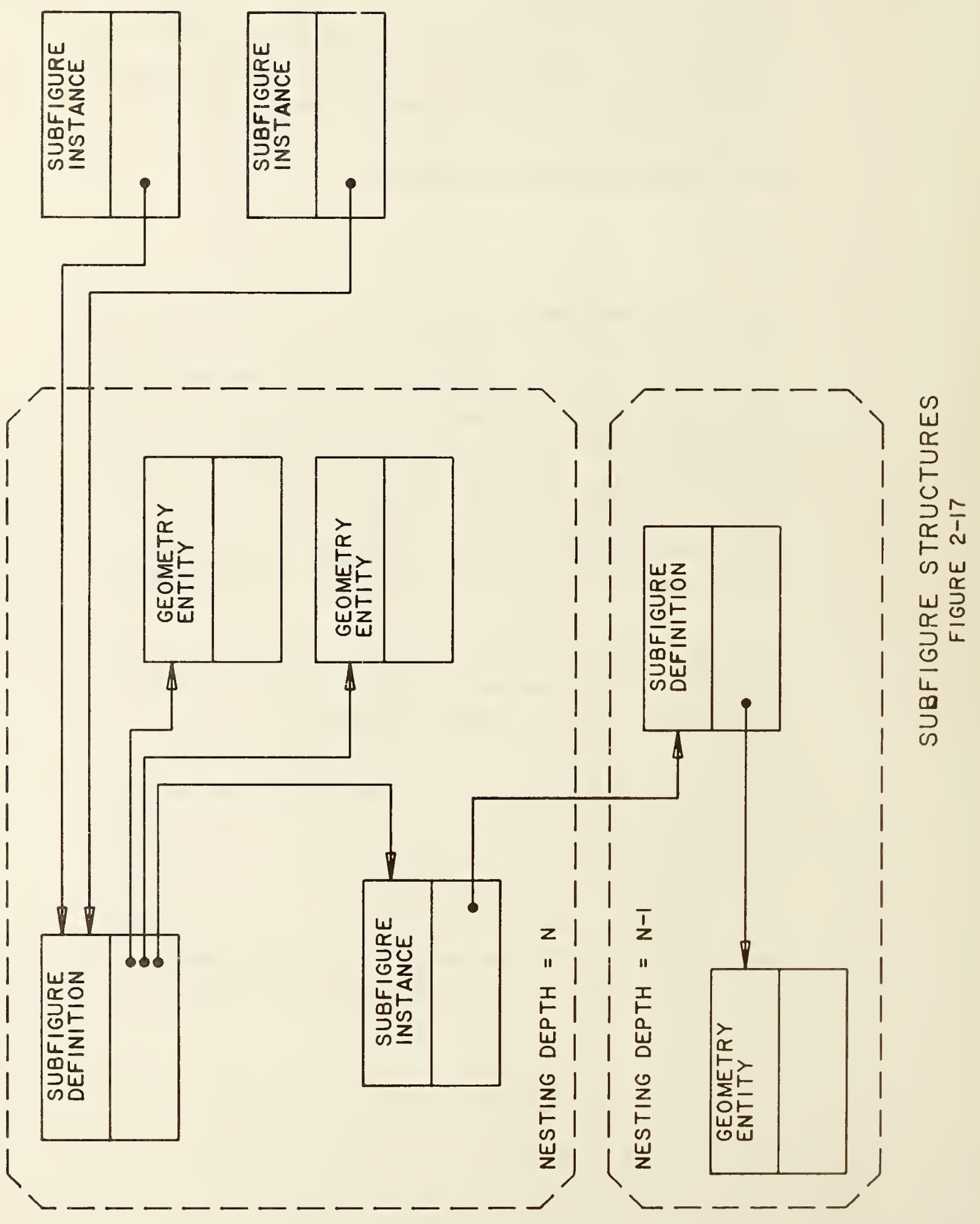




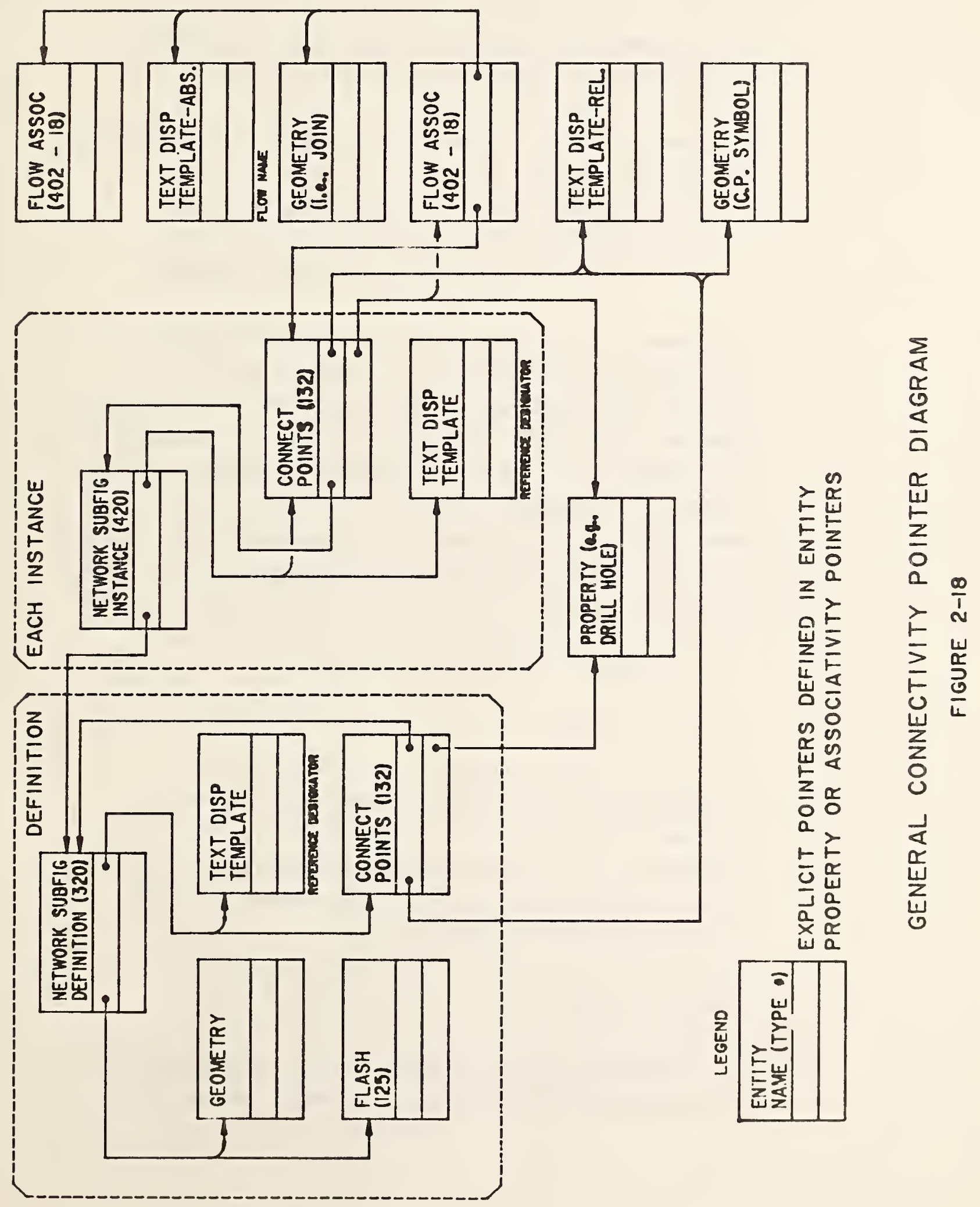


The following file structure shall be used to define logical (and the location for physical) connections between objects.

A formed connection between two or more objects requires the data to represent the following:

1. the exact location of each connection point

2. the flow path formed and its identification (if any)

3. the physical connection between the objects (if any)

These objects may include electrical or mechanical components such as transistors, pipes and valves, and air conditioning ductwork. Each connection formed defines a flow path between the objects, allowing a fluid (electricity, water, or air) to flow from one object to another. The Network Subfigure (Definition and Instance) entities are used to represent the objects to be connected. The Connect Point Entity is used to represent the exact location of connection. The term "link" will refer to the logical representation of the flow path (signal) formed, and "flow-name" will refer to the flow path identifier. The term "join" will refer to the file entity or entities which represent the physical connection (geometries between the items).

\subsubsection{Connectivity Entities}

The entities used to impleınent connectivity include the Network Subfigure Definition (type 320) and Network Subfigure Instance (type 420) entities, the Flow Associativity (type 402 form 18), the Connect Point Entity (type 132), and the Text Display Template (type 312; absolute $=$ form 0 , incrernental $=$ form 1 ).

\subsubsection{Entity Relationships}

A flow path (signal) may be formed between items by a link which references the items' connect points (entities) to be related. This 
creates an associativity among the connect points and thus the entities connected. The flow-name may be used to uniquely identify the particular signal formed. The join may be used to provide a graphical representation of the flow path. In electrical applications the join will be represented by geometric entities such as line, arc, subfigure, copious data, etc. In a piping application, an example of a join represented might be the section of pipe between a valve and a tank. The logical constructs (link and flow-name) shall be implemented by the Flow Associativity Entity which in turn identifies (by pointer) the entities which form the join.

In electrical applications, for example, the items to be connected are components (i.e., resistor, 16-pin dual in-line package, etc.), or integrated circuit cells, represented and instanced by Network Subfigures. Each pin (or signal port) is a potential connection point in a flow path, thus each Network Subfigure has a Connect Point for each pin (or por $t$ ). When such a subfigure is instanced, its connect points must also be instanced. An instanced Connect Point, when added to a flow path is different from its definition which shall not be a member of any flow path. See Figure 2-18 for the basic entity relationships. For more information see Appendix $B$.

\subsubsection{Information Display}

The Network Subfigures, representing electrical components for example, of ten contain text describing the component and its pins. The Text Display Template allows text, embedded in another entity to be displayed without redundant specification of the text string. The Text Display Template may be used to display reference designators and pin numbers. The absolute form, within a network subfigure, is recommended for the reference designator text. Each instance of the subfigure need only supply the text string. The pin number can be represented in the incremental form. All the pin numbers on a given side of a package outline having the same $X, Y$, and $Z$ of fsets relative to the pin whose number is to be displayed may use the same Text Display Template definition. 


\subsubsection{Additional Considerations}

The situation is exactly the same for both logical and physical product representations. The only differences arise in the subfigure and join entities used. One file may contain both schematic and physical representations of a product. The Flow Associativity Entity contains a type flag to indicate the connection type (logical or physical). In this case, one Flow Associativity would represent the logical connection and a second the physical connection. The two associativities would be related by the pointers provided in the Flow Associativity.

\subsubsection{MACROs}

A MACRO capability is provided for defining new entities in terms of other entities. See Section 4.3.6. Two specific applications for the MACRO capability are parametric designs and standard parts. The structure consists of MACRO definition entities which are pointed to by MACRO instance entities as shown in Figure 2-19. Each MACRO definition is assigned a unique MACRO entity type number. MACRO instance entities use this number as their entity type number. MACRO instance entities also use the third field of their directory entry to point to the directory entry of the MACRO definition (or to an external reference entity which points to a library file containing the MACRO definition).

\subsubsection{External Reference Linkage}

Linkages between entities can occur not only within a file, but also between entities in different files. Two entities shall be used in a referencing file to establish this linkage: the External Reference Entity (Type 416) which provides the actual linkage to the referenced file, and the External Reference File List Property Entity (Type 406 Form 12) which provides a list of the names of all the files referenced. Fur ther, only directly referenced files shall be in this property's parameter list. Each file name listed in the parameter data of this property must match the name in the fourth global parameter of a referenced file. 
DIRECTORY ENTRY SECTION

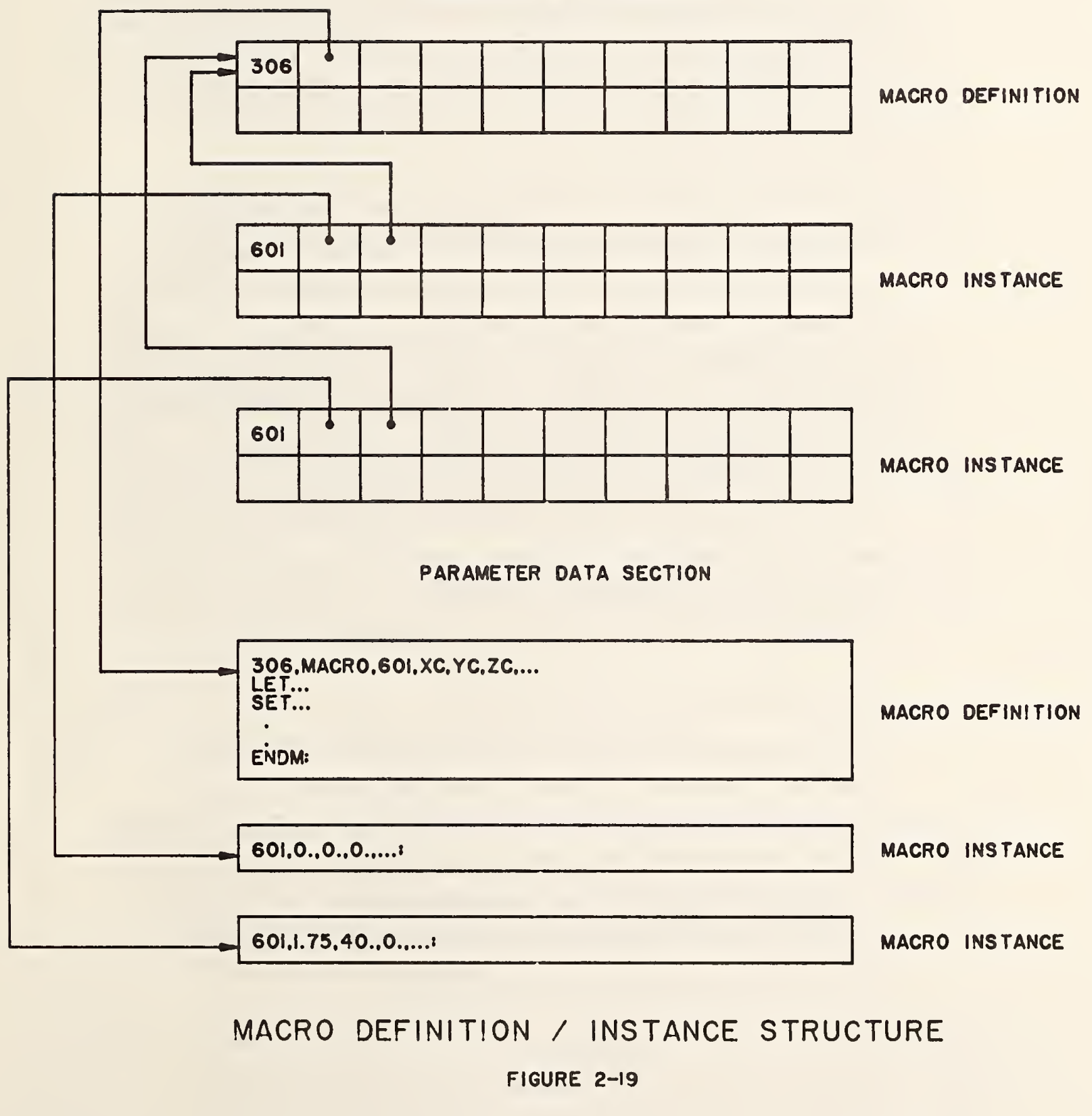

306.MACRO.601,XC.YC.ZC....

SET...

ENDN

$601.0 .0 .0 .0 . .$.

MACRO INSTANCE

MACRO INSTANCE 
An External Reference File Index associativity (type 402 form 12) is required in the referenced file when the type 416 Form 0 or 2 is used (i.e., more than one referenced entity in the referenced file).

This associativity provides a directory to the referenced entities within its file, and both relate a symbolic name to the directory entry of an entity within the file (see Figure 2-20). All symbolic names used within a set of files linked by references must be unique. Definitions may be nested, and a symbolic name used need be unique only on the nesting level on which it is used.

Because of the intricacy of the linkages, an example follows (refer to Figure 2-20). Consider a file containing a subfigure instance entity (type 408). The first item in its parameter data record is a pointer to the subfigure definition entry in the DE section of the file. In the case that the subfigure definition entity (type 308 ) is to be contained in a library file, this first parameter is a pointer to an external reference entity (type 416). The external reference entity so referenced will have in its parameter data record the name of the file which is to contain the definition and the symbolic name of the definition itself. The file name is the four th global parameter in the referenced file. The symbolic name is a string which identifies the appropriate referenced definition.

In the case of a library file which contains several definitions, each of which are expected to be referenced by other files, the External Reference Associativity (type 402 form 12) provides a "table of contents" of the available definitions in the file. The parameter data record of this associativity contains pairs of data: the symbolic name associated with the definition (the same one used in the Type 416 entity's parameter data record), and a pointer to the directory entry record which contains the desired definition. 
In the case that the entire external file is to be included (i.e., a supersubfigure), Form 1 of the Type 416 entity is used which does not contain a symbolic name in the parameter data record. In a similar manner, the referenced file does not contain an associativity Type 402 Form 2 or 12 entity; it is unneeded since the entire file is to be used.

In either case, the External Reference File Index Property (type 406 Form 12) will be found in the referencing file. The parameter data record contains a simple list of the file names of the various external files referenced by this file. Once again, the file name used is that in the fourth global parameter of the referenced file. Note that this list contains only those file names that are directly referenced; it gives no information about files which may be referenced in turn by those files used by this file.

A limitation of external referencing is that the backpointers (in the "backpointers to associativities" addition to an entity's parameters) cannot be used. If a pointer is required in each direction, separate external reference mechanisms must exist in each file (e.g., the double linkage between files A and B in Figure 2-20).

A preprocessor implementor should use the external logical reference mechanism with care because of the burden placed on the postprocessor.

2.5.5 Drawings and Views

This Specification provides a mechanism for associating models and drawings so that there is consistency between them. The mechanism is based on the existing practices of some CAD/CAM graphic systems to define the views of a part on a drawing in terms of a single 3dimensional (3-D) model.

The Drawing Entity (type 404) specifies a drawing of a given size within a special drawing space coordinate system. This entity can refer to one or more View entities (type 410) which will specify the projection from 


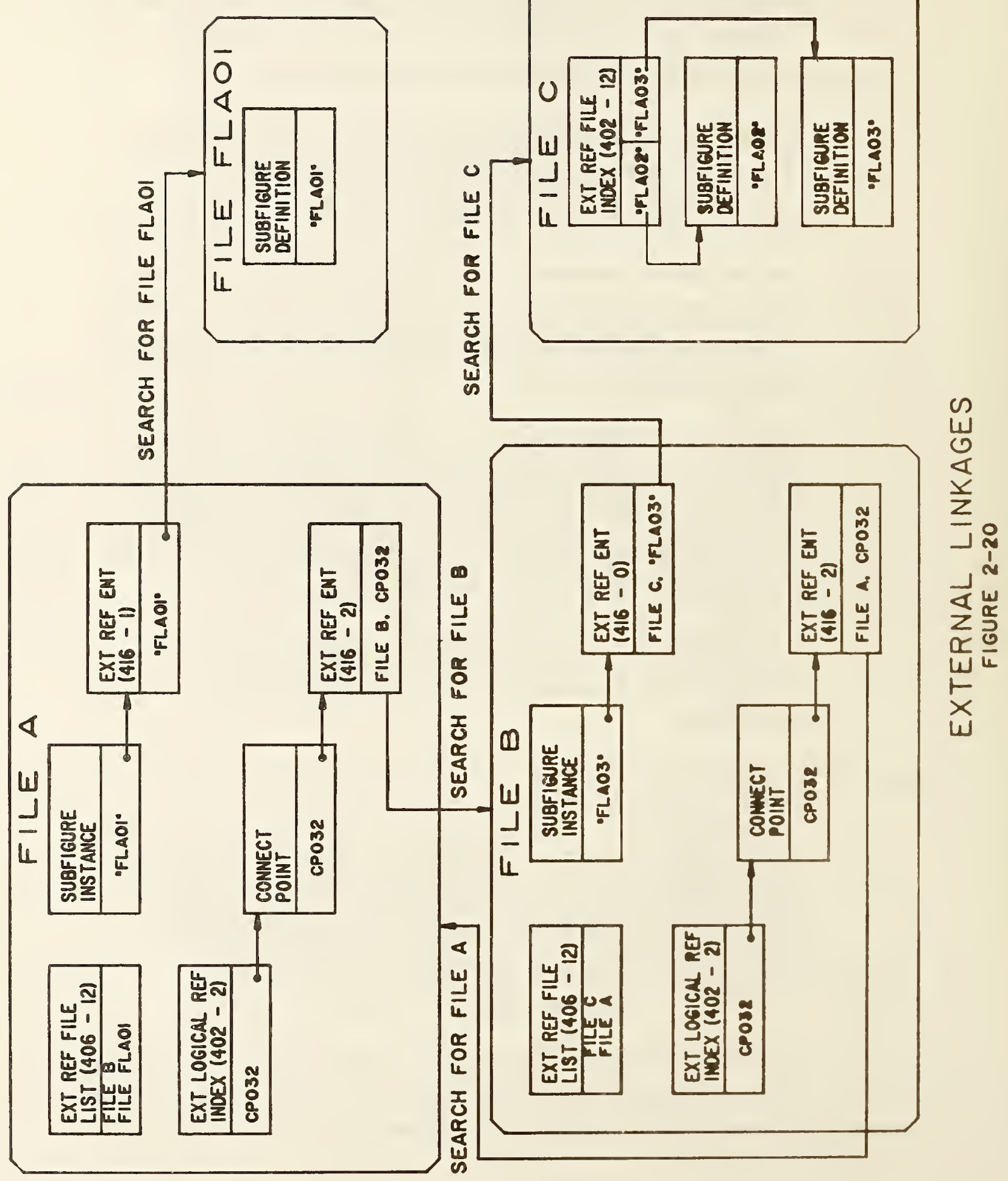


3-D model space to the 2-D drawing space. Annotation entities such as dimensioning can be defined directly in the drawing coordinate system, or can be defined in the 3-D model space and then be included in individual views. More than one drawing entity may be included in a file.

In addition to being used in conjunction with the Drawing entity, the view-specific display of parts of the model can be used to communicate hidden lines, phantom lines, etc.

Graphic systems which do not have the ability to define drawing and views of models in this manner are not required to preprocess this construct into a file, but all systems with postprocessors must be able to process the drawing and view entities in received files.

This Section defines the entities and their relationships (pointers) required to support the Finite Element Modeling (FEM) application and to display results of analysis on those systems which support finite element analysis postprocessing.

The entities available for exchanging FEM data are illustrated in Figures 2-21 and 2-22. The left side of Figure 2-21 illustrates the relationships between the entities that define the model's parametric attributes. The right side illustrates the addition of the analysis results. Figure 2-22 illustrates the FEM entities used to define an example beam structure with accompanying material properties, a load, and a constraint. The entities defined in support of such analysis are the Element, Node, Load/Constraint, Tabular Data Property, and Nodal Displacement and Rotation.

Element (type 136) defines a finite element to be used in the finite element model. Several finite elements are defined in the Specification. Examples of an element are: BEAM, CTRIA, and DAMP. Specifically, the element entity specifies the topology type, number of 
DATA FORM - $\begin{gathered}\text { Specific File } \\ \text { Structures }\end{gathered}$

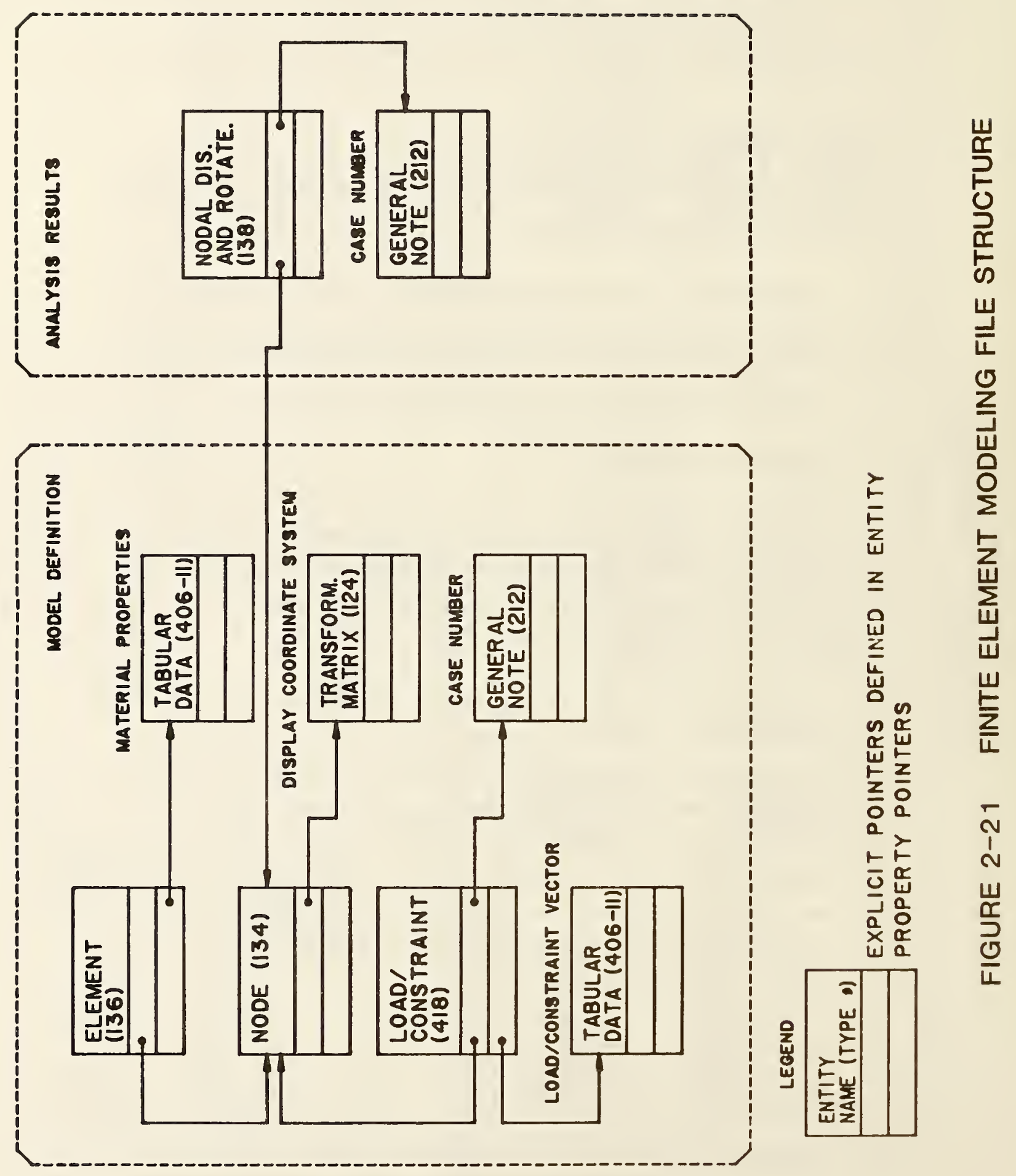



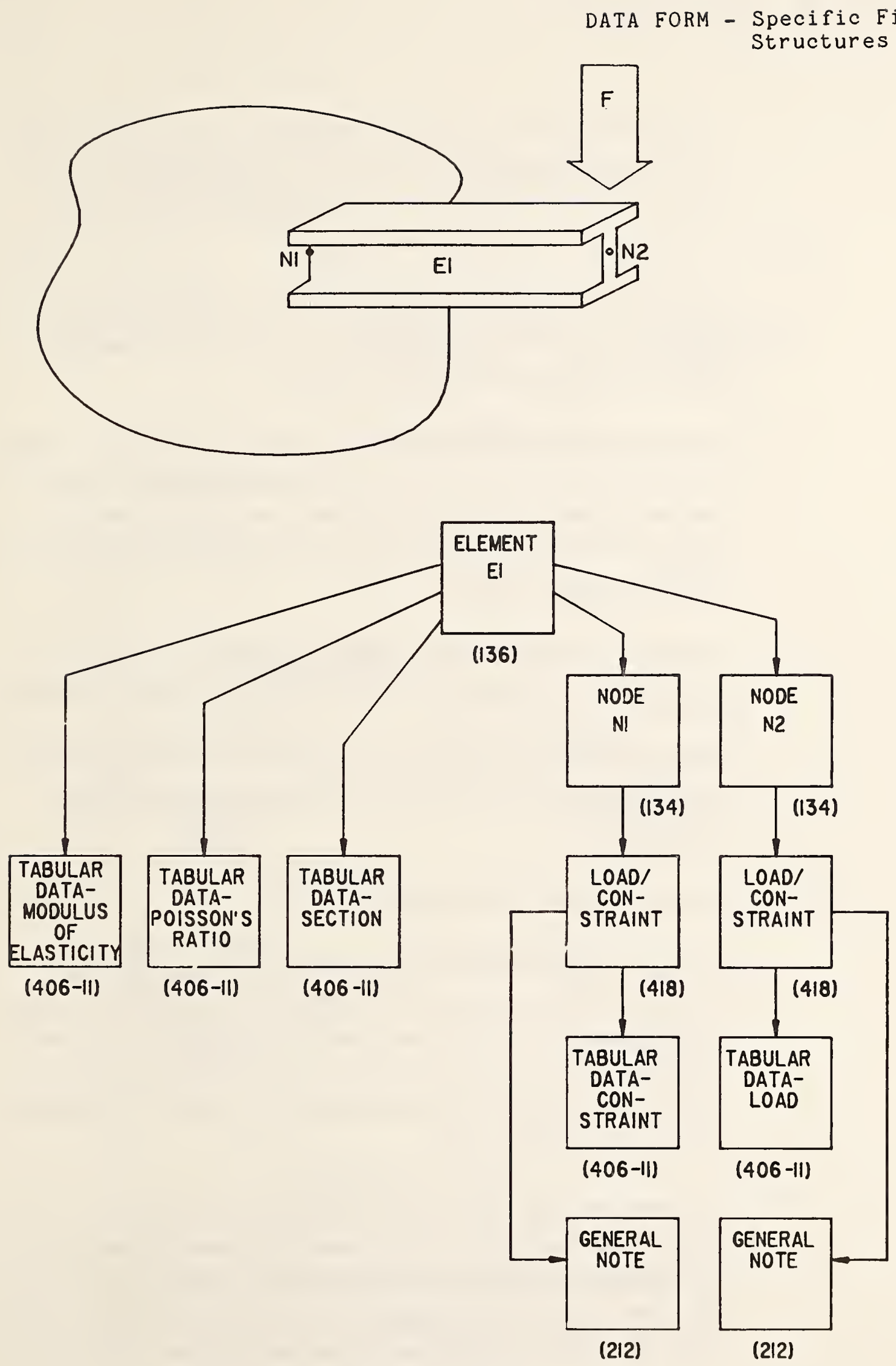

FINITE ELEMENT MODELING LOGICAL STRUCTURE

FIGURE 2-22 
nodes, and the element type name. Pointers locate the defining nodes and the material properties of the element. The connectivity of the nodes is implied in the order of the contained pointers and topology type.

Node (type 134) defines the grid points or nodes of the element. It contains the spatial values that define the node and a pointer to the coordinate system upon which it is defined.

Load/Constraint (type 418) is an entity that points to a node. It defines either a load or a constraint as applied to that node. It also contains a pointer to general note entities that define the load case. Property pointers point to the tabular data entity that contains the values of the load or constraint vector.

Tabular Data Property (type 406 form 11) contains the material property data of the elements and the load/constraint data as required.

Nodal Displacement and Rotation Entity (type 138) is used to contain the analysis results in the form of incremental variations in node position.

2.5.7 Multiple Transformation Entities

There are only two cases in which entities can be operated on by multiple transformation entities. The first is the explicit case in which an entity points to a transformation entity through its directory entry field 7 , and that transformation entity, in turn, points to an additional transformation entity through its directory entry field 7 . This structure is illustrated in Figure 2-23a.

The other case is an implicit one in which two entities are in a parent/child relationship, and each points to a transformation entity through its respective directory entry field 7. A parent/child relationship occurs when one entity (the parent) is pointing to another entity (the child). This structure is illustrated in Figure 2-23b. 


$$
\text { DATA FORM - } \begin{gathered}
\text { Specific File } \\
\text { Structures }
\end{gathered}
$$

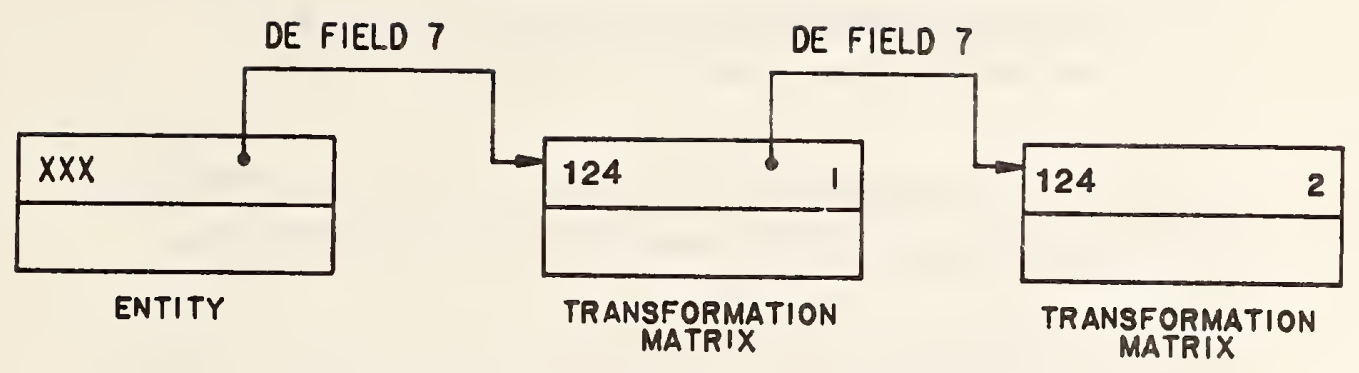

o) EXPLICIT CASE

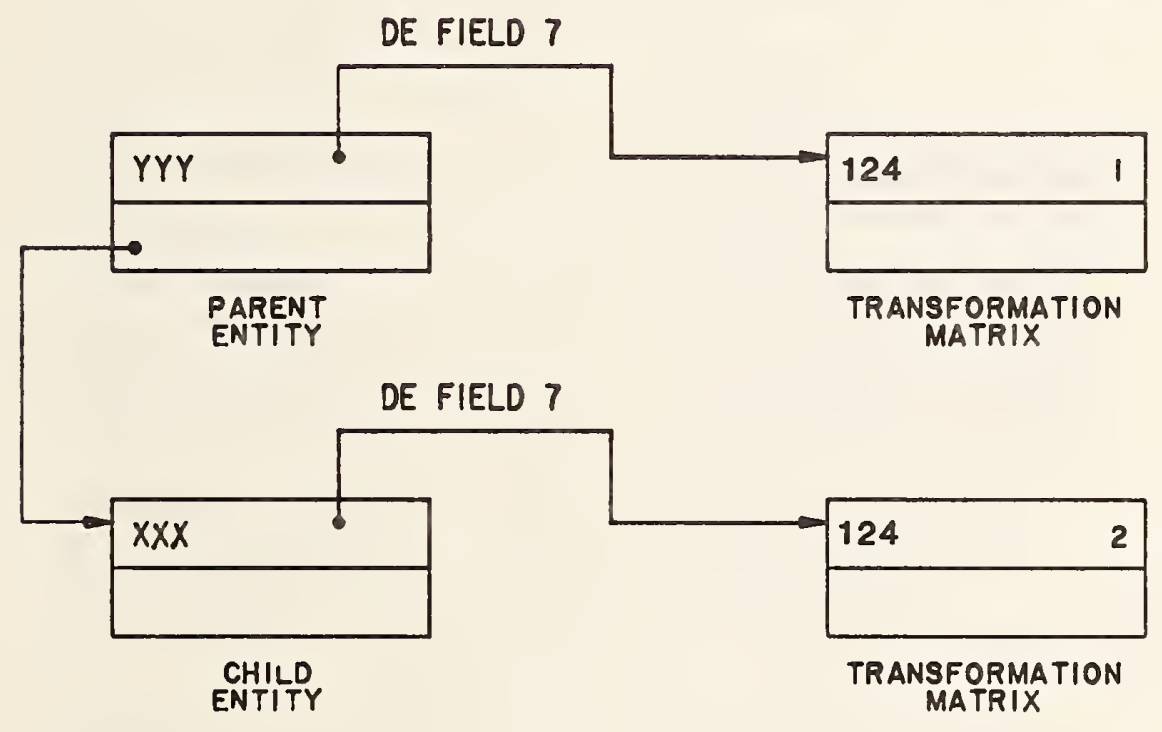

b) PARENT-CHILD CASE

MULTIPLE TRANSFORMATION CASES

FIGURE 2-23 
In the cases illustrated by Figure 2-23 the points represented by entity $X X X$ are operated upon by matrix 2 and from that point on are transformed like the points in entity YYY, using matrix 1.

A parent/child relationship between entities may also be created with a Single Parent Associativity instance entity (type 402, Form 9).

When the specific parent/child relationships shown in Table 2-4 occur the implicit relation rule shall apply. Each of the relationships in Table 2-4 ordinarily results in the subordinate entity switch of the child entity being set to 01 (physicaliy dependent). The exception is the case in which a preprocessor wishes to actually instance the child entity. In this case the child's subordinate entity switch is set to 02 (logically dependent), and the matrix pointed to by the parent has no effect on the location of the child (see Section 2.2.4.3.9.2). 
TABLE 2-4

PHYSICAL PARENT/CHILD RELATIONSHIPS

Parent

Composite Curve

Plane

Point

Ruled Surface

Flash

Surface of Revolution

Tabulated Cylinder

Offset Curve

Offset Surface

Trimmed Surface

Angular Dimension

Diameter Dimension

Flag Note

General Label

Linear Dimension

Ordinate Dimension

Point Dimension

Radius Dimension

General Symbol

Sectioned Area

Entity Label Display

Connect Point

Drawing

Subfigure Definition

Network Subfigure

Definition

Nodal Display and Rotation

any entity with entity use flag $=00$ or 01

\section{Child}

all constituents.

bounding curve

display symbol

rail curves

defining entity

axis, generatrix

directrix

base curve

surface

surface

all subordinate entities

all subordinate entities

all subordinate entities

all subordinate entities

all subordinate entities

all subordinate entities

all subordinate entities

all subordinate entities

all subordinate entities

all boundary curves

all leaders

display symbol, Text Display

Templates

all annotation entities

all associated entities

all associated entities,

Text Display Templates

and Connect Points

all General Notes and Nodes

all General Notes in text

pointer field 
THIS PAGE LEFT BLANK 
3.1 General

This section gives information concerning the geometry entity types available to be used in the entity-based product definition file. Descriptions of the various directory entry fields were given in Section 2.2.4.3. The meanings of these fields remain the same across all entities. In this section those entities making extended use of field 15 in the directory entry (Form Number) are indicated and the various options are listed. The parameter data record for each entity is also described in this section. The fields for this record vary from entity to entity.

\subsubsection{Coordinate Systems.}

This section introduces a model space concept and a definition space concept. Model space is three-dimensional Euclidean space, the space in which the "model" (or product) being represented resides. The model space $\mathrm{X}, \mathrm{Y}, \mathrm{Z}$ coordinate system is a right-handed Cartesian coordinate system. It is fixed relative to the model.

Definition space is also three-dimensional Euclidean space, but has its own right-handed Cartesian XT, YT, ZT coordinate system. In contrast to model space where a single fixed coordinate system exists, the definition space coordinate system may vary from entity to entity. The origin of a definition space coordinate system may be any point in model space, and the orientation may be arbitrary with respect to model space. It is assumed that the unit of length is always the same in both the model space and the definition space coordinate systems. 
The definition space concept allows the use of a temporary coordinate system in positioning certain geometric entities into model space. This concept plays a simplifying role that is most apparent in connection with those entities which can be contained within a single plane. Use of definition space entails initially describing an entity in definition space, and then converting this to a model space description. Thus, an or thogonal matrix and a translation vector are used to generate model space coordinates from definition space coordinates. The or thogonal matrix used for this purpose is called the defining matrix; both it and the translation vector are treated within the Transformation Matrix entity.

The value of the determinant of an or thogonal matrix is always plus or minus one. In case the determinant is one, there are two equivalent points of view that can be taken concerning how the geometric entity is related to model space from its definition space description. In order to simplify the discussion of these that follows, the translation vector is assumed to be the zero vector. This implies that the origin of the definition space coordinate system coincides with the origin in the model space coordinate system.

The first point of view imagines that the two coordinate systems are initially coincident (that is, $X$ axis to $X T$ axis, etc.) but that the $X T, Y T$, $Z T$ coordinate frame is free to rotate relative to the $X, Y, Z$ frame. The geometry entity is then considered to be defined relative to the $\mathrm{XT}, \mathrm{YT}$, $\mathrm{ZT}$ frame, and the defining matrix then rotates this frame, geometry included, so that the geometry entity is positioned as desired relative to the $X, Y, Z$ frame.

The second point of view imagines that the XT, YT, ZT frame is initially situated so that the geometry entity within definition space is positioned in the desired manner relative to model space. The defining matrix then leaves the geometry entity fixed, but rotates the XT, YT, ZT frame. At the completion of the rotation, the XT, YT, ZT frame becomes the $\mathrm{X}, \mathrm{Y}, \mathrm{Z}$ frame. The result is that the geometry entity is then positioned as desired relative to the $\mathrm{X}, \mathrm{Y}, \mathrm{Z}$ frame. 
It is to be emphasized that the discussion here pertains to a single defining matrix whose action in transforming coordinates can be viewed intuitively in two ways. Each point of view stresses the temporary nature of the XT, $\mathrm{YT}, \mathrm{ZT}$ system, insofar as what is ultimately of interest is the relationship of the geometry entity to the $X, Y, Z$ frame.

In a case when the geometry entity to be located within model space can be contained within a single plane, it can be seen that the definition space concept can be used in such a way that the geometry entity as initially described in definition space can be considered to lie in the XT, YT-plane (i.e., the plane $Z T=0$ ). From this, it is then convenient to also allow entities to be situated in definition space in any plane parallel to the XT, YT plane (i.e., ZT=arbitrary constant).

As indicated in 1.5.5, each entity in this section is acted upon by a transformation matrix. This implies that each entity makes use of the definition space concept, i.e., is defined initially in definition space, and then transformed into model space. Thus the complete definition of a geometry entity, with respect to model space, involves the Transformation Matrix entity. However, in some instances, it may very well be that the transformation matrix will leave all coordinates unchanged. This will be the case exactly when the defining matrix is the identity rotation matrix and the translation vector is the zero vector. (In this situation, a convention is provided to prevent unnecessary processing. See the explanation given in 2.2.4.3.7 for Field 7 of the directory entry.) 


\subsubsection{Directionality.}

Within model space, all curves are directed. Such curves have associated end points, i.e., start point and terminate point. For each entity type, the manner of assigning direction is discussed within the description of each individual entity.

Within the entity descriptions that follow, some refer to a "counterclockwise direction" with respect to a sense of rotation in the $\mathrm{XT}$, YT plane. Since the XT, YT plane is located within three dimensional XT, $\mathrm{YT}, \mathrm{ZT}$ space, this phrase is ambiguous unless a viewing direction is specified from which to view the rotation within the plane. The viewing direction is taken to be from the positive $\mathrm{ZT}$ axis looking "down" upon the $X T$, YT plane. Then, if a clock were imagined to be lying "face up" in the $\mathrm{XT}$, YT plane, i.e., so as to be readable from the chosen viewing direction along the ZT axis - the phrase "counterclockwise direction" refers to the sense of rotation which is opposite the sense of rotation of the hands of the clock. This same notion of the meaning of counterclockwise carries over to any plane that is parallel to the XT, YT plane. 


\subsubsection{Geometric Entities.}

Entity numbers from 100 through 199 are reserved for geometry entities. The following entity type numbers have been assigned:

$\begin{array}{ll}\text { Entity Type Number } & \text { Entity Type } \\ 100 & \\ 102 & \text { Circular Arc } \\ 104 & \text { Composite Curve } \\ 106 & \text { Conic Arc } \\ & \text { Copious Data } \\ & \text { Centerline } \\ & \text { Linear Path } \\ & \text { Section Line } \\ & \text { Simple Closed Area } \\ 108 & \text { Witness Line } \\ 110 & \text { Plane } \\ 112 & \text { Line } \\ 114 & \text { Parametric Spline Curve } \\ 116 & \text { Parametric Spline Surface } \\ 118 & \text { Point } \\ 120 & \text { Ruled Surface } \\ 122 & \text { Surface of Revolution } \\ 124 & \text { Tabulated Cylinder } \\ 125 & \text { Transformation Matrix } \\ 126 & \text { Flash } \\ 128 & \text { Rational B-Spline Curve } \\ 130 & \text { Rational B-Spline Surface } \\ 132 & \text { Offset Curve } \\ 134 & \text { Connect Point } \\ 136 & \text { Node } \\ 138 & \text { Finite Element } \\ 140 & \text { Nodal Displacement and Rotation } \\ 142 & \text { Offset Surface } \\ 144 & \text { Curve on a Parametric Surface } \\ & \text { Trimmed Parametric Surface } \\ & \end{array}$




\section{Circular Arc Entity}

A circular arc is a connected portion of a parent circle which consists of more than one point. The definition space coordinate system is always chosen so that the circular arc lies in a plane either coincident with or parallel to the XT, YT plane.

3.2.1 A circular arc determines unique arc end points and an arc center point (the center of the parent circle). By considering the arc end points to be enumerated and listed in an ordered manner, start point first, followed by terminate point, a direction with respect to definition space can be associated with the arc. The ordering of the end points corresponds to the ordering necessary for the arc to be traced out in a counterclockwise manner. This convention serves to distinguish the desired circular arc from its complementary arc (complementary with respect to the parent circle). Refer to Section 3.1.2 for information relating to use of the term counterclockwise.

3.2.2 The direction of the arc with respect to model space is determined by the original counterclockwise direction of the arc within definition space, in conjunction with the action of the transformation matrix on the arc.

3.2.3 In the event that a parameterization is required but not given, the default parameterization is:

$$
\begin{aligned}
C(t)= & (X 1+R * \cos t, Y 1+R * \sin t, Z T) \\
& \text { for } t 2 \leqslant t \leqslant t 3
\end{aligned}
$$

where, for $\mathrm{i}=2$ and 3 ,

(i) $\mathrm{R}=\operatorname{sqrt}((\mathrm{Xi}-\mathrm{X} 1) * * 2+(\mathrm{Yi}-\mathrm{Y} 1) * * 2)$

(ii) ti is such that $\left(R^{*} \cos t i, R * \sin t i\right)=(X i-X 1, Y i-Y l)$

and

$$
\begin{aligned}
& 0 \leqslant \mathrm{t} 2<2 * \mathrm{PI} \\
& 0 \leqslant \mathrm{t} 3-\mathrm{t} 2 \leqslant 2 * \mathrm{PI}
\end{aligned}
$$


3.2.4 Examples of the circular arc entity are shown in Figure 3-1. In Example 3 of Figure 3-1, the solid arc is defined using point $A$ as the start point and point $\mathrm{B}$ as the terminate point. If the complementary dashed arc were desired, the first endpoint listed in the parameter data entry would be $B$, and the second would be $A$.

\section{2 .5}

\section{Directory Data}

ENTITY TYPE NUMBER :

\subsubsection{Parameter Data}

\begin{tabular}{ccll} 
Index & Name & \multicolumn{1}{c}{ Type } & \multicolumn{1}{c}{ Description } \\
1 & Real & Real & $\begin{array}{l}\text { Parallel ZT displacement of } \\
\text { arc from XT, YT plane }\end{array}$ \\
2 & X1 & Real & Arc center abscissa \\
3 & Y1 & Real & Arc center ordinate \\
4 & X2 & Real & Start point abscissa \\
5 & Y2 & Real & Start point ordinate \\
6 & X3 & Real & Terminate point abscissa \\
7 & Y3 & & $\begin{array}{l}\text { Terminate point } \\
\text { ordinate }\end{array}$
\end{tabular}

Additional Pointers as required (see 2.2.4.4.2). 

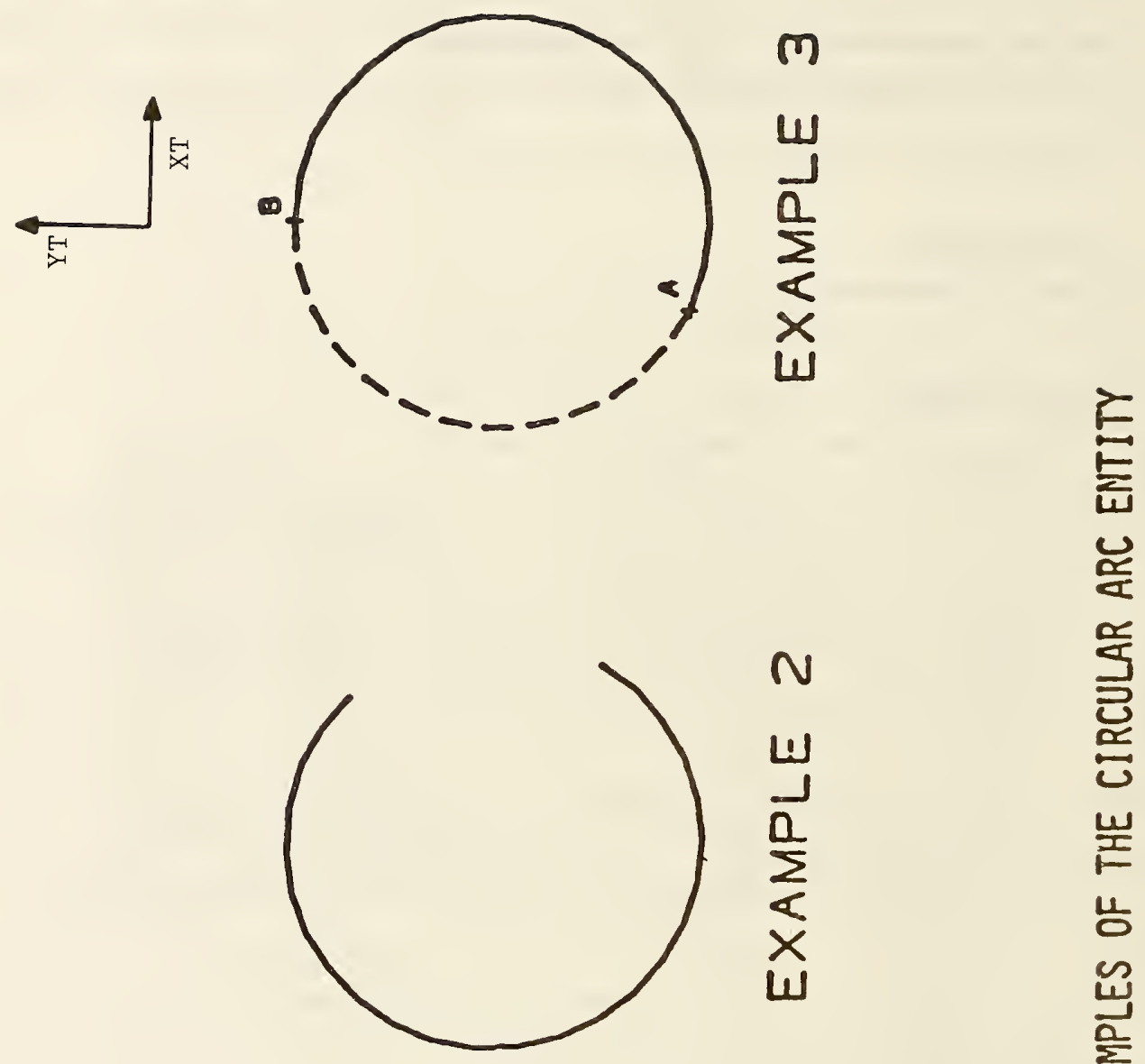

崖

害

崖

능

总

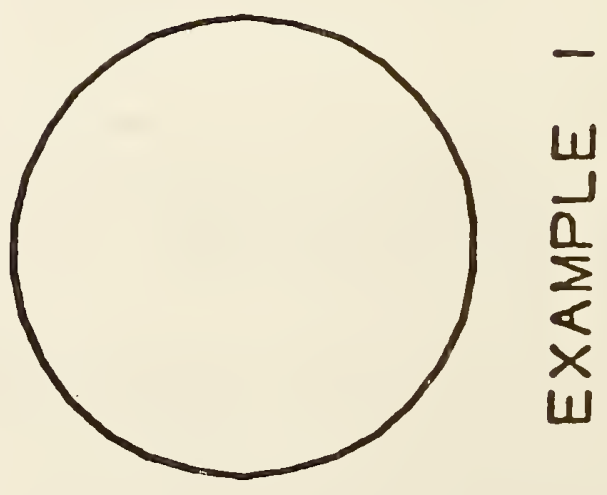

ํㅜㄹ 


\subsection{Composite Curve Entity}

A composite curve is a connected curve that results from the grouping of certain individual constituent entities into a logical unit.

3.3.1 A composite curve is defined as an ordered list of entities of the following types: point, line, circular arc, conic arc, parametric spline, rational B-spline, and connect point. The list of entities appears in the parameter data entry. There, each entity to appear in the defining list is indicated by means of a pointer to the directory entry of that entity. The order within the defining list is derived from the order of the listing of these pointers.

3.3.2 Each constituent entity has its own transformation matrix and display attributes. Each constituent entity may have text or properties associated with it. Because the constituent entities are subordinate to the composite entity, the Subordinate Entity Switch (digits 3-4 in directory entry field 9) of each constituent entity should indicate a physical dependency.

3.3.3 A composite curve is a directed curve, having a start point and a terminate point. The direction of the composite curve is induced by the direction of the constituent curve entities (i.e., those constituent entities other than the point entity) in the following way: The start point for the composite curve is the start point of the first curve entity appearing in the defining list. The terminate point for the composite curve is the terminate point of the last curve entity appearing in the defining list. Within the defining list itself, the terminate point of each constituent curve entity has the same coordinates as the start point of the succeeding curve entity.

3.3.4 The point and connect point entities are included as allowable entity types so that properties or general notes can be attached to either the start point or the terminate point of any constituent curve entities in the defining list.

A logical connection relationship can be indicated by having two composite curves or a composite curve and a network subfigure reference the connect point entity. For the special case of the logical connection of a connect point on one subfigure instance to a connect point on another subfigure instance a 


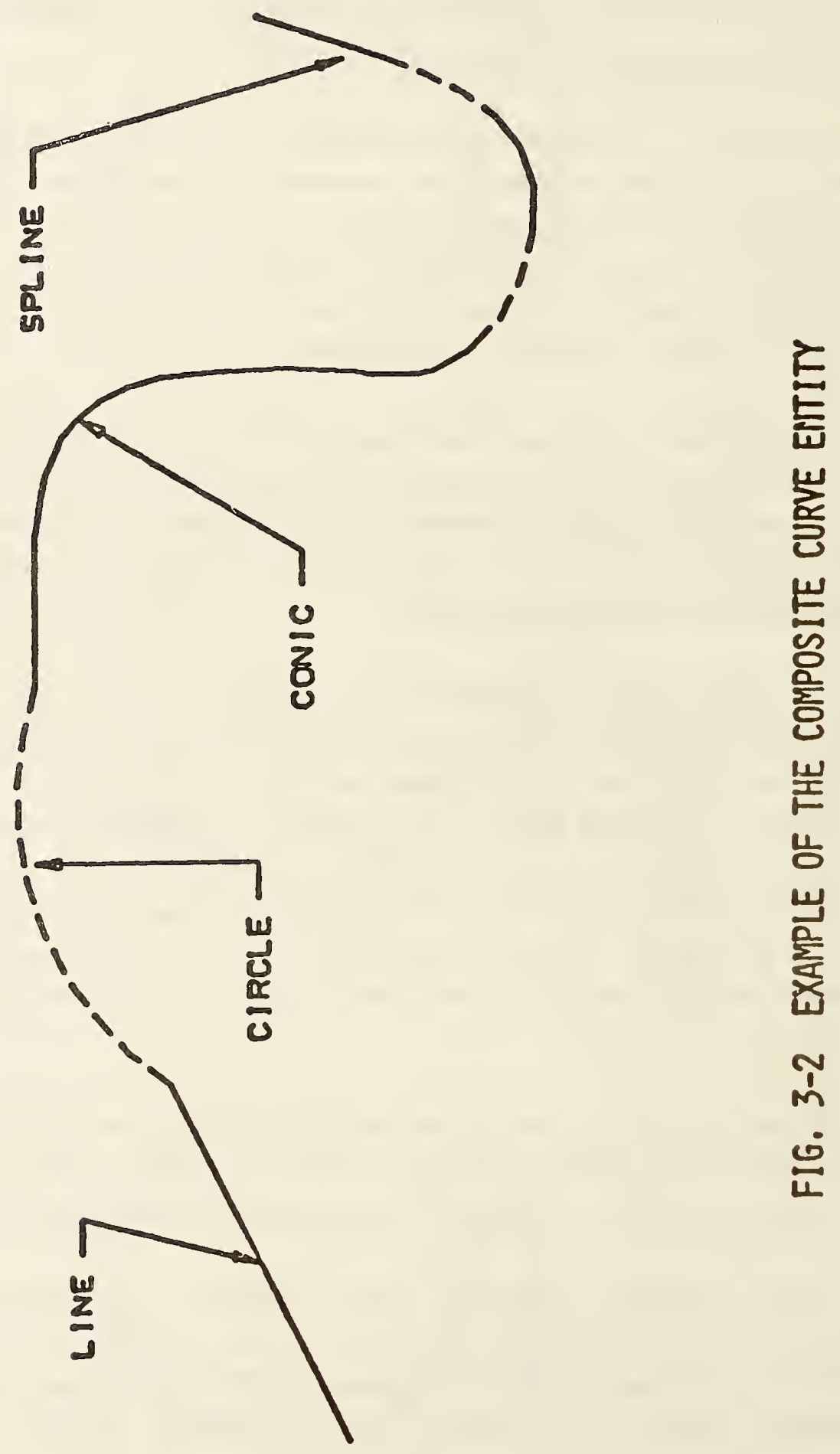


composite curve is allowed whose list contains only two connect point entities with no intervening curve entity. There are certain restrictions regarding the use of the point entity in a composite entity. They are:

a. Two point or connect point entities cannot appear consecutively in the defining list unless they are the only entities in the composite curve.

b. If a point or connect point entity and a curve entity are adjacent in the defining list, then the coordinates of the point or connect point entity must agree with the coordinates of the terminate point of the curve entity whenever the curve entity precedes the point or connect point entity, and must agree with the coordinates of the start point of the curve entity whenever the curve entity follows the point or connect point entity.

c. A composite curve cannot consist of a point entity alone or a single connect point.

3.3.5 In the event that a parametrization is required but not given, the default parametrization of the composite curve is obtained from the parametrization of the constituent curves as defined below. As point and connect point entities do not contribute to the parametrization of a composite curve, they are not considered in the definition below.

Let

C

$\mathrm{N}$

$\mathrm{CC}(\mathrm{i})$

PS(i)

PE(i)

$\mathrm{T}(0)$

$\mathrm{T}(\mathrm{i})$ be the composite curve;

be the number of constituent curves $(N \geqslant 1)$;

be the i-th constituent curve, for each $\mathrm{i}$ such that $1 \leqslant i \leqslant N$; be the parametric value of the start of CC(i) ; be the parametric value of the end of $\mathrm{CC}(\mathrm{i})$; be 0.0 ; be the sum from $j=1$ to $j=i$ of $(P E(j)-P S(j))$, for each $i$ such that $1 \leqslant i \leqslant N$. 
Then

(1) the parametric values of $\mathrm{C}$ range from $\mathrm{T}(0)$ to $\mathrm{T}(\mathrm{N})$; and

(2) $\quad \mathrm{C}(\mathrm{u})=\mathrm{CC}(\mathrm{i})(\mathrm{u}-\mathrm{T}(\mathrm{i}-1)+\mathrm{PS}(\mathrm{i}))$ where $\mathrm{u}$ is a parametric value such that $T(i-1) \leqslant u \leqslant T(i)$.

A composite curve consisting solely of point and/or connect point entities, will not be given a parametrization.

3.3.6 In this section, an example of a parametrization of a composite curve entity is given.

Let $\mathrm{N}=3$ and for each $\mathrm{i}$ such that $1 \leqslant \mathrm{i} \leqslant 3$, let $\mathrm{CC}(\mathrm{i})$ be the $\mathrm{i}$-th constituent curve of the composite curve $C$. Assume the parametric values of the start and end points of each $\mathrm{CC}(\mathrm{i})$ are given by the table

$\begin{array}{lll}\mathrm{i} & \mathrm{PS}(\mathrm{i}) & \mathrm{PE}(\mathrm{i}) \\ 1 & 0.0 & 0.4 \\ 2 & 3.3 & 3.5 \\ 3 & 0.0 & 0.3\end{array}$

Then $\mathrm{T}(0)=0.0, \mathrm{~T}(1)=0.4, \mathrm{~T}(2)=0.6, \mathrm{~T}(3)=0.9$, and the composite curve $\mathrm{C}$, is defined from 0.0 to 0.9 .

This situation described is illustrated by

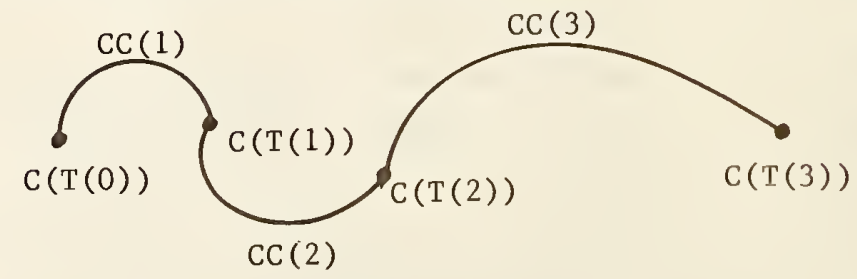

The curve combining $\mathrm{CC}(1), \mathrm{CC}(2)$, and $\mathrm{CC}(3)$ represents the composite curve $\mathrm{C}$. 
3.3.7 An example of a composite curve entity is shown in Figure 3-2

\subsubsection{Directory Data}

ENTITY TYPE NUMBER : 102

\subsubsection{Parameter Data}

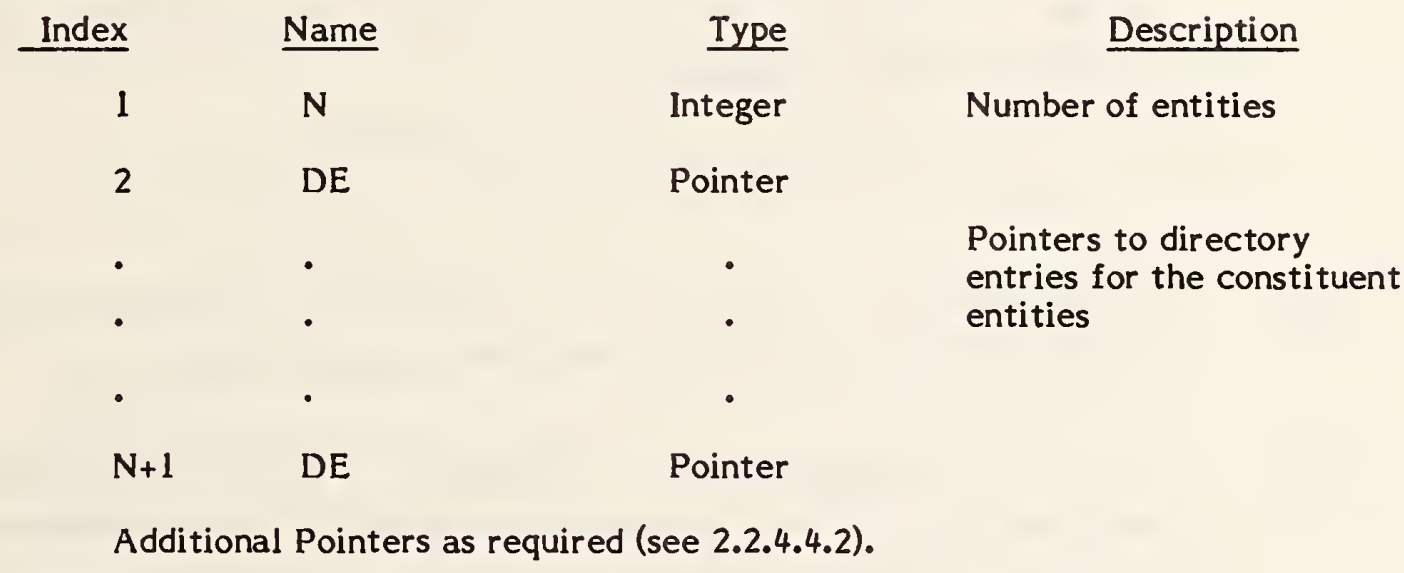




\section{Conic Arc Entity}

A conic arc is a bounded connected portion of a parent conic curve which consists of more than one point. The parent conic curve is either an ellipse, a parabola, or a hyperbola. The definition space coordinate system is always chosen so that the conic arc lies in a plane either coincident with or parallel to the XT, YT plane. Within such a plane, a conic is defined by the six coefficients in the following equation.

$$
A * X T^{2}+B * X T * Y T+C * Y T^{2}+D * X T+E * Y T+F=0
$$

3.4.1 Each coefficient is a real number. The definitions of ellipse, parabola, and hyperbola in terms of these six coefficients are given below.

3.4.2 A conic arc determines unique arc endpoints. A conic arc is defined within definition space by the six coefficients above and the two endpoints. By considering the conic arc endpoints to be enumerated and listed in an ordered manner, start point followed by terminate point, a direction with respect to definition space can be associated with the arc. In order for the desired elliptical arc to be distinquished from its complementary elliptical arc, the direction of the desired elliptical arc must be counterclockwise. In the case of a parabola or hyperbola, the parameters given in the parameter data section uniquely define a portion of the parabola or a portion of a branch of the hyperbola; therefore, the concept of a counterclockwise direction is not applied. (Refer to Section 3.1.2 for information concerning use of the term "counterclockwise".)

3.4.3 The direction of the conic arc with respect to model space is determined by the original direction of the arc within definition space, in conjunction with the action of the transformation matrix on the arc. 
3.4.4 The definitions of the terms ellipse, parabola, and hyperbola are given in terms of the quantities Q1, Q2, and Q3. These quantities are:

$$
\begin{aligned}
& Q 1=\text { determinant of }\left[\begin{array}{ccc}
A & B / 2 & D / 2 \\
B / 2 & C & E / 2 \\
D / 2 & E / 2 & F
\end{array}\right] \\
& Q 2=\text { determinant of }\left[\begin{array}{cc}
A & B / 2 \\
B / 2 & C
\end{array}\right] \\
& Q 3=A+C .
\end{aligned}
$$

3.4.5 A parent conic curve is

An ellipse if $\mathrm{Q} 2>0$ and $\mathrm{Q} 1 * \mathrm{Q} 3<0$.

A hyperbola if $\mathrm{Q} 2<0$ and $\mathrm{Q} 1 \neq 0$.

A parabola if $\mathrm{Q} 2=0$ and $\mathrm{Q} 1 \neq 0$.

An example of each type of conic arc is shown in Figure 3-3.

3.4.6 Those entities which can be represented as various degenerate forms of a conic equation (Point and Line) must not be put into the Entity Type 104; more appropriate Entity Types exist for these forms.

Because of the numerical sensitivity of the implicit form of the conic description, a receiving system not using that form as its internal representation for conics need not be expected to correctly process conics in this form unless they are put into a standard position in definition space. A conic arc entity is said to be in a standard position in definition space provided each of its axes is parallel to either the XT axis or YT axis and provided it is centered about the $\mathrm{ZT}$ axis. For a parabola, use the vertex as the origin. The conic is moved from this position in definition space to the desired position in space with a transformation matrix (Entity type 124).

The form number is regarded as purely informational by such a postprocessor.

Further details may be found in Appendix E. 

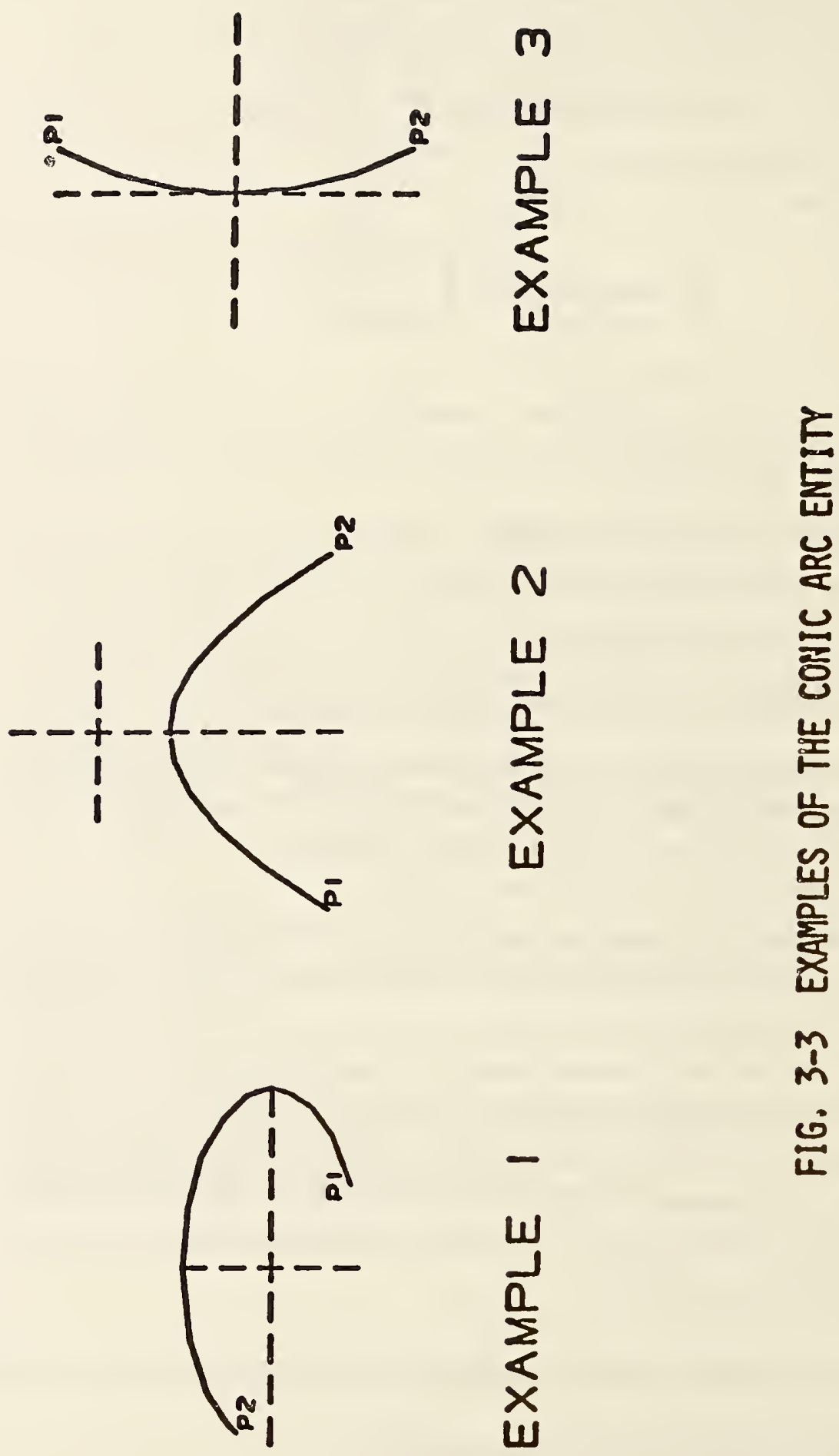

m

$\stackrel{0}{\pi}$ 
3.4.7 In the event that a parameterization is required but not given, the default parameterization is:

\section{$\underline{\text { Parabola }}$}

case $A$ and $E \neq 0.0$

if $\mathrm{X} 1<\mathrm{X} 2$

$$
\begin{aligned}
& C(t)=(t,-(A / E) * t * * 2, Z T) \\
& \text { where, for } i=1 \text { and } 2, t i=X i .
\end{aligned}
$$

if $\times 2<x 1$

$$
\begin{aligned}
& C(t)=(-t,-(A / E) * t * * 2, Z T) \quad \text { for } t 1 \leqslant t \leqslant t 2 \\
& \text { where, for } i=1 \text { and } 2, t i=-X i .
\end{aligned}
$$

case $C$ and $D \neq 0.0$

if $\mathrm{Y}_{1}<\mathrm{Y}_{2}$

$$
\begin{aligned}
& C(t)=(-(C / D) * t * *((2, t, Z T) \\
& \text { where, for } i=1 \text { and } 2, t i=Y i .
\end{aligned}
$$

if $\mathrm{Y} 2<\mathrm{Y} 1$

$$
\begin{aligned}
& C(t)=(-(C / D) * t * * 2,-t, Z T) \\
& \text { where, for } i=1 \text { and } 2, t i=-Y i .
\end{aligned}
$$

\section{Ellipse}

$C(t)=\left(a^{*} \cos t, b^{*} \sin t, Z T\right)$

$$
\text { for } t 1 \leqslant t \leqslant t 2
$$

where

$$
\begin{aligned}
& \mathrm{a}=\operatorname{sqrt}(-F / A) \\
& \mathrm{b}=\operatorname{sqr}(-F / C)
\end{aligned}
$$

and, for $\mathrm{i}=1$ and 2 , ti is such that
(i) $\left(\mathrm{a}^{*} \cos \mathrm{ti}, \mathrm{b}^{*} \sin \mathrm{ti}, \mathrm{ZT}\right)=(\mathrm{Xi}, \mathrm{Yi}, \mathrm{ZT})$
(ii) $0 \leqslant t 1 \leqslant 2 * \mathrm{PI}$
(iii) $0 \leqslant t 2-t 1 \leqslant 2 * P I$

\section{$\underline{\text { Hyperbola }}$}

case $F^{*} \mathrm{~A}<0.0$ and $\mathrm{F}^{*} \mathrm{C}>0.0$

let

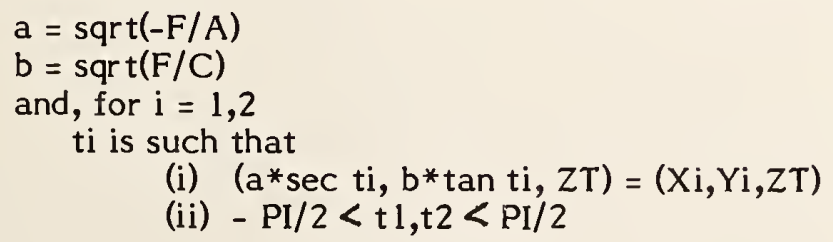


if $\mathrm{t} 1<\mathrm{t} 2$

$$
C(t)=\left(a^{*} \sec t, b^{*} \tan t, z T\right) \quad \text { for } 1 \leqslant t \leqslant t 2
$$

if $\mathrm{t} 2<\mathrm{tl}$

$$
C(t)=(a * \sec (-t), b * \tan (-t), z T) \quad \text { for }-t 1 \leqslant t \leqslant-t 2
$$

case $F^{*} \mathrm{~A}>0.0$ and $\mathrm{F} * \mathrm{C}<0.0$

let

$$
\begin{aligned}
& a=\operatorname{sqr} t(F / A) \\
& b=\operatorname{sqr} t(-F / C) \\
& \text { and, for } i=1,2 \\
& \text { ti is such that } \\
& \text { (i) }\left(a * \tan t i, b^{*} \sec t i, Z T\right)=(X i, Y i, Z T) \\
& \text { (ii) }-P I / 2<t 1, t 2<P I / 2
\end{aligned}
$$

if $\mathrm{t} 1<\mathrm{t} 2$

$$
C(t)=\left(a^{*} \tan t, b^{*} \sec t, z T\right) \quad \text { for } t 1 \leqslant t \leqslant t 2
$$

if $\mathrm{t} 2<\mathrm{t} 1$

$$
C(t)=\left(a^{*} \tan (-t), b^{*} \sec (-t), z T\right) \quad \text { for }-t 1 \leqslant t \leqslant-t 2
$$

3.4.8 Field 15 of the directory entry accommodates a Form Number. For this entity, the options are as follows:
FORM Meaning Form of parent conic curve must be determined from the general
equation.
1 Parent conic curve is an ellipse (See example 1, Figure 3-3).
2 Parent conic curve is a hyperbola (See example 2, Figure 3-3).
3 Parent conic curve is a parabola (See example 3, Figure 3-3). 
3.4.9 Directory Data

ENTITY TYPE NUMBER : 104

\subsubsection{Parameter Data}

\begin{tabular}{cccc} 
Index & Name & Type & \multicolumn{1}{c}{ Description } \\
1 & A & Real & Conic Coefficient \\
2 & B & Real & Conic Coefficient \\
3 & C & Real & Conic Coefficient \\
4 & D & Real & Conic Coefficient \\
5 & E & Real & Conic Coefficient \\
6 & F & Real & Conic Coefficient \\
7 & ZT & Real & ZT Coordinate of \\
plane of definition
\end{tabular}

Additional Pointers as required (see 2.2.4.4.2). 


\subsection{Copious Data Entity}

This entity stores data points in the form of pairs, triples, or sextuples. An interpretation flag value signifies which of these forms is being used. This value is one of the parameter data entries. The interpretation flag is abbreviated below by the letters IP.

Data points within definition space which lie within a single plane are specified in the form of XT, YT coordinate pairs. In this case, the common $\mathrm{ZT}$ value is also needed. Data points arbitrarily located within definition space are specified in the form of XT, YT, ZT coordinate triples. Data points within definition space which have an associated vector are specified in the form of sextuples; the XT, YT, ZT coordinates are specified first, followed by the $i, j, k$ coordinates of the vector associated with the point. (Note that, for an associated vector, no special meaning is implicit.)

Field 15 of the directory entry accommodates a Form Number. For this entity, the options are as follows:

\section{FORM Meaning}

1 Data points in the form of coordinate pairs. All data points lie in a plane $\mathrm{ZT}=$ constant. $(\mathrm{IP}=1)$

2 Data points in the form of coordinate triples. (IP=2)

3 Data points in the form of sextuples. (IP=3)

11 Data points in the form of coordinate pairs which represent the vertices of a planar, piecewise linear curve (piecewise linear string is sometimes used). All data points lie in a plane $\mathrm{ZT}=$ constant. $(\mathrm{IP}=1)$

Data points in the form of coordinate triples which represent the vertices of a piecewise linear curve (piecewise linear string is sometimes used). ( (IP=2)

Data points in the form of sextuples. The first triple of each sextuple represents the vertices of a piecewise linear curve (piecewise linear string is sometimes used). The second triple is an associated vector. (IP $=3$ )

Centerline Entity through points $(\mathrm{IP}=1)$ 
21 Centerline Entity through circle centers (IP=1)

31 Section Entity Form $31(\mathrm{IP}=1)$

32 Section Entity Form $32(\mathrm{IP}=1)$

33 Section Entity Form $33(\mathrm{IP}=1)$

34 Section Entity Form $34(\mathrm{IP}=1)$

35 Section Entity Form $35($ IP $=1)$

36 Section Entity Form $36($ IP $=1)$

37 Section Entity Form $37(\mathrm{IP}=1)$

38 Section Entity Form $38($ IP $=1)$

40 Witness Line Entity $(\mathrm{IP}=1)$

63 Simple Closed Area Entity $(\mathrm{IP}=1)$

The linear path is an ordered set of points in either 2- or 3-dimensional space. These points define a series of linear segments along the consecutive points of the path. The segments may cross or be coincident with each other. Paths may close, i.e., the first path point may be identical to the last.

The linear path is implemented as two forms of the copious data block (entity number 106). Form 11 is for 2-dimensional paths and form 12 is for 3dimensional paths. This entity will be closely associated with properties indicating functionality and fabrication parameters, such as Line Widening.

Refer to the centerline and witness line entities in Section 4 of this specification for examples of Form Numbers 20, 21 and 40. Each of these annotation entities contains a description of how the associated copious data are to be interpreted. Forms 31-38 provide for the transfer of graphical information and are defined here for compatability with previous versions of the specification. The Sectioned Area Entity (type 230) provides a more compact method for transferring this information.

A simple closed area is a bounded region of $X Y$ coordinate space represented by a set of points that forms a series of connected linear segments. These segments must form a closed loop, i.e., the first point of the boundary of the area and the last point must be identical. No segments of this entity are allowed to intersect or be coincident except for the closing of the entity at the initial and final 
points. This entity will be closely related to properties that indicate functionality of closed regions, such as Region Fill and Region Restriction.

The area is implemented as Form 63 of entity 106, the copious data block.

\subsubsection{Directory Data}

ENTITY TYPE NUMBER : 106

\subsubsection{Parameter Data}

\begin{tabular}{|c|c|c|c|c|}
\hline Index & Name & Type & & Description \\
\hline \multirow[t]{5}{*}{1} & IP & Integer & \multicolumn{2}{|c|}{ Interpretation Flag } \\
\hline & & & $\mathrm{IP}=1$ & $x, y$ pairs, common $z$ \\
\hline & & & $\mathrm{IP}=2$ & $x, y, z$ coordinates \\
\hline & & & $\mathrm{IP}=3$ & $x, y, z$ coordinates and \\
\hline & & & & $\mathrm{i}, \mathrm{j}, \mathrm{k}$ vectors \\
\hline 2 & N1 & Integer & \multicolumn{2}{|c|}{ Number of n-tuples } \\
\hline
\end{tabular}

For IP=1 ( $x, y$ pairs, common $z)$ :

$\begin{array}{lllc}3 & \text { ZT } & \text { Real } & \text { Common z displacement } \\ 4 & \text { XI } & \text { Real } & \text { First data point abscissa } \\ 5 & \text { Y1 } & \text { Real } & \text { First data point ordinate } \\ - & \text { - } & \cdot & - \\ - & \cdot & \cdot & - \\ \text { 3+2N } & \text { YN } & \text { Real } & \text { Last data point ordinate }\end{array}$

For IP $=2(x, y, z$ triples $):$

\begin{tabular}{|c|c|c|c|}
\hline 3 & $\times 1$ & Real & First data point $x$ value \\
\hline 4 & Yl & Real & First data point y value \\
\hline 5 & 21 & Real & First data point $\mathrm{z}$ value \\
\hline - & - & - & - \\
\hline • & - & - & - \\
\hline • & - & - & • \\
\hline $2+3 N$ & $\mathrm{ZN}$ & Real & Last data point $\mathrm{z}$ value \\
\hline
\end{tabular}


For IP $=3(x, y, z, i, j, k$ sextuples):

$\begin{array}{llll}3 & X 1 & \text { Real } & \text { First data point } x \text { value } \\ 4 & \text { Y } 1 & \text { Real } & \text { First data point } y \text { value } \\ 5 & \text { Z1 } & \text { Real } & \text { First data point } z \text { value } \\ 6 & \text { II } & \text { Real } & \text { First data point } i \text { value } \\ 7 & \text { J1 } & \text { Real } & \text { First data point } j \text { value } \\ 8 & \text { K1 } & \text { Real } & \text { First data point } k \text { value } \\ \text { - } & \text { - } & \text { - } & \\ \text { - } & \text { - } & \text { - } & \\ 2+6 \mathrm{~N} & \text { KN } & \text { Real } & \text { Last data point } k \text { value }\end{array}$

Additional pointers as required (see sec. 2.2.4.4.2). 
The plane entity can be used to represent an unbounded plane, as well as a bounded portion of a plane. In either of the above cases, the plane is defined within definition space by means of the coefficients $A, B, C, D$, where at least one of $A, B$, and $C$ is non-zero and

$$
A * X T+B * Y T+C * Z T=D
$$

for each point lying in the plane, and having definition space coordinates (XT, YT, ZT).

3.6.1 The definition space coordinates of a point, as well as a size parameter, can be specified in order to assist in defining a system-dependent display symbol. These values are parameter data entries six through nine, respectively. This information, together with the four coefficients defining the plane, provides sufficient information relative to definition space in order to be able to position the display symbol. (In Examples 1 and 3 of Figure 3-4, the dashed curve and the crosshair together constitute the display symbol.) Setting the size parameter to zero indicates that a display symbol is not intended.

3.6.2 The case of a bounded portion of a fixed plane is indicated by the existence of a pointer to a closed curve lying in the plane. This is parameter five. The only allowed coincident points for this curve are the start point and the terminate point. Setting this value to zero indicates the case of an unbounded plane.

3.6.3 The case of a bounded portion of a fixed plane minus some portion(s) of that plane, such as those shown in Figure 3-5, are expressed through the use of the Single Parent Associativity (Type 402, Form 9) where the outer closed curve defines the parent bounded plane and each internal closed curve defines some child bounded plane to be subtracted from the parent. Each of these planes (parent and child) is a separate plane entity in the IGES file and has a backpointer to the associativity structure. The child plane entity will have a subordinate entity switch class of 01 (Physically Dependent). 

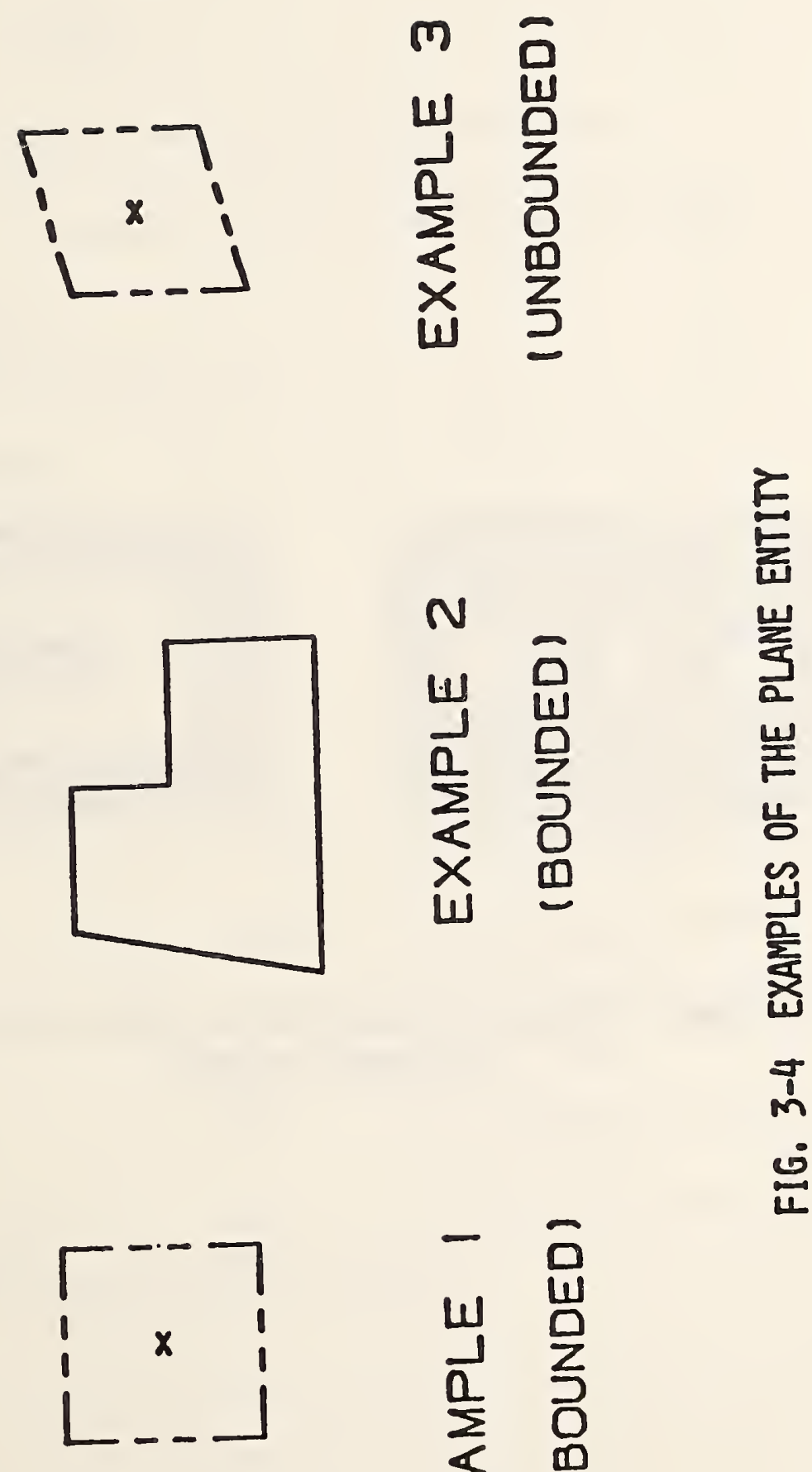

$\begin{array}{cc}- & 0 \\ w & w \\ 1 & z \\ 0 & 3 \\ \sum & 0 \\ < & \vdots \\ w & \vdots\end{array}$ 
108 - PLANE
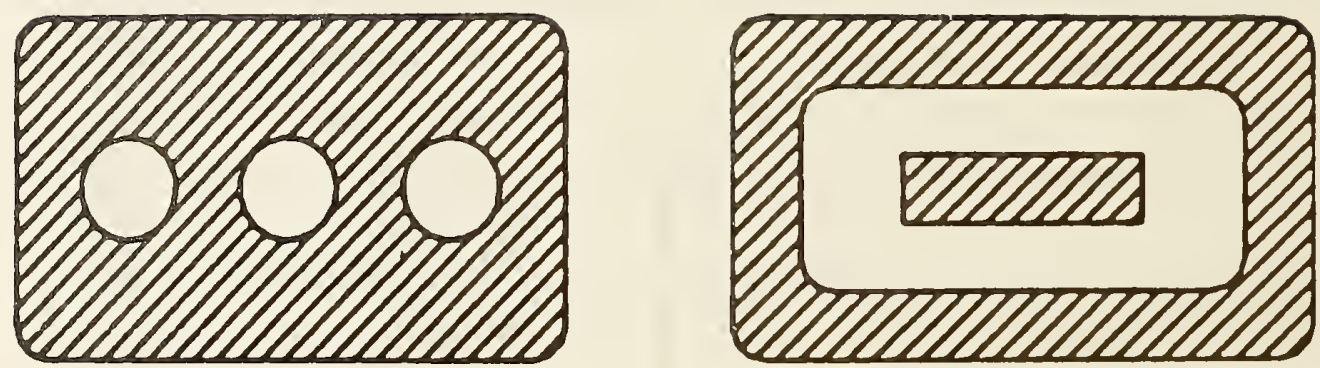

FIG. 3-5 SINGLE PARENT ASSOCIATIVITY AS USED WITH A COLLECTION OF BOUNDED PLANES 
3.6.4 Field 15 of the directory entry accommodates a Form Number. For this entity, the options are as follows:
FORM Meaning
+1 Bounded planar portion is considered positive.
$-1 \quad$ Bounded planar portion is considered negative (hole).

\subsubsection{Directory Data}

ENTITY TYPE NUMBER : 108

\subsubsection{Parameter Data}

\begin{tabular}{|c|c|c|c|}
\hline Index & Name & Type & Description \\
\hline 1 & A & Real & \\
\hline 2 & B & Real & \\
\hline 3 & C & Real & Coefficients of Plane \\
\hline 4 & D & Real & \\
\hline 5 & PTR & Pointer & $\begin{array}{l}\text { Pointer to directory entry of closed } \\
\text { curve entity or zero }\end{array}$ \\
\hline 6 & $\mathrm{x}$ & Real & $\begin{array}{l}\text { XT coordinate of location point for } \\
\text { display symbol }\end{array}$ \\
\hline 7 & $\mathrm{Y}$ & Real & $\begin{array}{l}\text { YT coordinate of location point for } \\
\text { display symbol }\end{array}$ \\
\hline 8 & $z$ & Real & $\begin{array}{l}\text { ZT coordinate of location point for } \\
\text { display symbol }\end{array}$ \\
\hline 9 & SIZE & Real & Size parameter for display symbol \\
\hline
\end{tabular}

Additional Pointers as required (see 2.2.4.4.2). 


\subsection{Line Entity}

A line is a bounded, connected portion of a parent straight line which consists of more than one point.

A line is defined by its end points. Each end point is specified relative to definition space by a triple of coordinates. With respect to definition space, a direction is associated with the line by considering the start point to be listed first and the terminate point second.

The direction of the line with respect to model space is determined by the original direction of the line within definition space, in conjunction with the action of the transformation matrix on the line. Examples of the line entity are shown in Figure 3-6.

3.7.1 In the event that a parameterization is required, the default parameterization is:

$$
C(t)=P_{1}+t\left(P_{2}-P_{1}\right) \text { for } 0 \leqslant t \leqslant 1
$$

\subsubsection{Directory Data}

ENTITY TYPE NUMBER :

\subsubsection{Parameter Data}

$\begin{array}{llll}\text { Index } & \text { Name } & \text { Type } & \text { Description } \\ 1 & \text { X1 } & \text { Real } & \text { Start Point P1 } \\ 2 & \text { Y1 } & \text { Real } & \\ 3 & Z 1 & \text { Real } & \\ 4 & X 2 & \text { Real } & \text { Terminate Point P2 } \\ 5 & \text { Y2 } & \text { Real } & \\ 6 & Z 2 & \text { Real } & \end{array}$

Additional Pointers as required (see 2.2.4.4.2). 

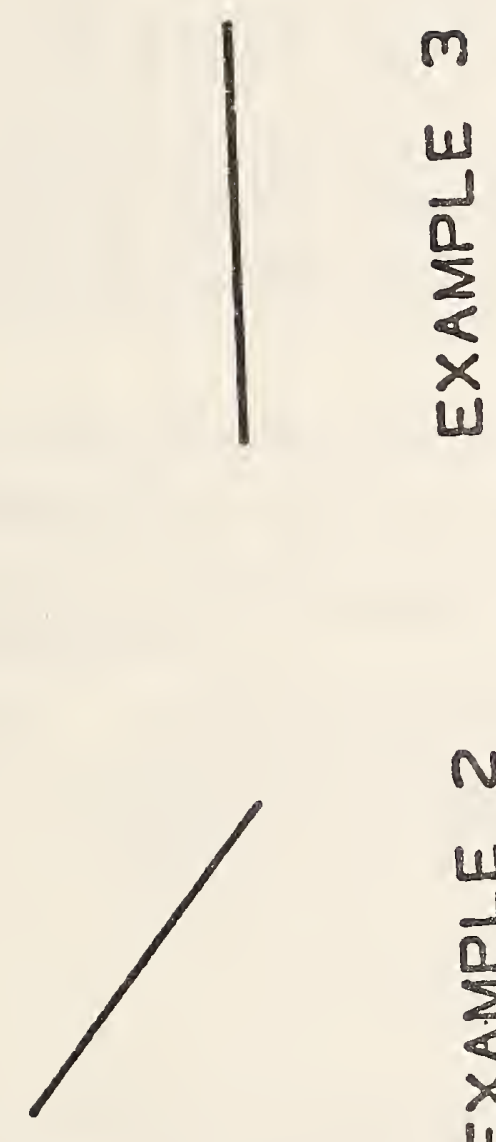

$N$

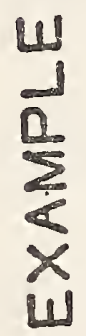

窑

$\underline{\square}$

L

농

थ

1

ก
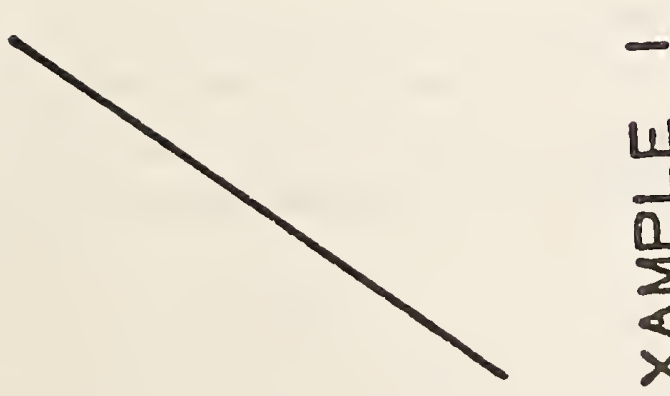

एँ

U)

2

$<$

us 


\subsection{Parametric Spline Curve Entity}

(Consult Appendix D for additional mathematical details)

The parametric spline curve is a sequence of parametric polynomial segments. The CTYPE value in Parameter 1 indicates the type of curve as it was represented in the sending (pre-processing) system before conversion to this entity.

3.8.1 The $\mathbf{N}$ polynomial segments are delimited by the breakpoints $\mathrm{T}(1), \mathrm{T}(2)$, $\ldots, \mathrm{T}(\mathrm{N}+1)$. The coordinates of the points in the $\mathrm{i}$-th segment of the curve are given by the following cubic polynomials (the coefficients $D$, or $C$ and $D$ will be zero if the polynomials are of degrees 2 or 1 , respectively):

$$
\begin{aligned}
& X(u)=A X(i)+B X(i) \cdot s+C X(i) s^{2}+D X(i) s^{3} \\
& Y(u)=A Y(i)+B Y(i) \cdot s+C Y(i) s^{2}+D Y(i) s^{3} \\
& Z(u)=A Z(i)+B Z(i) * s+C Z(i) s^{2}+D Z(i) \cdot s^{3}
\end{aligned}
$$

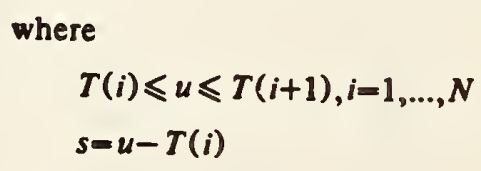

In order to avoid degeneracy, for each $i$ at least one of the nine real coefficients, BX(i), CX(i), DX(i), BY(i), CY(i), DY(i), BZ(i), CZ(i), and DZ(i) must be non-zero.

3.8.2 If the spline is planar, it must be parametrized in terms of the $X$ and $Y$ polynomials only. The $Z$ polynomial will then be zero except for each $i$, the $A Z(i)$ term which indicates the $Z$-depth in definition space.

3.8.3 The parameter $\mathrm{H}$ is used as an indicator of the smoothness of the curve. If $\mathrm{H}=0$, the curve is continuous at all breakpoints. If $\mathrm{H}=1$, the curve is continuous and has slope continuity (see section 6.3 of FAUX79) at all breakpoints. If $\mathrm{H}=2$, the curve is continuous and has both slope and curvature continuity at all breakpoints (see section 6.3 of Faux79). 
3.8.4 To enable determination of the terminate point and derivatives without computing the polynomials, the $\mathrm{N}$ th polynomials and their derivatives are evaluated at $u=T(N+1)$. These data are divided by appropriate factorials and stored following the polynomial coefficients. For example, the name TPY 3 will be used to designate $1 / 3$ ! times the third derivative of the $Y$ polynomial for the $\mathrm{N}$ th segment evaluated at $\mathrm{u}=\mathrm{T}(\mathrm{N}+1)$, the parameter value corresponding to the terminate point. Note that these data are redundant as they are derived from the data defining the $\mathrm{N}$ th polynomial segment.

3.8.5 An example of a parametric spline is shown in Figure 3-7. Additional examples are shown in Figure 3-8.

\subsubsection{Directory Data}

ENTITY TYPE NUMBER : 112

\subsubsection{Parameter Data}

\begin{tabular}{|c|c|c|c|}
\hline Index & $\underline{\text { Name }}$ & Type & Description \\
\hline 1 & CTYPE & Integer & $\begin{array}{l}\text { Spline Type } \\
\text { (1=Linear } \\
2=\text { Quadratic } \\
3=\text { Cubic } \\
\text { 4=Wilson-Fowler } \\
\text { 5=Modified } \\
\text { Wilson-Fowler } \\
\text { 6=B Spline) }\end{array}$ \\
\hline 2 & $\mathrm{H}$ & Integer & $\begin{array}{l}\text { Degree of con- } \\
\text { tinuity with } \\
\text { respect to arc } \\
\text { length }\end{array}$ \\
\hline 3 & NDIM & Integer & $\begin{array}{l}2=\text { =planar } \\
3=\text { non-planar }\end{array}$ \\
\hline 4 & $\mathbf{N}$ & Integer & $\begin{array}{l}\text { Number of seg- } \\
\text { ments }\end{array}$ \\
\hline $\begin{array}{c}5 \\
\dot{5} \\
\dot{5+N}\end{array}$ & $\begin{array}{l}\mathrm{T}(1) \\
\dot{0} \\
\dot{\mathrm{T}}(\mathrm{N}+1)\end{array}$ & Real & $\begin{array}{l}\text { Break points of } \\
\text { piecewise } \\
\text { polynomial }\end{array}$ \\
\hline
\end{tabular}



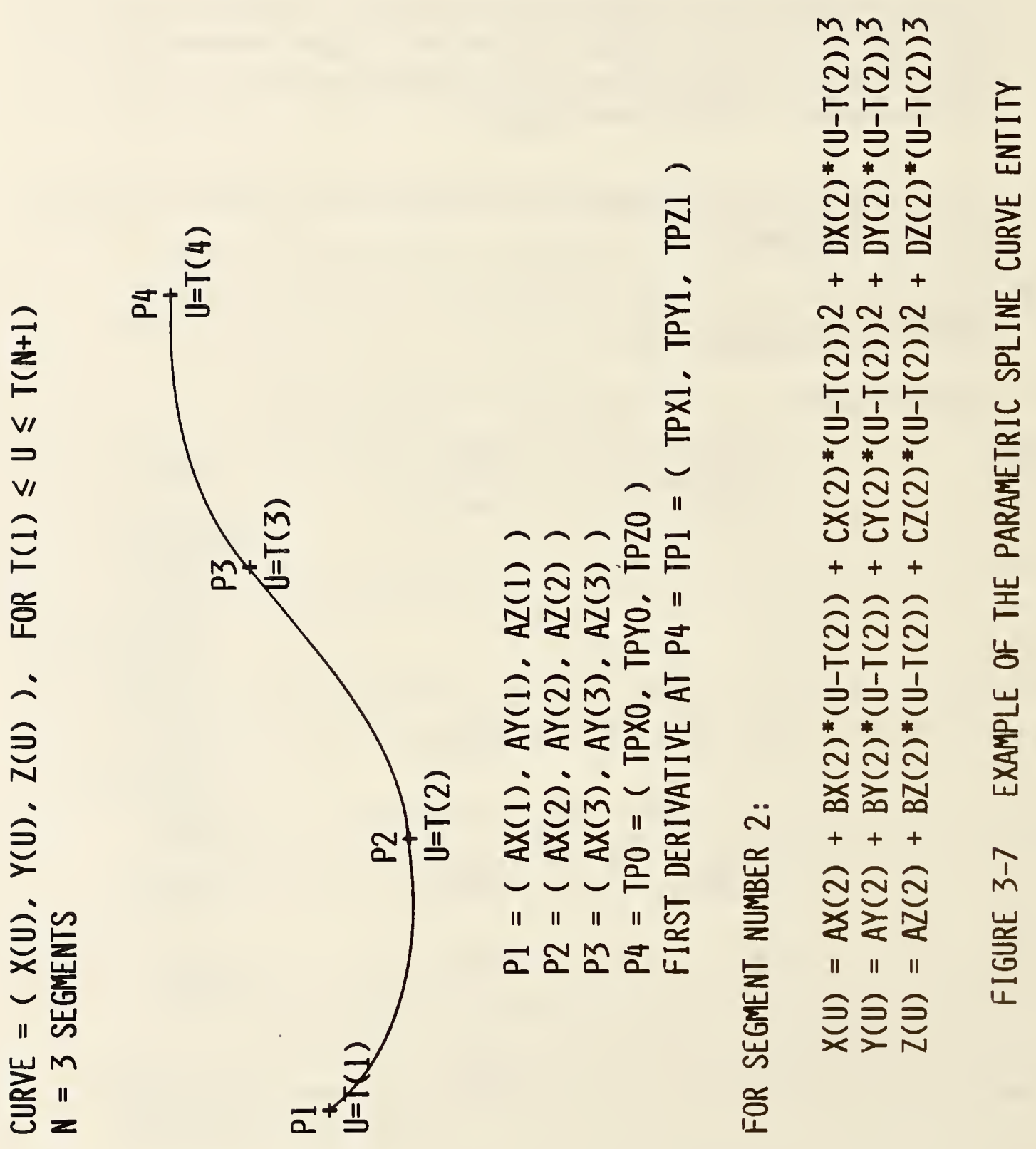


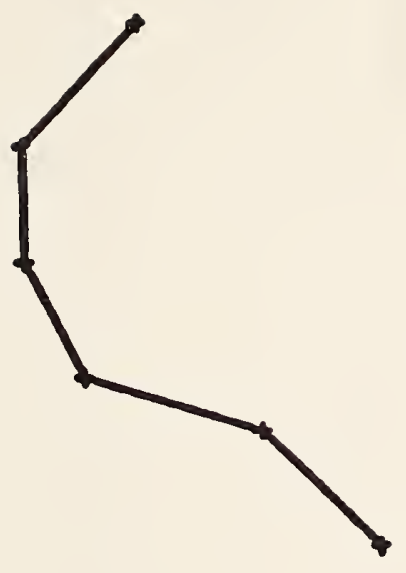

$m$
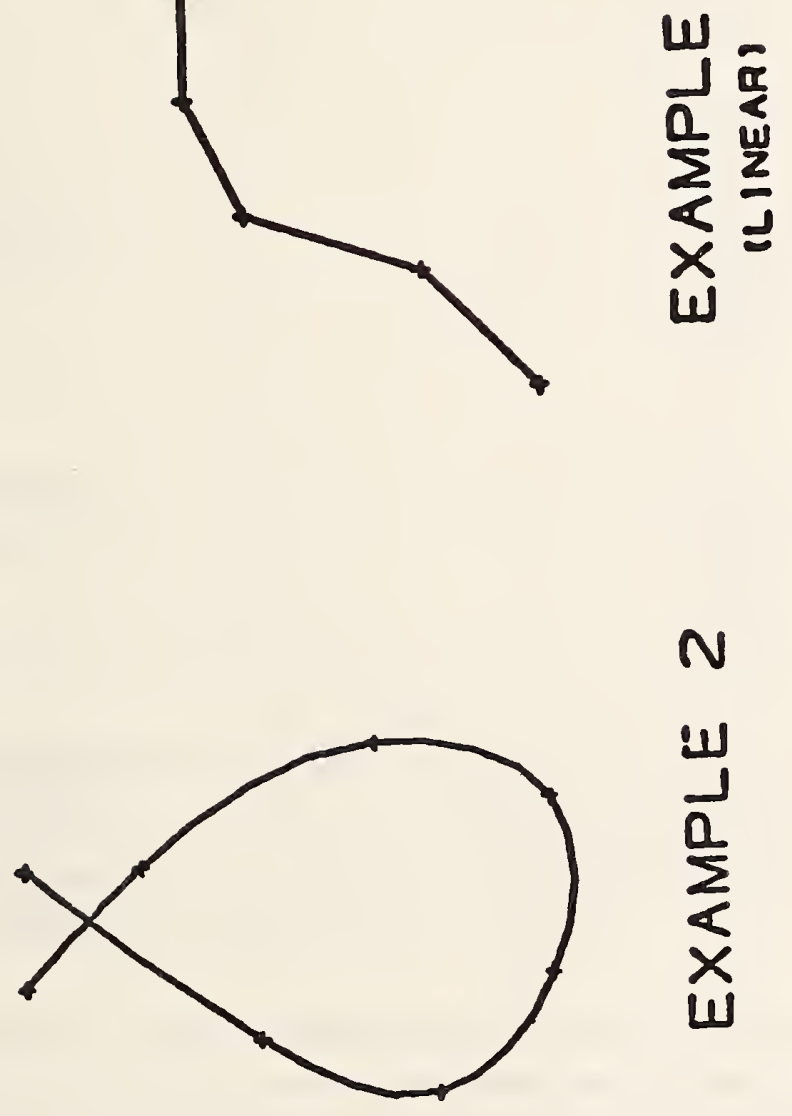

$\frac{1}{2}$
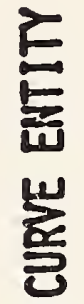

$N$

는 


\begin{tabular}{|c|c|c|c|}
\hline Index & Name & Type & Description \\
\hline $6+N$ & $A \times(1)$ & Real & $\begin{array}{l}\text { X coordinate } \\
\text { polynomial }\end{array}$ \\
\hline $7+\mathrm{N}$ & $\mathrm{BX}(1)$ & & \\
\hline $8+N$ & $\mathrm{CX}(1)$ & & \\
\hline $9+\mathrm{N}$ & $\mathrm{DX}(1)$ & & \\
\hline $10+\mathrm{N}$ & $A Y(1)$ & & $\mathrm{Y}$ coordinate \\
\hline $11+N$ & BY(1) & & polynomial \\
\hline $12+N$ & $C Y(1)$ & & \\
\hline $13+N$ & $\mathrm{DY}(1)$ & & \\
\hline $14+N$ & $A Z(1)$ & & $Z$ coordinate \\
\hline $15+N$ & $B Z(1)$ & & polynomial \\
\hline $16+N$ & $C Z(1)$ & & \\
\hline $17+N$ & $\mathrm{DZ}(1)$ & & \\
\hline & $\dot{\bullet}$ & & $\begin{array}{l}\text { Subsequent } X, Y, Z \\
\text { polynomials concluding } \\
\text { with the twelve } \\
\text { coefficients of the Nth } \\
\text { polynomial segment. }\end{array}$ \\
\hline
\end{tabular}

(The parameters that follow comprise the evaluations of the polynomials of the Nth segment and their derivatives at the parameter value $u=T(N+1)$ corresponding to the terminate point. Subsequently these evaluations are divided by appropriate factorials.) 


\begin{tabular}{|c|c|c|c|}
\hline \multirow[t]{12}{*}{$6+13 * N$} & TPXO & Real & $\mathrm{X}$ value \\
\hline & TPXI & & $X$ first derivative \\
\hline & TPX2 & & $\mathrm{X}$ second derivative/2! \\
\hline & TPX3 & & $\mathrm{X}$ third derivative/3! \\
\hline & TPYO & & $Y$ value \\
\hline & TPY 1 & & \\
\hline & TPY2 & & \\
\hline & TPY 3 & & \\
\hline & TPZO & & $Z$ value \\
\hline & TPZ1 & & \\
\hline & TPZ2 & & \\
\hline & TPZ3 & & \\
\hline
\end{tabular}

Additional Pointers as required (see 2.2.4.4.2)

Software to convert between parametric spline curves or surfaces and the corresponding rational B-spline curves or surfaces is available from the IGES office at the National Bureau of Standards. Materials provided include a magnetic tape of Pascal source code, a listing of the code, and accompanying documentation. 


\subsection{Parametric Spline Surface Entity}

(Consult Appendix $D$ for additional mathematical details)

The parametric spline surface is a grid of parametric polynomial patches. PTYPE in the Parameter Data Section indicates the type of patch under consideration.

3.9.1 The $M \times N$ grid of patches is defined by the $u$ breakpoints $T U(1), \ldots, T U(M+1)$ and the $v$ breakpoints TV $(1), \ldots, T V(N+1)$. The coordinates of the points in each of the patches are given by the general bicubic polynomials (given here for the $(i, j)$ Patch).

$$
\begin{aligned}
& X(u, v)=A X(i, j)+B X(i, j) * s+C X(i, j) * s^{2}+D X(i, j) * s^{3} \\
& +\operatorname{EX}(i, j) * t+F X(i, j) * v s+G X(i, j) * v^{2}+H X(i, j) \bullet * s^{3} \\
& +K X(i, j) \theta^{2}+L X(i, j) \bullet t^{2} s+M X(i, j) \bullet^{2} \cdot s^{2}+N X(i, j) \bullet t^{2} * s^{3} \\
& +P X(i, j) \theta^{3}+Q X(i, j) \bullet^{3} * s+R X(i, j) \bullet^{3} * s^{2}+S X(i, j) \bullet^{3} * s^{3} \\
& Y(u, v)=\cdots \\
& Z(u, v)=\cdots \\
& \text { where }
\end{aligned}
$$

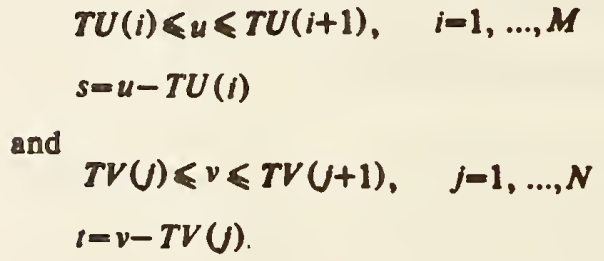

3.9.2 Parameters with the following indices shall be ignored by post-processors: for $k=1,2,3, \ldots, M$

$$
\begin{aligned}
& 7+M+N+48 *(k * N+(k-1)) \\
& \text { through } \\
& 6+M+N+48 *(k *(N+1))
\end{aligned}
$$

(i.e., the $(N+1)$ row of patches) 
and

$$
\begin{aligned}
& 7+M+N+48 *(M *(N+1)) \\
& \text { through } \\
& 6+\mathrm{M}+\mathrm{N}+48 *(\mathrm{M}+1) *(\mathrm{~N}+1) \\
& \text { (i.e., the }(M+1) \text { column of patches). }
\end{aligned}
$$

To maintain upward compatibility with previous versions of IGES, the preprocessors must either enter a real number for each of these parameters or a series of parameter delimiters (see 2.2.3). These values act as place holders in the parameter list. These parameters were intended to handle first, second, and third partial derivatives of the $\mathrm{N}^{\text {th }}$ row and $\mathrm{M}^{\text {th }}$ column of patches along the outer edge or boundary. However, these parameters can be computed by the receiving system, as needed, from the other parameter values contained in this entity, and therefore are not needed. 


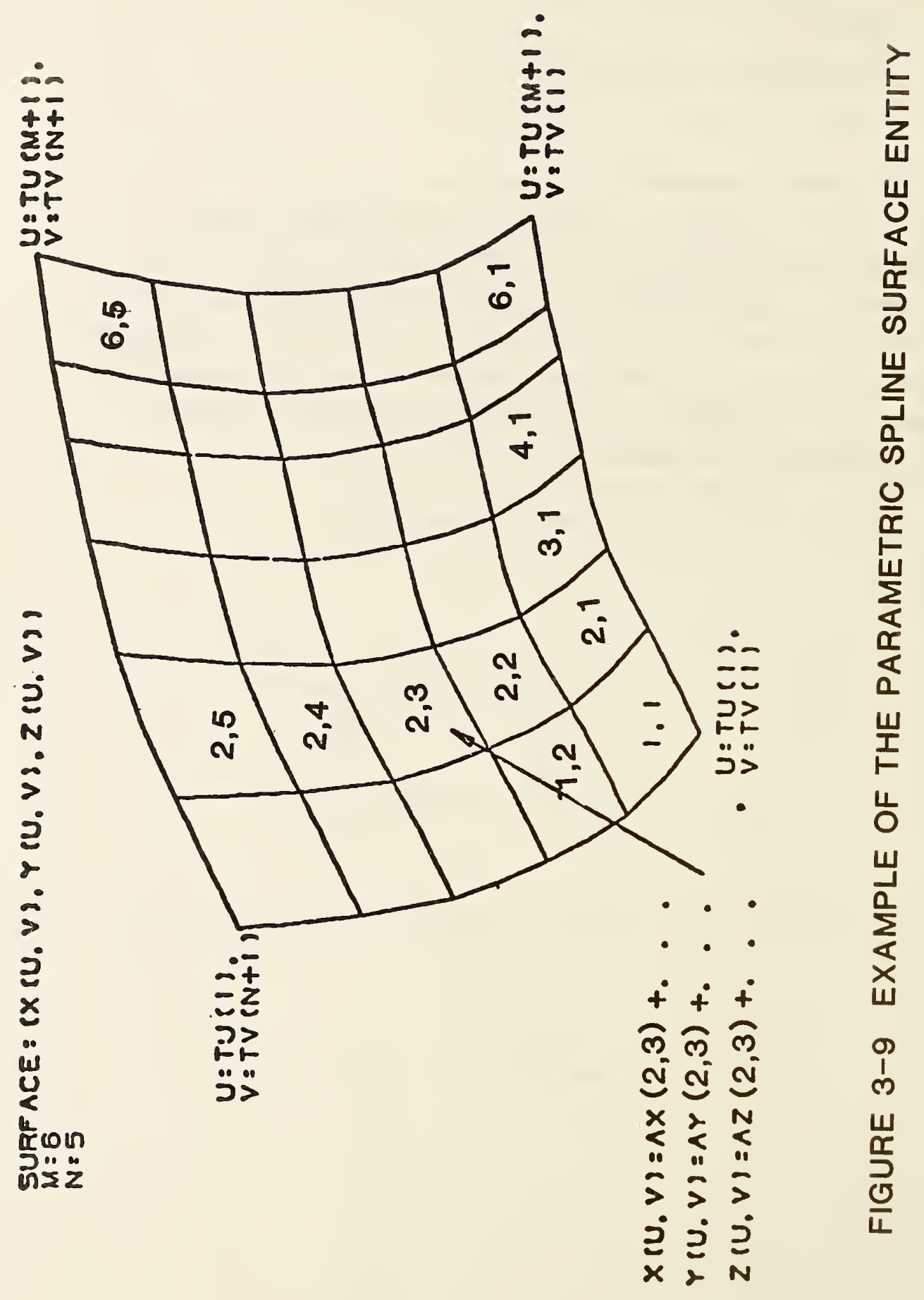


3.9.3 An example of the bicubic surface is shown in Figure 3-9.

\subsubsection{Directory Data}

ENTITY TYPE NUMBER : 114

\subsubsection{Parameter Data}

\begin{tabular}{|c|c|c|c|}
\hline Index & Name & Type & Description \\
\hline 1 & CTYPE & Integer & $\begin{array}{l}\text { Spline Boundary } \\
\text { Type } \\
\text { (1=Linear } \\
2=\text { Quadratic } \\
3=\text { Cubic } \\
\text { 4=Wilson-Fowler } \\
5=\text { Modified } \\
\text { Wilson-Fowler } \\
6=\text { B spline) }\end{array}$ \\
\hline 2 & PTYPE & Integer & $\begin{array}{l}\text { Patch Type } \\
\text { (1=Cartesian } \\
\text { Product } \\
0=\text { Unspecified) }\end{array}$ \\
\hline 3 & $M$ & Integer & $\begin{array}{l}\text { Number of } u \\
\text { segments }\end{array}$ \\
\hline 4 & $\mathbf{N}$ & Integer & $\begin{array}{l}\text { Number of } v \\
\text { segments }\end{array}$ \\
\hline 5 & $T U(1)$ & Real & $\begin{array}{l}\text { Breakpoints in } \\
u \text { ( } u \text { values of } \\
\text { grid lines) }\end{array}$ \\
\hline - & - & & \\
\hline - & • & & \\
\hline - & - & & \\
\hline $5+M$ & $\mathrm{TU}(\mathrm{M}+1)$ & & \\
\hline
\end{tabular}




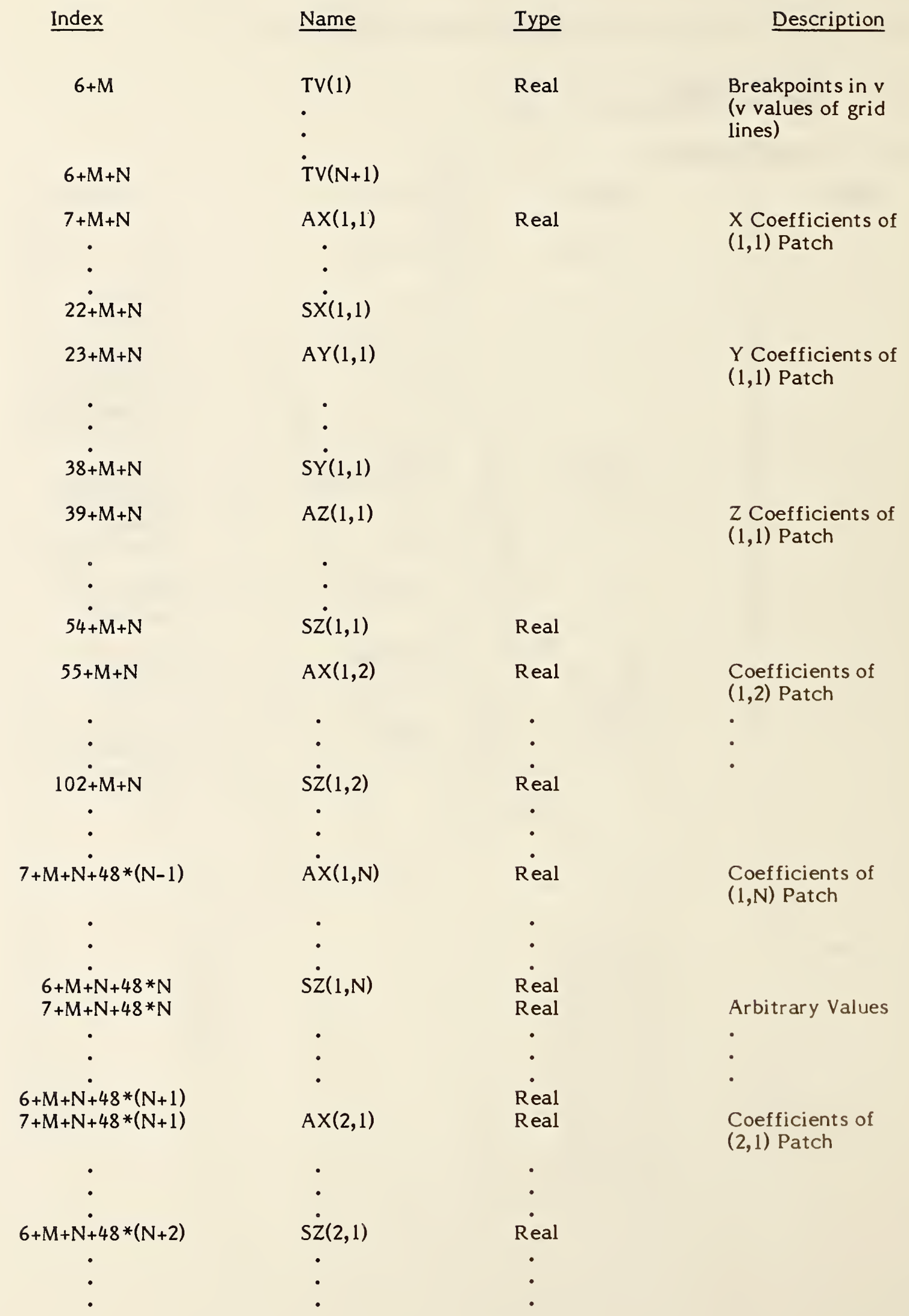




\begin{tabular}{|c|c|c|c|}
\hline Index & Name & Type & Description \\
\hline $7+M+N+48 *(2 * N)$ & $A \times(2, N)$ & Real & $\begin{array}{l}\text { Coefficients of } \\
(2, N) \text { Patch }\end{array}$ \\
\hline - & $\cdot$ & • & - \\
\hline - & - & - & - \\
\hline & & $\therefore$ & - \\
\hline $\begin{array}{l}6+M+N+48 *(2 * N+1) \\
7+M+N+48 *(2 * N+1)\end{array}$ & $S Z(2, N)$ & $\begin{array}{l}\text { Real } \\
\text { Real }\end{array}$ & Arbitrary Values \\
\hline$\cdot$ & & $\cdot$ & $\cdot$ \\
\hline$\cdot$ & & • & - \\
\hline $6+M+N+48 *(2 * N+2)$ & & Real & • \\
\hline$\cdot$ & & & \\
\hline$\dot{\bullet}$ & & & \\
\hline $7+\mathrm{M}+\mathrm{N}+48 *[(\mathrm{~J}-1) *(\mathrm{~N}+1)+\mathrm{K}-1]$ & $A \times(J, K)$ & Real & $\begin{array}{l}\text { Coefficients of } \\
(\mathrm{J}, \mathrm{K}) \text { Patch }\end{array}$ \\
\hline - & - & - & - \\
\hline$\cdot$ & $\cdot$ & $\cdot$ & - \\
\hline $6+\mathrm{M}+\dot{\mathrm{N}}+48 *[(\mathrm{~J}-1) *(\mathrm{~N}+1)+\mathrm{K}]$ & $\mathrm{SZ}(\mathrm{J}, \mathrm{K})$ & Real & • \\
\hline$\cdot$ & $\cdot$ & $\cdot$ & \\
\hline$\cdot$ & $\cdot$ & $\cdot$ & \\
\hline $7+\mathrm{M}+\mathrm{N}+\dot{48} *[(\mathrm{M}-1) *(\mathrm{~N}+1)+\mathrm{N}-1]$ & $\dot{A X}(M, N)$ & Real & $\begin{array}{l}\text { Coefficients of } \\
(M, N) \text { Patch }\end{array}$ \\
\hline • & $\cdot$ & $\cdot$ & $\cdot$ \\
\hline$\cdot$ & $\cdot$ & $\cdot$ & - \\
\hline $6+\mathrm{M}+\mathrm{N}+\dot{4} 8 *[(\mathrm{M}-1) *(\mathrm{~N}+1)+\mathrm{N}]$ & $\dot{s} \dot{Z}(M, N)$ & Real & • \\
\hline $7+\mathrm{M}+\mathrm{N}+48 *[(\mathrm{M}-1) *(\mathrm{~N}+1)+\mathrm{N}]$ & & Real & Arbitrary Values \\
\hline$\cdot$ & & $\cdot$ & $\cdot$ \\
\hline$\dot{\cdot}$ & & $\dot{\cdot}$ & $\dot{-}$ \\
\hline \multicolumn{2}{|l|}{$6+\mathrm{M}+\mathrm{N}+\dot{4} 8 *[(\mathrm{M}-1) *(\mathrm{~N}+1)+(\mathrm{N}+1)]$} & Real & \\
\hline \multicolumn{2}{|l|}{$7+M+N+48 *[M *(N+1)]$} & Real & Arbitrary Values \\
\hline \multirow{2}{*}{\multicolumn{2}{|c|}{$\cdot$}} & $\cdot$ & $\cdot$ \\
\hline & & $\dot{.}$ & $\cdot$ \\
\hline \multicolumn{2}{|l|}{$6+M+N+48 *[M *(N+1)+(N+1)]$} & Real & \\
\hline
\end{tabular}

Additional Pointers as required (see 2.2.4.4.2).

Software to convert between parametric spline curves or surfaces and the corresponding rational B-spline curves or surfaces is available from the IGES office at the National Bureau of Standards. Materials provided include a magnetic tape of Pascal source code, a listing of the code, and accompanying documentation. 


\subsection{Point Entity}

A point is defined by its coordinates in definition space. Examples of the point entity are shown in Figure 3-10.

\subsubsection{Directory Data}

ENTITY TYPE NUMBER: 116

\subsubsection{Parameter Data}

Index

1

2

3

4

4

Additional Pointers as required (see 2.2.4.4.2).

$\begin{array}{cll}\text { Name } & \text { Type } & \text { Description } \\ \mathrm{X} & \text { Real } & \text { Coordinates } \\ \mathrm{Y} & \text { Real } & \text { of point } \\ \mathrm{Z} & \text { Real } & \\ \text { PTR } & \text { Pointer } & \begin{array}{l}\text { Pointer to directory } \\ \text { entry of } \\ \text { subfigure instance } \\ \text { specifying the display } \\ \text { symbol. } \\ \text { If zero, no display } \\ \text { symbol specified. }\end{array}\end{array}$


$m$

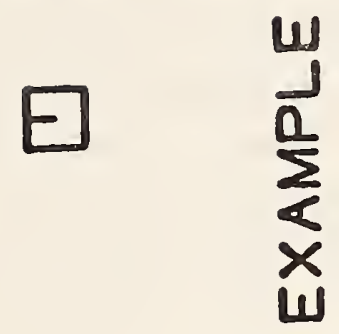

$\phi$

$N$

W

$\sum_{\frac{1}{2}} \frac{0}{2}$

W

岃

옴

-

W

1

$\sum_{x}^{2}$

w 


\subsection{Ruled Surface Entity}

A ruled surface is formed by moving a line connecting points of equal relative arc length (Form 0) or equal relative parametric value (Form 1) on two parametric curves from a start point to a terminate point on the curves. The parametric curves may be points, lines, circles, conics, parametric splines, rational B-splines, composite curves, or any parametric curves defined in this Specification (both planar and non-planar).

\subsection{1 .1}

Form 0.

In this case, DE1 and DE2 specify the defining rail curves, but their given parametrizations are not the ones used to generate the ruled surface. Instead, their arc length reparametrizations, $\mathrm{C} 1$ and $\mathrm{C} 2$ (respectively), are used.

Form 1 .

In this case, DE1 and DE2 specify the defining rail curves, $\mathrm{C} 1$ and $\mathrm{C} 2$ (respectively). Moreover, their given parametrizations are the ones used to generate the ruled surface.

For both Form 0 and Form 1 .

In either case, the two curves are expressed parametrically by the functions $\left(\mathrm{C} 1_{X}{ }^{(t), C 1} \mathrm{C}_{Y}(\mathrm{t}), \mathrm{C} 1_{Z}(\mathrm{t})\right)$ and $\left(\mathrm{C} 2 \mathrm{X}^{(\mathrm{s}), C 2} \mathrm{C}_{\mathrm{Y}}(\mathrm{s}), \mathrm{C} 2_{Z}(\mathrm{~s})\right)$, with range $a \leqslant t \leqslant b$ and $c \leqslant s \leqslant d$, then the coordinates of the points on the ruled surface can be written as

$$
\begin{aligned}
& X(u, v)=(1-v){ }^{\circ} \mathrm{C} 1_{X}(l)+v^{*} \mathrm{C} 2_{X}(s) \\
& Y(u, v)=(1-v){ }^{\circ} \mathrm{C} 1_{Y}(t)+v^{*} \mathrm{C} 2_{Y}(s) \\
& Z(u, v)=(1-v){ }^{\circ} \mathrm{C} 1_{Z}(t)+v^{*} \mathrm{C} 2_{Z}(s) \\
& \text { where } \\
& \quad 0 \leqslant u \leqslant 1, \\
& \quad 0 \leqslant v \leqslant 1, \\
& \mathrm{t}=\mathrm{a}+\mathrm{u}^{*}(\mathrm{~b}-\mathrm{a}) \\
& \mathrm{s}=\mathrm{C}+\mathrm{u}^{*}(\mathrm{~d}-\mathrm{c}), \text { if DIR FLG }=0 \\
& \mathrm{~s}=\mathrm{d}+\mathrm{u}^{*}(\mathrm{c}-\mathrm{d}), \text { if DIRFLG }=1
\end{aligned}
$$


$C 1(t)$ and $C_{2}(s)$ are said to be of equal relative parametric value if $t$ and $s$ are evaluated at the same $u$ value.

3.11.2 In case DIRFLG $=0$, then the first point of curve 1 is joined to the first point of curve 2 and the last point of curve ! to last point of curve 2. If DIRFLG=1, then the first point of curve 1 is joined to the last point of curve 2, the last point of curve 1 to the first point of curve 2 .

3.11.3 If DEVFLG $=1$, then the surface is a developable surface; if DEVFLG=0, the surface may or may not be a developable surface.

3.11.4 Field 15 of the directory entry accommodates a Form Number. For this entity the options are as follows:

FORM

0

1

\section{$\underline{\text { MEANING }}$}

Equal relative arc length

Equal relative parametric values.

The default is FORM 0.

3.11.5 An example of the Ruled Surface Entity is shown in Figure 3-11. Additional examples are shown in Figure 3-12. 


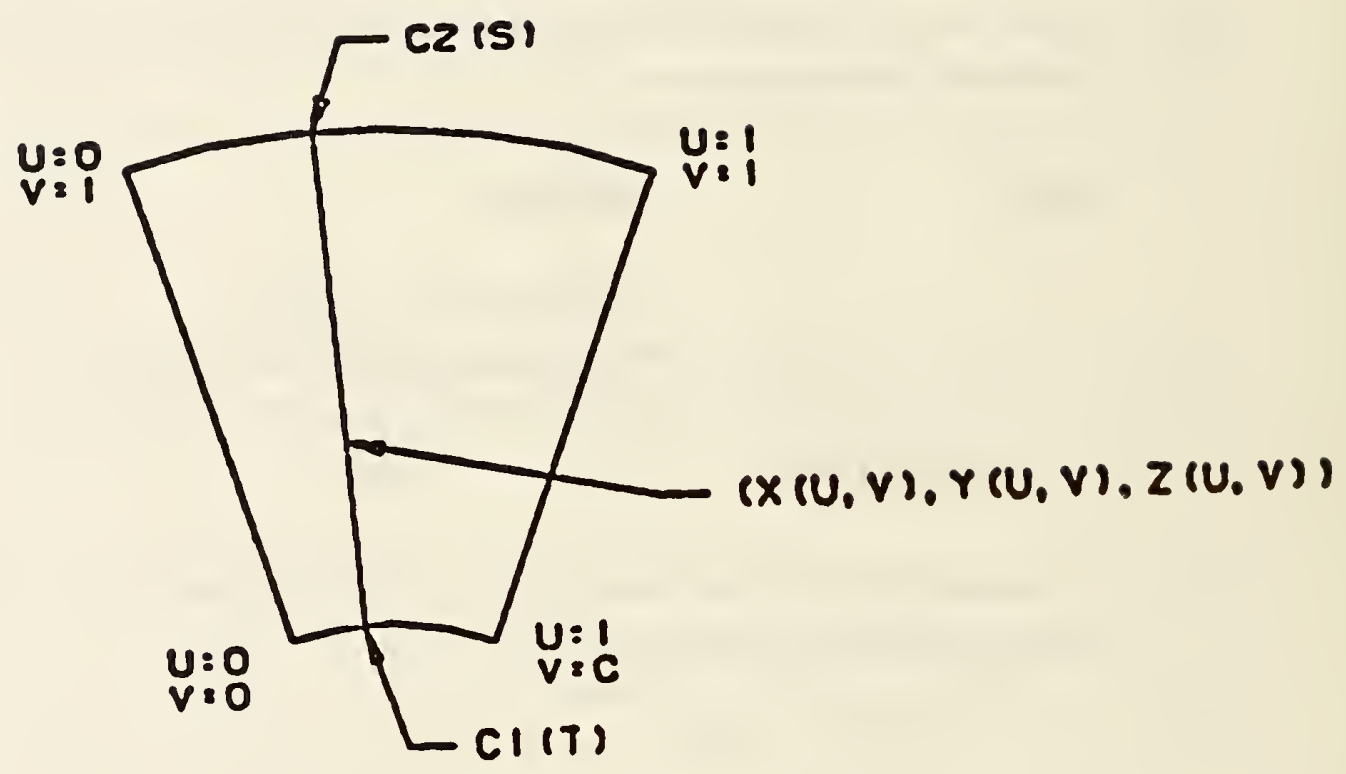

FIG. 3-11 EXAMPLE OF THE RULED SURFACE ENTITY 


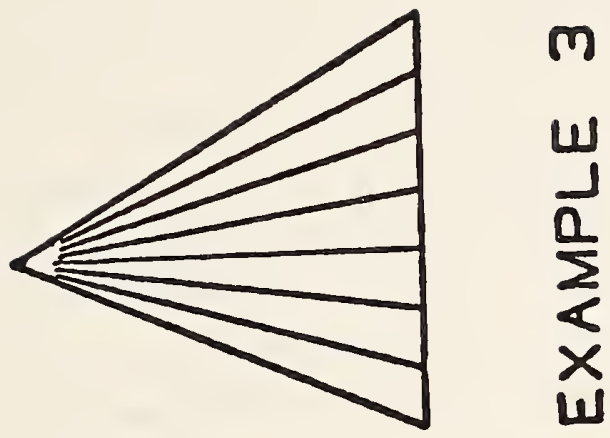

$E$

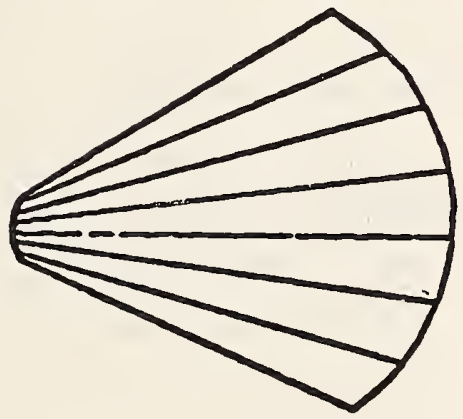

$N$

屴

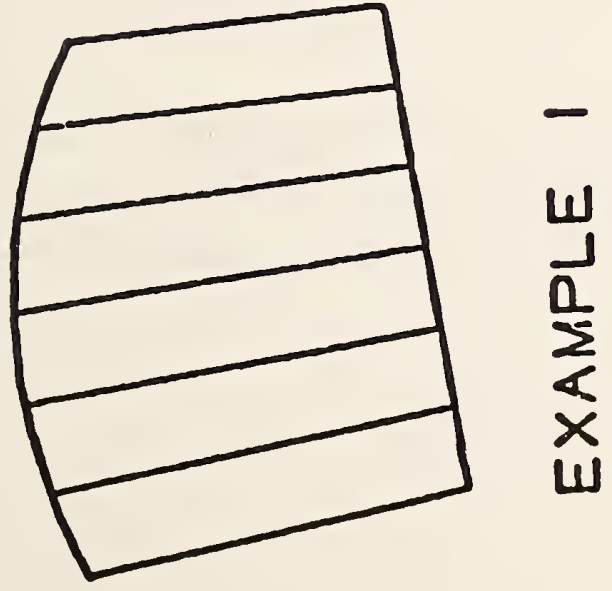

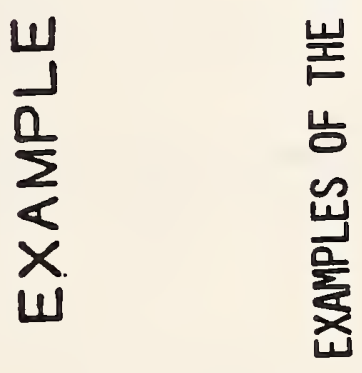

M

$\stackrel{5}{4}$ 


\subsubsection{Directory Data}

ENTITY TYPE NUMBER : 118

\subsubsection{Parameter Data}

\begin{tabular}{|c|c|c|c|}
\hline$\underline{\text { Index }}$ & Name & Type & Description \\
\hline 1 & DE1 & Pointer & $\begin{array}{l}\text { Pointer to first } \\
\text { curve }\end{array}$ \\
\hline 2 & DE2 & Pointer & $\begin{array}{l}\text { Pointer to } \\
\text { second curve }\end{array}$ \\
\hline 3 & DIRFLG & Integer & $\begin{array}{l}\text { Direction flag } \\
(0=\text { join first to } \\
\text { first, last to } \\
\text { last } \\
1=\text { join first to } \\
\text { last, last to } \\
\text { first) }\end{array}$ \\
\hline 4 & DEVFLG & Integer & $\begin{array}{l}\text { Developable } \\
\text { surface flag } \\
\text { ( } 1=\text { Developable, } \\
0=\text { Possibly not) }\end{array}$ \\
\hline
\end{tabular}




\subsection{Surface of Revolution Entity}

A surface of revolution is defined by an axis of rotation (which must be a line entity), a generatrix, and start and terminate rotation angles. The surface is created by rotating the generatrix about the axis of rotation through the start and terminating angles. Since the axis of rotation is a line entity, it contains in its parameter data section the coordinates of its start point first, followed by the coordinates of its terminate point. The angles of rotation are counterclockwise in the positive direction (See section 3.1.2). The generatrix may be a conic arc, line, circular arc, parametric spline curve, rational B-spline curve, or composite curve.

3.12.1 Examples of surface of revolution entities are shown in Figure 3-13.

3.12.2 The start and terminate angles of the surface can be explained by geometric construction. Refer to Figure 3-14 and the following:

a. Select a point on the generatrix which does not lie on the axis of rotation; label the point $\mathrm{Pl}$.

b. Construct a line through Pl such that it is perpendicular to the axis of rotation extended; label this line $\mathrm{Ll}$.

c. Construct a plane PN1 containing $\mathrm{L} 1$ and perpendicular to the axis of rotation.

d. All rotations in the plane PN1 about the axis of rotation are applied counterclockwise according to the method described in 3.12 .

e. Rotate counterclockwise the line $\mathrm{Ll}$ and the point selected from the generatrix the number of radians indicated in the start angle resulting in $\mathrm{LI}_{\mathrm{SA}}$. The location is labeled LOC 1 . 
f. Rotate counterclockwise the line $\mathrm{L} 1$ and the point $\mathrm{Pl}$ selected from the generatrix an additional number of radians given by the terminate angle minus the start angle resulting in $\mathrm{LI}_{\mathrm{TA}}$. The second location of the point is labeled LOC2.

g. The resulting surface is that generated by rotating the generatrix from LOC1 to LOC2.

\subsubsection{Directory Data}

ENTITY TYPE NUMBER : 120

\subsubsection{Parameter Data}

$\begin{array}{cc}\text { Index } & \text { Name } \\ 1 & \text { DE1 } \\ 2 & \text { DE2 } \\ 3 & \text { SA } \\ 4 & \text { TA }\end{array}$

Type

Pointer

Pointer

Real

Real

\section{Description}

Pointer to a line entity (axis of revolution)

Pointer to generatrix

Start angle in radians

Terminate angle in radians

Additional pointers as required (see 2.2.4.4.2). 


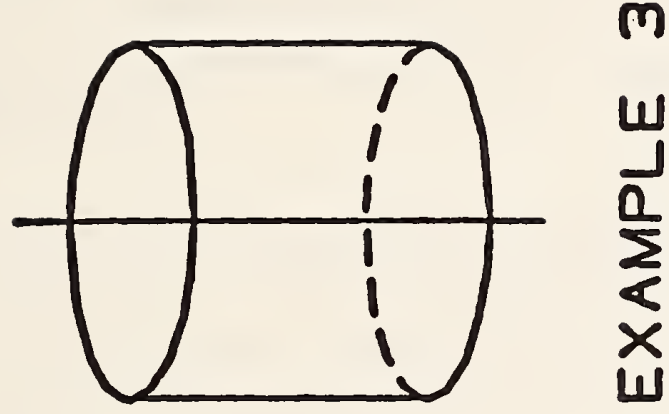

$m$

$\frac{\omega}{\frac{\omega}{2}}$

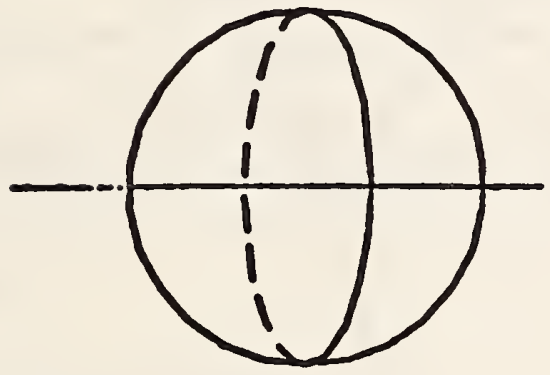

N

E

W

$\frac{1}{2}$

를

U1

놑

岁

岂

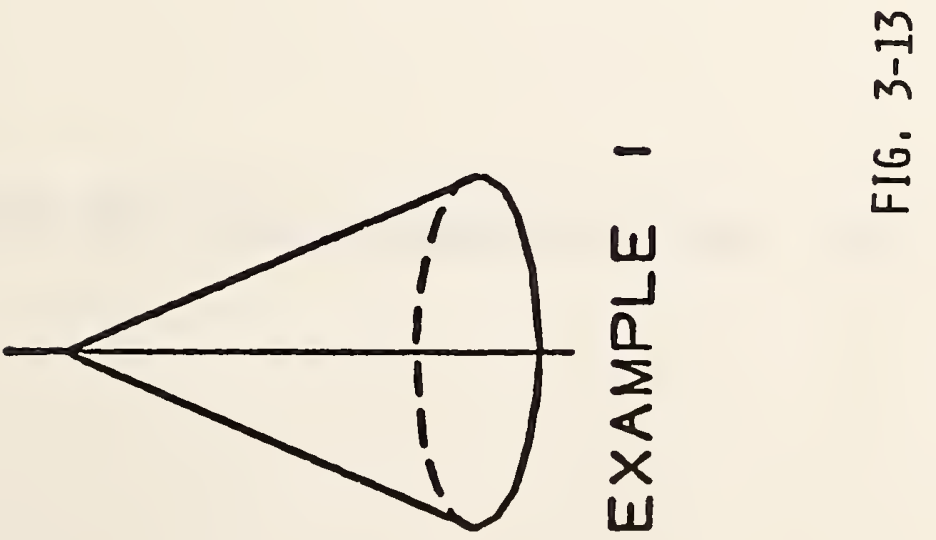




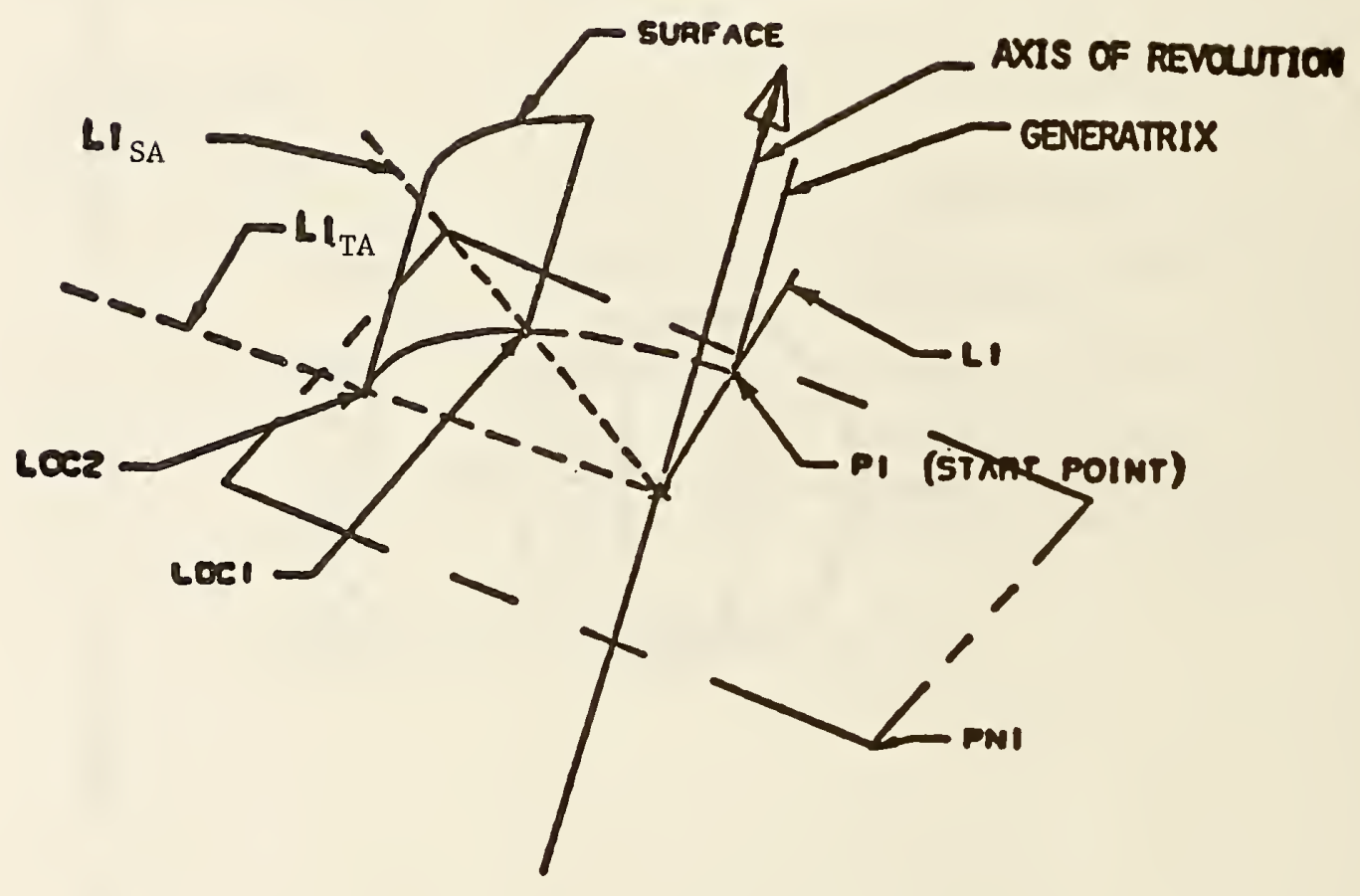

FIG. 3-14 SURFACE OF REVOLUTION START AND TERMINATING ANGLES 
3.13 Tabulated Cylinder Entity

A tabulated cylinder is a surface formed by moving a line segment called the generatrix parallel to itself along a curve called the directrix. This curve may be a line, circular arc, conic arc, parametric spline cürve, rational B-spline curve, or composite curve.

3.13.1 It must be pointed out that different parameterizations of the generating curves will produce different parameterized surfaces, but the underlying point set surface will still be the same. Assuming a parameterization $u$ on the directrix and $v$ on the generatrix, both of which run from 0 to 1 , we can express the points on the surface by

$$
\begin{aligned}
X(u, v) & =C X(u)+v^{*}(L X-C X(0)) \\
Y(u, v) & =C Y(u)+v^{*}(L Y-C Y(0)) \\
Z(u, v) & =C Z(u)+v^{*}(L Z-C Z(0))
\end{aligned}
$$

where $0 \leqslant u \leqslant 1,0 \leqslant v \leqslant 1$

and $\mathrm{CX}, \mathrm{CY}, \mathrm{CZ}$ represent the $\mathrm{X}, \mathrm{Y}, \mathrm{Z}$ components, respectively, along the directrix curve, while $(C X(0), C Y(0), C Z(0))$ and (LX, LY, LZ) represent the coordinates of the start and terminate points, respectively, of the generatrix.

3.13.2 An example of the tabulated cylinder is shown in Figure 3-15. 


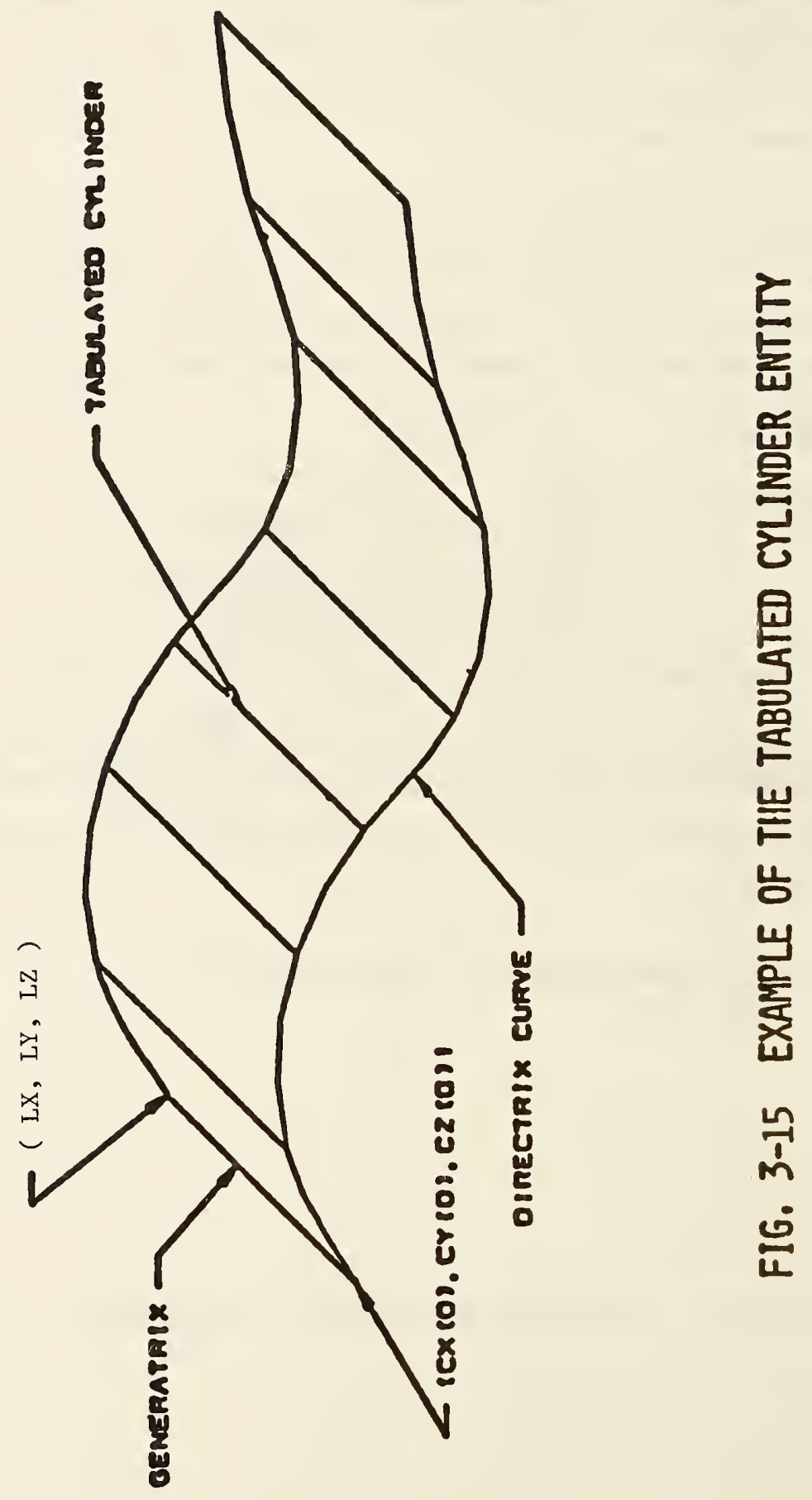




\subsubsection{Directory Data}

ENTITY TYPE NUMBER :

122

\subsubsection{Parameter Data}

$\begin{array}{ccc}\text { Index } & \frac{\text { Name }}{\text { DEI }} & \text { Type } \\ 1 & \text { LX } & \text { Point } \\ 2 & & \text { Real } \\ 3 & \text { LY } & \text { Real } \\ 4 & \text { LZ } & \text { Real }\end{array}$

Additional Pointers as required (see 2.2.4.4.2).

\begin{abstract}
Description
Pointer to directrix curve

Coordinates of the terminate point of the generatrix. The start point of the generatrix is identical with the start point of the directrix.
\end{abstract}




\subsection{Transformation Matrix Entity}

The Transformation Matrix entity transforms three-row column vectors by means of a matrix multiplication and then a vector addition. The notation for this transformation is

$$
\left[\begin{array}{lll}
R_{11} & R_{12} & R_{13} \\
R_{21} & R_{22} & R_{23} \\
R_{31} & R_{32} & R_{33}
\end{array}\right]\left[\begin{array}{l}
\text { XINPUT } \\
\text { YINPUT } \\
\text { ZINPUT }
\end{array}\right]+\left[\begin{array}{l}
T_{1} \\
T_{2} \\
T_{3}
\end{array}\right]=\left[\begin{array}{l}
\text { XOUTPUT } \\
\text { YOUTPUT } \\
\text { ZOUTPUT }
\end{array}\right]
$$

Here, col [XINPUT, YINPUT, ZINPUT] (i.e., the column vector) is the vector being transformed, and col [XOUTPUT, YOUTPUT, ZOUTPUT] is the column vector resulting from this transformation. $R=\left[R_{i j}\right]$ is a 3 row by 3 column matrix of real numbers, and $\mathrm{T}=\mathrm{col}[\mathrm{T} 1, \mathrm{~T} 2, \mathrm{~T} 3]$ is a three-row column vector of real numbers. Thus, 12 real numbers are required for a Transformation Matrix entity. This entity can be considered to be an "operator" entity in that it starts with the input vector, operates on it as described above, and produces the output vector.

3.14.1 Frequently, the input vector lists the coordinates of some point in one coordinate system, and the output vector lists the coordinates of that same point in a second coordinate system. The matrix $R$ and the translation vector $T$ then express a general relationship between the two coordinate systems. By considerating special input vectors such as $\operatorname{col}[1,0,0], \operatorname{col}[0,1,0]$, and $\operatorname{col}[0,0,1]$ and computing the corresponding output results, a geometric appreciation of the spatial relationship between the two coordinate system can be gained.

For example, for

$$
R=\left[\begin{array}{rrr}
0 & 0 & 1 \\
0 & 1 & 0 \\
-1 & 0 & 0
\end{array}\right] T=\left[\begin{array}{l}
0 \\
0 \\
0
\end{array}\right]
$$


the spatial relationship of the input and output cordinate systems is the following:

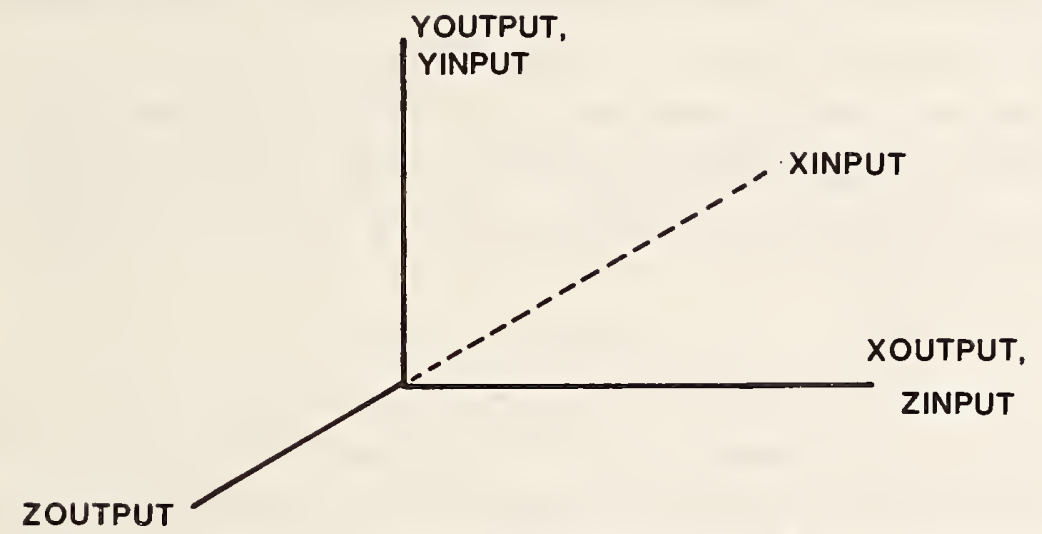

FIGURE $3-16$

All coordinate systems are assumed to be or thogonal, cartesian, and right-handed unless specifically noted otherwise.

3.14.2 Following are three specific areas where the Transformation Matrix entity is used to transform coordinates between coordinate systems. Each example area illustrates a specific choice of input and output coordinate system. Other choices of coordinate systems may be appropriate in other application areas.

The usual situation for this type of use of the Transformation Matrix entity is when the input vector refers to the definition space coordinate system for a certain entity, and the output vector refers to the model space coordinate system. (See Sect. 3.1.1) In this case, the matrix $R$ is referred to as the defining matrix, and the Transformation Matrix entity defining $R$ and $T$ is pointed to in field seven (transformation matrix field) of the directory entry of the entity. (See Sect. 2.2.4.3.7) In this use of the Transformation Matrix entity, the matrix $\mathrm{R}$ is subject to the restrictions given in Form 0 and Form 1 below. 
A second situation is the case when the input vector refers to the model space coordinate system and the output vector refers to a viewing coordinate system. In this case, the matrix $\mathrm{R}$ is referred to as a view matrix, and is subject to the restrictions given in Form 0 below. Note that when a planar entity is viewed at true length (i.e., the viewing plane is parallel to the plane containing the entity) then the rotation matrix pointed to by DE Field 7 of the planar entity will be the inverse (=matrix transpose) of the matrix pointed to by DE Field 7 of the View entity. (See Sect. 4.3.11)

A third situation involves finite element modeling applications. Here, it may be the case that an input coordinate system is related to an output coordinate system by a particular $\mathrm{R}$ and $\mathrm{T}$, and, in turn, the output coordinate system is then taken as an input coordinate system for a second $R$ and $T$ combination, and so on. These coordinate systems are frequently called local coordinate systems. Model space is frequently called the reference system. For example, the location of a finite element node may be given in one local coordinate system, which may serve as the input coordinate system for a second local coordinate system, which in turn serves as the input coordinate system for the model space coordinate system which is the reference system. Allowable forms of the matrix $\mathrm{R}$ for these applications are detailed in Forms 10, 11, and 12 below.

3.14.3 Whenever coordinate systems are related successively to each other as described above, a basic result is that the combined effect of the individual coordinate system changes can be expressed in terms of a single matrix $R$ and a single translation vector $T$. For example, if the coordinate system change involving the matrix R2 and the translation vector T2 is to be applied following the coordinate system change involving the matrix $\mathrm{R} 1$ and the translation vector $\mathrm{T} 1$, then the matrix $R$ and the translation vector $T$ expressing the combined changes are $R=(R 2)(R 1)$ and $T=(R 2)(T 1)+T 2$.

Here, (R2) (R1) denotes matrix multiplication of $3 \times 3$ matrices, where multiplication order is important. The matrix $R$ and the translation vector $T$ are computed similarly whenever more than two coordinate system changes are to be applied successively. 
Successive coordinate system changes are specified by allowing a Transformation Matrix entity to reference another Transformation Matrix entity through Field 7 of the Directory Entry. In the example above, the Transformation Matrix entity containing $\mathrm{R} \mathrm{I}$ and $\mathrm{T} 1$ would contain in its Directory Entry Field 7 a pointer to the Transformation Matrix entity containing R2 and T2. The general rule is that Transformation Matrix entities applied earlier in a succession will reference Transformation Matrix entities applied later. Note that the matrix product (R2) (R1) in the example above does not appear explicitly in the data, but, if needed, must be computed according to the usual rules of matrix multiplication.

A second example of coordinate systems being related successively (or "concatenated", or "stacked"), in addition to the finite element example mentioned above, involves one manner of locating into model space a çonic arc that is in standard position in definition space. In this case, RI and Tl move the conic arc from its standard position to an arbitrary location in any plane in definition space satisfying $\mathrm{ZT}=$ constant. (Therefore, $\mathrm{Rl}_{33}=1.0$, $\mathrm{R}_{131}=\mathrm{R} 1_{32}=\mathrm{R}_{13}=\mathrm{R}_{23}=0.0$. T 1 can be an arbitrary translation vector.) $\mathrm{R} 2$ and $\mathrm{T} 2$ then position the relocated conic arc into model space. ( $R 2$ can be an arbitrary defining matrix and $\mathrm{T} 2$ can be an arbitrary translation vector.) Note that for $\mathrm{Rl}$ and $\mathrm{Tl}$, both the input vector and the output vector refer to the same coordinate system, namely, the definition space for the conic arc.

3.14.4 A $3 \times 3$ matrix $R$ is called or thogonal provided $i$ ts transpose, $R^{t}$, about the main diagonal yields a matrix inverse for $R$. The columns of an orthogonal matrix considered as vectors form an or thogonal collection of unit vectors. As $(R)^{t}=R$, the transpose of an orthogonal matrix is again an orthogonal matrix. The determinant of an or thogonal matrix is equal to either plus one or minus one. In the event $R$ is an or thogonal matrix with determinant equal to positive one, $R$ can be expressed as a rotation about an axis passing through the origin. In this event, $R$ is referred to as a rotation matrix. In the event $R$ is an or thogonal matrix with determinant equal to negative one, $\mathrm{R}$ can be expressed as a rotation about an axis passing through the origin followed by a reflection about a plane passing through the origin perpendicular to the axis of rotation. 
3.14.5 Allowable Form Numbers. The defining matrix of an entity must use either Form zero or Form one. A defining matrix associated with a view entity must use Form zero.

Form 0: (default) $\mathrm{R}$ is an orthogonal matrix with determinant equal to positive one. $\mathrm{T}$ is arbitrary. The columns of $\mathrm{R}$ taken in order form a right-handed triple in the output coordinate system.

Form 1: $\mathrm{R}$ is an or thogonal matrix with determinant equal to negative one. $\mathrm{T}$ is arbitrary. The columns of $\mathrm{R}$ taken in order form a left-handed triple in the output coordinate system.

3.14.6 Forms 10, 11, 12. These form numbers indicate special matrices used in conjunction with the node entity (type number 134 ).

Form 10: This form number conveys special information when used in conjunction with the Node entity (type number 134) in Finite Element Applications.

Refer to Fig. 3-17(a) for notation. The matrix $R$ and the vector $T$ are used to transform coordinate data from the $u 1, u 2, u 3$ coordinate system to the $x, y, z$ local system.

The $u 1, u 2, u 3$ coordinate system has its origin at an arbitrary fixed point col XOFFSET, YOFFSET, ZOFFSET in the $x, y, z$ coordinate system and is assumed to be displaced parallel to that reference coordinate system. Thus,

$$
R=\left[\begin{array}{lll}
1 & 0 & 0 \\
0 & 1 & 0 \\
0 & 0 & 1
\end{array}\right], \quad T=\left[\begin{array}{c}
\text { XOFFSET } \\
\text { YOFFSET } \\
\text { ZOFFSET }
\end{array}\right]
$$


(a) Cartesian

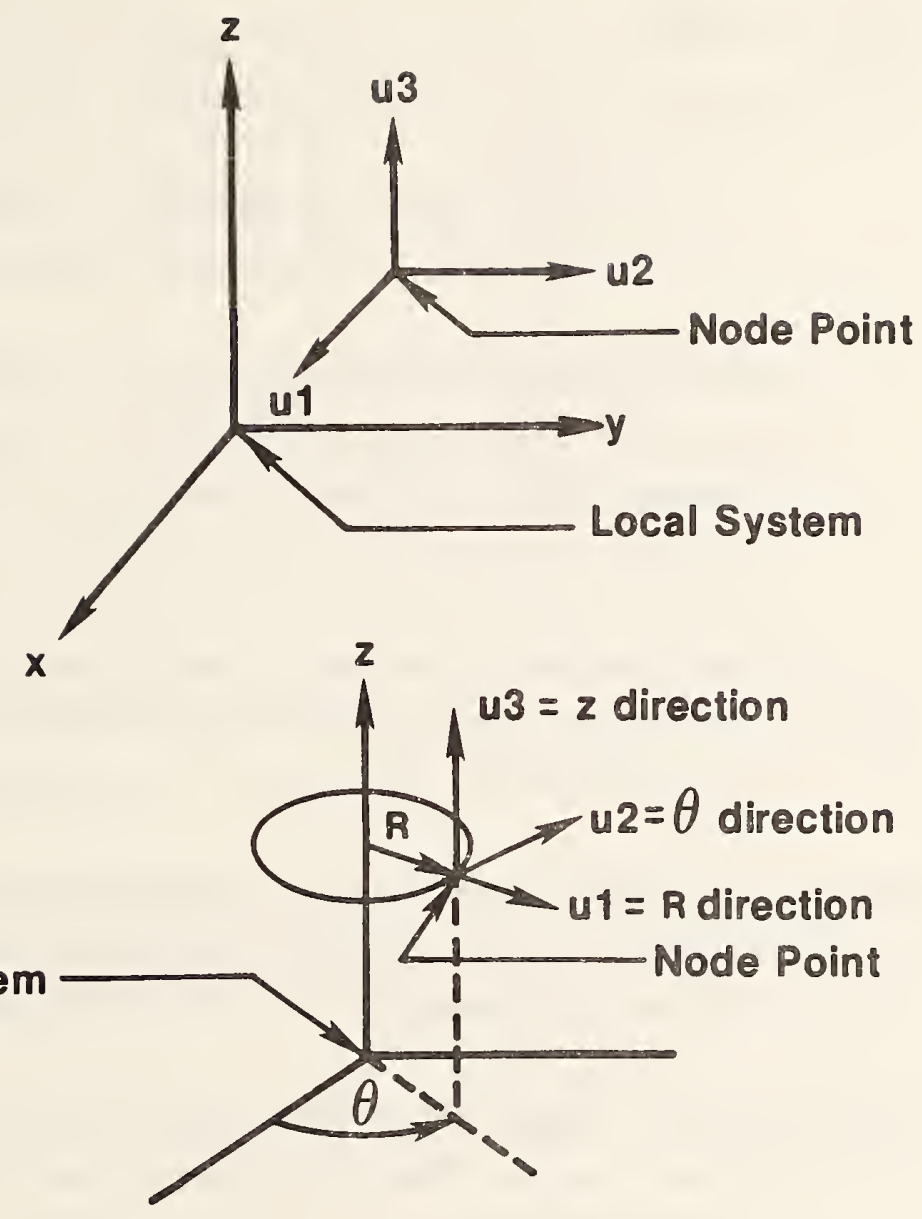

(b) Cylindrical

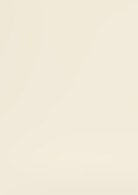


so that

$$
\left[\begin{array}{lll}
1 & 0 & 0 \\
0 & 1 & 0 \\
0 & 0 & 1
\end{array}\right]\left[\begin{array}{l}
u 1 \\
u 2 \\
u 3
\end{array}\right]+\left[\begin{array}{l}
\text { XOFFSET } \\
\text { YOFFSET } \\
\text { ZOFFSET }
\end{array}\right]=\left[\begin{array}{l}
\text { XLOCAL } \\
\text { YLOCAL } \\
\text { ZLOCAL }
\end{array}\right]
$$

Note that the orientation of the two coordinate systems can be described by saying that the ul,u2,u3 coordinate system is the system obtained by imposing or thogonal curvilinear coordinates onto the $x, y, z$ space and then constructing unit tangent vectors to the three curvilinear coordinate curves at the given fixed point to serve as basis vectors. In this special case of parallel displacement, the curvilinear coordinates imposed are identical to the existing $x, y, z$ coordinates.

Form 11: This form number conveys special information when used in conjunction with the Node entity (type number 134) in Finite Element applications.

Refer to Figure 3-17(b) for notation. The matrix $\mathrm{R}$ and the vector $\mathrm{T}$ are used to transform coordinate data from the u1, u2, u3 coordinate system to the $x, y, z$ local system.

The $u 1, u 2, u 3$ coordinate system has its origin at an arbitrary fixed point

$$
\begin{array}{rlrl}
\text { XOFFSET }= & r_{0} \cos \theta_{0} & r_{0} & 0 \\
\text { YOFFSET }= & r_{0} \sin \theta_{0} & 0 & \theta_{0} 360^{\circ} \\
\text { ZOFFSET }= & z_{0} & -\infty<z_{0}<\infty
\end{array}
$$

in the $x, y, z$ coordinate system. The $u 1, u 2, u 3$ system is the system obtained by imposing or thogonal curvilinear coordinates onto the $x, y, z$ space which are the cylindrical coordinates $(r, \theta, z)$ with

$$
\begin{aligned}
& x=r \cos \theta \\
& y=r \sin \theta \\
& z=z,
\end{aligned}
$$


and then constructing unit tangent vectors to the three curvilinear coordinate curves at the given fixed point to serve as basis vectors.

Thus, the relationship between the $u 1, u 2, u 3$ and the $x, y, z$ local coordinate system is given by

$$
\left[\begin{array}{ccc}
\cos \theta_{0} & -\sin \theta_{0} & 0 \\
\sin \theta_{0} & \cos \theta_{0} & 0 \\
0 & 0 & 1
\end{array}\right]\left[\begin{array}{l}
u 1 \\
u 2 \\
u 3
\end{array}\right]+\left[\begin{array}{l}
\text { XOFFSET } \\
\text { YOFFSET } \\
\text { ZOFFSET }
\end{array}\right]=\left[\begin{array}{l}
\text { XLOCAL } \\
\text { YLOCAL } \\
\text { ZLOCAL }
\end{array}\right]
$$

Form 12: This form number conveys special information when used in conjunction with the Node entity (type number 134) in Finite Element applications.

Refer to Fig. 3-17(c) for notation. The matrix $R$ and the vector $T$ are used to transform coordinate data from the ul, u2, u3 coordinate system to the $x, y, z$ local system.

The u1, u2, u3 coordinate system has its origin at an arbitrary fixed point

$$
\begin{array}{rlrl}
\text { XOFFSET } & =r_{0} \sin \theta_{0} \sin \emptyset_{0} & & r_{0} \geqslant 0 \\
\text { YOFFSET } & =r_{0} \sin \theta_{0} \cos \emptyset_{0} & & 0 \leqslant \theta_{0} \leqslant 180^{\circ} \\
\text { ZOFFSET } & =r_{0} \cos \theta_{0} & & 0 \leqslant \emptyset_{0}<360^{\circ} \\
\text { for } r_{0} & =0, \text { take } \theta_{0}=\emptyset_{0}=0^{\circ} & \\
\text { for } \theta_{0}=0^{\circ} \text { or } 180^{\circ}, \text { take } \emptyset_{0}=0^{\circ} &
\end{array}
$$

in the $x, y, z$ coordinate system. The $u 1, u 2, u 3$ system is the system obtained by imposing or thogonal curvilinear coordinates onto the $x, y, z$ space which are the spherical coordinates $(r, \theta, \emptyset)$ with

$$
\begin{aligned}
& X=r \sin \theta \cos \emptyset \\
& Y=r \sin \theta \sin \emptyset \\
& Z=r \cos \theta
\end{aligned}
$$


and then constructing unit tangent vectors to the three curvilinear coordinate curves at the given fixed point to serve as basis vectors.

Thus, the relationship between the $u 1, u 2, u 3$ and the $x, y, z$ local coordinate systems is given by

$$
\left[\begin{array}{ccccc}
\sin \theta_{0} & \cos \emptyset_{0} & \cos \theta_{0} & \cos \emptyset_{0} & -\sin \emptyset_{0} \\
\sin \theta_{0} & \sin \emptyset_{0} & \cos \theta_{0} & \sin \emptyset_{0} & \cos \emptyset_{0} \\
\cos \theta_{0} & -\sin \theta_{O} & 0
\end{array}\right]\left[\begin{array}{l}
u 1 \\
u 2 \\
u 3
\end{array}\right]+\left[\begin{array}{l}
\text { XOFFSET } \\
\text { YOFFSET } \\
\text { ZOFFSET }
\end{array}\right]=\left[\begin{array}{l}
\text { XLOCAL } \\
\text { YLOCAL } \\
\text { ZLOCAL }
\end{array}\right]
$$

See, Kaplan, (KAPL52) or Hildebrand, (HILD76) for a discussion of or thogonal curvilinear coordinate systems.

\subsubsection{Directory Data}

ENTITY TYPE NUMBER: 124

\subsubsection{Parameter Data}

$\begin{array}{llll}\text { Index } & \text { Name } & \text { Type } & \text { Description } \\ 1 & \text { R11 } & \text { Real } & \text { Top Row } \\ 2 & \text { R12 } & \text { Real } & \\ 3 & \text { R13 } & \text { Real } & \\ 4 & \text { T1 } & \text { Real } & \\ 5 & \text { R21 } & \text { Real } & \text { Second Row } \\ 6 & \text { R22 } & \text { Real } & \\ 7 & \text { R23 } & \text { Real } & \\ 8 & \text { T2 } & \text { Real } & \\ 9 & \text { R31 } & \text { Real } & \text { Third Row } \\ 10 & \text { R32 } & \text { Real } & \\ 11 & \text { R33 } & \text { Real } & \\ 12 & \text { T3 } & \text { Real } & \end{array}$

Additional Pointers as required (see 2.2.4.4.2). 


\subsection{Flash Entity}

A flash entity is a point in the $Z \mathrm{~T}=0$ plane that locates a specific instance of a particular closed area. That closed area can be defined in one of two ways. First, it can be an arbitrary closed area defined by any entity capable of defining a closed area. The points of this entity must all lie in the $Z T=0$ plane. Second, it can be a member of a predefined set of flash shapes.

In the latter case, parameters 3 through 5 of the fiash entity control the final size of the flash. Figure 3-18 indicates the usage of those parameters for the specific flash forms. Parameters 3 through 5 are ignored for form zero.

\subsubsection{Directory Data}

ENTITY TYPE NUMBER:

$\begin{array}{cl}\text { Form } & \text { Meaning } \\ 0 & \text { defined by referenced entity } \\ 1 & \text { circular } \\ 2 & \text { rectangle } \\ 3 & \text { donut } \\ 4 & \text { canoe }\end{array}$

\subsubsection{Parameter Data}

\begin{tabular}{|c|c|c|c|}
\hline Index & Name & Type & Description \\
\hline 1 & $x$ & Real & $\begin{array}{l}X \text { reference of } \\
\text { flash }\end{array}$ \\
\hline 2 & $\mathrm{Y}$ & Real & $\begin{array}{l}\text { Y reference of } \\
\text { flash }\end{array}$ \\
\hline 3 & DIM 1 & Real & $\begin{array}{l}\text { First flash sizing } \\
\text { parameter }\end{array}$ \\
\hline 4 & DIM2 & Real & $\begin{array}{l}\text { Second flash sizing } \\
\text { parameter }\end{array}$ \\
\hline 5 & ROT & Real & $\begin{array}{l}\text { Rotation of flash } \\
\text { about reference } \\
\text { point in radians }\end{array}$ \\
\hline 6 & $\mathrm{DE}$ & Pointer & $\begin{array}{l}\text { DE of referenced } \\
\text { entity or zero }\end{array}$ \\
\hline
\end{tabular}

Additional Pointers as required (see 2.2.4.4.2). 

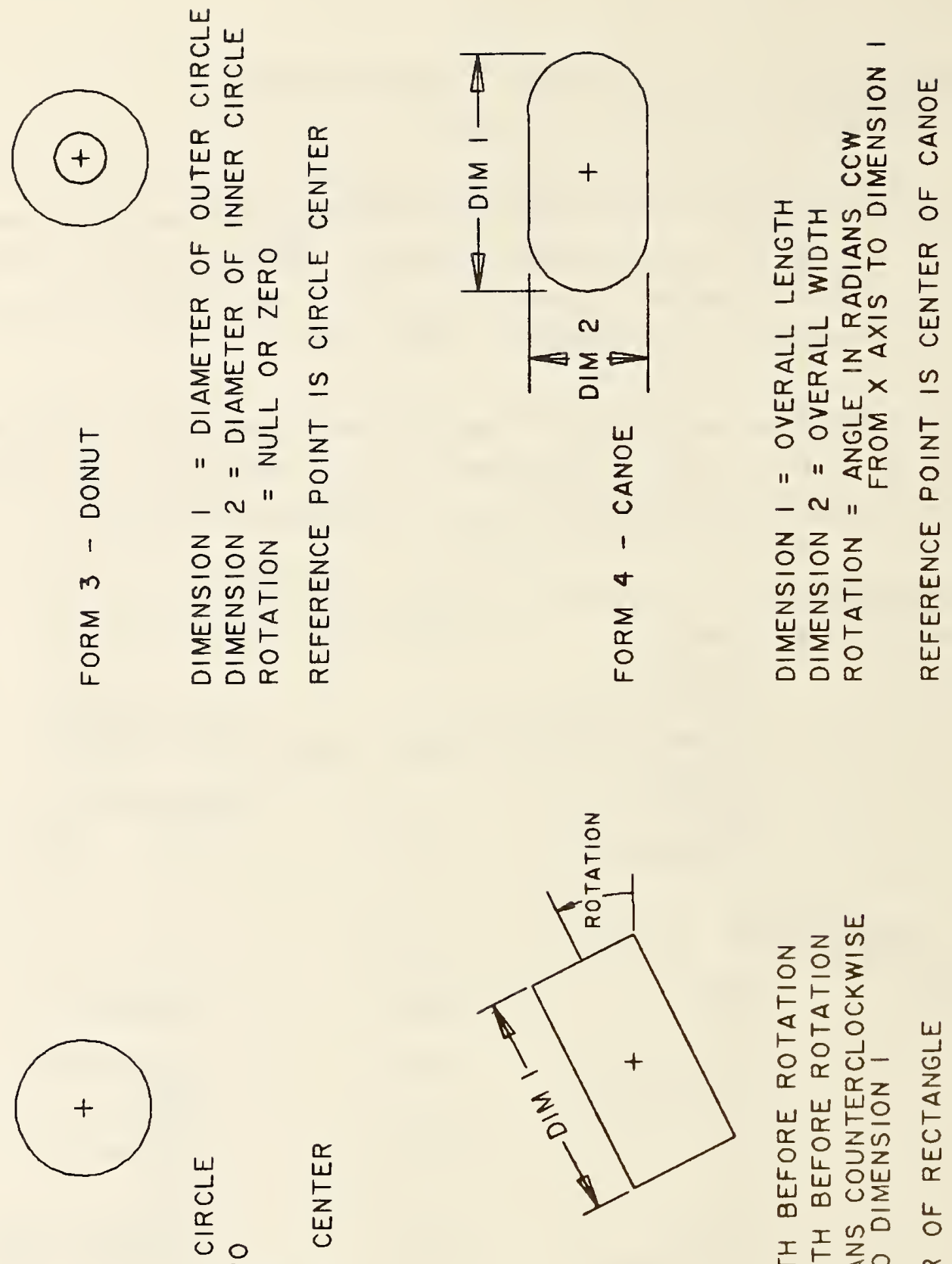

品
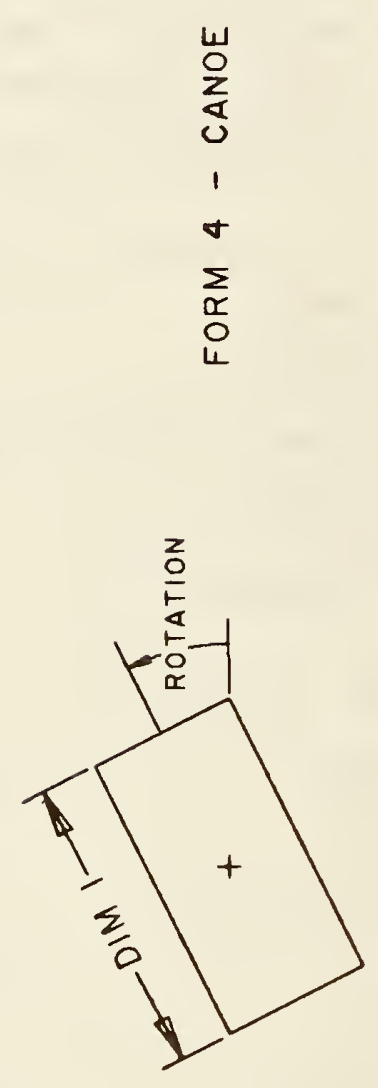

z

$\varangle \leftarrow 0$

山땀므

๙

u

嵌岕岂

$\infty$
$\frac{1}{n}$
$\frac{\pi}{\infty}$
$\frac{0}{0}$
$\frac{0}{4}$

岁䆓号耑

的㟔

IIZ

F

는

认 w

w $\frac{\pi}{x}$ u

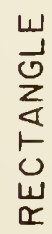

n $\frac{n}{x} z^{4}$

㞾品

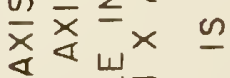

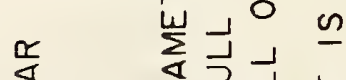

岁

$x>\overrightarrow{0}$

" "年品

选 $\quad-\infty$ " a

- $\sim "$

zzz $z$

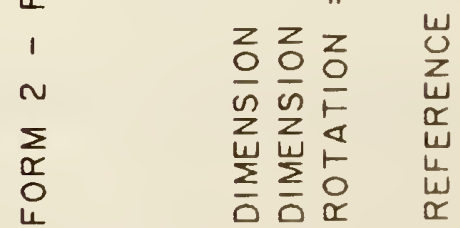


The rational B-spline curve may represent analytic curves of general interest. This information is important to both the sending and receiving systems. The directory entry form number parameter is provided to communicate this information. It should be emphasized that use of this curve form should be restricted to communications between systems operating directly on rational Bspline curves and not used as a replacement for the analytic forms for communication. For a brief description of a rational B-spline curves, see Section 4 of Appendix D.

If the rational B-spline curve represents a preferred curve type, the form number corresponds to the most preferred type. The preference order is from 1 through 5 followed by 0 . For example, if the curve is a circle or circular arc, the form number is set to 2 . If the curve is an ellipse with unequal major and minor axis lengths, the form number is set to 3. If the curve is not one of the preferred types, the form number is set to 0 .

If the curve lies entirely within a unique plane, the planar flag (PROPI) is set to 1 , otherwise it is set to 0 . If it is set to 1 , the plane normal (parameters $14+A+4 K$ through $16+A+4 K)$ contain a unit vector normal to the plane containing the curve. These fields exist but are ignored if the curve is non-planar. 
If the beginning and ending points on the curve are identical, PROP2 is set to 1 . If they are not equal, PROP2 is set to 0.

If the curve is rational (does not have all weights equal), PROP3 is set to 0 . If all weights are equal to each other, the curve is polynomial and PROP3 is set to 1. The curve is polynomial since in this case all weights cancel and the denominator sums to one (see Appendix D4).

If the curve is periodic with respect to its parametric variable, set $\mathrm{PROP} 4$ to 1 , otherwise set PROP 4 to 0.

\subsubsection{Directory Data}

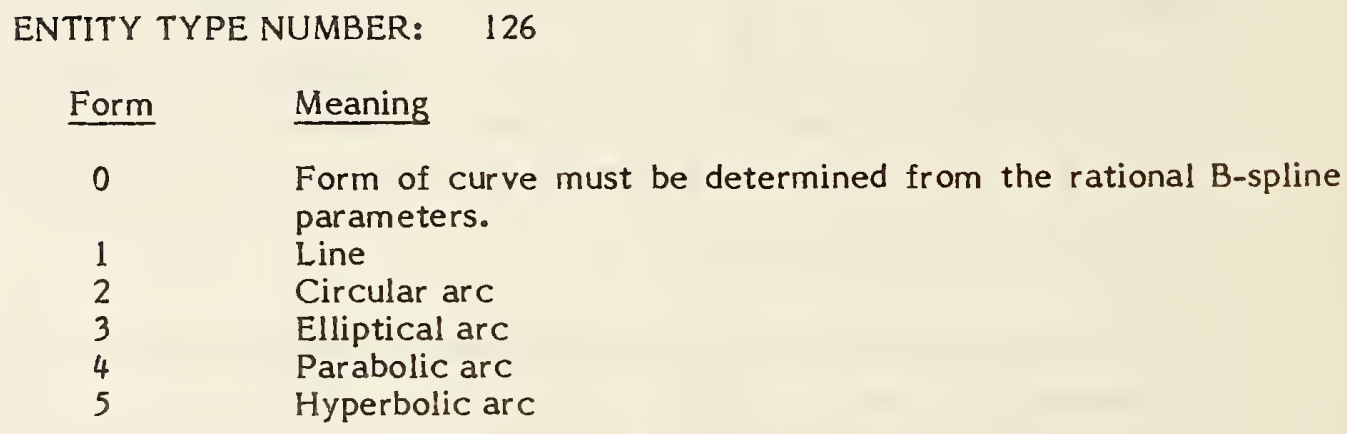

\subsubsection{Parameter Data}

\begin{tabular}{|c|c|c|c|}
\hline Index & Name & Type & Description \\
\hline 1 & K & Integer & $\begin{array}{l}\text { Upper index of } \\
\text { sum. See } \\
\text { Appendix D }\end{array}$ \\
\hline 2 & M & Integer & $\begin{array}{l}\text { Degree of basis } \\
\text { functions }\end{array}$ \\
\hline 3 & PROP 1 & Integer & $\begin{array}{l}=0-\text { non-planar } \\
=1-\text { planar }\end{array}$ \\
\hline 4 & PROP2 & Integer & $\begin{array}{l}=0-\text { open curve } \\
=1-\text { closed } \\
\text { curve }\end{array}$ \\
\hline 5 & PROP3 & Integer & $\begin{array}{l}=0-\text { rational } \\
=1-\text { polynomial }\end{array}$ \\
\hline 6 & PROP4 & Integer & $\begin{array}{l}=0-\text { non- } \\
\text { periodic } \\
=1-\text { periodic }\end{array}$ \\
\hline
\end{tabular}




\begin{tabular}{|c|c|c|c|}
\hline 7 & $T(-M)$ & Real & Knot Sequence \\
\hline$\dot{\bullet}$ & •. & & \\
\hline $7+A$ & $T(N+M)$ & & \\
\hline $8+A$ & $W(0)$ & Real & Weights \\
\hline • & - & & \\
\hline - & - & & \\
\hline - & - & & \\
\hline $8+A+K$ & $W(K)$ & & \\
\hline $9+A+K$ & xo & Real & Control Points \\
\hline $10+A+K$ & YO & & \\
\hline $11+A+K$ & 20 & & \\
\hline - & - & & \\
\hline - & - & & \\
\hline - & - & & \\
\hline $9+A+4 K$ & $\mathrm{XK}$ & & \\
\hline $10+A+4 K$ & YK & & \\
\hline $11+A+4 K$ & $\mathrm{ZK}$ & & \\
\hline $12+A+4 K$ & $v(0)$ & Real & $\begin{array}{l}\text { Starting para- } \\
\text { meter value }\end{array}$ \\
\hline $13+A+4 K$ & $\mathrm{~V}(1)$ & Real & $\begin{array}{l}\text { Ending para- } \\
\text { meter value }\end{array}$ \\
\hline $14+A+4 K$ & XNORM & Real & $\begin{array}{l}\text { Unit Normal (if } \\
\text { curve is planar) }\end{array}$ \\
\hline $15+A+4 K$ & YNORM & & \\
\hline $16+A+4 K$ & ZNORM & & \\
\hline
\end{tabular}

Additional Pointers as required (see 2.2.4.4.2).

Software to convert between parametric spline curves or surfaces and the corresponding rational B-spline curves or surfaces is available from the IGES office at the National Bureau of Standards. Materials provided include a magnetic tape of Pascal source code, a listing of the code, and accompanying documentation. 


\subsection{Rational B-Spline Surface Entity}

The rational B-spline surface represents various analytical surfaces of general interest. This information is important to both the generating and receiving system. The directory entry Form Number parameter is provided to communicate such information. For a brief description of rational Bspline surfaces, see Section 6 of Appendix D..

If the rational B-spline surface represents a preferred surface type, the form number corresponds to the most preferred type. The preference order is from 1 through 9 followed by 0 . For example, if the surface is a right circular cylinder, the form number is set to 2 . If the surface is a surface of revolution and also a torus, the form number is set to 5 . If the surface is not one of the preferred types, the form number is set to 0 .

If, for each fixed value of the second parametric variable the resulting curves which are functions of the first parametric variable are closed, set PROP1 to 1; otherwise set PROP1 to 0 . Similarly, if for each fixed value of the first parametric variable the resulting curves which are functions of the second parametric variable are closed, set PROP2 to 1; otherwise set PROP2 to 0.

If the surface is rational (does not have all weights equal) set PROP 3 to 0 . If all weights are equal to each other, the surface is polynomial and PROP3 is set to 1 . The surface is polynomial since in this case all weights cancel and the denominator sums to one (see Appendix D4).

If the surface is periodic with respect to the first parametric variable, set PROP4 to 1; otherwise set PROP4 to 0. If the surface is periodic with respect to the second parametric variable, set PROP5 to 1 ; otherwise set PROP5 to 0. 


\subsubsection{Directory Data}

ENTITY TYPE NUMBER: 128

Form Meaning

0 Form of the surface must be determined from the rational B-spline parameters

1 Plane

2 Right circular cylinder

3 Cone

4 Sphere

5 Torus

6 Surface of revolution

7 Tabulated cylinder

8 Ruled surface

9 General quadric surface

\subsubsection{Parameter Data}

\begin{tabular}{|c|c|c|c|c|}
\hline Index & Name & Type & & Description \\
\hline 1 & $\mathrm{~K} 1$ & Integer & \multicolumn{2}{|c|}{$\begin{array}{l}\text { Upper index of first sum. } \\
\text { See Appendix D }\end{array}$} \\
\hline 2 & $\mathrm{~K} 2$ & Integer & \multicolumn{2}{|c|}{$\begin{array}{l}\text { Upper index of second sum. } \\
\text { See Appendix D }\end{array}$} \\
\hline 3 & $\mathrm{M} \perp$ & Integer & \multicolumn{2}{|c|}{$\begin{array}{l}\text { Degree of first set of basis } \\
\text { functions }\end{array}$} \\
\hline 4 & M2 & Integer & \multicolumn{2}{|c|}{$\begin{array}{l}\text { Degree of second set of } \\
\text { basis functions }\end{array}$} \\
\hline 5 & PROP1 & Integer & $\begin{array}{l}=1 \\
=0\end{array}$ & $\begin{array}{l}\text { Closed in first } \\
\text { parametric variable } \\
\text { direction } \\
\text { Not closed }\end{array}$ \\
\hline 6 & PROP2 & Integer & $\begin{array}{l}=1 \\
=0\end{array}$ & $\begin{array}{l}\text { Closed in second } \\
\text { parametric variable } \\
\text { direction } \\
\text { Not closed }\end{array}$ \\
\hline 7 & PROP3 & Integer & & $\begin{array}{l}\text { Rational } \\
\text { Polynomial }\end{array}$ \\
\hline 8 & PROP4 & Integer & $\begin{array}{l}=0 \\
=1\end{array}$ & $\begin{array}{l}\text { Non-periodic in first } \\
\text { parametric variable } \\
\text { direction } \\
\text { Periodic in first } \\
\text { parametric variable } \\
\text { direction }\end{array}$ \\
\hline
\end{tabular}



direction

$=1$ Periodic in second parametric variable direction

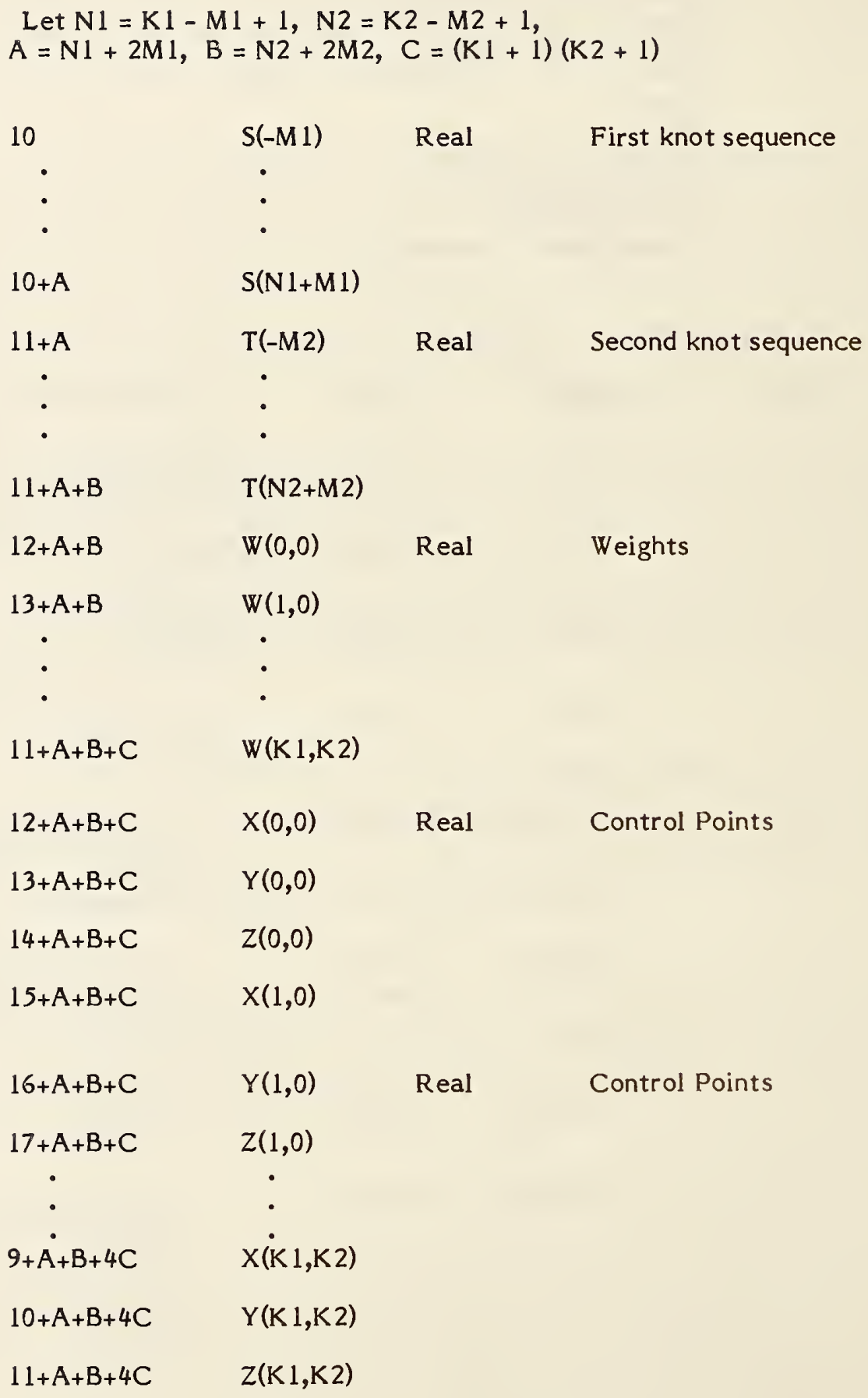




\begin{tabular}{|c|c|c|c|}
\hline Index & Name & Type & Description \\
\hline $12+A+B+4 C$ & $\mathrm{U}(\mathrm{O})$ & Real & $\begin{array}{l}\text { Starting value for first } \\
\text { parametric direction }\end{array}$ \\
\hline $13+A+B+4 C$ & $\mathrm{U}(1)$ & Real & $\begin{array}{l}\text { Ending value for first } \\
\text { parametric direction }\end{array}$ \\
\hline $14+A+B+4 C$ & $\mathrm{~V}(\mathrm{O})$ & Real & $\begin{array}{l}\text { Starting value for second } \\
\text { parametric direction }\end{array}$ \\
\hline $15+A+B+4 C$ & $\mathrm{~V}(1)$ & Real & $\begin{array}{l}\text { Ending value for second } \\
\text { parametric direction }\end{array}$ \\
\hline
\end{tabular}

Additional Pointers as required (see 2.2.4.4.2).

Software to convert between parametric spline curves or surfaces and the corresponding rational B-spline curves or surfaces is available from the IGES office at the National Bureau of Standards. Materials provided include a magnetic tape of Pascal source code, a listing of the code, and accompanying documentation. 
The Offset Curve entity contains the data necessary to determine the offset of a given curve $C$. This entity points to the base curve to be of fset and contains the of fset distance and additional pertinent information. No restriction is placed on the entity types of curves. Any parametric curve may be offset.

It is the intent of this Specification to limit the applicability of offsets to curves which are planar and slope continuous. The of fset curve lies in the plane which contains the base curve as follows:

let $\mathrm{C}$ denote a curve in definition space which is defined by $r=r(t)$;

let $\mathrm{T}(\mathrm{t})$ denote the unit tangent at $\mathrm{r}(\mathrm{t})$;

(See FAUX79)

let $\mathrm{V}$ be a unit vector normal to the plane which contains $\mathrm{C}$.

Then the offset curve is a curve defined as:

$$
\begin{aligned}
& O(t)=r(t)+f(s) *(V X T(t)) \quad \text { TT } 1 \leqslant t \leqslant T T 2 \\
& f(s)=D 1 \\
& f(s)=D 1+(D 2-D 1) *(s-T D 1) /(T D 2-T D 1) \text { with } \\
& \text { case (i) PTYPE = } 1 \\
& s=\text { arc length along } r \text { from } r(T T 1) \text { to } r(t) \text {, } \\
& \text { DI = the offset at arc length value TDI, } \\
& \mathrm{D} 2 \text { = the offset at arc length value TD2 } \\
& \text { case (ii) PTYPE = } 2 \\
& \mathrm{~S}=\mathrm{t} \text {, } \\
& \text { DI = the offset at parametric value TD 1, } \\
& \text { D2 = the offset at parametric value TD2 }
\end{aligned}
$$


c) if $F L A G=3$, an offset distance defined by a function, $\mathrm{f}(\mathrm{s})$ is the NDIM-th cordinate function of the curve pointed to by $\mathrm{DE} 2$, with

$$
\begin{aligned}
& \text { case (i) PTYPE }=1 \\
& \mathrm{~s}=\text { arc length along } \mathrm{r} \text { from } \mathrm{r}(\mathrm{TT} \mathrm{l}) \text { to } \mathrm{r}(\mathrm{t}) \text {, } \\
& \text { case (ii) PTYPE }=2 \\
& \mathrm{~s}=\mathrm{t}
\end{aligned}
$$

Note that TT1 and TT2 must be chosen to be in the domain of the base curve $r(t)$.

\begin{tabular}{|c|c|c|c|}
\hline Index & Name & Type & Description \\
\hline 1 & DE 1 & Pointer & $\begin{array}{l}\text { Pointer to curve entity to } \\
\text { be offset. }\end{array}$ \\
\hline 2 & FLAG & Integer & $\begin{aligned} \text { Offset distance flag: } & \\
1= & \text { Single value offset, } \\
& \text { uniform distance } \\
2= & \text { Offset distance varying } \\
& \text { linearly } \\
3= & \text { Offset distance as a } \\
& \text { specified function. }\end{aligned}$ \\
\hline 3 & DE2 & $\begin{array}{l}\text { Pointer } \\
\text { or } 0\end{array}$ & $\begin{array}{l}\text { Pointer to curve, one } \\
\text { coordinate of which } \\
\text { describes the offset as a } \\
\text { function of its parameter. } \\
(0 \text { unless FLAG }=3)\end{array}$ \\
\hline 4 & NDIM & Integer & $\begin{array}{l}\text { Pointer of particular } \\
\text { coordinate of DE } 2 \text { which } \\
\text { describes offset as a } \\
\text { function of its parameter. } \\
\text { (only used if FLAG = 3) }\end{array}$ \\
\hline
\end{tabular}

\subsubsection{Directory Data}

ENTITY TYPE NUMBER: 130

\subsubsection{Parameter Data}




\begin{tabular}{|c|c|c|c|}
\hline Index & Name & Type & Description \\
\hline 5 & PTYPE & Integer & $\begin{array}{l}\text { Tapered of fset type flag: } \\
1=\text { Function of arc length } \\
2=\text { Function of parameter } \\
\text { (only used if FLAG }=2 \text { or } 3 \text { ) }\end{array}$ \\
\hline 6 & D1 & Real & $\begin{array}{l}\text { First offset distance. } \\
\text { (only used if FLAG }=1 \text { or } 2 \text { ) }\end{array}$ \\
\hline 7 & TD 1 & Real & $\begin{array}{l}\text { Arc length or parameter } \\
\text { value, depending on PTYPE, } \\
\text { of first of fset distance. } \\
\text { (only used if FLAG=2) }\end{array}$ \\
\hline 8 & D2 & Real & Second offset distance. \\
\hline 9 & TD2 & Real & $\begin{array}{l}\text { Arc length or parameter } \\
\text { value, depending on PTYPE, } \\
\text { of second offset distance. } \\
\text { (only used if FLAG } 2 \text { ) }\end{array}$ \\
\hline 10 & $\mathrm{vX}$ & Real & $\begin{array}{l}\text { X-component of unit vector } \\
\text { normal to plane containing } \\
\text { curve to be offset. }\end{array}$ \\
\hline 11 & VY & Real & $\begin{array}{l}\text { Y-component of unit vector } \\
\text { normal to plane containing } \\
\text { curve to be offset. }\end{array}$ \\
\hline 12 & $v z$ & Real & $\begin{array}{l}\text { Z-component of unit vector } \\
\text { normal to plane containing } \\
\text { curve to be offset. }\end{array}$ \\
\hline 13 & TT 1 & Real & $\begin{array}{l}\text { Offset curve star ting } \\
\text { parameter value. }\end{array}$ \\
\hline 14 & TT2 & Real & $\begin{array}{l}\text { Offset curve ending } \\
\text { parameter value. }\end{array}$ \\
\hline
\end{tabular}

Additional Pointers as required (see 2.2.4.4.2).

Parameter data not required for a particular case should be given zero values. For example, if the value of parameter 2 is not 3 , then parameters 3 and 4 should be given zero values. 


\subsection{Connect Point Entity}

A Connect Point Entity describes a point of connection for zero, one or more entities. These entities include those required in piping diagrams, electrical and electronic schematics, and physical designs (e.g., printed wiring board). The Connect Point Entity is referenced from either the Network Subfigure Definition, Network Subfigure Instance, or the Flow Associativity Instance; or it may stand alone in a file. The connect point may be displayed by the receiving system using default display parameters and/or symbol. Also see 2.5.2.

\subsubsection{Directory Data}

ENTITY TYPE NUMBER:

\subsubsection{Parameter Data}

$\begin{array}{lll}\frac{\text { Index }}{1} & \frac{\text { Name }}{X} & \frac{\text { Type }}{\text { Real }} \\ 2 & Y & \text { Real } \\ 3 & Z & \text { Real } \\ 4 & \text { PTR } & \end{array}$

Description

$X$ coordinate of the connection point

$Y$ Coordinate of the connection point

$Z$ coordinate of the connection point

Pointer to directory entry of the display symbol geometry 
$\underline{\text { Index }}$

5

6

7

8

9

10

11

12

13

14
Name

TF

FF

CID

PTTCID

CFN

PTTCFN

CPID

FC

SF

PSFI
Type

Integer

Integer

String

Pointer

String

Pointer

Integer

Integer

Integer

Pointer
Description

Type flag

$0=$ not specified

$1=$ logical

2 = physical

Function Flag:

$0=$ not specified

1 = electrical signal

2 = fluid flow path

Connect Point

Function Identifier

(e.g., Pin Number or

Nozzle Label)

Pointer to Text

Display Template

Entity for CID.

Connection Point

Function Name

Pointer to Text

Display Template

Entity for CFN

Unique Connect

Point Identifier

Connect Point

Function Code:

$0=$ Unspecified

(default)

1 = Input

2 = Output

3 = Bidirectional

$4=$ Power

$5=$ Ground

Swap Flag

$0=$ Connect point may be swapped

(default)

1 = Connect point may not be swapped

Pointer to "owner" Network Subfigure Instance, or Network Subfigure Definition, or zero.

Additional Pointers as required (see 2.2.4.4.2). 
3.20 Node Entity

The node entity is a geometric point used in the definition of a finite element. Directory entry field 7 points to a labeled definition coordinate system Transformation Matrix. The form number of the Transformation Matrix indicates the definition coordinate system type. Coordinate angles for the cylindrical and spherical coordinate systems are specified in degrees.

3.20.1 Every node has a nodal displacement coordinate system associated with it. This is form 10, 11, or 12 of the Transformation Matrix Entity which locates translational and rotational directions for load, restraint and displacement results. Again, the form number of the Transformation Matrix indicates the coordinate system type.

The origin of the nodal displacement coordinate system is always the location of the node. However, the orientation of the nodal displacement axes depends on the location of the node and the type of displacement coordinate system being referenced. Cartesian (Rectangular), cylindrical and spherical are the three possible types.

If the displacement coordinate system is Cartesian, then the nodal displacement axes are parallel to the respective referenced coordinate system. This is illustrated in Figure 3-19(a) Cartesian.

For the cylindrical type displacement coordinate system, the orientation of the nodal displacement axes depends on the coordinate value of the node as defined in the referenced displacement coordinate system. The nodal displacement axes are respectively in the radial, tangential and axial directions as illustrated in Figure 3-19(b) Cylindrical.

Finally, for spherical, the orientation of the nodal displacement axes depend on both the $\theta$ and $\emptyset$ coordinates of the node as defined in the referenced displacement coordinate system. The nodal displacement axes are respectively in the radial, meridional and azimuthal directions as indicated in Figure 3-19(c) Spherical. 
If a node lies on the polar axis of either the cylindrical or spherical coordinate system, the nodal displacement axes are defined parallel to the referenced displacement coordinate system axes. For cylindrical the first axis is the $\theta=0$ axis and the third axis is the $z$ axis. For spherical the first axis is the $\emptyset=0$ axis while the third axis is the $\theta=0$ axis. The remaining axis of both systems is defined by the appropriate cross product of the previously defined axes.

\subsubsection{Directory Data}

\section{ENTITY TYPE NUMBER: 134}

Entity Label: Node Label (Optional)

Entity Subscript: Node Number (Required)

\subsubsection{Parameter Data}

\begin{tabular}{|c|c|c|}
\hline Index & Name & Type \\
\hline 1 & $X / R / R$ & Real \\
\hline 2 & $Y / \theta / \theta$ & Real \\
\hline 3 & $Z / Z / \emptyset$ & Real \\
\hline 4 & NDCSP & Pointer \\
\hline
\end{tabular}

\section{Description}

First nodal coordinate

Second nodal coordinate

Third nodal coordinate

Pointer to the

Transformation Matrix form 10,11 or 12 which defines the Nodal

Displacement Coordinate System entity. Default (zero) is Global Cartesian Coordinate System.

Additional Pointers as required (see 2.2.4.4.2).

Figure 3-19 illustrates the definition of a node in the three coordinate systems. 


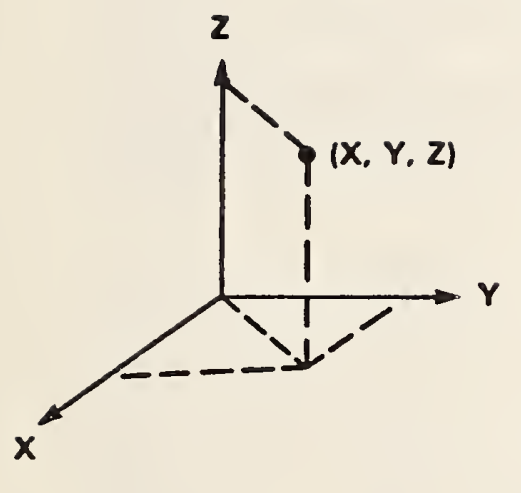

CARTESIAN

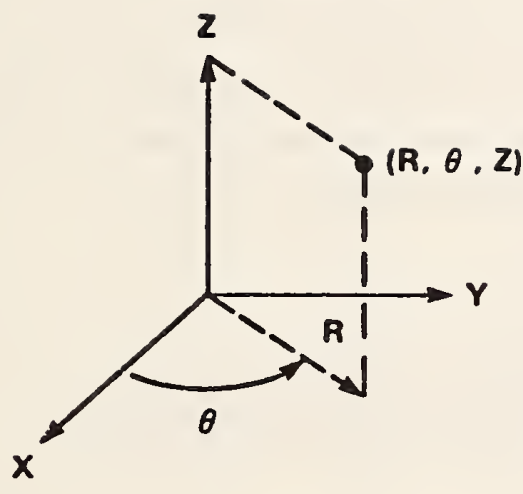

CYLINDRICAL

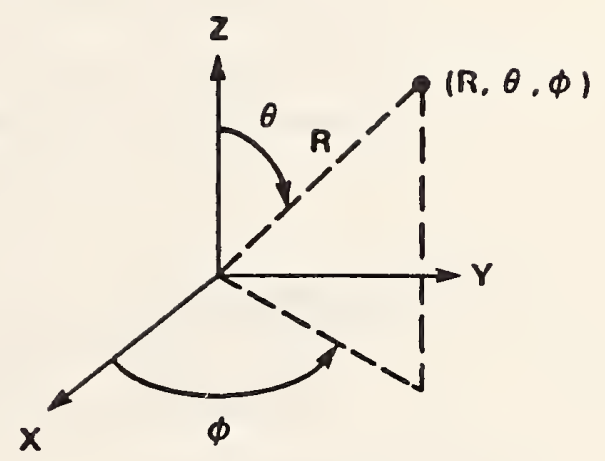

SPHERICAL

FIGURE 3-19 NODE DEFINITION IN EACH COORDINATE SYSTEM 


\subsection{Finite Element Entity}

A finite element is defined by an element topology (i.e., node connectivity) along with physical and material properties.

3.21.1 Table 3-1 lists the data to define the element topology. Figure 3-20 illustrates the node connectivity for each element topology.

3.21.2 In Table 3-1 the element name is an English abbreviation or acronym describing the element. The element topology type is an integer number which will appear as the first parameter of the parameter data. The order is an integer identifying the order of an edge where $0=$ not applicable, $1=$ linear, $2=$ parabolic and 3 =cubic. The number of nodes from Table 3-1 will appear as the second parameter of the finite element parameter data. A missing node in the connectivity sequence will have its corresponding pointer value equal to zero.

\subsubsection{Directory Data}

ENTITY TYPE NUMBER: 136

Entity Label: $\quad$ Element Label (Optional)

Entity Subscript: Element Number (Required)

\subsubsection{Parameter Data}

\begin{tabular}{|c|c|c|c|}
\hline Index & Name & Type & Description \\
\hline 1 & ITOP & Integer & Topology Type. \\
\hline 2 & N1 & Integer & $\begin{array}{l}\text { Number of Nodes de- } \\
\text { fining Element. See } \\
\text { 3.23.2. }\end{array}$ \\
\hline 3 & $\mathrm{DE}$ & Pointer & $\begin{array}{l}\text { Pointer to first node } \\
\text { defining element. See } \\
\text { 3.23.2. }\end{array}$ \\
\hline . & • & • & \\
\hline$\cdot$ & • & - & \\
\hline$\dot{\mathrm{N}}+2$ & $\dot{D E}$ & Pointer & $\begin{array}{l}\text { Pointer to last node } \\
\text { defining element }\end{array}$ \\
\hline $\mathrm{N} 1+3$ & ETYP & String & Element type name \\
\hline
\end{tabular}


TABLE 3-1 FINITE ELEMENT TOPOLOGY

\section{ELEMENT DATA CHART}

\section{Element Data}

\begin{tabular}{|c|c|c|c|c|c|}
\hline $\begin{array}{l}\text { Element } \\
\text { Name }\end{array}$ & $\begin{array}{l}\text { Element } \\
\text { Topology } \\
\text { Type }\end{array}$ & Order & $\begin{array}{l}\text { Number } \\
\text { of } \\
\text { Nodes }\end{array}$ & $\begin{array}{l}\text { Number } \\
\text { of } \\
\text { Edges }\end{array}$ & $\begin{array}{l}\text { Number } \\
\text { of } \\
\text { Faces }\end{array}$ \\
\hline $\begin{array}{l}\text { BEAM } \\
\text { LTRIA } \\
\text { PTRIA } \\
\text { CTRIA } \\
\text { LQUAD } \\
\text { PQUAD } \\
\text { CQUAD }\end{array}$ & $\begin{array}{l}1 \\
2 \\
3 \\
4 \\
5 \\
6 \\
7\end{array}$ & $\begin{array}{l}1 \\
1 \\
2 \\
3 \\
1 \\
2 \\
3\end{array}$ & $\begin{array}{r}2 \\
3 \\
6 \\
9 \\
4 \\
8 \\
12\end{array}$ & $\begin{array}{l}1 \\
3 \\
3 \\
3 \\
4 \\
4 \\
4\end{array}$ & $\begin{array}{l}0 \\
1 \\
1 \\
1 \\
1 \\
1 \\
1\end{array}$ \\
\hline $\begin{array}{l}\text { PTSW } \\
\text { CTSW }\end{array}$ & $\begin{array}{l}8 \\
9\end{array}$ & $\begin{array}{l}2 \\
3\end{array}$ & $\begin{array}{l}12 \\
18\end{array}$ & $\begin{array}{l}9 \\
9\end{array}$ & $\begin{array}{l}5 \\
5\end{array}$ \\
\hline $\begin{array}{l}\text { PTS } \\
\text { CTS } \\
\text { LSOT } \\
\text { PSOT } \\
\text { LSOW } \\
\text { PSOW } \\
\text { CSOW } \\
\text { LSO } \\
\text { PSO } \\
\text { CSO } \\
\text { ALLIN } \\
\text { APLIN } \\
\text { ACLIN } \\
\text { ALTRIA } \\
\text { APTRIA } \\
\text { ALQUAD } \\
\text { APQUAD } \\
\text { SPR } \\
\text { GSPR } \\
\text { DAMP } \\
\text { GDAMP } \\
\text { MASS } \\
\text { RBDY } \\
\text { TBEAM }\end{array}$ & $\begin{array}{l}10 \\
11 \\
12 \\
13 \\
14 \\
15 \\
16 \\
17 \\
18 \\
19 \\
20 \\
21 \\
22 \\
23 \\
24 \\
25 \\
26 \\
27 \\
28 \\
29 \\
30 \\
31 \\
32 \\
33\end{array}$ & $\begin{array}{l}2 \\
3 . \\
1 \\
2 \\
1 \\
2 \\
3 \\
1 \\
2 \\
3 \\
1 \\
2 \\
3 \\
1 \\
2 \\
1 \\
2 \\
0 \\
0 \\
0 \\
0 \\
0 \\
0 \\
1\end{array}$ & $\begin{array}{r}16 \\
24 \\
4 \\
10 \\
6 \\
15 \\
24 \\
8 \\
20 \\
32 \\
2 \\
3 \\
4 \\
3 \\
6 \\
4 \\
8 \\
2 \\
1 \\
2 \\
1 \\
1 \\
2 \\
3\end{array}$ & $\begin{array}{r}12 \\
12 \\
6 \\
6 \\
9 \\
9 \\
9 \\
12 \\
12 \\
12 \\
1 \\
1 \\
1 \\
3 \\
3 \\
4 \\
4 \\
0 \\
0 \\
0 \\
0 \\
0 \\
0 \\
1\end{array}$ & $\begin{array}{l}6 \\
6 \\
4 \\
4 \\
5 \\
5 \\
5 \\
6 \\
6 \\
6 \\
0 \\
0 \\
0 \\
0 \\
0 \\
0 \\
0 \\
0 \\
0 \\
0 \\
0 \\
0 \\
0 \\
0\end{array}$ \\
\hline
\end{tabular}


1. BEAM

$$
E l=1,2
$$

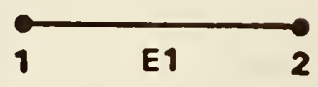

2. LTRIA - Linear Triangle

$$
\begin{aligned}
& E 1=1,2 \\
& E 2=2,3 \\
& E 3=3,1
\end{aligned}
$$

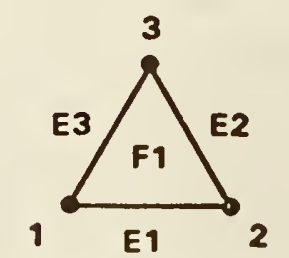

3. PTRIA - Parabolic Triangle

$$
\begin{aligned}
& E 1=1,2,3 \\
& E 2=3,4,5 \\
& E 3=5,6,1
\end{aligned}
$$
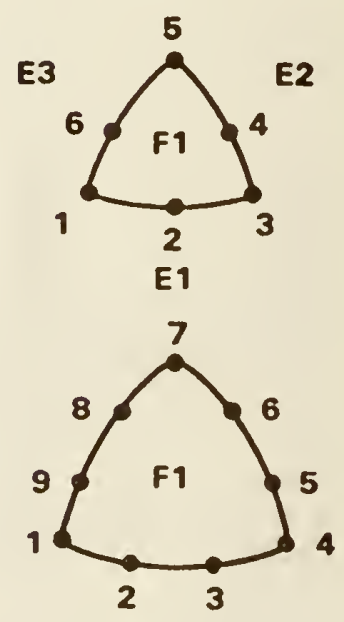

5. LQUAD - Linear Quadrilateral

$$
\begin{aligned}
& E 1=1,2 \\
& E 2=2,3 \\
& E 3=3,4 \\
& E 4=4,1
\end{aligned}
$$

$F 1=1,2,3,4,5,6,7,8,9$

$E 1=1,2,3,4$

$E 3=7,8,9,1$

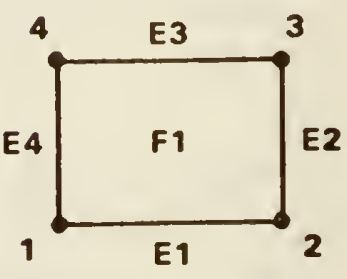

6. PQUAD - Parabolic Quadrilaterial

$$
\begin{aligned}
& E 1=1,2,3 \\
& E 2=3,4,5 \\
& E 3=5,6,7 \\
& E 4=7,8,1
\end{aligned}
$$

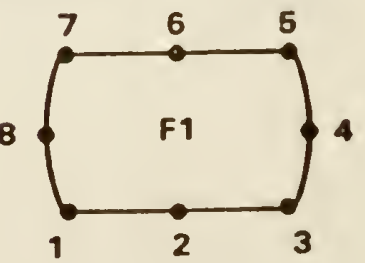


7. CQUAD - Cubic Quadrilateral

$$
\begin{aligned}
& E 1=1,2,3,4 \\
& E 2=4,5,6,7 \\
& E 3=7,8,9,10 \\
& E 4=10,11,12,1 \\
& F 1=1,2,3,4,5,6,7,8,9,10,11,12
\end{aligned}
$$

8. PTSW - Parabolic Thick Shell Wedge

$$
\begin{array}{lll}
E 1=1,2,3 & E 4=7,8,9 & E 7=1,7 \\
E 2=3,4,5 & E 5=9,10,11 & E 8=3,9 \\
E 3=5,6,1 & E 6=11,12,7 & E 9=5,11 \\
F 1=1,2,3,4,5,6 & & \\
F 2=7,8,9,10,11,12 & & \\
F 3=1,2,3,9,8,7 & & \\
F 4=3,4,5,11,10,9 & & \\
F 5=5,6,1,7,12,11 & &
\end{array}
$$
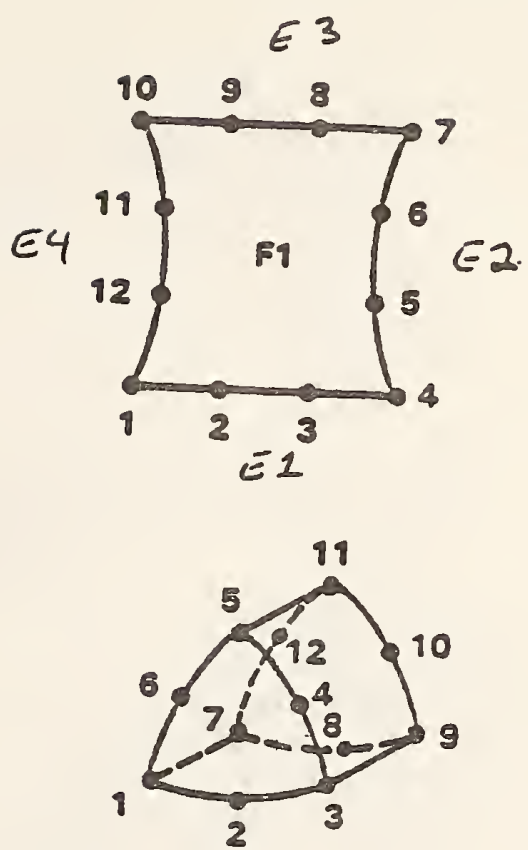

$E 7=1,10$

$\mathrm{E} 8=4.13$

$\mathrm{E} 9=7,16$

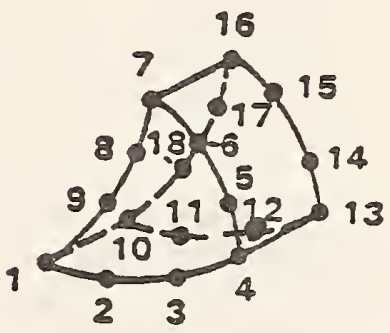

$\mathrm{E} 4=10,11,12,13$

$\mathrm{E} 5=13,14,15, \mathrm{i} 6$

$\mathrm{E} 6=16,17,18,10$

E3 $=7,8,9,1$

$F 1=1,2,3,4,5,6,7,8,9$

$\mathrm{F} 2=10,11,12,13,14,15,16,17,18$

$F 3=1,2,3,4,13,12,11,10$

$\mathrm{F} 4=4,5,6,7,16,15,14,13$

$F 5=7,8,9,1,10,18,17,16$

10. PTS - Parabolic Thick Shell
$E 1=1,2,3$
$E 2=3,4,5$
$E 5=9,10,11 \quad E 9=1,9$
$E 2=3,4,5$
$E 3=5,6,7$
$E 6=11,12,13 \quad E 10=3,11$
E3 $=5,6,7$
E4 $-7,8,1$
E7 $=13,14,15 \quad$ E1 $1=5,13$
$E 8=15,16,9 \quad E 12=7,15$
$F 1=1,2,3,4,5,6,7,8$
$F 2=9,10,11,12,13,14,15,16$
F $3=1,2,3,11,10,9 \quad$ F $5=5,6,7,15,14,13$
$\mathrm{F} 4=3,4,5,13,12,11 \quad \mathrm{~F} 6=7,8,1,9,16,15$

11. CTS - Cubic Thick Shell
$\mathrm{E} 1=1,2,3,4$
$\mathrm{E} 2=4,5,6,7$
$E 5=13,14,15,16$
E3 $=7,8,9,10$
$\mathrm{E} 6=16,17,18,19$
$\mathrm{E} 7=19,20,21,22$
$E 4=10,11,12,1$
$E 8=22,23,24,13$

$F 1=1,2,3,4,5,6,7,8,9,10,11,12$

$F 2=13,14,15,16,17,18,19,20,21,22,23,24$

$F 3=1,2,3,4,16,15,14,13$

$F 4=4,5,6,7,19,18,17,16$

$F 5=7,8,9,10,22,21,20,19$

$F 6=10,11,12,1,13,24,23,22$

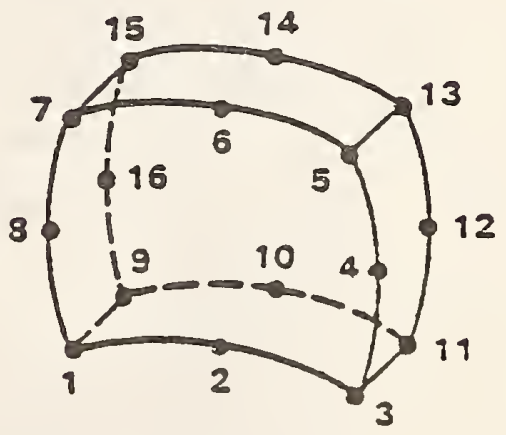

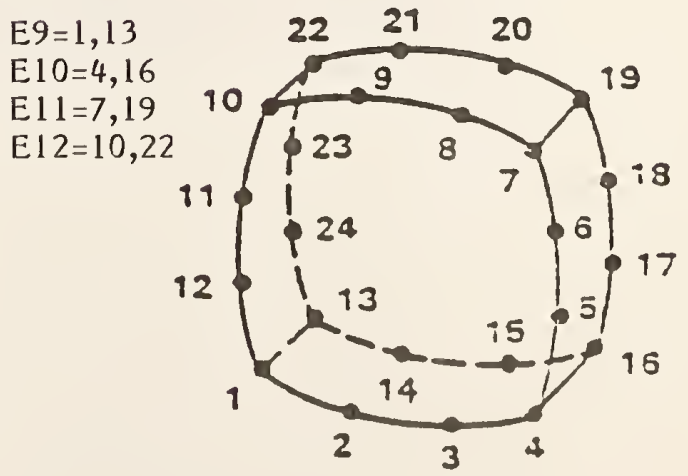


12. LSOT - Linear Solid Tetrahedron
$\mathrm{El}=\mathrm{l}, 2$
$\mathrm{E} 2=2,3$
$E 3=3,1$
$F 1=1,2,3$
$\mathrm{F} 2=1,2,4$
$\mathrm{F} 3=2,3,4$
$\mathrm{F} 4=3,1,4$

$$
\begin{aligned}
& E 4=1,4 \\
& E 5=2,4 \\
& E 6=3,4
\end{aligned}
$$

SOT - Parabolic Solid Tetrahedron
$\mathrm{E} 1=1,2,3$
$\mathrm{E} 2=3,4,5$
E3 $=5,6,1$
$F 1=1,2,3,4,5,6$
$F 2=1,2,3,8,10,7$
$\mathrm{F} 3=3,4,5,9,10,8$
$F \cdot 4=5,6,1,7,10,9$
$\mathrm{E} 4=1,7,10$
$\mathrm{E} 5=3,8,10$
$\mathrm{E} 6=5,9,10$

OW - Linear Solid Wedge
$\mathrm{E} 1=1,2$
$E 2=2,3$
$\mathrm{E} 3=3,1$
$\mathrm{F} 1=1,2,3$
$\mathrm{F} 2=4,5,6$
$\mathrm{F} 3=1,2,5,4$
$\mathrm{F}_{4}=2,3,6,5$
$\mathrm{F} 5=3,1,4,6$
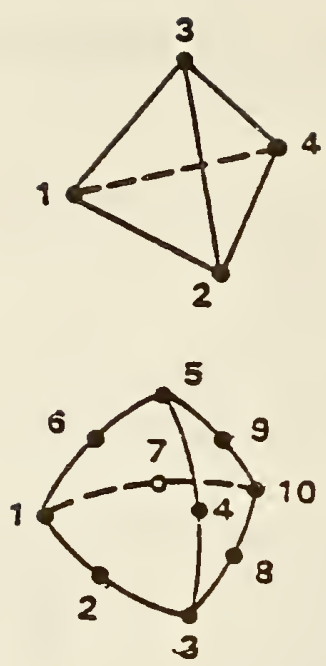

$\mathrm{E} 7=1,4$

$\mathrm{E} 8=2,5$

$\mathrm{E} 9=3,5$

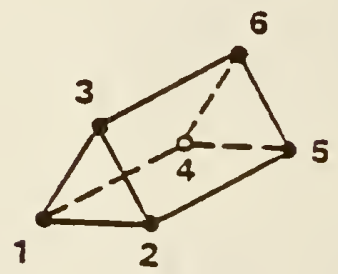

$\mathrm{E} 7=1,7,10$

$E 8=3,8,12$

$\mathrm{E} 9=5,9,14$

$$
\begin{aligned}
& E 1=1,2,3 \\
& E 2=3,4,5 \\
& E 3=5,6,1 \\
& F 1=1,2,3,4,5,6 \\
& F 2=10,11,12,13,14,15 \\
& F 3=1,2,3,8,12,11,10,7 \\
& F 4=3,4,5,9,14,13,12,8 \\
& F 5=5,6,1,7,10,15,14,9
\end{aligned}
$$

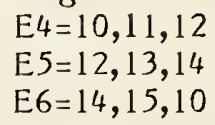

16. CSOW - Cubic Solid Wedge
$\mathrm{E} 1=1,2,3,4$
$E 4=16,17,18,19$
$\mathrm{E} 2=4,5,6,7$
$E 5=19,20,21,22$
E $3=7,8,9,1$
$E 6=22,23,24,16$
$\mathrm{F} 1=1,2,3,4,5,6,7,8,9$
$\mathrm{F} 2=16,17,18,19,20,21,22,23,24$
$\mathrm{F} 3=1,2,3,4,11,14,19,18,17,16,13,10$
$\mathrm{F}_{4}=4,5,6,7,12,15,22,21,20,19,14,11$
$F 5=7,8,9,1,10,13,16,24,23,22,15,12$

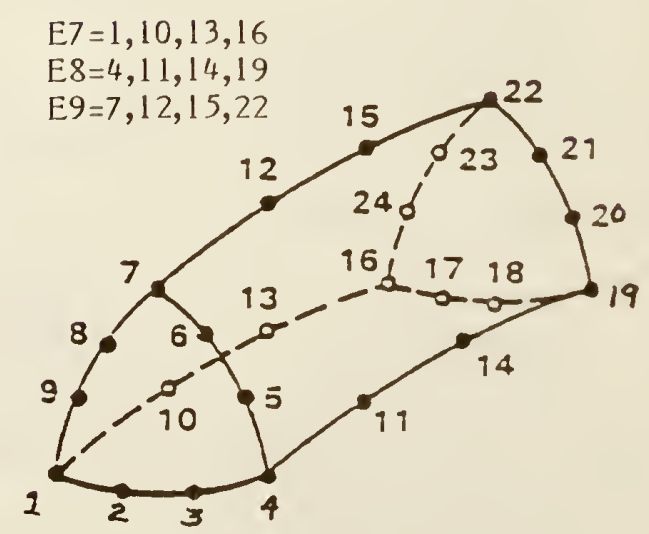


17. LSO - Linear Solid
$E 1=1, ?$
$\mathrm{E} 2=2,3$
$\mathrm{E} 3=3,4$
$E 4=4,1$
$\mathrm{F} 1=1,2,3,4$
$\mathrm{F} 2=5,6,7,8$
$\mathrm{F} 3=1,2,6,5$
$\mathrm{F} 4=2,3,7,6$
$F 5=3,4,8,7$
$\mathrm{F} 6=4,1,5,8$

$\begin{array}{ll}\text { E5 }=5,6 & \text { E9 }=1,5 \\ \text { E6 }=6,7 & \text { E1 } 0=2,6 \\ \text { E7 }=7,8 & \text { E1 } 1=3,7 \\ \text { E8 }=8,5 & \text { E12 }=4,8\end{array}$

$E 5=5,6$

$E 7=7,8$

E12 $=4,8$

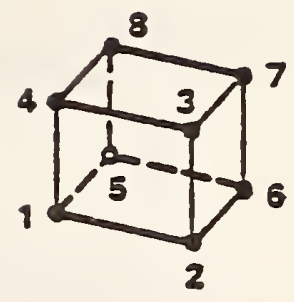

18. PSO - Parabolic Solid
$\mathrm{E} 1=1,2,3$
$\mathrm{E} 2=3,4,5$
$E 3=5,6,7$
$E 7=17,18,19$
$E 8=19,20,13$
$\mathrm{E} 4=7,8,1$
$\mathrm{E} 9=1,9,13$
E $5=13,14,15$
E $10=3,10,15$
E $11=5,11,17$
$\mathrm{E} 6=15,16,17$
E 12=7,12,19

$F 1=1,2,3,4,5,6,7,8$

$F 2=13,14,15,16,17,18,19,20$

$F 3=1,2,3,10,15,14,13,9$

$F_{4}=3,4,5,11,17,16,15,10$

$F 5=5,6,7,12,19,18,17,11$

$\mathrm{F} 6=7,8,1,9,13,20,19,12$

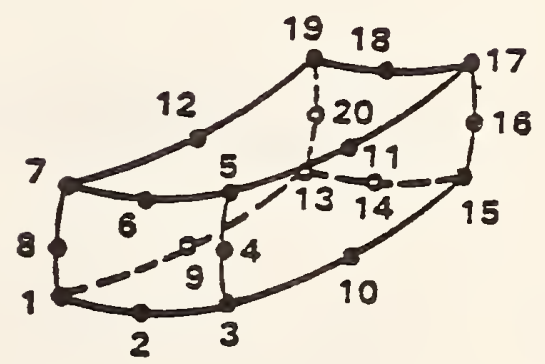

19. CSO - Cubic Solid
$\mathrm{E} 1=1,2,3,4$
$\mathrm{E} 2=4,5,6,7$
E $3=7,8,9,10$
$\mathrm{E} 4=10,11,12,1$
$\mathrm{E} 5=21,22,23,24$
$\mathrm{E} 6=24,25,26,27$
E7 $=27,28,29,30$
$\mathrm{E} 8=30,31,32,21$
$\mathrm{E} 9=1,13,17,21$
E $10=4,14,18,24$
$E 11=7,15,19,27$
$E_{12}=10,16,20,30$
$F 1=1,2,3,4,5,6,7,8,9,10,11,12$
$F 2=21,22,23,24,25,26,27,28,29,30,31,32$
F $3=1,2,3,4,14,18,24,23,22,21,17,13$
$\mathrm{F} 4=4,5,6,7,15,19,27,26,25,24,18,14$
$F 5=7,8,9,10,16,20,30,29,28,27,19,15$
$F 6=10,11,12,1,13,17,21,32,31,30,20,16$

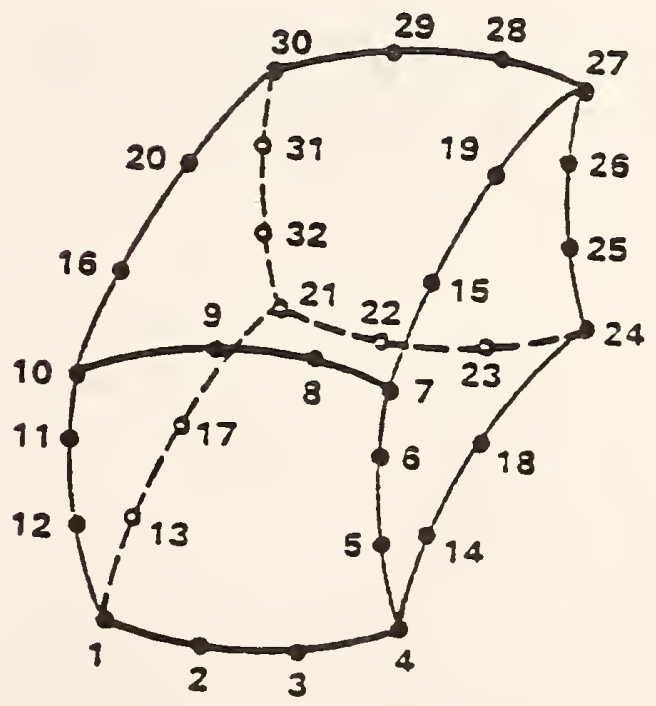


20. ALLIN - Axisymmetric Linear Line

$E_{1}=1,2 \quad$ No Faces

21. APLIN - Axisymmetric Parabolic Line $\mathrm{El}=1,2,3 \quad$ No Faces

22. ACLIN - Axisymmetric Cubic Line

$\mathrm{El}=1,2,3,4 \quad$ No Faces

23. ALTRIA - Axisymmetric Linear Triangle

$\mathrm{E} 1=1,2$

$\mathrm{E} 2=2,3 \quad$ No Faces

$\mathrm{E} \hat{\jmath}=3, \hat{i}$

24. APTRIA - Axisymmetric Parabolic Triangle

$\mathrm{E}_{1}=1,2,3$

$\mathrm{E} 2=3,4,5 \quad$ No Faces

$\mathrm{E} 3=5,6,1$

25. ALQUAD - Axisymmetric Linear Quadrilateral

$\mathrm{E}_{1}=1,2$

$\mathrm{E} 2=2,3$

$\mathrm{E} 3=3,4 \quad$ No Faces

$\mathrm{E} 4=4,1$

26. APQUAD - Axisymmetric Parabolic Quadrilateral

$\mathrm{E} 1=1,2,3$

$\mathrm{E} 2=3,4,5$

$\mathrm{E} 3=5,6,7$

$\mathrm{E} 4=7,8,1$

No Faces
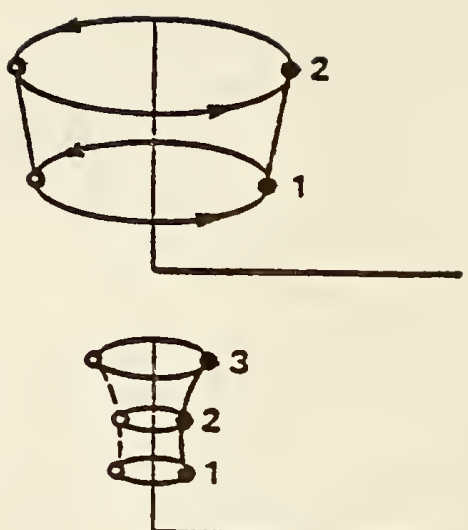

3

(1)
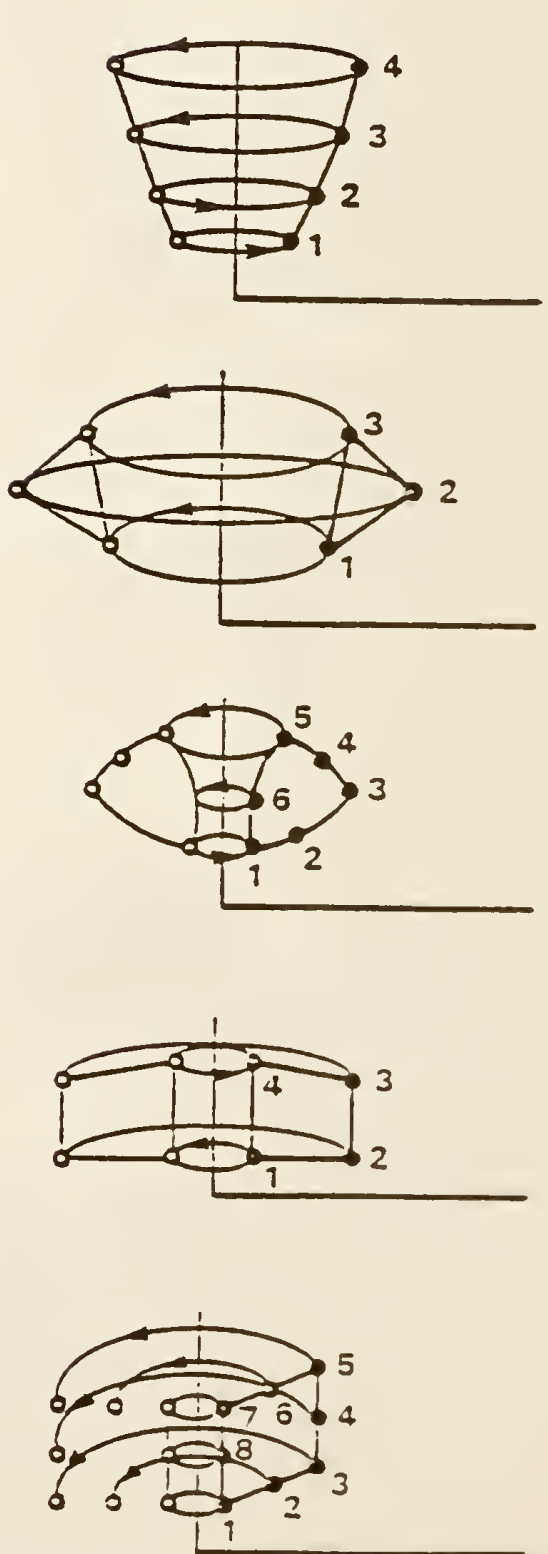
27. SPR - Spring

No edges or faces

28. GSPR - Grounded Spring

29. DAMP - Damper

30. GDAMP - Grounded damper

31. MASS - Mass

32. RBDY - Rigid Body

33. TBEAM - three noded beam (no faces)

$\mathrm{E} 1=1,2$

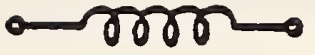

0202
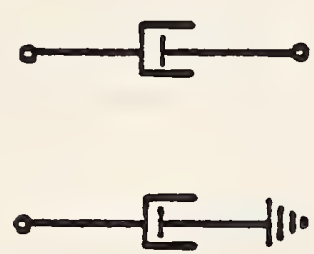

0

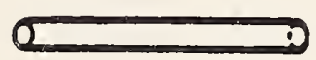

- 3

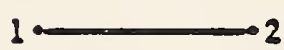

91 


\subsection{Nodal Displacement and Rotation Entity}

The FEM Nodal Displacement and Rotation Entity is used to communicate finite element post processing data. It contains the incremental displacements and rotations expressed in radians for each load case and each node in the model. It also contains a pointer to a general note entity for a description of the load cases. For each node it contains the node number identifier and the node DE pointer. The node number identifier is equivalent to the node number in the directory entry subscript field of the node entity.

\subsubsection{Directory Data}

ENTITY TYPE NUMBER: 138

\subsubsection{Parameter Data}

\begin{tabular}{|c|c|c|c|}
\hline Index & Name & Type & Description \\
\hline 1 & NC & Integer & $\begin{array}{l}\text { Number of analysis } \\
\text { cases }\end{array}$ \\
\hline 2 & GP1 & Pointer & $\begin{array}{l}\text { Pointer to general } \\
\text { note that describes } \\
\text { the first analysis } \\
\text { case }\end{array}$ \\
\hline • & - & • & - \\
\hline - & - & • & • \\
\hline • & - & • & • \\
\hline $1+N C$ & GPNC & Pointer & $\begin{array}{l}\text { Pointer to } \\
\text { general note that } \\
\text { describes the last } \\
\text { analysis case }\end{array}$ \\
\hline $2+N C$ & NN & Integer & Number of nodes \\
\hline $3+N C$ & NO 1 & Integer & $\begin{array}{l}\text { Node number } \\
\text { identifier for first } \\
\text { node }\end{array}$ \\
\hline $4+\mathrm{NC}$ & NP1 & Pointer & $\begin{array}{l}\text { Pointer to Node } \\
\text { Directory Entry }\end{array}$ \\
\hline
\end{tabular}




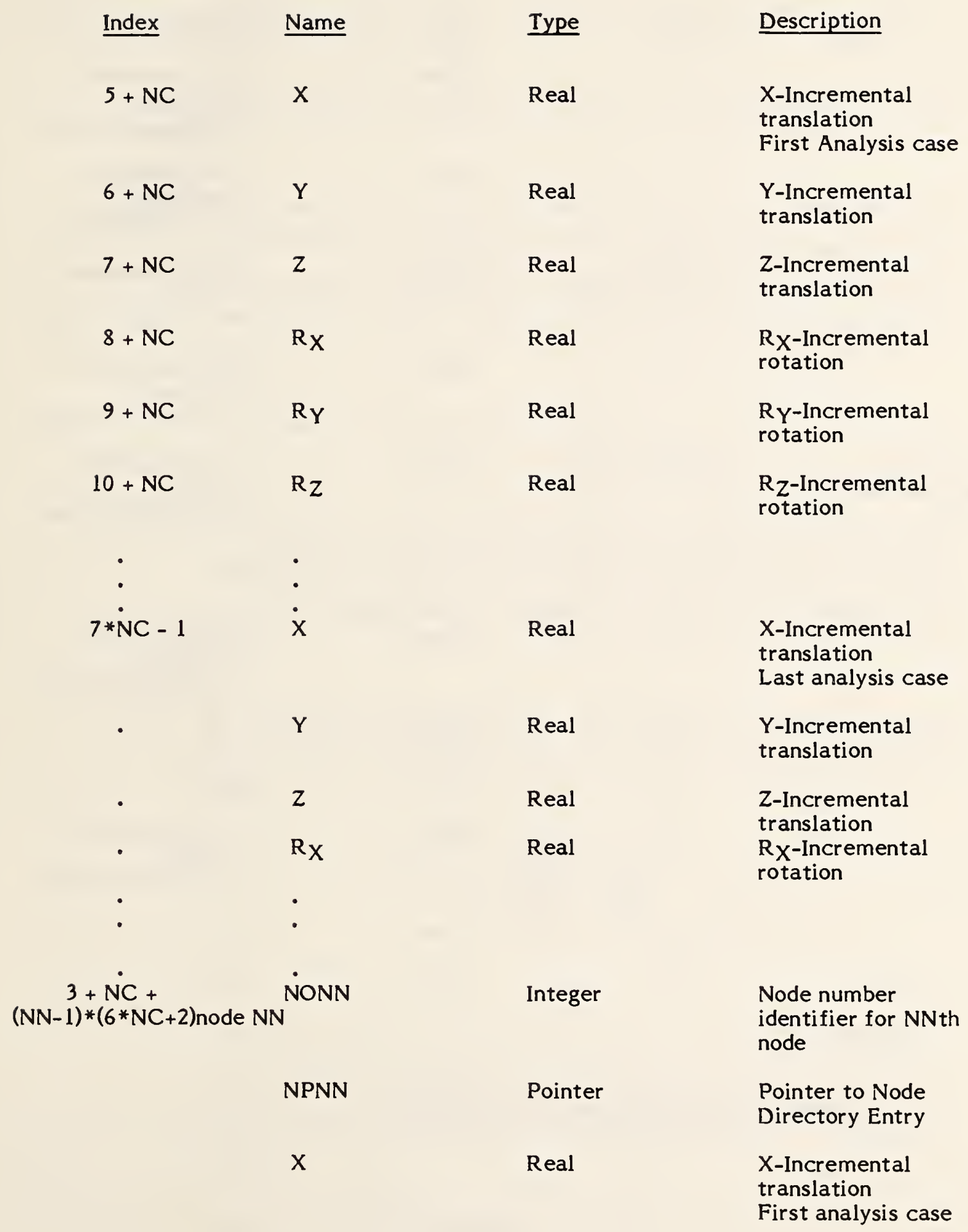




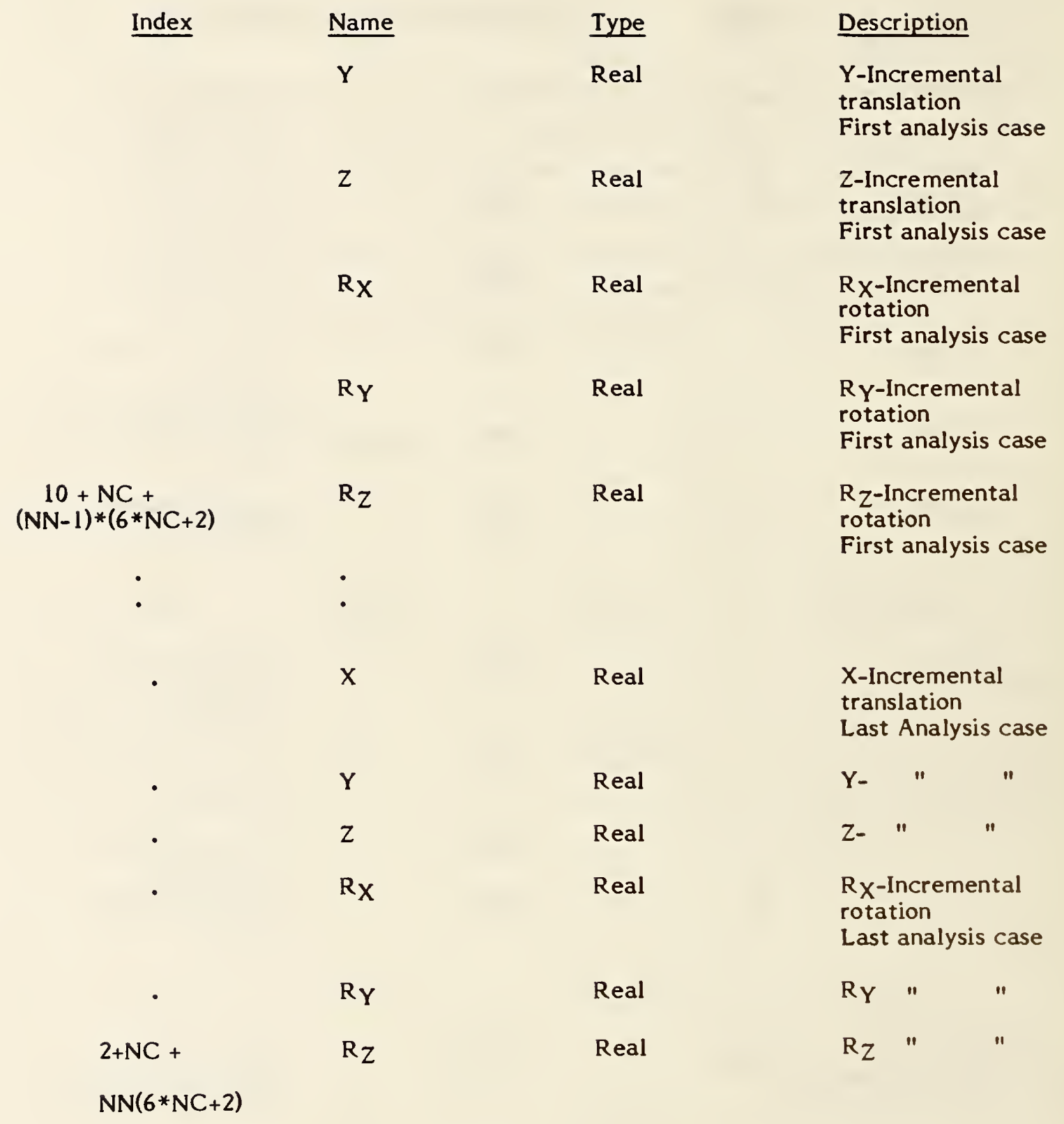

Additional Pointers as required (see 2.2.4.4.2). 
The offset surface is a surface defined in terms of an already existing surface.

1. Let $\mathrm{S}=\mathrm{S}(\mathrm{u}, \mathrm{v})$ be a regular surface defined by this Specification parametrized and oriented by $\mathrm{N}(\mathrm{u}, \mathrm{v})$, a differentiable field of unit normal vectors defined on the whole surface, and $d$ a fixed non-zero real number. An offset surface to $S$ is a parametrized surface $S(u, v)$ given by:

$$
\begin{aligned}
& O(u, v)=S(u, v)+d * N(u, v) ; \quad u l \leqslant u \leqslant u 2 \\
& v 1 \leqslant v \leqslant v 2
\end{aligned}
$$

The base surface $S(u, v)$ is referenced by a pointer in the parameter data section, while $\mathrm{N}(\mathrm{u}, \mathrm{v})$ is found from $\mathrm{S}(\mathrm{u}, \mathrm{v})$ as defined below. The value of $d$ is provided as a parameter value in the parameter data section.

2. To determine which one of the two orientations of the orientable regular surface $S(u, v)$ the offset surface will be used to define $O$, define

$$
N(u, v)=\frac{\frac{\partial S}{\partial u} \times \frac{\partial S}{\partial v}}{\left\|\frac{\partial S}{\partial u} \times \frac{\partial S}{\partial v}\right\|}
$$

In order to avoid confusion connecting the orientation of the base surface S(u,v), an additional offset indicator is included. That indicator shown in Figure 3-21 consists of the vector $(\mathrm{Nx}, \mathrm{Ny}, \mathrm{Nz}$ ) defined by

$(N x, N y, N z)=N(U m, V m) /\|N(U m, V m)\|$

(This is the unit normal vector at the parameter values $(\mathrm{Um}, \mathrm{Vm})$ ) 
where, if the surface is bounded,

$$
\mathrm{Um}=\frac{\mathrm{U} 1+\mathrm{u} 2}{2} \text { and } \mathrm{Vm}=\frac{\mathrm{v} 1+\mathrm{v} 2}{2}
$$

or, if the surface is unbounded,

$$
\mathrm{Um}=0.0 \quad \text { and } \quad \mathrm{Vm}=0.0
$$

This indicates the direction in which the offset distance, $d$, is measured positive at $(\mathrm{Um}, \mathrm{Vm})$.

CAUTION: the vector $(\mathrm{Nx}, \mathrm{Ny}, \mathrm{Nz})$ is just an indicator of the direction with respect to the base surface $S(u, v)$ where the offset distance, $d$, is measured positively; this vector does not participate in the evaluation of the offset surface as is evident from formula for $O$ that defines the of fset surface.

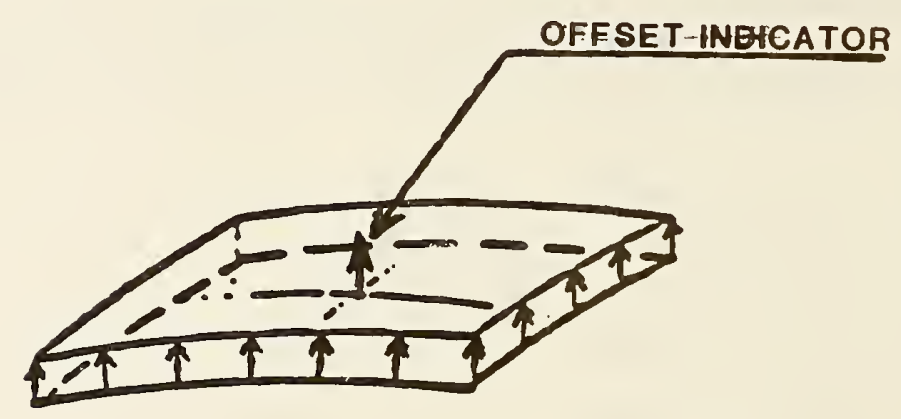

FIGURE 3-21 OFFSET SURFACE IN 3-D EUCLIDEAN SPACE 


\subsubsection{Directory Data}

ENTITY TYPE NUMBER:

140

\subsubsection{Parameter Data}

\begin{tabular}{|c|c|c|c|}
\hline Index & Name & Type & Description \\
\hline 1 & $N x$ & Real & $\begin{array}{l}\text { The } x \text {-coordinate of the offset } \\
\text { indicator } N(U m, V m) \text {. }\end{array}$ \\
\hline 2 & $\mathrm{Ny}$ & Real & $\begin{array}{l}\text { The } y \text {-coordinate of the offset } \\
\text { indicator } \mathrm{N}(\mathrm{Um}, \mathrm{Vm}) \text {. }\end{array}$ \\
\hline 3 & $\mathrm{Nz}$ & Real & $\begin{array}{l}\text { The } z \text {-coordinate of the offset } \\
\text { indicator } N(U m, V m) \text {. }\end{array}$ \\
\hline 4 & d & Real & $\begin{array}{l}\text { The distance by which the surface is } \\
\text { normally offset on the side of the } \\
\text { of fset indicator if } d>0 \text { and on the } \\
\text { opposite side if } d<0 \text {. }\end{array}$ \\
\hline 5 & $\mathrm{DE}$ & Pointer & $\begin{array}{l}\text { Pointer to the surface entity to be } \\
\text { of fset. }\end{array}$ \\
\hline
\end{tabular}

Additional Pointers as required (see 2.2.4.4.2). 


\subsection{Curve On A Parametric Surface Entity}

The Curve on a Parametric Surface entity associates a given curve with a surface and identifies the curve as lying on the surface.

Let $S=S(u, v)=(x(u, v), y(u, v), z(u, v))$

be a regular parametrized surface whose domain is a rectangle defined by

$$
D=((u, v) \mid u 1 \leqslant u \leqslant u 2 \text { and } v 1 \leqslant v \leqslant v 2)
$$

Let $B=B(t)$ be a curve defined by

$$
\begin{aligned}
& B(t)=(u(t), v(t)) \quad \text { for } a \leqslant t \leqslant b \\
& \text { taking its values in } D \text {. }
\end{aligned}
$$

A curve $C(t)$ on the surface $S(u, v)$ is the composition of two mappings, $\mathrm{S}$ and $\mathrm{B}$ defined as follows:

$$
\begin{aligned}
C(t) & =S o B(t) \\
& =S(B(t)) \\
& =S(u(t), v(t)) \\
& =(x(u(t), v(t)), y(u(t), v(t)), z(u(t), v(t))) \quad a \leqslant t \leqslant b
\end{aligned}
$$

The curve $B$ lies in the two dimensional space which is the domain of the surface $\mathrm{S}$. Therefore, the representation used for $\mathrm{B}$ which has been derived from a curve defined in this Specification must be two dimensional: the $X$ and $\mathrm{Y}$ coordinates of this curve pointed to by BPTR are used.

The "Entity Use Flag" (DE field 9) of the entity B is set to 05, indicating that $B$ is in the parameter space of the surface. As a consequence of that, $B$ cannot be scaled and if a transformation matrix is to be applied on $B$ it has to map it within the parameter space $D$ in which it resides. 
Hence, a curve on a parametric surface is given by:

(a) the mapping $C$ and an indication that the curve lies on the surface S(u,v),

and

(b) the mappings $\mathrm{B}$ and $\mathrm{S}$ whose composition gives the curve $\mathrm{C}$.

A curve on a surface may have been created in one of a number of various ways:

(a) As the projection on the surface of a given curve in model space in a prescribed way, for example, parallel to a given fixed vector.

(b) As the intersection of two given surfaces.

(c) By a prescribed functional relation between the surface parameters " $u$ " and "v".

(d) By a special curve, such as a geodesic, emanating from a given point in a certain direction, a principal curve (line of curvature) emanating from a certain point, an asymptotic curve emanation from a certain point, an isoparametric curve for a given value, or any other kind of special curve.

The parameter data section contains three pointers:

(a) A pointer to the curve from which $B(t)$ is derived;

(b) A pointer to the surface $S(u, v)$;

(c) A pointer to the mapping $C(t)$.

It also contains:

(d) A flag to indicate how the curve was created;

(e) A flag to indicate which of the two alternate representations was preferred by the sending system. 
3.24.1 Directory Data

ENTITY TYPE NUMBER:

\subsubsection{Parameter Data}

\begin{tabular}{|c|c|c|c|}
\hline Index & Name & Type & Description \\
\hline 1 & CRTN & Integer & $\begin{array}{l}\text { Indicates the way the curve on the sur- } \\
\text { face has been created: } \\
0=\text { Unspecified. } \\
1=\text { Projection of a given curve on the } \\
\text { surface. } \\
2=\text { Intersection of two surfaces. } \\
3=\text { Isoparametric curve, i.e. either a } \\
\text { u-parametric or a v-parametric } \\
\text { curve. }\end{array}$ \\
\hline 2 & SPTR & Pointer & $\begin{array}{l}\text { Pointer to the surface on which } \\
\text { the curve lies. }\end{array}$ \\
\hline 3 & $B * P T R$ & Pointer & $\begin{array}{l}\text { Pointer to the entity that contains the } \\
\text { definition of the curve } C^{*} \text { in the para- } \\
\text { metric space }(u, v) \text { of the surface } S \text {. }\end{array}$ \\
\hline 4 & CPTR & Pointer & Pointer to the curve $\mathrm{C}$. \\
\hline 5 & PREF & Integer & $\begin{array}{l}\text { Indicates preferred representation } \\
\text { in the sending system: } \\
0=\text { Unspecified. }\end{array}$ \\
\hline & & & $\begin{array}{l}1=\mathrm{SoB} * \text { is preferred. } \\
2=C \text { is preferred. } \\
3=C \text { and SoB } B^{*} \text { are equally preferred. }\end{array}$ \\
\hline
\end{tabular}

Additional pointers as required (see 2.2.4.4.2). 
A simple closed curve in the Euclidean plane divides the plane into two disjoint open connected components; one bounded and one unbounded. The bounded one is called the interior region to the curve (herein called, "interior"). The unbounded component is called the exterior region to the curve (herein called, "exterior").

The domain of the trimmed surface is defined as the common region of the interior of the outer boundary and the exterior of each of the inner boundaries and includes the boundary curves. Note that the trimmed surface has the same mapping $S(u, v)$ as the original (untrimmed surface) but different domain. The curves that delineate either the outer or the inner boundary of the trimmed surface are curves on the surface $S$, and are to be exchanged by means of the Curve on a Parametric Surface (Entity Type 142).

Let $S(u, v)$ be a regular parametrized surface, whose untrimmed domain is a rectangle $D$ consisting of those points $(u, v)$ such that $a \leqslant u \leqslant b$ and $c \leqslant v \leqslant d$ for given constants $a, b, c$, and $d$ with $a<b$ and $c<d$. Assume that $S$ takes its values in three dimensional Euclidean space so that it can be expressed as

$S=S(u, v)=\left[\begin{array}{c}x(u, v) \\ y(u, v) \\ z(u, v)\end{array}\right]$ for each ordered pair $(u, v)$ in D.

Also let the mapping $S$ be subject to the following regularity conditions:

- It has continuous normal vector in the interior of D.

- It is one-to-one in D.

- There are no singular points in D, i.e., the vectors of the first partial derivatives of $S$ at any point in $D$ are linearly independent. 
Two types of simple closed curves are utilized to define the domain of the trimmed (parametric) surface.

The first type is called the outer boundary. There is exactly one. It lies in $\mathrm{D}$, and in particular, it can be the boundary curve of D.

The second type is called the inner boundary. There can be any number of them including zero. The set of inner boundaries satisfies two criteria:

1. The curves as well as their interiors are mutually disjoint.

2. Each curve lies in the interior of the outer boundary.

If the outer boundary of the surface being defined is the boundary of D and there are no inner boundaries, then the trimmed surface being defined is, in fact, untrimmed.

\subsubsection{Directory Data}

ENTITY TYPE NUMBER: 144

\subsubsection{Parameter Data}

Index Name Type Description

1 PTS Pointer Pointer to the surface entity that is to be trimmed

2 N1 Integer $=0$, if the outer boundary is the boundary of D. $=1$, otherwise.

$3 \quad$ N2 Integer

This number indicates the number of simple closed curves which constitute the inner boundary of the trimmed surface. In case no inner boundary is introduced, this is set equal to zero.

3+N1 PTO1 Pointer Pointer to the simple closed curve (Curve on a Parametric Surface Entity), that constitutes the outer boundary of the trimmed surface. 


$\begin{array}{llll}\text { Index } & \text { Name } & \text { Type } & \text { Description } \\ 4+\mathrm{N} 1 & \text { PTIl } & \text { Pointer } & \begin{array}{l}\text { Pointer to the first simple closed inner } \\ \text { boundary curve (Curve on a Parametric } \\ \text { Surface entity) according to some arbitrary } \\ \text { ordering of these entities. }\end{array} \\ \text {. }\end{array}$

3+N1+N2 PTIN2 Pointer Pointer to the last simple closed inner boundary curve (Curve on a Parametric Surface entity).

Additional pointers as required (see 2.2.4.4.2) 
THIS PAGE LEFT BLANK 


\subsection{General}

This section contains capabilities for representing non-geometry and includes:
- Annotation Entities
- Structure Entities

Entity numbers from 200 through 499 are reserved for this Section. In addition, some non-geometric entities make use of entity type number 106 (copious data). 
4.2 Annotation Entities

4.2.1 Entity Type/Type Number.

The following entities are defined in this section:

Entity Type Number Entity Type

106

202

206

208

210

212

214

216

218

220

222

228

230
Copious Data

Centerline

Section

Witness Line

Angular Dimension

Diameter Dimension

Flag Note

General Label

General Note

Leader (Arrow)

Linear Dimension

Ordinate Dimension

Point Dimension

Radius Dimension

General Symbol

Sectioned Area

\subsubsection{Construction.}

Many annotation entities are constructed by using other entities. For example, the dimension entities may have 0,1 , or 2 pointers to witness line entities ( $a$ form of copious data), 0, 1, or 2 pointers to leader (arrow) entitites and a pointer to a general note entity.

For some annotation entities, a witness line or leader may not exist. For these cases the parameter data field pointer value can be set zero. If any constructive entity exists, but its display is suppressed, it can be set to blank status or, if allowed, the pointer value can be set to zero. 


\subsubsection{Definition Space.}

An annotation entity may be defined in XT, YT, ZT definition space (see the discussion in Section 3.1) or in a two-dimensional space associated with a Drawing Entity (type 404). In the case of XT, YT, ZT definition space, a transformation matrix is applied to locate the annotation entity within model space.

Within the XT, YT, ZT definition space, subordinate entities to an annotation entity may have different $Z T$ displacements. For example, within the linear dimension, a different $Z T$ value may be found in each of: general note, leader, and witness lines (which are pointed to in the linear dimension parameter data). An example showing the use of ZT displacement (DEPTH) is shown in Figure 4-1.

While the option of having dimensions occupy different planes exists, it is expected that only a single plane will be used. The reason for its existence is due to the structure of annotation entities. As each dimension may be comprised of several subordinate entities, each subordinate entity by its definition has the ability to stand alone and may require its own $\mathrm{ZT}$ displacement. When used in conjunction with other entities as a subordinate to a primary entity, it is likely, though not necessary, that each $Z T$ is identical. 
NON-GEOMETRY - Annotation Entities

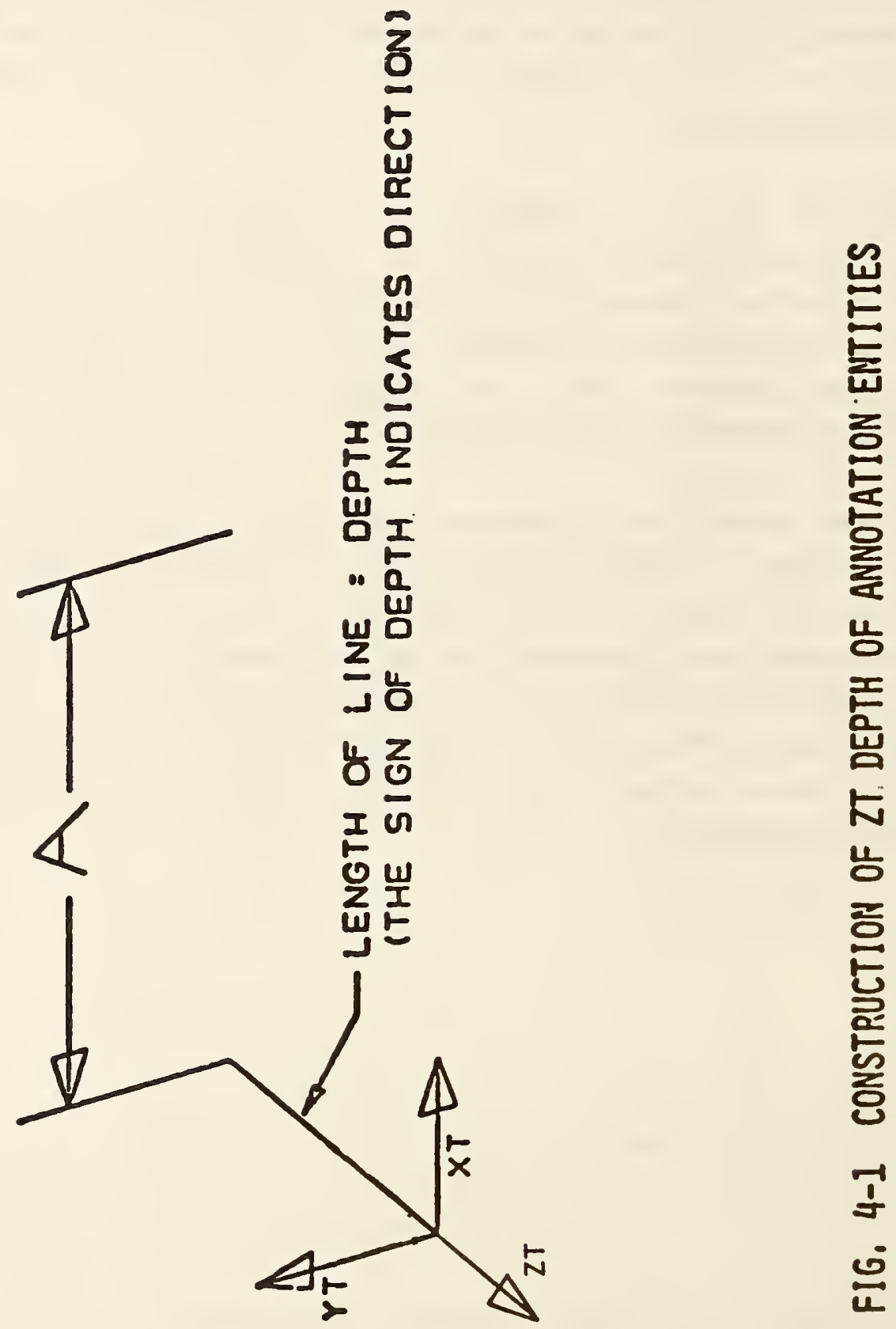


4.2.4 Angular Dimension Entity.

An angular dimension entity consists of a general note; zero, one, or two witness lines; two leaders; and an angle vertex point. Figure 4-2 indicates the construction used. Refer to Figure 4-3 for examples of angular dimensions. If two witness lines are used, each is contained in its own copious data entity.

Each leader consists of at least one circular arc segment w: th an arrowhead at one end. The leader pointers are ordered such that the first circular arc segment of the first leader is defined in a counterclockwise manner from arrowhead to terminate point, and the first circular arc segment of the second leader is defined in a clockwise manner. (Refer to 3.1.2 for information relating to the use of the term counterclockwise).

4.2.4.1 Section 4.2.10 contains a discussion of multi-segment leaders. For those leaders in angular dimension entities consisting of more than one segment, the first two segments are circular arcs with a center at the vertex point. The second circular arc segment is defined in the opposite direction from the first circular arc segment. . Remaining segments, if any, are straight lines. Any leader segment in which the start point is the same as the terminate point is to be ignored. This convention arises to facilitate the definition of the second circular arc segment such as in the bottom leader in Figure 4-2. Example 1 in Figure 4-3 illustrates a leader with three segments. 


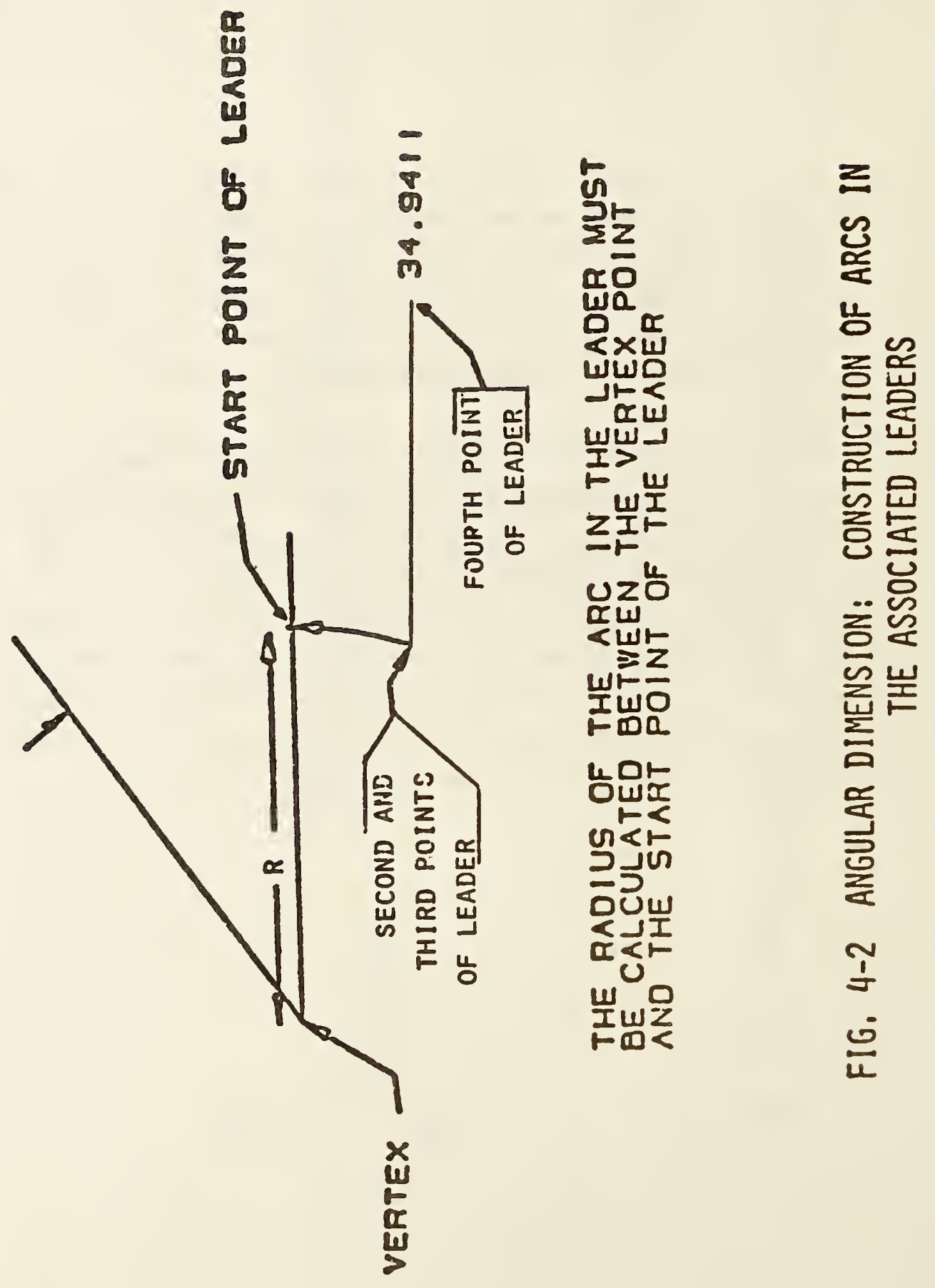


$m$
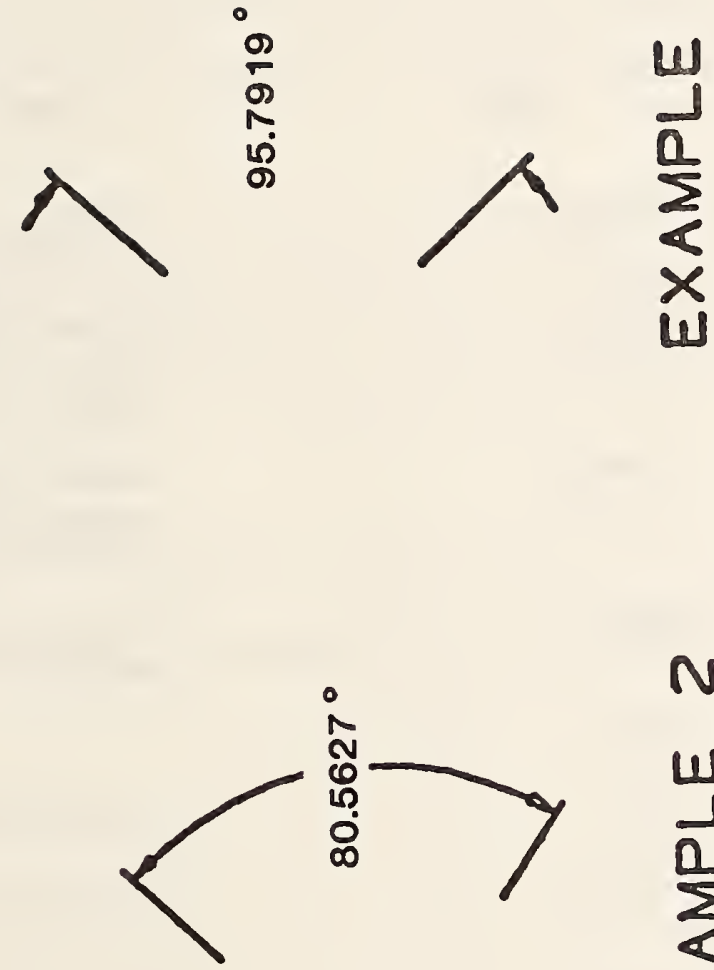

$u$
$\frac{1}{0}$
$\frac{1}{u}$
$w$

N

$\frac{1}{3}$

$E$<smiles>CCC[Pb]</smiles>

胥

出

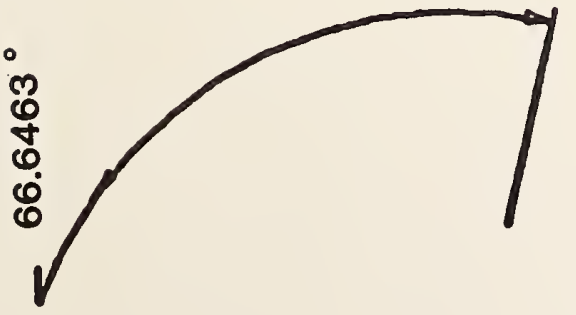

$\frac{1}{2}$

m

$\stackrel{5}{4}$ 


\subsubsection{Directory Data}

ENTITY TYPE NUMBER : 202

\subsubsection{Parameter Data}

\begin{tabular}{|c|c|c|c|}
\hline Index & Name & Type & Description \\
\hline 1 & GN & Pointer & $\begin{array}{l}\text { Pointer to general note } \\
\text { directory entry }\end{array}$ \\
\hline 2 & W 1 & Pointer & $\begin{array}{l}\text { Pointer to first wit- } \\
\text { ness line directory } \\
\text { entry or } 0\end{array}$ \\
\hline 3 & W2 & Pointer & $\begin{array}{l}\text { Pointer to second } \\
\text { witness line directory } \\
\text { entry or } 0\end{array}$ \\
\hline 4 & $\mathrm{XT}$ & Real & $\begin{array}{l}\text { Coordinates of ver- } \\
\text { tex point }\end{array}$ \\
\hline 5 & YT & Real & \\
\hline 6 & $\mathrm{R}$ & Real & Radius of leader arcs \\
\hline 7 & Al & Pointer & $\begin{array}{l}\text { Pointer to 1st leader } \\
\text { directory entry }\end{array}$ \\
\hline 8 & A2 & Pointer & $\begin{array}{l}\text { Pointer to } 2 \text { nd leader } \\
\text { directory entry }\end{array}$ \\
\hline
\end{tabular}

Additional Pointers as required (see 2.2.4.4.2). 


\subsubsection{Centerline Entity}

The centerline entity takes one of two forms. The first, as illustrated in Figure 4-4, Examples 1 and 2, appears as crosshairs and is normally used in conjunction with circles. The second type (Example 3) is a construction between 2 positions.

The Centerline entities are defined as form 20 or 21 of copious data. The associated matrix transforms the XT-YT plane of the centerline into model space. The coordinates of the centerline points describe the centerline display symbol. The display symbol is described by line segments where each line is from

$\left(X_{n}, Y_{n}, Z_{n}\right)$, to $\left(X_{n+1}, Y_{n+1}, Z_{n+1}\right)$ where $n=1,3,5, \ldots, N-1$

4.2.5.1 See section 3.5 for parameters of the Centerline Entity. 
106, Forms 20,21 - CENTERLINE
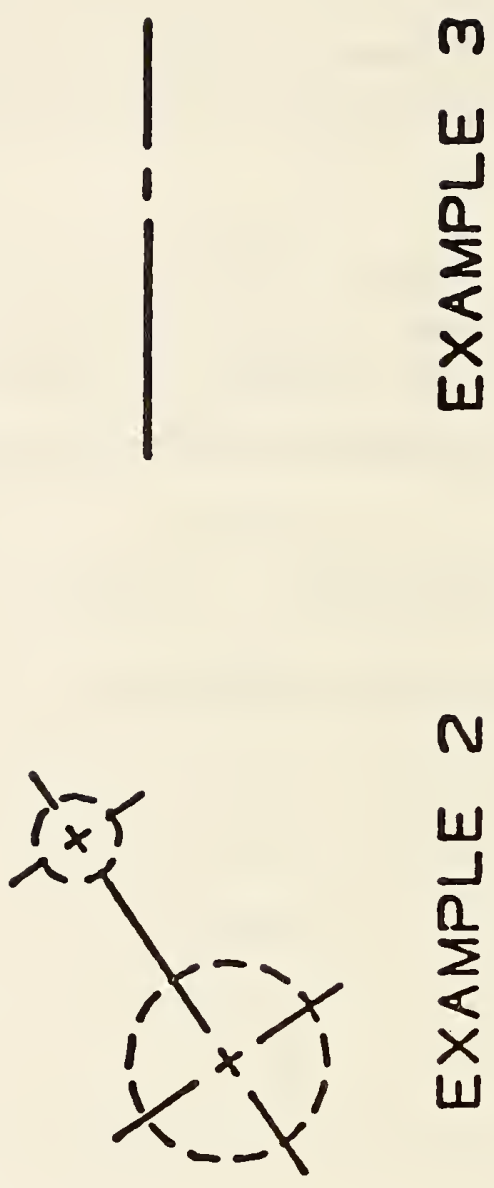

$N$

u

1

E

$\sum_{0}$

这

는

岁

U

us

$\stackrel{1}{1}$

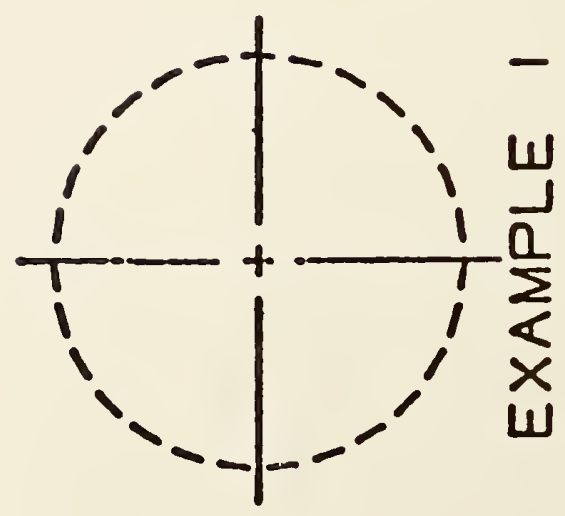

芒 


\subsubsection{Diameter Dimension Entity}

A diameter dimension consists of a general note, one or two leaders, and an arc center point. Refer to Figure 4-5 for examples of the diameter dimension entity. The arc center coordinates are used for positioning the diameter dimension line relative to the arc being dimensioned.

\subsubsection{Directory Data}

\section{ENTITY TYPE NUMBER : 206}

\subsubsection{Parameter Data}

\begin{tabular}{|c|c|c|c|}
\hline Index & Name & Type & Description \\
\hline 1 & NE & Pointer & $\begin{array}{l}\text { Pointer to general } \\
\text { note directory entry }\end{array}$ \\
\hline 2 & Al & Pointer & $\begin{array}{l}\text { Pointer to first lead- } \\
\text { er directory entry }\end{array}$ \\
\hline 3 & A2 & Pointer & $\begin{array}{l}\text { Pointer to second } \\
\text { leader directory } \\
\text { entry or zero }\end{array}$ \\
\hline 4 & $\mathrm{XT}$ & Real & $\begin{array}{l}\text { Arc center coordin- } \\
\text { ates }\end{array}$ \\
\hline 5 & YT & Real & \\
\hline
\end{tabular}

Additional Pointers as required (see 2.2.4.4.2). 

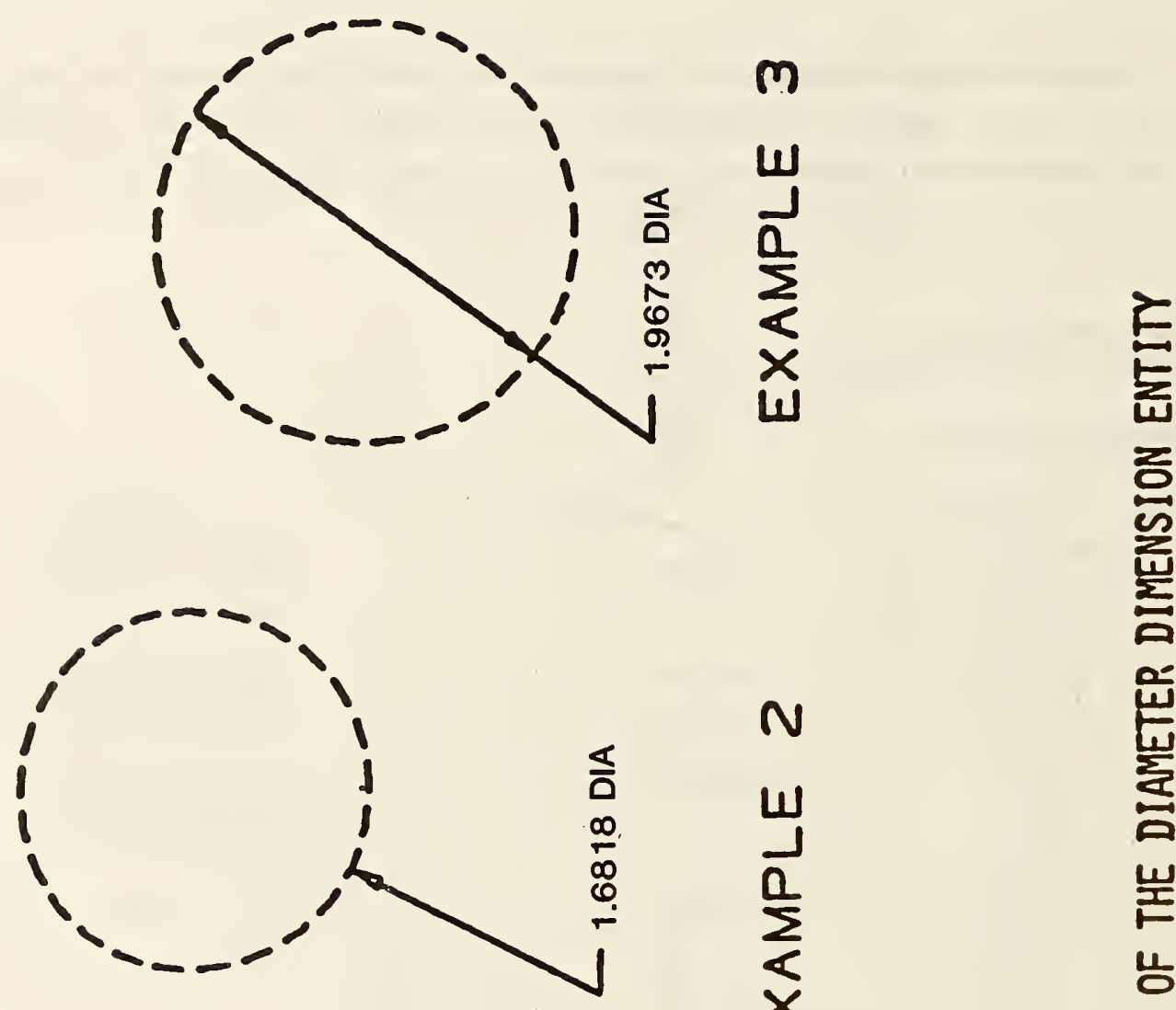

$N$

山 究

$\sum^{0}$ 본

능

뜰

!n

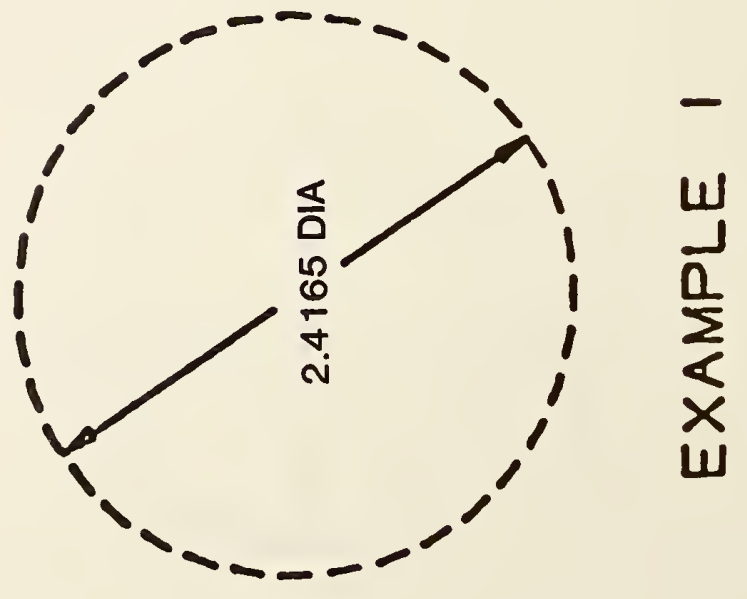

ப் 


\subsubsection{Flag Note Entity}

A flag note entity is label information formatted as shown in Figure 4-6. The rotation angle overrides the general note rotation angle and placement. Additional examples of the flag note entity are shown in Figure 4-7.

The flag note entity may be defined with or without associated leaders.

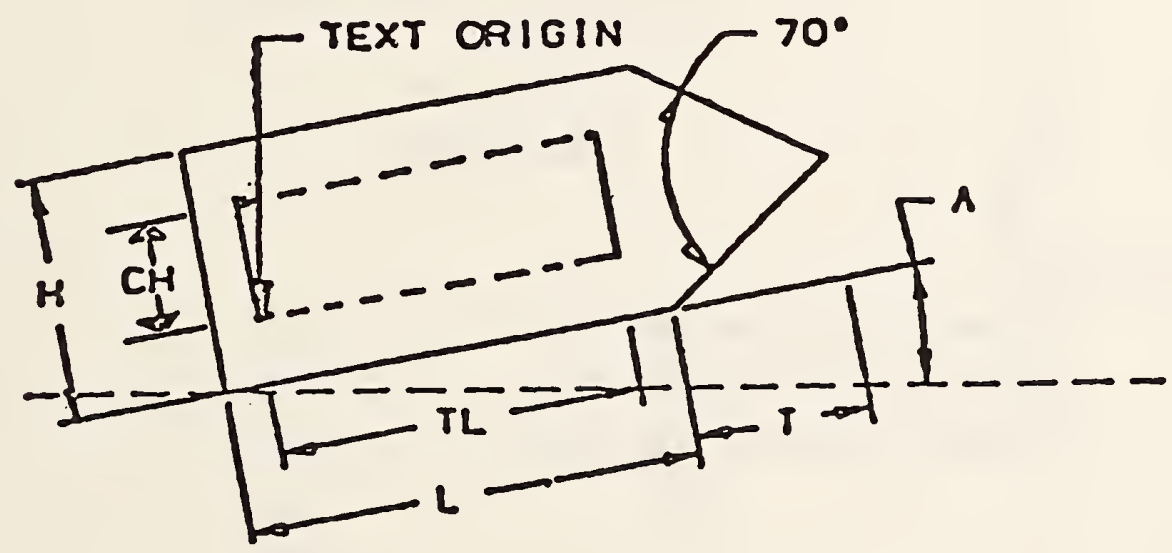

NOTE: Box outlined within flag illustrates bounds of text and should not be interpreted as a sub-symbol.

Figure 4-6 FLAG NOTE

4.2.7.1 The flag note is constructed from information defined in the general note entity. This data is the character height and number of characters. For this reason, no geometric definition is explicit within the definition of the flag note entity.

The following specifications apply to Figure 4-6. 
Variables:
$\mathrm{H}=$ Height
$\mathrm{CH}=$ Character height
$L=$ Length
$\mathrm{NC}=$ Number of characters
$\mathrm{TL}=$ Text Length
(in general note)
$T=$ Tip Length
$A=$ Rotation angle in radians

Formulas:

$\begin{array}{lll}\mathrm{TL} & = & (.8)(\mathrm{CH})(\mathrm{NC})+(.4)(\mathrm{CH})(\mathrm{NC}-1) \\ \mathrm{H} & = & (2)(\mathrm{CH}) \\ \mathrm{L} & = & (\mathrm{TL})+(.4)(\mathrm{CH}) \\ \mathrm{T} & = & (.5)(\mathrm{H}) / \mathrm{TAN}^{\circ} 5^{\circ}\end{array}$

Restrictions:

$\mathrm{H}$ shall never be less than .3 in.

L shall never be less than .6 in.

$T$ shall never be less than .214 in. 

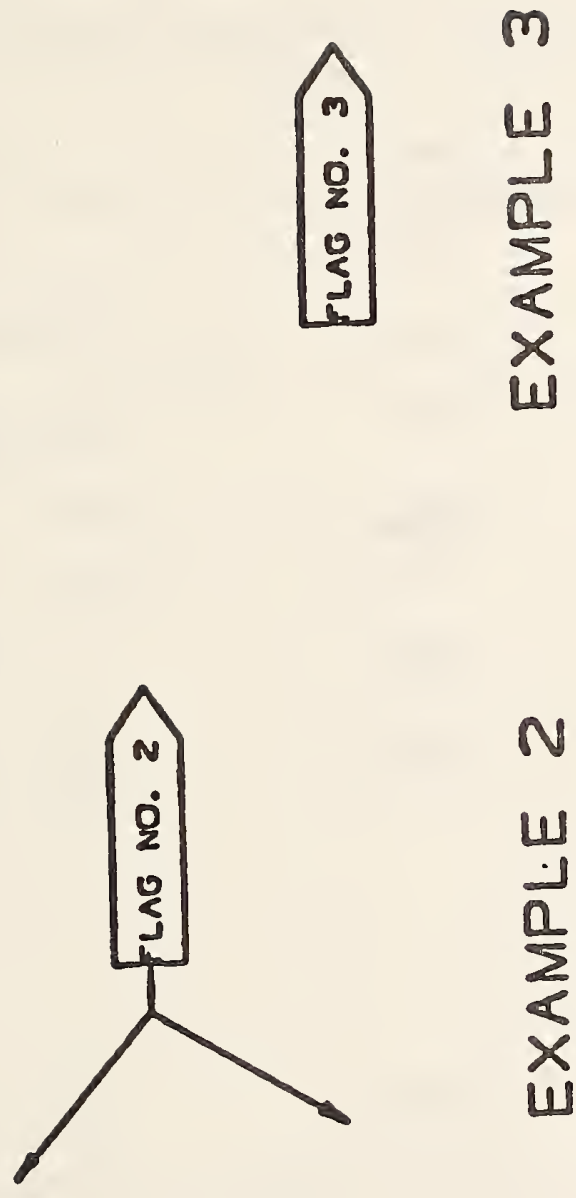

$N$

U

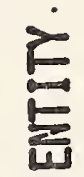

a 崖

$\sum_{<}^{\infty}$ 항

$x$

U

㟧

I

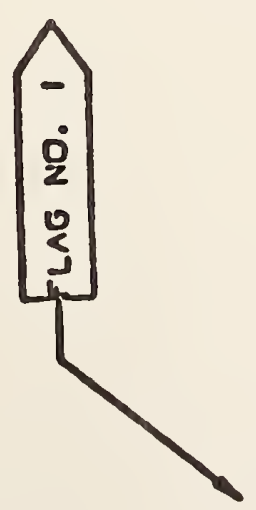

$\stackrel{5}{11}$

$\frac{1}{a}$ 


\subsubsection{Directory Data}

\section{ENTITY TYPE NUMBER : 208}

\subsubsection{Parameter Data}

\begin{tabular}{|c|c|c|c|}
\hline Index & $\underline{\text { Name }}$ & Type & Description \\
\hline 1 & XT & Real & \multirow{3}{*}{$\begin{array}{l}\text { Lower left corner } \\
\text { coordinate of the } \\
\text { flag }\end{array}$} \\
\hline 2 & $\mathrm{YT}$ & Real & \\
\hline 3 & ZT & Real & \\
\hline 4 & A & Real & $\begin{array}{l}\text { Rotation angle in } \\
\text { radians }\end{array}$ \\
\hline 5 & DENOTE & Pointer & $\begin{array}{l}\text { Pointer to general } \\
\text { note directory entry }\end{array}$ \\
\hline 6 & $\mathrm{~N}$ & Integer & $\begin{array}{l}\text { Number of arrows } \\
\text { (leaders) or zero }\end{array}$ \\
\hline 7 & DE1 & Pointer & $\begin{array}{l}\text { Pointers to } \\
\text { associated leaders }\end{array}$ \\
\hline \multicolumn{4}{|l|}{ - } \\
\hline \multicolumn{4}{|l|}{ • } \\
\hline \multicolumn{4}{|l|}{ • } \\
\hline $6+N$ & DE 1 & Pointer & Pointer to last leader \\
\hline
\end{tabular}




\subsubsection{General Label Entity.}

A general label entity consists of a general note with one or more associated leaders.

Examples of general label entities are shown in Figure 4-8

\subsubsection{Directory Data}

ENTITY TYPE NUMBER : 210

\subsubsection{Parameter Data}

\begin{tabular}{|c|c|c|c|}
\hline Index & $\underline{\text { Name }}$ & Type & Description \\
\hline 1 & DENOTE & Pointer & $\begin{array}{l}\text { Pointer to associated } \\
\text { general note }\end{array}$ \\
\hline 2 & $\mathbf{N}$ & Integer & Number of leaders \\
\hline 3 & $\mathrm{DE}$ & Pointer & $\begin{array}{l}\text { Pointers to } \\
\text { associated leaders }\end{array}$ \\
\hline • & • & - & \\
\hline • & • & • & \\
\hline • & • & $\cdot$ & \\
\hline $\mathrm{N}+2$ & $\mathrm{DE}$ & Pointer & Pointer to last leader \\
\hline
\end{tabular}

Additional Pointers as required (see 2.2.4.4.2). 
210 - GENERAL LABEL
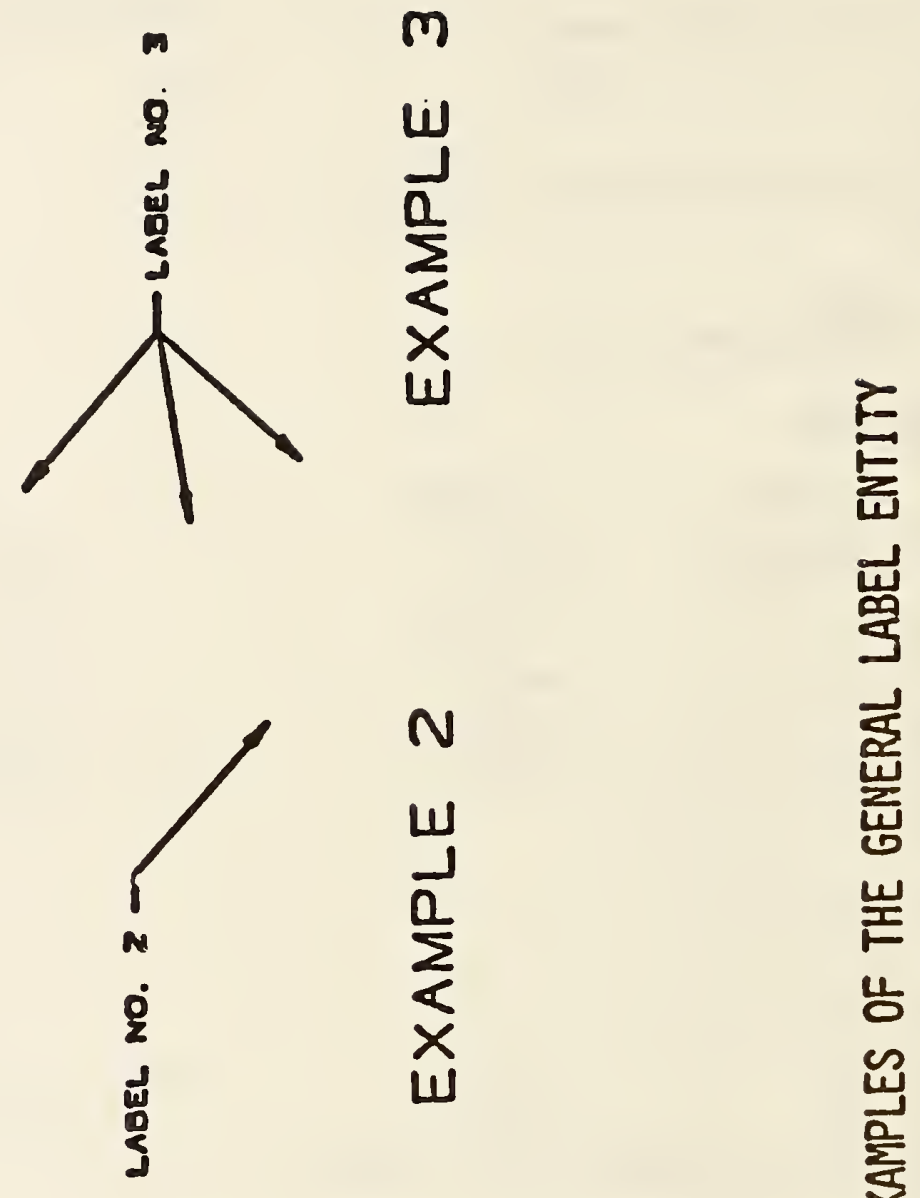

$\frac{1}{x}$

岸

岁

崫

$\infty$
1

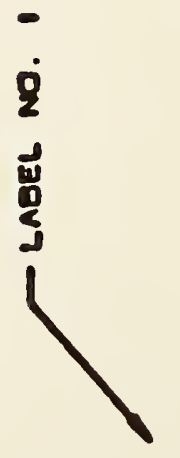

늠

$\sum_{\omega}^{\frac{1}{2}}$ 


\subsubsection{General Note Entity.}

A general note entity consists of one or more text strings. Each text string contains text, a starting point, text size, and angle of rotation of the text. Examples of general notes are shown in Figure 4-9. The FC value indicates the font number and is an integer. Positive values are pre-defined fonts. Negative values point to user defined fonts or modifications to a pre-defined font.

The following fonts will be defined:

0. Symbol Font (use no longer recommended)

1. Standard Block

2. LeRoy

3. Futura

4. Fastfont

5. Calcomp

6. Comp 80

7. Micro-Film Standard

8. ISO Standard

9. DIN Standard

10. Military Standard

11. Gothic

12. News Gothic

13. Lightline Gothic

14. Simplex Roman

15. Italic

16. APL

17. Century Schoolbook

18. Helvetica

1001. Symbol Font 1

1002. Symbol Font 2

Fonts in the 1000 series display symbols mapped onto ASCII characters as shown in Figures $4-10$ and 4 - 11. They do not specify a character display font. 


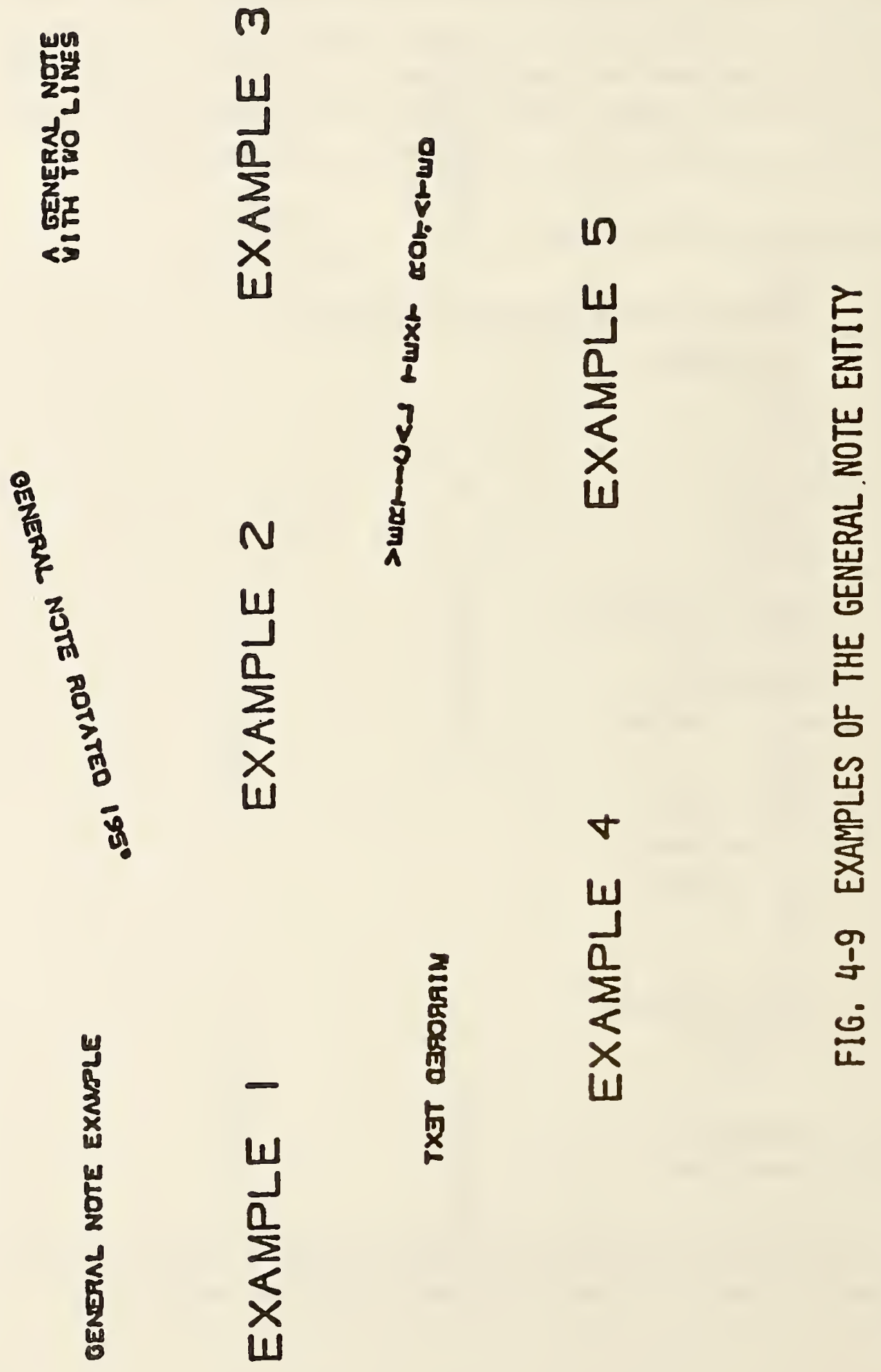




\begin{tabular}{|c|c|c|c|c|c|c|c|c|c|c|c|}
\hline $8 r^{4}$ & & 0 & 0 & - & (c) & $P$ & $P$ & • & 1 & $P$ & (P) \\
\hline 1 & $!$ & 1 & 1 & A & $A$ & 0 & $Q$ & $\mathbf{a}$ & $<$ & $q$ & $\Phi$ \\
\hline - & 10 & 2 & 2 & B & $B$ & $\mathbf{R}$ & $R$ & $b$ & 中 & $r$ & (2) \\
\hline 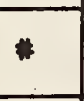 & $\#$ & 3 & 3 & C & $\mathrm{C}$ & $s$ & $S$ & $c$ & $\square$ & s & (S) \\
\hline 8 & $\$$ & 4 & 4 & $D$ & $D$ & $T$ & $T$ & d & $\curvearrowright$ & $t$ & $\square$ \\
\hline$x$ & $\%$ & 5 & 5 & $E$ & $E$ & $u$ & $U$ & e & 0 & $u$ & (1) \\
\hline 8 & 8 & 6 & 6 & $F$ & $F$ & $v$ & $V$ & $f$ & II & $v$ & $\triangle$ \\
\hline . & 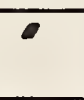 & 7 & 7 & G & $G$ & $w$ & $W$ & 9 & $\phi$ & $v$ & 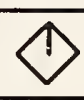 \\
\hline c & $C$ & 8 & 8 & $H$ & $H$ & $x$ & $X$ & h & $\not$ & $x$ & 4 \\
\hline J & ) & 9 & 9 & I & $I$ & $Y$ & $Y$ & $\mathbf{i}$ & $\equiv$ & $y$ & 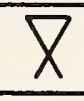 \\
\hline w & 米 & : & $:$ & $J$ & $J$ & Z & 2 & $\mathbf{j}$ & $\phi$ & $z$ & $Y$ \\
\hline+ & + & : & : & $K$ & $K$ & [ & {[} & $k$ & $\curvearrowleft$ & $c$ & \{ \\
\hline - &. & $<$ & $<$ & $L$ & $L$ & 1 & $\backslash$ & 1 & $\perp$ & i & 1 \\
\hline - & - & - & $=$ & $M$ & $M$ & J & ] & $m$ & (1) & 3 & \} \\
\hline . & . & $>$ & $>$ & $N$ & $N$ & $\wedge$ & $\wedge$ & $n$ & $\varnothing$ & $\sim$ & $\sim$ \\
\hline 1 & 1 & $?$ & $?$ & 0 & 0 & - & - & 0 & 0 & & \\
\hline
\end{tabular}

FIGURE 4-10 
212 - GENERAL NOTE

\begin{tabular}{|c|c|c|c|c|c|c|c|c|c|c|c|}
\hline $8^{47^{4}}$ & & 0 & 0 & - & @ & $P$ & $\mathrm{P}$ & $\cdot$ & 1 & $p$ & 4 \\
\hline 1 & $!$ & 1 & 1 & A & $A$ & 0 & $\bar{Q}$ & $\exists$ & 8 & a & $\downarrow$ \\
\hline • & 10 & 2 & 2 & B & $B$ & R & $R$ & b & $\div$ & $r$ & $\rightarrow$ \\
\hline . & \pm & 3 & 3 & C & C & $s$ & $S$ & $c$ & $\leq$ & s & $\leftarrow$ \\
\hline 8 & 0 & 4 & 4 & $D$ & $D$ & $T$ & $\mathrm{~T}$ & d & $\geq$ & $t$ & $\phi$ \\
\hline$x$ & $\%$ & 5 & 5 & $E$ & $E$ & u & $U$ & e & $\Delta$ & $u$ & $\theta$ \\
\hline 8 & $\bar{\alpha}$ & 6 & 6 & $F$ & $F$ & $v$ & $V$ & $f$ & $\sqrt{ }$ & $v$ & $\Upsilon$ \\
\hline$\cdot$ & 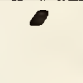 & 7 & 7 & G & $G$ & $w$ & $W$ & 9 & $x$ & $v$ & $\psi$ \\
\hline$c$ & $C$ & 8 & 8 & $H$ & $\mathrm{H}$ & $x$ & $X$ & $h$ & $\equiv$ & $x$ & $\omega$ \\
\hline 3 & $J$ & 9 & 9 & I & $I$ & $Y$ & $Y$ & $i$ & $\neq$ & $y$ & $\lambda$ \\
\hline * & 米 & $:$ & : & $J$ & $J$ & $z$ & $\bar{Z}$ & $\mathrm{j}$ & $\int$ & $z$ & $\propto$ \\
\hline - & + & $:$ & : & $k$ & $\mathrm{~K}$ & c & {[} & $k$ & $\Rightarrow$ & $c$ & $\delta$ \\
\hline . & . & $<$ & $<$ & L & $L$ & 1 & 1 & 1 & V & $i$ & $\mu$ \\
\hline- & - & - & $=$ & $M$ & $M$ & J & ] & m & $\wedge$ & ) & $\pi$ \\
\hline . & . & $>$ & $>$ & $N$ & $N$ & A & $\Lambda$ & $n$ & $\approx$ & $\sim$ & - \\
\hline 1 & 1 & 3 & ? & 0 & 0 & - & - & 0 & $\sum$ & & \\
\hline
\end{tabular}

FIGURE 4-11 
Font 1 does not have a defined display. Use of Font 1 implies the receiving system may use any font which displays the appropriate ASCII characters. The intent of this font is for usage when the actual display of the characters is not critical for the application.

Font 0 is an old symbol font and should no longer be used. Figure $4-12$ is a mapping symbol definition for font 0 .

If the FC number is not sufficient to describe the font, a text font definition entity may be used to define the font. If a text font definition is being used, the negative of the pointer value for the directory entry of the text font definition entity is placed in the FC parameter. The use of the values WT, HT, SL, A, and text start point are shown in Figure 4-13.

Within definition space, the parameters for the text block are applied in the following order (See Figure 4-14):

1) Define the box height (HT) and box width (WT).

The rotate internal text flag indicates whether the text box is filled with horizontal text or vertical text. The box width is measured from the text start point in the positive XT direction and the box height is measured in the positive YT direction from the text start point, before the rotation angle (A) is applied.

2) The slant angle is then applied to each individual character. For horizontal text it is measured from the XT axis in a counterclockwise direction. For vertical text the slant angle is measured from the YT axis.

3) The rotation angle is then applied to the text block. This rotation is applied in a counterclockwise direction about the text start point. The plane of rotation is the XT, YT plane at the depth ZSn (where ZSn is the value given for the text start point). 


\begin{tabular}{|c|c|c|c|c|c|c|c|c|c|c|c|}
\hline 0 & 8 & 26 & $\psi$ & 54 & . & 102 & $B$ & 130 & $X$ & 156 & $\varnothing$ \\
\hline 1 & $\div$ & 27 & $\omega$ & 55 & - & 103 & $C$ & 131 & $Y$ & 157 & 0 \\
\hline 2 & $\leq$ & 30 & $\lambda$ & 56 & $\therefore$ & 104 & $D$ & 132 & 2 & 160 & (P) \\
\hline 3 & $\geq$ & 31 & $\propto$ & 57 & $/$ & 105 & $E$ & 133 & {[} & 161 & $\Phi$ \\
\hline 4 & $\Delta$ & 32 & $\delta$ & 60 & 0 & 106 & $F$ & 134 & $\backslash$ & 162 & (2) \\
\hline 5 & $\sqrt{ }$ & 33 & $\mu$ & 61 & 1 & 107 & $G$ & 135 & ] & 163 & (5) \\
\hline 6 & $x$ & 34 & $\pi$ & 62 & 2 & 110 & $H$ & 136 & $\wedge$ & 164 & $\square$ \\
\hline 7 & $\equiv$ & 35 & - & 63 & 3 & 11111 & $I$ & 137 & - & 165 & (1) \\
\hline 10 & $\neq$ & 36 & \pm & 64 & 4 & 112 & $J$ & 140 & 1 & 166 & $\triangle$ \\
\hline 11 & $\int$ & 37 & 0 & 65 & 5 & 1113 & $K$ & 141 & $<$ & 167 & $\diamond$ \\
\hline 12 & $\supset$ & 40 & & 66 & 6 & 114 & $L$ & 142 & 中 & 170 & 4 \\
\hline 13 & V & 41 & $!$ & 67 & 7 & 115 & $M$ & 143 & $\square$ & 171 & 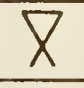 \\
\hline 14 & $\wedge$ & 42 & $\|$ & 70 & 8 & 116 & $N$ & 144 & $\curvearrowright$ & 172 & $Y$ \\
\hline 15 & $\approx$ & 43 & $\#$ & 71 & 9 & 117 & 0 & 145 & 0 & 173 & \{ \\
\hline 16 & $\sum$ & 44 & $\$$ & 72 & : & 120 & $P$ & 146 & II & 174 & 1 \\
\hline 17 & 4 & 45 & $\%$ & 73 & : & 121 & $Q$ & 147 & od & 175 & \} \\
\hline 20 & $\downarrow$ & 46 & 8 & 74 & $<$ & 122 & $R$ & 150 & 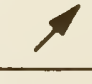 & 176 & $\sim$ \\
\hline 21 & $\rightarrow$ & 47 & $\cdot$ & 75 & $=$ & 123 & $S$ & 151 & $\equiv$ & 177 & $z$ \\
\hline 22 & $\leftarrow$ & 50 & $C$ & 76 & $>$ & 124 & $T$ & 152 & $\phi$ & & \\
\hline 23 & $\phi$ & 51 & $J$ & 77 & $?$ & 125 & $U$ & 153 & $\cap$ & & \\
\hline 24 & $\theta$ & 52 & 米 & 100 & (C) & 126 & V & 154 & $\perp$ & & \\
\hline 25 & $\Upsilon$ & 53 & + & 101 & $A$ & 127 & $W$ & 155 & (M) & & \\
\hline
\end{tabular}

FIGURE 4-12 CHARACTER SET \& OCTAL CODE FOR FONT CODE ZERO 
4) The mirror operation is performed next. The value 1 indicates the mirror axis is the (rotated) line perpendicular to the text base line and through the text start point. The value 2 indicates the mirror axis is the (rotated) test base line.

Finally, the Transformation Matrix entity is used to specify the relative position of definition space within model space.

The number of characters $(\mathrm{NCn})$ must always be equal to the character count in its corresponding text string (TEXTn). 


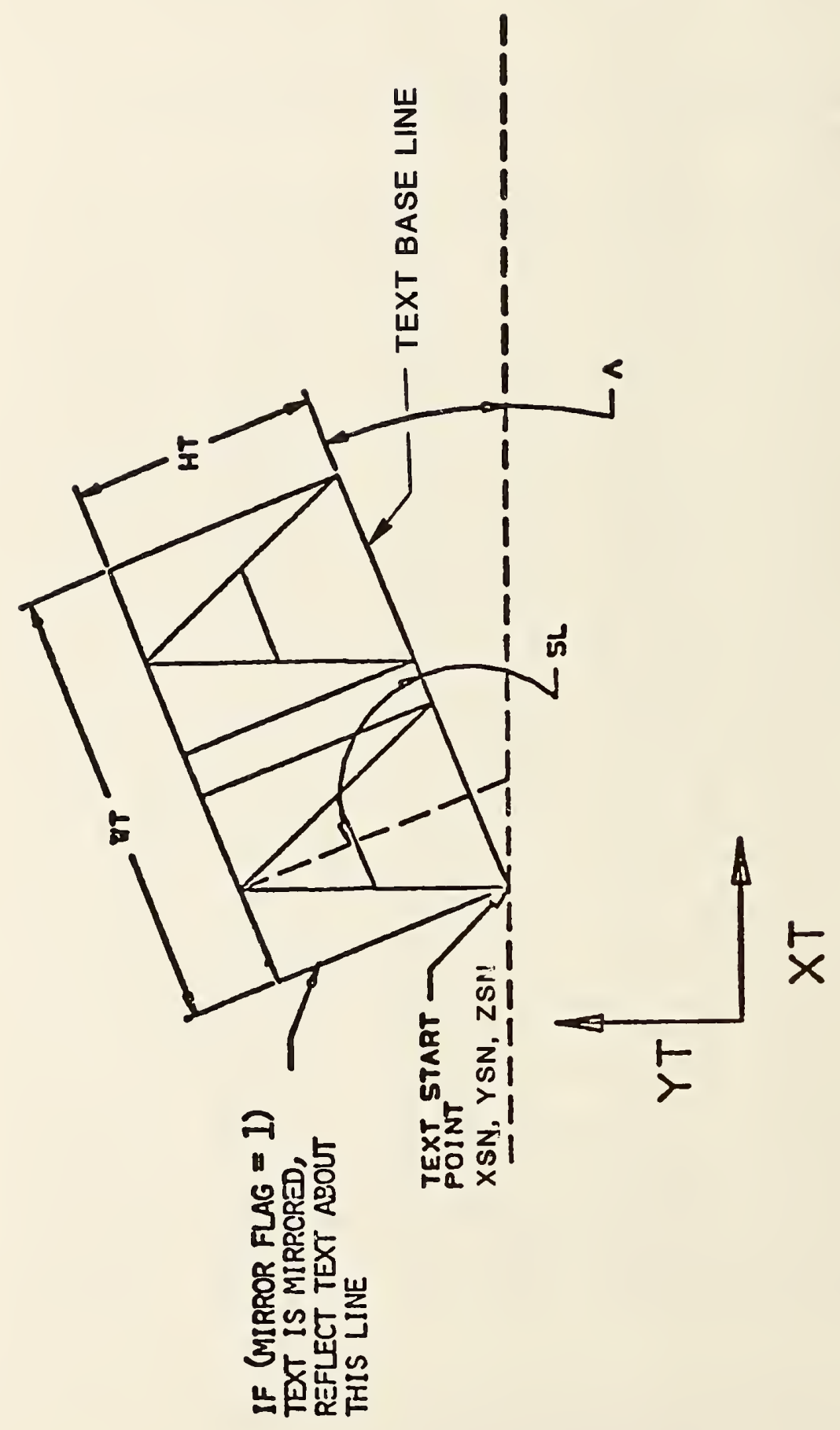

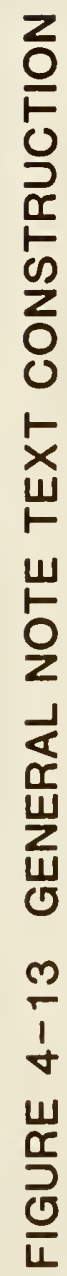




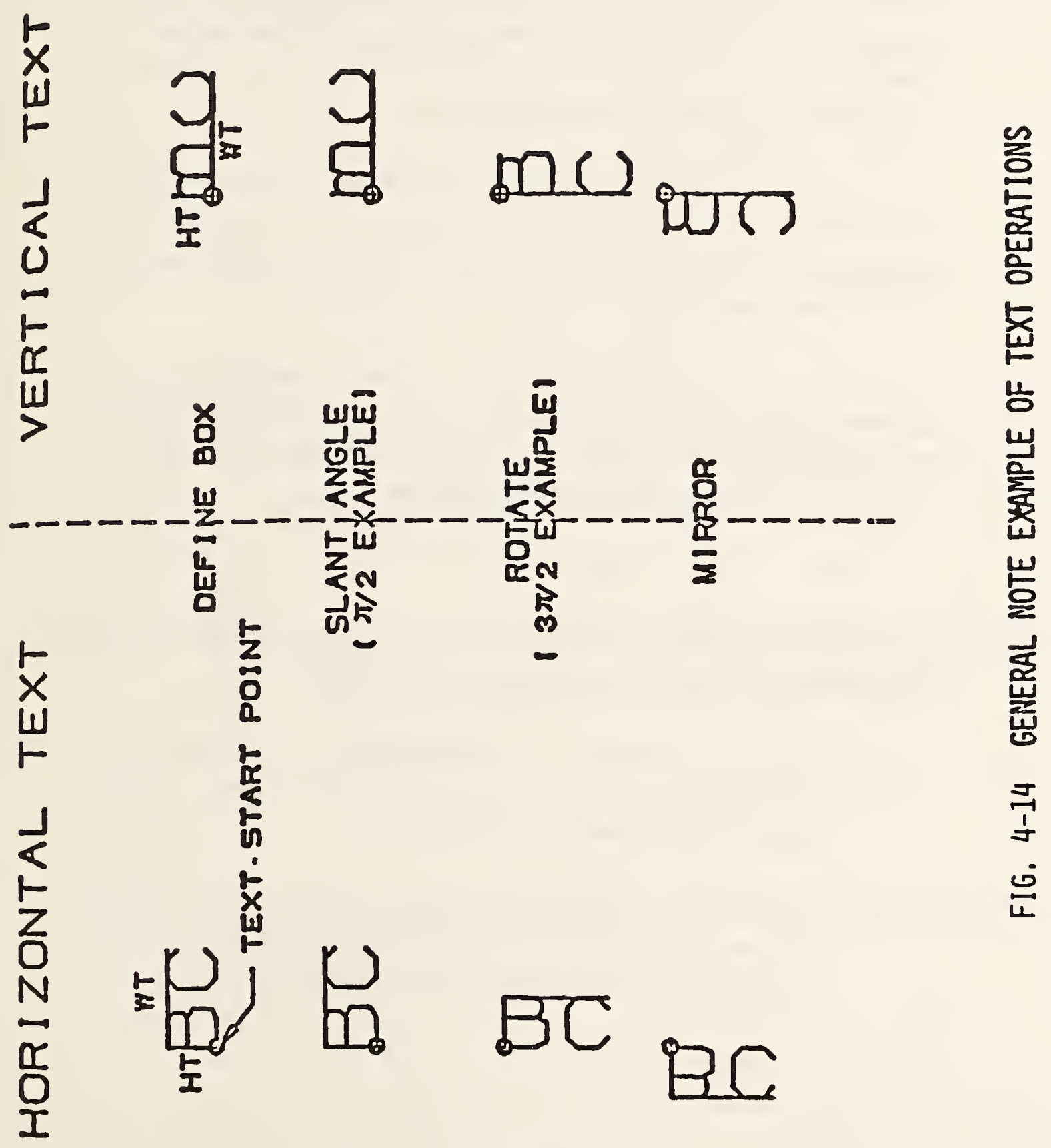


4.2.9.1 The graphical representation and recreation of notes with a special structure are handled by the use of the Form Number in Field 15 of the Directory Entry for this entity. A system to accommodate these notes is outlined below. Any strings after those specified by the form number are considered additional, appended strings that are not related in any particular manner to the previously referenced strings.

4.2.9.2 In the event that a string necessary for the defined structure is not present in the originating system's note, a null string shall be inserted in the general note entity to take the place of the non-existent string to maintain the structure of the data.

4.2.9.3 Notes that contain fractional notation will be represented as mixed numerals. This is done through the use of four consecutive strings representing the whole number, the numerator, the denominator, and the divisor bar. These are examples of the divisor bar string:

$1 \mathrm{H} / \quad \mathrm{H}-\quad 2 \mathrm{H}-\mathrm{H}_{-}$

4.2.9.4 The following form numbers for the general note are used to maintain the graphical representation of the originating system's note:

4.2.9.4.1 Form 0: Simple Note (default) - A general note of one or more strings such that a text string is not related in any manner to another string in the same general note entity.

4.2.9.4.2 Form 1: Dual Stack - A general note of two or more strings where the first two are related in a manner such that they are both left justified and the second string is displayed 'below' the first.

$\operatorname{xxxxxx}$

yyyyy 
4.2.9.4.3 Form 2: Imbedded Font Change - A general note of two or more strings that is intended as a single string but was divided to accommodate a font change in the string.

$\operatorname{xxxx}$ yyy $x x x x$

4.2.9.4.4 Form 3: Superscript - A general note of two or more strings where the second string is a superscript of the first string.

$$
\text { yyyxxx }
$$

4.2.9.4.5 Form 4: Subscript - A general note of two or more strings where the second string is a subscript of the first string.

$$
\text { xxxyyy }
$$

4.2.9.4.6 Form 5: Super-/Sub-script - A general note of three or more strings where the second string is a superscript of the first string and the third string is a subscript of the first string.

$$
\begin{array}{r}
\text { yyyxxx } \\
z z z
\end{array}
$$

4.2.9.4.7 Form 6: Multiple Stack/Left Justified - A general note where all strings are left justified to a common margin. These strings originated as a "paragraphed" note.

$x \times x x x x x x x x$

yyyyyyyyy

ZZZZZZZZZZZ

4.2.9.4.8 Form 7: Multiple Stack/Center Justified - A general note where all strings are center justified to a common axis.

$x x x x x x x$

yyyy

zzzzzzz 
4.2.9.4.9 Form 8: Multiple Stack/Right Justified - A general note where all strings are right justified to a common margin.

$x x x x x x x x x$

yуyуууy

zzzzzzzz

4.2.9.4.10 Form 100: Simple Fraction - A general note of four or more strings where the first four strings define a mixed numeral as defined in 4.2.9.3.

$$
\begin{array}{r}
\text { yyy } \\
\text { xx --- } \\
z z z
\end{array}
$$

4.2.9.4.11 Form 101: Dual Stack Fraction - A general note of eight or more strings which represent two mixed numerals as defined in 4.2.9.3. These mixed numerals are related such that the fifth through the eighth strings are displayed below the first through the fourth strings respectively.

$$
\begin{array}{r}
y y \\
x \times-2 \\
z z \\
\mathrm{jij}_{\mathrm{ji}}--
\end{array}
$$

4.2.9.4.12 Form 102: Imbedded Font Change/Double Fraction - This general note originated as a single string but was split to accommodate a font change for a special character in the fifth string. This is a general note of nine or more strings where the first and sixth strings represent the whole number string of a mixed numeral as defined in 4.2.9.3. The fifth string is a character (or characters) that was set apart to accommodate the font change.

$$
x x \frac{y y}{z z}-\text { ii } \frac{j j}{\overline{k k}}
$$


4.2.9.4.13 Form 105: Super-/Subscript Fraction - A general note of twelve or more strings where the first, fifth, and ninth strings represent the whole number string of a mixed numeral as defined in 4.2.9.3. The second and third mixed numerals are the superscript and subscript respectively of the first mixed numeral.

$$
\mathrm{ii}_{\mathrm{kk}}^{\mathrm{jj}}
$$

$x x \frac{y y}{z z}$

sS

$$
r r \frac{-}{t t}
$$




\subsubsection{Directory Data}

ENTITY TYPE NUMBER : 212

\subsubsection{Parameter Data}

\begin{tabular}{cll} 
Index & Name & \multicolumn{1}{c}{ Type } \\
1 & NS & Integer \\
2 & NC1 & \\
& & \\
3 & WT 1 & Real \\
4 & HT 1 & Real \\
5 & FC & Integer or Pointer \\
6 & SL1 & Real \\
8 & M1 & \\
7 & Al & Real \\
& &
\end{tabular}

Number of text strings in general note

Number of characters in first string (TEXT1) or zero. The number of characters $(\mathrm{NCn})$ must always be equal to the character count of its corresponding text string (TEXTn)

Box width

Box height

Font characteristic (Default = 1)

Slant angle of TEXT 1 in radians (pi/ 2 is the value for no slant angle and is the default value)

Rotation angle in radians for TEXT 1

Mirror flag 0 -no mirroring 1 - inirror axis is perpendicular to text base line

2 - mirror axis is text base line

9

VH1 Integer

Rotate internal text flag (0-text horizontal, 1-text vertical)

10

Real

First text start point

Real 
12

ZSI

Real

13

TEXT 1

String

14

$\mathrm{NC2}$

Integer

$1+N S * 12$ TEXTNS

String

$2+N S * 12$

NCNS
Integer
$Z$ depth from XT, YT plane

First text string

Number of characters in second text string

Last text string

Number of characters in last text string

Additional Pointers as required (see 2.2.4.4.2) 


\subsubsection{Leader (Arrow) Entity.}

A leader consists of one or more line segments except when the leader is part of an angular dimension (see 4.2.4). The first segment begins with an arrowhead. Remaining segments successively link to a presumed text item. An individual segment is assumed to extend from the end point of its predecessor in the segment list to its defined end point. Examples of leaders are shown in Figure 4-15.

In the use of angular, diameter, and linear dimension, there are instances where the text is exterior to the line or arc lying between the two arrows. In these situations, it remains the case that the appearance of two arrows implies the use of two leaders. These are formed by dividing the line or arc lying between the two arrows into two non-overlapping segments. Refer to Figure 4-16.

Some leaders (for example, the leader involved with the radius dimension in Figure 4-16) give the appearance of locating an arrow interior to a segment. There are two overlapping segments. The first segment begins at the arrow and, in the radius dimension example, ends at the center of the arc or circle being dimensioned. The second segment then retraces the first in the opposite direction and extends it. Leaders of this type for other types of dimensions are constructed similarly. For cases involving angular dimension, the first two segments are arcs.

4.2.10.1 Forms. There are eleven arrowhead types defined (see figure 4-17) and selection is made by entering the form number in directory entry field 15 . 

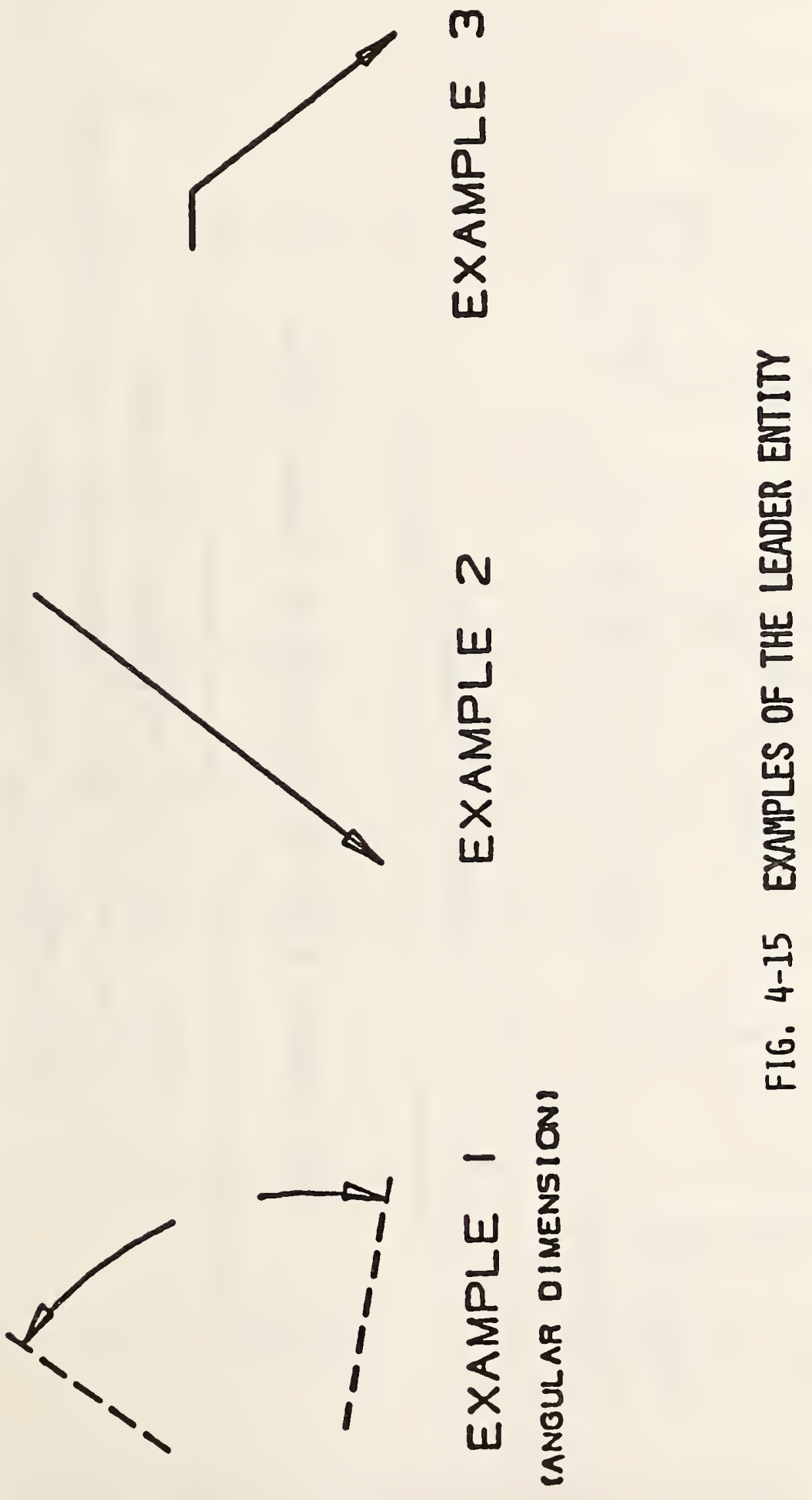

$N$

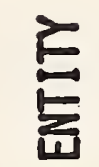



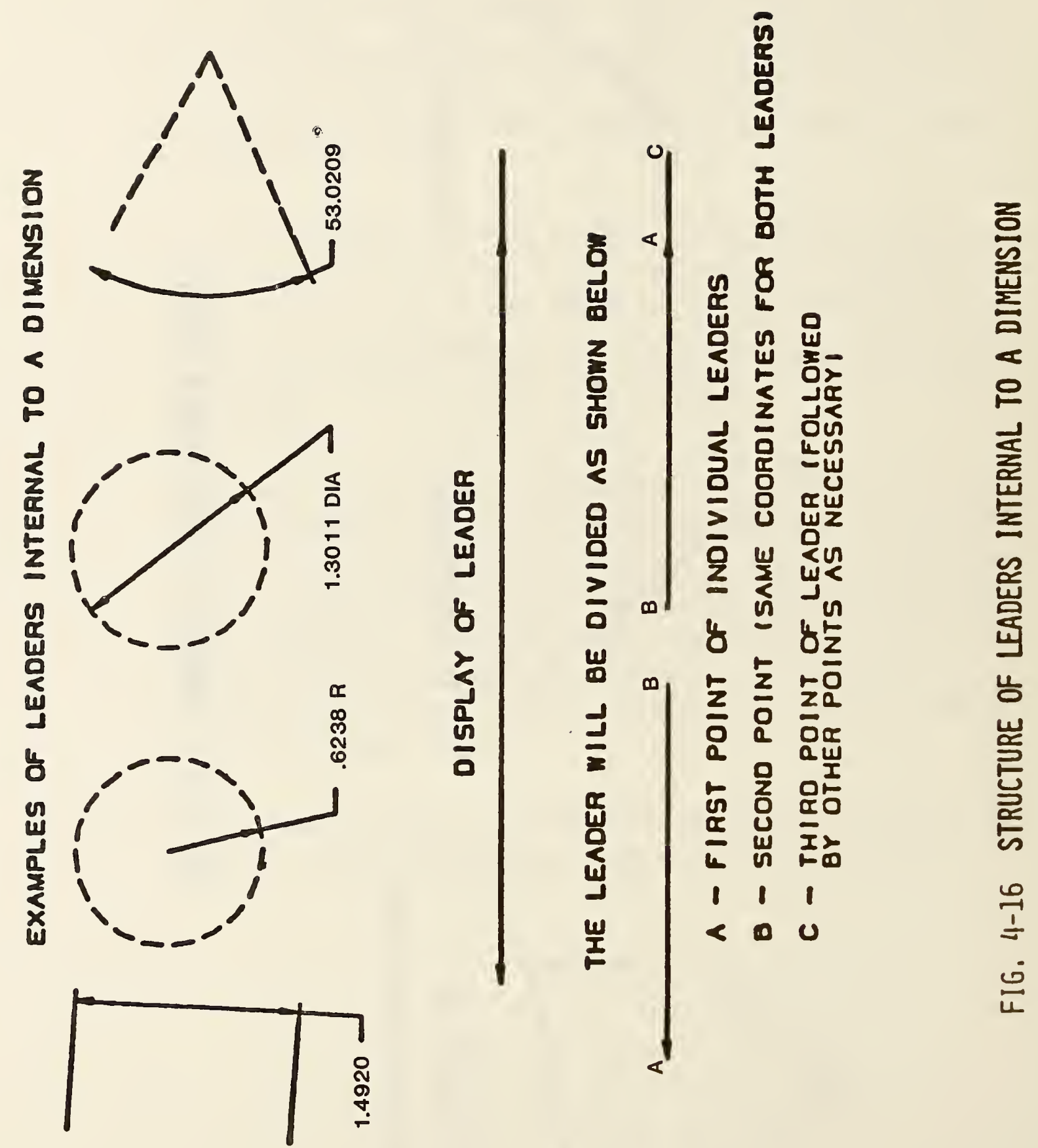
FORM I: WEDGE

FORM 2: TRIANGLE

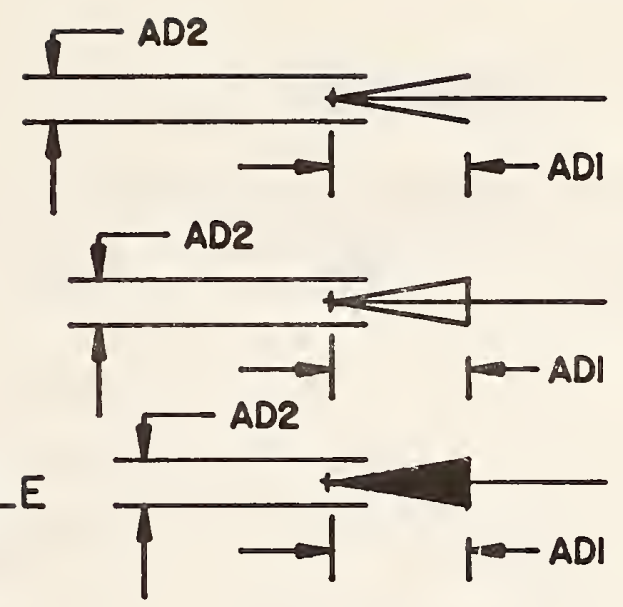

FORM 4: NO ARROWHEAD

FORM 5: CIRCLE

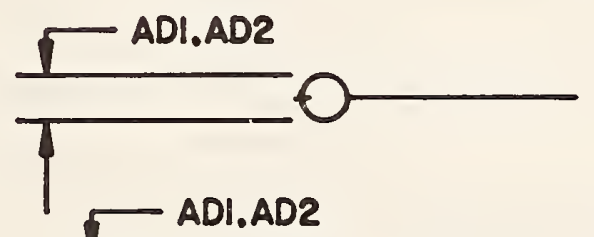

FORM 6: FILLED CIRCLE

FORM 7: RECTANGLE

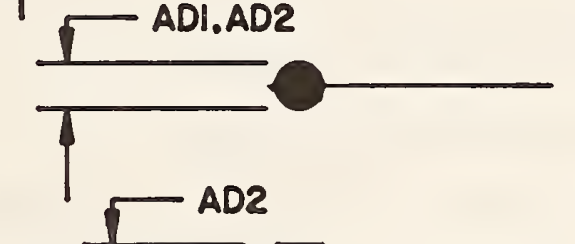

FORM 8: FILLED RECTANGLE

FORM 9: SLASH

FORM 1O: INTEGRAL SIGN

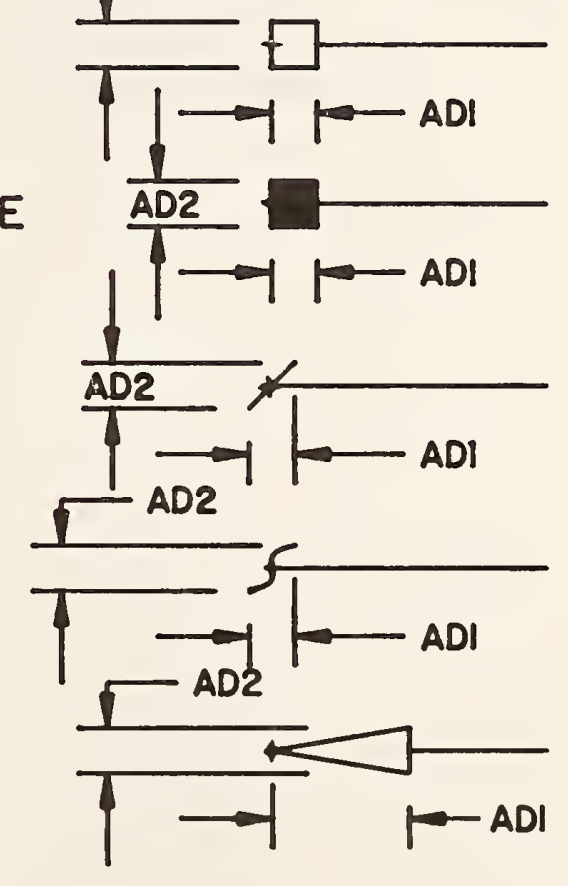

FORM $1 / 3$ OPEN TRIANGLE

NOTE: - IMDICATES ORIGIN (XH, YH) NND IS MOT PART OF ARROWHEAD

FIG. 4-17 ARROWHEAD DEFINITIONS 


\subsubsection{Directory Data}

ENTITY TYPE NUMBER: 214

\subsubsection{Parameter Data}

$\begin{array}{clll}\text { Index } & \text { Name } & \text { Type } & \text { Description } \\ 1 & \mathrm{~N} & \text { Integer } & \text { Number of segments } \\ 2 & \text { ADI } & \text { Real } & \text { Arrowhead height } \\ 3 & \text { AD2 } & \text { Real } & \text { Arrowhead width } \\ 4 & \text { ZT } & \text { Real } & \text { Z depth } \\ 5 & \text { XH } & \text { Real } & \text { Arrowhead coordinates } \\ 6 & \text { YH } & \text { Real } & \\ 7 & \text { X } & \text { Real } & \text { Segment tail } \\ \cdot & \text { Y } & \text { Real } & \text { coordinate pairs } \\ \cdot & & & \text { Last Segment } \\ 6+2 \mathrm{~N} & \mathrm{Y} & \text { Real } & \text { Coordinate }\end{array}$

Additional Pointers as required (see 2.2.4.4.2). 
4.2.11 Linear Dimension Entity.

A linear dimension consists of a general note; two leaders; and zero, one or two witness lines. Refer to Figure 4-18 for examples of linear dimensions.

\subsubsection{Directory Data}

ENTITY TYPE NUMBER : 216

\subsubsection{Parameter Data}

\begin{tabular}{|c|c|c|c|}
\hline Index & Name & Type & Description \\
\hline 1 & DENOTE & Pointer & $\begin{array}{l}\text { Pointer to general note direc- } \\
\text { tory entry }\end{array}$ \\
\hline 2 & DEARR W 1 & Pointer & $\begin{array}{l}\text { Pointer to first leader directory } \\
\text { entry }\end{array}$ \\
\hline 3 & DEARR W 2 & Pointer & $\begin{array}{l}\text { Pointer to second leader direc- } \\
\text { tory entry }\end{array}$ \\
\hline 4 & DEWIT I & Pointer & $\begin{array}{l}\text { Pointer to witness line direc- } \\
\text { tory entry, } 0 \text { if not defined }\end{array}$ \\
\hline 5 & DEWIT2 & Pointer & $\begin{array}{l}\text { Pointer to witness line direc- } \\
\text { tory entry or } 0\end{array}$ \\
\hline
\end{tabular}

Additional Pointers as required (see 2.2.4.4.2). 

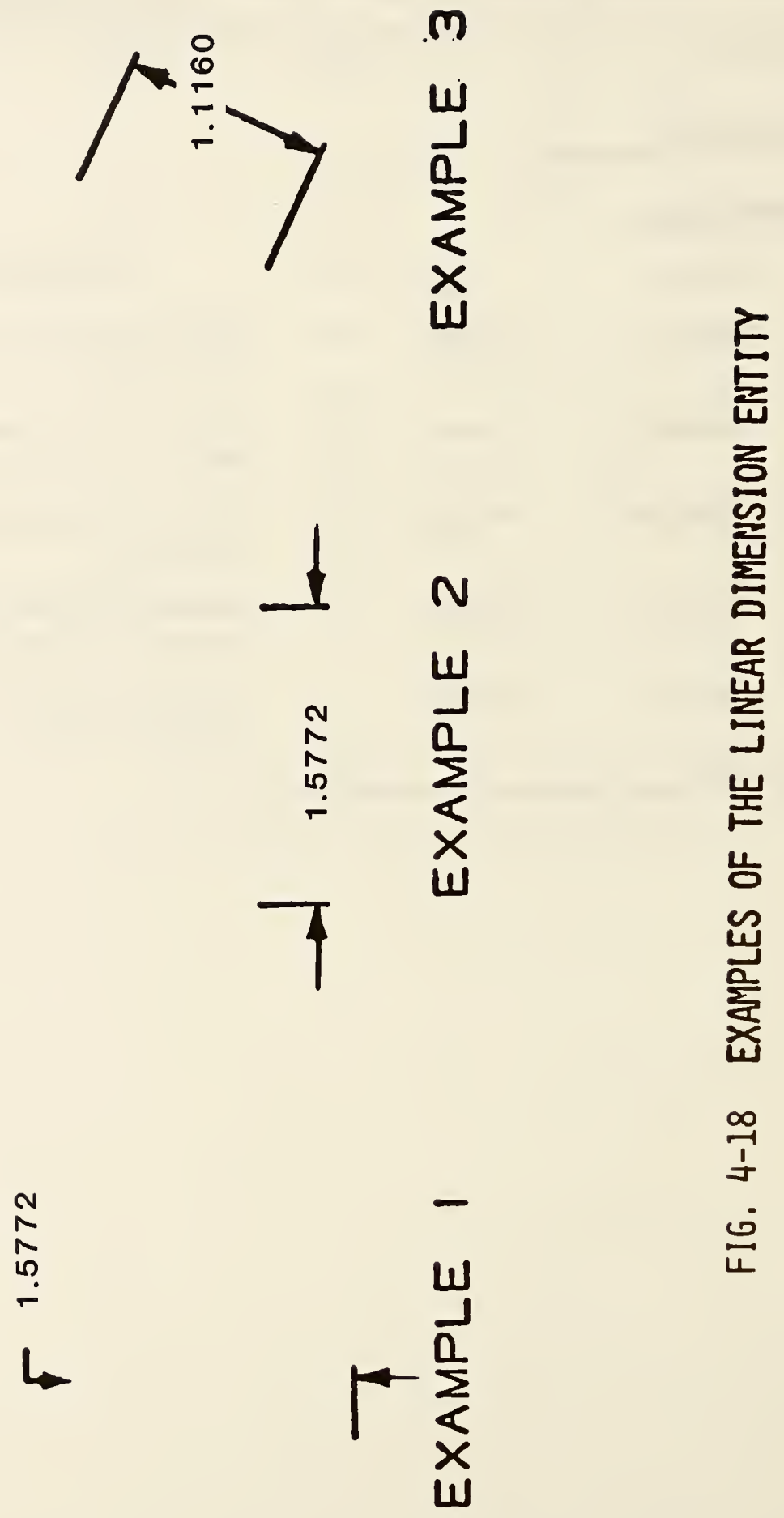


\subsubsection{Ordinate Dimension Entity.}

The ordinate dimension entity is used to indicate dimensions from a common base line. Dimensioning is only permitted along the XT or YT axis.

4.2.12.1 An ordinate dimension consists of a general note and a witness line or leader. The values stored are pointers to the directory entry for the associated note and witness line. Examples of ordinate dimensions are shown in Figure 4-19.

\subsubsection{Directory Data}

ENTITY TYPE NUMBER : 218

\subsubsection{Parameter Data}

\begin{tabular}{|c|c|c|c|}
\hline Index & Name & Type & Description \\
\hline 1 & DENOTE & Pointer & $\begin{array}{l}\text { Pointer to general note } \\
\text { directory entry }\end{array}$ \\
\hline 2 & DEWIT & Pointer & $\begin{array}{l}\text { Pointer to wi thess line direc- } \\
\text { tory entry or leader directory } \\
\text { entry }\end{array}$ \\
\hline
\end{tabular}

Additional Pointers as required (see 2.2.4.4.2). 


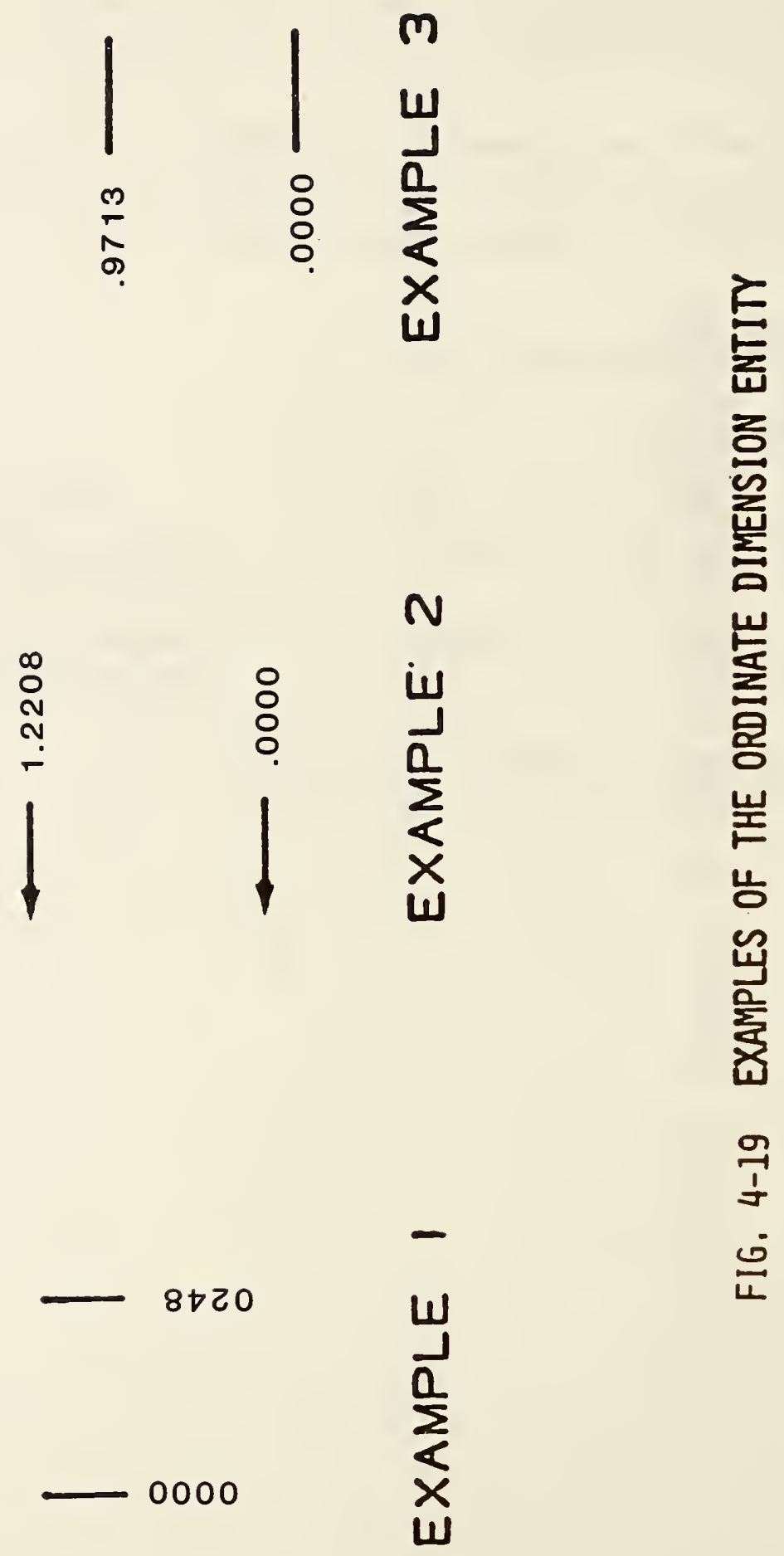




\subsubsection{Point Dimension Entity.}

A point dimension consists of a leader, text, and an optional circle or hexagon enclosing the text.

4.2.13.1 The leader will always contain three segments, and its first and last segments are always horizontal or vertical. If a hexagon encloses the text, it will be described by a Composite Curve entity. If a circle or hexagon does not enclose the text, the last segment of the leader will be horizontal and it will underline the text.

4.2.13.2 Examples are shown in Figure 4-20.

\subsubsection{Directory Data}

\section{ENTITY TYPE NUMBER :220}

\subsubsection{Parameter Data}

\begin{tabular}{|c|c|c|c|}
\hline Index & Name & Type & Description \\
\hline 1 & DE I & Pointer & $\begin{array}{l}\text { Pointer to general note di- } \\
\text { rectory entry }\end{array}$ \\
\hline 2 & DE2 & Pointer & $\begin{array}{l}\text { Pointer to leader directory } \\
\text { entry }\end{array}$ \\
\hline 3 & DE3 & Pointer & $\begin{array}{l}\text { Pointer to circular arc, com- } \\
\text { posite curve, or } 0 .\end{array}$ \\
\hline
\end{tabular}

Additional Pointers as required (see 2.2.4.4.2). 


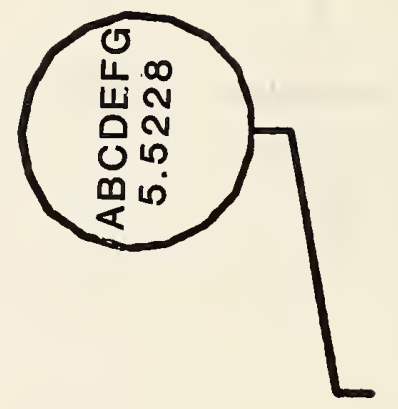

$\frac{1}{2}$

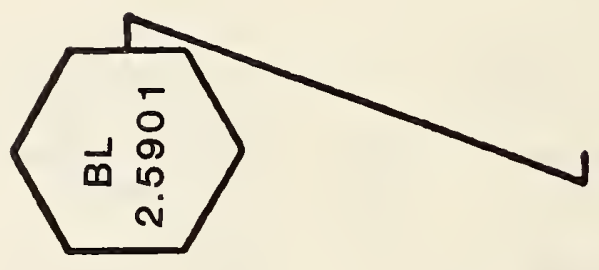

N

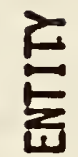

$\sum_{\substack{x \\ \frac{1}{x}}}^{\frac{1}{u}}$

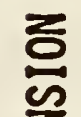




\subsubsection{Radius Dimension Entity.}

A radius dimension consists of a general note, a leader, and an arc center point, (XT, YT). Refer to Figure 4-21 for examples of radius dimensions.

4.2.14.1 The arc center coordinates are used for positioning the radius dimension line relative to the arc being dimensioned.

\subsubsection{Directory Data}

\section{ENTITY TYPE NUMBER: 222}

\subsubsection{Parameter Data}

\begin{tabular}{|c|c|c|c|}
\hline Index & Name & Type & Description \\
\hline 1 & DEN & Pointer & $\begin{array}{l}\text { Pointer to general note di- } \\
\text { rectory entry }\end{array}$ \\
\hline 2 & DEP & Pointer & $\begin{array}{l}\text { Pointer to leader directory } \\
\text { entry }\end{array}$ \\
\hline 3 & $\mathrm{XT}$ & Real & Arc center coordinates \\
\hline 4 & YT & Real & \\
\hline
\end{tabular}

Additional Pointers as required (see 2.2.4.4.2). 


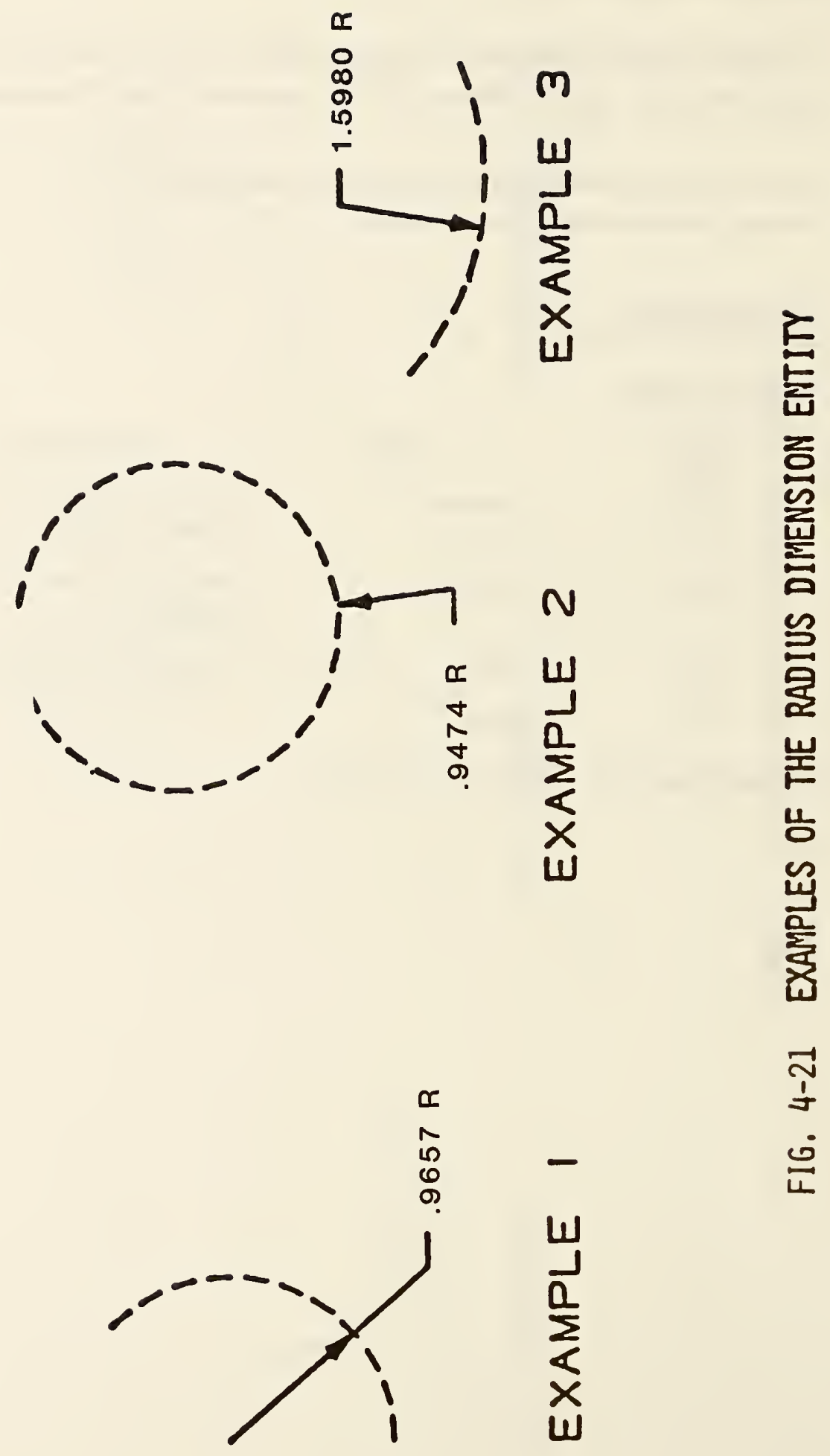


4.2.15 Section Entity.

A section entity is defined as a copious data entity (Type 106 forms 31 to 38). The form number describes how the data are to be interpreted. These descriptions are included for compatability with previous versions of the specification. The Sectioned Area Entity (Type 230) provides a more compact method for transferring this information.

4.2.15.1 The point data contains a list of points $(X n, Y n), n=1,2, \ldots, N$, (The $Z$ value is constant and $\mathrm{N}$ is an even integer.)

The display of the lines consists of solid line segments between the points $(X n, Y n, Z)$ and $(X n+1, Y n+1, Z)$ where $n=1,3,5, \ldots N-1$.

A portion of collinear line segments which appear to be a dashed line shall consist of point pairs for each dash.

4.2.15.2 The defined line patterns are described below and illustrated in Figure 4-22. (ANSI79).

\section{Form number $\quad$ Description}

Parallel line segments from section edge to edge. (Cast or malleable iron and general use for all materials)

Parallel line segments in pairs with a gap between pairs. (Steel)

Alternating pattern of a solid line and a set of collinear dash segments. (Bronze, brass, copper, and compositions)

Parallel lines in quadruples with a gap between groups. (Rubber, plastic, and electrical insulation) 
106, Forms 31-38 SECTION
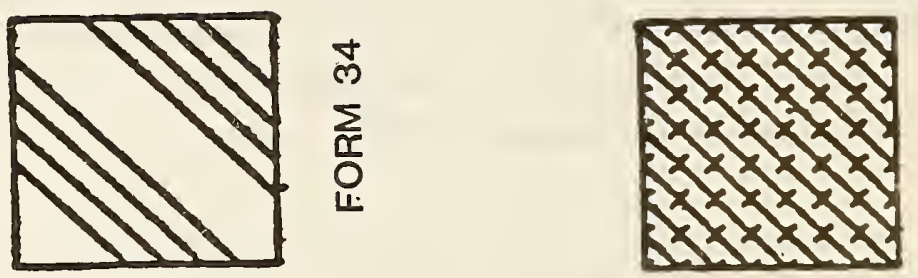

$\sum_{\substack{\infty \\ \hdashline}}^{\infty}$
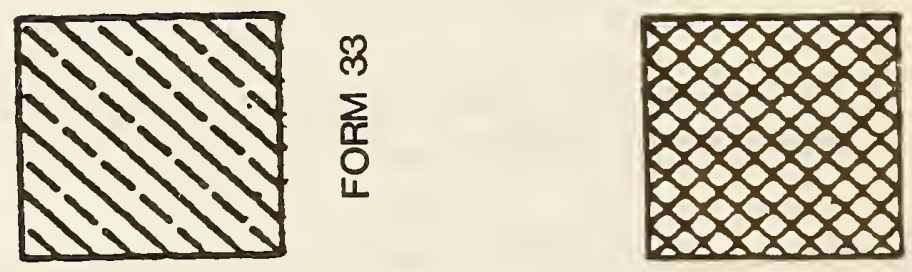

$\sum_{1}^{n}$
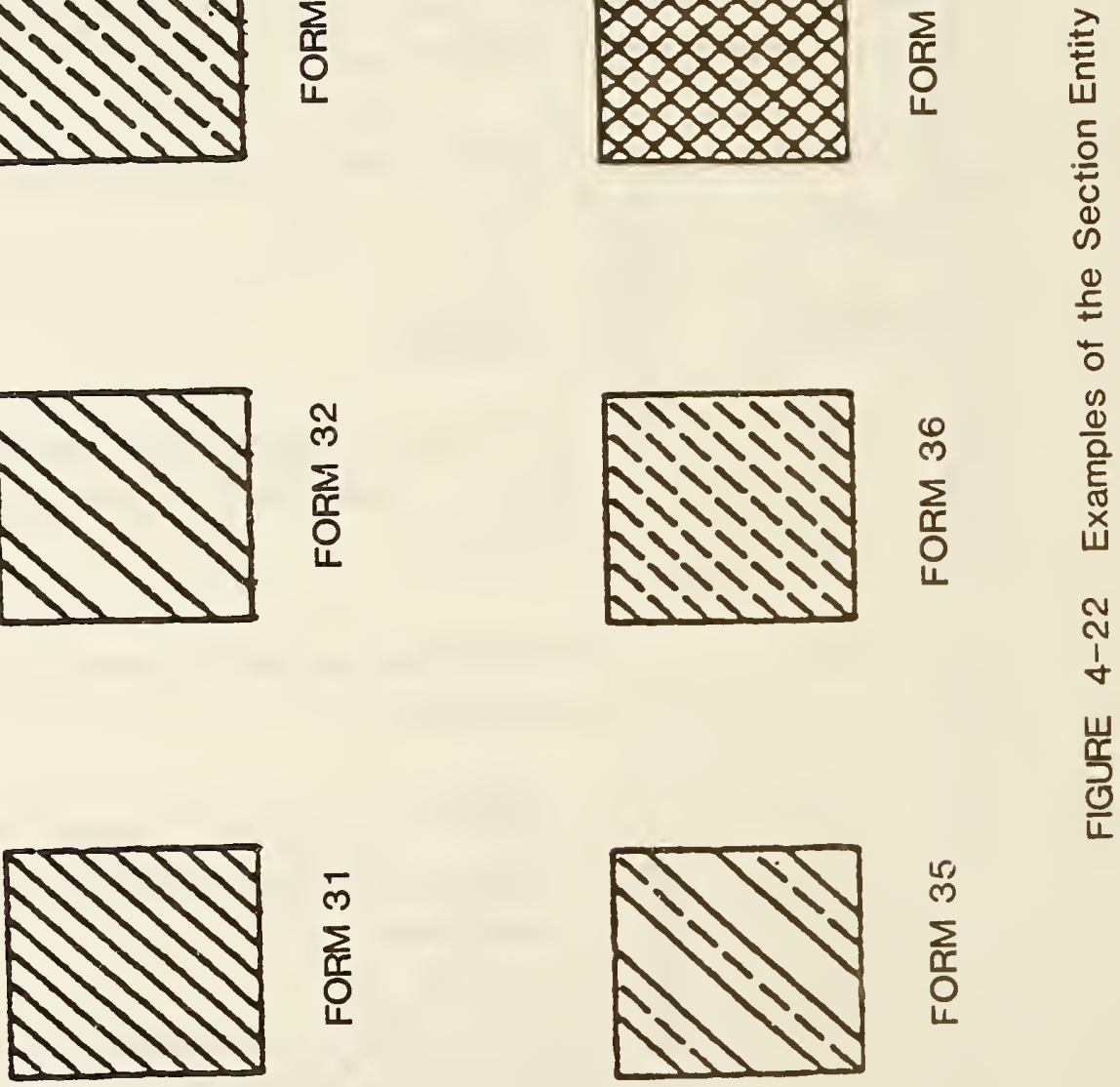

$\sum_{1}^{\infty}$

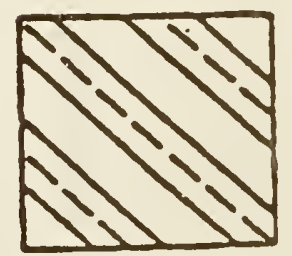

$\sum_{0}^{n}$ 
Form number

35

36

37

38

\section{Description}

Triples of parallel lines consisting of two solid lines and a set of collinear dash segments between them with a gap between triples. (Titanium and refractory material)

Parallel sets of collinear dash segments. (Marble, slate, glass, porcelain)

Two perpendicular sets of parallel lines. (White metal, zinc, lead, babbitt, and alloys)

Two perpendicular sets of lines with the principal set solid from edge to edge and the second set consisting of collinear dash segments alternating on the solid lines. (Magnesium, aluminum, and aluminum alloys)

4.2.15.3 See Section 3.5 for parameters of the Section Entity. 
4.2.16 General Symbol Entity.

A general symbol entity consists of a general note; one or more geometric entities which define a symbol; and zero, one or more associated leaders. Examples of general symbol entities are shown in Figure 4-23.

Any geometric entity used to create the symbol will have a subordinate entity switch of 01 and an entity use flag of 01 in field 9 of its Directory Entry section.

\subsubsection{Directory Data}

ENTITY TYPE NUMBER: 228

\subsubsection{Parameter Data}

\begin{tabular}{|c|c|c|c|}
\hline Index & $\underline{\text { Name }}$ & Type & Description \\
\hline 1 & DENOTE & Pointer & $\begin{array}{l}\text { Pointer to associated general } \\
\text { note }\end{array}$ \\
\hline 2 & $\mathrm{~N}$ & Integer & $\begin{array}{l}\text { Number of pointers to } \\
\text { geometry }\end{array}$ \\
\hline 3 & GPNT1 & Pointer & Pointer to defining geometry \\
\hline - & $\cdot$ & - & \\
\hline - & - & $\cdot$ & \\
\hline$N+2$ & GPNTN & Pointer & \\
\hline $\mathrm{N}+3$ & $\mathrm{~L}$ & Integer & Number of Leaders or zero \\
\hline$N+4$ & LPNTI & Pointer & $\begin{array}{l}\text { Pointers to associated } \\
\text { leaders }\end{array}$ \\
\hline$\cdot$ & - & • & \\
\hline$\dot{N}+3+L$ & LPNTL & Pointer & \\
\hline
\end{tabular}

Additional Pointers as required (see 2.2.4.4.2). 
228 - GENERAL SYMBOL
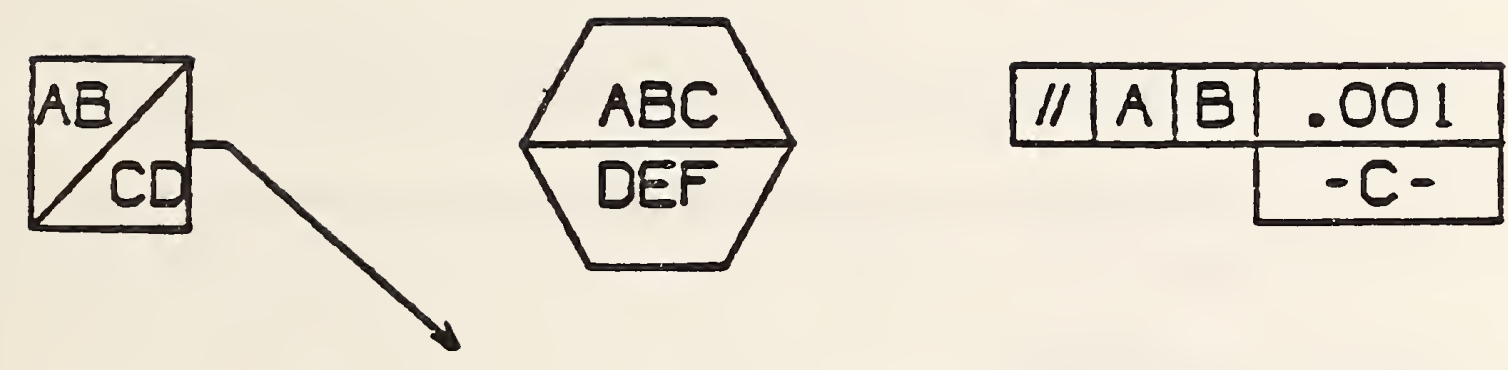

FIGURE 4-23 GENERAL SYMBOL ENTITIES 


\subsubsection{Sectioned Area Entity}

A sectioned area is a portion of a design which is to be filled with a pattern of lines. It consists of a pointer to a boundary curve, a specification of the pattern of lines, the coordinates of a point on the lines, the distance between lines, the angle between the lines and the $\mathrm{X}$-axis of the definition space, and the specification of any enclosed boundary curves (islands).

4.2.17.1 The XT and YT coordinates, which may be specified, indicate a location which is on one the lines. This point allows applications which require specific placements of the lines to constrain them appropriately. If not specified, i.e., indicated by default, the lines need only be within the bounding curve.

4.2.17.2 The angle of the lines has a default value of $\mathrm{pi} / 4$, measured in radians.

4.2.17.3 The line pattern is specified according to predefined definitions illustrated in Figure 4-24.

\subsubsection{Directory Data}

ENTITY TYPE NUMBER: 230

\subsubsection{Parameter Data}

$\begin{array}{lll}\text { Index } & \text { Name } & \text { Type } \\ 1 & \text { BNDP } & \text { Pointer } \\ 2 & \text { PATRN } & \text { Integer } \\ 3 & \text { XT } & \text { Real } \\ 4 & \text { YT } & \text { Real } \\ 5 & \text { ZT } & \text { Real } \\ 6 & \text { DIST } & \text { Real }\end{array}$

\section{$\underline{\text { Description }}$}

Pointer to boundary curve-a single closed curve or a composite curve

Line pattern code

$X$ coordinate through which a line should pass

$Y$ coordinate through which a line should pass

$Z$ depth of lines

Normal distance between adjacent lines 


$\begin{array}{ccc}7 & \text { ANGLE } & \text { Real } \\ 8 & \text { N } & \text { Integer } \\ 9 & \text { ISLPT 1 } & \text { Pointer } \\ . & \cdot & \cdot \\ \cdot & \cdot & \cdot \\ & \cdot & \cdot \\ 8+\mathrm{N} & \text { ISLPTN } & \text { Pointer }\end{array}$

Angle measured in radians from the $X T$ axis to the lines of the sectioning

Number of island curves or zero

Pointer to a boundary curve for an island

Pointer to last boundary curve for an island

Additional pointers as required (see 2.2.4.4.2). 

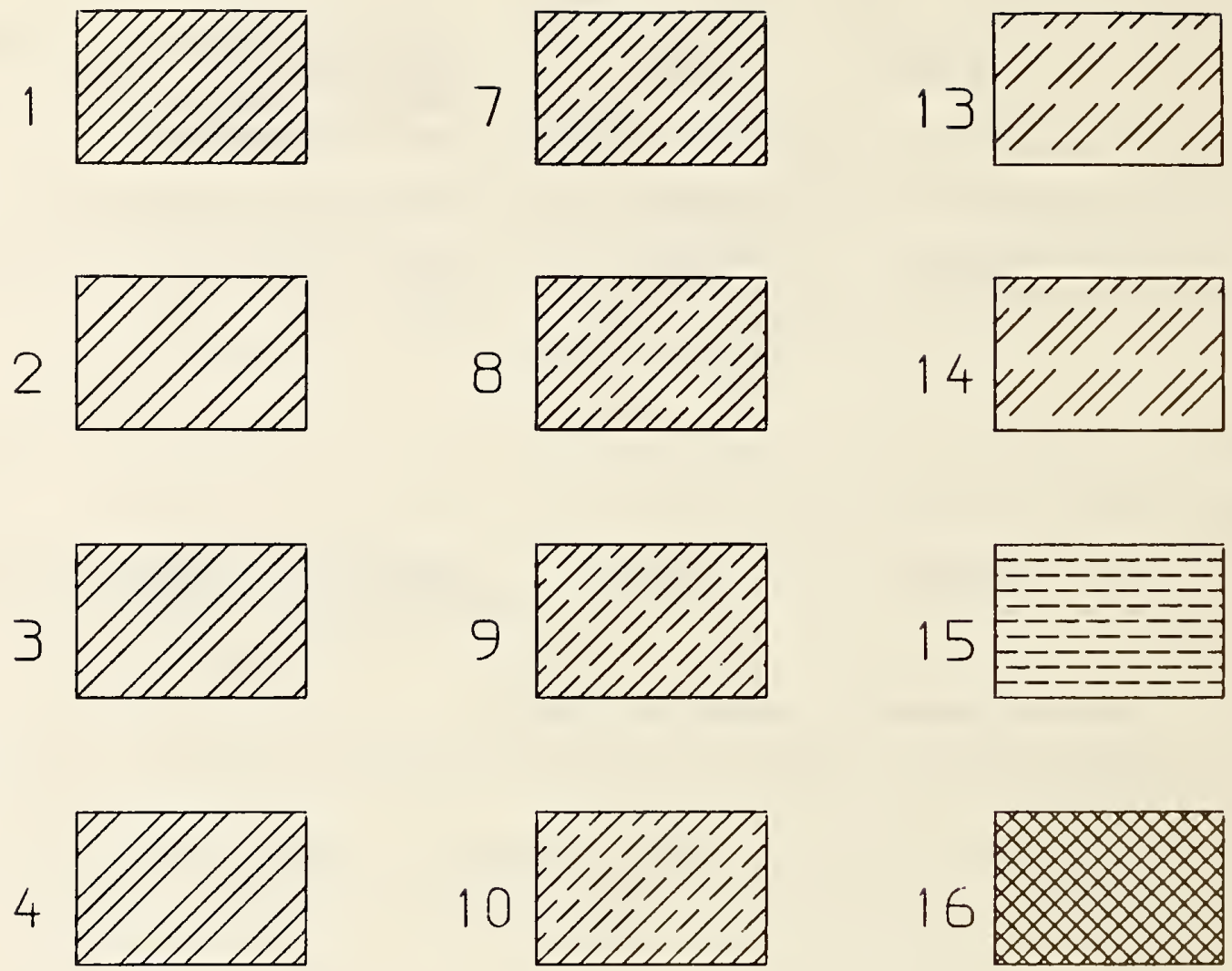

16
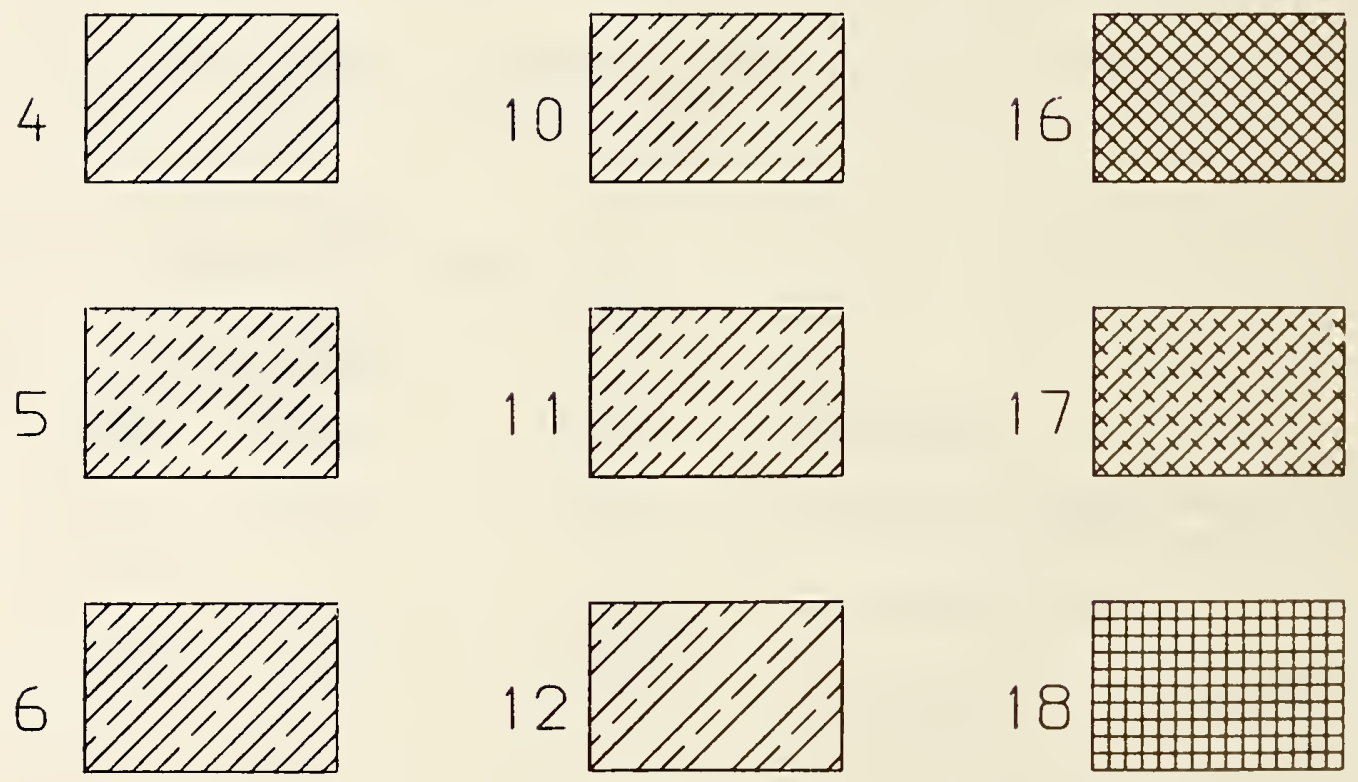

\section{LINE PATTERN CODES}

FIGURE 4-24 SECTION LINE PATTERNS 


\subsubsection{Witness Line Entity.}

A witness line is a form number 40 of a copious data block that contains one or more straight line segments associated with drafting entities of various types. Each line segment may be visible or invisible. Refer to Figure 4-25 for examples.

4.2.18.1 If the witness line is suppressed, this is indicated by a 0 in the pointer field of the drafting entity pointing to a witness line, or by setting the blank status of the directory entry of the copious data entity for the witness line.

4.2.18.2 Within the copious data, there will be the location from which the witness line gap must be maintained. This point is indicated in the figure as Pl. The location will be the first point in the copious data. Pl will be coincident with the geometry being dimensioned or equal to P2 when the location of the geometry is unknown. Note: for those annotation methods that do not allow drafting entities to be displaced from the plane of annotation, coincident with the geometry indicates that a line normal to the plane of annotation connects $\mathrm{Pl}$ and the point on the geometry being dimensioned. Note that all points must be collinear, and that the number of points will be odd and at least 3 ( 3,5 , $7, \ldots$ ), with alternating blank and displayed segments. The examples in Figure 4-25 show the blanking of segments and the order of points stored in the copious data.

4.2.18.3 See Section 3.5 for parameters of the Witness Line Entity. 

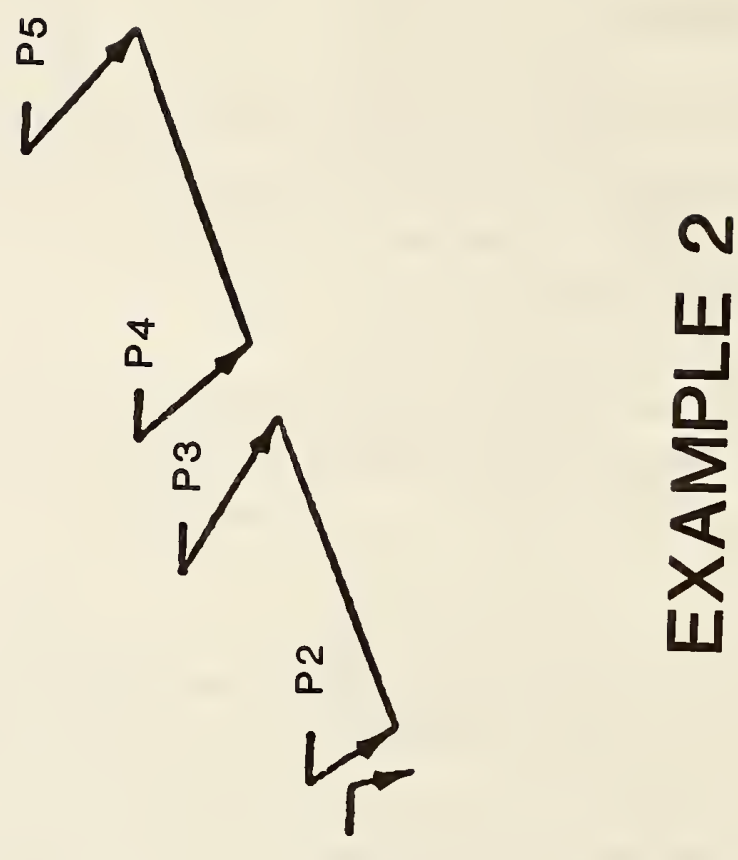

0

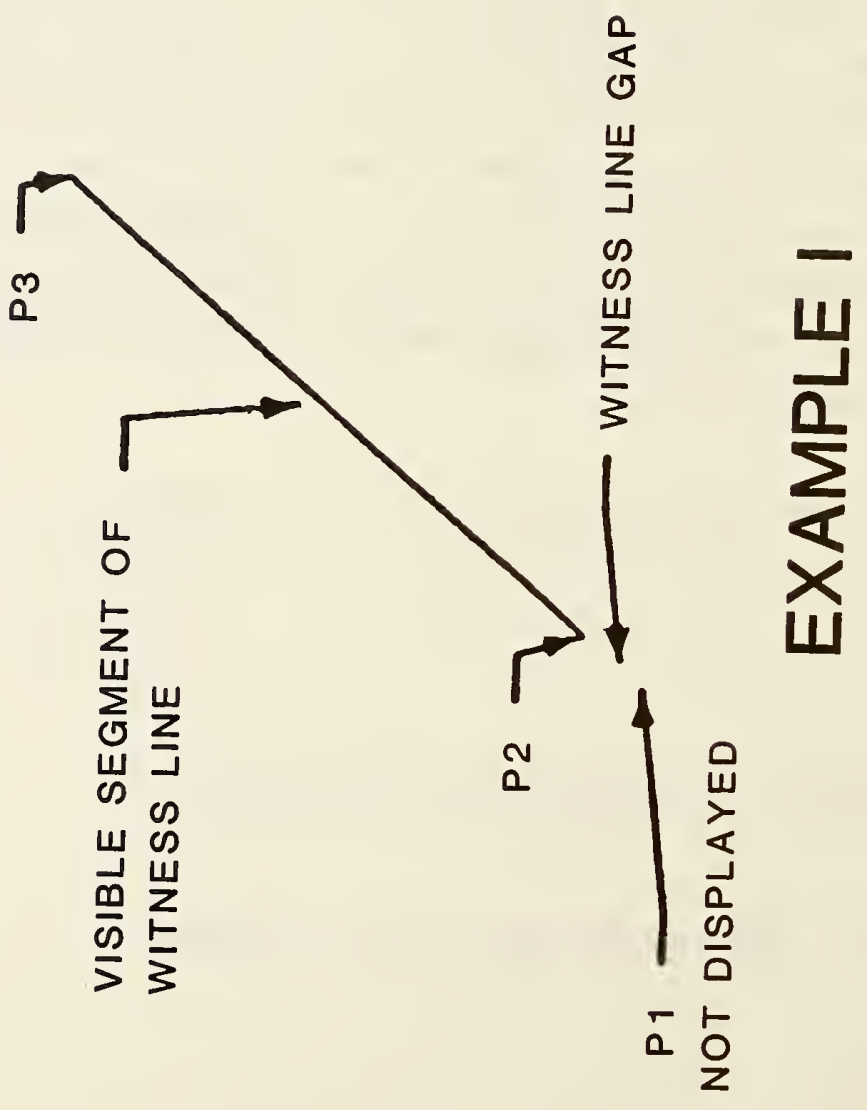




\subsection{Structure Entities}

\subsubsection{Entity Type/Type Number}

The following entities are defined in this section:

Entity Type Number

302

304

306

308

310

312

314

320

402

404

406

408

410

412

414

416

418

420

600-699 or

10000-99999 as

specified by user
Entity Type

Associativity Definition Entity

Line Font Definition Entity

MACRO Definition Entity

Subfigure Definition Entity

Text Font Definition Entity

Text Display Template

Color Definition

Network Subfigure Definition

Associativity Instance Entity

Drawing Entity

Property Entity

Singular Subfigure Instance Entity

View Entity

Rectangular Array Subfigure Instance Entity

Circular Array Subfigure Instance Entity

External Reference

Nodal Load/Constraint

Network Subfigure Instance

MACRO Instance Entity 
The associativity definition entity permits the preprocessor to define an associativity schema. That is, by using the associativity definition, the preprocessor defines the type of relationship. It is important to note that this mechanism specifies the syntax of such a relationship and not the semantics.

4.3.2.1 Schema. The definition schema allows the specification of multiple groups of data which are called classes. A class is considered to be a separate list, and the existence of several classes implies an association among the classes as well as among the contents of each class.

For each class, the schema has provision to specify whether or not back pointers are required. A back pointer being required implies that an entity which is a member of this associativity (when it is instanced) has a pointer to the directory entry of the associativity instance in its back pointer parameter section.

The provision in the schema to specify whether or not a class is ordered is used to state whether the order of appearance of entries in the class is significant.

In the schema, "ENTRIES" are the members of the class. However, each entry could be composed of several items. If multiple items are required, they will be ordered. For example, if the entries were locations, each entry might have three items to specify $X, Y$, and $Z$ values.

The associativity definition will fix the number of classes for an associativity and the number of items per entry in a particular class. Each associativity instance will have a variable number of entries per class. In order to help decode instances of the definition, each item is specified as a pointer (to an entity directory entry) or a data value. 
4.3.2.2 Form. Two kinds of associativity are permitted within the file. Pre-defined associativities will have form numbers in the range of 1 to 5000 and are defined in 4.3.3.3. The second kind of associativity is defined in the file by a preprocessor (Form numbers 5001-9999). These definitions appear once in the file for each form of associativity defined, and allow the preprocessor to fill in the definition according to a schema which defines the details of the associativity.

The definition includes the associativity form, the number of class definitions, the number and type of items in each entry, and whether back pointers (from the entity to the associativity) are required. Each set of values (BP, Order, $\mathrm{N}$, and Item type) is considered a class. See 4.3.3.3.1 for a complete example of associativity.

\subsubsection{Directory Data}

ENTITY NUMBER : 302

\subsubsection{Parameter Data}

\begin{tabular}{|c|c|c|c|}
\hline Index & Name & Type & Description \\
\hline 1 & $\mathrm{~K}$ & Integer & Number of class definitions \\
\hline 2 & BPI & Integer & $\begin{array}{l}\text { 1- back pointers required } \\
2 \text { - back pointers not required }\end{array}$ \\
\hline 3 & OR 1 & Integer & $\begin{array}{l}\text { 1-ordered class } \\
\text { 2-unordered class }\end{array}$ \\
\hline 4 & $\mathrm{NI}$ & Integer & Number of items per entry \\
\hline 5 & IT 1(1) & $\begin{array}{l}\text { if parameter } \\
\text { if parameter }\end{array}$ & $\begin{array}{l}\text { 1-pointer to a directory entry } \\
\text { 2-value } \\
\text { 3-parameter is a value or a } \\
\text { pointer } \\
\geqslant 0 \text { it is a value } \\
<0 \text { it is a pointer }\end{array}$ \\
\hline
\end{tabular}

The items in parameters 2 through $4+\mathrm{Nl}$ are repeated for each of the $\mathrm{K}$ classes. 


\section{3 .3}

Associativity Instance Entity.

Each time an associativity relation is needed in the file an associativity instance entity is used.

The form number of the associativity instance will identify the meaning of the entity. If the form number is between 1 and 5000, the definition is specified as described in 4.3.2.2. If the form number is between 5001 and 9999, an associativity definition will occur in the file and the structure field of the instance (DE field 3) will contain a pointer to the directory entry of the associativity definition.

Each entity that is a member of an associativity instance can contain a back pointer to the associativity instance (see 2.2.4.4.2).

The parameters $\mathrm{K}$ and $\mathrm{N1}, \mathrm{N} 2, \ldots \mathrm{NK}$ are specified in the associativity definition. (see 4.3.2.4)

\subsubsection{Directory Data}

ENTITY TYPE NUMBER :402

\subsubsection{Parameter Data}

$\begin{array}{cccc}\text { Index } & \text { Name } & \text { Type } & \text { Description } \\ 1 & \text { NE1 } & \text { Integer } & \text { Number of class one entries } \\ 2 & \text { NE2 } & \text { Integer } & \text { Number of class two entries } \\ \therefore & & & \\ \text { K } & \text { NEK } & \text { Integer } & \text { Number of class K entries }\end{array}$


For $\mathrm{K}$ classes with $(\mathrm{NE} 1, \ldots, \mathrm{NEK})$ entries with $(\mathrm{N} 1, \ldots, \mathrm{NK})$ items per entry

\begin{tabular}{|c|c|c|c|}
\hline Index & Name & Type & Description \\
\hline \multirow[t]{7}{*}{$\mathrm{K}+1$} & $I 1,1,1$ & $\begin{array}{l}\text { Variable } \\
\dot{:} \\
\dot{\cdot} \\
\text { Variable }\end{array}$ & $\begin{array}{l}\text { Class 1, Entry 1, Item } 1 \\
\text { Item 2 } \\
: \\
: \\
\cdot \\
\text { Item N1 }\end{array}$ \\
\hline & & $\begin{array}{l}\text { Variable } \\
\dot{ } \\
\text { Variable } \\
\dot{ } \\
\dot{\cdot}\end{array}$ & $\begin{array}{c}\text { Entry } 2, \text { Item } 1 \\
\cdot \\
\dot{-} \\
\text { Item N1 } \\
\dot{\cdot} \\
.\end{array}$ \\
\hline & & $\begin{array}{l}\text { Variable } \\
\text { • } \\
\dot{\cdot} \\
\text {. }\end{array}$ & $\begin{array}{c}\text { Entry NE1, Item } 1 \\
\cdot \\
\dot{\text { Item N1 }}\end{array}$ \\
\hline & $\mathrm{I} 2,1, \mathrm{I}$ & $\begin{array}{l}\text { Variable } \\
\cdot \\
\dot{ } \\
\text { Variable }\end{array}$ & $\begin{array}{c}\text { Class 2, Entry 1, Item } 1 \\
\cdot \\
\dot{\text { Item N2 }}\end{array}$ \\
\hline & & $\begin{array}{l}\text { Variable } \\
\dot{ } \\
\dot{ } \\
\dot{ } \\
\dot{ } \\
\text { Variable }\end{array}$ & $\begin{array}{c}\text { Entry 2, Item } 1 \\
\dot{\cdot} \\
\dot{\text { Item N2 }} \\
\dot{\cdot} \\
\dot{0} \\
\text { Entry NE2, Item N2 }\end{array}$ \\
\hline & $\dot{.}$ & : & \\
\hline & IK $, 1,1$ & Variable & $\begin{array}{l}\text { Class } \mathrm{K} \text {, Entry 1, Item } \\
1\end{array}$ \\
\hline $\mathrm{x}$ & IK,NEK,NK & Variable & $\begin{array}{l}\text { Class K, Entry NEK, } \\
\text { Item NK }\end{array}$ \\
\hline
\end{tabular}

Additional Pointers as required (see 2.2.4.4.2). 
4.3.3.3 Pre-defined Associativities. As defined in 4.3.2.2, the associativity definition entity will only occur for Form Numbers 5001 through 9999. The following paragraphs contain the definitions of the pre-defined associativities as they would appear if they were defined by a user. Also included in this Section are the descriptions of each associativity's parameters in a manner similar to other entities in this Specification.

\subsection{FORM NUMBER: 1 Group}

The Group Associativity allows a collection of a set of entities to be maintained as a single, logical entity. Figure 4-26 is an example.

There are four form numbers which specify Group associativities:

$\begin{array}{cl}\text { Form Number } & \text { Description } \\ 1 & \text { Unordered group with backpointers required } \\ 7 & \begin{array}{l}\text { Unordered group with backpointers not } \\ \text { required } \\ 14\end{array} \\ 15 & \text { Ordered group with backpointers required }\end{array}$

The first $($ Form $=1)$ is defined here; the others are defined in subsections 4.3.3.3.6 (Form=7), 4.3.3.3.10 (Form $=14)$, and 4.3.3.3.11 (Form=15), respectively.

\section{DEFINITION}

$\begin{array}{ccl}\text { Index } & \text { Set Value } & \\ 1 & 1 & \text { Meaning } \\ 2 & 1 & \text { One class } \\ 3 & 2 & \text { Back pointers required } \\ 4 & 1 & \text { Onordered } \\ 5 & 1 & \text { The item per entry }\end{array}$




\section{DESCRIPTION}

Directory Data

ENTITY NUMBER: 402

FORM NUMBER: 1

\section{Parameter Data}

\begin{tabular}{cllr} 
Index & Name & \multicolumn{1}{c}{ Type } & Description \\
1 & $\mathrm{~N}$ & Integer & Number of entries \\
2 & $\mathrm{DE}$ & Pointer & Pointer to entity 1 \\
3 & $\mathrm{DE}$ & Pointer & Pointer to entity 2 \\
- & - & - & \\
$\mathrm{N}+1$ & $\mathrm{DE}$ & Pointer & Pointer to entity N
\end{tabular}




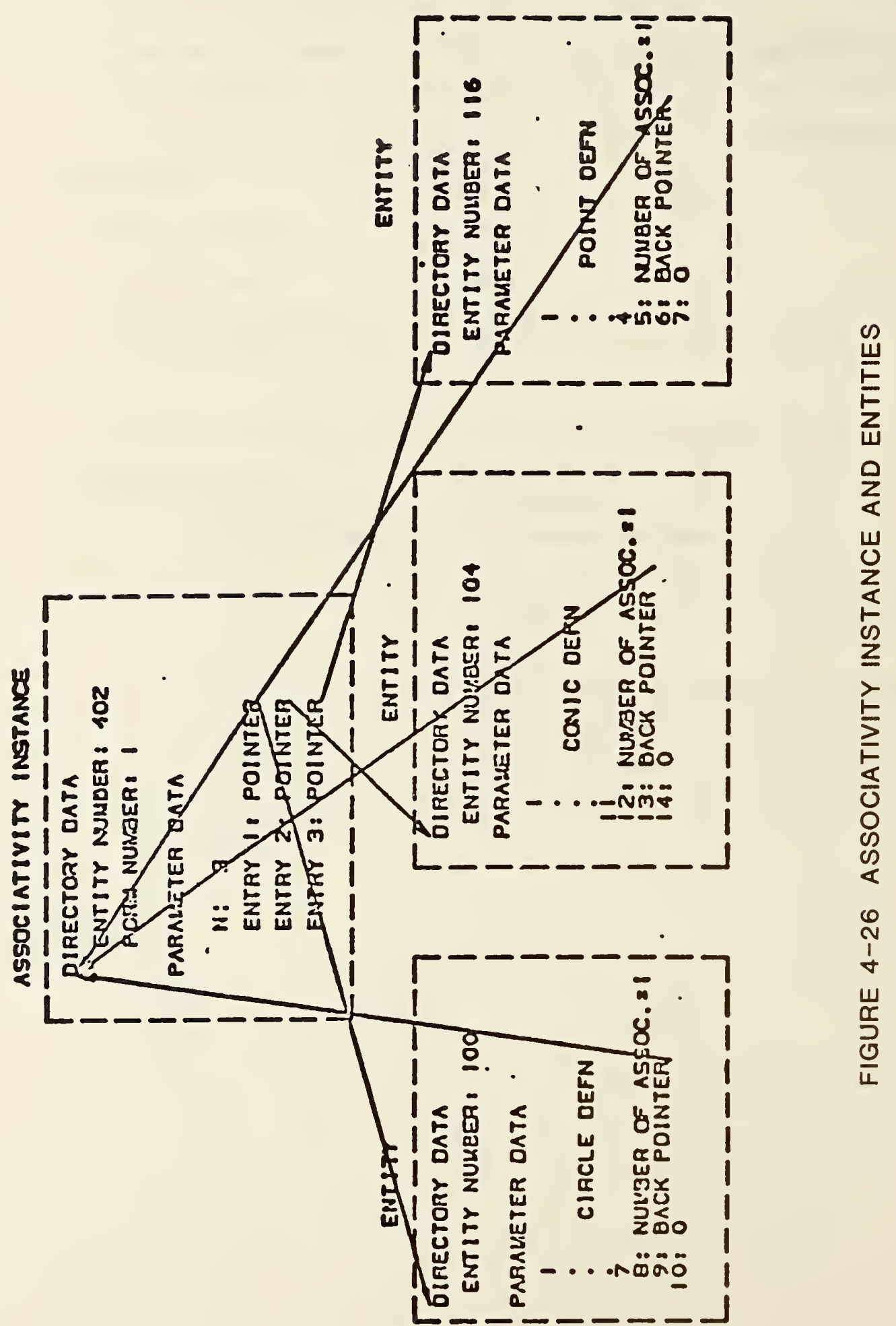




\subsection{FORM NUMBER: 2 External Logical Reference File Index}

The External Logical Reference File Index appears in one file which contains references from another file. It contains a list of the symbolic names used by the referencing files and the DE pointers to the corresponding definitions within the referenced file. See section 2.5.4 and the External Reference Entity (type 416) for more detail.

\section{DEFINITION}

$\begin{array}{cc}\text { Index } & \text { Set Value } \\ 1 & 1 \\ 2 & 2 \\ 3 & 2 \\ 4 & 2 \\ 5 & 2 \\ 6 & 1\end{array}$

Meaning

One class (externally referenced entities)

Backpointers not required

Unordered list of entries in a class

Number of items in an entry

First item is a value (External Reference Entity Symbolic Name)

Second item is a pointer (Internal Entity DE Pointer)

\section{DESCRIPTION}

\section{Directory Data}

ENTITY NUMBER: $\quad 402$

FORM NUMBER: 2

\section{Parameter Data}

\begin{tabular}{|c|c|c|}
\hline Index & Name & Type \\
\hline 1 & $N$ & Integer \\
\hline 2 & NAME 1 & String \\
\hline 3 & PTR 1 & Pointer \\
\hline$\cdot$ & - & • \\
\hline$\bullet$ & • & • \\
\hline
\end{tabular}

\section{Description}

Number of Index Entries

External Reference Entity Symbolic Name

Internal Entity DE Pointer 


\begin{tabular}{|c|c|c|c|}
\hline $2 \mathrm{~N}$ & NAMEN & String & $\begin{array}{l}\text { Last External Reference } \\
\text { Entity Symbolic Name }\end{array}$ \\
\hline $2 \mathrm{~N}+1$ & PTRN & Pointer & $\begin{array}{l}\text { Last Internal Entity DE } \\
\text { Pointer }\end{array}$ \\
\hline
\end{tabular}

Additional Pointers as Required (see 2.2.4.4.2)

\subsection{FORM NUMBER : 3 Views Visible}

When an entity is to be displayed in a single view, a pointer to that view entity is entered in parameter 6 of the entity's DE.

If an entity is to be displayed in more than one view, parameter 6 of its DE contains a pointer to an instance of a Form 3 associativity. This form of the associativity contains two classes of information. The first class contains the number of views visible followed by pointers to each of the view entities visible in the specific associativity instance. The second class contains the number of entities whose display is specified by this instance, followed by pointers to each of the entities.

\section{DEFINITION}

Index

1

2

3

4

5
Set Value

2

Class 1

1

2

1

1

Class 2

2

2

1

1
Meaning

Two classes

Back pointers required

Unordered

One item per entry

Item is a pointer

(to view entity)

Back pointers not required

Unordered

One item per entry

Item is a pointer

(to o ther entity) 
DESCRIPTION

\section{Directory Data}

ENTITY TYPE NUMBER: 402

FORM NUMBER: 3

\section{Parameter Data}

\begin{tabular}{|c|c|c|}
\hline Index & Name & Type \\
\hline 1 & N1 & Integer \\
\hline 2 & N2 & Integer \\
\hline 3 & DEV1 & Pointer \\
\hline - & - & - \\
\hline . & • & - \\
\hline $\mathrm{N} 1+2$ & DEVN1 & Pointer \\
\hline $\mathrm{N} 1+3$ & $\mathrm{DE} 1$ & Pointer \\
\hline - & - & - \\
\hline - & - & - \\
\hline $\mathrm{N} 1+\mathrm{N} 2+2$ & DEN2 & Pointer \\
\hline
\end{tabular}

\section{Description}

Number of views visible

Number of entities displayed in these views

Pointer to view entity

Pointer to entity whose display is being specified by this associativity instance

Pointer to entity N2 


\subsection{FORM NUMBER: 4 Views Visible, Color, Line Weight}

This associativity is an extension of Form Number 3. For those entities that are visible in multiple views, but must have a different line font, color number, or line weight in each view, there will be an occurrence of the associativity instance Form Number 4.

In the parameter data portion of the associativity instance, the parameter $\mathrm{N} 1$ will indicate the number of blocks containing the view visible, line font, color number, and line weight specifications. Each block will contain a pointer to the view entity, a line font value or 0 , a pointer to a line font definition entity if the line font value was 0 , a color value or pointer to a color definition entity, and a line weight value. Parameter N2 will contain the number of entities which are members of this associativity (i.e., entities which have this particular display characteristic).

Note that N2 may often be 1. If more than one entity appears in Class 2 the complete set of display characteristics in Class 1 apply to each entity in Class 2.

\begin{tabular}{|c|c|c|}
\hline \multicolumn{3}{|l|}{ DEFINITION } \\
\hline Index & $\underline{\text { Set Value }}$ & Meaning \\
\hline \multirow[t]{2}{*}{1} & 2 & Two classes \\
\hline & Class & \\
\hline 2 & 1 & Back pointers required \\
\hline 3 & 2 & Unordered \\
\hline \multirow[t]{2}{*}{4} & 5 & Five items per entry \\
\hline & (Entr & \\
\hline 5 & 1 & $\begin{array}{l}\text { Pointer to view directory } \\
\text { entry }\end{array}$ \\
\hline 6 & 2 & Line font value \\
\hline 7 & 1 & $\begin{array}{l}\text { Pointer to line font directory } \\
\text { entry }\end{array}$ \\
\hline 8 & 3 & $\begin{array}{l}\text { Color number (value) or } \\
\text { pointer }\end{array}$ \\
\hline
\end{tabular}


Line weight (value)

Class 2 (entity)

Back pointers not required

11

2

Unordered

12

1

One item per entry

13

\section{DESCRIPTION}

\section{Directory Data}

ENTITY TYPE NUMBER: 402

FORM NUMBER:

4

\section{$\underline{\text { Parameter Data }}$}

\begin{tabular}{|c|c|c|c|}
\hline Index & $\underline{\text { Name }}$ & Type & Description \\
\hline 1 & N1 & Integer & $\begin{array}{l}\text { Number of blocks containing } \\
\text { the view visible, line font, } \\
\text { color number, and line weight } \\
\text { information }\end{array}$ \\
\hline 2 & N2 & Integer & $\begin{array}{l}\text { Number of entities which } \\
\text { have this particular set of } \\
\text { display characteristics }\end{array}$ \\
\hline 3 & DEVI & Pointer & Pointer to view entity 1 \\
\hline 4 & LFI & Integer & Line font value or 0 \\
\hline 5 & DEF 1 & Pointer & $\begin{array}{l}\text { If parameter } 4=0 \text {, pointer to } \\
\text { a line font definition. Other- } \\
\text { wise }=0 \text {. }\end{array}$ \\
\hline 6 & CNI & $\begin{array}{l}\text { Integer or } \\
\text { Pointer }\end{array}$ & Color number value 1 \\
\hline 7 & LW 1 & Integer & Line weight value 1 \\
\hline 8 & DEV2 & Pointer & Pointer to view entity 2 \\
\hline
\end{tabular}


402, Form 4 - VIEWS VISIBLE, COLOR, LINE WEIGHT

$\begin{array}{clll}5 * \mathrm{~N} 1+2 & \text { LWN1 } & \text { Integer } & \text { Line weight value N1 } \\ 5 * \mathrm{~N} 1+3 & \text { DE1 } & \text { Pointer } & \text { Pointer to entity } 1 \\ - & - & - & \\ 5 * \mathrm{~N} 1+\mathrm{N} 2+2 & \text { DEN2 } & \text { Pointer } & \text { Pointer to entity N2 } \\ & \text { Additional pointers as required (see } 2.2 .4 .4 .2) .\end{array}$




\subsection{FORM NUMBER: 5 Entity Label Display}

Some entities may have one or more possible displays for their entity labels, depending on the view in which they are being displayed. For those entities, there will be an occurrence of the associativity instance Form Number 5.

In the parameter data portion of the associativity instance, the parameter $\mathrm{N}$ will indicate the number of blocks containing label placement information. Each block will contain a pointer to a view entity which specifies the view of visibility. The remaining information (text location, leader, and level number) applies to the label for that view.

\section{DEFINITION}

$\begin{array}{cc}\text { Index } & \text { Set Value } \\ 1 & 1 \\ 2 & 2 \\ 3 & 1 \\ 4 & 7 \\ 5 & 1 \\ 6 & \\ 7 & 2 \\ 8 & 2 \\ 9 & 2 \\ 10 & 1 \\ 11 & 2\end{array}$

Meaning

One class

Back pointers not required

Ordered

Seven items per entry

Pointer to view directory entry

$\mathrm{XT}$ of text location

$\mathrm{YT}$ of text location

ZT of text location

Pointer to leader directory entry

Entity label level number

Pointer to entity 
DESCRIPTION

Directory Data

ENTITY TYPE NUMBER: 402

FORM NUMBER:

Parameter Data

\begin{tabular}{|c|c|c|c|}
\hline$\underline{\text { Index }}$ & $\underline{\text { Name }}$ & Type & Description \\
\hline 1 & $\mathrm{~N}$ & Integer & Number of label placements \\
\hline 2 & DEV1 & Pointer & Pointer to a first view entity \\
\hline 3 & $\mathrm{XT1}$ & Real & $\begin{array}{l}\text { XT coordinate of text } \\
\text { location in first view }\end{array}$ \\
\hline 4 & YT 1 & Real & $\begin{array}{l}\text { YT coordinate of text } \\
\text { location in first view }\end{array}$ \\
\hline 5 & ZT 1 & Real & $\begin{array}{l}\text { ZT coordinate of text } \\
\text { location in first view }\end{array}$ \\
\hline 6 & DEL 1 & Pointer & $\begin{array}{l}\text { Pointer to leader in first } \\
\text { view }\end{array}$ \\
\hline 7 & LLN 1 & Integer & $\begin{array}{l}\text { Entity label level number in } \\
\text { first view }\end{array}$ \\
\hline 8 & DE 1 & Pointer & $\begin{array}{l}\text { Pointer to first entity being } \\
\text { displayed }\end{array}$ \\
\hline 9 & DEV2 & Pointer & Pointer to second view entity \\
\hline • & - & - & - \\
\hline - & - & - & - \\
\hline$\cdot$ & $\cdot$ & - & $\cdot$ \\
\hline 15 & DE2 & Pointer & $\begin{array}{l}\text { Point er to second entity } \\
\text { being displayed }\end{array}$ \\
\hline - & • & • & $\cdot$ \\
\hline - & - & - & - \\
\hline - & - & - & • \\
\hline $7 * N-5$ & DEVN & Pointer & Pointer to NIth view entity \\
\hline . & XTN & Real & $\begin{array}{l}\text { XT coordinate of text } \\
\text { location in Nth view }\end{array}$ \\
\hline • & YTN & Real & $\begin{array}{l}\text { YT coordinate of text } \\
\text { location in Nth view }\end{array}$ \\
\hline
\end{tabular}




\begin{tabular}{|c|c|c|c|}
\hline Index & $\underline{\text { Name }}$ & Type & Description \\
\hline - & ZTN & Real & $\begin{array}{l}\text { ZT coordinate of text } \\
\text { location in Nth view }\end{array}$ \\
\hline - & DELN & Pointer & Pointer to leader in Nth view \\
\hline - & LLNN & Integer & $\begin{array}{l}\text { Entity label level } \\
\text { number in Nth view }\end{array}$ \\
\hline $7 * N+1$ & DEN & Pointer & $\begin{array}{l}\text { Pointer to } N \text { th entity being } \\
\text { displayed }\end{array}$ \\
\hline
\end{tabular}

Additional pointers as required (see $2 \cdot 2.4 .4 .2$ ) 


\subsection{FORM NUMBER: 7 Group Without Back Pointers}

DEFINITION

$\begin{array}{cc}\text { Index } & \text { Set Value } \\ 1 & 1 \\ 2 & 2 \\ 3 & 2 \\ 4 & 1 \\ 5 & 1\end{array}$

Meaning

One class

Back pointers not required

Unordered

One item per entry

The item is a pointer

DESCRIPTION

Directory Data
ENTITY TYPE NUMBER: 402
FORM NUMBER:

\section{$\underline{\text { Parameter Data }}$}

\begin{tabular}{clll} 
Index & Name & \multicolumn{1}{c}{ Type } & Description \\
\cline { 2 - 4 } & $\mathrm{N}$ & Integer & Number of entries \\
2 & $\mathrm{DEl}$ & Pointer & Pointer to entity 1 \\
- & - & - & \\
$\mathrm{N}+1$ & DEN & Pointer & Pointer to entity N \\
& Additional Pointers as required (see 2.2.4.4.2).
\end{tabular}




\subsection{FORM NUMBER: 9 Single Parent Associativity}

This associativity defines a logical structure of one independent (parent) entity and one or more subordinate (children) entities.

Both parent and child entities require back pointers to this instance. Any necessary display parameters are governed by the parent entity.

\section{DEFINITION}

$\underline{\text { Index }}$

1

2

3

4

5

6

7

8

9

6
Set Value

2

Class 1 (parent)

1
2
1
1

Class 2 (children)
1

1

1

1
Meaning

Two classes

Back pointers required

Unordered

One item per entry

Item is pointer

to parent entity

Back pointers required

Ordered

One item per entry

Item is pointer

to child entity 
DESCRIPTION

Directory Data

ENTITY TYPE NUMBER: 402

FORM NUMBER:

\section{Parameter Data}

\begin{tabular}{|c|c|c|c|}
\hline Index & Name & Type & Description \\
\hline 1 & NP & Integer & $\begin{array}{l}\text { Number of Parent } \\
\text { Entities }(\mathrm{NP}=1)\end{array}$ \\
\hline 2 & $\mathrm{NC}$ & Integer & Number of Children \\
\hline 3 & DE & Pointer & $\begin{array}{l}\text { Pointer to Parent } \\
\text { Entity }\end{array}$ \\
\hline 4 & DE 1 & Pointer & $\begin{array}{l}\text { Pointer to Child } \\
\text { Entity } 1\end{array}$ \\
\hline$\dot{\circ}$ & $\dot{\circ}$ & $\dot{\bullet}$ & \\
\hline $2+\mathrm{NC}$ & DENC & Pointer & $\begin{array}{l}\text { Pointer to Child } \\
\text { Entity NC }\end{array}$ \\
\hline
\end{tabular}

Additional Pointers as required (see 2.2.4.4.2). 


\subsection{FORM NUMBER: $12 \quad$ External Reference File Index}

The External Reference File Index appears in one file which contains definitions referenced by another file. It contains a list of the symbolic names used by the referencing files and the DE pointers to the corresponding definitions within the referenced file. See section 2.5.4 and the External Reference Entity (Type 416) for more detail.

\section{DEFINITION}

\begin{tabular}{|c|c|c|}
\hline Index & Set Value & Meaning \\
\hline 1 & 1 & $\begin{array}{l}\text { One class (externally referenced } \\
\text { entities) }\end{array}$ \\
\hline 2 & 2 & Backpointers not required \\
\hline 3 & 2 & Unordered list of entries in a class \\
\hline 4 & 2 & Number of items in an entry \\
\hline 5 & 2 & $\begin{array}{l}\text { First item is a value (External Reference } \\
\text { Entity Symbolic Name) }\end{array}$ \\
\hline 6 & 1 & $\begin{array}{l}\text { Second item is a pointer (Internal Entity } \\
\text { DE Pointer) }\end{array}$ \\
\hline
\end{tabular}

\section{DESCRIPTION}

\section{Directory Data}

ENTITY TYPE NUMBER: 402

FORM NUMBER:

\section{Parameter Data}

\begin{tabular}{|c|c|c|c|}
\hline Index & Name & Type & Description \\
\hline 1 & N & Integer & Number of Index Entries \\
\hline 2 & NAME 1 & String & $\begin{array}{l}\text { External Reference Entity } \\
\text { Symbolic Name }\end{array}$ \\
\hline 3 & PTR 1 & Pointer & Internal Entity DE Pointer \\
\hline . & • & $\dot{.}$ & \\
\hline $2 \mathrm{~N}$ & NAMEN & String & $\begin{array}{l}\text { Last External Reference } \\
\text { Entity Symbolic Name }\end{array}$ \\
\hline $2 \mathrm{~N}+1$ & PTRN & Pointer & $\begin{array}{l}\text { Last Internal Entity DE } \\
\text { Pointer }\end{array}$ \\
\hline
\end{tabular}

Additional Pointers as required (see 2.2.4.4.2) 


\subsection{FORM NUMBER: 13 Dimensioned Geometry Associativity}

This associativity links a dimension entity with the geometry entities it is dimensioning. The pointers to the entities being dimensioned have interpretations related to the type of dimension entity. See Figure 4-27.

\section{DEFINITION}

\begin{tabular}{|c|c|c|}
\hline Index & Set Value & Meaning \\
\hline 1 & 2 & Two classes \\
\hline \multicolumn{3}{|c|}{ Class 1 (Dimension Entity) } \\
\hline 2 & 1 & Back pointers required \\
\hline 3 & 2 & Unordered \\
\hline 4 & 1 & One item (pointer to dimension) \\
\hline 5 & 1 & Item is pointer \\
\hline \multicolumn{3}{|c|}{ Class 2 (Related Geometry) } \\
\hline 6 & 2 & Back pointers not required \\
\hline 7 & 2 & Unordered \\
\hline 8 & 1 & $\begin{array}{l}\text { One item per entry (pointers } \\
\text { to geometry) }\end{array}$ \\
\hline 9 & 1 & Item is pointer \\
\hline
\end{tabular}




$$
\begin{aligned}
\text { 402, Form } 13 \text { - DIMENSIONED GEOMETRY } \\
\text { ASSOCIATIVITY }
\end{aligned}
$$

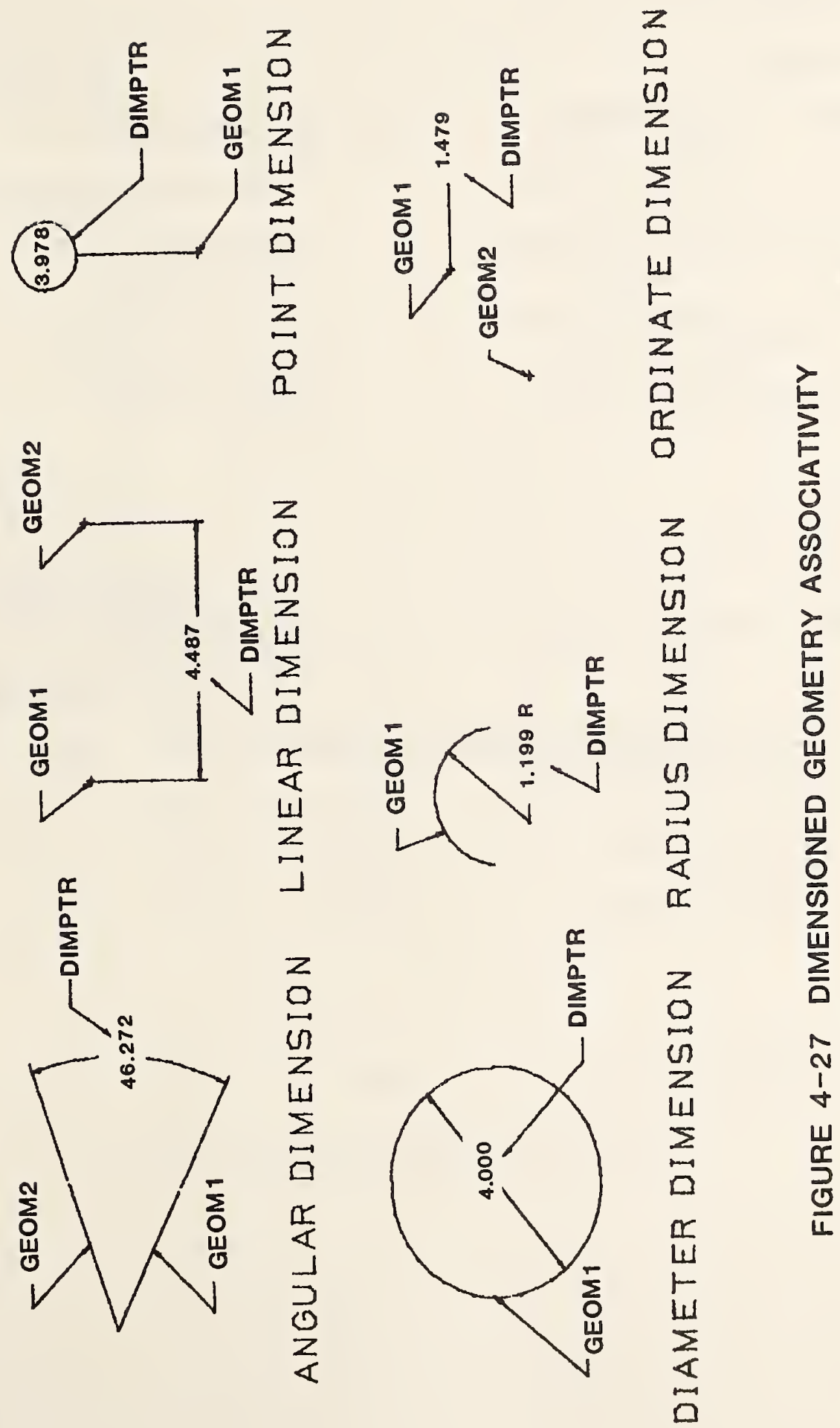


402, Form 13 - DIMENSIONED GEOMETRY ASSOCIATIVITY

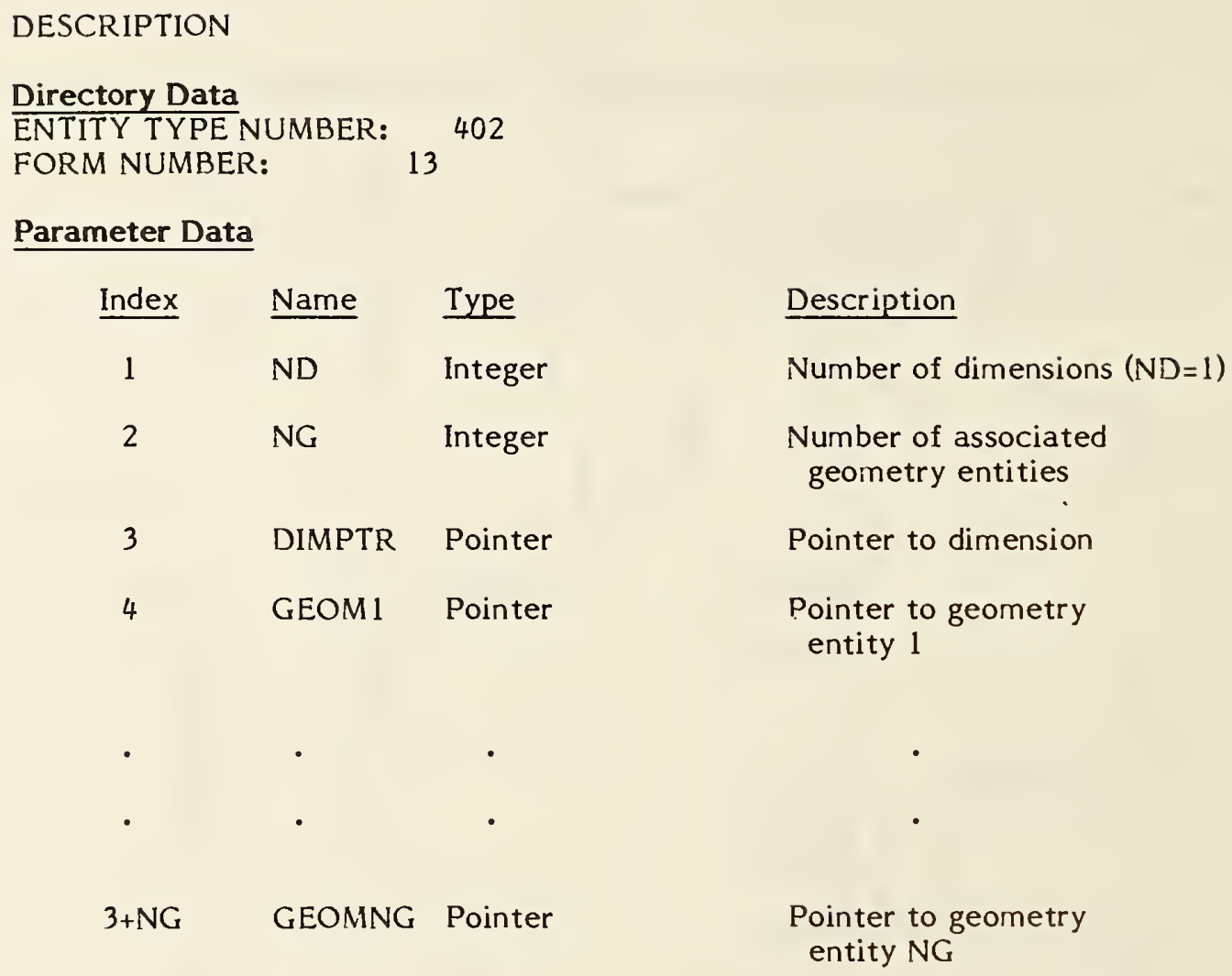

Additional Pointers as required (see 2.2.4.4.2) 
4.3.3.3.10 FORM NUMBER: 14 Ordered Group with Backpointers

DEFINITION

$\begin{array}{ccl}\text { Index } & \text { Set Value } & \text { Meaning } \\ 1 & 1 & \text { One class } \\ 2 & 1 & \text { Back pointers required } \\ 3 & 1 & \text { Ordered } \\ 4 & 1 & \text { One item per entry } \\ 5 & 1 & \text { The item is a pointer }\end{array}$

DESCRIPTION

Directory Data

ENTITY NUMBER: 402

FORM NUMBER: 14

Parameter Data

$\begin{array}{clll}\text { Index } & \text { Name } & \text { Type } & \text { Description } \\ 1 & \mathrm{~N} & \text { Integer } & \text { Number of entries } \\ 2 & \mathrm{DE} & \text { Pointer } & \text { Pointer to entity 1 } \\ 3 & \mathrm{DE} & \text { Pointer } & \text { Pointer to entity 2 } \\ \cdot & - & - & \\ \mathrm{N}+1 & \text { DE } & \text { Pointer } & \text { Pointer to entity N }\end{array}$

Additional Pointers as required (see 2.2.4.4.2). 
402, Form 15 - ORDERED GROUP
WITHOUT BACK POINTER

\subsection{FORM NUMBER: 15 Ordered Group without Backpointers}

DEFINITION

$\begin{array}{ccl}\text { Index } & \text { Set Value } & \text { Meaning } \\ 1 & 1 & \text { One class } \\ 2 & 2 & \text { Back pointers not required } \\ 3 & 1 & \text { Ordered } \\ 4 & 1 & \text { One itern per entry } \\ 5 & 1 & \text { The item is a pointer }\end{array}$

DESCRIPTION

Directory Data

ENTITY NUMBER: 402

FORM NUMBER: 15

\section{Parameter Data}

$\begin{array}{clll}\text { Index } & \text { Narne } & \text { Type } & \text { Description } \\ 1 & \mathrm{~N} & \text { Integer } & \text { Number of entries } \\ 2 & \mathrm{DE} & \text { Pointer } & \text { Pointer to entity 1 } \\ 3 & \mathrm{DE} & \text { Pointer } & \text { Pointer to entity 2 } \\ \cdot & - & - & \\ \text { - } & \text { - } & \text { - } & \\ \mathrm{N}+1 & \mathrm{DE} & \text { Pointer } & \text { Pointer to entity N }\end{array}$

Additional Pointers as required (see 2.2.4.4.2). 


\subsection{FORM NUMBER: 16 Planar Associativity}

This associativity is used to indicate that a collection of entities is coplanar. They may be geometric, annotative, and/or structural. In the case of an entity containing subordinate entities, these must also be coplanar.

The first class contains the pointer to the transformation matrix indicating the plane the entities have been moved to. The plane in question is the image under this transformation of the $X Y$ plane. As noted in the description for DE field 7 , the value 0 may be used to indicate the identity transformation matrix. This matrix is informational only for the associativity; the constituent entities must properly position themselves in model space.

The second class contains the pointers to the coplanar entities. 


\section{DEFINITION}

\begin{tabular}{ccl} 
Index & \multicolumn{1}{c}{ Set Value } & Meaning \\
1 & 2 & Two classes \\
2 & Class 1 (Transformation Matrix) & \\
3 & 2 & No back pointers \\
4 & 1 & Ordered class \\
5 & 1 & Number of items per entry \\
6 & 1 & Pointer to directory entry \\
7 & Class 2 (Coplanar Entities) & \\
8 & 1 & Back pointers required \\
9 & 2 & Unordered class \\
& 1 & Number of items per entry
\end{tabular}

\section{Directory Data}

ENTITY TYPE NUMBER: 402

FORM NUMBER: 16

\section{Parameter Data}

\begin{tabular}{|c|c|c|}
\hline Index & Name & Type \\
\hline 1 & 1 & Integer \\
\hline 2 & $N$ & Integer \\
\hline 3 & DETR & Pointer \\
\hline 4 & DE 1 & Pointer \\
\hline - & - & - \\
\hline - & - & - \\
\hline - & - & - \\
\hline $\mathrm{N}+3$ & DEN & Pointer \\
\hline
\end{tabular}

\section{Description}

One transformation matrix

Number of entities in this plane pointed to by this associativity

Pointer to transformation matrix moving data from $X Y$ plane into space or zero

Pointer to first entity on plane specified

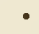

$\cdot$

Pointer to last entity on plane on specified

Additional Pointers as required (see 2.2.4.4.2) 


\subsection{FORM NUMBER: 18 Flow}

The Flow Associativity represents a single signal or a single fluid flow path. The associativity contains seven classes.

Class one contains the type and function flags:

\section{Type Flag Meaning}

$\begin{array}{ll}0 & \text { Not specified (Default) } \\ 1 & \text { Logical } \\ 2 & \text { Physical }\end{array}$

The use of the Type Flag is mandatory when both the logical (e.g., schematic) and physical (e.g., printed board) product definitions are in the same file. In such a file, the type flag shall not be zero.

The function flag differentiates between a fluid path and an electrical conductor:

$\begin{array}{cl}\text { Function Flag } & \text { Meaning } \\ & \\ 0 & \text { Not specified (Default) } \\ 1 & \text { Electrical signal } \\ 2 & \text { Fluid flow path }\end{array}$

A fluid flow path is a single path from a starting connect point entity. The path may include additional intermediate connect points but separate flow entities will be required to describe the branch flow paths. Class four, the Join, lists the elements of the path. For fluid flow paths the elements are ordered as they occur along the flow path and the Connect Points (class three) lists the connect points in the same sense as the order of the Join. That is, the start of the fluid flow path is the one listed first; the end of the fluid flow path is listed last.

Class two contains pointers to other associated flow associativities. These other associativities may implement alternative flow representations. The obvious example of this is the file containing both the schematic and physical product definitions. The corresponding Flow associativities of each type would be paired. 
Class three is the Link, which contains the list of pointers to the Connect Point entities involved in the signal/flow.

Class four is the Join, which contains the list of pointers to the entities representing the graphical implementation of the signal/flow.

Class five contains the flow names which are associated with the signal/flow.

Class six contains a list of pointers to the Text Display Template entities which are to be used to display the first flow name listed in class five. The Text Display Templates provide the locations and attributes for the signal name display.

The text string for display is obtained from the first flow name listed in class five.

Class seven contains a list of pointers to flow paths which branch from the current flow path. This is an ordered list and the "main" continuation of the path, if any, is always listed last. A null pointer is used if there is no continuation of the main path. 
DEFINITION

Index

Set Value

Meaning

1

2

3

4

5

6
7
8
9

10

11

12

13

14

15

16

17

18

19

20

21

22

23

24

25

26

27

28

29
7

Class 1 (Context Flag)

2

1

1

2

Class 2 (Associated Flows)

2

1

1

Class 3 (Connect Points (Link))

Class 4 (Join)

$$
\begin{aligned}
& 1 \\
& 1 \\
& 1
\end{aligned}
$$

Class 5 (Flow Name)

2
2
1
2

Class 6 (Flow Name Display)

$$
2
$$$$
2
$$

1

1

Class 7 (Flow Continuations)
Seven classes

Back pointers not required

Ordered

One item per entry

Item is value

Back pointers not required

Unordered

One item per entry

Pointer to Flow Associativity

Back pointers required

Ordered

One item per entry

Pointer to Connect Point Entity

Back pointers required

Ordered

One item per entry

Pointer to geometry or Subfigure instance

Back pointers not required

Unordered

One item per entry

Item is value

Back pointers not required

Unordered

One item per entry

Pointer to Text Display Template Entity

Back pointers required

Ordered

One item per entry

Item is a pointer 
The Status Flag values should be set as follows:

Subordinate

Entity Use

Hierarchy
To be set by sending system

03

00

\section{DESCRIPTION}

Directory Data

ENTITY TYPE NUMBER: 402

FORM NUMBER:

18

\section{Parameter Data}

\begin{tabular}{|c|c|c|c|}
\hline Index & Name & Type & Description \\
\hline 1 & NCF & Integer & Count of context flags $(=2)$ \\
\hline 2 & NF & Integer & Count of associated Flow associativities \\
\hline 3 & NC & Integer & Count of Connect Point entities \\
\hline 4 & NJ & Integer & Count of Join entities (geometry of subfigure) \\
\hline 5 & NN & Integer & Count of Flow names \\
\hline 6 & NT & Integer & $\begin{array}{l}\text { Count of Text Display Templates for Flow } \\
\text { name display }\end{array}$ \\
\hline 7 & NP & Integer & Count of continuation flow associativities \\
\hline 8 & TF & Integer & $\begin{array}{l}\text { Type of Flow } \\
\qquad \begin{array}{l}0=\text { not specified (Default) } \\
1=\text { logical } \\
2=\text { physical } \\
\text {. other as required } \\
\text {. }\end{array}\end{array}$ \\
\hline 9 & $\mathrm{FF}$ & Integer & $\begin{array}{l}\text { Function flag: } \\
\qquad \begin{array}{l}0=\text { not specified (Default) } \\
1 \text { = electrical signal } \\
2 \text { = fluid flow path } \\
\text {. other as required }\end{array}\end{array}$ \\
\hline
\end{tabular}




\begin{tabular}{|c|c|c|c|}
\hline Index & Name & Type & Description \\
\hline 10 & SPTR 1 & Pointer & $\begin{array}{l}\text { Pointer to directory entry for Flow } \\
\text { Associativity } 1\end{array}$ \\
\hline$\cdots$ & $\cdots$ & $\cdots$ & \\
\hline $\mathrm{NF}+9$ & SPTRNF & Pointer & $\begin{array}{l}\text { Pointer to directory entry for Flow } \\
\text { Associativity NF }\end{array}$ \\
\hline $\mathrm{NF}+10$ & CPTR 1 & Pointer & $\begin{array}{l}\text { Pointer to directory entry for Connect } \\
\text { Point } 1\end{array}$ \\
\hline$\cdots$ & $\cdots$ & $\cdots$ & \\
\hline $\mathrm{NF}+\mathrm{NC}+9$ & CPTRNC & Pointer & $\begin{array}{l}\text { Pointer to directory entry for Connect } \\
\text { Point NC }\end{array}$ \\
\hline $\mathrm{NF}+\mathrm{NC}+10$ & JPTR 1 & Pointer & Pointer to Join entity 1 \\
\hline ... & $\cdots$ & ... & \\
\hline $\mathrm{NF}+\mathrm{NC}+\mathrm{NJ}+9$ & JPTRNJ & Pointer & Pointer to Join entity NJ \\
\hline $\mathrm{NF}+\mathrm{NC}+\mathrm{NJ}+10$ & NAME 1 & String & Flow name 1 \\
\hline$\cdots$ & $\cdots$ & $\cdots$ & \\
\hline $\mathrm{NF}+\mathrm{NC}+\mathrm{NJ}+\mathrm{NN}+9$ & NAMENN & String & Flow Name NN \\
\hline $\mathrm{NF}+\mathrm{NC}+\mathrm{NJ}+\mathrm{NN}+10$ & GPTR 1 & Pointer & $\begin{array}{l}\text { Pointer to directory entry for Text } \\
\text { Template } 1\end{array}$ \\
\hline$\cdots$ & ... & $\cdots$ & \\
\hline $\mathrm{NF}+\mathrm{NC}+\mathrm{NJ}+\mathrm{NN}+\mathrm{NT}+9$ & GPTRNT & Pointer & $\begin{array}{l}\text { Pointer to directory entry for } \\
\text { Text Template NT }\end{array}$ \\
\hline $\mathrm{NF}+\mathrm{NC}+\mathrm{NJ}+\mathrm{NN}+\mathrm{NT}+10$ & CFPTR 1 & Pointer & $\begin{array}{l}\text { Pointer to continuation flow } \\
\text { associativity entity } 1\end{array}$ \\
\hline $\mathrm{NF}+\mathrm{NC}+\mathrm{NJ}+\mathrm{NN}+\mathrm{NT}+\mathrm{NP}+9$ & CFPTRNP & Pointer & $\begin{array}{l}\text { Pointer to continuation flow } \\
\text { associativity entity NP (the "main" } \\
\text { continuation) }\end{array}$ \\
\hline
\end{tabular}

Additional Pointers as required (see 2.2.4.4.2) 
THIS PAGE INTENTIONALLY LEFT BLANK

294 


\subsection{Obsolete Associativity Forms}

The addition of new entities and associativities which greatly increase the capability for transfer of specific data constructs has given cause to deprecate associativity forms published in previous versions of this Specification. The parameter lists for these forms are included herein to provide for interpretation of files created under an earlier version. The new associativity forms or entities which are valid for the previous forms are as follows:

OBSOLETE FORM 非

$\begin{array}{cl}6 & \text { View List } \\ 8 & \text { Signal String } \\ 10 & \text { Text Node } \\ 11 & \text { Connect Node }\end{array}$
VALID FORM \# OR ENTITY TYPE 非

3 Views Visible

18 Flow

312 Text Template

132 Connect Point

The parameter lists for these following associativities may have additional parameters as specified in section 2.2.4.4.2: 


\subsection{FORM NUMBER: 6 View List}

\section{DEFINITION}

Ind

$\frac{\text { Set Value }}{2}$

2

3

4

5

1

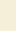

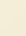

5

6

7

8

9

DESCRIPTION

Directory Data

ENTITY TYPE NUMBER:

FORM NUMBER:

6

402

\section{$\underline{\text { Parameter Data }}$}

$\begin{array}{llll}\text { Index } & \text { Name } & \text { Type } & \text { Description } \\ 1 & 1 & \text { Integer } & \text { Single entry in first class } \\ 2 & \text { N1 } & \text { Integer } & \text { Number of entities in second class } \\ 3 & \text { DEV } & \text { Pointer } & \text { Pointer to view entity } \\ 4 & \text { DE1 } & \text { Pointer } & \begin{array}{l}\text { Pointers to entities visible in } \\ \text { view specified in parameter } 3\end{array} \\ : & \text { - } & \text { - } & \text { - } \\ \text { N1+3 } & \text { DEN1 } & \text { Pointer } & \end{array}$

Class 1 (View)

Meaning

Two classes

Back pointers required

Unordered

One item per entry

Pointer to view directory entry

Class 2 (Entities)

Back pointers required

Unordered

One item per entry

Pointer to directory entry of entity visible in view 


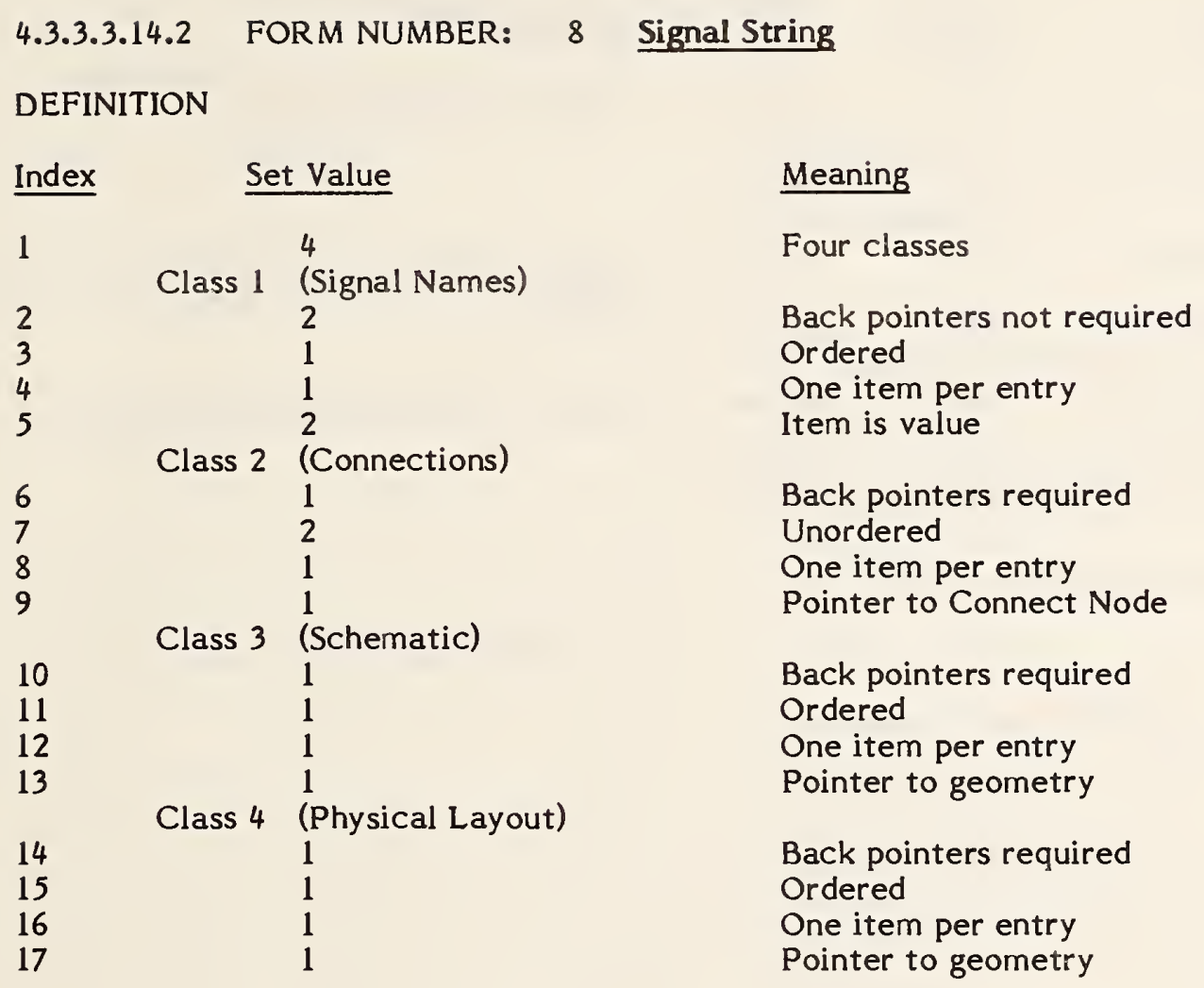

\section{DESCRIPTION}

Directory Data

ENTITY TYPE NUMBER: 402

FORM NUMBER:

8

\section{Parameter Data}

$\begin{array}{llll}\text { Index } & \text { Name } & \text { Type } & \text { Description } \\ 1 & \text { NS } & \text { Integer } & \text { Number of signal names } \\ 2 & \text { N1 } & \text { Integer } & \text { Number of Connection Nodes } \\ 3 & \text { N2 } & \text { Integer } & \text { Number of Entities in schematic signal string } \\ 4 & \text { N3 } & \text { Integer } & \text { Number of Entities in physical signal string } \\ 5 & \text { SIG1 } & \text { String } & \text { Signal name } \\ \text { NS } & \text { S } & & \\ & \text { SIGNS } & & \end{array}$


402 - OBSOLETE ASSOCIATIVITY FORMS

\begin{tabular}{|c|c|c|c|}
\hline Index & Name & Type & Description \\
\hline NS +5 & PC1 & Pointer & Pointer to Connect Nodes \\
\hline$\cdot$ & $\cdot$ & $\cdot$ & \\
\hline $\mathrm{NS}+\mathrm{N} 1+4$ & PCN1 & Pointer & \\
\hline $\mathrm{NS}+\mathrm{N} 1+5$ & PS 1 & Pointer & $\begin{array}{l}\text { Pointer to Entity in schematic logical signal } \\
\text { string }\end{array}$ \\
\hline $\mathrm{NS}+\mathrm{N} 2+\mathrm{N} 1+4$ & PSN2 & Pointer & \\
\hline $\begin{array}{l}\mathrm{NS}+\mathrm{N} 2+\mathrm{N} 1+5 \\
\dot{N} S+N 3+N 2+N\end{array}$ & $\begin{array}{l}\text { PP1 } \\
\dot{P P N 3}\end{array}$ & $\begin{array}{l}\text { Pointer } \\
\text { Pointer }\end{array}$ & Pointer to entity in physical signal string \\
\hline
\end{tabular}




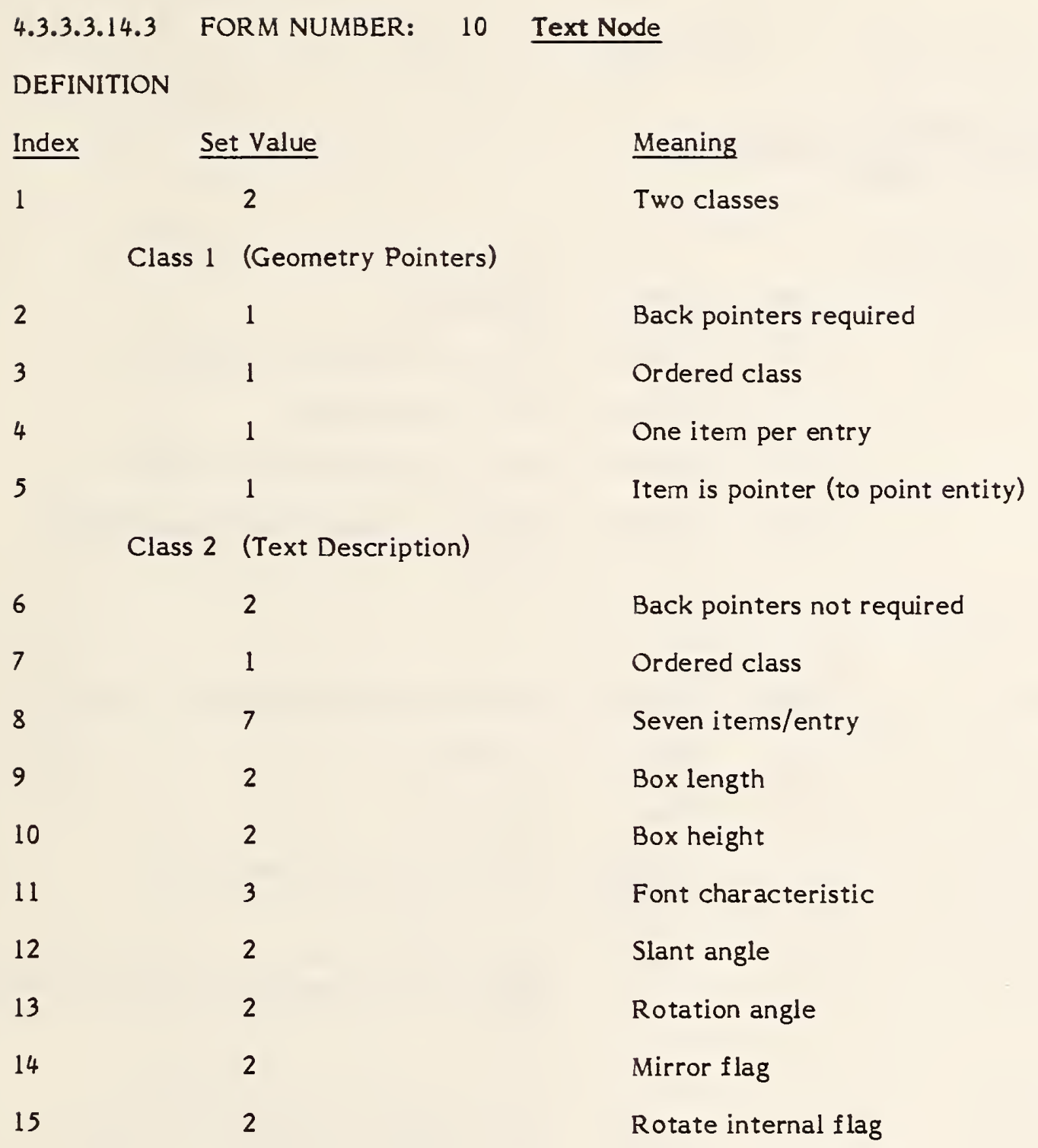




\section{DESCRIPTION}

\section{Directory Data}

ENTITY TYPE NUMBER:
FORM NUMBER:

\section{Parameter Data}

\begin{tabular}{|c|c|c|c|}
\hline Index & Name & Type & Description \\
\hline 1 & NP & Integer & Number of geometry pointers \\
\hline 2 & 1 & Integer & One Text Description \\
\hline 3 & GP1 & Pointer & Pointer to point (original location) \\
\hline 4 & GP2 & Pointer & Pointer to instance point (first instance) \\
\hline • & • & • & \\
\hline • & • & • & \\
\hline $\mathrm{NP}+2$ & GPNP & Pointer & Pointer to instance point (NP- 1 instance) \\
\hline$N P+3$ & WT & Real & Box length \\
\hline $\mathrm{NP}+4$ & HT & Real & Box height \\
\hline $\mathrm{NP}+5$ & FC & $\begin{array}{l}\text { Integer } \\
\text { or pointer }\end{array}$ & Font characteristic (default $=1)$ \\
\hline $\mathrm{NP}+6$ & $\mathrm{SL}$ & Real & $\begin{array}{l}\text { Slant angle of text in radians. pi/ } 2 \text { is the } \\
\text { value for no slant angle and is the default } \\
\text { value. }\end{array}$ \\
\hline $\mathrm{NP}+7$ & A & Real & Rotation angle in radians for text. \\
\hline $\mathrm{NP}+8$ & M & Integer & $\begin{array}{l}\text { Mirror flag }(0=\text { no mirror, } 1=Y T \text { mirror } \\
\text { axis, } 2=X T \text { mirror axis. })\end{array}$ \\
\hline $\mathrm{NP}+9$ & VH & Integer & $\begin{array}{l}\text { Rotate internal text flag } \\
(0=\text { text horizontal, } \\
1=\text { text vertical })\end{array}$ \\
\hline
\end{tabular}




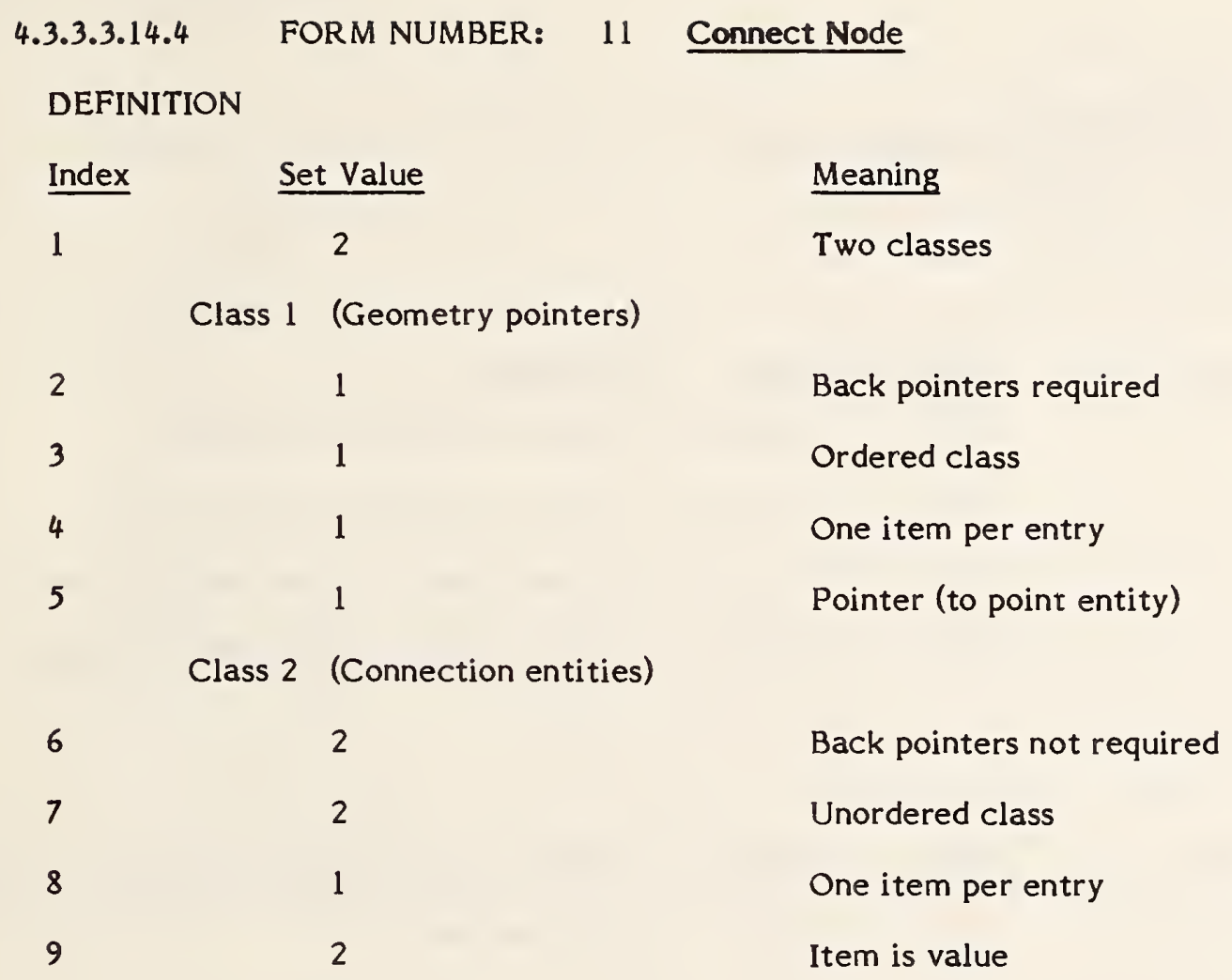


DESCRIPTION

\section{Directory Data}

ENTITY TYPE NUMBER:

FORM NUMBER:

11

\section{Parameter Data}

$\begin{array}{llll}\text { Index } & \text { Name } & \text { Type } & \text { Description } \\ 1 & \text { NC } & \text { Integer } & \text { Number of pointers (to points) } \\ 2 & \text { NP } & \text { Integer } & \text { Number of entries in second class } \\ 3 & \text { PT1 } & \text { Pointer } & \text { Pointer to defining point (original location) } \\ 4 & \text { PT2 } & \text { Pointer } & \text { Pointer to instance point (first instance) } \\ \cdot & \text { - } & \text { - } & \\ \text { NC+2 } & \text { PTNC } & \text { Pointer } & \text { Pointer to last instance point (NC-1 instance) } \\ \text { NC+3 } & \text { DT1 } & \text { Data } & \text { First data entry } \\ \cdot & \text { - } & \text { - } & \\ \cdot & \text { D } & \text { - } & \\ \text { NC+NP+2 } & \text { DTNP } & \text { Data } & \text { Last data entry }\end{array}$




\subsubsection{Drawing Entity.}

The Drawing entity specifies a drawing as a collection of annotation entities (i.e., any entity with use flag set to 01 ) defined in drawing space, and views (i.e., projections of model space data in view space) which, together, constitute a single representation of a part, in the sense that an engineering drawing constitutes a single representation of a part in standard drafting practice. Views are specified by referring to the View Entity. If desired, multiple drawings can be included in a single file, referring to the same model space.

Drawings are located in drawing space as illustrated in Figure 4-28, with sides coincident with the drawing coordinate system axes, and with the lower left corner at the origin $(0,0)$. The drawing space coordinate system (XD, YD) is a special 2-dimensional coordinate system used for view origin locations in the Drawing entity and for annotation entities referenced by the Drawing entity. Any $Z$ coordinates are ignored in the referenced annotation entities and any transformation matrix from definition space to drawing space must be 2dimensional (i.e., in entity $124, \mathrm{~T}_{3}=\mathrm{R}_{13}=\mathrm{R}_{31}=\mathrm{R}_{32}=\mathrm{R}_{23}=0.0$ and $\mathrm{R}_{33}=1.0$ ).

Annotation entities can be defined in drawing space and be referenced by the Drawing entity directly, or can be defined in model space and appear in individual views. When defined in drawing space, the subordinate entity switch should be set to physically dependent (01). The subordinate entity switch for a View entity referenced by the Drawing entity should be set to logically dependent (02).

The transformation of a view from view space to drawing space is controlled by the view scale factor $S$, specified in the View entity, and the view origin drawing locations, specified in the Drawing entity. In the case of or thographic parallel projection the transformation is:

$$
\left[\begin{array}{c}
X D \\
Y D
\end{array}\right]=\left[\begin{array}{ccc}
S & 0 & 0 \\
0 & S & 0
\end{array}\right]\left[\begin{array}{c}
X V \\
Y V \\
Z V
\end{array}\right]+\left[\begin{array}{l}
\text { XORIGIN } \\
\text { YORIGIN }
\end{array}\right]
$$


where $\left[\begin{array}{l}X V \\ Y V \\ Z V\end{array}\right]$

denotes the view space coordinates

and $\left[\begin{array}{l}\text { XORIGIN } \\ \text { YORIGIN }\end{array}\right]$

denotes the drawing space coordinates of the origin of the transformed view (see 4.3.11).

If known, the name of the drawing may be provided by using the Name Property (406, form 15). If not provided, a receiving system may default the name of the drawing.

The size of the drawing may be communicated by using the Drawing Size Property (406, form 16).

The units for drawing space may be different from the model space units specified in the Global section. This is accomplished by use of the Drawing Units Property (406, form 17). When this property is not provided, the drawing units will be the same as the model units.

The following values are given in drawing units:

- view origin drawing locations

- drawing size

- coordinates of annotation entities referenced directly

Refer to Figures 4-28 and 4-29 for examples of the use of the drawing entity. 

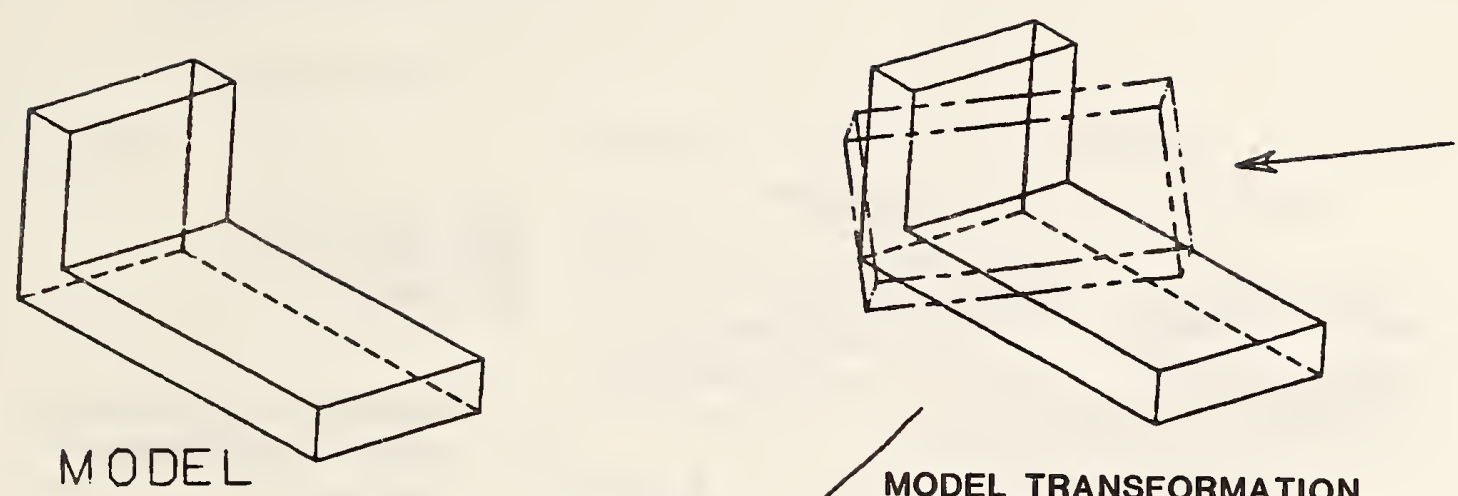

MODEL TRANSFORMATION

TO VIEW COORDINATES

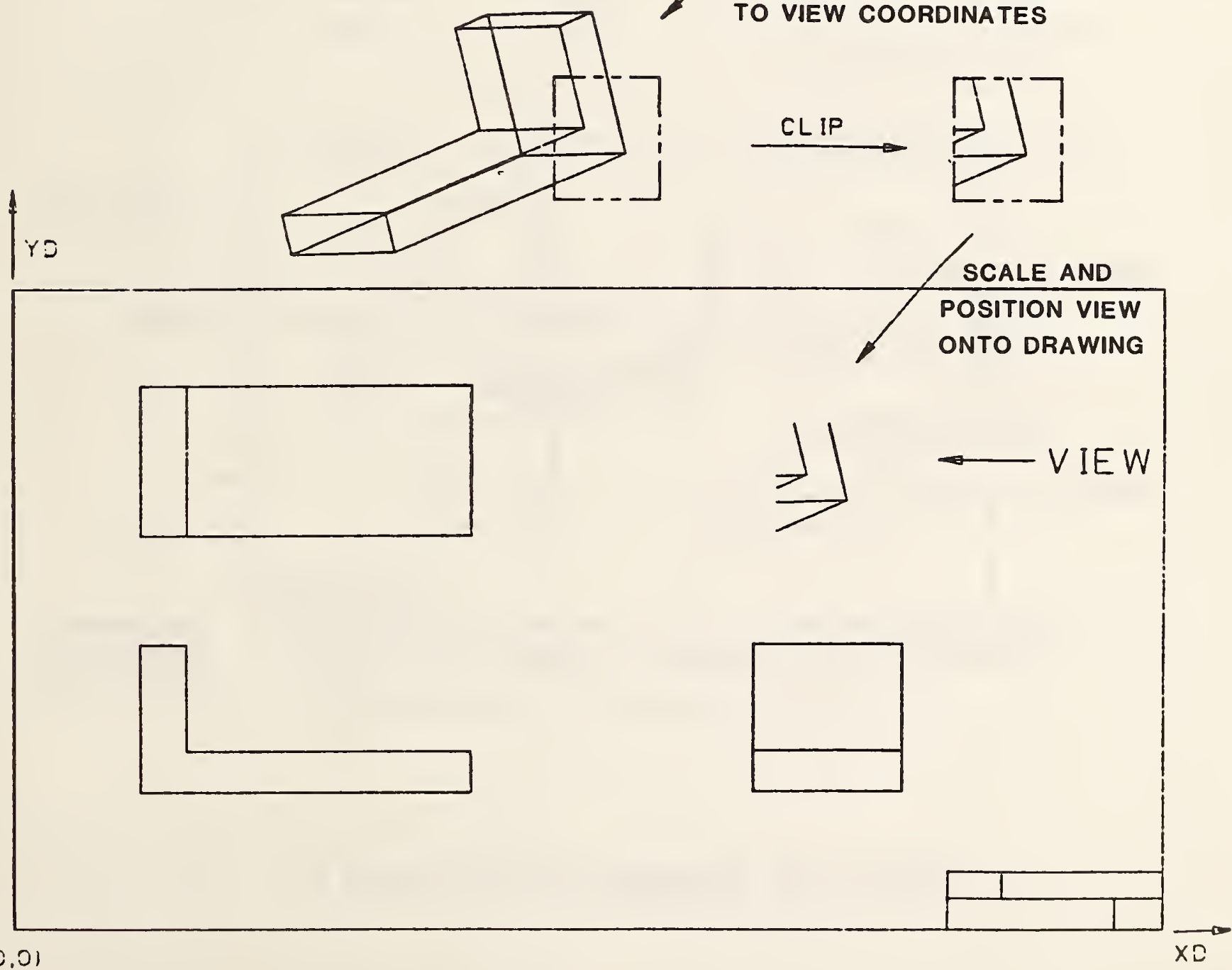

FIGURE 4-28 DRAWING ENTITY EXAMPLE 1 


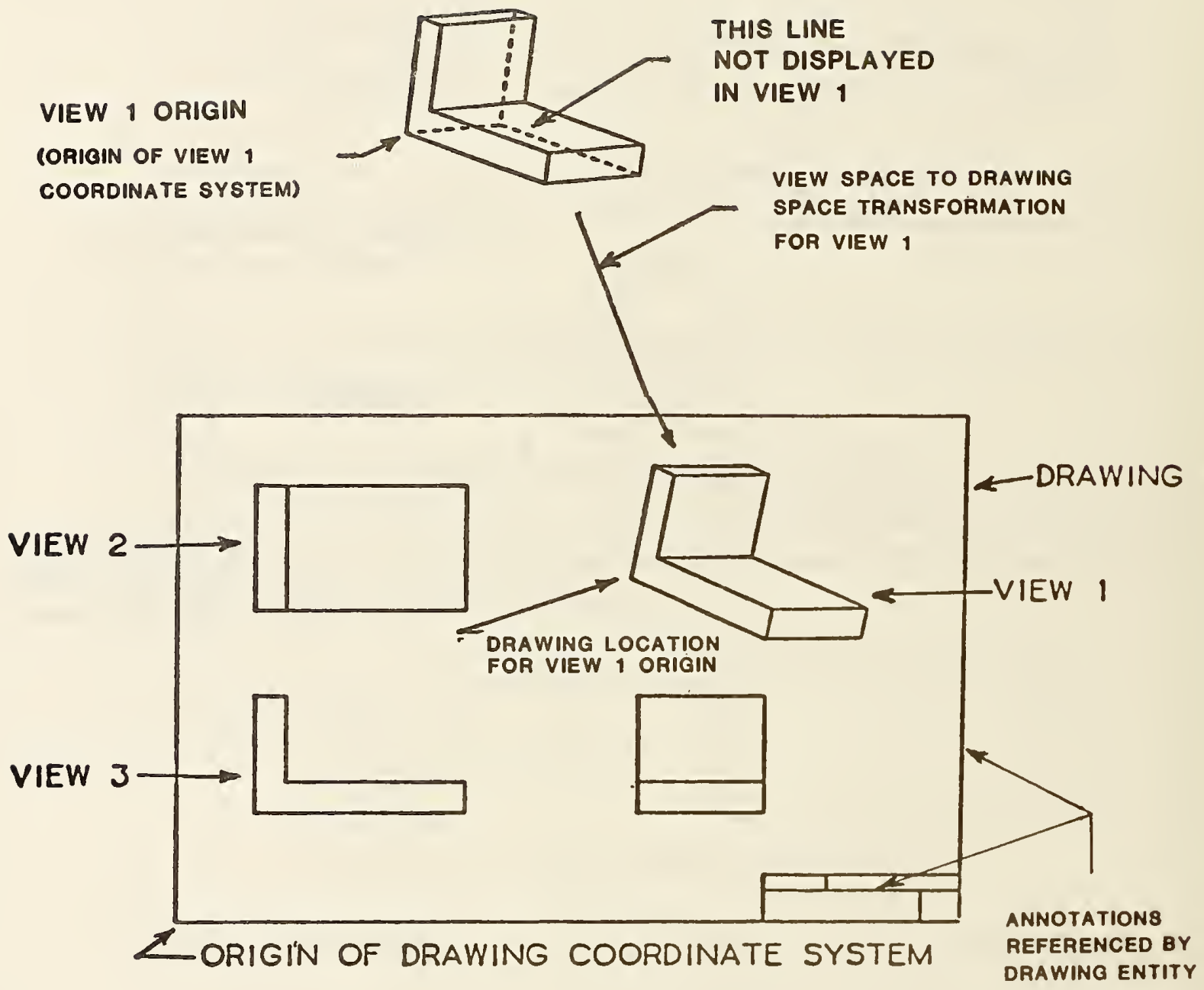

FIGURE 4-29 DRAWING ENTITY EXAMPLE 2 
4.3.4.1 Directory Data

ENTITY TYPE NUMBER : 404

\subsubsection{Parameter Data}

\begin{tabular}{|c|c|c|c|}
\hline Parameter & Value & Format & Comment \\
\hline 1 & $\mathbf{N}$ & Integer & Number of view pointers \\
\hline 2 & VPTR 1 & Pointer & $\begin{array}{l}\text { Pointer to directory entry of } \\
\text { first view entity }\end{array}$ \\
\hline 3 & XORIGINI & Real & $\begin{array}{l}\text { Drawing space coordinate of } \\
\text { the origin of the first } \\
\text { transformed view }\end{array}$ \\
\hline 4 & YORIGINI & Real & $\begin{array}{l}\text { Drawing space coordinate of } \\
\text { the origin of the first } \\
\text { transformed view }\end{array}$ \\
\hline 5 & VPTR2 & Pointer & $\begin{array}{l}\text { Pointer to directory entry of } \\
\text { second view entity }\end{array}$ \\
\hline$\cdot$ & $\cdot$ & & \\
\hline $3 N+2$ & M & Integer & $\begin{array}{l}\text { Number of annotation } \\
\text { entities (may be zero) }\end{array}$ \\
\hline $3 N+3$ & DPTR 1 & Pointer & $\begin{array}{l}\text { Pointer to first annotation } \\
\text { entity in this drawing }\end{array}$ \\
\hline - & • & 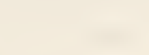 & \\
\hline$\cdot$ & $\cdot$ & & \\
\hline $3 N+M+2$ & DPTRM & Pointer & $\begin{array}{l}\text { Pointer to Mth annotation } \\
\text { entity in this drawing }\end{array}$ \\
\hline
\end{tabular}

Additional Pointers as required (see 2.2.4.4.2) 


\subsubsection{Line Font Definition Entity.}

Two types of line fonts may be defined. One type considers a line font as a repetition of a basic pattern of visible-blanked (or, on-off) segments superimposed on a line or a curve. The line or curve is then displayed according to the basic pattern. The other type considers a line font as a repetition of a template figure that is displayed at regularly spaced locations along a planar anchoring curve. The anchoring curve itself has no visual purpose.

Any line or curve geometry entity type may reference a Line Font Definition entity by inserting a pointer to that entity in its Directory Entry field 4, the line font pattern field. The type of line font being specified is then indicated by a Form Number in the Line Font Definition entity.

Setting the Form Number to 1 in a Line Font Definition entity specifies that the line font type is to be a repetition of template figure displays along the referencing anchoring curve. The template figure is specified as a subfigure definition entity. In this case, four Index values specify the entity as follows:

The first parameter specifies the orientation of the template displays. This may remain constant, or it may vary with the direction of the anchoring curve at the point of each template figure display location. The second parameter is a pointer to the subfigure definition entity containing the template display. The third parameter specifies display locations on the anchoring curve by giving the common arc length distance between corresponding points on successive template figure displays. The fourth parameter gives a scale factor to be applied to the template subfigure at each display location.

Figure 4-30 illustrates two examples of a line font with Form Number equal 1. In each case, the anchoring curve is a straight line. 

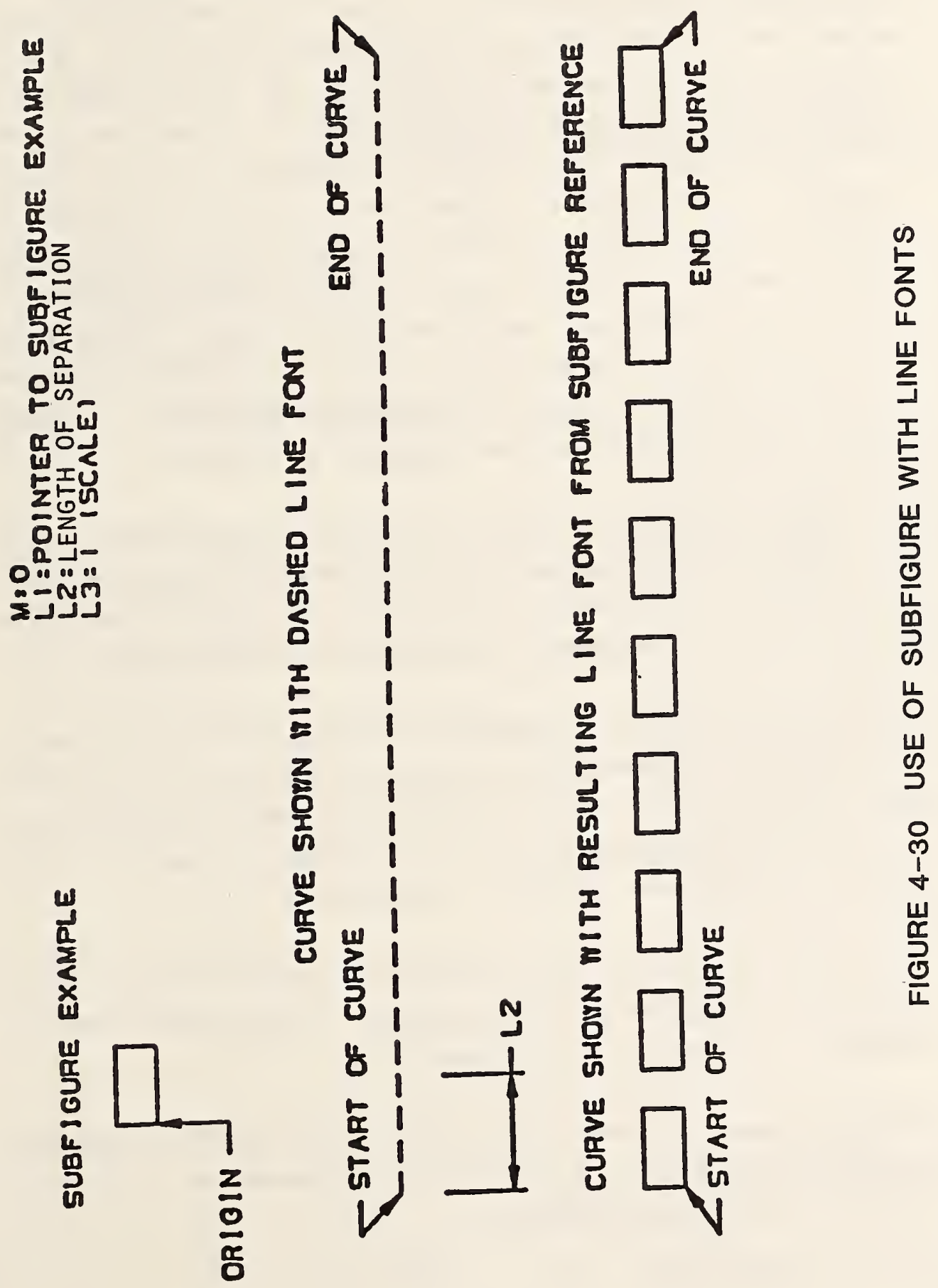
Setting the Form Number to 2 in a Line Font Definition entity specifies that the line font type is to be a repetition of a basic visible-blank pattern superimposed on the referencing line or curve. An arbitrary number $M$ of segments may be used in the basic pattern. When the basic pattern is laid out horizontally, the "first" segment is the leftmost one, the "Mth" segment is the rightmost one. The length (in the units of the curve on which the pattern is being superimposed) of each segment of the pattern may be specified individually. This allows the visible-blank sequence of the pattern to alternate between "visible" and "blank" regardless of the lengths of the segments, but does not prohibit adjacent segments from being either both visible or both blanked when unequal lengths are employed. Another option for some patterns is to hold the length constant across segments, and achieve variation in the lengths of the visible and blanked segments by making the "visible" and/or the "blank" segments be adjacent as required.

For example, a basic pattern whose left two-thirds is visible, and whose right third is blanked may be described either by the sequence "visible-blank" with the length of the first segment twice that of the second, or else by the sequence "visible-visible-blank", with the lengths of all three segments equal.

The visible-blank sequence is specified by correlating it with the rightmost $M$ bits in the binary representation of a string of hexadecimal digits, the $M$ th segment being associated with the units bit of the binary representation of the rightmost hexadecimal digit. A "0" represents a "blank", or "off" segment, a "l" represents a "visible", or "on" segment.

For this line font type, the first parameter is the positive integer $M$ giving the number of segments in the basic pattern. Then, parameter values 2 through $M+2$ specify the entity as follows:

Parameter values 2 through $M+1$ give the lengths of the $M$ segments. Parameter value $M+2$ is the minimal string of hexadecimal digits whose significance has been described above. 
Figure 4-31 shows an example of the Form Number 2. Shown is a font made up of five segments of unequal length. Two repetitions of the basic font are illustrated.

Form

1

2
Meaning

Line font specified by a repeating template subfigure

Line font specified by a repeating visible-blanked pattern

4.3.5.1 Directory Data

ENTITY TYPE NUMBER: 304

FORM NUMBER:

\subsubsection{Parameter Data}

Index

1

Name

M

Type

Integer

2

L 1

L2

Real

L3

Real

4

\section{Description}

$\mathrm{M}=0$ :Each template display is oriented by aligning the axes of the subfigure definition coordinate system with the axes of the definition space of the anchoring curve.

$\mathrm{M}=\mathrm{l}$ : Each template display is oriented by aligning the $\mathrm{X}$ axis of the subfigure definition coordinate system with the tangent vector of the anchoring curve at the point of incidence of the curve and the origin of the subfigure, and the $Z$ axis of the subfigure definition coordinate system with the $Z$ axis of the definition space of the anchoring curve.

Pointer to the subfigure definition for the template displays.

Common arc length distance between correspon " "ng points on successive template figure displays.

Scale factor to be applied to the subfigure.

Additional Pointers as Required (see 2.2.4.4.2) 
4.3.5.3 Directory Data ENTITY TYPE NUMBER: 304

FORM NUMBER: 2

\subsubsection{Parameter Data}

$\begin{array}{ccc}\text { Index } & \begin{array}{ccc}\text { Name } \\ 1\end{array} & \begin{array}{l}\text { Type } \\ \text { Integer }\end{array} \\ 2 & \text { L1 } & \text { Real } \\ 3 & \text { L2 } & \text { Real } \\ 4 & \text { L3 } & \text { Real } \\ & \text { : } & \text { : } \\ \text { M+1 } & \text { LM } & \text { Real } \\ \text { M+2 } & \text { B } & \text { String }\end{array}$

\section{Description}

Number of segments in the basic pattern of visibleblanked segments.

Length of the first segment of the basic pattern.

Length of the second segment of the basic pattern.

Length of the third segment of the basic pattern.

Length of the Mth segment of the basic pattern.

$$
\left\lfloor\left(\frac{M-1}{4}\right)+1\right\rfloor
$$

hexadecimal digits indicating which segments of the basic pattern are visible and which are blanked, where

represents the greatest integer result. E.g., "5" indicates that segments $\perp$ and 3 are visible. Bits are right justified.

Additional Pointers as Required (see 2.2.4.4.2) 


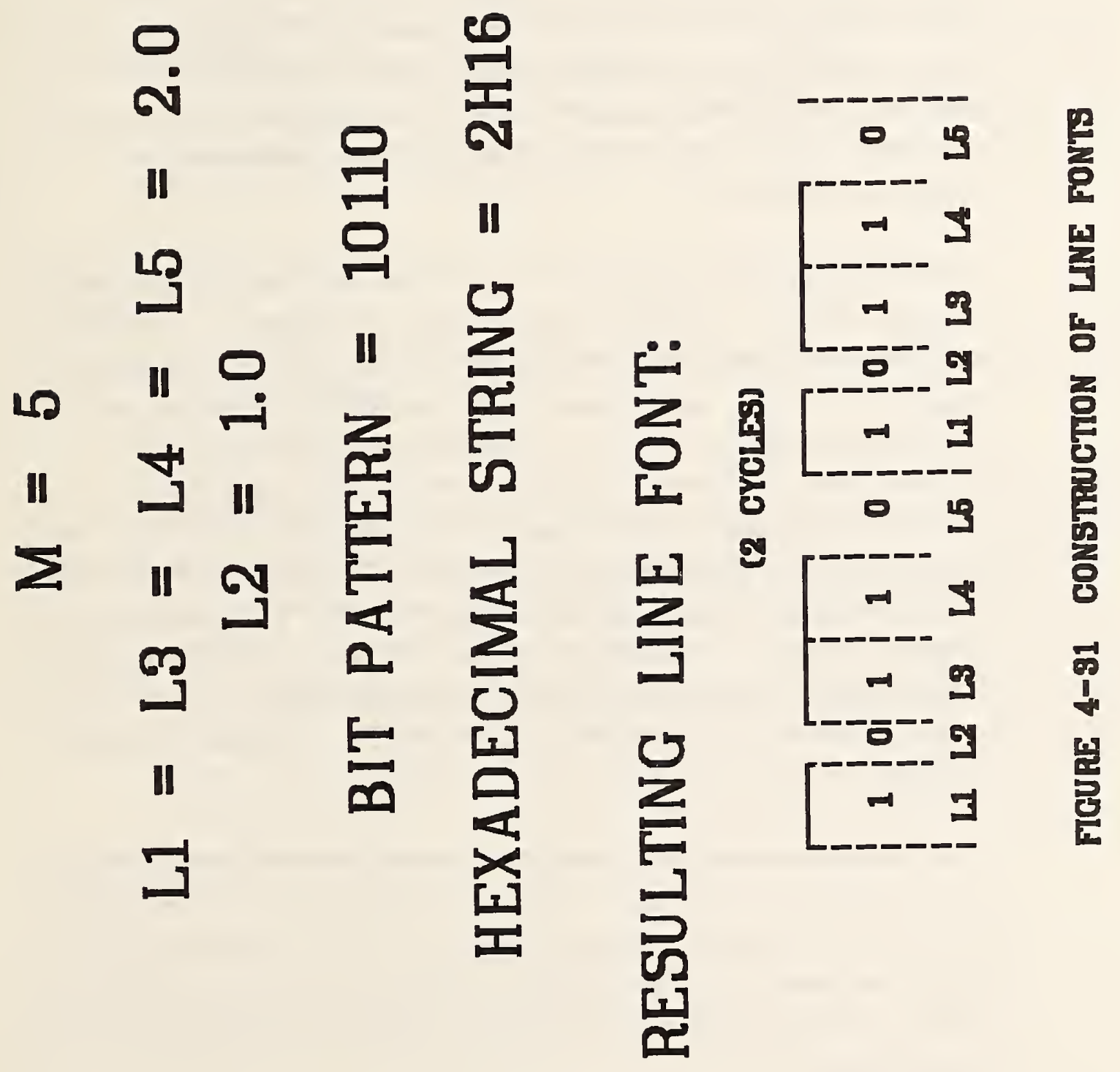




\subsubsection{MACRO Capability}

4.3.6.1 General. This Specification provides a means for communicating 3-dimensional and 2-dimensional geometric models and drawings. The Specification, however, does not provide a format for every geometric or drafting entity available on all currently used CAD/CAM systems, and is thus a common subset of such entities. To allow exchange of a larger subset of entities - a subset containing some of the entities not defined in this Specification but which can be defined in terms of the basic entities, the MACRO capability is provided. This capability allows the use of the Specification to be extended beyond the common entity subset, utilizing a formal mechanism which is a part of the Specification.

The MACRO capability provides for the definition of a "new" entity in terms of other entities. The "new" entity schema is provided in a MACRO definition which occurs once for every "new" entity in the file. Instances of these "new" entities are replaced during the MACRO processing by the constituent entities specified in the corresponding MACRO definition.

A MACRO definition is written using the MACRO definition entity. The parameter section of the entity contains the MACRO body. In the MACRO body, eleven types of language statements are usable. The statements are LET, SET, REPEAT, CONTINUE, BREAK, IF, LABEL, GOTO, MACRO, ENDM, MREF. The details of the MACRO syntax are given in 4.3.6.2. Each of the statements in a MACRO definition entity is terminated by a record delimiter.

In order to use a "new" entity defined by the MACRO definition, a MACRO instance is placed in the file. The directory entry portion of an instance specifies the new entity type number in field 1 and 11 of the directory entry record and refers to the definition by a pointer in the structure field (DE field 3). The parameters for the instance are placed in the parameter record of the instance. 
The directory entry record of a MACRO definition has a standard form.

The attributes 4 through $9,12,13,15,18$, and 19 have no significance. The default values for these attributes are taken from the directory entry record of the MACRO instance (described in 4.3.6.4).

The parameter data records of a MACRO definition consist of MACRO language statements. The statements are not in Hollerith form, i.e., they have no preceding " $\mathrm{H}$ " specification. The statements are free format and may branch over record boundaries (see 2.2.3). Every statement is terminated by a record delimiter.

\subsubsection{MACRO Syntax}

Constants. Constants may be integer, real, double precision real, pointer or string (See 2.2.2).

Variables. Variable names may be from one to six characters in length. The first character must be one of the characters listed below. This character determines the variable type. It is not possible to override the conventions. The six character limitation includes the first character. Upper and lower case letters are recognized as distinct, i.e., $X$ is different from $\mathrm{x}$. Variable names longer than six characters may be used; however, only the first six characters will be significant. Variable names may contain imbedded blanks. These blanks are NOT taken as part of the name; therefore " $A$ B" is equivalent to "AB." Except for the first character, as outlined below, all characters must be alphabetic ( $A-Z$ or $a-z)$, or numeric (0-9). 
Variable type

Integer

Real

Double precision real

String

Pointer

Label

\section{First character}

I-N, i-n

A-H, O-Z a-h, o-z

$\$$

非

\&

Examples of valid variable names are:

Integer: IJK ICOUNT K101 NTIMES max

Real: $\quad X Y Z \quad X 1 \quad$ y2 QrsTul

Double precision real:

$\begin{array}{lllll} & \text { !h } & \text { !xi } & \text { !Y2 } & \text { !12341 } \\ \text { String: } & \text { \$str } & \text { \$TITLE } & \text { \$label } & \\ \text { Pointer: } & \text { \#lline } & \text { \#note } & \text { \#REF } & \text { \#XYXI }\end{array}$

Some invalid variable names:
\$\$\$
(\$ not permitted after first character)
$1 \times 43 \mathrm{~B}$
(1 may not be first character)
A.BC
(. is illegal)

Note that there are no "reserved" words. Thus a variable name such as "MACRO", which is identical to a statement keyword (described below), will not confuse the interpreter, although it may confuse the user of such a MACRO. It is suggested that these words be avoided.

Functions. Functions similar to FOR TRAN library functions are provided. The rules for mixed mode have been relaxed so that it is not necessary to use SQRT(2. ) instead of SQRT(2). While this assists the preprocessor writer in preparing MACROs, it places a responsibility on the writer of a processor for the MACRO language in handling the mixed mode. While the arguments can be mixed mode, the functions do have a specific type of value that they return, i.e., integer, real, double precision real, or string. The functions are listed here by the type of value returned. The type of argument usually used is also noted for clarity. For example, either IDINT(!d) or INT(!d) will work 
equally well, although the meaning might be a little clearer with IDINT(!d). Functions are only recognized in upper case.

FUNCTIONS RETURNING INTEGER VALUES:

IABS(i)

Returns the absolute value of $\mathrm{i}$.

IDINT(!d)

Returns integer part of !d.

$\operatorname{IFIX}(x)$ or INT(x)

Returns the integer part of $x$.

ISIGN(i)

Returns 1 if $i$ is positive, 0 if it is zero, or -1 if it is negative.

FUNCTIONS RETURNING REAL VALUES:

$\operatorname{ABS}(\mathrm{x})$

Returns absolute value of $x$. $\operatorname{AINT}(\mathrm{x})$

Returns integer part of $x$, in real form.

$\operatorname{ALOG}(\mathrm{x})$

Returns natural logarithm of $x$.

ALOG 10(x)

Returns common (base 10) logarithm of $x$. $\operatorname{ATAN}(x)$

Returns arctangent of $\mathrm{x}$; angle returned in radians. $\cos (x)$

Returns cosine of angle $x$; angle in radians. $\operatorname{EXP}(\mathrm{x})$

Returns natural anti-logarithm of $x$ ("e to the $\left.x^{\prime \prime}\right)$.

FLOAT(i)

Returns a real (floated) value for i, e.g., FLOAT(2) returns "2." $\operatorname{SIGN}(x)$

Returns 1. if $\mathrm{x}$ is positive, 0 . if $\mathrm{x}$ is zero, and -1 . if $\mathrm{x}$ is negative. 
$\operatorname{SIN}(\mathrm{x})$

Returns sine of angle $x$; angle in radians.

SNGL(!d)

Returns single precision (real) value of double precision variable !d. As many significant digits of $! d$ as possible are used in the returned value. SQRT $(x)$

Returns square root of $x$. TAN(x)

Returns tangent of angle $\mathrm{x}$; angle in radians.

FUNCTIONS RETURNING DOUBLE PRECISION REAL VALUES:

\section{DABS(!d)}

Returns absolute value of !d.

DATAN(!d)

Returns arctangent of !d; value returned in radians.

\section{$\operatorname{DBLE}(x)$}

Returns double precision real value of $x$. Note that this is merely a conversion, not an extension. Thus, DBL(.333333333) will return $.333333333 \mathrm{D} 0$, but not $.333333333333333333333333 \mathrm{DO}$. Thus, $\operatorname{DBLE}(1 . / 3$.$) is not necessarily equal to IDO/3DO.$

$\operatorname{DCOS}(! d)$

Returns cosine of angle !d; angle in radians.

$\operatorname{DEXP}(! d)$

Returns natural anti-logarithm of !d(i.e., e to the !d).

DLOG(!d)

Returns natural logarithm of !d.

DLOGIO(!d)

Returns common (base 10) logarithm of !d. DSIGN(!d)

Returns IDO if !d is positive, ODO if zero, - IDO if negative. $\operatorname{DSIN}(! d)$

Returns sine of angle !d; angle in radians. DSQRT(!d)

Returns square root of !d. 
DTAN(!d)

Returns tangent of angle !d; angle in radians.

FUNCTIONS RETURNING STRING VALUES:

STRING (expression, format)

Returns the character representation of the argument "expression". See section 4.3.6.3.3 for a description of the argument "format".

Expressions. Expressions may be formed using the above functions, variables and constants, and the following operators:

Function

Symbol

addition

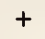

Subtraction

multiplication

division

I

exponentiation

The operators are evaluated in normal algebraic order, i.e., first exponentiation, then unary negation, then multiplication or division, then addition or subtraction. Within any one hierarchy, operators evaluate left to right. Parentheses may be used to override the normal evaluation order, as in the expression " $A *(B+C)$," which is different from " $A * B+C$." Extra parentheses do not alter the value of the expression; it is a good idea to use them, even if not truly necessary. Examples of expressions include:

$\mathrm{X}+1.0$

$-B+\operatorname{SQRT}(B * 2-4 * A * C)$

$\mathrm{I}+1$

$3.14159 / 2$.

$-X$

!DEL * (!ALPHA-!BET A) 
Except for the ** operator, it is never permissable to have two operators next to each other, i.e., not $2 *-2$, but $-2 * 2$ or $2^{*}(-2)$. Multiplication may not be implied by parentheses, e.g., $(A+B)(C+D)$ is invalid, and $A B$ does not imply $A * B$, but rather the separate variable $A B$.

Mode of expression evaluation. Mixed mode (integer mixed with real, etc.) is permitted. Whenever two different types are to be operated upon, the calculation is performed in the "higher" type. Integer is the lowest type, real is next, and double precision real is the highest. Note, however, that this decision is made for each operation, not once for the entire expression. Thus $1 / 3+1.0$ evaluates to 1.0 , because the $1 / 3 "$ is done first, and it is done in integer mode. Integer mode truncates fractions, and does not round. Therefore, the expression " $2 / 3+2 / 3+2 / 3 "$ has a value of zero.

\section{Conditional expressions}

Conditional expressions may be formed using functions, variables and constants and the following six standard relational operators:

Function

$\begin{array}{lll}\text { less than or equal } & (\leq) & \text {.LE. } \\ \text { less than } & (<) & \text {.LT. } \\ \text { equal } & (=) & \text {.EQ. } \\ \text { greater than or equal } & (\geq) & \text {.GE. } \\ \text { greater than } & (>) & \text {.GT. } \\ \text { not equal } & (\neq) & \text {.NE. }\end{array}$

Examples of conditional expressions include:

X.GT.3

SQRT $(A+B) \cdot N E \cdot I+1$

(!A-!B).GE.3.14159

\section{Symbol}

LE.

LT.

EQ.

GE

NE. 
4.3.6.3 MACRO Definition Entity. The MACRO definition entity specifies the action of a specific MACRO. After having been specified in a definition entity, the MACRO can be used as often as necessary by means of the MACRO instance entity.

The MACRO definition entity differs from other entity structures in this Specification by consisting of only language statements in the parameter data. The character strings constituting the language statements in the MACRO definition are not set off by means of the $\mathrm{nH}$ structure of string constants but rather consist of only the actual character string terminated by a record delimiter (see 2.2.2.5).

4.3.6.3.1 Directory Data

ENTITY TYPE NUMBER: 306

\subsection{Parameter Data}

\begin{tabular}{|c|c|c|c|}
\hline Index & Name & Type & Description \\
\hline 1 & ID & Literal & MACRO \\
\hline 2 & NE & Integer & Entity Type ID \\
\hline 3 & TEXT & Language statement & First statement \\
\hline 4 & TEXT & Language statement & Second Statement \\
\hline - & • & • & $\cdot$ \\
\hline • & $\cdot$ & • & - \\
\hline - & - & - & - \\
\hline $\mathrm{N}+2$ & TEXT & Language statement & Last statement \\
\hline$N+3$ & $\mathrm{~T}$ & Literal & ENDM \\
\hline
\end{tabular}

Additional parameters as required (see 2.2.4.4.2). 
4.3.6.3.3 There are eleven language statements that can be used. They are:

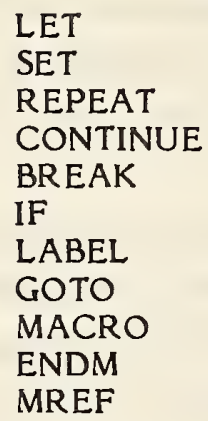

These "keywords" are recognized only in upper case, and every statement must begin with one of these keywords. Statements are free format; blanks and tabs are ignored except within strings. Statements may extend over several lines, or more than one statement may be present on a line. All statements are terminated by a record delimiter which must be present.

\section{$\underline{\text { LET statement (Arithmetic) }}$}

This is the assignment statement and is equivalent to the LET statement of BASIC. The format of a LET statement is:

\section{LET variable = expression;}

The expression and the variable may be integer, real, or double precision; they need not be of the same type. Note that this is an assignment statement and not an algebraic equality. All of the variables on the right hand side of the expression must have been previously defined; it cannot be assumed that variables will default to zero if they are undefined. Some examples of valid LET statements:

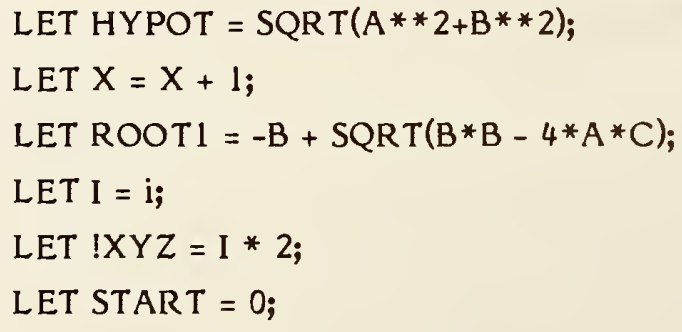




\section{LET Statement (String)}

String variables allow characters to be manipulated. String variables may be used in statements almost anywhere that any other variable type may be used; exceptions are noted below.

String variables may be used in LET statements. Note that they shall not be mixed with any other type of variable in a LET statement. Also note that arithmetic operations (i.e., $+,-, *, l^{*} *$ ) are not possible with string variables. Two forms of LET statements for string variables are possible:

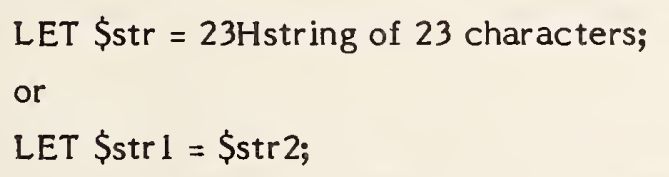

In the first case, the 23 characters following the $\mathrm{H}$ are assigned to the string variable \$str. In the second case, the string "\$str2" is copied into "\$str1." Examples of these statements include:

$$
\begin{aligned}
& \text { LET \$title = 3HBox; } \\
& \text { LET \$subti = 6HBottom; } \\
& \text { LET \$x = \$subti; }
\end{aligned}
$$

Note that if a string variable appears on the right hand side of the statement, it must have been previously defined. Spaces are not ignored within a string constant; they become part of the string. Any printable ASCII character may be part of a string.

There is one other form for setting up a string. It involves the STRING function. The STRING function may only appear in this form. Specifically, it shall not appear in SET statement argument lists, MACRO statements, or MREF statements. However, string constants, such as "6Hstring," and variables, such as "\$x", may appear in SET statements and MACRO statements. 
The form of a LET statement including string function is:

\section{LET \$str = STRING(expression, format);}

where "expression" is any normal integer, real or double precision real expression, "\$str" is a string variable name, and "format" is a format specification as used in a FORTRAN FORMAT statement. The allowable format specifications are:
Iw
Fw.d
Ew.d
Dw.d

The effect of this statement is to convert the numeric value of the expression into characters, e.g., the statement:

$$
\begin{aligned}
& \text { LET \$PI = STRING(3.14159,F7.5); } \\
& \text { will result in the same thing as } \\
& \text { LET \$PI = 7H3.14159; }
\end{aligned}
$$

Of course, the usefulness of the STRING function is that expressions can be converted, rather than constants. Thus:

$$
\begin{aligned}
& \text { LET } x=1 ; \\
& \text { LET } y=2 \text {; } \\
& \text { LET \$xyz = STRING }(x+y+1, F 5.0) \text {; } \\
& \text { will result in the same thing as } \\
& \text { LET \$xyz = 5H 4.; }
\end{aligned}
$$

The rules for the format specifications follow the standard FORTRAN convention. "Iw" causes integer conversion, resulting in " $w$ " characters. "Fw.d" causes real conversion, resulting in " $w$ " characters, with " $d$ " characters after the decimal point. "Ew. $d$ " results in real conversion, but using an exponent form. "Dw.d" is the same as "E" but for double precision real values. Note that this is one place where mixed mode is not allowed. The type of format specification and the type of the expression's result must be identical. 


\title{
LET statements (Attributes)
}

Attributes (those appearing in the directory entry record for the MACRO instance) may be set using the LET statement. The format is:

\author{
LET / at tribute name = expression; \\ or \\ LET / at tribute name = / HDR;
}

The first form allows an attribute to be set to any constant value, including numeric expressions. Attributes may also be set to string constants or string variables, but not to the result of a STRING function.

\section{Examples:}

$$
\begin{aligned}
& \text { LET / LEV = 1; } \\
& \text { LET / VIEW = 3; } \\
& \text { LET / LABEL = 6HBottom; } \\
& \text { LET / LABEL = \$X; }
\end{aligned}
$$

The second form allows restoring an attribute to its default value.

Examples:

$$
\begin{aligned}
& \text { LET / LEV = /HDR; } \\
& \text { LET / LABEL = /HDR; }
\end{aligned}
$$

The word "/HDR" is the only non-constant that is allowed on the right side of an attribute assignment statement. The effect is to restore the value of the attribute to what it was in the directory entry for the instance or, in some cases, to a specified default value. The defaults are described below.

Attributes may not be mixed with any other variable type nor may they appear anywhere but in the above two forms of LET statements. 
The allowable attribute names and their defaults are given here. A default of /HDR indicates that the attribute defaults to the value in the directory entry of the instance.

Attribute

Line font pattern

Level

View

Transformation matrix

Label display associativity

Blank status

Subordinate entity

Entity use

Hierarchy

Line weight

Color

Form

Entity label

Entity subscript
Name

/LFP

/LEV

/VIEW

/MTX

/CE

/BS

/SE

/ET

/HF

/LW

/PN

/FORM

/LABEL

ISUB
Default

/HDR

/HDR

/HDR

/HDR

0

/HDR

/HDR

/HDR

/HDR

/HDR

/HDR

0

blanks

0

\section{SET statement}

The SET statement establishes directory and parameter data entries for the specified entity. The form is:

SET 非ptr = entity type number, argument list;

"非tr" is a pointer variable, such as "\#XYZ"; "entity type number" is an IGES entity type number, such as "110"; and "argument list" is a group of variables which is the parameter data of the entity.

Examples of this type of SET statement:

SET \#LINE = 110,X1,Y1,Z,X2,Y2,Z,0,0;

SET \# $A B C=828, Z, A+B / C, Y 1, X 2, Y 2+1,0,0$;

SET 非we $=864,15 \mathrm{Hstrings}$ allowed, $\mathrm{X}, \mathrm{Y}, \$$ this 2 ; 
The argument list may contain expressions and may spread over more than one line. At least one argument must be present, i.e., the argument list may not be null. The entity type number may not be an expression; i.e., it must be an integer constant. The pointer variable will be assigned a value corresponding to the sequence number of the directory entry of the entity created.

"Forward referencing" of pointers is valid in the argument list of a SET statement. A pointer may appear in the argument list of a SET statement that comes before the SET statement defining the pointer. The only restriction is that any pointer so referenced must appear on the left hand side of one SET statement.

Pointers which appear on the left hand side of more than one SET statement or those which are located inside of REPEAT loops should not be forward referenced.

Note that the STRING function is not allowable in a SET statement -- use a separate LET statement with a string variable instead.

\section{REPEAT statement}

The REPEAT statement causes a group of statements terminated by a CONTINUE statement to be repeated a specified number of times. The form of a REPEAT statement is:

REPEAT expression; 
The expression is evaluated, and the resulting value is the number of times the statements will be repeated. The expression may be of integer, real or double precision real type; in the case of real or double precision real expressions, the result is truncated to determine the repeat count. If the repeat count is zero or negative, the group of statements is still executed one time.

Examples of REPEAT statements:

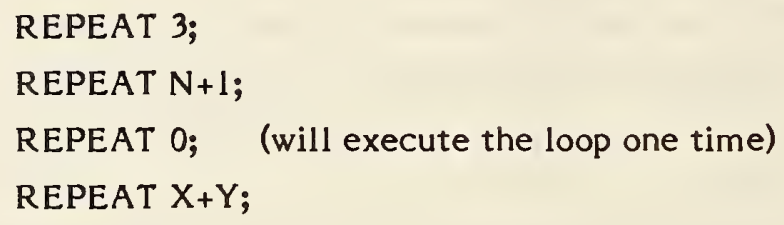

REPEAT statements may be nested only to a depth of ten.

After a REPEAT statement, such as REPEAT $N$, it is valid to alter the value of $N$. This does not affect the repeat count. Also note that REPEAT is unlike a FORTRAN DO statement because there is no variable being incremented on every pass.

\section{CONTINUE statement}

The CONTINUE statement marks the end of a REPEAT group. The form of a CONTINUE statement is:

\section{CONTINUE;}

When a CONTINUE statement is encountered, the repeat count is decremented by one and checked to see if it is greater than zero. If it is, the interpreter goes back to the first statement after the most recent REPEAT. If not, then the next statement is processed. The number of REPEAT statements and CONTINUE statements in a MACRO shall be the same. A CONTINUE statement(s) is not implied by ENDM. 


\section{$\underline{\text { BREAK statement }}$}

A BREAK statement may be used within a REPEAT construct to terminate the processing of statements of the REPEAT construct before the completion of the specified number of loops, such as upon detection of a condition during the processing. The form of a BREAK statement is:

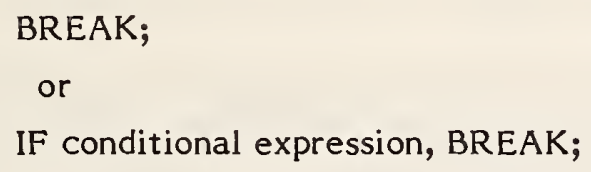

When a BREAK statement is encountered, processing of MACRO statements resumes with the statement immediately following the CONTINUE statement marking the end of the affected REPEAT construct.

\section{$\underline{\text { IF statement }}$}

The IF statement causes a single language statement to be executed if a cer tain condition is true.

The form of an IF statement is:

IF conditional expression, language statement;

where "conditional expression" is a conditional expression as described in section 4.3.6.2. "language statement" can be any statement allowed in a MACRO except:

\section{MACRO \\ ENDM \\ IF \\ LABEL}

Examples of IF statements:

IF A.LT.3,LET $A=3$;

IF B.EQ.0,SET \#LIN1=110,...;

IF SWITCH.EQ.1, GOTO \&A; 


\section{$\underline{\text { LABEL statement }}$}

The LABEL statement is used to mark a position in a MACRO where the execution control is transferred to using a GOTO statement.

The form of a LABEL statement is:

LABEL label name;

where "label name" is any character string beginning with an ampersand (\&). It should be from one to six characters long (including the $\&$ ). Within a single MACRO definition, all label names must be unique.

Examples:

\section{LABEL \&loop; \\ LABEL \&end;}

\section{GOTO statement}

This statement is used to transfer the execution control to a particular point which is marked by a LABEL statement.

The form of a GOTO statement is:

\section{GOTO label name;}

where 'label name' is any label name specified in a LABEL statement.

The GOTO statement can be used to jump both forward and backward, but both the GOTO statement and the target LABEL must be at the same nesting level and within the same REPEAT construct.

\section{Examples:}

\section{GOTO \&start; \\ GOTO \&end;}




\section{MACRO statement}

The MACRO statement is used to signify the start of a MACRO definition. The first statement in every MACRO definition must be a MACRO statement.

The form of a MACRO statement is:

306,MACRO, entity type number, argument list;

where "entity type number" is the assigned entity number of the MACRO, and "argument list" is a list of parameters that are to be assigned values at execution time. Entity type numbers in the range of 600 to 699 and 10000 to 99999 will be used.

The argument list may not be null. The parameters in the argument list take the form of the variables described in Section 4.3.6.2. Note that the argument list may not contain expressions, only symbolic variable names.

One additional type of variable, the "class variable" can be used in an argument list. The class variable takes the form:

$$
\text { size_variable (class_var_1, class_var_2, ..., class_var_n) }
$$

where size_variable precedes the occurrence of the class variable in the argument list and class_var_i members are referenced in the MACRO body by means of a subscript:

$$
\text { class_var_i ( J) }
$$


In the MACRO statement, the size_variable is used to identify the class variable collection being defined. In the MACRO body, the size_variable indicates the number of sets of class variable members that are included (i.e. the number of times the class variable collection is to be repeated).

A simple class variable example is:

\section{N(ITEM1, ITEM2, ITEM3, ITEM4)}

which specifies a class variable collection with four members. For an instance of the MACRO, using the example class variable with $\mathrm{N}$ equal to 3, three sets of class variable data for the collection are available to the macro body statements. The parameter list for the associated MACRO instance is:

$\operatorname{ITEM}(1), \operatorname{ITEM} 2(1), \operatorname{ITEM} 3(1), \operatorname{ITEM} 4(1), \ldots, \operatorname{ITEM} 3(3), \operatorname{ITEM} 4(3)$

Each value for each member of the class variable may be referenced individually:

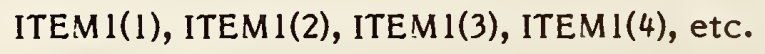

in any order, or implicitly, by using an index variable, e.g., J:

$\operatorname{ITEM} 3(\mathrm{~J})$ with $\mathrm{J}$ ranging from 1 to 3

Use of the class variable to represent a MACRO with a parameter list identical to the Views Visible Associativity, Form 4, would be specified as:

$$
\begin{aligned}
& \text { 306,MACRO,681,N1,N2,N1(非DEV,LF, 非DEF,IPN,LW), } \\
& \text { N2(非DE),N,N(\#DEA),M,M(非DEP); }
\end{aligned}
$$

where NI(\#DEV,LF,\#DEF,ICN,LW) indicates the blocks of views visible, line font, color number, and line weight information;

$\mathrm{N} 2($ \#DE) contains the pointers to the entities included in the view;

$\mathrm{N}($ \#DEA) contains the back pointers/text pointers; and

$M(\# D E P)$ contains the pointers to properties. 
Note that zero is a valid value for a size_variable in a MACRO instance.

\section{ENDM statement}

ENDM signifies the end of a MACRO. The form of an ENDM statement is:

\section{ENDM;}

All MACROs must have an ENDM statement as their last statement. ENDM is not implied by the end of the parameter data section.

\section{MREF statement}

The MREF statement is used to reference another MACRO from inside a MACRO definition. The form of an MREF statement is:

MREF, ptr, entity type number, argument list;

where "ptr" may be either a pointer variable or an integer expression. The value is the sequence number of the directory entry record of the MACRO definition being referenced. "Entity type number" is the assigned entity number of the MACRO being referenced. "Argument list" is exactly like that of a SET statement. The effect of the argument list is to replace the symbolic names found in the MACRO definition with the values of the expressions contained in the MREF statement, so that execution of the referenced MACRO will start with the appropriate values. Note that MREF does not start expansion of the referenced MACRO. Rather it creates an entity entry which may later be expanded. It is thus not possible for a MACRO being referenced to have access to any of those values except for those in the argument list. (All variables not in the argument list are treated as local variables.) Even then, it is not possible for the occurrence of a MREF statement to alter any of those values. 
Examples of MREF statements:

MREF, 非ac $1,600, X 1, Y 1, Z 1, X 2, Y 2,3.1$;

MREF,33,621,A,B,3+X/W+1,6*W,3.,0,6Hstring,\$x;

It is not strictly necessary for the values in a MREF statement to be of the same type as the values in the definition MACRO, within certain limitations. Integer, real, and double precision real values may be freely mixed, although it might be considered a good idea not to do so. String values may only appear where string variables appear in the definition.

4.3.6.4 MACRO Instance Entity. A MACRO instance entity is used to invoke a MACRO. The parameter data records of the instance contain values for the arguments to the MACRO. This is similar to a standard entity entry.

The directory entry for a MACRO instance entity contains the attribute values that are to be used as the default values during the expansion of the MACRO. The only special field is the structure field (directory entry field 3), which contains a pointer to the directory entry of the MACRO definition. The pointer is preceded by a minus sign.

\subsection{Directory Data}

\section{ENTITY TYPE NUMBER:}

Structure field:

Other attributes:
As defined for each MACRO in the range 600 to 699 or 10000 to 99999 .

Pointer to directory entry of MACRO definition, preceded by a minus sign.

Default values to be used during expansion of the MACRO. Attributes listed as defaulting to /HDR obtain their values from here. (See discussion of LET statement (attributes) in Section 4.3 .6 .3 .3$. 


\subsection{Parameter Data}

The parameter data section for an instance has the following form:

(Note that, as with all parameter data entities, the first record begins with the entity type number as defined in the MACRO.)

$\underline{\text { Index }}$ $1, \ldots \mathrm{K}$
Name

As appropriate for the particular MACRO
Type

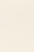

Description

The values for the arguments to the MACRO. They must agree in format and number with the arguments in the MACRO statement of the definition.

Additional pointers as required (See 2.2.4.4.2)

\subsubsection{Examples of MACRO usage.}

Five examples are given to illustrate some of the capabilities of a MACRO.

1. Isosceles Triangle

2. Repeated Parallelograms

3. Concentric Circles

4. Ground Symbol

5. Useful Features

\section{Example 1:}

The following MACRO definition creates an isosceles triangle, given a vertex point, a base width, a height and a scale.

Directory Data

ENTITY TYPE NUMBER: 306 


\section{Parameter Data}

306, MACRO, 621, X1, Y1, A1, A2, K;

LET $Z=0$;

SET 非Line $=110, \mathrm{X} 1, \mathrm{Y} 1, \mathrm{Z}, \mathrm{X} 1+(\mathrm{K} * \mathrm{~A} 1), \mathrm{Y} 1+(\mathrm{K} * \mathrm{~A} 2 / 2),. \mathrm{Z}, 0,0$;

SET 非Line2 $=110, X 1+(K * A 1), Y 1+(K * A 2 / 2),$.$Z ,$ $X 1+(K * A 1), Y 1-(K * A 2 / 2), Z, 0,$.0 ;

SET 非Line3 $=110, X 1+(K * A 1), Y 1-(K * A 2 / 2),$. $\mathrm{X} 1, \mathrm{Y} 1, \mathrm{Z}, 0,0$;

ENDM;

The MACRO can be used to create a triangle by using a MACRO instance which supplies the needed values for $X 1, Y 1, A 1, A 2$, and $K$. The parameter data section for the MACRO instance would have the following format:

$\begin{array}{ccll}\text { Index } & \text { Name } & \text { Type } & \underline{\text { Description }} \\ 1 & \mathrm{X} & \text { Real } & \text { X coordinate of vertex } \\ 2 & \mathrm{Y} & \text { Real } & \text { Y coordinate of vertex } \\ 3 & \text { A1 } & \text { Real } & \text { Height of triangle } \\ 4 & \text { A2 } & \text { Real } & \text { Base of triangle } \\ 5 & \text { K } & \text { Integer } & \text { Scaling factor }\end{array}$


In particular, to create a triangle shown in Figure 4-32 with a base of 5. and a height of 17 ., a vertex at $(0,0)$, and a scale factor 1 , the following instance could be placed into the file:

\section{Directory Data:}

\section{ENTITY TYPE NUMBER: 621}

Structure: -nnn, where "nnn" is the sequence number of the directory entry of the definition.

Other attributes: As desired for default values during MACRO expansion.

Parameter data:

$$
621,0 ., 0 ., 17 ., 5 ., 1 \text {; }
$$

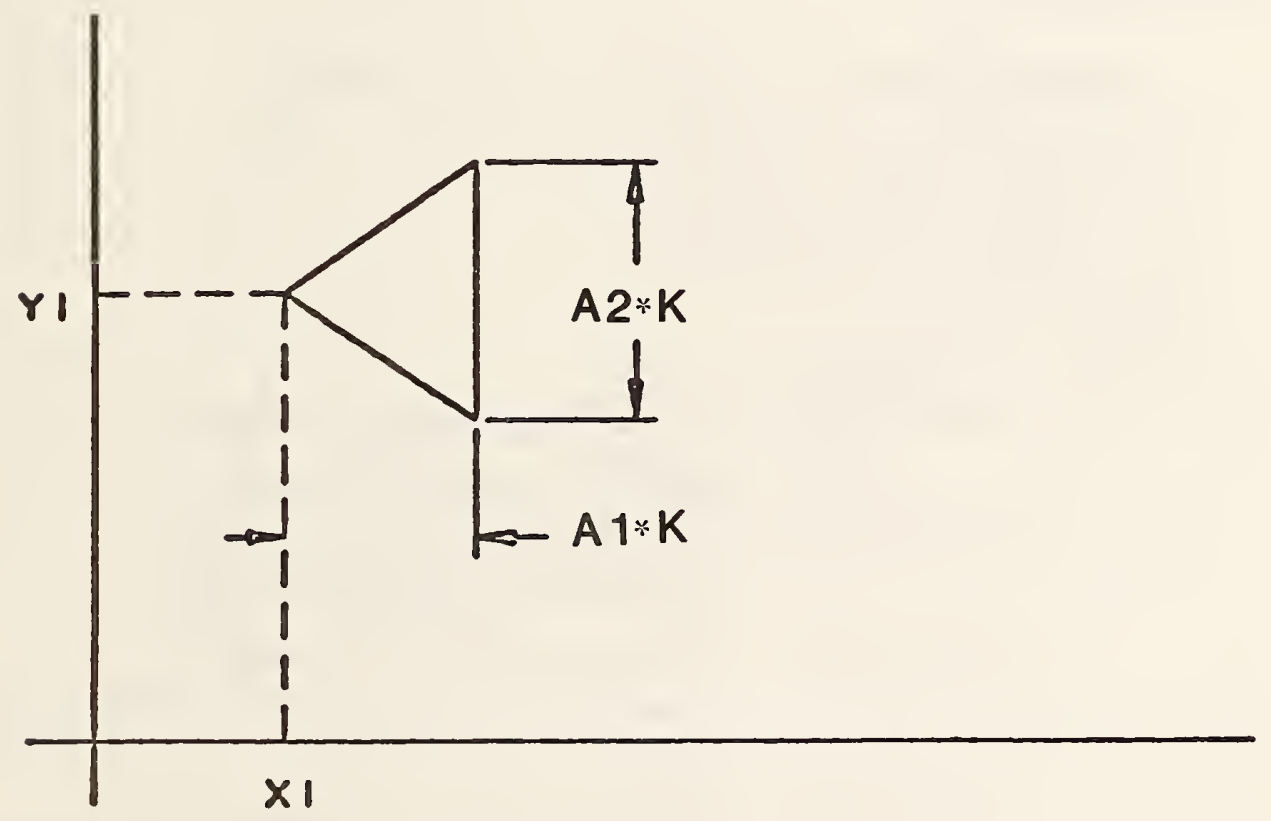

FIGURE 4-32 EXAMPLE OF TRIANGLE MACRO 
Example 2: Repeated parallelograms

The following MACRO takes the coordinates of three points and a repetition number as arguments and creates a pattern of repeated parallelograms as shown in Figure 433. The three points represent the vertices of the initial parallelogram. The repetition number argument (NTANG) controls how many additional parallelograms will be drawn offset in the positive $X$ and $Y$ direction from the initial one. For simplicity, the points have been constrained to all lie in a plane parallel to the $X-Y$ plane.

Directory Data

ENTITY TYPE NUMBER: 306

\section{Parameter Data}

306,MACRO, 600, X1, Y 1, X2, Y2, X3, Y3, Z, NTANG;

IF NTANG.EQ.0, GOTO \&END;

LET XDEL $=(X 2-X 1) / N T A N G$;

LET YDEL $=(Y 3-Y 1) / N T A N G$;

LET $K=0$;

REPEAT NTANG +1;

SET \#VLINE $=\quad 110, X 1+K * X D E L, Y 1+K * Y D E L, Z$,

$X 2+K * X D E L, Y 2+K * Y D E L, Z, 0,0$;

SET 非HLINE $=110, X 1+K * X D E L, Y 1+K * Y D E L, Z$,

$X 3+K * X D E L, Y 3+K * Y D E L, Z, 0,0$;

SET \#VLINE $=110, X 3+K * X D E L, Y 3+K * Y D E L, Z$,

$X 3+(X 2-X 1)+K * X D E L, Y 2+(Y 3-Y 1)+K * Y D E L$,

$\mathrm{Z}, 0,0$;

SET 非HLINE $=110, X 2+K * X D E L, Y 2+K * Y D E L, Z$, $X 3+(X 2-X 1)+K * X D E L, Y 2+(Y 3-Y 1)+K * Y D E L$, Z, 0,0;

LET $K=K+1$;

CONTINUE;

LABEL \&END;

ENDM; 
Parameter Data for an instance of this MACRO looks like this: $600,1 ., 1 ., 2 .$, 5., 5., 2., 1., 3;

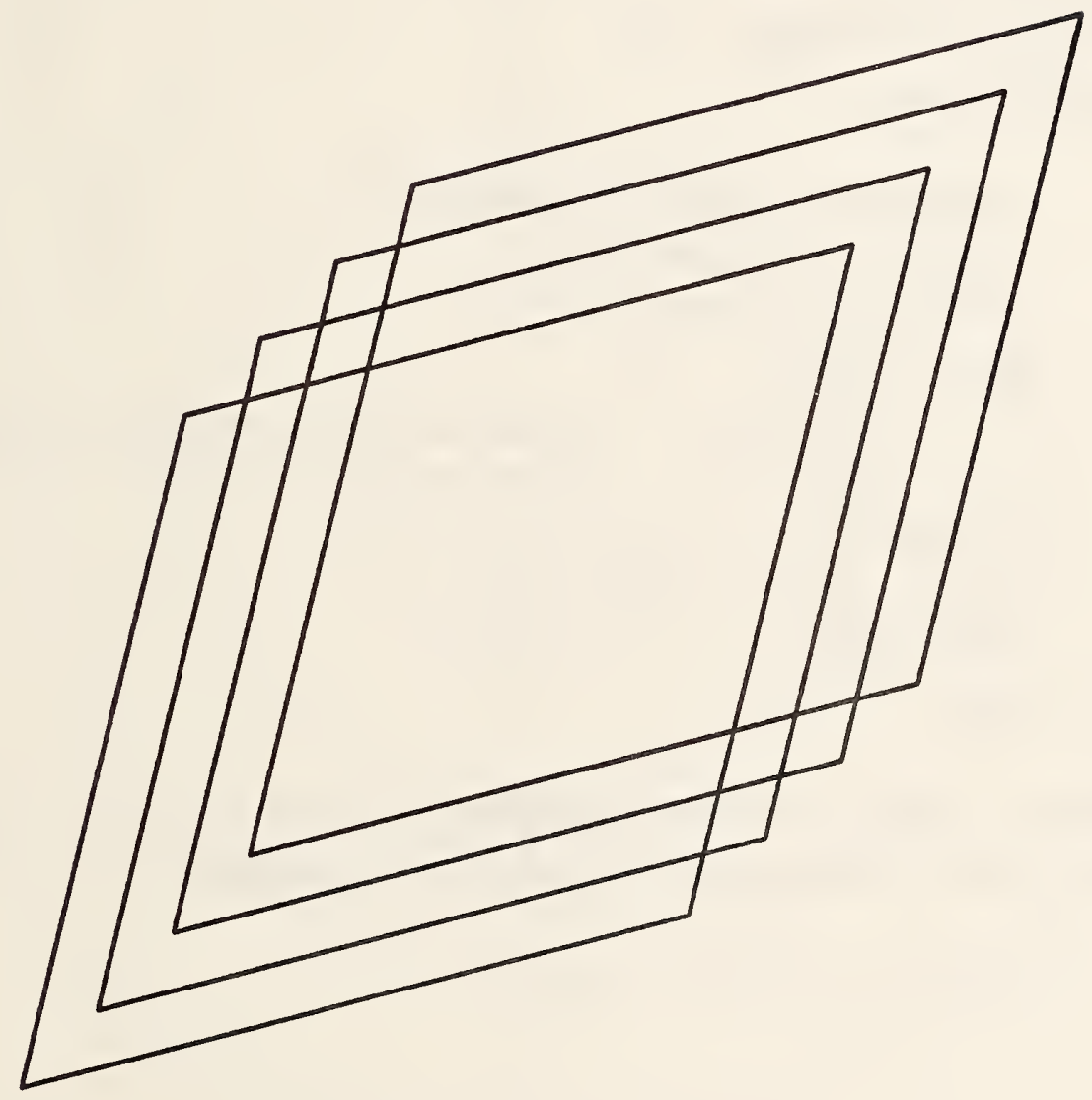

FIGURE 4-33 REPEATED PARALLELOGRAMS 
Example 3: Concentric circles

The following MACRO, given a coordinate, a radius, and a number, creates concentric circles out to the radius. A point is put into the center. Figure 4-34 shows the result.

Directory Data

ENTITY TYPE NUMBER: 306

Parameter Data

306,MACRO,601,XC,YC,ZC,R,NCIRC;

IF NCIRC .EQ. 0, GOTO \&END;

LET DELTR = R/NCIRC;

REPEAT NCIRC;

SET 非CIR $=\quad 100, Z C, X C, Y C, X C, Y C+R, X C, Y C+R, 0,0$;

LET R = $\quad R-D E L T R ;$

CONTINUE;

SET 非T $=116, X C, Y C, Z C, 0,0,0$;

LABEL \&END;

ENDM;

Parameter Data for an instance of the MACRO which would create four concentric circles around the origin out to a radius of 20 looks like this:

$601,0 ., 0 ., 0 ., 20 ., 4$; 


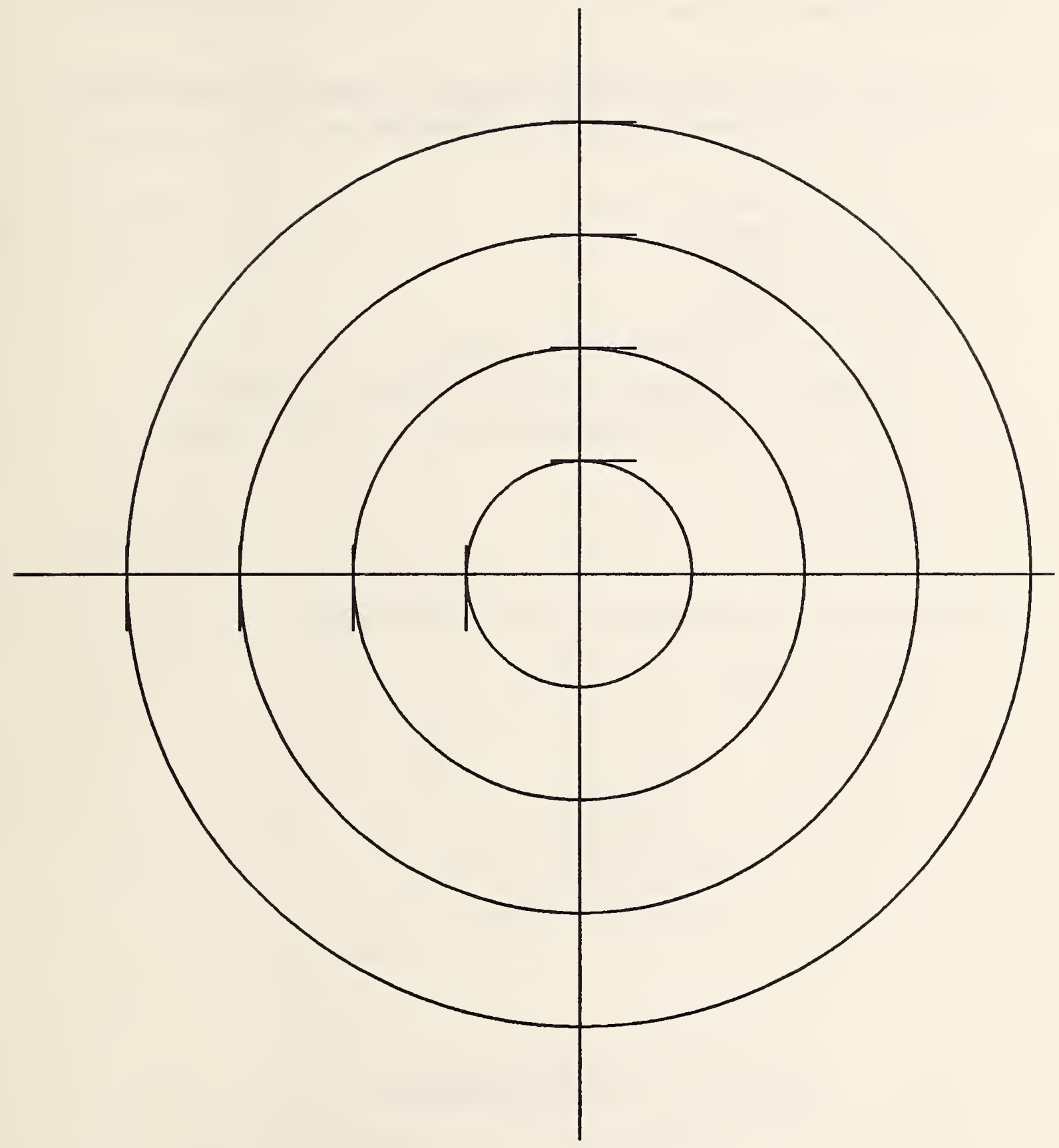

FIGURE 4-34 CONCENTRIC CIRCLES 
Example 4: Electrical ground symbol

This MACRO takes a point and a base length and constructs a ground symbol (horizontally) at that point. Figure 4-35 shows the result.

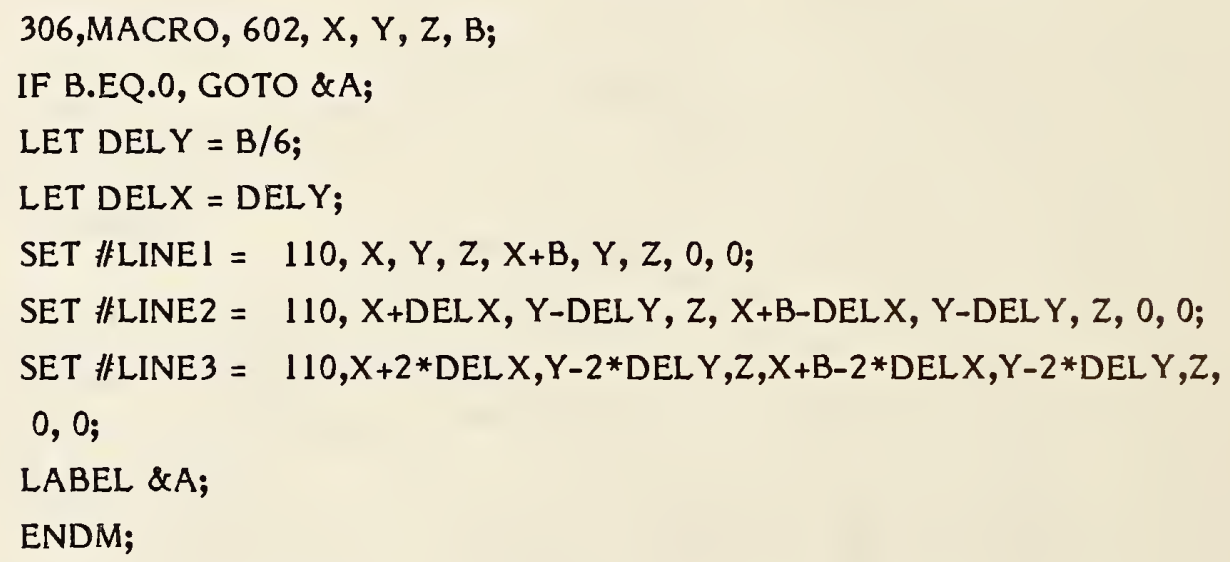

Parameter Data for an instance of this MACRO looks like this:

602, 1., 6., 2., 1.3;

FIGURE 4-35 GROUND SYMBOL 
Example 5: Useful features

This last example demonstrates the use of various MACRO features. It is not meant as an example of a "useful" MACRO.

\section{Directory Data}

ENTITY TYPE NUMBER: 306

\section{Parameter Data}

306,MACRO,613,NROW,NCOL,VDIST,HDIST,!ANGLE;

LET/LABEL = 6HPOINTS;

LET !SIN=DSIN(!ANGLE); LET !COS=DCOS(!ANGLE);

LET YHD=HDIST *!SIN;

LET $X H D=H D I S T *$ !COS;

LET YVD=VDIST * !COS;

LET XVD=VDIST * $(-!$ SIN $)$;

LET IRC=0; LET ICC $=0$;

REPEAT NROW;

LET $X C O L=I R C * X V D$;

LET YCOL=IRC*YVD;

REPEAT NCOL;

LET $X=X C O L+I C C * X H D ;$

LET Y = YCOL + ICC*YHD;

SET 非PT $=116, \mathrm{X}, \mathrm{Y}, 0 ., 0,0,0$;

LET ICC = ICC + 1;

CONTINUE;

LET IRC = ICC + 1;

CONTINUE;

LET \$NPTS = STRING(NROW *NCOL,I7);

LET $/$ LABEL = \$NPTS;

SET 非INE = 110, 0., 0., 0., 10., 0., 0.;

SET 非CIRC = 100, 0., 0., 0., 10., 0., 10., 0.;

MREF, 22, 601, 0., 0., 0., 10., 5;

ENDM;

Parameter Data for an instance of this MACRO looks like this:

$613,4,5,0.2,0.1,7.85398 \mathrm{D}-01$; 
4.3.7 Property Entity.

The property entity contains numerical or textual data. It also has a form number to indicate its meaning. Certain generic Property form numbers are described in the following sections and are expected to be augmented by others in future versions of this Specification. Form numbers in the range $5001-9999$ are left undefined for users.

Note that properties can also point to other properties, as well as participate in associativities, point to related general notes, or display text by pointing to a Text Display Template.

Property instances are usually referenced by the presence of a pointer to the instance in the second group of additional pointers as described in section 2.2.4.4.2; however, as stated in section 1.5.1, when an instance is independent it applies to all entities on the same leel as the instance.

\subsubsection{Directory Data}

ENTITY TYPE NUMBER : 406

\subsubsection{Parameter Data}

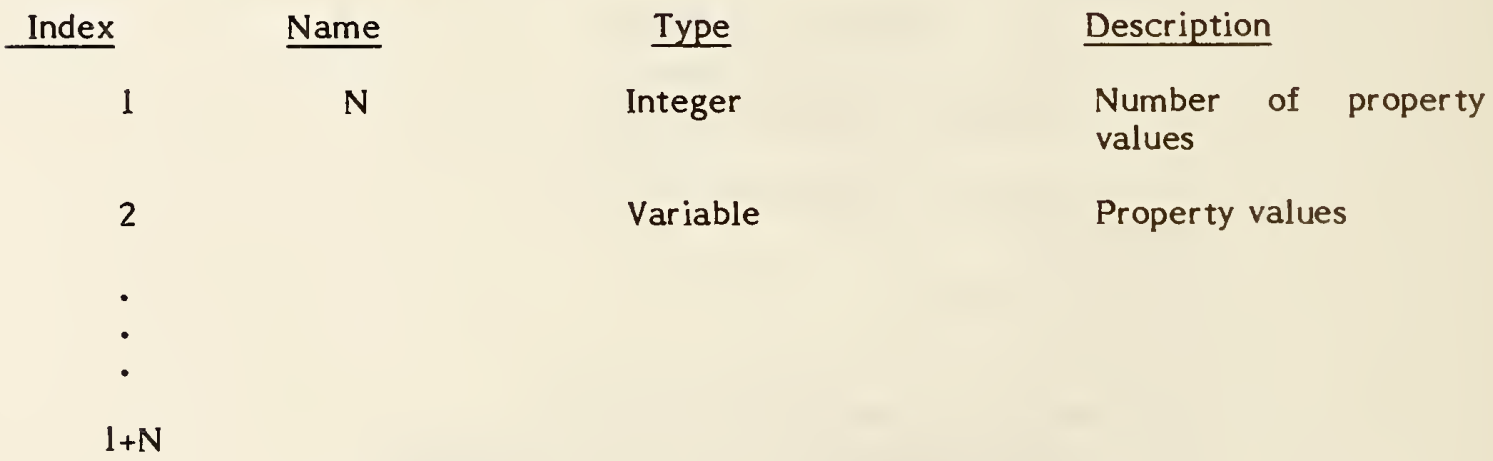

Additional Pointers as required (see 2.2.4.4.2). 


\subsubsection{Defined Properties}

\subsection{FORM NUMBER: 1 Definition levels}

\section{DESCRIPTION}

For one or more entities in the file that are defined on a set of multiple levels, there will be an occurrence of the property instance (Form 1). In the parameter data portion of the property instance, the first parameter, NI, will contain the number of multiple levels followed by a list of those levels. Each entity that is defined on this set of levels will contain a pointer (in the level field of the directory entry) to this property instance. A different set of multiple levels will result in a different property instance.

$\begin{array}{cccl}\text { Index } & \text { Name } & \text { Type } & \text { Description } \\ 1 & \text { N1 } & \text { Integer } & \text { Number of property values } \\ 2 & \text { L } & \text { Integer } & \text { Level number } \\ \text { - } & \text { - } & - & \\ \text { - } & \text { - } & - & \\ \text { - } & \text { - } & - & \end{array}$

Additional Pointers as required (see 2.2.4.4.2) 


\section{DESCRIPTION}

This property allows entities that can define regions to set an applications restriction over that region. The restrictions will indicate whether a given applications item must lie completely within regions with this property or completely outside such regions. The restriction applies to all points of entities used to represent the applications item and to all points within the effect of the item when all properties, such as line widening, are applied.

Each of the property values in this property will have one of three values indicating the region restriction relevant to the application's item.

Property Value
0
1
2

$\begin{array}{cll}\text { Index } & \text { Name } & \text { Type } \\ 1 & \mathrm{NP} & \text { Integer } \\ 2 & \text { EVR } & \text { Integer } \\ 3 & \text { ECPR } & \text { Integer } \\ 4 & \text { ECRR } & \text { Integer }\end{array}$

\author{
Description \\ No Restriction \\ Item must be inside region \\ Item must be outside region
}

Additional Pointers as required (see 2.2.4.4.2) 


\section{DESCRIPTION}

This property is used to transfer the meaning or intended use of a level in the sending system. An instance of this property shall apply to all entities in the same file with the same DE level value (field 5), without the requirement of a pointer to it (see section 1.5.1). Parameter 2 is used to record an integer code number when the sending system uses a level-use index or table. Parameter 3 is used to record the level-use text, whether such text is obtained from the index which provided parameter 2, or exists independently. Either parameter 2 or parameter 3 may have a default value. This property may be readily added to a file (by edit or data merge) when level-use information is required by the receiving system or archive. The parameter (2 and 3 ) values of an instance of this property shall apply to multiple levels if the instance's level value is a pointer to an instance of Property Form 1. Note that parameter 3 was an integer value for "source level" in Version 2. The source level (for this Version) shall be the level value for the instance of this property.

\begin{tabular}{cccc}
\hline Index & Name & Type & Description \\
1 & NP & Integer & $\begin{array}{c}\text { Number of property values } \\
(\mathrm{NP}=2)\end{array}$ \\
2 & FC & Integer & $\begin{array}{c}\text { Function description code } \\
\text { (Default }=0)\end{array}$ \\
3 & FD & String & $\begin{array}{c}\text { Function Description } \\
\text { (Default }=\text { null string) }\end{array}$
\end{tabular}

Additional Pointers as required (see 2.2.4.4.2) 


\subsection{FORM NUMBER: $4 \quad$ Region Fill Property}

\section{DESCRIPTION}

This property helps define the functional value of any closed region. It classifies the region as to its "filled" status. It will be used most of ten to identify which region-defining entities are defining a functional region (or a gap in that region) and which have other purposes. The actual function of the region will likely be determined in conjunction with level or subfigure membership.

\section{$\underline{\text { Index }}$}

1

2

FC

Name

NP

3
Integer

Type

Integer
O Pointer

\section{Description}

Number of property values $(\mathrm{NP}=2)$

Fill code:

0 solid fill

1 unfill (i.e., a gap in solid fill)

2 meshed fill (indicates that an associativity is used to link the fill area with its fill mesh description). Using the associativity will allow the implementation of this obsolete method. The recommended method of mesh fill is to use Sectioned Area entity (230).

Additional pointers as required (see 2.2.4.4.2).
Obsolete. Note: a previous implementation of this parameter was as a pointer to the DE of a section entity defining linear segments of meshed fill. This previous implementation would be indicated by a non-zero value. 


\subsection{FORM NUMBER: 5 Line Widening}

\section{DESCRIPTION}

This property defines the characteristics of entities when they are used to define the location of items such as strips of metalization on printed wiring boards.

The justification flag terminology is interpreted as follows: right justified means that a defining line segment forms the right edge of the widened line in the direction from first defining point to second. Left justified is the opposite while center justified indicates that the defining line segment splits the widening exactly in half. Figure 4-36 indicates the measurement of the property values.

\begin{tabular}{|c|c|c|c|}
\hline Index & $\underline{\text { Name }}$ & Type & Description \\
\hline 1 & NP & Integer & $\begin{array}{l}\text { Number of property values } \\
\qquad(\mathrm{NP}=5)\end{array}$ \\
\hline 2 & WM & Real & Width of metalization \\
\hline 3 & $\mathrm{CC}$ & Integer & $\begin{array}{l}\text { Cornering codes } \\
0 \text { rounded } \\
1 \text { squared }\end{array}$ \\
\hline 4 & $\mathrm{EF}$ & Integer & $\begin{array}{l}\text { Extension flag } \\
\text { O No extension } \\
1 \text { One-half width extension } \\
2 \text { Extension set by } \\
\text { parameter }\end{array}$ \\
\hline 5 & $\mathrm{JF}$ & Integer & $\begin{array}{l}\text { Justification flag } \\
0 \text { center justified } \\
1 \text { left justified } \\
2 \text { right justified }\end{array}$ \\
\hline 6 & $E$ & Real & $\begin{array}{l}\text { Extension value } \\
\text { parameter } 4=2 \text { (Note: this value } \\
\text { may be negative) }\end{array}$ \\
\hline
\end{tabular}




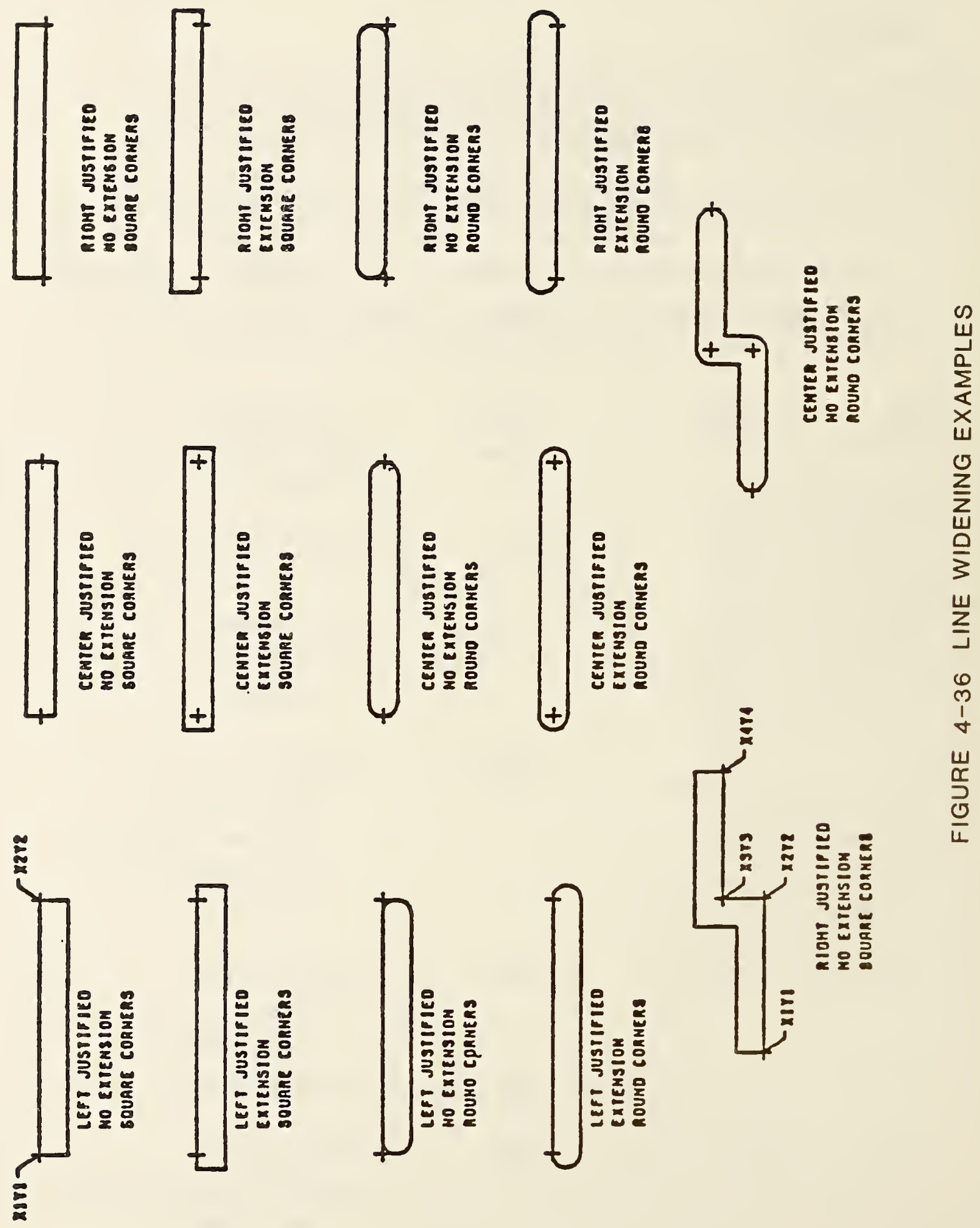




\subsection{FORM NUMBER: 6 Drilled Hole \\ DESCRIPTION}

The Drilled Hole property identifies an entity representing a drilled hole through a printed circuit board. The parameters of the property define the characteristics of the hole necessary for actual machining. The layer range indicated by parameters 5 and 6 refers to physical layers of the assembled printed circuit board.

$\begin{array}{cccc}\frac{\text { Index }}{1} & \frac{\text { Name }}{\text { NP }} & \begin{array}{c}\text { Type } \\ \text { Integer }\end{array} & \begin{array}{c}\text { Description } \\ \text { Number of property values } \\ (\mathrm{NP}=5)\end{array} \\ 2 & \text { RDS } & \text { Real } & \text { Drill diameter size } \\ 3 & \text { FDS } & \text { Feal } & \text { Finish diameter size } \\ 4 & \text { Integer } & \begin{array}{c}\text { Plating indication flag } \\ (0=\text { no, } 1=\text { yes })\end{array} \\ 5 & \text { LNL } & \text { Integer } & \text { Lower numbered layer } \\ 6 & \text { HNL } & \text { Integer } & \text { Higher numbered layer }\end{array}$

Additional Pointers as required (2.2.4.4.2) 


\subsection{FORM NUMBER: 7 Reference Designator}

\section{DESCRIPTION}

The Reference Designator property attaches a text string containing the value of a component reference designator to an entity being used to represent a component. This property is not to be used for the primary reference designator when a component is represented by a Network Subfigure Instance as that value is included in the subfigure parameters.

$\begin{array}{clll}\frac{\text { Index }}{1} & \frac{\text { Name }}{\text { NP }} & \frac{\text { Type }}{\text { Integer }} & \begin{array}{l}\text { Description } \\ \text { Number of property values } \\ (\mathrm{NP}=1)\end{array} \\ 2 & \text { RD } & \text { String } & \text { Reference designator text }\end{array}$

Additional Pointers as required (see 2.2.4.4.2) 


\section{DESCRIPTION}

The Pin Number property attaches a text string representing a component pin number to an entity being used to represent an electrical component's pin. This property is not to be used when a pin is represented by a Connect Point entity as the pin number is included in one of the Connect Point parameters.

$\begin{array}{clll}\frac{\text { Index }}{1} & \frac{\text { Name }}{\mathrm{NP}} & \frac{\text { Type }}{\text { Integer }} & \begin{array}{l}\text { Description } \\ \text { Number of property values } \\ (\mathrm{NP}=1)\end{array} \\ 2 & \text { PN } & \text { String } & \text { Pin Number Value }\end{array}$

Additional Pointers as required (see 2.2.4.4.2) 


\section{DESCRIPTION}

The Part Number property attaches a set of text strings that define the common part numbers to an entity being used to represent a physical component. Null text values in any parameter will imply that the missing value is not relevant to the transferred data.

$\begin{array}{clll}\frac{\text { Index }}{1} & \frac{\text { Name }}{\mathrm{NP}} & \frac{\text { Type }}{\text { Integer }} & \begin{array}{l}\frac{\text { Description }}{\text { Number of property values }} \\ (\mathrm{NP}=4)\end{array} \\ 2 & \text { GPN } & \text { String } & \begin{array}{l}\text { Generic part number or } \\ \text { name }\end{array} \\ 3 & \text { MPN } & \text { String } & \text { MIL-STD part number } \\ 4 & \text { VPN } & \text { String } & \begin{array}{l}\text { Vendor part number or } \\ \text { name }\end{array} \\ 5 & \text { IPN } & \text { String } & \text { Internal part number }\end{array}$

Additional Pointers as required (see $2 \cdot 2 \cdot 4.4 .2$ ) 


\section{DESCRIPTION}

The hierarchy property provides the ability to control the hierarchy of each directory entry attribute. This property is referenced when the directory entry status digits 7 and 8 are 02 .

$\begin{array}{llll}\frac{\text { Index }}{1} & \frac{\text { Name }}{\mathrm{NP}} & \begin{array}{c}\text { Type } \\ \text { Integer }\end{array} & \begin{array}{c}\text { Description } \\ \text { Number of property values } \\ (\mathrm{NP}=6)\end{array} \\ 3 & \text { LF } & \text { Integer } & \text { Line font } \\ 4 & \text { VU } & \text { Integer } & \text { View } \\ 5 & \text { LAB } & \text { Integer } & \text { Entity level } \\ 6 & \text { BL } & \text { Integer } & \text { Blank status } \\ 7 & \text { LW } & \text { Integer } & \text { Line weight } \\ & \text { CO } & \text { Integer } & \text { Color number }\end{array}$

Additional Pointers as required (see. 2.2.3.4.2).

Acceptable values for parameters 2 through 7 are 0 and 1 .

See definition in section $2 \cdot 2 \cdot 4 \cdot 3.9 .4$. 


\subsection{FORM NUMBER: 11 Tabular Data}

\section{DESCRIPTION}

The tabular data property provides a structure to accommodate point form data. The basic structure is a two dimensional array organized in column row order. In the simplified form this structure can contain a single list of values. The more complex forms contain multiple lists of independent and dependent variables.

The PROPERTY TYPE is the key used to define the dependent variable data values.

PROPERTY TYPES 1 to 5000 are reserved for defining finite element material properties.

The units used in these properties are specified in the MKSA (Meters, Kilograms, Seconds, Ampere) system according to the following table:

$\begin{array}{ll}\text { Length } & =\text { meters } \\ \text { Mass } & =\text { kilograms } \\ \text { Time }= & \text { seconds } \\ \text { Current }= & \text { amperes } \\ \text { Energy }= & \text { joules } \\ \text { Force }= & \text { newtons }\end{array}$

DE Form Number $\quad 11=$ Tabular Data Property

$\underline{\text { Index }}$

1

2
Name

N

PTYPE
Type

Integer

Integer

\section{Description}

\# of Property values

Property Type

1 = Young's Modulus

$\mathrm{ND}=3$

2 = Poisson's Ratio $\mathrm{ND}=3$ 


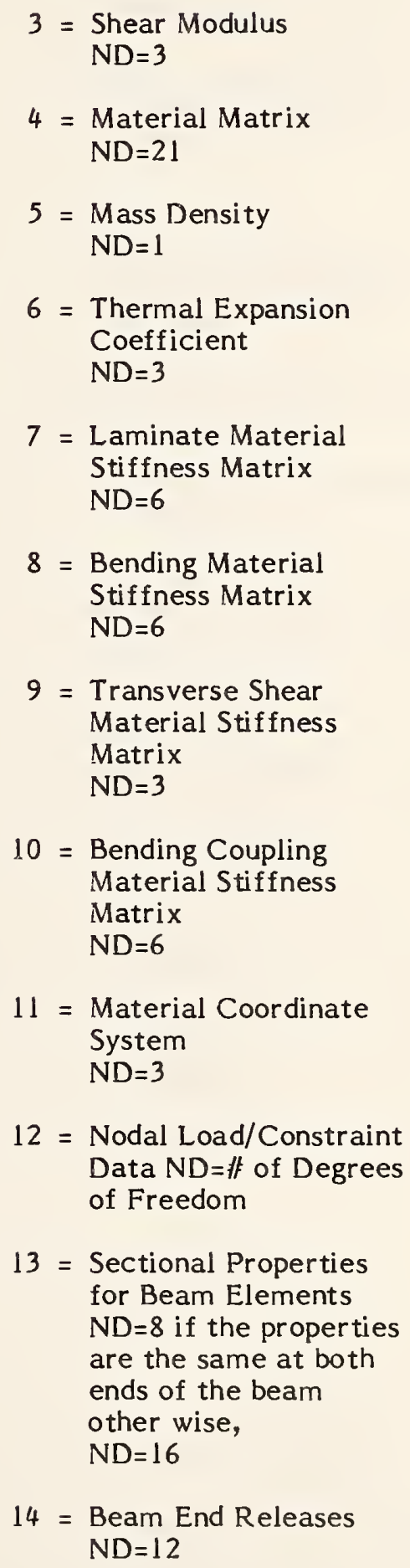




$$
\begin{aligned}
& 15 \text { = Offsets } \\
& \mathrm{ND}=9 \text { or } 18 \\
& 16 \text { = Stress Recovery Infor- } \\
& \text { mation } \\
& \mathrm{ND}=12 \text { or } 24 \\
& 17 \text { = Element Thickness } \\
& \mathrm{ND}=1 \text { or } \mathrm{n} \\
& 18=\text { Non-Struct:ural Mass } \\
& \mathrm{ND}=1 \\
& 19=\text { Thermal Conductivity } \\
& \mathrm{ND}=3 \\
& 20 \text { = Heat Capacity } \\
& \mathrm{ND}=1
\end{aligned}
$$

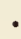

3

4

5
ND

NI

TYP 1
Integer

Integer

Integer

Integer

TYPNI

\# of dependent variables

\# of independent variables

Type of first independent

$$
\text { variable }
$$

1 = Temperature

2 = Pressure

3 = Relative humidity

4 = Rate of Strain

$5=$ Velocity

6 = Acceleration

7 = Time

$8=$ Strain

$\mathrm{n}=$ Open Ended

Type of last independent variable 


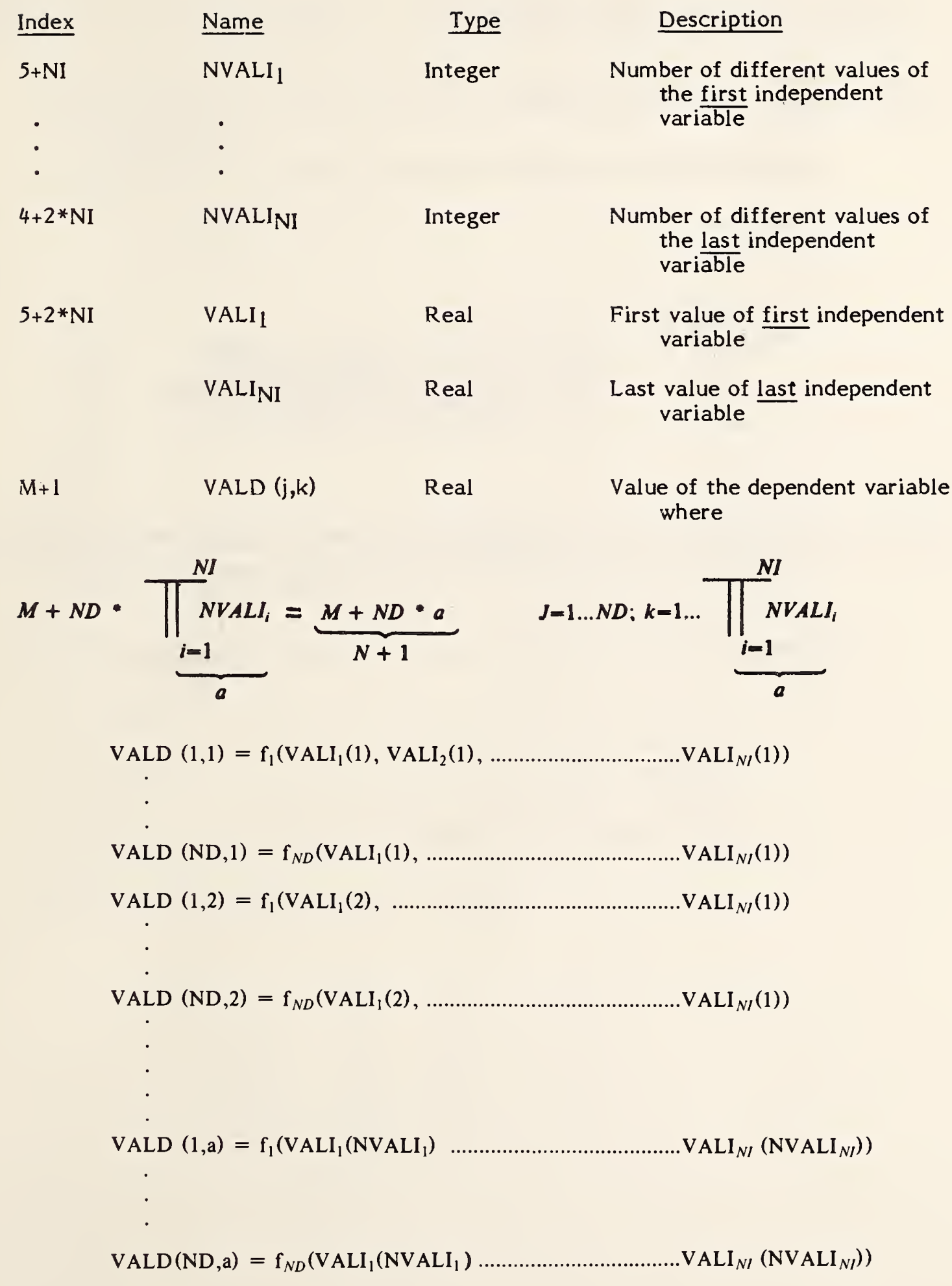

Additional Pointers as required (see 2.2.4.4.2). 


\section{MATERIAL PROPERTY TYPE DEFINITIONS:}

PTYPE $=1 \quad$ Young's Modulus

Young's modulus relates stress to strain in materials. In the simple case:

$$
\bar{\sigma}=E \bar{\epsilon}
$$

$$
\text { Where } \begin{aligned}
\bar{\sigma} & =\text { Stress Vector (Row of Elements } \sigma_{x}, \sigma_{y}, \sigma_{z} \text { ) } \\
\bar{\epsilon} & \left.=\text { Strain Vector (Row of Elements } \epsilon_{x}, \epsilon_{y}, \epsilon_{z}\right) \\
E & =\text { Young's Modulus Matrix of Diagonal Elements } \\
& E_{x x}, E_{y y}, \text { and } E_{z z}
\end{aligned}
$$

The modulus is a vector with three principal values $E_{x x}, E_{y y}$, and $E_{z z}$. This implies that ND (Number of Dependent Variables) is equal to three.

In matrix form:

$$
\left|\begin{array}{c}
\sigma_{x} \\
\sigma_{y} \\
\sigma_{z}
\end{array}\right|=\left|\begin{array}{lll}
E_{x x} & 0 & 0 \\
0 & E_{y y} & 0 \\
0 & 0 & E_{z z}
\end{array}\right|\left|\begin{array}{c}
\epsilon_{x} \\
\epsilon_{y} \\
\epsilon_{z}
\end{array}\right|
$$


PTYPE $=2 \quad$ Poisson's Ratio

Poisson's ratio is the ratio of transverse strain in the j-direction when stressed in the i-direction, i.e.,

$$
\nu_{i j}=\frac{\epsilon_{j}}{\epsilon_{i}}
$$

Where

$$
\begin{aligned}
& \nu=\text { Poisson's Ratio } \\
& \epsilon=\text { Strain } \\
& i=\text { One Orthogonal Direction } \\
& j=\text { Another Orthogonal Direction }
\end{aligned}
$$

The Poisson's Ratio is a vector consisting of matrix elements with the three principal values, $v_{X Y}, v_{X Z}, v_{Y Z}$

The other off diagonal matrix values are reciprocals of the principal values.

$$
\text { i.e., } \quad \nu_{x y}=\frac{1}{\nu_{y x}}
$$

This implies that ND (Number of Dependent Variables) is equal to three.

In matrix form for an or thotropic material:

$$
\left|\begin{array}{l}
\epsilon_{x} \\
\epsilon_{y} \\
\epsilon_{z}
\end{array}\right|=\left|\begin{array}{ccc}
\frac{1}{E_{x x}} & -\frac{\nu_{y x}}{E_{y y}} & -\frac{\nu_{z x}}{E_{z z}} \\
-\frac{\nu_{x y}}{E_{x x}} & \frac{1}{E_{y y}} & -\frac{\nu_{z y}}{E_{z z}} \\
-\frac{\nu_{x z}}{E_{x x}} & -\frac{\nu_{y z}}{E_{y z}} & \frac{1}{E_{z z}}
\end{array}\right| \quad\left|\begin{array}{c}
\sigma_{x} \\
\sigma_{y} \\
\sigma_{z}
\end{array}\right|
$$


406, Form 11 - TABULAR DATA

PTYPE = $3 \quad$ Shear Modulus

Shear Modulus - The ratio of shear stress to shear strain.

$$
G=\frac{\tau_{s}}{\gamma}
$$

Where $G$ is the Shear Modulus,

$\tau_{s}$ is the Shear Stress, and

$\gamma$ is the Shear Strain

The Shear Modulus is a vector with three princpal values:

$$
G_{x y}, G_{y x} \text {, and } G_{x z} \text {. }
$$

This implies that the ND (Number of Dependent Variables) is equal to three.

In matrix form for or thotropic materials:

$$
\left|\begin{array}{c}
\gamma_{x y} \\
\gamma_{y z} \\
\gamma_{z x}
\end{array}\right|=\left|\begin{array}{ccc}
\frac{1}{G_{x y}} & 0 & 0 \\
0 & \frac{1}{G_{y z}} & 0 \\
0 & 0 & \frac{1}{G_{z x}}
\end{array}\right|\left|\begin{array}{c}
\tau_{s_{x y}} \\
\tau_{s_{y z}} \\
\tau_{s_{z x}}
\end{array}\right|
$$




\section{PTYPE $=4 \quad$ Material Matrix}

Material matrix defines the tensor qualitites of the material. For example:

$$
\bar{\sigma}=|C| \bar{\epsilon}
$$

$$
\begin{aligned}
& \text { Where } \begin{aligned}
\overline{\bar{\sigma}} & \text { is the Stress Vector, } \\
\bar{\epsilon} & \text { is the Strain Vector, and } \\
|C| & \text { is the Material Matrix }
\end{aligned}
\end{aligned}
$$

Because of symmetry, the elements $C_{j i}=C_{i j}$. Therefore, 21 elements define the material matrix.

$\begin{array}{lll}c_{11} & c_{44} & c_{46} \\ c_{12} & c_{15} & c_{56} \\ c_{22} & c_{25} & c_{66} \\ c_{13} & c_{35} & \\ c_{23} & c_{45} & \\ c_{33} & c_{55} & \\ c_{14} & c_{16} & \\ c_{24} & c_{26} & \\ c_{34} & c_{36} & \end{array}$

This implies that $\mathrm{ND}=21$.

In Matrix form:

$$
\left|\begin{array}{c}
\sigma_{x} \\
\sigma_{y} \\
\sigma_{z} \\
\tau_{x y} \\
\tau_{y 2} \\
\tau_{z x}
\end{array}\right|=\left|\begin{array}{llllll}
C_{11} & C_{12} & C_{13} & C_{14} & C_{15} & C_{16} \\
C_{21} & C_{22} & C_{23} & C_{24} & C_{25} & C_{26} \\
C_{31} & C_{32} & C_{33} & C_{34} & C_{35} & C_{36} \\
C_{41} & C_{42} & C_{43} & C_{44} & C_{45} & C_{46} \\
C_{51} & C_{52} & C_{53} & C_{54} & C_{55} & C_{56} \\
C_{61} & C_{62} & C_{63} & C_{64} & C_{65} & C_{66}
\end{array}\right|\left|\begin{array}{c}
\epsilon_{x} \\
\epsilon_{y} \\
\epsilon_{z} \\
\gamma_{x y} \\
\gamma_{y 2} \\
\gamma_{z x}
\end{array}\right|
$$


406, Form 11 - TABULAR DATA

PTYPE $=5 \quad$ Mass Density

Mass density is the Mass/Unit Volume.

This implies that ND $=1$.

Mass Density $=\rho$

PTYPE $=6$ Thermal Expansion Coefficient

THERMAL EXPANSION COEFFICIENT is a material property that computes the strain given a temperature differential, i.e.,

$$
\epsilon=\alpha \Delta T \text { or } \alpha=\frac{\epsilon}{\Delta T}
$$

Where

$$
\begin{aligned}
\epsilon & =\text { strain } \\
\alpha & =\text { thermal expansion coefficient } \\
\Delta T & =\text { the temperature differential }
\end{aligned}
$$

The THERMAL EXPANSION COEFFICIENT may be represented as a vector with three principal values:

$$
a_{x x}, a_{y y} \text { and } a_{z z}
$$

This implies that ND (number of Dependent Variables) is equal to three. 
PTYPES $7-11 \quad$ Composite Materials

Composite materials will be represented with linkages to Tabular Data Property 11 as described in Figure 4-37. PTYPES required are:

\section{PTYPE DESCRIPTION}

7. Laminate material stiffness matrix

8. Bending material stiffness matrix

9. Transverse shear material stiffness matrix.

10. Bending coupling material stiffness matrix.

11. Material Coordinate System.

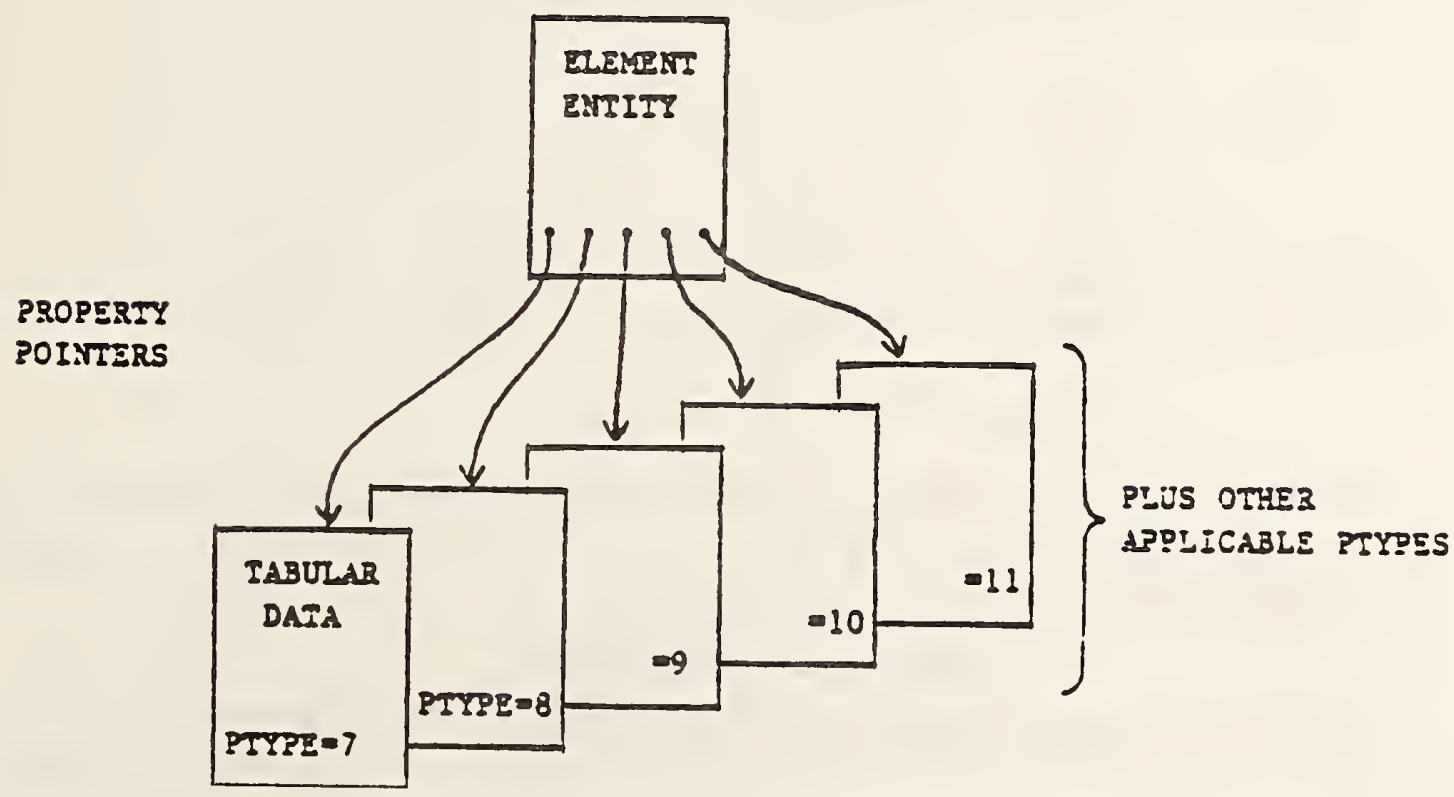

FIGURE 4-37 COMPOSITE MATERIAL CONFIGURATION 
PTYPE = $7 \quad$ Laminate Material Stiffness Matrix

The membrane material stiffness matrix defines anisotropic material properties for shell =membrane action. For example:

$$
\bar{f}=t[M] \bar{\epsilon}
$$

Where $\quad \bar{f}=$ Forces per unit length row of elements $\left(f_{x}, f_{y}, f_{x y}\right)$

$\bar{\epsilon}=$ Midplane strains row of elements $\left(\epsilon_{x}, \epsilon_{y}, \epsilon_{x y}\right)$

$t=$ Shell thickness - see element property

$[M]=$ Membrane material stiffness matrix

$\bar{F}$ and $\bar{\epsilon}$ Defined in shell material coordinate system, PTYPE $=11$

Because of symmetry, the elements $\mathrm{Mji}=\mathrm{Mij}$. Therefore, six elements define the membrane material stiffness matrix. This implies ND $=6$.

$M_{11}$
$M_{12}$
$M_{13}$
$M_{22}$
$M_{23}$
$M_{33}$

The matrix [M] is a laminate material stiffness matrix which is calculated from lamina stress strain matrices $[G]_{n}$. One method for calculating $[M]$ for a laminate containing $m$ plys is:

$$
[M i j]=\frac{1}{t} \sum_{n=1}^{m}[G i j]_{n} \Delta t_{n}
$$

$$
\begin{aligned}
& \Delta I_{n} \text { is thickness of } n^{\text {th }} \\
& t \text { is total thickness of laminate }
\end{aligned}
$$

Where $[G]_{n}$ is stress strain matrix for $n^{\text {th }}$ ply of laminate, defined in the material coordinate system 
PTYPE $=8$ Bending Material Stiffness Matrix

The bending material stiffness matrix defines the anisotropic material properties for shell bending. For example:

$$
\bar{m}=\frac{r^{3}}{12}[B] \bar{x}
$$

Where $\bar{m} \quad$ Shell bending moments per unit length (Row of elements $M_{x}, M_{y}, M_{x y}$

$\bar{x}=$ Shell curvature (Row of elements $\left(\chi_{x}, x_{y}, \chi_{x y}\right)$

$t$ = Shell thickness - see element property

$[B]=$ Bending material stiffness matrix $\bar{M}$ and $\bar{\chi}$ are defined in shell material coordinate system, PTYPE $=11$

Because of symmetry the elements $\mathrm{Bij}=\mathrm{Bji}$. Therefore, six elements define the bending stress strain matrix. This implies ND $=6$

$B_{11}$
$B_{12}$
$B_{13}$
$B_{22}$
$B_{23}$
$B_{33}$

The matrix $[B]$ is a laminate matrix for bending, which is calculated from lamina matrices $[G]_{n}$. One method for calculating $[B]$ for a laminate containing $m$ plys is:

$$
[B i j]=\frac{12}{t^{3}} \sum_{n=1}^{m}\left(Z_{n}^{2}[G i j]_{n} \Delta t_{n}\right)
$$

Where $[G]_{n}$ is stress strain matrix of lamina for $\mathbf{n}^{\text {th }}$ ply of laminate, defined in the shell material coordinate system

$\Delta t_{n} \quad$ is thickness of $\mathrm{n}^{\text {th }}$ ply

$Z_{n} \quad$ is the normal distance from midplane of shell to centroid of ply

$t$ is total thickness of shell 
406, Form 11 - TABULAR DATA

\section{PTYPE $=9 \quad$ Transverse Shear Material Stiffness Matrix}

The transverse shear material stiffness matrix defines anisotropic material properties for transverse shear flexibility in shell structure. For example:

$$
\bar{V}=t_{s}[S] \bar{\gamma}
$$

Where $\quad \bar{V}=$ transverse shear force per unit length (row of elements $V_{x}, V_{y}$ )

$\bar{\gamma}=$ transverse shear strains, dimensionless

(Row of elements $\left(\gamma_{x}, \gamma_{y}\right)$

$t_{s}=\frac{5}{6}$,effective transverse shear thickness

$[S]=$ transverse shear material stiffness matrix

$\bar{V}$ and $\bar{\gamma}$ are defined in material coordinate system

Because of symmetry $\mathrm{Sij}=\mathrm{Sji}$. Therefore, three elements define the transverse shear material stiffness matrix. This implies ND $=3$.

$$
\begin{aligned}
& s_{11} \\
& s_{12} \\
& s_{22}
\end{aligned}
$$

The matrix $[\mathrm{s}]$ is a laminate material stiffness matrix for transverse shear flexibility. If the matrix is not defined, deflections normal to the shell do not include contributions from transverse shear strain. 
PTYPE $=10 \quad$ Bending Coupling Material Stiffness Matrix

The membrane - bending coupling material stiffness matrix defines the anisotropic material properties for shell structure with the neutral axis for bending offset from the midplane of the shell. For example:

$$
\begin{gathered}
\bar{f}=t^{2}[C] \bar{\chi} \\
\text { and } \\
\bar{m}=t^{2}[C]^{T} \bar{\epsilon}
\end{gathered}
$$

Where $\bar{f}=$ Forces per unit length (row of elements $f_{x}, f_{y}, f_{x y}$ )

$\bar{m}=$ Bending moments per unit length (row of elements $m_{x}, m_{y}, m_{x y}$ )

$\bar{\chi}=$ Curvature (measurements of bending strain), units (meter) $)^{-1}$ (Row of elements $X_{x}, X_{y}, X_{x y}$ )

$\bar{\epsilon}=$ Midplane strains (row of elements $\epsilon_{x}, \epsilon_{y}, \epsilon_{x y}$ )

$t=$ Shell thickness

$\bar{f}, \bar{m}, \bar{\chi}$ and $\bar{\epsilon}$ are defined in the shell material coordinate system

Because of symmetry, the elements $C_{i j}=C_{j i}$. Therefore, six elements define the membrane bending coupling material matrix. This implies ND $=6$.
$\mathrm{C}_{11}$
$\mathrm{C}_{12}$
$\mathrm{C}_{13}$
$\mathrm{C}_{22}$
$\mathrm{C}_{23}$
$\mathrm{C}_{33}$ 


\section{PTYPE $=10$ (Continued)}

The matrix [C] is a laminate matrix for membrane bending coupling, which is calculated from lamina stress strain matrices $[G]_{n}$. One method for calculating $[C]$ for a laminate containing $m$ plys is:

$$
[C i j]=\frac{1}{t^{2}} \sum_{n=1}^{m}\left(Z_{n}[G i j]_{n} \Delta t_{n}\right)
$$

Where $[G]_{n}$ is stress strain matrix for $\mathrm{n}^{\text {th }}$ ply, defined in shell material coordinate system

$\Delta t_{n} \quad$ is thickness of $\mathrm{n}^{\text {th }} \mathrm{ply}$

$Z_{n} \quad$ is the normal distance from midplane of shell to centroid of ply

$t$ is thickness of shell 
PTYPE = $11 \quad$ Material Coordinate System

The orientation of the element material coordinate system is specified by a set of direction cosines defining a vector $D$. The use of the vector $D$ depends upon the element type.

For element topology type 1 and 33 of entity type 136, the following Figure 4-38 illustrates the use of the vector $D$ to define the element material coordinate system. For topology type 33 the vector D is defined by the reference node 3.

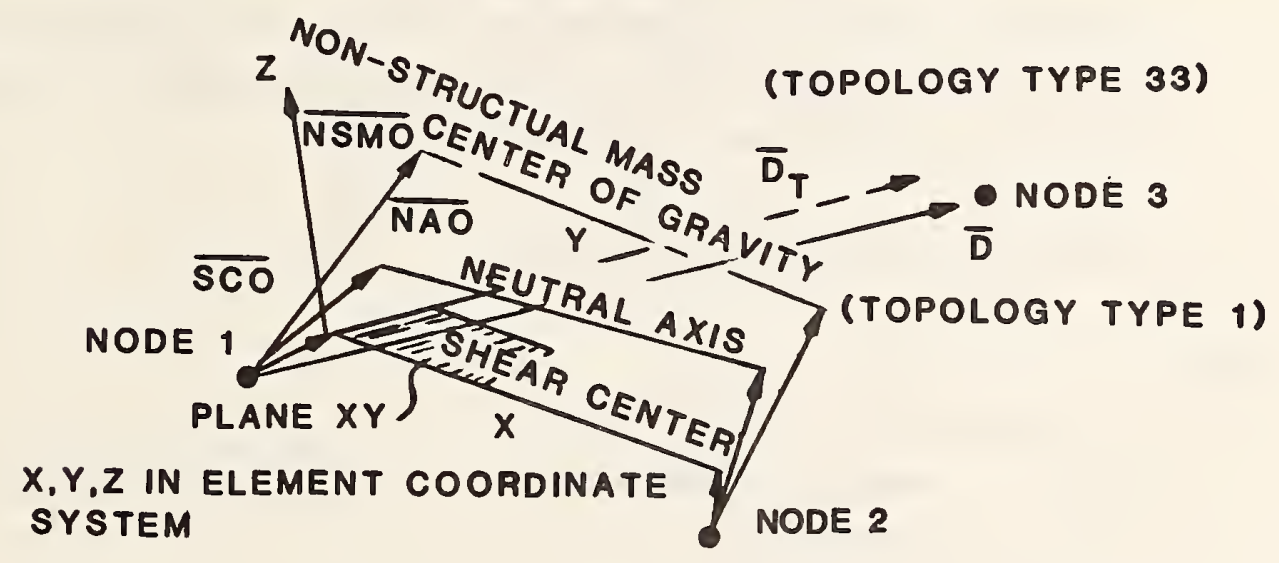

\section{FIGURE 4-38 Elemental and Materlal Coordinate System}

The cosines for vector $D$ are translated to the location of the shear center offset to establish the reference planes for material property definition (vector DT).

For element topology types 2 through 26 of the entity type 136, the following paragraphs discuss the use of vector $D$ to define the material coordinate system.

The projection of $\mathrm{D}$ on the plane of the element face (F1) (outward normal $\mathrm{N}$ ) defines a vector in the $\mathrm{X}$ direction of the material coordinate system.

$$
\begin{aligned}
\bar{x}=\bar{N} \times \bar{D} \times \bar{N} \quad \bar{N}=\begin{array}{l}
\text { positive outward normal of the element face (F1), and is } \\
\text { defined by nodal connection of the element, i.e., } \\
\bar{N}=\overline{S_{1}} \times \overline{S_{2}}
\end{array} \\
\bar{S}_{1}=\text { vector from first to second corner of element face (F1) } \\
\bar{S}_{2}=\text { vector from second to third corner of element face (F1) }
\end{aligned}
$$


PTYPE = 11 (Continued)

Three direction cosines are required to define the Vector $D$ in the global coordinate system. Therefore, ND $=3$.

$$
\begin{aligned}
& D_{1} \\
& D_{2} \\
& D_{3}
\end{aligned}
$$

The vectors $\bar{D}$ and $\bar{N}$ define the element material $X$ and $Z$ axes, respectively. The internal load and strain sign conventions must be described to ensure consistent definition of material types 7-10. See Figure 4-39.

INTERNAL LOAD SIGN CONVENTION:

where:
$x, y, z$
are material coordinate
system axes
u, v, w
are displacements of a
point in the material
coordinate system. 


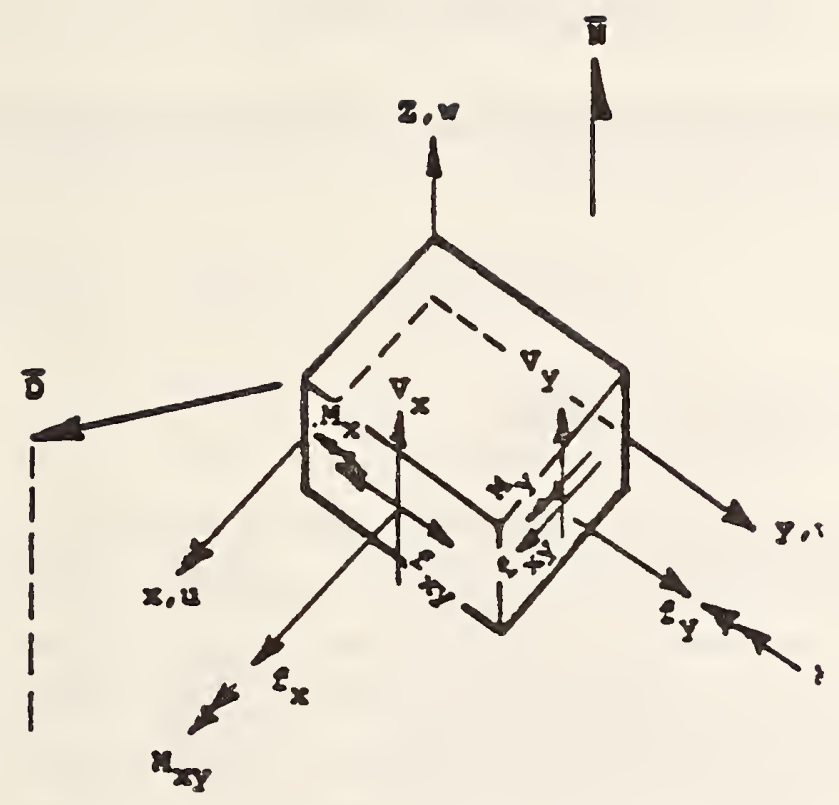

$$
\begin{aligned}
& \left\{\begin{array}{l}
f_{x} \\
f_{y} \\
f_{x y}
\end{array}\right\}=\bar{f} \quad \text { forces per unit length } \\
& \left\{\begin{array}{l}
\epsilon_{x} \\
\epsilon_{y} \\
\epsilon_{x y}
\end{array}\right\}=\epsilon \text { midplane strains } \\
& \left\{\begin{array}{l}
M_{x} \\
M_{y} \\
M_{x y}
\end{array}\right\}=\bar{M} \text { moments per unit length } \\
& \left.\mid \begin{array}{l}
x_{x} \\
x_{y} \\
x_{x y}
\end{array}\right\}=c \text { bending curvatures } \\
& \left\{\begin{array}{l}
v_{x} \\
V_{y}
\end{array}\right\}=\bar{V} \quad \begin{array}{c}
\text { transverse shear forces } \\
\text { per unit length }
\end{array} \\
& \left\{\begin{array}{l}
\gamma_{x} \\
y_{y}
\end{array}\right\}=\gamma \text { transverse shear strains }
\end{aligned}
$$

\section{STRAIN DISPLACEMENT RELATIONSHIPS}

$$
\begin{array}{lll}
\epsilon_{x}=\frac{\partial u}{\partial x}, & \epsilon_{y}=\frac{\partial v}{\partial y}, & \epsilon_{x y}=\frac{\partial u}{\partial y}+\frac{\partial v}{\partial x} \\
\chi_{x}=\frac{\partial^{2} u}{\partial x^{2}}, & \chi_{y}=\frac{\partial^{2} w}{\partial^{2} y}, & \chi_{x y}=2 \frac{\partial^{2} w}{\partial x \partial y} \\
\gamma_{x}=\frac{\partial w}{\partial x}, & \gamma_{y}=\frac{\partial w}{\partial y} &
\end{array}
$$

FIGURE 4-39 INTERNAL LOAD SIGN 


\section{PTYPE $=12 \quad$ Nodal Loads/Constraints Data}

The nodal load/constraint data will be stored in the tabular data property form number in the following manner:

PTYPE $=12$

$\mathrm{ND}=$ \# of degrees of freedom

For example, if the load vector has an $X, Y, Z M_{X}, M_{y}$, and $M_{Z}$ components; the $N D=6$. (Note $M_{X}, M_{y}, M_{z}$ refer to moments). If the load.vector has an $X$ and $Y$ component, the $N D=2$. If the load vector has only a $Z$-component, then $\mathrm{ND}=3$. In other words the $\mathrm{X}$-component is the lst degree, the $\mathrm{Y}$-component is the 2nd degree, and the $\mathrm{Z}$-component is the 3rd degree. Other components are treated in a similar manner starting in the 4 th degree for the rotation $X$ component.

The constraint vector has an $X, Y, Z, M_{X}, M_{y}$, and $M_{Z}$ constraints. These constraints are represented by a $0=$ No Constraint and $1=$ Constraint. $N D=$ 6 always for constraints. 


\section{PTYPE $=13 \quad$ Sectional Properties for Beam Elements}

Sectional properties for beam elements define the structural characteristics of the beam. These properties are:

\begin{tabular}{|c|c|c|}
\hline PROPERTY & $\underline{\text { UNITS }}$ & DESCRIPTION \\
\hline Area & $M^{2}$ & Area of section \\
\hline $\mathrm{I}_{\mathbf{X}}$ & $M^{4}$ & $\begin{array}{l}\text { Area moment of inertia about the element } x \text { - } \\
\text { axis }\end{array}$ \\
\hline $\mathrm{I}_{\mathrm{y}}$ & $M^{4}$ & $\begin{array}{l}\text { Area moment of inertia about the element } y \text { - } \\
\text { axis }\end{array}$ \\
\hline$I_{x y}$ & $M^{4}$ & Product of inertia \\
\hline J & $M^{4}$ & Torsional stiffness parameter \\
\hline $\mathrm{S}_{\mathrm{Ry}}$ & Unitless & Shear stiffness ratio \\
\hline $\mathrm{S}_{\mathrm{Rz}}$ & Unitless & Shear stiffness ratio \\
\hline WC & $M^{6}$ & Warping coefficient \\
\hline
\end{tabular}

If the properties are the same at both ends of the beam then ND=8, otherwise $\mathrm{ND}=16$. If $\mathrm{ND}=16$, two sets of section properties are specified. They are stated in order of the topology set grid number scheme. 
PTYPE $=14 \quad$ Beam End Releases

Beam end releases specify whether the ends of the beam are constrained or free to move. If free to move then both translation and rotational freedoms of the end are considered.

\section{PROPERTY DESCRIPTION}

FOR $\left[\begin{array}{cc}\mathrm{X} & \mathrm{X} \text { direction translation freedom/constraint } \\ \mathrm{Y} & \mathrm{Y} \text { direction translation freedom/constraint } \\ Z & \mathrm{Z} \text { direction translation freedom/constraint } \\ \mathrm{M}_{\mathrm{X}} & \mathrm{X} \text { direction rotational freedom/constraint } \\ \mathrm{M}_{\mathrm{y}} & \mathrm{Y} \text { direction rotational freedom/constraint } \\ \mathrm{M}_{\mathrm{Z}} & \mathrm{Z} \text { direction rotational freedom/constraint }\end{array}\right.$

The value for each property $X, Y, Z, M_{X}, M_{y}, M_{Z}$, is either a 0 =unconstrained; $+1=$ constrained to the global coordinate system; or $-1=$ constrained to the element coordinate system.

The beam release must be specified at both ends. Therefore, ND=12.

The beam ends are defined by the Topology Set grid number scheme. 
Offsets are global $x, y, z$ values used to define the location of the shear center axis, neutral axis, and non-structural center of mass relative to the element end nodes.

Figure 4-38 shows SCO - "Shear Center Offset";

NAO - "Neutral Axis Offset"; and

NSMO - "Non-Structural Mass Offset".

These offsets are vectors in the global coordinate system (model space) relative to the end of the beam.

\begin{tabular}{|c|c|c|}
\hline \multicolumn{2}{|c|}{ PROPERTY } & DESCRIPTION \\
\hline & $\mathrm{SCO}_{\mathrm{x}}$ & Shear Center Offset in global $\mathrm{x}$ direction \\
\hline & $\mathrm{SCO}_{y}$ & Shear Center Offset in global y direction \\
\hline & $\mathrm{SCO}_{\mathrm{Z}}$ & Shear Center Offset in global $\mathrm{z}$ direction \\
\hline & $\mathrm{NAO}_{\mathrm{X}}$ & Neutral Axis Offset in global x direction \\
\hline & $\mathrm{NAO}_{y}$ & Neutral Axis Offset in global y direction \\
\hline & $\mathrm{NAO}_{z}$ & Neutral Axis Offset in global $\mathrm{z}$ direction \\
\hline & $\mathrm{NSMO}_{\mathbf{x}}$ & Non-Structural Mass Offset in global $\mathrm{x}$ direction \\
\hline & $\mathrm{NSMO}_{\mathrm{y}}$ & Non-Structural Mass Offset in global y direction \\
\hline & $\mathrm{NSMO}_{Z}$ & Non-Structural Mass Offset in global z direction \\
\hline
\end{tabular}

$\mathrm{ND}=9$ or $\mathrm{ND}=18$ depending on whether or not both ends must be specified. They are stated in order of the topology set grid number scheme. 
406, Form 11 - TABULAR DATA

PTYPE $=16 \quad$ Stress Recovery Information

Stress Recovery Information is used to define up to four offset points at each beam end at which stress levels will be recovered from the finite element analysis program.

These offset points are described as global $x, y, z$ offsets from each end node.

All offset points are in a plane which is normal to thebeam element axis.

These points occur in pairs from one end of the beam to the other. 
PTYPE $=16 \quad$ (Continued)

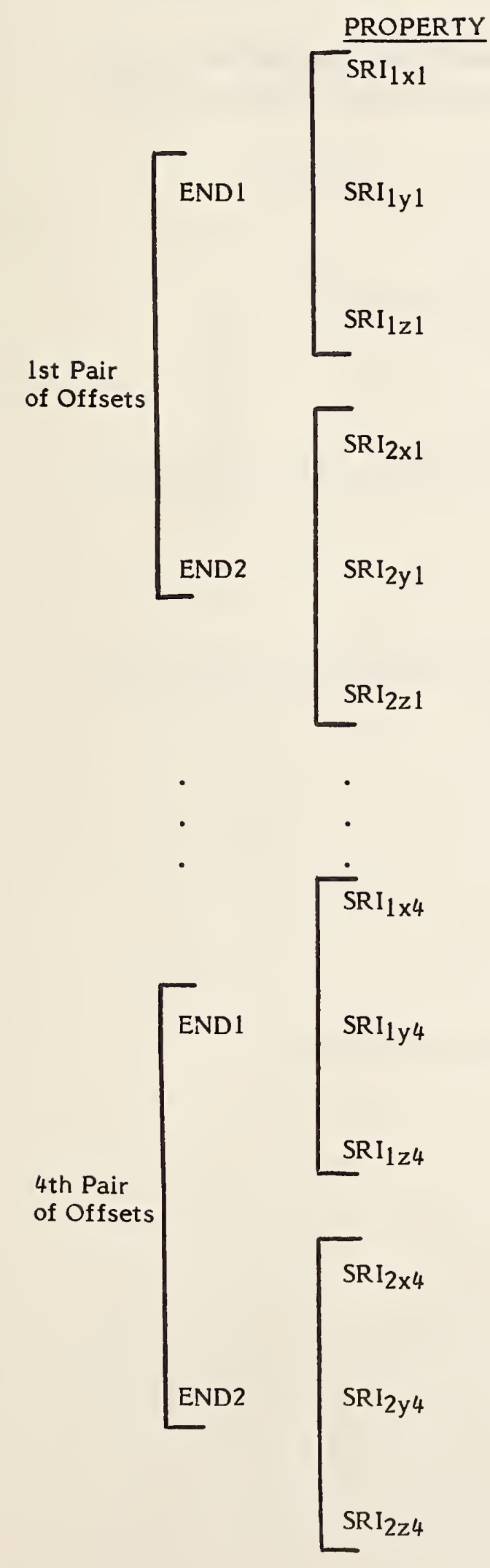

\section{DESCRIPTION}

Stress Recovery Information beam end $1 \mathrm{x}$ direction pair 1

Stress Recovery Information beam end $1 \mathrm{y}$ direction pair 1

Stress Recovery Information beam end $1 \mathrm{z}$ direction pair 1

Stress Recovery Information beam end $2 \mathrm{x}$ direction pair 1

Stress Recovery Information beam end $2 y-$ direction pair 1

Stress Recovery Information beam end $2 \mathrm{z}$ direction pair 1

Stress Recovery Information beam end $1 \mathrm{x}$ direction pair 4

Stress Recovery Information beam end 1 ydirection pair 4

Stress Recovery Information beam end $1 \mathrm{z}$ direction pair 4

Stress Recovery Information beam end $2 \mathrm{x}$ direction pair 4

Stress Recovery Information beam end $2 \mathrm{y}$ direction pair 4

Stress Recovery Information beam end $2 \mathrm{z}$ direction pair 4 


\section{PTYPE $=17 \quad$ Element Thickness}

Element Thickness defines the net section thickness for a homogeneous element or individual plate thickness for a laminate or sandwich plate. $\mathrm{ND}=1$ or $\mathrm{n}$ and is defined as follows:

\section{$\mathrm{ND}=1$ Scalar thickness of element}

$\mathrm{ND}=\mathrm{n} \quad$ Thickness of the nth plate of a sandwich or laminate

The thickness is in the order from 1 to $n$,

The thicknesses are measured in the positive $Z$ direction in order of increasing $z$ in local element coordinate system.

\section{PROPERTY DESCRIPTION}

$t_{1}$ Thickness for homogeneous plate or the thickness for the first lamina of the plate

$t_{n} \quad$ Thickness for the nth lamina of the plate. 
PTYPE $=18 \quad$ Non-Structural Mass

Non-Structural Mass is defined as the mass not accounted for in volume and density information for the structural elements. $\mathrm{ND}=1$.

\section{PROPERTY $\quad$ DESCRIPTION}

$\begin{array}{ll}\text { NSM } & \text { Mass/unit length or } \\ \text { Mass/unit area or } \\ \text { Mass/unit volume }\end{array}$

this depends on type of element. 
PTYPE $=19 \quad$ Thermal Conductivity

Thermal Conductivity relates heat flow across a surface as a function of temperature. The heat balance equation shows this relationship:

$$
\begin{aligned}
\frac{\partial}{\partial x}\left(k_{x} \frac{\partial T}{\partial x}\right) & +\frac{\partial}{\partial y}\left(k_{y} \frac{\partial T}{\partial y}\right) \\
& +\frac{\partial}{\partial z}\left(k_{z} \frac{\partial T}{\partial z}\right)=\rho C_{p} \frac{\partial T}{\partial t}-Q_{I}
\end{aligned}
$$

Where

$$
\begin{aligned}
& k_{n}=\text { Thermal conductivity coefficient where } n=x, y, z \\
& C_{p}=\text { Heat capacity at constant pressure } \\
& \rho=\text { Material Density } \\
& T=\text { Temperature } \\
& t=\text { Time } \\
& Q_{I}=\text { Energy converted to heat internally }
\end{aligned}
$$

$x, y, z$ are defined in the local element coordinate system.

If we consider $k$ (thermal conductivity coefficient) as independent of direction, then $k$ can be represented as constant in the $x, y, z$ directions:

i.e.,

$$
\frac{\partial}{\partial x}\left(k_{x} \frac{\partial T}{\partial x}\right)=\frac{\partial k_{x}}{\partial x} \frac{\partial T}{\partial x}+k_{x} \frac{\partial^{2} T}{\partial x^{2}}
$$

Now assuming a constancy in the $\mathrm{x}$ direction:

$$
\frac{\partial}{\partial x}\left(k_{x} \frac{\partial T}{\partial x}\right)=k_{x} \frac{\partial^{2} T}{\partial x^{2}}
$$

Therefore the thermal conductivity is a vector with three principal values, $k_{x}, k_{y}$, and $k_{z}$. This implies that ND (Number of Dependent Variables) is equal to three. 
PTYPE $=19$ Thermal Conductivity (Continued)

In matrix form for constraint conductivity:

$$
k_{x} k_{y} k_{z}\left(\begin{array}{l}
\frac{\partial^{2} T}{\partial x^{2}} \\
\frac{\partial^{2} T}{\partial y^{2}} \\
\frac{\partial^{2} T}{\partial z^{2}}
\end{array}\right)=\rho C_{p} \frac{\partial T}{\partial t}-Q_{I}
$$

Now assuming no internal heat sources $Q_{I}$ and the steady state where $\frac{\partial T}{\partial t}=0$

Consequently, integrating in one direction

$$
Q=k_{x} A \frac{\partial T}{\partial x}
$$

Where $Q$ is the heat flux in the $x$ direction across a surface of area A normal to the $x$-direction. Likewise solutions may be found in the $y$ and $z$ direction.

\section{PROPERTY $\quad \underline{\text { DESCRIPTION }}$}

$\begin{array}{ll}\mathrm{K}_{\mathrm{x}} & \text { Thermal Conductivity coefficient } \mathrm{x} \text { direction } \\ \mathrm{K}_{\mathrm{y}} & \text { Thermal Conductivity coefficient } \mathrm{y} \text { direction } \\ \mathrm{K}_{\mathrm{z}} & \text { Thermal Conductivity coefficient } \mathrm{z} \text { direction }\end{array}$


Heat Capacity is a material's ability to store heat. The heat balance equation shows the relationship of heat capacity to the spatial variation and time variation of temperature.

$$
\frac{\partial}{\partial x}\left(k_{x} \frac{\partial T}{\partial x}\right)+\frac{\partial}{\partial y}\left(k_{y} \frac{\partial T}{\partial y}\right)+\frac{\partial}{\partial z}\left(k_{z} \frac{\partial T}{\partial z}\right)=\rho C_{p} \frac{\partial T}{\partial t}-Q_{I}
$$

Where

$$
\begin{aligned}
& k_{n}=\text { Thermal conductivity coefficient where } n=x, y, z \\
& C_{p}=\text { Heat capacity at constant pressure } \\
& \rho=\text { Material Density } \\
& T=\text { Temperature } \\
& t=\text { Time } \\
& Q_{I}=\text { Energy converted to heat internally }
\end{aligned}
$$

If we consider constant pressure, the heat capacity can be considered a constant.

Consequently, ND=1 to define the constant.

\section{PROPERTY DESCRIPTION}

$\mathrm{CP}_{\mathrm{p}} \quad$ Heat capacity at constant pressure 
Convective film coefficient relates to the amount of heat flux that is conveted to adjacent materials at the interface boundary of a heat source.

$$
Q=-h_{c} A \Delta T
$$

Where $Q=$ the heat flux

$h_{c}=$ the convective film coefficient

$A=$ the surface area through which the heat llows

$\Delta T=$ the Temperature differential between the materials.

The convective film coefficient may be represented as a constant. This implies that $\mathrm{ND}=1$.

\section{PROPERTY $\quad \underline{\text { DESCRIPTION }}$}

$h_{C} \quad$ Convective Film Coefficient

PTYPE $=22 \quad$ Electromagnetic Radiation Parameters

Properties for Absorptivity, Transmissivity, Reflectivity, and Emissivity are defined for structural elements using four values. $N D=4$.

\section{PROPERTY DESCRIPTION}

a

$\mathrm{t}$

$\mathbf{R}$

E
Absorptivity Constant

Transmissivity Constant

Reflectivity Constants

Emissivity Constant 
406, Form 11 - TABULAR DATA

\section{Examples:}

Consider the representation of the mass density as a function of pressure:

\begin{tabular}{llc} 
INDEX & NAME & RECORDED VALUE \\
\cline { 2 - 3 } 1 & & 9 \\
2 & N & 5 \\
3 & PTYPE & 1 \\
4 & ND & 1 \\
5 & NI & 2 \\
6 & TYPI & 2 \\
7 & NVALI & 50 \\
8 & VALI 1 & 25 \\
9 & VALI 2 & 33 \\
10 & VALD $(1,1)$ & 46
\end{tabular}

Additional pointers as required (see $2 \cdot 2 \cdot 4.4 .2$ ) 
406, Form 11 - TABULAR DATA

\section{Examples:}

Consider the representation of Young's modulus for a linear, static, independent case:

$\begin{array}{llc}\text { INDEX } & \text { NAME } & \text { RECORDED VALUE } \\ 1 & \text { N } & 6 \\ 2 & \text { PTYPE } & 1 \\ 3 & \text { ND } & 3 \\ 4 & \text { NI } & 0\end{array}$

$\begin{array}{rlr}4+2 * N I+1=5 & E_{X x} & 30 \times 10^{6} \\ 6 & E_{y y} & 30 \times 10^{6} \\ 7 & E_{Z z} & 30 \times 10^{6}\end{array}$

Additional pointers as required (see 2.2.4.4.2) 


\subsection{FORM NUMBER: $12 \quad$ External Reference File List}

\section{DESCRIPTION}

The External Reference File List appears in an IGES file which references definitions that reside in another IGES file. It contains a list of the names of the IGES files directly referenced by entities within this IGES file. See section 2.5.4 and the External Reference Entity (Type 416) for more detail.

\begin{tabular}{ccll} 
Index & Name & Type & \multicolumn{2}{l}{ Description } \\
1 & $\mathrm{~N}$ & Integer & Number of List Entries \\
2 & NAME1 & String & $\begin{array}{l}\text { External Reference File } \\
\text { Name }\end{array}$ \\
\hdashline$\ddot{N}+1$ & NAMEN & String & $\begin{array}{l}\text { Last External Reference } \\
\text { File Name }\end{array}$
\end{tabular}

Additional pointers as required (see 2.2.4.4.2). 


\subsection{FORM NUMBER: $13 \quad$ Nominal Size}

\section{DESCRIPTION}

A nominal size consists of a value, a name and optionally a reference to an engineering standard. The nominal size value is a real value in the units appropriate for the specified name. The name is a string constant, but the following values are predefined:

Nominal Size Name
3HAWG
3HIPS
2HOD

$\underline{\text { Index }}$

1

2

3

4

Name
NP
SZ
NM
SP

Name

Type

Integer

Real

String constant

String constant
Meaning

American Wire Gauge

Iron Pipe Size

Outside Diameter schedule, i.e., tubing

\section{Description}

Number of property values $(\mathrm{NP}=2$ or 3$)$

Nominal size value

Nominal size name

Name of relevant engineering standard (optional)

Additional pointers as required (see 2.2.4.4.2) 


\subsection{FORM NUMBER: 14 Flow Line Specification DESCRIPTION}

The flow line specification property attaches one or more text strings to entities being used to represent a flow line.

$\begin{array}{llll}\text { Index } & \text { Name } & \text { Type } & \text { Description } \\ 1 & \text { NP } & \text { Integer } & \text { Number of property values } \\ 2 & \text { L1 } & \text { String constant } & \begin{array}{l}\text { Primary flow line } \\ \text { specification name }\end{array} \\ 3 & \text { L2 } & \text { String constant } & \text { Modifier (optional) } \\ \cdot & \text { - } & \text { - } & \text { - } \\ \text { - } & \text { - } & \text { - } & - \\ \text { NP+1 } & \text { LN } & \text { String constant } & \text { Modifier (optional) }\end{array}$

Additional Pointers as required (see 2.2.4.4.2) 


\subsection{FORM NUMBER: 15 Name \\ DESCRIPTION}

This property contains a string which specifies a user-defined name. It can be used for any entity that does not have a name explicity specified in the parameter data for the entity.

$\begin{array}{llll}\text { Index } & \text { Name } & \text { Type } & \text { Description } \\ 1 & \text { NP } & \text { Integer } & \begin{array}{l}\text { No. of property } \\ \text { Values (NP=1) }\end{array} \\ 2 & \text { NAME } & \text { String } & \text { Entity Name }\end{array}$

Additional Pointers as required (see 2.2.4.4.2) 
406, Form 16 - DRAWING SIZE

\subsection{FORM NUMBER: 16 Drawing Size \\ DESCRIPTION}

This property specifies the size of the drawing in drawing units. The origin of the drawing is defined to be $(0,0)$ in drawing space.

$\begin{array}{clll}\text { Index } & \text { Name } & \text { Type } & \text { Description } \\ 1 & \text { Integer } & \begin{array}{l}\text { No. of property Values } \\ (\mathrm{NP}=2)\end{array} \\ 2 & \text { XS } & \text { Real } & \begin{array}{l}\text { X Size (Extent of Drawing } \\ \text { along positive XD axis) }\end{array} \\ 3 & \text { YS } & \text { Real } & \begin{array}{l}\text { Y Size (Extent of Drawing } \\ \text { along positive YD AXIS) }\end{array}\end{array}$

Additional Pointers as required (see 2.2.4.4.2) 
406, Form 17 - DRAWING UNITS

\subsection{FORM NUMBER: 17 Drawing Units}

\section{DESCRIPTION}

This property specifies the drawing space units as outlined in the drawing entity (Type 404). The drawing units are given in the same form as the model space units in the global section (see 2.2.4.2.14).

$\begin{array}{clll}\text { Index } & \text { Name } & \text { Type } & \text { Description } \\ 1 & \text { NP } & \text { Integer } & \begin{array}{l}\text { No. of Property Values } \\ (\mathrm{NP}=2)\end{array} \\ 2 & \text { FLAG } & \text { Integer } & \text { Units Flag } \\ 3 & \text { UNIT } & \text { String } & \text { Units Name }\end{array}$

Additional Pointers as required (see 2.2.4.4.2). 
The following definition entities are used to provide a "template" for the instances of subfigures (see 2.5.1).

\subsubsection{Subfigure Definition Entity.}

The subfigure definition entity is designed to support the concept of a subpicture (if one equates drawing creation with graphics picture processing). This entity permits a single definition of a detail to be utilized in multiple instances in the creation of the whole picture. The contents of the subfigure include a set of pointers to any combination of entities and other subfigures. DEPTH indicates the actual nesting of the subfigures. If $\mathrm{DEPTH}=0$, the subfigure has no references to any subfigure instances. A subfigure cannot reference a subfigure instance that has equal or greater depth. A DEPTH $=\mathrm{N}$ indicates there is a reference to an instance of a subfigure definition with DEPTH $\mathrm{N}-1$.

\subsubsection{1}

Directory Data

ENTITY TYPE NUMBER : 308

\subsection{Parameter Data}

\begin{tabular}{|c|c|c|c|}
\hline Index & Name & Type & Description \\
\hline 1 & DEPTH & Integer & $\begin{array}{l}\text { Depth of subfigure (indicat- } \\
\text { ing the amount of nesting) }\end{array}$ \\
\hline 2 & NAME & String & Subfigure name \\
\hline 3 & $N$ & Integer & $\begin{array}{l}\text { Number of entities in the } \\
\text { subfigure }\end{array}$ \\
\hline 4 & $\mathrm{DE}$ & Pointer & $\begin{array}{l}\text { Pointers to the directory en- } \\
\text { tries for the associated }\end{array}$ \\
\hline • & • & - & entities \\
\hline - & - & - & \\
\hline $\mathrm{N}+3$ & - & Pointer & $\begin{array}{l}\text { Pointer to the last directory } \\
\text { entry }\end{array}$ \\
\hline
\end{tabular}

Additional Pointers as required (see 2.2.4.4.2). 
4.3.8.2 Network Subfigure Definition Entity

The Network Subfigure Definition entity permits a single definition of a detail to be used in many instances in the file, similar to the Subfigure Definition entity (Type 308). It differs from the ordinary Subfigure Definition in that it defines a specialized subfigure, one whose instances may participate in networks. To participate in a network, points of connection (Connect Point entity Type 132) must be defined (see indices NA+7 and $\mathrm{NA}+\mathrm{NC}+7)$ and instanced along with the subfigure. Often, products which contain networks are designed first as schematics (showing the logical connections or relationships), which are then converted into the designs of the physical products. Whenever both a logical design and a physical design are present in the same file, the processor needs a way to determine which entities belong in which design. The Type Flag field (index NA+4) implements this distinction. Other fields, such as NAME and DEPTH, function in exactly the same manner as the Subfigure Definition (308).

Note: the depth of the subfigure is inclusive of both Network Subfigures entity (Type 320) and the ordinary subfigure entity (Type 308). Thus, the two may be nested but must indicate that in the depth parameter.

\subsection{Directory Data}

ENTITY TYPE NUMBER: 320

\subsection{Parameter Data}

\begin{tabular}{|c|c|c|c|}
\hline Index & $\underline{\text { Name }}$ & Type & Description \\
\hline 1 & DEPTH & Integer & $\begin{array}{l}\text { Depth of subfigure } \\
\text { (indicating the amount of } \\
\text { nesting) }\end{array}$ \\
\hline 2 & NAME & String & Subfigure name \\
\hline
\end{tabular}




\begin{tabular}{|c|c|c|c|}
\hline Index & Name & Type & Description \\
\hline 3 & NA & Integer & $\begin{array}{l}\text { Number of associated (child) } \\
\text { entities in the subfigure } \\
\text { exclusive of Primary } \\
\text { Reference Designator and } \\
\text { connect points. }\end{array}$ \\
\hline 4 & APTR 1 & Pointer & $\begin{array}{l}\text { Pointer to the directory } \\
\text { entry of the first associated } \\
\text { entity. }\end{array}$ \\
\hline$\dot{.}$ & & & \\
\hline $\mathrm{NA}+3$ & APTRNA & Pointer & $\begin{array}{l}\text { Pointer to the directory } \\
\text { entry for the associated } \\
\text { entity NA. }\end{array}$ \\
\hline $\mathrm{NA}+4$ & TF & Integer & $\begin{array}{l}\text { Type Flag: } \\
\begin{aligned} 0 & =\text { not specified } \\
1 & =\text { logical } \\
2 & =\text { physical }\end{aligned}\end{array}$ \\
\hline $\mathrm{NA}+5$ & PRD & String & Primary reference designator \\
\hline $\mathrm{NA}+6$ & DPTR & Pointer & $\begin{array}{l}\text { Pointer to the directory } \\
\text { entry of the primary } \\
\text { reference designator Text } \\
\text { Display Template }\end{array}$ \\
\hline $\mathrm{NA}+7$ & NC & Integer & $\begin{array}{l}\text { Number of associated (child) } \\
\text { Connect Point entities }\end{array}$ \\
\hline $\begin{array}{l}\mathrm{NA}+8 \\
\dot{\cdot}\end{array}$ & $\begin{array}{l}\text { CPTR } 1 \\
\cdot \\
\cdot\end{array}$ & $\begin{array}{l}\text { Pointer } \\
\text {. }\end{array}$ & $\begin{array}{l}\text { Pointer to the directory } \\
\text { entry for the associated } \\
\text { Connect Point } 1 \text { Connect }\end{array}$ \\
\hline $\mathrm{NA}+\mathrm{NC}+7$ & CPTRNC & Pointer & $\begin{array}{l}\text { Pointer to the directory } \\
\text { entry for the associated } \\
\text { Connect Point NC }\end{array}$ \\
\hline
\end{tabular}

Additional Pointers as required (see 2.2.4.4.2). 


\subsubsection{Subfigure Instances}

4.3.9.1 Singular Subfigure Instance Entity

This entity defines the occurrence of a single instance of the defined subfigure (Type 308). See Figure 4-40 and Section 2.5.1.

\subsection{Directory Data}

ENTITY TYPE NUMBER: 408

\subsection{Parameter Data}

\begin{tabular}{|c|c|c|c|}
\hline Index & Name & Type & Description \\
\hline 1 & $\mathrm{DE}$ & Pointer & $\begin{array}{l}\text { Pointer to subfigure } \\
\text { definition entry }\end{array}$ \\
\hline 2 & $\mathrm{X}$ & Real & $\begin{array}{l}\text { Translation data relative to } \\
\text { either model space or to the } \\
\text { definition space of a } \\
\text { referring entity }\end{array}$ \\
\hline 3 & $\mathrm{Y}$ & Real & $"$ \\
\hline 4 & Z & Real & $"$ \\
\hline 5 & $\mathrm{~S}$ & Real & Scale factor (default $=1.0$ ) \\
\hline
\end{tabular}

Additional Pointers as required (see 2.2.4.4.2).

4.3.9.2 Rectangular Array Subfigure Instance Entity.

The rectangular array produces copies of an object called the base entity, arranging them in equally spaced rows and columns. The following types of entities are valid for use as a base entity: group associativity, point, line, circular arc, conic arc, parametric spline curve, rational B-spline curve, any annotation entity, rectangular array instance, circular array instance, or subfigure definition. The number of columns and rows of the rectangular array, together with their respective horizontal and vertical displacements are given. Also, the coordinates of the lower left hand corner for the entire array is indicated. This is where the first entity in the reproduction process is placed and is called position No. 1. The successive positions are counted vertically up the first column, then vertically up the second column to the right, and so on. 
The array of instance locations for the base entity is rotated about the line through the point $(X, Y)$, parallel to the $Z T$-axis. The angle of rotation is specified in radians counterclockwise from the positive XT-axis. The instances of the base entity are not rotated from their original orientation.

A DO-DON'T flag enables one to display only a portion of the array. If the DO flag is chosen, half or fewer of the elements of the rectangular array are to be defined. If the DON'T flag is chosen, half or more of the elements of the rectangular array are to be defined.

\subsection{Directory Data}

ENTITY TYPE NUMBER: $\quad 412$

\subsection{Parameter Data}

$\begin{array}{ccl}\text { Index } & \text { Name } & \text { Type } \\ 1 & \text { DE } & \text { Pointer } \\ 2 & \text { S } & \text { Real } \\ 3 & X & \text { Real } \\ 4 & \text { Y } & \text { Real } \\ 5 & Z & \text { Real } \\ 6 & \text { NC } & \text { Integer } \\ 7 & \text { NR } & \text { Integer } \\ 8 & \text { DX } & \text { Real } \\ 9 & \text { DY } & \text { Real } \\ 10 & \text { AX } & \text { Real } \\ 11 & \text { LC } & \text { Integer }\end{array}$

\section{Description}

Pointer to base entity

Scale factor (default $=$ 1.0)

Coordinates of point

to be used as lower

left hand corner of

array

Number of columns

Number of rows

Horizontal distance between columns

Vertical distance between rows

Rotation angle in radians

DO-DON'T list count $=\mathrm{L}$.

( $L=0$ indicates all to be displayed.) 


\begin{tabular}{|c|c|c|c|}
\hline Index & $\underline{\text { Name }}$ & Type & Description \\
\hline 12 & DDF & Integer & $\begin{array}{l}\text { DO-DON'T flag } \\
(D O=0 ; D O N ' T=1)\end{array}$ \\
\hline 13 & N1 & Integer & $\begin{array}{l}\text { Number of first } \\
\text { position to be processed } \\
\text { (DO), or to be pro- } \\
\text { cessed (DON'T) }\end{array}$ \\
\hline $12+L C$ & NLC & Integer & Number of last position \\
\hline
\end{tabular}




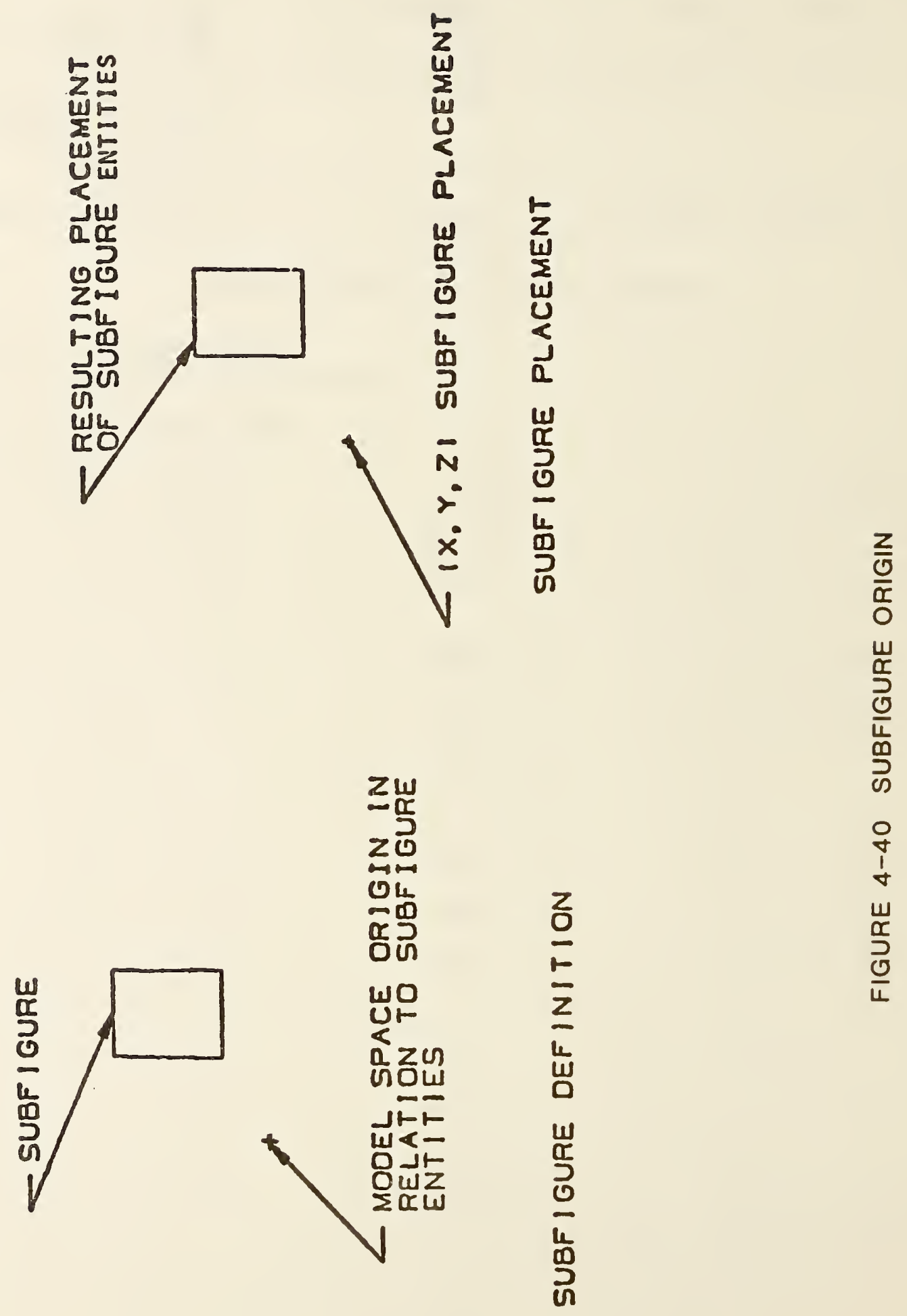


4.3.9.3 Circular Array Subfigure Instance Entity

The circular array produces copies of an object called the base entity, arranging them around the edge of an. imaginary circle whose center and radius are specified. The following types of entities are valid for use as a base entity: group associativity, point, line, circular arc, conic arc, parametric spline curve, rational B-spline curve, any annotation entity, rectangular array instance, circular array instance, or subfigure definition. The number of possible instance locations for the base entity is specified and the location of the first instance position is specified in terms of a radius and a start angle measured positive, counterclockwise in radians from the line through the point $(\mathrm{X}, \mathrm{Y})$, parallel to the $\mathrm{ZT}$-axis. The successive positions follow a counterclockwise direction around the imaginary circle and are distributed according to a given delta angle.

A DO-DON'T flag enables one to display only a portion of the array. If the DO-flag is chosen, half or fewer of the elements of the circular array are to be defined. If the DON'T-flag is chosen, half or more of the elements of the circular array are to be defined.

\subsection{Directory Data}

ENTITY TYPE NUMBER: $\quad 414$

\subsection{Parameter Data}

\begin{tabular}{|c|c|c|c|}
\hline Index & Name & Type & Description \\
\hline 1 & $\mathrm{DE}$ & Pointer & Pointer to base entity \\
\hline 2 & $\mathrm{NE}$ & Integer & $\begin{array}{l}\text { Total number of } \\
\text { possible instance } \\
\text { locations }\end{array}$ \\
\hline $\begin{array}{l}3 \\
4\end{array}$ & $\begin{array}{l}X \\
Y\end{array}$ & $\begin{array}{l}\text { Real } \\
\text { Real }\end{array}$ & $\begin{array}{l}\text { Coordinates of center } \\
\text { of imaginary circle }\end{array}$ \\
\hline $\begin{array}{l}5 \\
6\end{array}$ & $\begin{array}{l}Z \\
\mathrm{R}\end{array}$ & $\begin{array}{l}\text { Real } \\
\text { Real }\end{array}$ & $\begin{array}{l}\text { Radius of imaginary } \\
\text { circle }\end{array}$ \\
\hline 7 & AS & Real & Start angle in radians \\
\hline 8 & AD & Real & Delta angle in radians \\
\hline
\end{tabular}


414 - CIRCULAR ARRAY

SUBFIGURE INSTANCE

\begin{tabular}{|c|c|c|c|}
\hline Index & $\underline{\text { Name }}$ & Type & Description \\
\hline 9 & LC & Integer & $\begin{array}{l}\text { DO-DON'T list count }= \\
\mathrm{L} \text {. } \\
(\mathrm{L}=0 \text { indicates all repli- } \\
\text { cated entities to be dis- } \\
\text { played })\end{array}$ \\
\hline 10 & DDF & Integer & $\begin{array}{l}\text { DO-DON'T Flag } \\
(\mathrm{DO}=0 ; \mathrm{DON} T=1)\end{array}$ \\
\hline 11 & N1 & & $\begin{array}{l}\text { Number of first } \\
\text { position to be processed } \\
\text { (DO), or to be not pro- } \\
\text { cessed (DON'T) }\end{array}$ \\
\hline - & • & • & • \\
\hline - & - & • & - \\
\hline - & $\cdot$ & - & - \\
\hline $10+L C$ & NLC & Integer & Number of last position \\
\hline
\end{tabular}


4.3.9.4 Network Subfigure Instance Entity

Each instance of a Network Subfigure Definition Entity is specified by a Network Subfigure Instance Entity. It functions and may be used as described in section 2.5.1.

In addition though, the points of connection (connect Point Entity Type 132) specified by the Network Subfigure Definition Entity must be instanced and associated with each Network Subfigure Instance (see indices 11 and 12).

The Type Flag field (index 8) implements the distinction between logical design and physical design data if both may be present in the file.

The Network Subfigure Instance Entity allows different scale factor in the $x$, $y$, and $z$ axes. This scaling is performed before the translation from $X, Y$, and $Z$ and before the Transformation matrix entity pointed to in the directory entry (if any) is applied. The scaling does not apply to the model space placement coordinates $(X, Y, Z)$.

\subsection{Directory Data}

ENTITY TYPE NUMBER: $\quad 420$

\subsection{Parameter Data}

$\begin{array}{cccc}\text { Index } & \text { Name } & \text { Type } & \text { Description } \\ 1 & \text { PE } & \text { Real } & \begin{array}{l}\text { Pointer to network } \\ \text { definition entry }\end{array} \\ 3 & Y & \begin{array}{l}\text { Translation data } \\ \text { relative to either model } \\ \text { space or to the } \\ \text { definition space of a } \\ \text { referring entity }\end{array} \\ 4 & \mathrm{Z} & \text { Real } & \text { " }\end{array}$




\begin{tabular}{|c|c|c|c|}
\hline Index & Name & Type & Description \\
\hline 5 & $\mathrm{XS}$ & Real & $\begin{array}{l}\text { Scale factor in } \\
\text { definition space } x \text { axis } \\
\text { (default } 1.0 \text { ) }\end{array}$ \\
\hline 6 & YS & Real & $\begin{array}{l}\text { Scale factor in } \\
\text { definition space y axis } \\
\text { (default XS) }\end{array}$ \\
\hline 7 & ZS & Real & $\begin{array}{l}\text { Scale factor in } \\
\text { definition space } z \text { axis } \\
\text { (default XS) }\end{array}$ \\
\hline 8 & TF & Integer & $\begin{array}{l}\text { Type flag: } \\
0 \text { - not specified } \\
1 \text { - logical } \\
2 \text { - physical }\end{array}$ \\
\hline 9 & PDR & String & $\begin{array}{l}\text { Primary reference } \\
\text { designator }\end{array}$ \\
\hline 10 & DPTR & Pointer & $\begin{array}{l}\text { Pointer to the directory } \\
\text { entry of the primary } \\
\text { reference designator } \\
\text { Text Display Template }\end{array}$ \\
\hline 11 & NC & Integer & $\begin{array}{l}\text { Number of associated } \\
\text { (child) Connect Point } \\
\text { entities }\end{array}$ \\
\hline $\begin{array}{c}12 \\
.\end{array}$ & $\begin{array}{l}\text { CPTR } 1 \\
. \\
\end{array}$ & $\begin{array}{l}\text { Pointer } \\
\text {. }\end{array}$ & $\begin{array}{l}\text { Pointer to the directory } \\
\text { entry for associated } \\
\text { Connect Point } 1\end{array}$ \\
\hline $\mathrm{NC}+11$ & CPTRNC & Pointer & $\begin{array}{l}\text { Pointer to the directory } \\
\text { entry for associated } \\
\text { Connect Point NC }\end{array}$ \\
\hline
\end{tabular}

Additional Pointers as required (see 2.2 .4 .4 .2 ) 
This entity defines the appearance of characters in a text font. The data.describing the appearance of a character may be located by the Font Code (FC) and the ASCII character code. This entity may describe any or all the characters in a character set. Thus, this entity may be used to describe a complete font or a modification to a subset of characters in another font. Font Number and Font Name are the number and name used to reference the font on the originating system. When this entity is a modification to another font, the Supersedes Font value (Parameter 3) indicates which font the entity modifies. This value is an integer which indicates the font number to be modified or the negative of the pointer value to the directory entry of another text font definition entity. When this entity modifies another font, i.e., Parameter 3 references another font, the definitions in this entity supersede the definition in the original font. For example, a complete set of characters may have their font definition specified by this entity. Another text font definition entity could reference the first definition and modify a subset of the characters.

Each character is defined by overlaying an equally spaced square grid over the character. The character is decomposed into straight line segments which connect grid points. Grid points are referenced by standard Cartesian coordinates. The position of the character relative to the grid is defined by two points. The character's origin point is placed at the origin $(0,0)$ of the grid and defines the position of the character relative to the text origin of that character. The second point defines the origin point of the character following the character being defined. This allows the spacing between characters to be specified. Construction of text strings consists of placing the character origin of the first character at the text string origin and placing subsequent character origins at the location specified in the previous character as the location of the next character's origin. 
The parameterization of the character appearance is described by the motion of an imaginary pen moving between grid points. Commands to move the pen reference the grid location to which the pen is to move. The pen may be "lifted" such that its movement is not displayed. The representation of the movement of the pen is a sequence of pen commands and grid locations. Each movement of the pen is represented by a pen updown flag and a pair of integer grid coordinates. The pen up/down flag defaults to pen down. A flag value of 1 means the pen is to be lifted (i.e., display off) and moved to the next location in the sequence. Upon arrival at this location the pen is returned to a "down" position (i.e., display on)

The grid size is related to the text height through the scale parameter. This parameter defines how many grid units equal one text height unit.

\subsubsection{Directory Data}

ENTITY TYPE NUMBER : $\quad 310$

\subsubsection{Parameter Data}

\begin{tabular}{|c|c|c|c|}
\hline Index & Name & Type & Description \\
\hline 1 & FC & Integer & Font Number \\
\hline 2 & FNAME & String & Font Name \\
\hline 3 & SF & Integer & $\begin{array}{l}\text { Number of the font which } \\
\text { this definition supersedes }\end{array}$ \\
\hline 4 & SCALE & Integer & $\begin{array}{l}\text { Number of grid units which } \\
\text { equal one text height unit }\end{array}$ \\
\hline 5 & $\mathrm{~N}$ & Integer & $\begin{array}{l}\text { Number of characters in this } \\
\text { definition }\end{array}$ \\
\hline 6 & $\mathrm{ACl}$ & Integer & $\begin{array}{l}\text { ASCII code for first } \\
\text { character }\end{array}$ \\
\hline 7 & NX1 & Integer & $\begin{array}{l}\text { Grid location of the next } \\
\text { character's origin }\end{array}$ \\
\hline
\end{tabular}


$\underline{\text { Index }}$

Name

8

9

10

11

$X 1_{1}$

NY 1

NM1

Intege

Integer

Integer

12

$\mathrm{Y}_{1}$

Integer

$9+\mathrm{NM} 1 * 3$

AC2

NX2

NY2

NM2

$12+\mathrm{NM} 1 * 3$

$5+4 \mathrm{~N}+\sum_{\mathrm{i}=1}^{\mathrm{N}} 3 * \mathrm{NMi}$

Type

Integer

Integer

Integer
Description

Number of pen motions for first character

Pen up flag $0=$ Down, 1 = Up

Grid location to which the pen is to move

ASCII code for second character

Grid location of the next character origin

Number of pen motions for second character
Last grid location of last character

Additional Pointers as required (see 2.2.4.4.2). 
An example of this entity using the character in Figure 4-41 is

\begin{tabular}{|c|c|}
\hline $\mathrm{FC}$ & 1 \\
\hline FNAME & 8H STANDARD \\
\hline \multicolumn{2}{|l|}{$\mathrm{SF}$} \\
\hline SCALE & 8 \\
\hline $\mathrm{N}$ & 60 \\
\hline $\mathrm{ACl}$ & 65 \\
\hline NX1 & 11 \\
\hline NY 1 & 0 \\
\hline NM 1 & 4 \\
\hline PF 1 & 0 \\
\hline $\mathrm{X} 1$ & 4 \\
\hline Y1 & 8 \\
\hline PF2 & 0 \\
\hline $\mathrm{x} 2$ & 8 \\
\hline Y2 & 0 \\
\hline PF 3 & 1 \\
\hline$\times 3$ & 2 \\
\hline Y3 & 4 \\
\hline PF4 & 0 \\
\hline $\mathrm{X} 4$ & 6 \\
\hline$Y 4$ & 4 \\
\hline • & \\
\hline - & \\
\hline . & \\
\hline
\end{tabular}

In the parameter section of the IGES file it would look like: 1,8HST ANDARD, , 8,60,65, $11,0,4,4,4,8,8,0,1,2,4,, 6,4 \ldots$.

Figure 4-42 provides another example. 


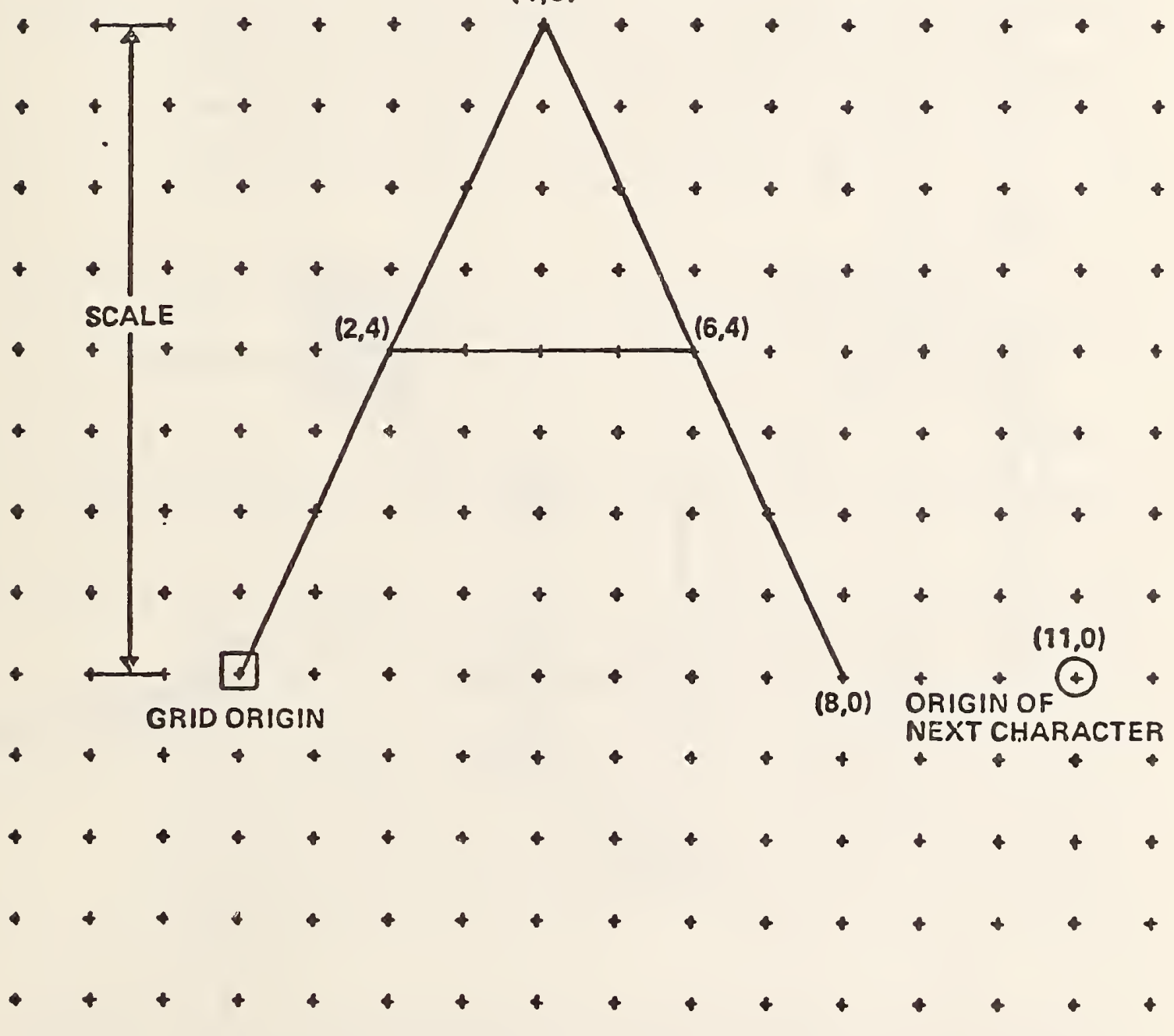

FIGURE 4-41 EXAMPLE OF A CHARACTER DEFINITION 


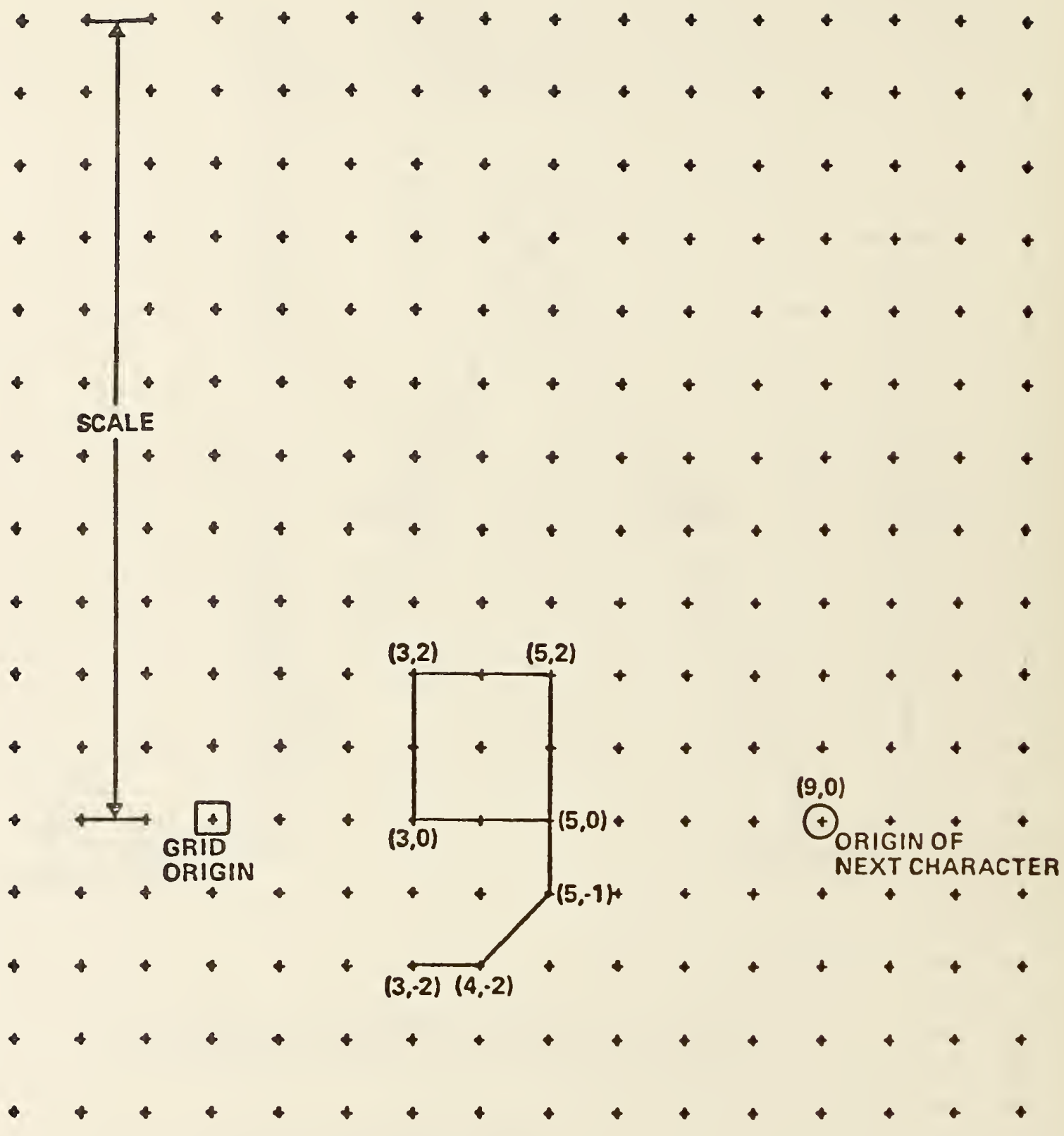

FIGURE 4-42 SECOND CHARACTER DEFINITION EXAMPLE 


\subsubsection{View Entity.}

The View Entity defines a framework for specifying a viewing orientation of an object in three dimensional model space $(X, Y, Z)$. The framework is also used to support the projection of all or part of model space onto a view plane. One type of projection, an orthographic parallel projection, can be specified.

4.3.11.1 Orthographic Parallel Projection. An orthographic parallel projection onto a view plane of an object in model space is formed by passing rays normal to the view plane through each point of the object and finding the intersection with the view plane as shown in Figure 4-43.

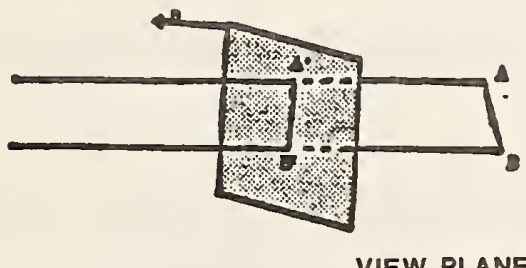

VIEW PLANE

Figure 4-43 Orthographic Parallel Projection

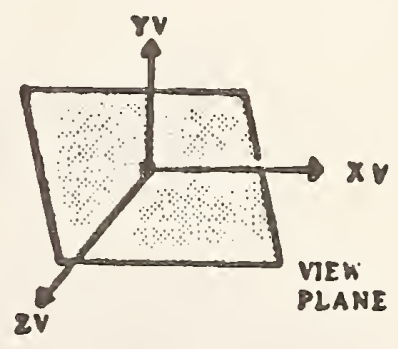

Figure 4-44 View Origin

4.3.11.2 View Coordinate System. The view plane can be described by introducing a right-handed view coordinate system, (XV, YV, ZV) into model space. The view plane is, the $X V, Y V$ plane, i.e., the plane $Z V=0$. The view direction is along the positive $Z V$ axis toward the view plane, i.e., in the direction of the vector $(0,0,-1)$. The positive $Y V$ axis points in the "up" direction in the resulting view. The point $(0,0,0)$ in the view coordinate system as shown in Figure 4-44 is called the view origin. Thus a complete viewing orientation is specified by a view coordinate system.

4.3.11.3 View Coordinates Obtained from Model Coordinates. View coordinates are obtained from model coordinates through translation and rotation. There are several ways that systems use to specify the data required to transfer from model to view coordinates. However, in each case, the data can be recorded using Form 0 of the Transformation Matrix entity such that the model coordinates are taken as input and the view coordinates are produced as output, as follows, where $\mathrm{R}$ denotes the rotation matrix and $\mathrm{T}$ the translation vector (see 3.14). 


$$
\left[\begin{array}{l}
X V \\
Y V \\
Z V
\end{array}\right]=[R]\left[\begin{array}{l}
X \\
Y \\
Z
\end{array}\right]+[T]
$$

In this situation, $\mathrm{R}$ is called the view matrix.

The View entity specifies the view matrix and the translation vector by use of a pointer to a Transformation Matrix entity in field 7 of the Directory Entry. In the special case when the view matrix is the identity matrix and there is zero translation, a zero value in field 7 may be used.

Example 1: (View coordinates obtained from model coordinates by a translation and then a rotation.)

The system defines a viewing orientation by specifying a view origin $(\mathrm{XO}, \mathrm{YO}, \mathrm{ZO})$ in model space and a rotation matrix so that

$$
\left[\begin{array}{l}
X V \\
Y V \\
Z V
\end{array}\right]=\left[\begin{array}{l}
3 \times 3 \text { rotation } \\
\text { Matrix }
\end{array}\right] \quad\left(\left[\begin{array}{l}
X \\
Y \\
Z
\end{array}\right]-\left[\begin{array}{l}
X O \\
Y O \\
Z O
\end{array}\right]\right)
$$

or,

$$
\left[\begin{array}{l}
\mathrm{XV} \\
\mathrm{YV} \\
\mathrm{ZV}
\end{array}\right]=\left[\begin{array}{l}
3 \times 3 \text { rotation } \\
\text { Matrix }
\end{array}\right]\left[\begin{array}{l}
\mathrm{X} \\
\mathrm{Y} \\
\mathrm{Z}
\end{array}\right]-\left[\begin{array}{l}
3 \times 3 \text { rotation } \\
\text { Matrix }
\end{array}\right]\left[\begin{array}{l}
\mathrm{XO} \\
\mathrm{YO} \\
\mathrm{ZO}
\end{array}\right] .
$$

Therefore, the rotation matrix is the view matrix, and in the Transformation Matrix entity,

$$
R=\left[\begin{array}{l}
3 \times 3 \text { rotation } \\
\text { Matrix }
\end{array}\right], T=-\left[\begin{array}{l}
3 \times 3 \text { rotation } \\
\text { Matrix }
\end{array}\right]\left[\begin{array}{l}
x O \\
\text { Yo } \\
z O
\end{array}\right]
$$


Example 2: (View coordinates obtained from model coordinates by a rotation and then a translation.)

The system defines a viewing orientation by specifying a rotation matrix and a translate or pan vector $(X L, Y L, Z L)$ expressed in the rotated coordinate system, so that

$$
\left[\begin{array}{l}
\mathrm{XV} \\
\mathrm{YV} \\
\mathrm{ZV}
\end{array}\right]=\left[\begin{array}{l}
3 \times 3 \text { rotation } \\
\text { Matrix }
\end{array}\right]\left[\begin{array}{l}
\mathrm{X} \\
\mathrm{Y} \\
\mathrm{Z}
\end{array}\right]-\left[\begin{array}{c}
\mathrm{XL} \\
\mathrm{YL} \\
\mathrm{ZL}
\end{array}\right] \text {. }
$$

Therefore, the rotation matrix is the view matrix, and in the Transformation Matrix entity,

$R=\left[\begin{array}{l}3 \times 3 \text { rotation } \\ \text { Matrix }\end{array}\right], \quad T=-\left[\begin{array}{c}\mathrm{XL} \\ \mathrm{YL} \\ \mathrm{ZL}\end{array}\right]$

4.3.11.4 Simple Form of the View. Entity. The View entity provides a view number for the purpose of identifying differing view orientations. However, no standard indexing scheme is presumed to exist.

In its simplest form, the View entity consists of a pointer to the Transformation Matrix entity (in field 7 or the Directory Entry), and a view number. The Transformation Matrix entity specifies a view matrix $\mathrm{R}$ and a translation vector $\mathrm{T}$ as given in the preceeding section.

4.3.11.5 Projection of a View Volume. In some cases, a view volume and a scale factor may be required to control the projection of the view into a two dimensional drawing space specified by a Drawing entity (see 4.3.4).

The view volume bounds that portion of the data which will be projected after clipping is performed. The view volume is a rectangular parallelepiped with limits specified by Plane entities defined in the model coordinate system. The absence of a clipping in a particular direction may be included by setting the pointer for the appropriate Plane entity equal to zero. 
The Plane entities used to define the view volume shall not be artibrary planar definitions. After transformation from model coordinates to view coordinates each plane must result in a plane perpendicular to the appropriate view coordinate system axis (e.g., the left side of the view volume must transform into a plane $X V=$ constant). In addition, only the coefficients of the Plane entity are required, i.e., the bounding curve and display symbol are not used. See Figure 4-45.

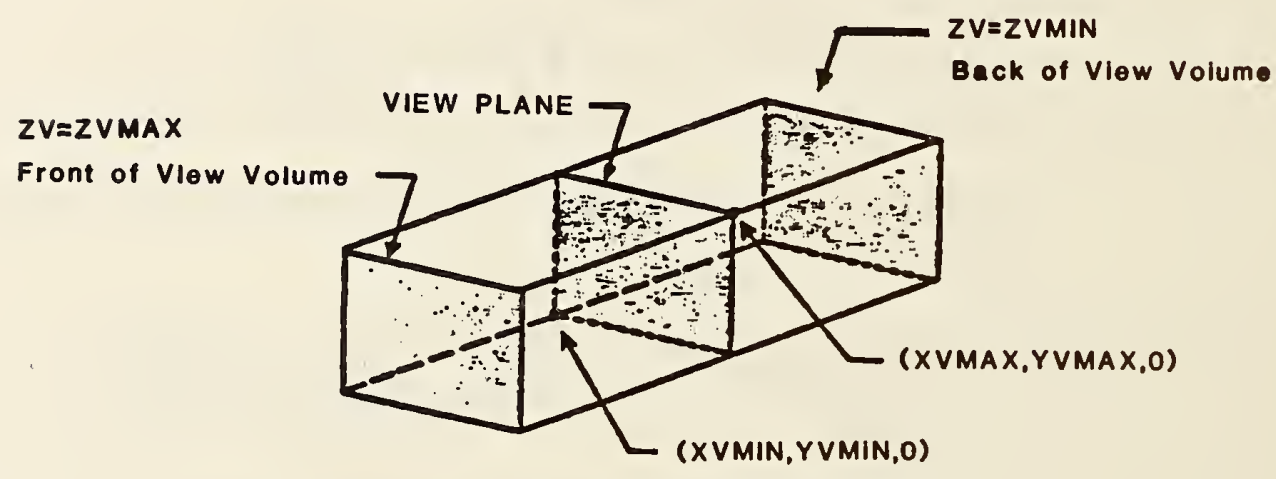

FIGURE 4-45 VIEW VOLUME

4.3.11.6 Projection Operations. The order of operations for the view entity is as follows:

1. Transform from model to view space.

2. Perform clipping (if included).

3. Perform projection onto the view plane.

4. Transform from view space to drawing space.

The projection onto the view plane and the transform from view space to drawing space can be controlled by the following equation in the case of or thographic parallel projection, where $S$ is the scale factor and XORIGIN and YORIGIN are defined in the Drawing Entity (see 4.3.4).

$$
\left[\begin{array}{c}
X D \\
Y D
\end{array}\right]=\left[\begin{array}{lll}
\text { S O } & 0 \\
\text { O } & S & O
\end{array}\right]\left[\begin{array}{l}
X V \\
Y V \\
Z V
\end{array}\right]+\left[\begin{array}{l}
\text { XORIGIN } \\
\text { YORIGIN }
\end{array}\right]
$$


4.3.11.7 Entity Display. The display of an entity in a particular view is controlled by the use of the view value in field 6 of the Directory Entry for the entity. If this value is zero or undefined, the entity is displayed with its own characteristics in all views unless display is controlled by other parameters (e.g., pointed to by another entity such as Subfigure Definition or Drawing). If this value is a pointer to a View entity, the entity is displayed with its own characteristics in only the one view.

The selection of multiple views and/or display characteristics for an entity may be made by using one of the Views Visible associativity entities (type number 402 , form 3 or 4 ). The view value for the entity then is a pointer to this associativity instead of to a View entity.

\subsubsection{Directory Data}

ENTITY TYPE NUMBER: 410

\subsubsection{Parameter Data}

\begin{tabular}{|c|c|c|c|}
\hline Index & Name & Type & Description \\
\hline 1 & VNO & Integer & View number \\
\hline 2 & SCALE & Real & $\begin{array}{l}\text { Scale factor } \\
(\text { Default }=1.0)\end{array}$ \\
\hline 3 & XVMINP & Pointer & $\begin{array}{l}\text { Pointer to left } \\
\text { side of view } \\
\text { volume (XVMIN } \\
\text { plane) or zero }\end{array}$ \\
\hline 4 & YVMAXP & Pointer & $\begin{array}{l}\text { Pointer to top of } \\
\text { view volume } \\
\text { (YVMAX plane) or } \\
\text { zero }\end{array}$ \\
\hline 5 & XVMAXP & Pointer & $\begin{array}{l}\text { Pointer to right } \\
\text { side of view } \\
\text { volume (XVMAX } \\
\text { plane) or zero }\end{array}$ \\
\hline 6 & YVMINP & Pointer & $\begin{array}{l}\text { Pointer to bottom } \\
\text { of view volume } \\
\text { (YVMIN plane) or } \\
\text { zero }\end{array}$ \\
\hline
\end{tabular}


410 - VIEW

7
ZVMINP

ZVMAXP

Pointer

Additional Pointers as required (see 2.2.4.4.2)
Pointer to back of view volume (ZVMIN plane) or zero

Pointer to front of view volume (ZVMAX plane) or zero 


\subsubsection{External Reference Entity}

The External Reference Entity provides a link between an entity in a referencing file and the definition or a logically related entity in referenced file. Further, in keeping with the concept of treating this entity as the definition which it replaces the subordinate entity switch should be set as it would be on the definition replaced. See section 2.5.4 for the entities used in the linkage.

Three forms of the External Reference Entity are defined. Two of these forms are used to reference a definition and one form is a logical reference. Form 0 is used when a single definition from the referenced file is desired. This would be the case where the referenced file contained a collection of definitions. Form 1 is used when the entire file is to be instanced. This would be the case where the referenced file contained a complete sub-assembly. Form 2 is used for external logical references where an entity in one file relates to an entity in a separate file (e.g., when each sheet of a drawing is a separate file, and a flange on one sheet is also depicted on, or mates with, a flange on another sheet). Forms 0 and 2 require an entity-unique symbolic name. The following entities and the parameter which supplies the symbolic name are identified for use:

Entity

132

302

304

306

308

310

312

314

320
Name

Connect Point

Associativity Definition Line Font Definition MACRO Definition Subfigure Definition Text Font Definition Text Display Template Color Definition Network Subfigure Definition
Parameter Index

\section{Description}

CP Function Name (unique)

Implementor assigned

Implementor assigned Entity Type Identification Subfigure name (unique)

Font name Implementor assigned Implementor assigned Subfigure name (unique) 
Possible alternatives for the entity-unique symbolic name for those entities marked "Implementor assigned" could be: (1) the property REFERENCE DESIGNATOR (Type 406, Form 7), or (2) the ENTITY LABEL, ENTITY SUBSCRIPT (directory entry fields 18 and 19.)

\subsubsection{Directory Data \\ ENTITY TYPE NUMBER: 416}

\subsubsection{Parameter Data (Form 0 and 2)}

\begin{tabular}{|c|c|c|c|}
\hline Index & Name & Type & Description \\
\hline 1 & EXTFID & String & $\begin{array}{l}\text { External Reference File } \\
\text { Identifier (contained } \\
\text { as Global Parameter } \\
\text { Number } 4 \text { in the referenced } \\
\text { file) }\end{array}$ \\
\hline 2 & EXTNAM & String & $\begin{array}{l}\text { External Reference Entity } \\
\text { Symbolic Name }\end{array}$ \\
\hline
\end{tabular}

Additional Pointers as required (see $2 \cdot 2.4 .4 .2)$

\subsubsection{Parameter Data (Form 1)}

\begin{tabular}{|c|c|c|c|}
\hline Index & Name & Type & Description \\
\hline 1 & EXTFID & String & $\begin{array}{l}\text { External Reference File } \\
\text { Identifier (contained } \\
\text { as Global Parameter } \\
\text { Number } 4 \text { in the referenced } \\
\text { file) }\end{array}$ \\
\hline
\end{tabular}

Additional Pointers as required (see 2.2.4.4.2) 
This entity relates loads and/or constraints to specific nodes in the Finite Element Model. This is accomplished by creating a relation between node entities and the tabular data property that contains the load or constraint data. Each load and constraint case will require a nodal load/constraint entity and a tabular data property Form 11 with PTYPE $=12$.

Figure 4-45 shows the relationship or linkage between the nodal load/constraint entity and the tabular data which carries the load or constraint vector. The relationship or linkage is also shown on the general note entity which described the load/constraint test case being performed. There is a one to one correspondence between the load case description and the general note entity and the pointer to the tabular data containing the load case magnitudes (see also section 2.5.6).

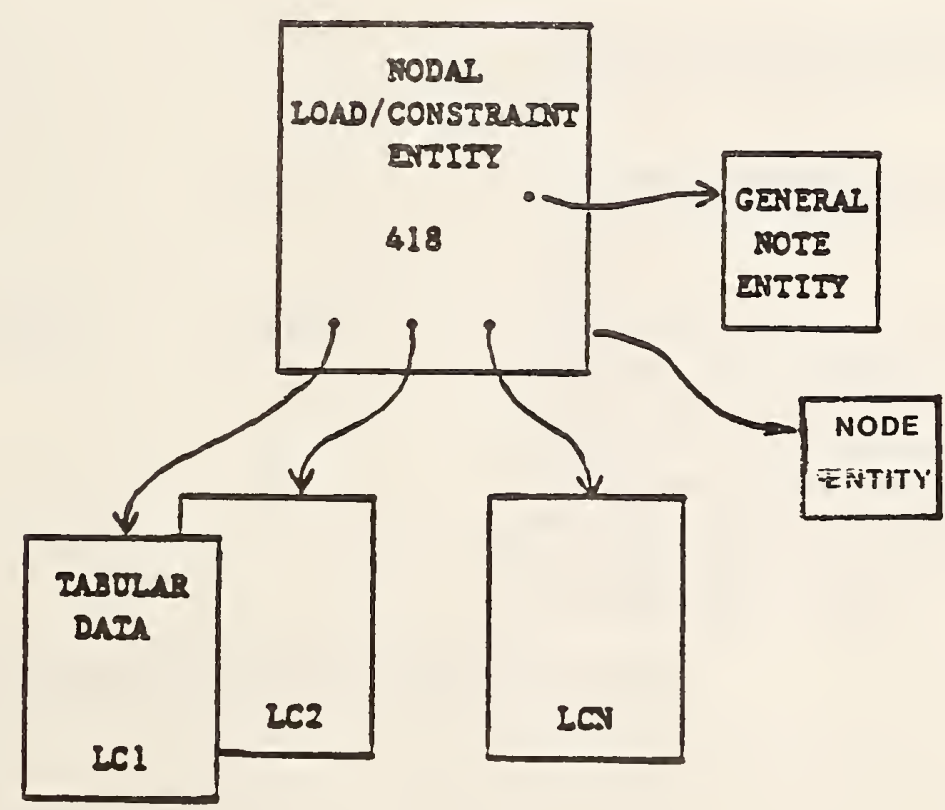

FGURE 4-46 NODAL LOAD / CONSTRAINT 
418 - NODAL LOAD/CONSTRAINT

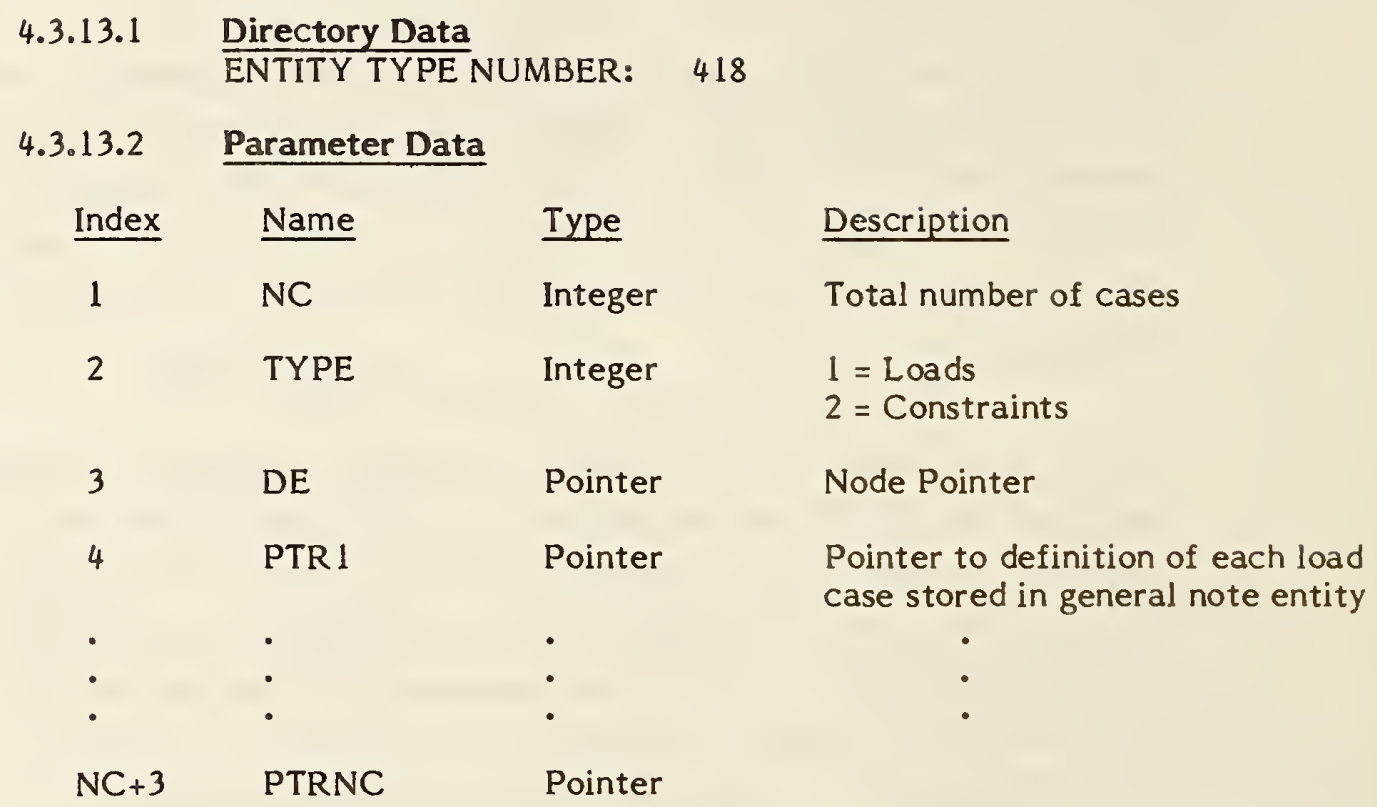

Additional Pointers as required (see 2.2.4.4.2). 


\subsubsection{Color Definition Entity}

The Color Definition Entity is used to communicate the relationship of the primary (red, green, and blue) colors to the intensity level of the respective graphics devices as a percent of the full intensity range.

These red, green, blue coordinates (RGB) can be readily transformed to cyan, magenta, yellow (CMY) and to hue, lightness, saturation (HLS) using transformations that are given in Appendix F.

\subsubsection{Directory Data}

\section{ENTITY TYPE NUMBER: $\quad 314$}

\subsubsection{Parameter Data}

\begin{tabular}{|c|c|c|c|}
\hline Index & Name & Type & Description \\
\hline 1 & $\mathrm{CCl}$ & Real & $\begin{array}{l}\text { First color coordinate (red) as a } \\
\text { percent of full intensity (range } 0 . \\
\text { to } 100 . \text {.) }\end{array}$ \\
\hline 2 & $\mathrm{CC} 2$ & Real & $\begin{array}{l}\text { Second color coordinate (green) as } \\
\text { a percent of full intensity (range } 0 . \\
\text { to } 100 . \text {. }\end{array}$ \\
\hline 3 & $\mathrm{CC} 3$ & Real & $\begin{array}{l}\text { Third color coordinate (blue) as a } \\
\text { percent of full intensity (range } 0 \text {. } \\
\text { to } 100 . \text {.) }\end{array}$ \\
\hline 4 & CNAME & String & $\begin{array}{l}\text { Color name; this is an optional } \\
\text { character string which may contain } \\
\text { some verbal description of the } \\
\text { color. If the color name is not } \\
\text { provided and additional pointers } \\
\text { are required, a placekeeper must } \\
\text { be supplied between CC } 3 \text { and the } \\
\text { first additional pointer. }\end{array}$ \\
\hline
\end{tabular}

Additional pointers as required (see 2.2.4.4.2). 


\section{3 .15}

\section{Text Display Template Entity}

The Text Display Template Entity is used to display self-contained information such as numbers and/or text strings according to the template's display parameters. There are two forms of the Text Display Template; Absolute and Incremental.

Note: For a more detailed description of the parameters, see the General Note Entity (type 212)

4.3.15.1 Absolute Text Display Template (Form 0)

This form of the Text Display Template specifies the parameters for the text block at the specified starting point. The text to be displayed are taken from the entity pointing to this instance of the Text Display Template. If the text string to be displayed is not explicitly pointed to then the first text string specified in the pointing entity will be displayed.

\subsubsection{Directory Data}

ENTITY TYPE NUMBER: 312

FORM NUMBER: 0

\subsubsection{Parameter Data}

\begin{tabular}{|c|c|c|c|}
\hline Index & Name & Type & Description \\
\hline 1 & CBW & Real & Character box width \\
\hline 2 & $\mathrm{CBH}$ & Real & Character box height \\
\hline 3 & FC & $\begin{array}{l}\text { Integer or } \\
\text { Pointer }\end{array}$ & $\begin{array}{l}\text { Font characteristic (or pointer if } \\
\text { negative) (Default }=1 \text { ) }\end{array}$ \\
\hline 4 & SL & Real & $\begin{array}{l}\text { Slant angle (Radians) (Default }= \\
\mathrm{pi} / 2 \text { ) }\end{array}$ \\
\hline 5 & A & Real & Rotation angle (Radians) \\
\hline
\end{tabular}




$\begin{array}{ccll}6 & M & \text { Integer } & \text { Mirror Flag } \\ 7 & \text { VH } & \text { Integer } & \text { Rotate internal text flag } \\ 8 & \text { XS } & \text { Real } & \text { Real } \\ 9 & \text { YS } & \text { Real } & \begin{array}{l}\text { coordinates of lower left } \\ \text { corner of first character } \\ \text { box; see form for } \\ \text { coordinate type }\end{array}\end{array}$

Additional pointers as required (see paragraph 2.2.4.4.2).

4.3.15.4 Incremental Text Display Template (Form 1)

This form of the Text Display Template specifies the parameters for the text block at a starting point specified incrementally from one taken from the entity point to the given instance of the Text Display Template. The text to be displayed is taken from the entity pointing to this instance of the Text Display Template. If the text string to be displayed is not explicitly pointed to, then the first string specified in the pointing entity will be displayed.

\subsubsection{Directory Data \\ ENTITY TYPE NUMBER: $\quad 312$ \\ FORM NUMBER: 1}

\subsubsection{Parameter Data}

\begin{tabular}{|c|c|c|c|}
\hline Index & Name & Type & Description \\
\hline 1 & CBW & Real & Character box width \\
\hline 2 & $\mathrm{CBH}$ & Real & Character box height \\
\hline 3 & FC & $\begin{array}{l}\text { Integer or } \\
\text { Pointer }\end{array}$ & $\begin{array}{l}\text { Font characteristic (or pointer if } \\
\text { negative) (Default = 1). }\end{array}$ \\
\hline 4 & SL & Real & $\begin{array}{l}\text { Slant angle (Radians) (Default = } \\
\mathrm{pi} / 2 \text { ) }\end{array}$ \\
\hline 5 & A & Real & Rotation angle (Radians) \\
\hline 6 & M & Integer & Mirror Flag \\
\hline 7 & VH & Integer & Rotate internal text flag \\
\hline 8 & DXS & Real & $\begin{array}{l}\text { Increment in } X \text { from } X \text { coordinate } \\
\text { found in parent entity }\end{array}$ \\
\hline
\end{tabular}


9

10

DZS

Real

Increment in $Y$ from $Y$ coordinate found in parent entity

Increment in $Z$ from $Z$ coordinate found in parent entity

Additional pointers as required (see 2.2.4.4.2). 


\section{APPENDIX A}

\section{PART FILE EXAMPLES}

This appendix contains three sample parts encoded in the Specification ASCII format. These files are included herein to provide a guide to the usage of IGES and, as such, do not represent all design application uses. The files are a two-dimensional application using structure entities, a three-dimensional mechanical part with dimensioning, and a threedimensional part with 2-D drawing views defined.

Example file 1 is an integrated circuit (IC) cell. The IC application was selected because of the predominance of 2-D geometry used in electrical designs. The geometry used in the cell in Figure A-1 consists of simple closed area, linear path entities and line widening property. The structure entities are nested subfigures using a network subfigure definition, array subfigure instance. A connect point is included to identify the signal port. The geometry is on five different levels, each representing a process mask. The entity label field of each directory entry record has optional text included to describe the entity's use. The entities in this file would be typical of those used in an IC application to transfer either cell libraries or a complete design between design systems. The file of a design prepared for pattern generation, with subfigures resolved and the geometry fractured, would use the flash entity exclusively. The cell file was adapted from a cell library in A Guide to LSI Implementation with kind permission from author Carlos Sequin.

Example file 2 is a three-dimensional mechanical part containing geometry entities and annotation entities typically found on engineering drawings. Included as geometry are points, lines, circular arcs and conics. For annotation the file includes linear dimensions, angular dimensions, radius dimensions, ordinate dimensions, a general label and general notes. Figure A-2 shows the mechanical part which was used during one of the early public demonstrations of intersystem data exchange.

Example file 3 is included to show the use of view entities and drawing entities in conjunction with a three-dimensional part model to convey a drawing to the receiving system. Figure A-3 shows the example drawing. In this way model geometry and viewing parameters are logically separate. A 3-D model as well as the drawing is received enabling additional views to be created if necessary, and changes to the part model are reflected in all views. 

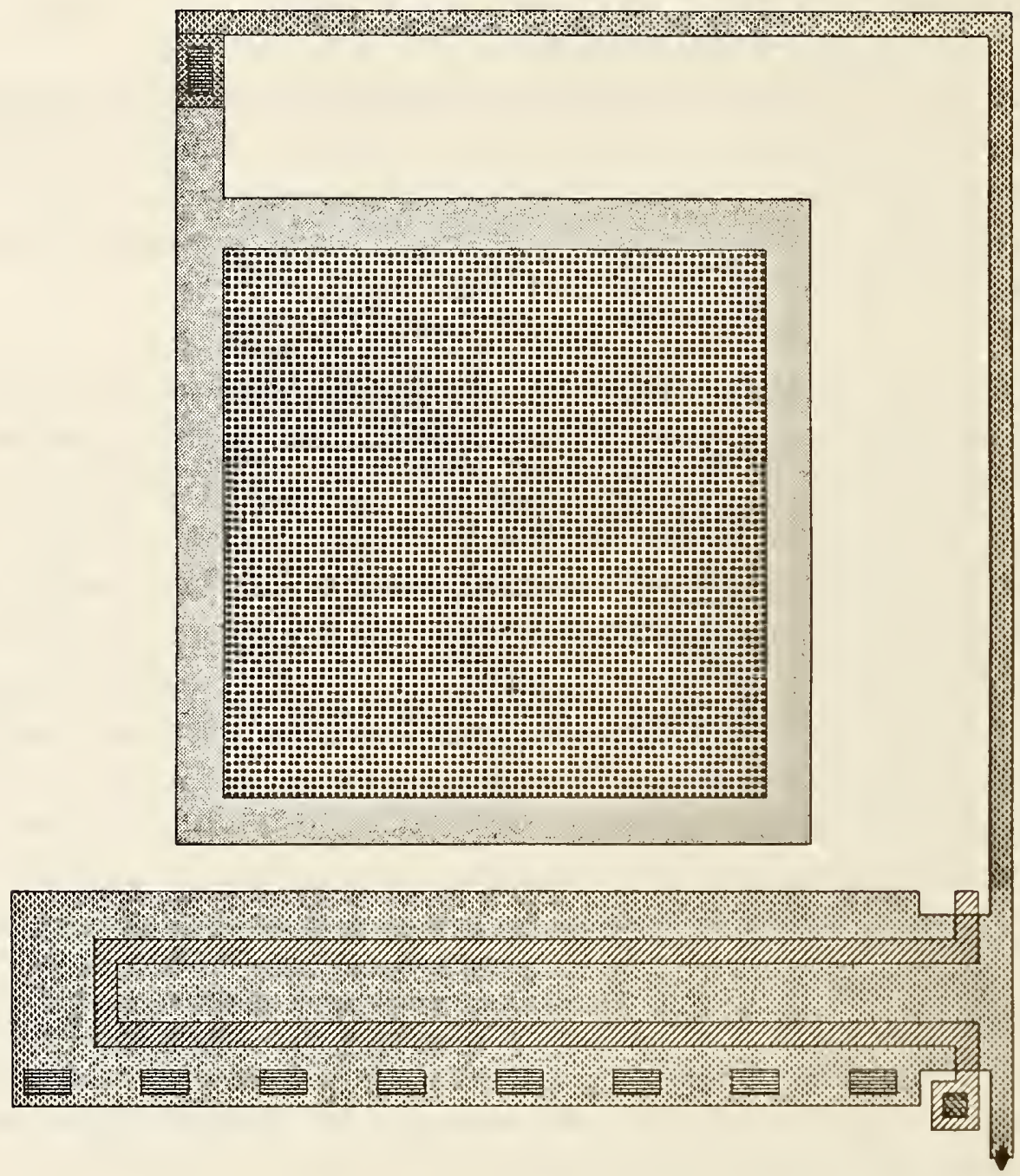

FIGURE A -1 ELECTRICAL PART EXAMPLE 
INTEGRATED CIRCUIT SEMICUSTOM CELL (ONE PART OF A IIBRARY FILE) $1 \mathrm{H}, 1 \mathrm{H} ;, 10 \mathrm{H} 5 \mathrm{MICRONL}$ IB, 5 HPADIN, 9 HEXAMPL $\mathrm{E} 1,4 \mathrm{HIGES}, 16,38,06,38,13$, 10HIC.LIBRARY, 1 .0,9,2HUM, 1, 13 H851128.090000,0 .01,265.0,9 BC.B.PARKS。 23 BGENERAL DYNAMICS/POMONA, 4, 0;

\begin{tabular}{|c|c|c|c|}
\hline 308 & 01 & & 1 \\
\hline 308 & 0 & & I \\
\hline 106 & 02 & & 1 \\
\hline 106 & 0 & 4 & 1 \\
\hline 106 & 03 & & 1 \\
\hline 106 & 0 & 4 & 1 \\
\hline 106 & 04 & & 1 \\
\hline 106 & 0 & 4 & 1 \\
\hline 106 & 05 & & 1 \\
\hline 106 & 0 & 5 & 1 \\
\hline 320 & 06 & & 1 \\
\hline 320 & 0 & & $I$ \\
\hline 408 & 07 & & 1 \\
\hline 408 & 0 & & 1 \\
\hline 106 & 08 & & 1 \\
\hline 106 & 0 & 3 & 2 \\
\hline 106 & 10 & & 1 \\
\hline 106 & 0 & 3 & 1 \\
\hline 106 & 11 & & 1 \\
\hline 106 & 0 & 3 & 1 \\
\hline 106 & 12 & & 1 \\
\hline 106 & 0 & 3 & 2 \\
\hline 132 & 14 & & 1 \\
\hline 132 & 0 & & 1 \\
\hline 106 & 15 & & 1 \\
\hline 106 & 0 & 8 & 2 \\
\hline 106 & 17 & & 1 \\
\hline 106 & 0 & 8 & 2 \\
\hline 308 & 19 & & 1 \\
\hline 308 & 0 & & 1 \\
\hline 106 & 20 & & 1 \\
\hline 106 & 0 & 8 & 1 \\
\hline 412 & 21 & & 1 \\
\hline 412 & 0 & & 1 \\
\hline 106 & 22 & & 1 \\
\hline 106 & 0 & 2 & 2 \\
\hline 106 & 24 & & 1 \\
\hline 106 & 0 & 2 & 2 \\
\hline 106 & 26 & & 1 \\
\hline 106 & 0 & 4 & 1 \\
\hline 406 & 27 & & 1 \\
\hline 406 & 0 & & 1 \\
\hline
\end{tabular}
(20) 


\section{APPENDIX A - PART FILE EXAMPLES}

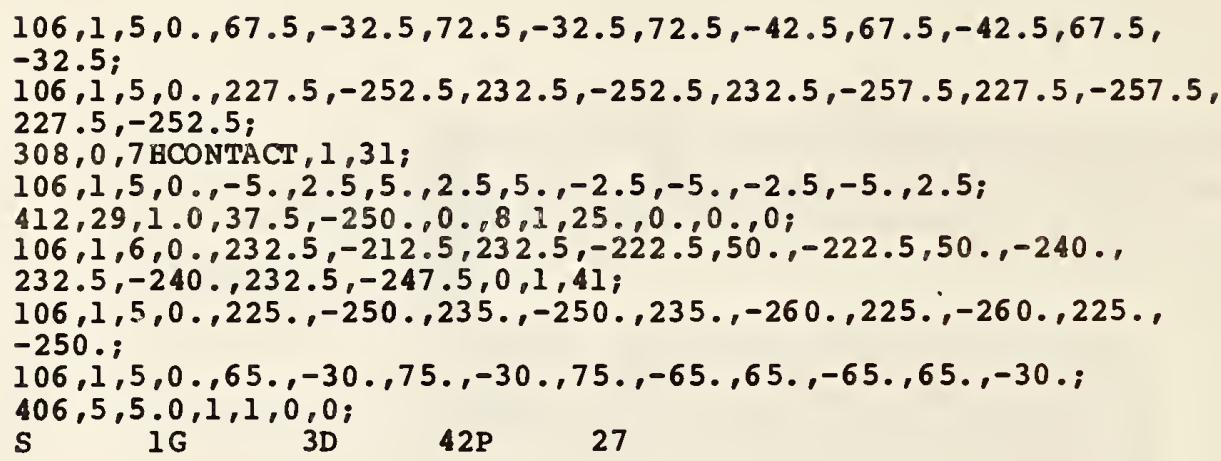

$\begin{array}{rr}25 \mathrm{P} & 15 \\ 25 \mathrm{P} & 16 \\ 27 \mathrm{P} & 17 \\ 27 \mathrm{P} & 18 \\ 29 \mathrm{P} & 19 \\ 31 \mathrm{P} & 20 \\ 33 \mathrm{P} & 21 \\ 35 \mathrm{P} & 22 \\ 35 \mathrm{P} & 23 \\ 37 \mathrm{P} & 24 \\ 37 \mathrm{P} & 25 \\ 39 \mathrm{P} & 26 \\ 41 \mathrm{P} & 27 \\ \mathrm{~T} & 1\end{array}$




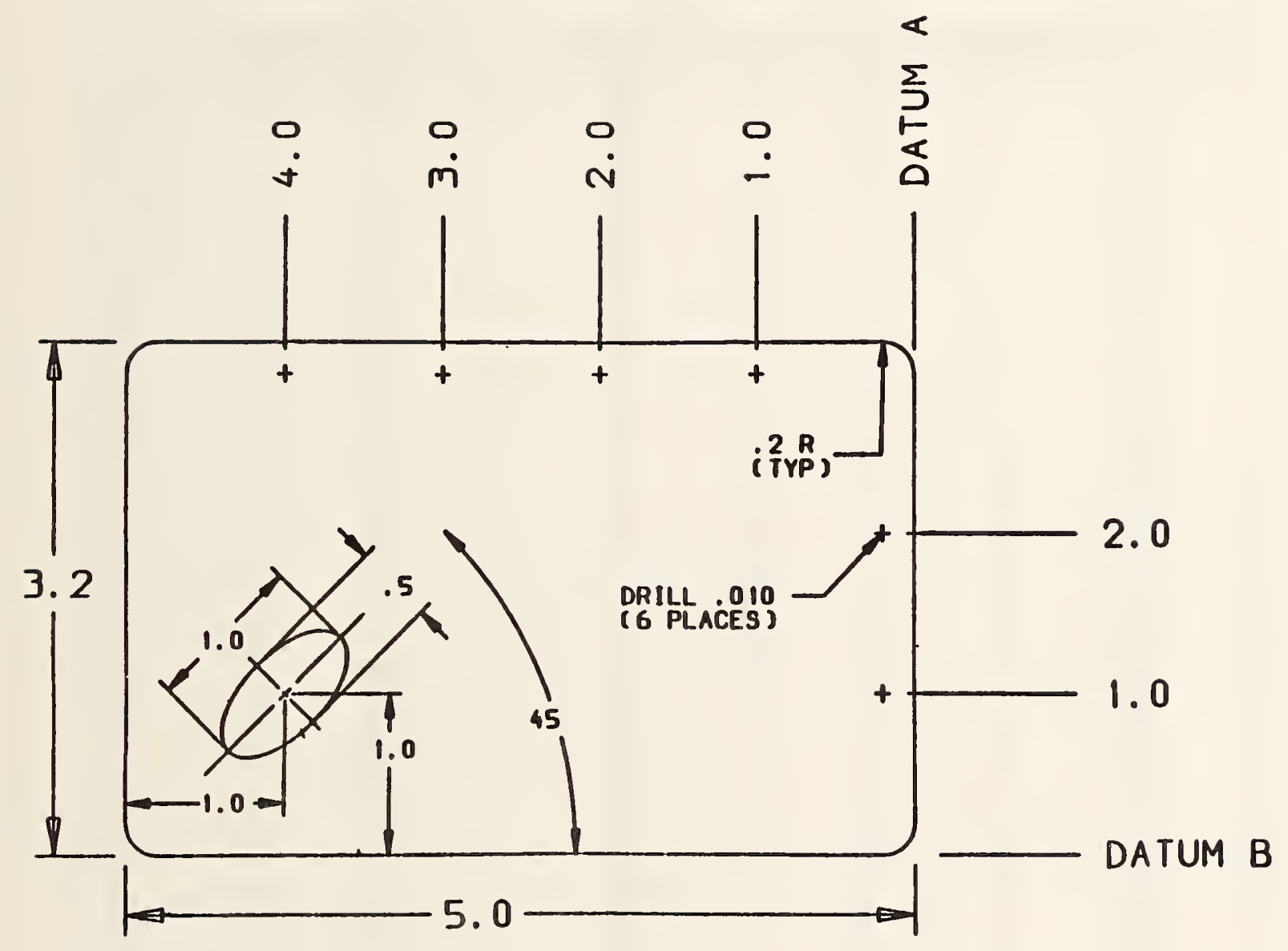

FIGURE A - 2 MECHANICAL PART EXAMPLE 
EXAMPLE 2 MECHANICAL PART

THIS IS A SAMPLE PART FOR USE IN THE AUTOFACT 82 IGES DEMONSTRATION , 8 HPANEL $123,10 \mathrm{HPANEL}$. IGES, 4 HEX 2,4 HIGES, $16,38,7,38,14,8$ HPANEL $123,1.0$, $1,4 \mathrm{HINCH}, 1,0.028,13 \mathrm{B8} 50816.144004,0.0005,100.0,9 \mathrm{HD}$. BRIGGS, $6 \mathrm{HBOEING}, 4,0$; G

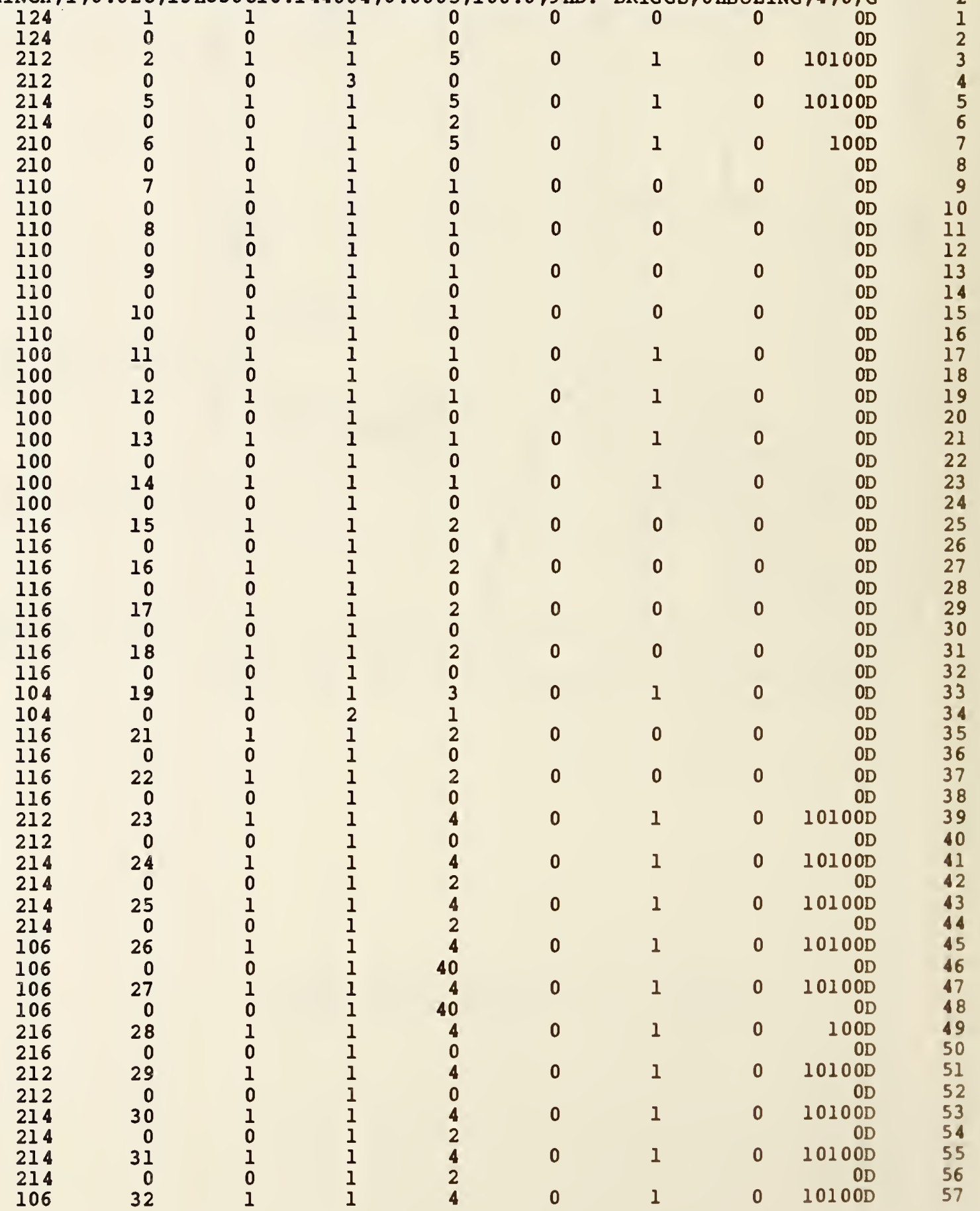




\begin{tabular}{|c|c|c|c|c|c|c|c|c|c|}
\hline 306 & & & & & & & & & \\
\hline 106 & 0 & 0 & 1 & 40 & & & & OD & $\begin{array}{l}58 \\
59\end{array}$ \\
\hline 106 & $\begin{array}{r}33 \\
0\end{array}$ & 0 & $\frac{1}{1}$ & $\begin{array}{r}4 \\
40\end{array}$ & 0 & 1 & 0 & $\begin{array}{r}10100 \mathrm{D} \\
\text { OD }\end{array}$ & 60 \\
\hline 216 & 34 & 1 & 1 & 4 & 0 & 1 & 0 & $100 \mathrm{D}$ & 61 \\
\hline 216 & 0 & 0 & $\overline{1}$ & 0 & & & & OD & 62 \\
\hline 212 & 35 & 1 & 1 & 5 & 0 & 1 & 0 & $10100 \mathrm{D}$ & 63 \\
\hline 212 & 0 & 0 & 1 & 0 & & & & OD & 64 \\
\hline 106 & 36 & 1 & 1 & 5 & 0 & 1 & 0 & $10100 D$ & 65 \\
\hline 106 & 0 & 0 & 1 & 40 & & & & $O D$ & 66 \\
\hline 218 & 37 & 1 & 1 & 5 & 0 & 1 & 0 & $100 \mathrm{D}$ & 67 \\
\hline 218 & 0 & 0 & 1 & 0 & & & & OD & 68 \\
\hline 212 & 38 & 1 & 1 & 5 & 0 & 1 & 0 & $10100 D$ & 69 \\
\hline 212 & 0 & 0 & 1 & 0 & & & & OD & \\
\hline 106 & 39 & 1 & 1 & 5 & 0 & 1 & 0 & $10100 \mathrm{D}$ & 71 \\
\hline 106 & 0 & 0 & 1 & 40 & & & & OD & 72 \\
\hline 218 & 40 & 1 & 1 & 5 & 0 & 1 & 0 & $100 \mathrm{D}$ & 73 \\
\hline 218 & 0 & 0 & 1 & 0 & & & & OD & 74 \\
\hline 212 & 41 & 1 & 1 & 5 & 0 & 1 & 0 & $10100 \mathrm{D}$ & \\
\hline 212 & 0 & 0 & 1 & 0 & & & & OD & 76 \\
\hline 106 & 42 & 1 & $\overline{1}$ & 5 & 0 & 1 & 0 & $10100 D$ & \\
\hline 106 & 0 & 0 & 1 & 40 & & & & OD & 8 \\
\hline 218 & 43 & 1 & 1 & 5 & 0 & 1 & 0 & $100 \mathrm{D}$ & 79 \\
\hline 218 & 0 & 0 & 1 & 0 & & & & OD & 80 \\
\hline 212 & 44 & 1 & 1 & 5 & 0 & 1 & 0 & $10100 \mathrm{D}$ & 81 \\
\hline 212 & 0 & 0 & 1 & 0 & & & & OD & 82 \\
\hline 106 & 45 & 1 & 1 & 5 & 0 & 1 & 0 & $10100 D$ & 83 \\
\hline 106 & 0 & 0 & 1 & 40 & & & & OD & 4 \\
\hline 218 & 46 & 1 & 1 & 5 & 0 & 1 & 0 & $100 D$ & 8 \\
\hline 218 & 0 & 0 & 1 & 0 & & & & OD & 86 \\
\hline 212 & 47 & 1 & 1 & 5 & 0 & 1 & 0 & $10100 D$ & 87 \\
\hline 212 & 0 & 0 & 1 & 0 & & & & OD & 88 \\
\hline 106 & 48 & 1 & 1 & 5 & 0 & 1 & 0 & 10100D & \\
\hline 106 & 0 & 0 & 1 & 40 & & & & $O D$ & 9 \\
\hline 218 & 49 & 1 & 1 & 5 & 0 & 1 & 0 & $100 \mathrm{D}$ & 91 \\
\hline 218 & 0 & 0 & 1 & 0 & & & & OD & 92 \\
\hline 212 & 50 & 1 & 1 & 5 & 0 & 1 & 0 & $10100 \mathrm{D}$ & 93 \\
\hline 212 & 0 & 0 & 1 & 0 & & & & OD & \\
\hline 106 & 51 & 1 & 1 & 5 & 0 & 1 & 0 & 101000 & 9 \\
\hline 106 & 0 & 0 & 1 & 40 & & & & OD & \\
\hline 218 & 52 & 1 & 1 & 5 & 0 & 1 & 0 & $100 D$ & 9 \\
\hline 218 & 0 & 0 & 1 & 0 & & & & OD & \\
\hline 212 & 53 & 1 & 1 & 5 & 0 & 1 & 0 & $10100 \mathrm{D}$ & 9 \\
\hline 212 & 0 & 0 & 2 & 0 & & & & OD & 100 \\
\hline 106 & 55 & 1 & 1 & 5 & 0 & 1 & 0 & $10100 D$ & 10. \\
\hline 106 & 0 & 0 & 1 & 40 & & & & OD & 10 \\
\hline 218 & 56 & 1 & 1 & 5 & 0 & 1 & 0 & $100 D$ & 103 \\
\hline 218 & 0 & 0 & 1 & 0 & & & & OD & 104 \\
\hline 212 & 57 & 1 & 1 & 5 & 0 & 1 & 0 & $10100 D$ & 105 \\
\hline 212 & 0 & 0 & 2 & 0 & & & & OD & 106 \\
\hline 106 & 59 & 1 & 1 & 5 & 0 & 1 & 0 & $10100 \mathrm{D}$ & 10 \\
\hline 106 & 0 & 0 & 1 & 40 & & & & OD & 108 \\
\hline 218 & 60 & 1 & 1 & 5 & 0 & 1 & 0 & $100 \mathrm{D}$ & 10 \\
\hline 218 & 0 & 0 & 1 & 0 & & & & OD & 110 \\
\hline 212 & 61 & 1 & 1 & 5 & 0 & 1 & 0 & $10100 D$ & 111 \\
\hline 212 & 0 & 0 & 2 & 0 & & & & OD & 1 \\
\hline 214 & 63 & 1 & 1 & 5 & 0 & 1 & 0 & $10100 D$ & \\
\hline 214 & 0 & 0 & 1 & 2 & & & & OD & $\bar{l}$ \\
\hline 222 & 64 & 1 & 1 & 5 & 0 & 1 & 0 & $100 \mathrm{D}$ & \\
\hline 222 & 0 & 0 & 1 & 0 & & & & OD & 116 \\
\hline 212 & 65 & 1 & 1 & 6 & 0 & 1 & 0 & $10100 \mathrm{D}$ & 1 \\
\hline
\end{tabular}




\begin{tabular}{|c|c|c|c|c|c|c|c|c|c|}
\hline 212 & & & & & & & & & \\
\hline 214 & 56 & 0 & 1 & 0 & & & & OD & 118 \\
\hline 214 & 0 & 0 & 1 & 2 & 0 & 1 & 0 & $\begin{array}{r}10100 \mathrm{D} \\
\mathrm{OD}\end{array}$ & $\begin{array}{l}119 \\
120\end{array}$ \\
\hline 214 & 67 & 1 & $\overrightarrow{1}$ & 6 & 0 & 1 & 0 & $10100 \mathrm{D}$ & 121 \\
\hline 214 & 0 & 0 & 1 & 2 & & & & OD & 122 \\
\hline 106 & 68 & 1 & 1 & 6 & 0 & 1 & 0 & $1010100 \mathrm{D}$ & 123 \\
\hline 106 & 0 & 0 & 1 & 40 & & & & OD & 124 \\
\hline 106 & 69 & 1 & 1 & 6 & 0 & 1 & 0 & 101000 & 125 \\
\hline 106 & 0 & 0 & 1 & 40 & & & & OD & 126 \\
\hline 216 & 70 & 1 & 1 & 6 & 0 & 1 & 0 & $100 \mathrm{D}$ & 127 \\
\hline 216 & 0 & 0 & 1 & 0 & & & & OD & 128 \\
\hline 212 & 71 & 1 & 1 & 6 & 0 & 1 & 0 & $10100 \mathrm{D}$ & 129 \\
\hline 212 & 0 & 0 & 1 & 0 & & & & OD & 130 \\
\hline 214 & 72 & 1 & 1 & 6 & 0 & 1 & 0 & 101000 & 131 \\
\hline 214 & 0 & 0 & 1 & 2 & & & & OD & 132 \\
\hline 214 & 73 & 1 & 1 & 6 & 0 & 1 & 0 & 101000 & 133 \\
\hline 214 & 0 & 0 & 1 & 2 & & & & OD & 134 \\
\hline 106 & 74 & 1 & 1 & 6 & 0 & 1 & 0 & 101000 & 135 \\
\hline 106 & 0 & 0 & 1 & 40 & & & & OD & 136 \\
\hline 106 & 75 & 1 & 1 & 6 & 0 & 1 & 0 & $1010100 \mathrm{D}$ & 137 \\
\hline 106 & 0 & 0 & 1 & 40 & & & & OD & 138 \\
\hline 216 & 76 & 1 & 1 & 6 & 0 & 1 & 0 & $100 D$ & 139 \\
\hline 216 & 0 & 0 & 1 & 0 & & & & OD & 140 \\
\hline 212 & 77 & 1 & 1 & 6 & 0 & 1 & 0 & 101000 & 141 \\
\hline 212 & 0 & 0 & 1 & 0 & & & & OD & 142 \\
\hline 214 & 78 & 1 & 1 & 6 & 0 & 1 & 0 & $10100 \mathrm{D}$ & 143 \\
\hline 214 & 0 & 0 & 1 & 2 & & & & OD & 144 \\
\hline 214 & 79 & 1 & 1 & 6 & 0 & 1 & 0 & $10100 \mathrm{D}$ & 145 \\
\hline 214 & 0 & 0 & 1 & 2 & & & & OD & 146 \\
\hline 106 & 80 & 1 & 1 & 6 & 0 & 1 & 0 & $10100 \mathrm{D}$ & 147 \\
\hline 106 & 0 & 0 & 1 & 40 & & & & OD & 148 \\
\hline 106 & 81 & 1 & 1 & 6 & 0 & 1 & 0 & $10100 \mathrm{D}$ & 149 \\
\hline 106 & 0 & 0 & 1 & 40 & & & & OD & 150 \\
\hline 216 & 82 & 1 & 1 & 6 & 0 & 1 & 0 & $100 D$ & 151 \\
\hline 216 & 0 & 0 & 1 & 0 & & & & OD & 152 \\
\hline 212 & 83 & 1 & 1 & 6 & 0 & 1 & 0 & 101000 & 153 \\
\hline 212 & 0 & 0 & 1 & 0 & & & & OD & 154 \\
\hline 214 & 84 & 1 & 1 & 6 & 0 & 1 & 0 & 101000 & 155 \\
\hline 214 & 0 & 0 & 1 & 2 & & & & OD & 156 \\
\hline 214 & 85 & 1 & 1 & 6 & 0 & 1 & 0 & $10100 \mathrm{D}$ & 157 \\
\hline 214 & 0 & 0 & 1 & 2 & & & & OD & 158 \\
\hline 106 & 86 & 1 & 1 & 6 & 0 & 1 & 0 & $10100 \mathrm{D}$ & 159 \\
\hline 106 & 0 & 0 & 1 & 40 & & & & OD & 160 \\
\hline 106 & 87 & 1 & 1 & 6 & 0 & 1 & 0 & $10100 \mathrm{D}$ & 161 \\
\hline 106 & 0 & 0 & 1 & 40 & & & & OD & 162 \\
\hline 216 & 88 & 1 & 1 & 6 & 0 & 1 & 0 & $100 \mathrm{D}$ & 163 \\
\hline 216 & 0 & 0 & 1 & 0 & & & & OD & 164 \\
\hline 110 & 89 & 1 & 4 & 6 & 0 & 0 & 0 & $100 \mathrm{D}$ & 165 \\
\hline 110 & 0 & 0 & 1 & 0 & & & & OD & 166 \\
\hline 110 & 90 & 1 & 4 & 6 & 0 & 0 & 0 & $100 \mathrm{D}$ & 167 \\
\hline 110 & 0 & 0 & 1 & 0 & & & & OD & 168 \\
\hline 212 & 91 & 1 & 1 & 6 & 0 & 1 & 0 & 101000 & 169 \\
\hline 212 & 0 & 0 & 1 & 0 & & & & OD & 170 \\
\hline 214 & 92 & 1 & 1 & 6 & 0 & 1 & 0 & $10100 \mathrm{D}$ & 171 \\
\hline 214 & 0 & 0 & 1 & 2 & & & & OD & 172 \\
\hline 214 & 93 & 1 & 1 & 6 & 0 & 1 & 0 & $10100 \mathrm{D}$ & 173 \\
\hline 214 & 0 & 0 & 1 & 2 & & & & OD & 174 \\
\hline 106 & 94 & 1 & 1 & 6 & 0 & 1 & 0 & $1010100 \mathrm{D}$ & 175 \\
\hline 106 & 0 & 0 & 1 & 40 & & & & OD & 176 \\
\hline 106 & 95 & 1 & 1 & 6 & 0 & 1 & 0 & $1010100 \mathrm{D}$ & 177 \\
\hline
\end{tabular}




$\begin{array}{lccccccc}106 & 0 & 0 & 1 & 40 & & & 0 \\ 202 & 96 & 1 & 1 & 6 & 0 & 1 & 0 \\ 202 & 0 & 0 & 1 & 0 & & 0\end{array}$
$124,1.000,0.0,0.0,0,0,0.0,1,000,0,0,0,0,0,0,0,0,1,000,0,0,0,0 ;$ $212,2,10,0.98,0.10,1,1.571,0.0,0,0,3.210,1.656,0.0,10$ HDR ILL .010 $, 10,1.02,0.100,1,1.571,0.0,0,0,3.210,1.506,0.0,10 \mathrm{H}(6$ PLACES), 0 , $0 ;$

$214,2,0.150,0.050,0.0,4.800,2.000,4.562,1.546,4.262,1.546,0,0$;

$210,3,1,5,0,0$;

$110,0.0,0.200,0.0,0.0,3.000,0.0,0,0$

$110,0.200,0.0,0.0,4.800,0.0,0.0,0,0$;

$110,5.000,0.200,0.0,5.000,3.000,0.0,0,0$ :

$110,0.200,3.200,0.0,4.800,3.200,0.0,0,0$;

$100,0.0,4.800,3.000,5.000,3.000,4.800,3.200,0,0$ :

$100,0.0,0.200,3.000,0.200,3.200,0.0,3.000,0,0$;

$100,0.0,0.200,0.200,0.0,0.200,0.200,0.00,0,0$;

$100,0.0,4.800,0.200,4.800,0.0,5.000,0.200,0,0$;

$116,4.000,3.000,0.0,0,0,0$;

$116,3.000,3.000,0.0,0,0,0$;

$116,2.000,3.000,0.0,0,0,0$;

$116,1.000,3.000,0.0,0,0,0$;

$104,10.000,-12.000,10.000,-8.000,-8.000,7.000,0.0,1.3538,1.3538$,

$1.3538,1.3538,0,0$;

$116,4.800,1.000,0.0,0,0,0$;

$116,4.800,2.000,0.0,0,0,0$;

$212,1,3,0.421,0.156,1,1.571,0.0,0,0,-0.639,1.614,0.0,3$ 83 .2,0,0;

$214,1,0.150,0.050,0.0,-0.454,3.200,-0.454,1.870,0,0$;

$214,1,0.150,0.050,0.0,-0.454,0.0,-0.454,1.514,0,0$;

$106,1,3,0.0,0.0,3.200,-0.094,3.200,-0.579,3.200,0,0$;

$106,1,3,0.0,0.0,0.0,-0.094,0.0,-0.579,0.0,0,0$;

$216,39,41,43,45,47,0,0$

$212,1,3,0.437,0.156,1,1.571,0.0,0,0,2.032,-0.447,0.0,3 \mathrm{~B} 5.0,0,0$;

$214,1,0.150,0.050,0.0,0.0,-0.369,1.932,-0.369,0,0$;

$214,1,0.150,0.050,0.0,5.000,-0.369,2.519,-0.369,0,0$;

$106,1,3,0.0,0.0,0.0,0.0,-0.094,0.0,-0.494,0,0$;

$106,1,3,0.0,5.000,0.0,5.000,-0.094,5.000,-0.494,0,0$;

$216,51,53,55,57,59,0,0$ :

$212,1,3,0.374,0.156,1,1.571,1.571,0,0,4.078,4.181,0.0,3 \mathrm{Bl} .0,0,0$;

$106,1,3,0.0,4.000,3.094,4.000,3.188,4.000,3.993,0,0$;

$218,63,65,0,0$;

$212,1,3,0.421,0.156,1,1.571,1.571,0,0,3.078,4.183,0.0,3 \mathrm{H} 2.0,0,0$;

$106,1,3,0.0,3.000,3.094,3.000,3.188,3.000,3.996,0,0$;

$218,69,71,0,0$;

$212,1,3,0.437,0.156,1,1.571,1.571,0,0,2.078,4.177,0.0,383.0,0,0$;

$106,1,3,0.0,2.000,3.094,2.000,3.188,2.000,3.989,0,0$;

$218,75,77,0,0$;

$212,1,3,0.437,0.156,1,1.571,1.571,0,0,1.078,4.177,0.0,3 \mathrm{H} 4.0,0,0$;

$106,1,3,0.0,1.000,3.094,1.000,3.188,1.000,3.989,0,0$;

$218,81,83,0,0$;

$212,1,3,0.374,0.156,1,1.571,0.0,0,0,6.211,0.922,0.0,3 \mathrm{H1} .0,0,0$;

$106,1,3,0.0,4.894,1.000,4.988,1.000,6.024,1.000,0,0$;

$218,87,89,0,0$;

$212,1,3,0.421,0.156,1,1.571,0.0,0,0,6.211,1.922,0.0,3 \mathrm{~B} 2.0,0,0$;

$106,1,3,0.0,4.894,2.000,4.988,2.000,6.024,2.000,0,0$;

$218,93,95,0,0$;

$212,1,7,1.248,0.156,1,1.571,0 ., 0,0,6.23,-0.078,0.0,7$ HDATUM B , 0 , 0 ;

$106,1,3,0.0,5.094,0.0,5.188,0.0,6.042,0.0,0,0$;

$218,99,101,0,0$;

$212,1,7,1.232,0.156,1,1.571,1.571,0,0,5.078,4.193,0 ., 7$ HDATUM A,

\begin{tabular}{|c|c|}
\hline \multicolumn{2}{|r|}{178} \\
\hline $100 D$ & 179 \\
\hline OD & 180 \\
\hline 1P & 1 \\
\hline 3P & \\
\hline 3P & \\
\hline $3 P$ & \\
\hline $5 P$ & \\
\hline $7 P$ & 6 \\
\hline $9 \mathrm{P}$ & \\
\hline IIP & \\
\hline $13 P$ & \\
\hline $15 \mathrm{P}$ & 10 \\
\hline 17P & 11 \\
\hline $19 \mathrm{P}$ & 12 \\
\hline $21 P$ & 13 \\
\hline 23P & 14 \\
\hline $25 \mathrm{P}$ & 15 \\
\hline $27 P$ & 16 \\
\hline $29 \mathrm{P}$ & 17 \\
\hline 3 IP & 18 \\
\hline $\begin{array}{l}33 \mathrm{P} \\
23 \mathrm{P}\end{array}$ & 19 \\
\hline $\begin{array}{l}33 P \\
35 P\end{array}$ & $\begin{array}{l}20 \\
2]\end{array}$ \\
\hline $37 P$ & 22 \\
\hline $39 P$ & 2 \\
\hline 4IP & \\
\hline $43 P$ & 25 \\
\hline $45 \mathrm{P}$ & 26 \\
\hline 47P & 27 \\
\hline $49 P$ & 28 \\
\hline 5IP & 2 \\
\hline 53P & 3 \\
\hline 55P & 3 \\
\hline 57P & \\
\hline 59P & \\
\hline 6IP & \\
\hline $\begin{array}{l}63 P \\
65 P\end{array}$ & 3 \\
\hline $67 P$ & 3 \\
\hline $69 P$ & 3 \\
\hline 7 IP & 3 \\
\hline $73 P$ & 4 \\
\hline $75 P$ & 4 \\
\hline $77 P$ & 4 \\
\hline $79 P$ & 4 \\
\hline $81 P$ & 4 \\
\hline $83 P$ & 4 \\
\hline $85 P$ & 46 \\
\hline $87 P$ & 4 \\
\hline $89 \mathrm{P}$ & 4 \\
\hline 9IP & 4 \\
\hline $\begin{array}{l}93 \mathrm{P} \\
95 \mathrm{P}\end{array}$ & \\
\hline $97 P$ & \\
\hline $\begin{array}{l}97 \mathrm{P} \\
99 \mathrm{P}\end{array}$ & \\
\hline 99P & \\
\hline 101P & \\
\hline $103 P$ & \\
\hline & \\
\hline
\end{tabular}


0,0 ;

$106,1,3,0.0,5.000,3.094,5.000,3.187,5.000,4.006,0,0$;

$218,105,107,0,0$;

$212,2,4,0.35,0.100,1,1.571,0,0,0,4.029,2.611,0 ., 4 \mathrm{H} .2 \mathrm{R}, 5,0.500$, $0.100,1,1.571,0.0,0,0,4.029,2.461,0.0,5$ H (TYP) $, 0,0$;

$214,2,0.150,0.050,0.0,4.877,3.185,4.862,2.511,4.562,2.511,0,0$;

$222,111,113,4.800,3.000,0,0$;

$212,1,3,0.240,0.100,1,1.571,0.0,0,0,1.559,0.602,0.0,3 \mathrm{H1} .0,0,0$;

$214,1,0.150,0.050,0.0,1.663,0.0,1.663,0.538,0,0$;

$214,1,0.150,0.050,0.0,1.663,1.000,1.663,0.766,0,0$;

$106,1,3,0.0,0.200,0.0,0.294,0.0,1.788,0.0,0,0$;

$106,1,3,0.0,1.000,1.000,1.094,1.000,1.788,1.000,0,0$;

$216,117,119,121,123,125,0,0$;

$212,1,3,0.240,0.100,1,1.571,0.0,0,0,0.537,0.275,0.0,3 \mathrm{Hl} .0,0,0$;

$214,1,0.150,0.050,0.0,1.000,0.325,0.809,0.325,0,0$;

$214,1,0.150,0.050,0.0,0.0,0.325,0.473,0.325,0,0$;

$106,1,3,0.0,1.000,1.000,1.000,0.906,1.000,0.200,0,0$;

$106,1,3,0.0,0.0,0.012,0.0,0.106,0.0,0.200,0,0$;

$216,129,131,133,135,137,0,0$;

$212,1,3,0.240,0.100,1,1.571,0.0,0,0,0.470,1.289,0.0,3 \mathrm{H1} .0,0,0$;

$214,1,0.150,0.050,0.0,0.971,1.736,0.688,1.453,0,0$;

$214,1,0.150,0.050,0.0,0.264,1.029,0.459,1.225,0,0$;

$106,1,3,0.0,1.354,1.354,1.287,1.420,0.882,1.825,0,0$;

$106,1,3,0.0,0.646,0.646,0.580,0.713,0.175,1.118,0,0$;

$216,141,143,145,147,149,0,0$;

$212,1,2,0.170,0.100,1,1.571,0.0,0,0,1.631,1.622,0.0,2$ B. $5,0,0$;

$214,1,0.150,0.050,0.0,1.863,1.509,2.146,1.226,0,0$;

$214,1,0.150,0.050,0.0,1.509,1.863,1.226,2.146,0,0$;

$106,1,3,0.0,1.177,0.823,1.243,0.890,1.951,1.598,0,0$;

$106,1,3,0.0,0.823,1.177,0.890,1.243,1.598,1.951,0,0$;

$216,153,155,157,159,161,0,0$;

$110,0.500,0.500,0.0,1.500,1.500,0.0,0,0$;

$110,1 \cdot 225,0.775,0.0,0.775,1.225,0.0,0,0$;

$212,1,2,0.220,0.100,1,1.571,0.0,0,0,2.566,0.786,0.0,2$ B4 $5,0,0$ :

$214,1,0.150,0.050,0.0,2.847,0.0,2.754,0.722,0,0$;

$214,1,0.150,0.050,0.0,2.013,2.013,2.683,0.951,0,0$;

$106,1,3,0.0,4.988,0.0,4.894,0.0,2.972,0.0,0,0$;

$106,1,3,0.0,2.000,2.000,2.066,2.066,2.101,2.101,0,0$;

$202,169,175,177,0.0,0.0,2.847,171,173,0,0$;

$\mathrm{S}$

$1 \mathrm{G}$

2D $180 \mathrm{P}$ 


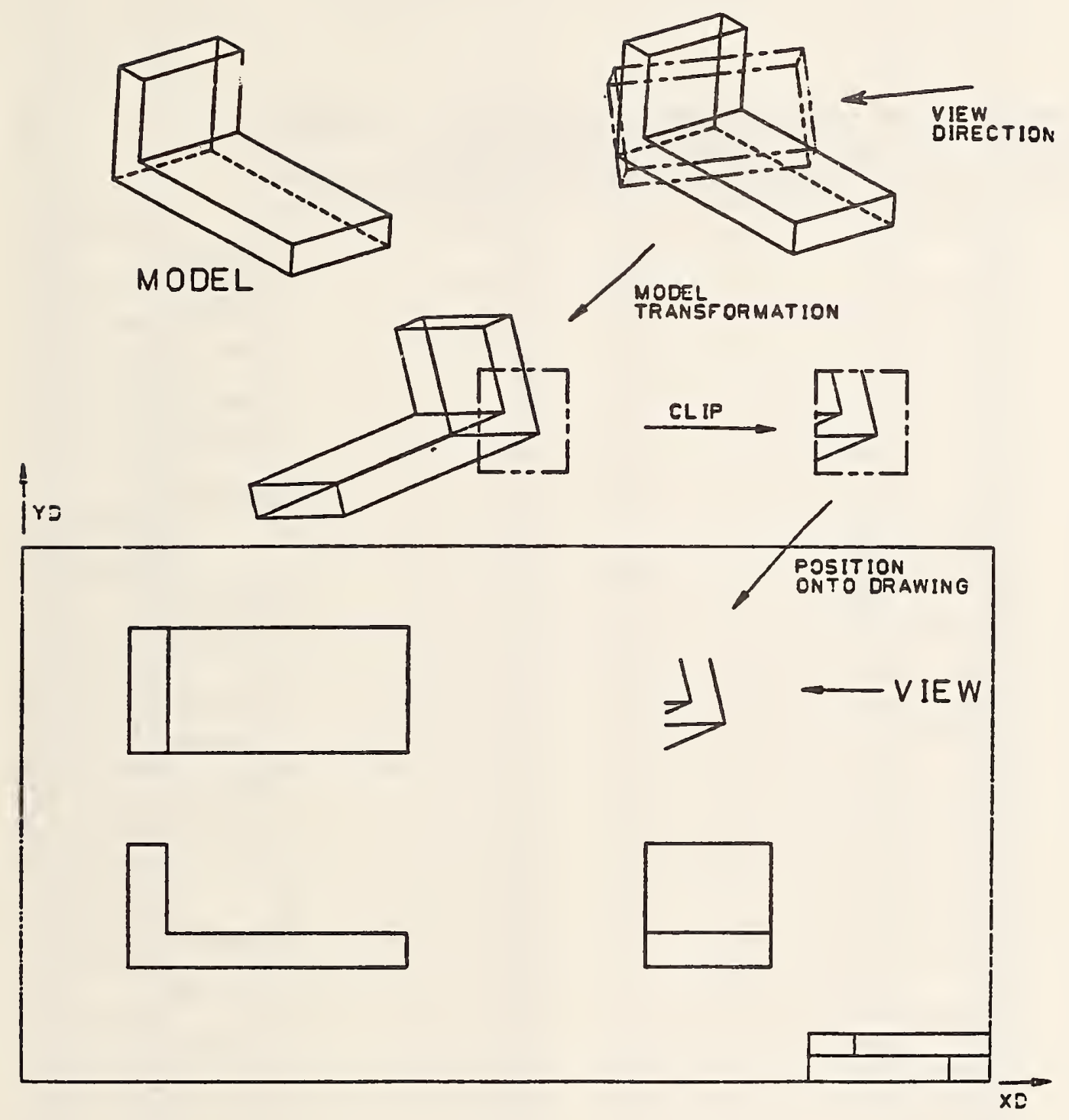

FIGURE A - 3 DRAWING AND VIEW EXAMPLE 
EXAMPLE 3 VIEW AND DRAWING PART'

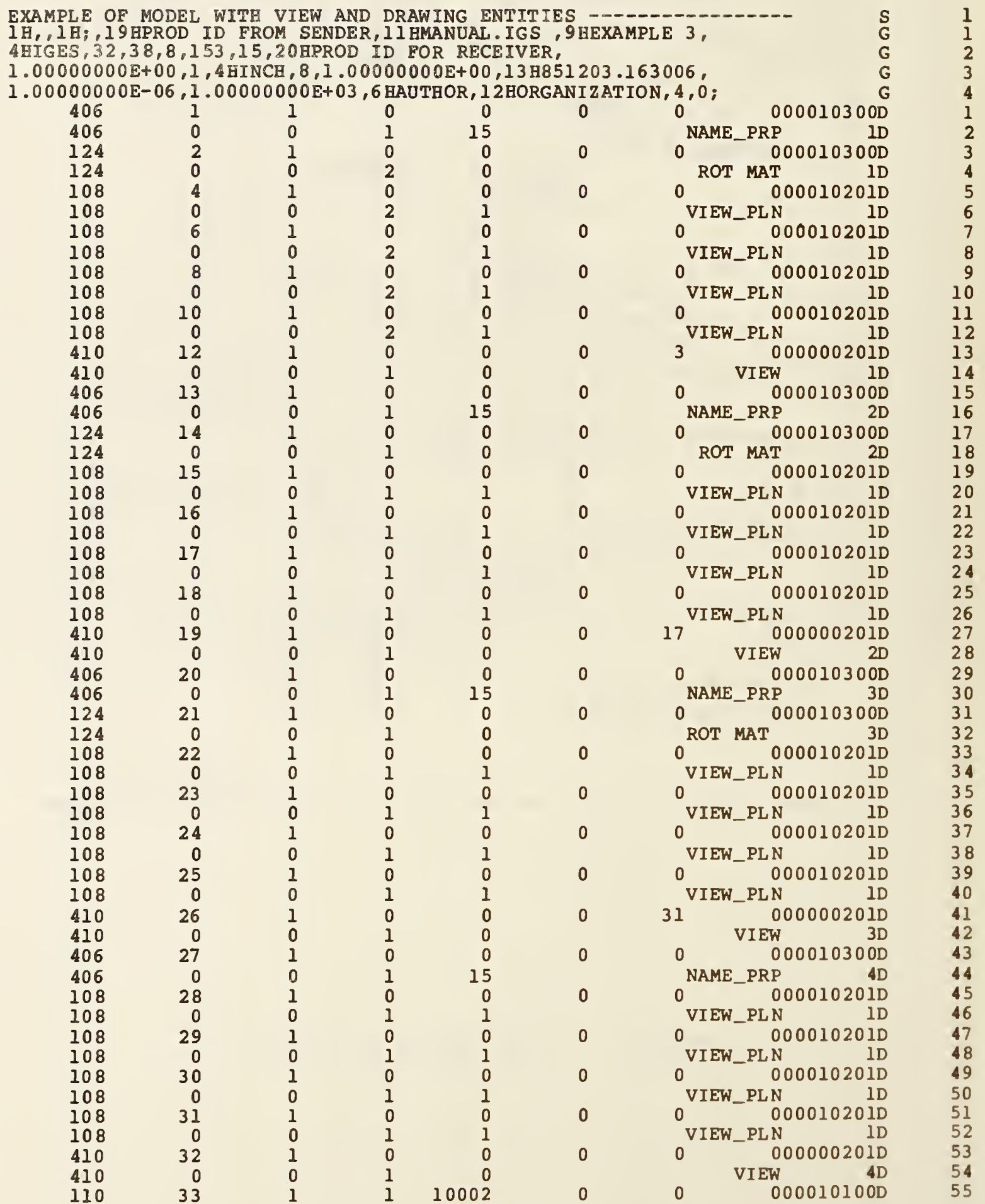




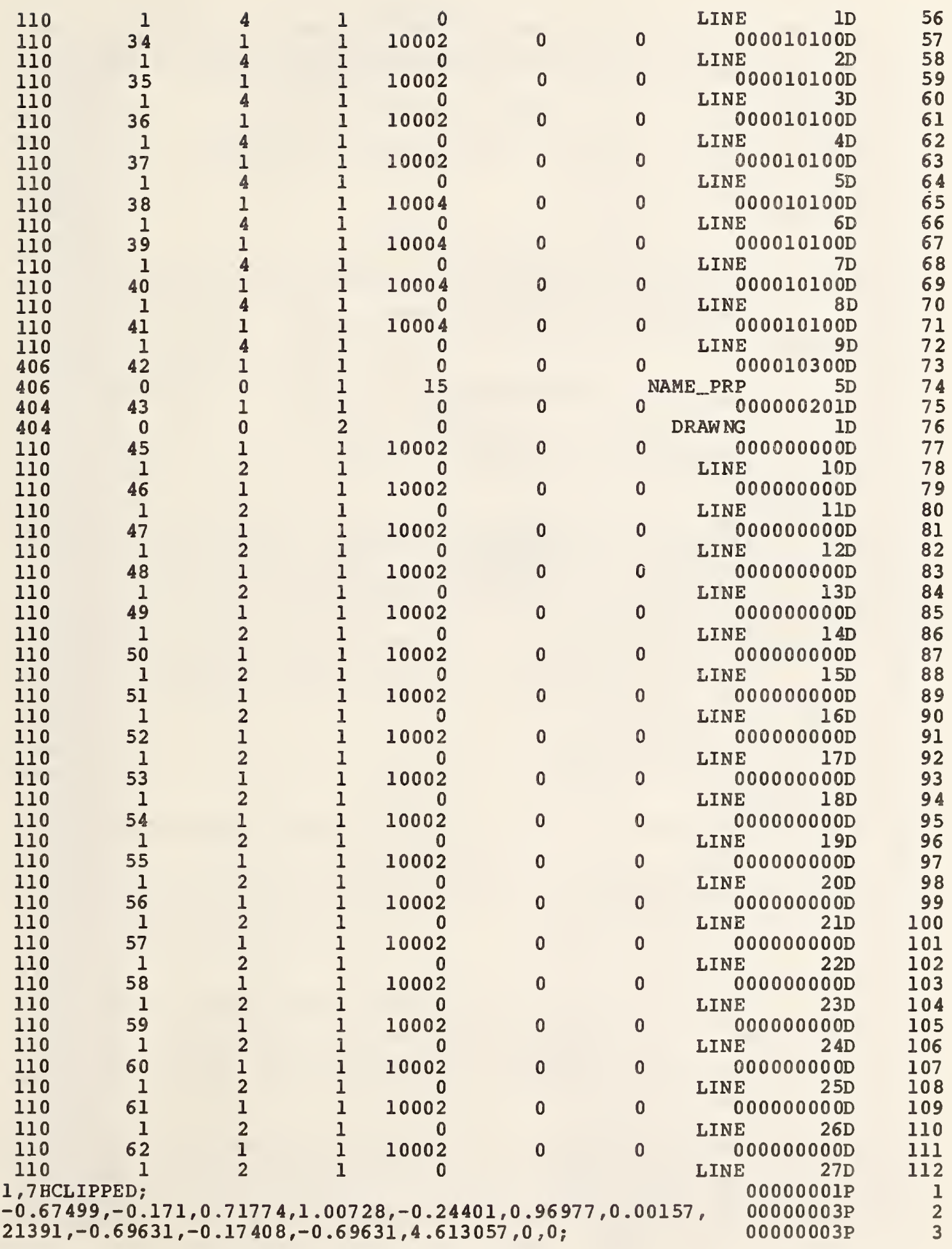


$108,0.67499,0.171,-0.71774,5.0055,0,6.390347,2.455541,-0.379235,00000005 \mathrm{P}$ $0.0,0$; $108,-0.24401,0.96977,0.00157,3.55308,0,3.025032,4.420965$, $2.494731,0,0,0$; $108,-0.67499,-0.171,0.71774,2.99094,0,0.992841,1.088152$, $5.360119,0,0,0$; $108,0.24401,-0.96977,-0.00157,1.9103,0,4.358156,-0.877273$, $2.486153,0,0,0$; $410,4,1,5,7,9,11,0,0,0,1,1 ;$

$406,1,5$ HRIGHT;

$124,0,0,-1,0,0,1,0,0,1,0,0,0,0,0$;

$108,0 \ldots, 0 \ldots 1,36,0,0 \ldots, 0,36,0 \ldots, 0,0$;

$108,0 \ldots 1 \ldots, 0 \ldots 24.59627,0,0,24.59627,0 \ldots, 0,0,0$;

$108,0 \ldots, 0,-1,36,0,0 \ldots, 0,-36,0,0,0$;

$108,0 \ldots-1,0,24.59627,0,0 \ldots,-24.59627,0,0,0,0$;

$410,3,1,19,21,23,25,0,0,0,1,15$;

$406,1,3$ HTOP;

$124,1 \ldots, 0 \ldots, 0,0 \ldots, 0,0 \ldots, 1,0,0,1,0,0,0,0$;

$108,-1,0,0,0,36,0,-36,0,0,0,0,0$;

$108,0 \ldots, 0 \ldots-1,24.59627,0,0 \ldots 0 \ldots-24.59627,0 \ldots, 0$;

$108,1 \ldots, 0 \ldots, 0,36,0,36,0 \ldots, 0 \ldots, 0,0,0$;

$108,0 \ldots, 0 \ldots 1 \ldots, 24.59627,0,0 \ldots, 0 \ldots, 24.59627,0,0,0$;

$410,2,1,33,35,37,39,0,0,0,1,29$;

$406,1,5$ HFRONT:

$108,-1,0,0,0,36,0,-36,0,0,0,0,0$;

$108,0 \ldots, 1,0,24.59627,0,0,24.59627,0 \ldots, 0,0,0$;

$108,1 \ldots, 0,0,36,0,36 \ldots, 0 \ldots, 0,0 \ldots, 0,0$;

$108,0 \ldots,-1 \ldots, 0,24.59627,0,0,-24.59627,0,0 \ldots, 0,0$;

$410,1,1,45,47,49,51,0,0,0,1,43$;

$110,67 \ldots, 0,0,67 \ldots, 2 \ldots, 0,0,0$;

$110,60 \ldots, 4 \ldots, 0,60 \ldots, 2 \ldots, 0,0,0$;

$110,57 \ldots, 4,0,57,0,0,0,0$ :

$110,70 \ldots, 40,0,57, .40,0,0,0$;

$110,70 \ldots, 2,0,057,2,0,0,0$;

$110,0 \ldots, 40,0 \ldots, 0,0 \ldots, 0,0,0 ;$

$110,70 \ldots 40,0 \ldots, 0,40 \ldots, 0,0,0$;

$110,70 \ldots, 0 \ldots, 0,70,40 \ldots, 0,0,0$;

$110,0 \ldots, 0,0 \ldots 70,0 \ldots, 0,0,0 ;$

$406,1,7$ HDRAW ING;

$404,4,13,51 \ldots, 29,27,46,8 \ldots, 41,7 \ldots, 24 \ldots, 53,7 \ldots, 8 \ldots, 9,55,57,59,61,63$, $65,67,69,71,0,1,73$;

$110,0,9,0 \ldots, 0,9,,-9,0,0$;

$110,2.5,9 \ldots 0 \ldots 2.5,9 \ldots,-9 ., 0,0$;

$110,2.5,2.5,0,2.5,2.5,-9 ., 0,0$;

$110,20 \ldots, 2.5,0,20 \ldots, 2.5,-9 \ldots, 0,0$;

$110,20 \ldots, 0,0,20 \ldots, 0,-9,0,0$;

$110,0 \ldots, 0,0,0 \ldots, 0 \ldots,-9,0,0$;

$110,0 \ldots, 9,-9,0,0,0,-9,0,0$;

$110,2.5,9 \ldots,-9,0,0,9,-9,0,0$;

$110,2.5,2.5,-9 ., 2.5,9,,-9 ., 0,0$;

$110,20 \ldots, 2.5,-9 ., 2.5,2.5,-9 ., 0,0$;

$110,20 \ldots, 0,-9,20,2.5,-9,0,0$;

$110,0 \ldots, 0 \ldots-9,20 \ldots, 0 \ldots-9,0,0$;

$110,0 \ldots, 9 \ldots, 0,0,0,0,0,0$;

$110,2.5,9 \ldots, 0,0 \ldots, 9 \ldots, 0,0,0$;

$110,2.5,2.5,0.2 .5,9,0,0,0$;

$110,20 \ldots, 2.5,0,2.5,2.5,0 \ldots, 0,0$;

$110,20 \ldots, 0,0,20 \ldots, 2.5,0 \ldots, 0,0$;

$110,0 \ldots, 0,0 \ldots, 20,0 \ldots, 0,0,0$;

$\mathrm{S} 2 \mathrm{G} \cdot 4 \mathrm{D}$ il $2 \mathrm{P}$ 


\section{APPENDIX B \\ ELECTRICAL/ELECTRONIC PRODUCT REPRESENTATION}

\section{INTRODUCTION}

Purpose

The purpose of this appendix is to provide IGES implementors and users with a roadmap of the IGES representation of electrical/electronic product designs. The topics of discussion will include (but are not limited to) design, engineering, manufacturing, testing, and inspection.

\section{Assumptions}

The reader should have a basic understanding of electrical/electronic product design using CAD/CAM/CAE tools, including (but not limited to) connectivity, component descriptions, placement and routing, and the manufacturing interface. Each topic will be discussed in general, but these discussions are not intended to provide a tutorial in the subject.

\section{CONNECTIVITY}

In General

Forming a connection between two or more items requires the ability to represent the following:

(1) the exact location of each connection point

(2) the signal formed and its identification (if any)

(3) the physical connection between the items (if any)

The term "connect node" will refer to a database entity which represents the exact location of connection. The term "link" will refer to the representation of the signal formed, and "signal name" will refer to the signal identifier. The 


\section{APPENDIX B - ELECTRICAL ELECTRONIC \\ PRODUCT REPRESENTATION}

term "join" will refer to the database entity or entities which represent the physical connection between the iterns.

Each item to be connected requires a connect node to represent each possible connection point of the item. A signal may be formed between any such items by a link which references the connect nodes to be connected. This creates an associativity between the connect nodes, and thus the connected items. The signal name may be used to uniquely identify the particular signal formed. The join may be used to provide a graphical representation of the signal. In electrical applications the join will most often be represented by a line (schematic) or a widened line (printed wiring board).

In electrical applications, the items to be connected are typically electrical components (i.e., resistor, 16-pin DIP, etc.). Most often, these components are represented by subfigures which are defined once, then referenced (instanced) in the database for each occurrence of the component. Each pin of the component is a potential connection point in a signal; thus each subfigure has a connect node defined for each pin. When such a subfigure is instanced, its connect nodes must also be instanced. This allows each connect node to participate in the unique signal to which it belongs. An instanced connect node, when added to a signal, is different from its definition which is not a member of any signal.

These subfigures, representing electrical components, often contain text describing the component and its pins. In some cases (e.g., part number), this text is fixed and unchanging. In other cases (e.g., reference designator), the text may be variable, and thus may not be filled in until the subfigure is instanced. This text (sometimes called a "text node"), like the connect node, is instanced along with its parent subfigure. In some cases, a connect node and a text node are related (e.g., the connect node represents a component pin and the text node represents the pin number). 
In IGES, the connect node is represented by the Connect Point entity. The text node is represented by the Text Display Template entity. The Flow Associativity entity represents a signal and contains the link, signal name, and pointers to the join entities. The Network Subfigure entities (definition and instance) represent electrical components which participate in signals. A number of property entities will also be used, as mentioned below.

Network Subfigure Construction

A component is constructed using the Network Subfigure Definition entity. The graphics representing the component geometry are referenced as for the Subfigure Definition entity. In addition, a separate set of pointers to defining Connect Point entities is provided. These Connect Point entities define the locations and characteristics of the component's pins. Properties, for example the Part Name property, may be attached to the Network Subfigure Definition entity.

\section{Connect Points}

A component pin (or surface mounted device pad, IC I/O port, lead frame, schematic symbol lead, etc.) is represented by the Connect Point entity. The Connect Point entity is used in both logical and physical product designs. The exact location in model space is specified, along with several characteristic flags (connection type, function type, $1 / 0$ direction). There is a pointer to the parent Network Subfigure entity (definition or instance), which provides a much-needed association for signal processing. An additional Subfigure Instance pointer is provided for Connect Point display. This allows a graphical symbol to be displayed, representing the Connect Point. The pin number is provided in the Function Connect Point Identifier field, along with a pointer to a Text Display Template for pin number display. A pin function name is provided in the Connect Point Function Name field, along with a pointer to a Text Display Template for its display. 
Signal Construction

A signal, representing one set of electrically common Connect Points, is constructed using the Flow Associativity entity. It contains pointers to other associated Flow Associativity entities, the Connect Point entities participating in the signal (this is the Link), and the Join entities representing the geometry of the signal (either logical or physical). Also contained is a list of signal names which may be used to identify the signal, along with a set of pointers to Text Display Template entities which may be used to display the first signal name in a number of locations. Two characteristic flags determine the signal type (logical or physical), and the function type (fluid flow or electrical signal).

A signal, then, is represented by one Flow Associativity entity pointing to a set of electrically common Connect Points. This is the Link. The Join entities represent the physical display geometry of the signal. For a schematic (logical), a line without width is typically used. For a printed board (physical), a line with the Line Widening property is typically used. A number of signal names may be associated with the signal. Multiple displays of the first, or primary, name are possible.

The components participating in a signal are represented by the Network Subfigure Instance entity. Note that the Connect Point entities "belonging" to a component are instanced along with the subfigure. This is necessary to allow a subfigure to participate in a number of different signals, while retaining unique component/pin identification. Each component is usually identified by a reference designator. This is supplied by the Primary Reference Designator field of the Network Subfigure. Any alternate reference designators may be designated with the Reference Designator property, attached through the normal property pointer mechanism.

\section{Information Display}

Throughout the above discussion, references to the Text Display Template entity have been made. This entity allows text, embedded in an entity, to be displayed without the redundant specification of the text string. There are 
two reasons for this feature. First, it eliminates a possible source of error by allowing the information to be specified in only one place. Second, it reduces the file size overhead. This entity exists in two forms, absolute and incremental. The absolute form provides an exact location for display of the information, as in the display of a reference designator. The incremental form provides an of fset to be applied to the parent entity's location to provide the exact location for display of information like pin numbers. When a direct pointer for information display is provided, the base location is readily determined from the parent entity's location such as when displaying a pin number. In the case of property value display, the base location must be determined by "remembering" the location of the property entity's parent entity. This would occur when displaying the Part Name. Also in this case, the pointer to the Text Display Template entity is located in the additional pointers section of the property entity parameters:

\section{Additional Considerations}

The situation is exactly the same for both logical and physical representations. The only differences arise in the subfigure and Join entities used. In fact, a file may contain representations for both the schematic and the board. The Flow Associativity entity contains a type flag to indicate the connection type (logical or physical). In this case, one Flow Associativity would represent the logical connection and a second the physical connection. The two associativities would be related by the pointers provided in the Flow Associativity.

\section{Figures}

The following figures illustrate certain aspects of the above discussions. Figure B-1 illustrates the basic entity relationships. Figure B-2 and Table B-1 illustrate the usage of the Text Display Template. Figure B-3 illustrates an actual implementaiton. Figure B-4 shows an example of logical and physical signals and their relationships in the same file. 


\section{APPENDIX B - ELECTRICAL ELECTRONIC \\ PRODUCT REPRESENTATION}

\section{IGES ELECTRICAL ENTITY DESCRIPTIONS}

The following entities (in entity number order) are the subset of IGES entities which have particular meanings when used for electrical product data.

100 Circular Arc Entity

The electrical use of this entity is in the geometric representation of component parts and their symbolic representations. In such usage, it is generally part of a subfigure. It may be used as a join entity. Its use may be defined by a Level Function property or DE Level field.

\section{Composite Curve Entity}

The electrical use of this entity is in the geometric representation of component parts and their symbolic representations. In such usuage, it is generally part of a subfigure. It may be used as a join entity. Its use may be defined by a Level Function property or DE Level field.

\section{Copious Data Entity}

Forms 11, 12, and 13 of this entity may be used to implement the electrical join (i.e., schematic or wiring diagram circuit connection lines). Any of these forms may point to a Line Widening property. Examples of this entityproperty combination are circuit paths on a PC board or IC metalization, or as a bus in a schematic. Form 63, Simple Closed Area, may be used to define an auto-router restriction area or a PC (or IC) defined area having special attributes.

\section{Plane Entity}

Certain layers of PC design are designated as "ground", "power", or "heat sink", and as such are large conductive areas. These layers, as well as larger curved or rounded conductive areas on other layers, are best defined by the Plane entity. Note that the form number indicates whether the bounded region is positive or void (i.e., copper clad area or cutout). 
The Line entity has several important uses in the electrical application. It may be used to construct component outlines, and to represent both logical and physical connections (as a join entity). As a physical join entity, the Line Widening property will most often be attached, giving the width of metalization to be etched on the board. As a logical join entity, the line will most typically be used without the Line Widening property.

116 Point Entity

The point entity is used to locate features that do not participate in electrical connectivity, for example, a mounting hole.

124 Transformation Matrix Entity

A Transformation Matrix may be used to rotate subfigures to other than normal (top up) positions. Generally, rotations are about the $Z$ axis for PC and IC constructs, but may be about any axis for 3D cabling files.

125 Flash Entity

The Flash entity may be used to represent a repetitive artwork feature which is usually produced by photo-optical means. Examples include PC pads, targets, clearances, and IC features.

\section{Connect Point Entity}

The Connect Point entity is used to represent a point of connection. A subfigure defining an electrical component typically uses the Connect Point entity to represent a pin of the logical or physical component or symbol. A Connect Point may also be used in a "stand-alone" mode, representing a via hole, for example. 


\section{General Note Entity}

A General Note is used to display constant text. Design notes would require a General Note, for example.

\section{Associativity Definition Entity}

When the originating system provides for a relationship not included among the IGES predefined associativities, this entity is required. Possible uses are to relate subfigures to entities in other databases (e.g., circuit analysis or text requirements) or for back-annotation purposes.

\section{Text Display Template Entity}

Form 0: absolute, Form 1: incremental

The Text Display Template may be used to display text which may be unique in each instance of the defined entity (A pin number, for example).

\section{Network Subfigure Definition Entity}

For PC and Cable usage, a subfigure usually represents a component and its required PC constructs. This entity is normally a library physical or logical figure in the originating system.

402 Associativity Instance Entity

This entity relates other entities within a file to provide a "set" with a common meaning. Those associativities which are predefined by IGES are identified by IGES form numbers (e.g., form 18: Flow). The user defined associativities are defined by an entity 302 and its form number.

\section{Property Entity}

The use of a property to indicate the meaning or purpose of a geometric entity applies to electrical constructs as well as general graphics. A Connect Point 
entity may point to the Drilled Hole property. A Plane entity or Simple Closed Area entity may point to the Region Restriction property. Any property, however, may point to the Text Display Template, with the text string specified in the property. In this case, the Text Display Template locates the text display. This entity is an open-ended list allowing for expansion to address future needs such as simulation, testing, inspection applications, and extensions into electrical/electronic systems hierarchical design.

412 Rectangular array Subfigure Instance Entity

414 Circular Array Subfigure Instance Entity

These entities may be used to instance multiple Network Subfigure Instances, but must not be used for instancing Connect Points.

420 Network Subfigure Instance Entity

This entity allows an electrical component to be instanced in a number of unique locations. Note that "owned" Connect Point entities must be instanced with this entity. 


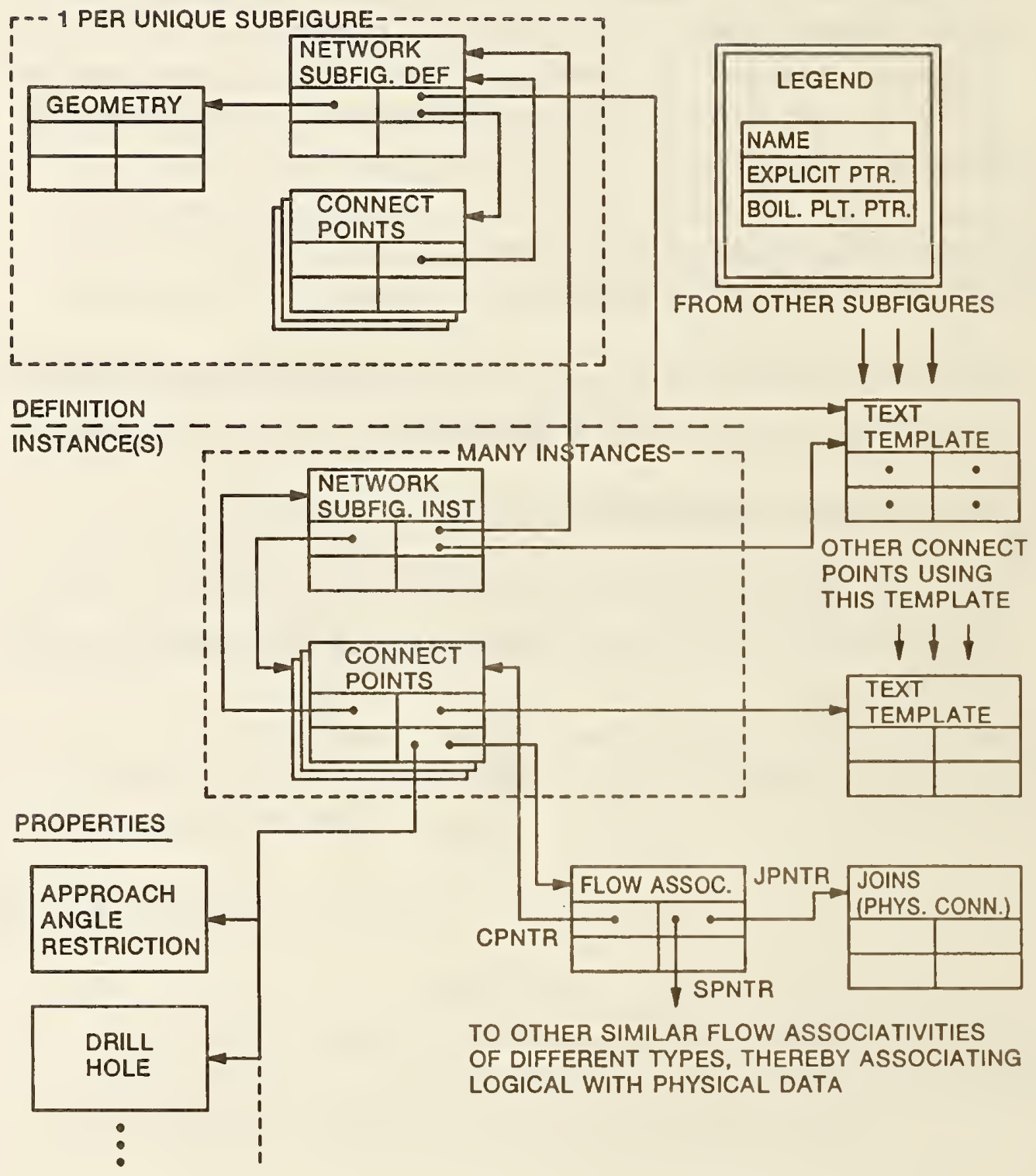

Figure B-1 General Pointer and Entity Model 
TABLE 1. TEXT TEMPLATE VALUES FOR SAMPLE SCHEMATIC

\begin{tabular}{|c|c|c|c|c|}
\hline TEMPLATE & TLEFT & TRIGHT & TTOP & TBOT \\
\hline $\begin{array}{l}\text { WIDTH } \\
\text { HEIGHT } \\
\text { SLANT ANG. } \\
\text { ROTN. ANG. } \\
\text { MIRR. FLG } \\
\text { VRT/HORZ } \\
\text { DE FORM NO. } \\
\text { X (DX) } \\
\text { Y (DY) } \\
\text { Z (DZ) }\end{array}$ & $\begin{array}{l}.10 \\
.13 \\
0 . \\
0 . \\
0 \\
0 \\
21 \\
-.09 \\
+.03 \\
0 .\end{array}$ & $\begin{array}{l}.10 \\
.15 \\
0 . \\
0 . \\
0 \\
0 \\
21 \\
-.03 \\
+.03 \\
0 .\end{array}$ & $\begin{array}{l}.10 \\
.13 \\
0 . \\
0 . \\
0 \\
0 \\
21 \\
+.03 \\
-.15 \\
0 .\end{array}$ & $\begin{array}{l}.10 \\
.13 \\
0 . \\
0 . \\
0 \\
0 \\
21 \\
+.03 \\
+.1 \\
0 .\end{array}$ \\
\hline $\begin{array}{l}\text { U1 } \\
\text { U2 } \\
\text { U3 }\end{array}$ & $\begin{array}{l}\text { P1-P8 } \\
\text { P1-P8 } \\
\text { P1-P4 }\end{array}$ & $\begin{array}{l}\text { P9-P16 } \\
\text { P9-P16 } \\
\text { P9-P12 }\end{array}$ & $\begin{array}{l}----- \\
--- \\
\text { P13-P16 }\end{array}$ & P5-P8 \\
\hline
\end{tabular}

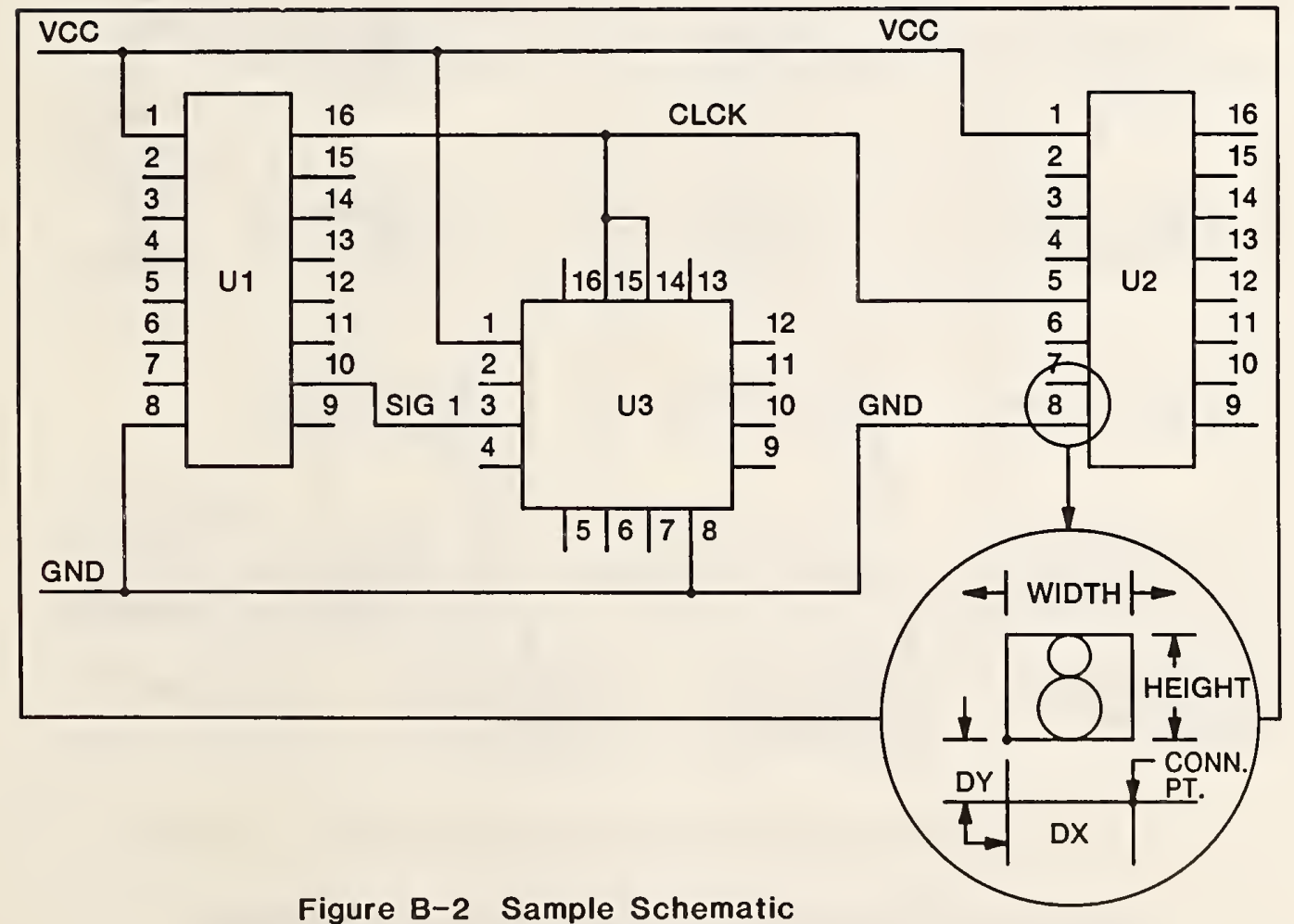




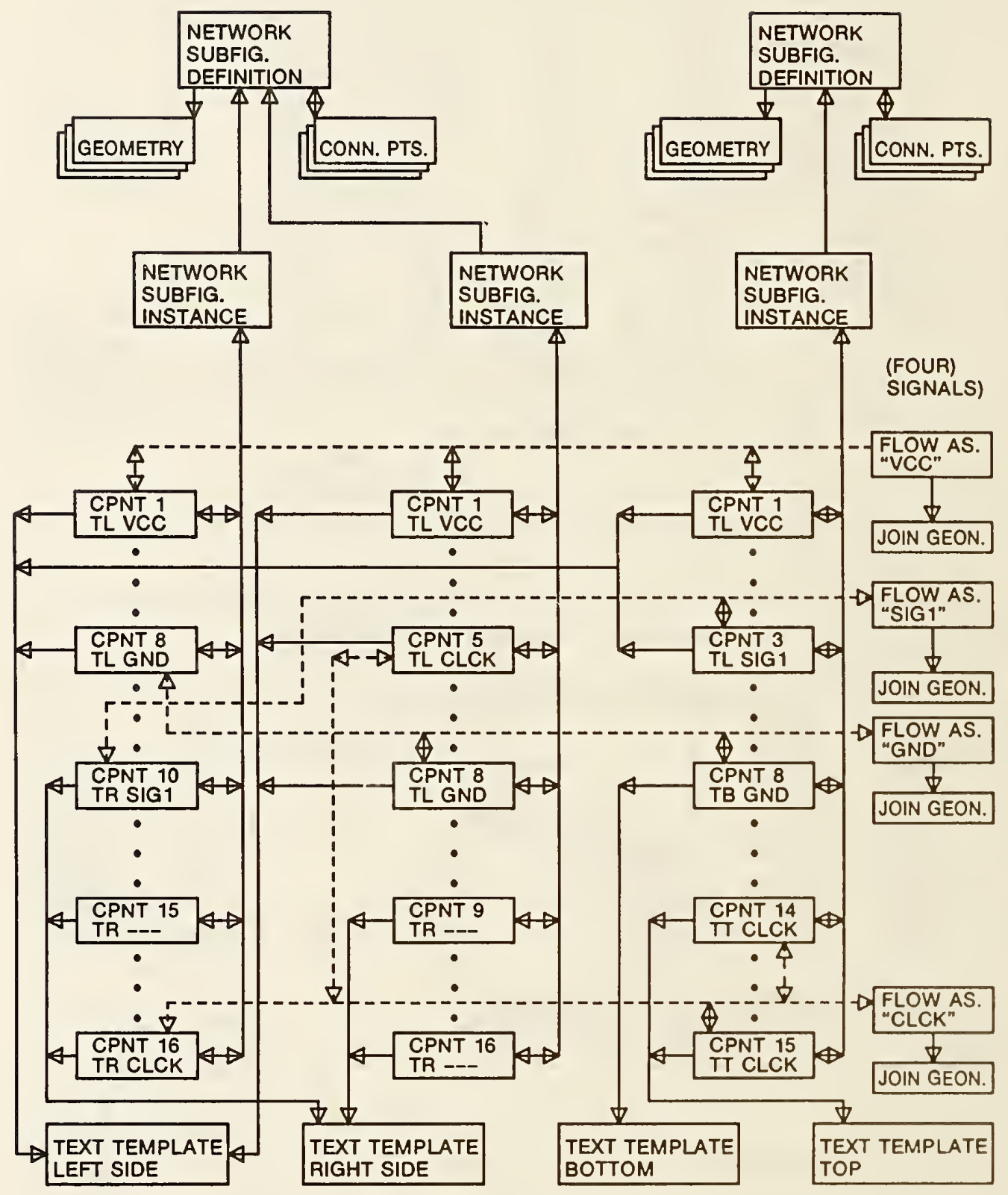

Figure B-3 Entity Relations Chart for Sample Schematic 


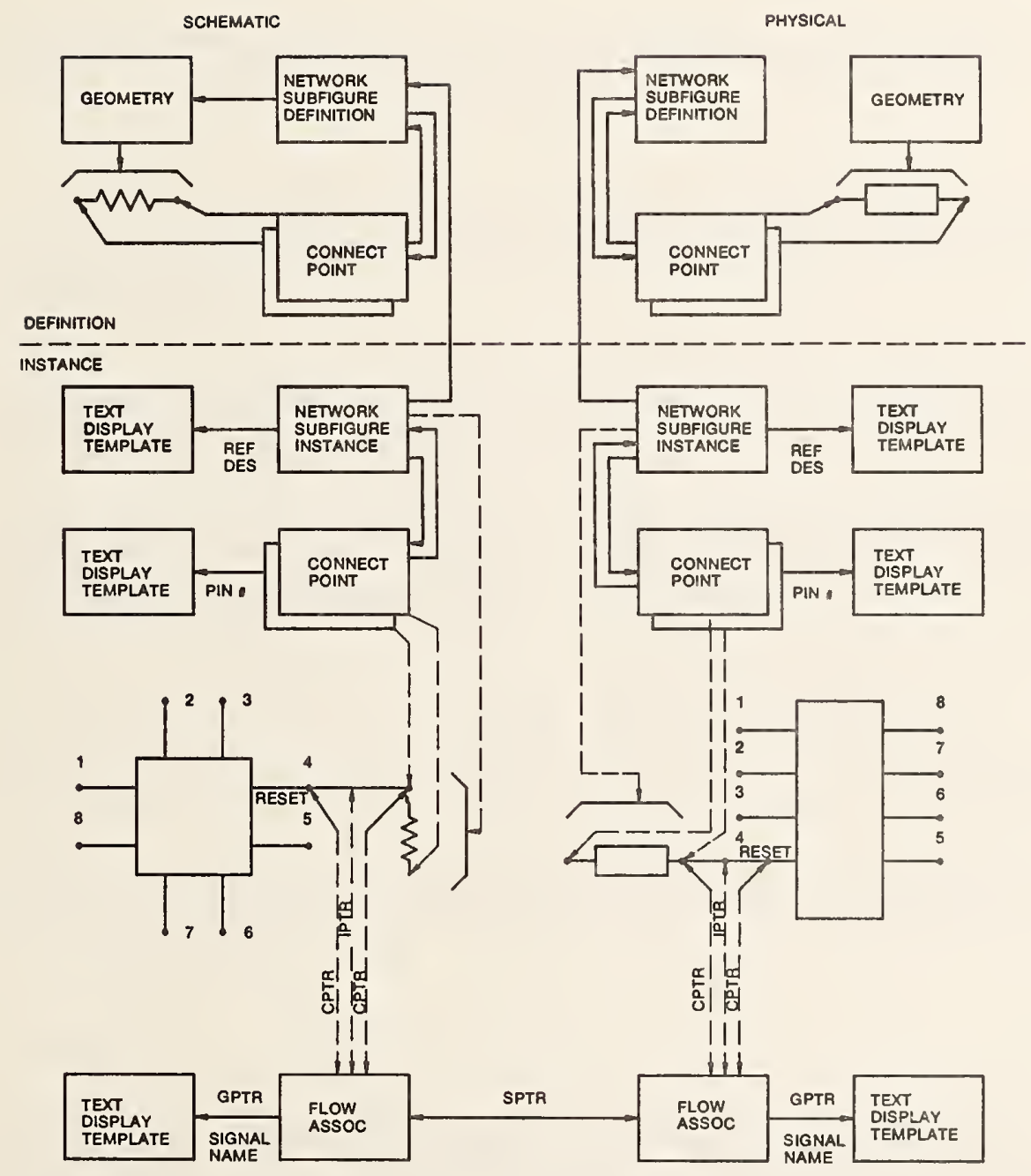

Figure B-4 Schematic/Physical Diagram for Sample Schematic 
THIS PAGE LEFT BLANK 


\section{APPENDIX C \\ PLANT FLOW SHEET REPRESENTATION}

\section{Flqwsheet Characteristics}

Process flowsheets carry several important types of information which determine what data structures must be present. They must show:

1. The identity of the process streams or lines flowing through the plant. Process and instrumentation diagrams typically show line designation, size, spec, and stream direction.

2. The identity of "nozzles" on equipment to which line connections are made, and into and out of which process streams flow.

3. The identity of equipment (i.e., valves, columns, pumps) which control, mix, pump and process the stream.

4. The association of "nozzles" with the equipment to which they belong.

5. The association of the beginning and end of each stream with unique nozzles.

6. Indication of where a line is continued if it is too large for a single sheet and is continued elsewhere.

\section{Support for Flowsheets}

The graphics of a piping and instrument diagram will typically be composed of lines, arcs, and text. Components will consist of symbols (subfigure defintions) which are defined once by a set of lines and arcs and then referenced (as subfigure instances) as many times as needed. 
A piping or instrument line is described by a path associativity entity which lists the from and to nozzles (points to their connect point entities) and then lists the in-line components. Properties can be attached to any entity and Pipe Nominal Size and Line Spec Name Properties would be attached to pipe line path entities.

\section{Plant Flow Sheet Example}

A simple flowsheet is shown in Figure $\mathrm{C}-1$. The way the flowsheet would appear on paper is shown in Figure $\mathrm{C}-\mathrm{la}$. The recommended assignment of connection points is shown in Figure $\mathrm{C}-\mathrm{lb}$.

An exploded view of the flowsheet showing the conceptual entities to be used to describe the flowsheet is given in Figure C-2a. These include two subfigures, "tank" and "valve", and four connect points belonging to them. Subfigures are defined at one time in the file for multiple use. When subfigures are used, each use is an "instance" of previously defined subfigure definitions. Two additional connect points are also shown to permit flow paths to begin and end properly. Five line entities appear to visually indicate how things are connected.

Logically the process designer has assigned these entities to pipelines or streams depending on what is being described. The logical flow paths for two process streams " $\mathrm{A}$ " and "B" are shown in Figure C-2b. Pipelines and streams are ordered sequences of equipment. When the line or stream reaches a point where two continuing paths are possible it must be told how to proceed. In some practical cases, both continuing paths may belong logically to the line or stream. A mechanism to indicate these continuations is provided by the flow path entity.

Describing how to encode a flowsheet involves:

(1) How to define the subfigure,

(2) How to instance the subfigure and the connect points it owns,

(3) How to define graphic lines which give pipelines and streams their appearance, and

(4) How to define the pipelines and streams themselves.

The entities which encode this flowsheet are given in Table C-1. The entity numbers given are the DE pointers to the example file shown in Table C-2. 


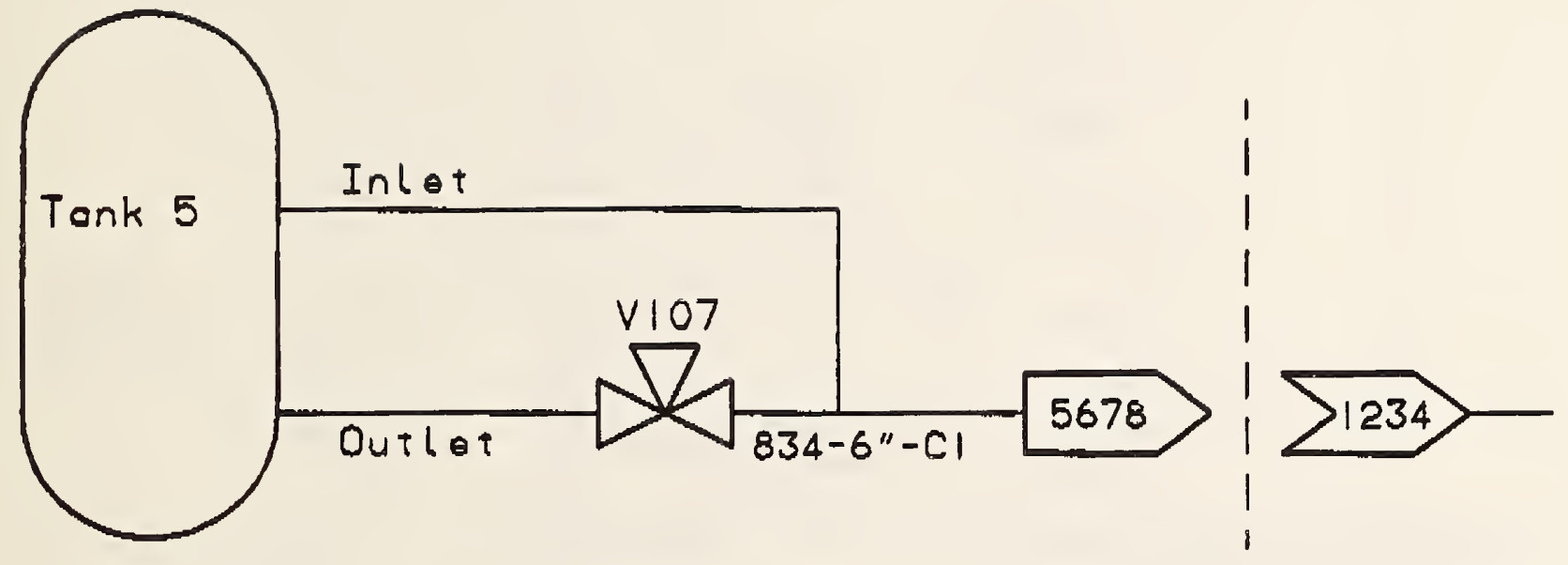

FIGURE C-1a FLOWSHEET APPEARANCE

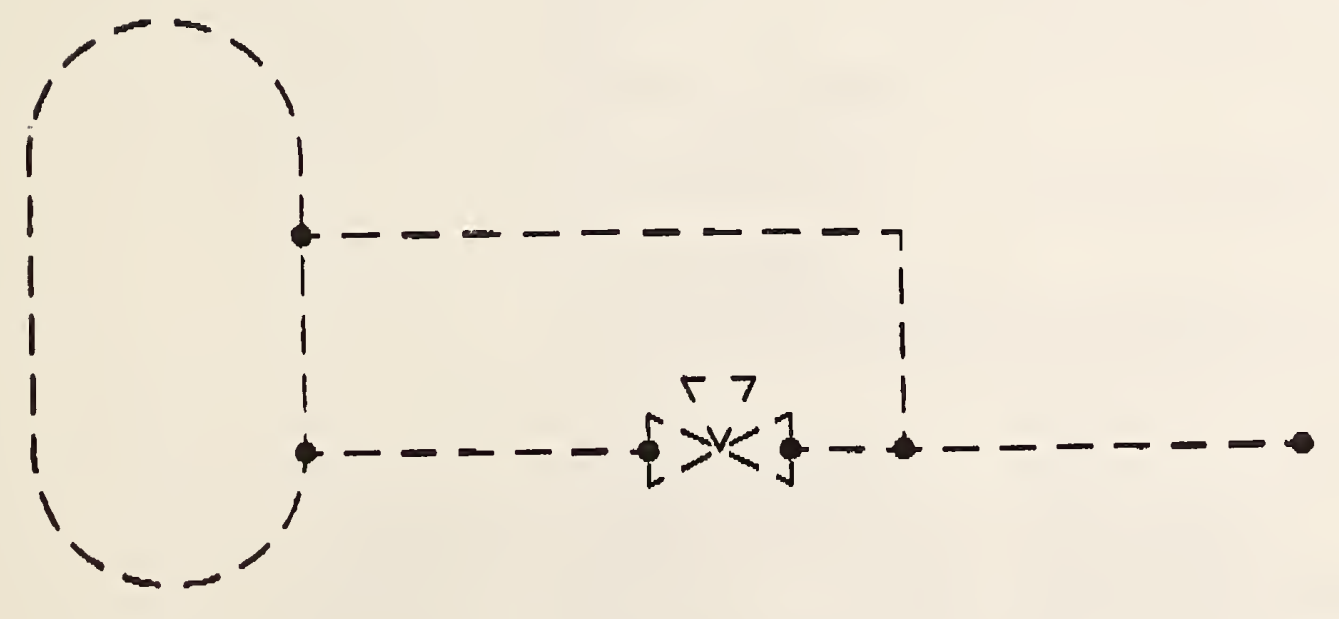

FIGURE C-1b REQUIRED CONNECTION POINTS 


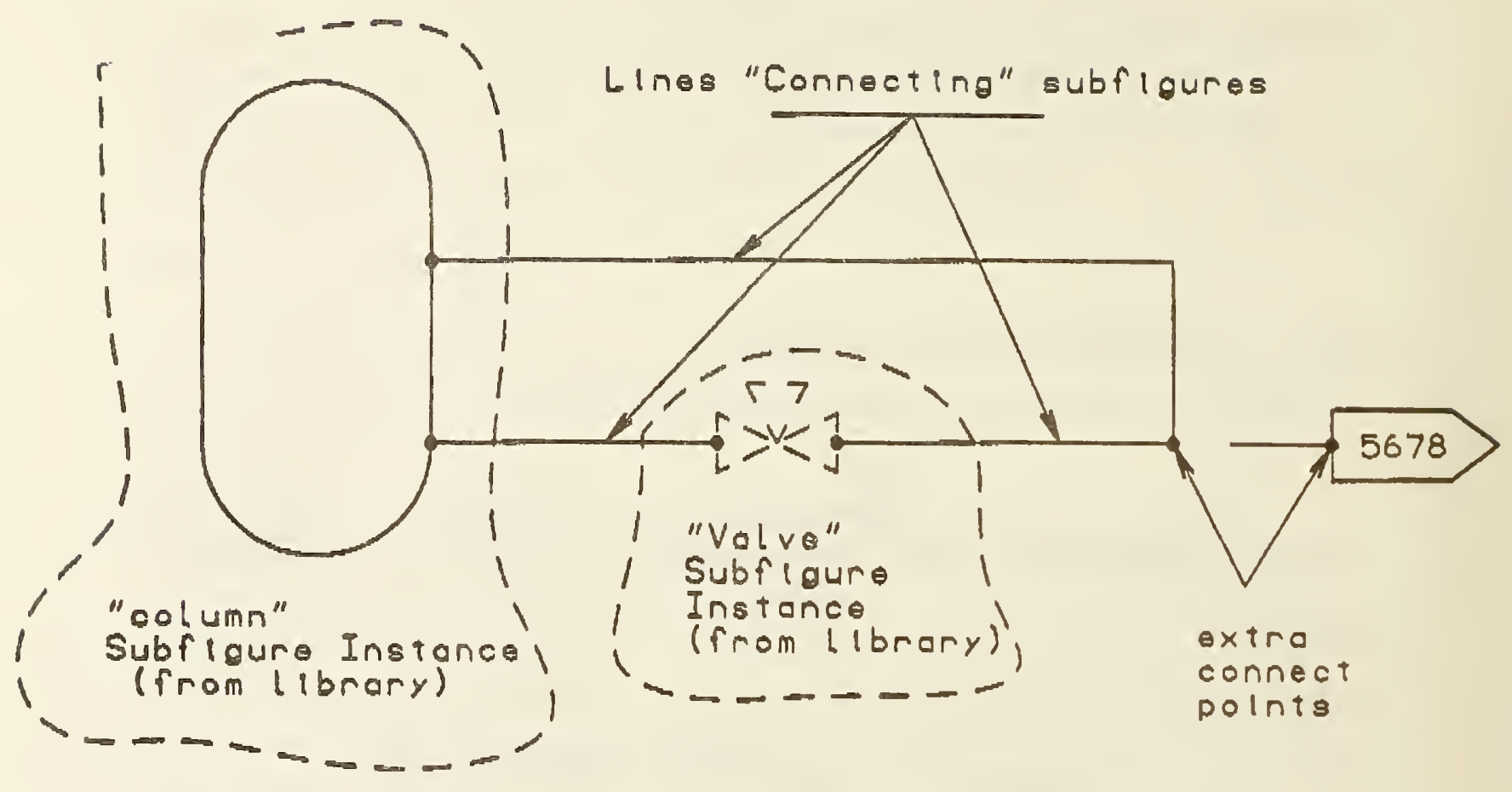

FIGURE C-2a CONCEPTUAL FLOWSHEET ENTITIES

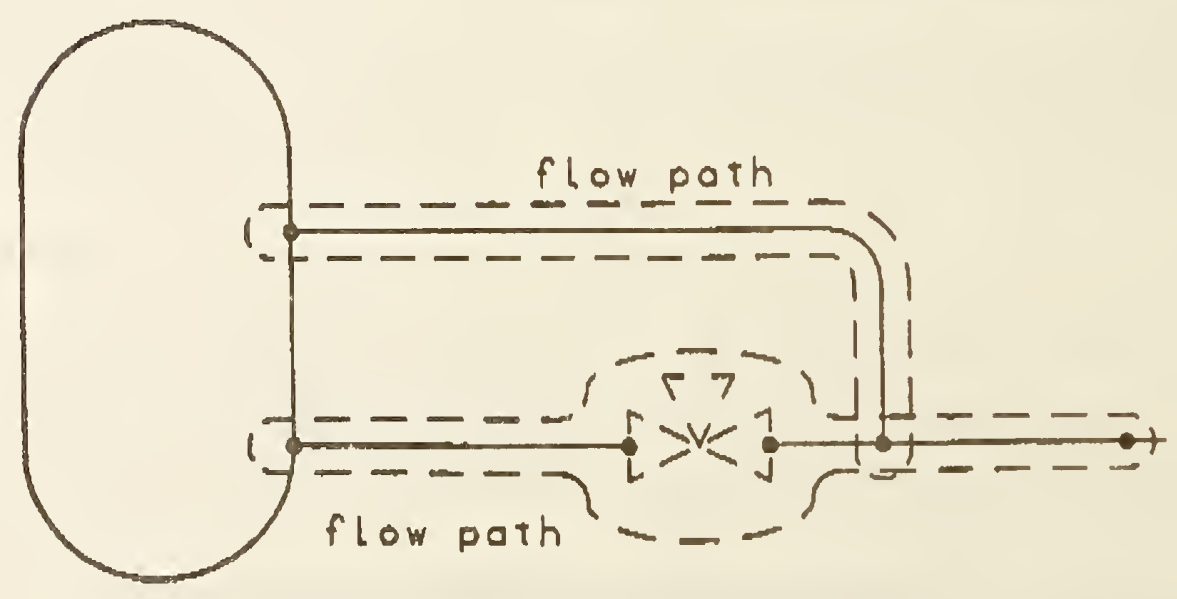


TABLE C-1

ENTITIES IN PI \& D TEST CASE

\begin{tabular}{|c|c|c|}
\hline Entity 非 & Type & $\underline{\text { Remarks }}$ \\
\hline 1 & Network subfigure def & Column \\
\hline 3 & Network subfigure def & Valve \\
\hline 5 & Line & Part of column \\
\hline 7 & Circular arc & $"$ \\
\hline 9 & Line & $"$ \\
\hline 11 & Circular arc & $"$ \\
\hline 13,15 & Connect point & Valve nozzle \\
\hline $17-29$ & Lines & Part of valve \\
\hline 31 & General note & Miscellaneous drawing text \\
\hline 33 & Text display template & Flow path name display \\
\hline 35 & Network subfigure inst. & Instance of column \\
\hline 37 & Network subfigure inst. & Instance of valve \\
\hline 39,41 & Connect point & Instance of tank nozzles \\
\hline 43,45 & Connect point & Instance of valve nozzles \\
\hline 47 & Connect point & Branch point \\
\hline 49 & Connect Point & Flow path end \\
\hline $51-59$ & Lines & Geometry of connecting path \\
\hline $61,63,65$ & Nominal size property & \\
\hline 67 & Part number property & \\
\hline 69 & External reference & Continuation of flow path \\
\hline 71,73 & Flow associativity & \\
\hline 75,77 & Text display template & $\begin{array}{l}\text { Templates for subfigure } \\
\text { name reference designator } \\
\text { display }\end{array}$ \\
\hline 79 & Text display template & $\begin{array}{l}\text { Template for display of } \\
\text { nozzle names }\end{array}$ \\
\hline $81-89$ & Line & Continuation symbol \\
\hline 91 & Line spec property & A 100 \\
\hline 93 & External reference file list & \\
\hline 95 & External reference file indes & \\
\hline
\end{tabular}




\section{Network Subfigure Definitions}

The definitions of the tank and valve subfigures are shown in Figures C-3a and C-3b. Each subfigure consists of the Network Subfigure Definition entity, geometry entities, and connect point entities. The tank is defined by entities 1,5,7,9 and 11 . The Network Subfigure Definition entity 1 contains lists of the geometry entities $($ 非 $5,7,9$, and 11$)$. This is merely a definition which is "instanced" when actually needed.

The valve has a similar form. The valve Network Subfigure Definition entity 非 points to geometric entities 17 through 29, and to Connect Point entities 13 and 15.

\section{Network Subfigure Instances}

When the tank and valve network subfigures are instanced, the subfigure's connect points will be duplicated and associated with the instance. The geometric entities will not be duplicated. Figure $\mathrm{C}-4 \mathrm{a}$ shows the subfigures as instanced in the flowsheet with instance entities 非35 ("tank") and 非 37 ("valve"). Note that the subfigures may be scaled when they are instanced. In the "valve" example different scale factors are used in the $x$ and $y$ axes. Independent $x$ and $y$ axis scaling is often used by CAD vendors to "fit" their symbols to the drawing. Network subfigure instance $\$ 35$ points to subfigure definition 1 非 1 and to the new duplicated connect points 非39 and 非1. Similarly, Network Subfigure Instance 非 37 points to Subfigure Definition $\mathbb{k}^{3}$ and to duplicate connect points 非 43 and 45.

\section{Geometric Entities for Pipeline or Stream}

Lines will appear on the drawing to indicate graphically how symbols are connected into pipelines and streams. These geometric entities alone do not logically connect symbols. This is done by Flow Path Associativity instance entities. The geometric entities are lines 非 51 through 非 59. 


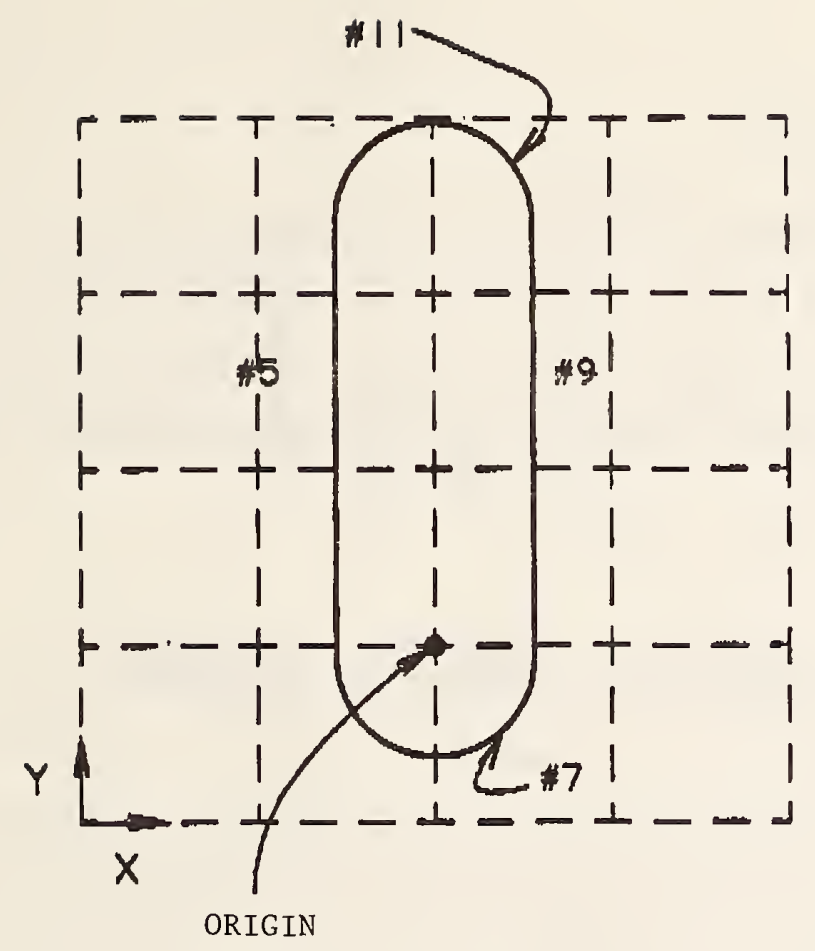

FIGURE C-3a ENTITY \#1 TANK

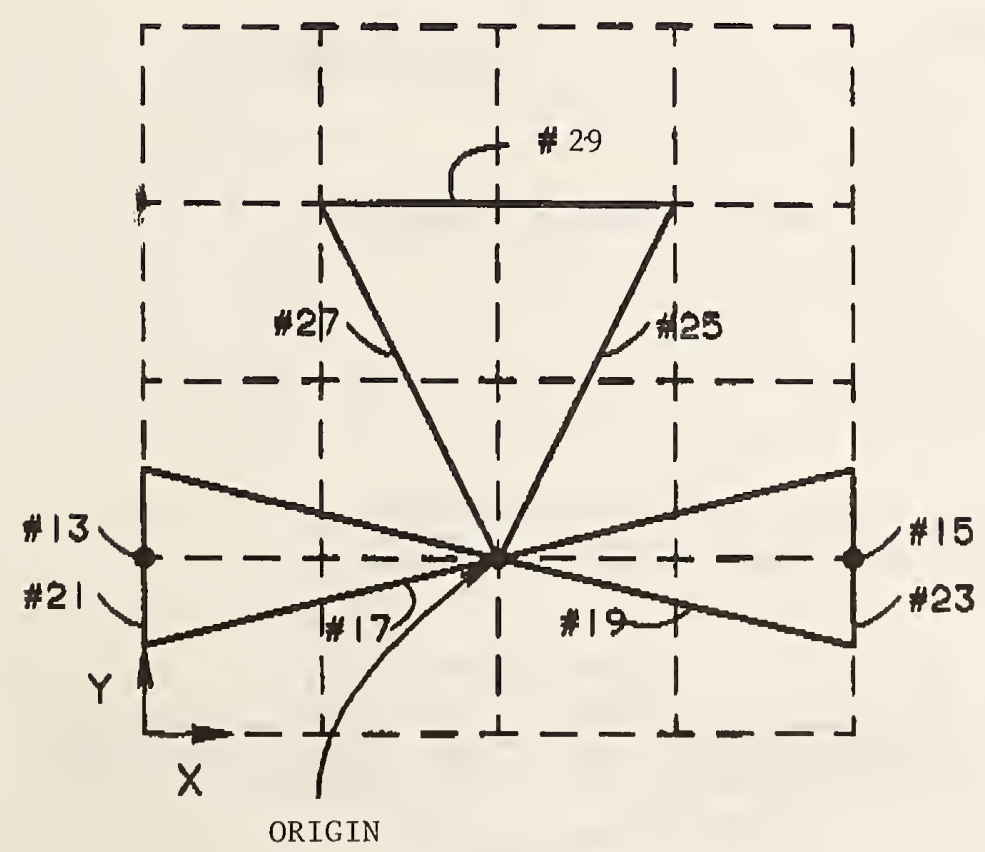

FIGURE $\mathrm{C}-3 \mathrm{~b}$ ENTITY \#3 VALVE 


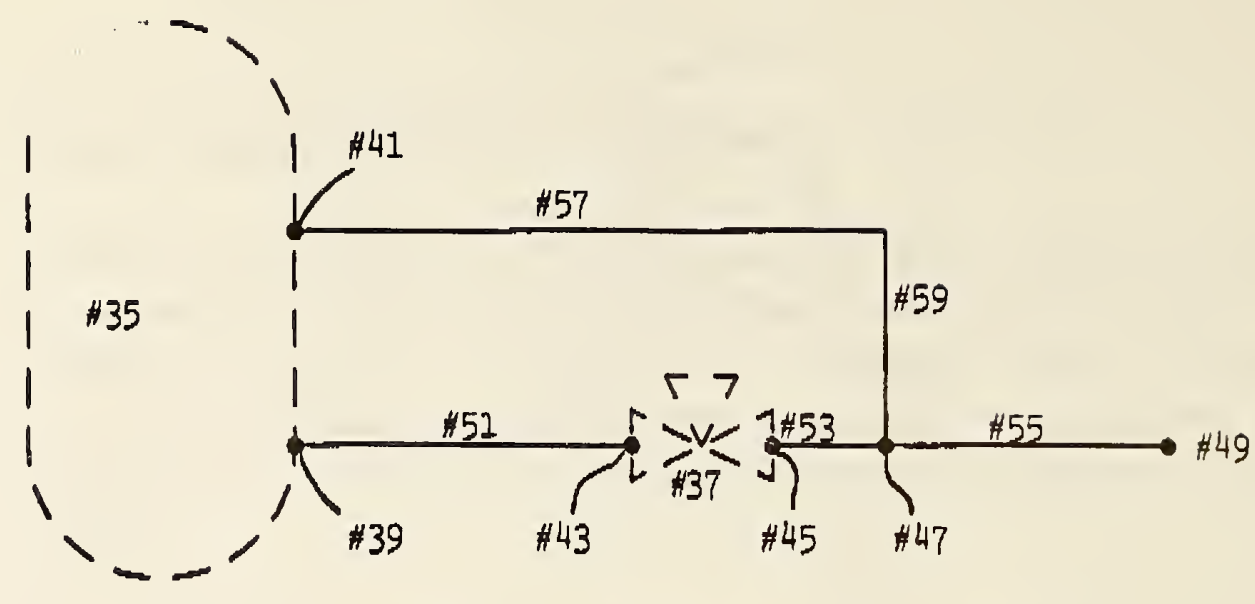

FIGURE C-4a GEOMETRY ENTITIES

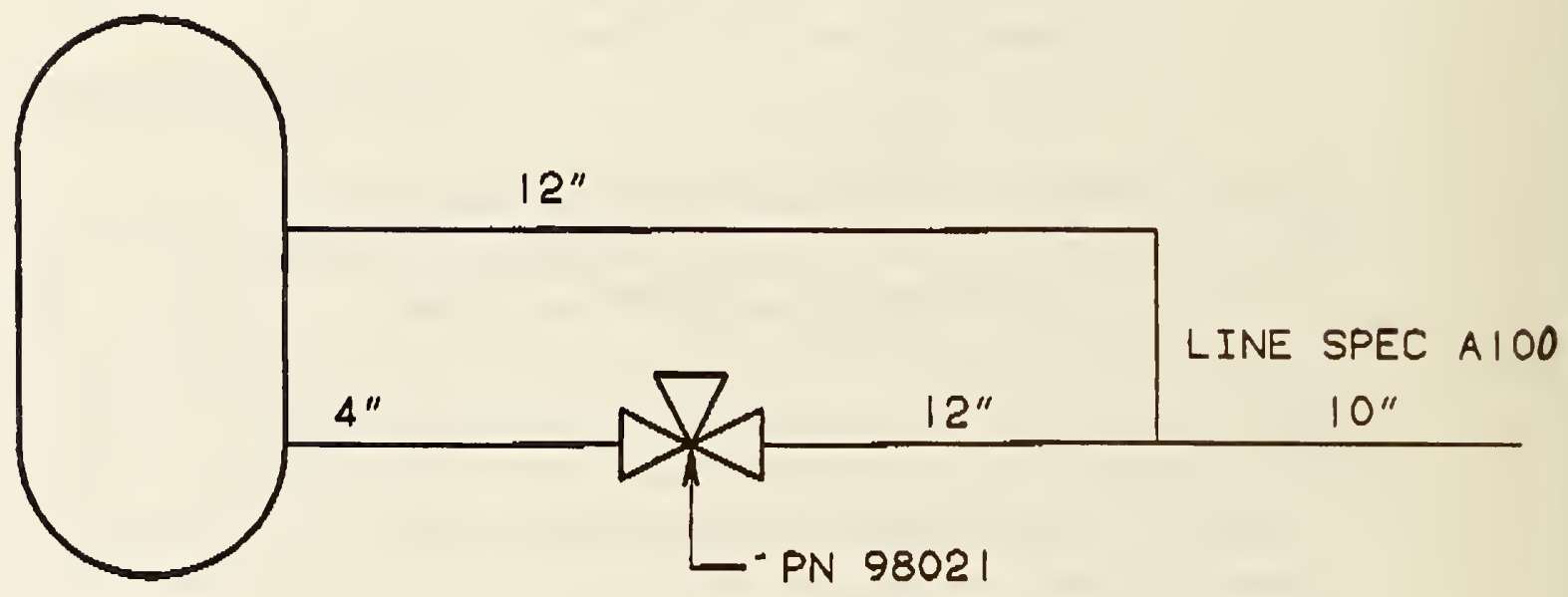

FIGURE $\mathrm{c}-4 \mathrm{~b}$ ATTRIBUTES

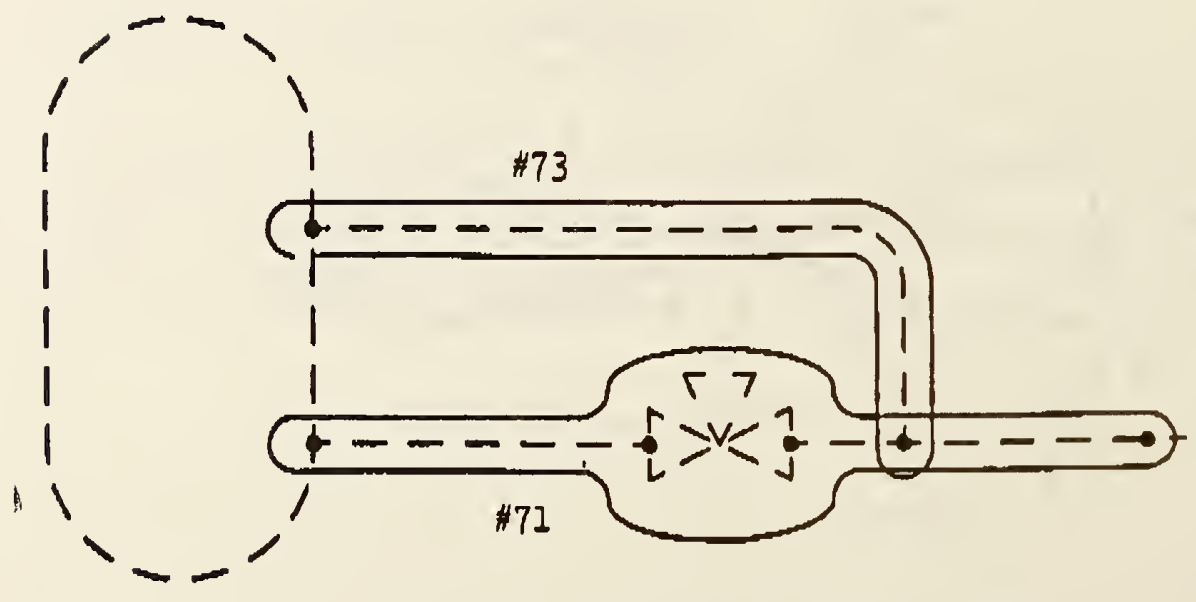

FIGURE C-4C FLOW PATHS 


\section{Attributes}

Three specific properties (attributes) are provided for flowsheets. These are part number, nominal size, and (flow) line specification. Figure C-4b shows the properties used for the test case. These are implemented by three forms of the property entity. The entities in the test case are 非 61 through 67 and 非 91 .

\section{Flow Paths}

Figure $\mathrm{C}-4 \mathrm{C}$ shows an assignment of flow paths. Two flow path entities are required, 非 1 and 73. Each Flow Path Associativity entity contains an ordered list of the connect points along the path and a separate list of the geometry entities representing the path. Where a branch occurs one path may point to one or more

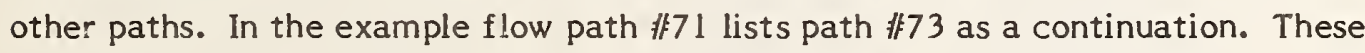
points are bi-directional, so flow path 1 作 73 contains a list of the entities (1k71) which point to it. The flow path is also the entity to which the Line Specification property is attached. Note that a rather arbitrary flow path assignment was made by the process engineer. Other assignments are possible and could be encoded.

\section{Text Templates}

Text templates are used to control display of the text which is actually contained in the Subfigure Instance and connect point entities. There are two uses of Text Template entities in this example. The first type of use is to control display of the names (reference designators) of the tank and valve instances. Absolute templates \#75 and \#77 are used for this. The tank nozzles (connect points \#39 and 非1) both have their nozzle names (connect point function identifier) displayed with the same text size and location offset relative to the connect point location. Both connect point entities point to the same Incremental Text Template entity \#79. 


\section{External References}

Flow path $\# 71$ continues offpage, i.e., the path is logically continued on another flowsheet. Three pieces of information must be matched to make this continuation:

1. Line designation or number.

2. Drawing on which the path is continued, and

3. "Incoming" or "outgoing" connectors which match to the start or end of the path on the page.

This is handled by two mechanisms:

1. The External Reference entity which points (using a unique symbolic name) to the next connect point of the path upon which continuation is made, and to the file in which the drawing is contained. The connect point function identifier field is used to carry the drawing name and a text template is used to display it. File names are recommended to be constructed from the drawing name by the pre-processor.

2. The position of connect point within a flow path to the external flow path on the external flowsheet will determine whether it is logically "incorning" or "outcoming.

We will now implement these pointer structures for the example (see figure C-5.)

Flow path \# 71 ends at external reference entity \#69. It points by a unique name 'I834-6"-C121' to a connect point in file 'XYZOIL.PROCESS.5678'. These external reference names must be unique. Typical CAD systems that do not maintain a globally unique name for a connect point may construct one using some set of conventions. The unique name ' $1834-6$ "'-C121' is typical of what can be constructed by a pre-processor using ' I' for incoming and ' $834-6$ "'-C121', the line number, size, and spec. The file name 'XYZOIL.PROCESS.5678' is constructed from the connect point function identifier string '5678' using the root string file name entry in the External Reference Entity. The External Reference File List entity 133 carries this file name also, in the referencing file. 


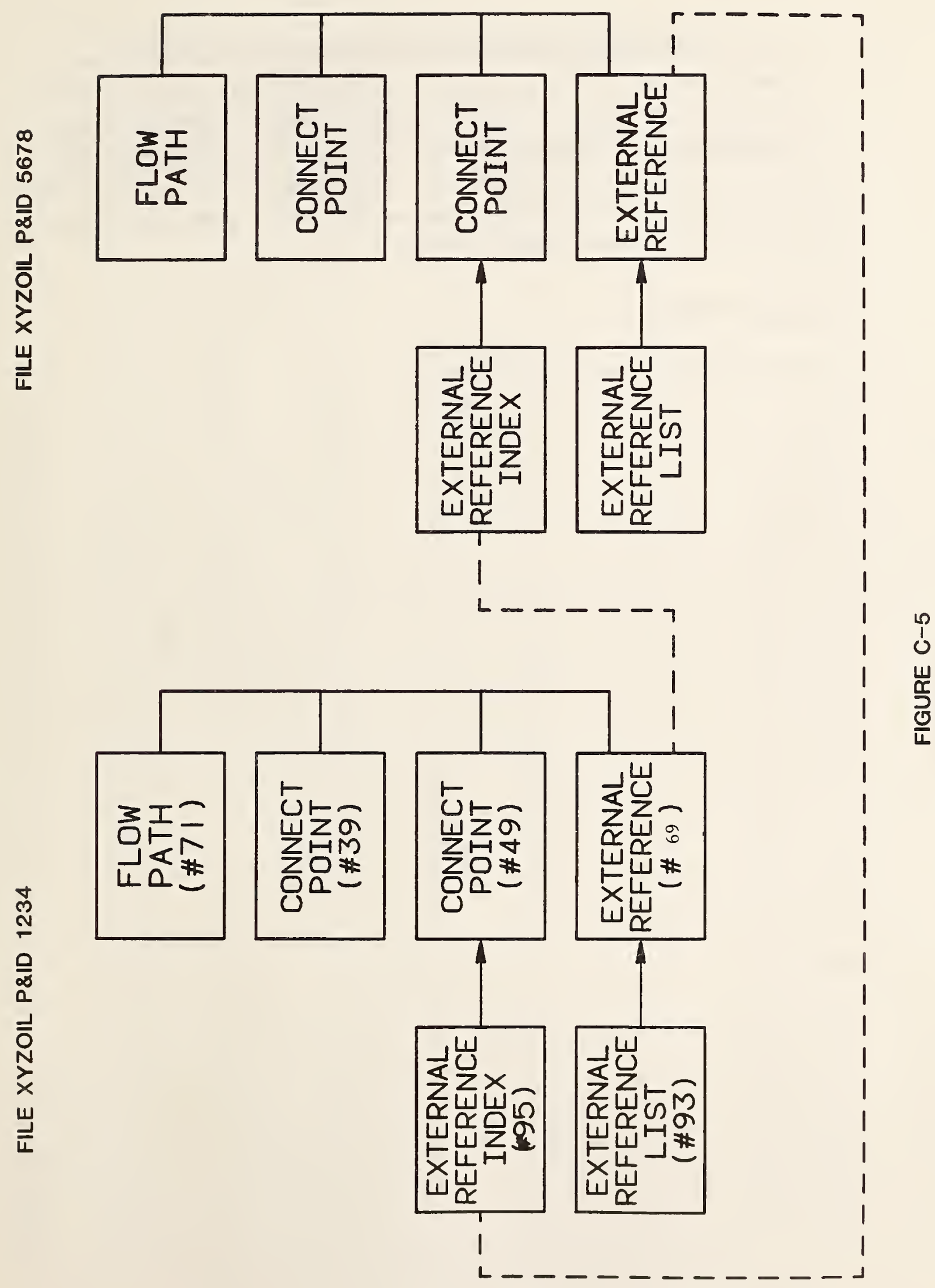


In the external flowsheet, an External Reference File Index entity will list the symbolic name '1834-6-C121' and reciprocal entities also exist to allow the external flowsheet to continue back into the referencing flowsheet. The flow path begins with an external reference entity '0834-6"C121' in file 'XYZOIL.PROCESS.1234'. It will also contain an external reference file list repeating this file name. There will also be an External Reference File Index entity $\$ 95$ containing the symbolic name '0834-6-C121' a pointer to connect point 非9.

\section{Encoded Flowsheet}

The encoded flowsheet is shown in Table C-2. 


\section{TABLE C-2 PLANT FLOWSHEET EXAMPLE}

PIPING AND INSTRUMENTATION DIAGRAM IGES EXAMPLE

$1 \mathrm{H}, 1 \mathrm{H} ;, 12 \mathrm{HPI} \& \mathrm{D}$ EXAMPLE, $19 \mathrm{HXYZOIL}$.PROCESS. $1234,10 \mathrm{HHYBRID} 1.0,1 \mathrm{H} 2,32,38, \mathrm{G}$ $6,308,15,12 \mathrm{HPI} \& \mathrm{D}$ EXAMPLE $, 0.100000 \mathrm{E}+01,1,4 \mathrm{HINCH}, 3, .06,13 \mathrm{H} 851115.233700, \mathrm{G}$ $0.0001,10000$., 10HP.W.ROURKE , 3HORG , 4, 0;

\begin{tabular}{|c|c|c|c|}
\hline 320 & & 1 & 0 \\
\hline 320 & 0 & 1 & 1 \\
\hline 320 & 2 & 1 & 0 \\
\hline 320 & 0 & 1 & 1 \\
\hline 110 & 3 & 1 & 0 \\
\hline 110 & 0 & 1 & 1 \\
\hline 100 & 4 & 1 & 1 \\
\hline 100 & 2 & 1 & 1 \\
\hline 110 & 5 & 1 & 1 \\
\hline 110 & 2 & 1 & 1 \\
\hline 100 & 6 & 1 & 1 \\
\hline 100 & 2 & 1 & 1 \\
\hline 132 & 7 & 1 & 1 \\
\hline 132 & 2 & 1 & 1 \\
\hline 132 & 8 & 1 & 1 \\
\hline 132 & 2 & 1 & 1 \\
\hline 110 & 9 & 1 & 1 \\
\hline 110 & 2 & 1 & 1 \\
\hline 110 & 10 & 1 & 1 \\
\hline 110 & 2 & 1 & 1 \\
\hline 110 & 11 & 1 & 1 \\
\hline 110 & 2 & 1 & 1 \\
\hline 110 & 12 & 1 & 1 \\
\hline 110 & 2 & 1 & 1 \\
\hline 110 & 13 & 1 & 1 \\
\hline 110 & 2 & 1 & 1 \\
\hline 110 & 14 & 1 & 1 \\
\hline 110 & 2 & 1 & 1 \\
\hline 110 & 15 & 1 & 1 \\
\hline 110 & 2 & 1 & 1 \\
\hline 212 & 16 & 1 & 1 \\
\hline 212 & 2 & 1 & 1 \\
\hline 312 & 18 & 1 & 1 \\
\hline 312 & 2 & 1 & 1 \\
\hline 420 & 19 & 1 & 1 \\
\hline 420 & 2 & 1 & 1 \\
\hline 420 & 20 & 1 & 1 \\
\hline 420 & 2 & 1 & 1 \\
\hline 132 & 21 & 1 & 1 \\
\hline 132 & 2 & 1 & 1 \\
\hline 132 & 22 & 1 & 1 \\
\hline 132 & 2 & 1 & 1 \\
\hline 132 & 23 & 1 & 1 \\
\hline 132 & 2 & 1 & 1 \\
\hline 132 & 24 & 1 & 1 \\
\hline 132 & 2 & 1 & 1 \\
\hline 132 & 25 & 1 & 1 \\
\hline 132 & 2 & 1 & 1 \\
\hline 132 & 26 & 1 & 1 \\
\hline 132 & 2 & 1 & 1 \\
\hline
\end{tabular}

\begin{tabular}{|c|c|c|}
\hline 0 & 0 & $00010200 \mathrm{D}$ \\
\hline & & D \\
\hline 0 & 0 & $00010200 \mathrm{D}$ \\
\hline & & D \\
\hline 0 & 0 & $00010000 \mathrm{D}$ \\
\hline 5 & 0 & $00010000 \mathrm{D}$ \\
\hline & & D \\
\hline 5 & 0 & $00010000 \mathrm{D}$ \\
\hline & & D \\
\hline 5 & 0 & $00010000 \mathrm{D}$ \\
\hline & & $D$ \\
\hline 5 & 0 & $00020400 D$ \\
\hline & & D \\
\hline 5 & 0 & $00020400 D$ \\
\hline 5 & 0 & D \\
\hline כ & 0 & $\begin{array}{r}00010000 \mathrm{D} \\
\mathrm{D}\end{array}$ \\
\hline 5 & 0 & $00010000 \mathrm{D}$ \\
\hline & & D \\
\hline 5 & 0 & $00010000 \mathrm{D}$ \\
\hline & & D \\
\hline 5 & 0 & $00010000 \mathrm{D}$ \\
\hline & & D \\
\hline 5 & 0 & $00010000 \mathrm{D}$ \\
\hline 5 & 0 & $00010000 \mathrm{D}$ \\
\hline & & D \\
\hline 5 & 0 & $00010000 \mathrm{D}$ \\
\hline & & D \\
\hline 5 & 0 & $\begin{array}{r}00000100 \mathrm{D} \\
\mathrm{D}\end{array}$ \\
\hline 5 & 0 & $00010201 \mathrm{D}$ \\
\hline & & D \\
\hline 5 & 0 & $00000300 \mathrm{D}$ \\
\hline & & D \\
\hline 5 & 0 & $00000300 \mathrm{D}$ \\
\hline & & D \\
\hline 5 & 0 & $00020400 \mathrm{D}$ \\
\hline & & D \\
\hline 5 & 0 & $00020400 \mathrm{D}$ \\
\hline & & D \\
\hline 5 & 0 & $00020400 \mathrm{D}$ \\
\hline & & $\mathrm{D}$ \\
\hline 5 & 0 & $00020400 \mathrm{D}$ \\
\hline & & D \\
\hline 5 & 0 & $00020400 \mathrm{D}$ \\
\hline & 0 & $00020400 D$ \\
\hline & & D \\
\hline
\end{tabular}




\begin{tabular}{|c|c|c|c|c|c|c|c|c|}
\hline 110 & 27 & 1 & 1 & 2 & \multirow[t]{2}{*}{5} & \multirow[t]{2}{*}{0} & $00000000 D$ & 51 \\
\hline 110 & 2 & 1 & 1 & 0 & & & & 52 \\
\hline 110 & 28 & 1 & 1 & 2 & 5 & \multirow[t]{2}{*}{0} & $00000000 D$ & $5 \overline{3}$ \\
\hline 110 & 2 & 1 & 1 & 0 & \multirow[b]{2}{*}{5} & & D & 54 \\
\hline 110 & 29 & 1 & 1 & 1 & & \multirow[t]{2}{*}{0} & $00000000 \mathrm{D}$ & 55 \\
\hline 110 & 2 & 1 & 1 & 0 & \multirow{3}{*}{5} & & D & 56 \\
\hline 110 & 30 & 1 & 1 & 2 & & \multirow[t]{2}{*}{0} & $00000000 D$ & 57 \\
\hline 110 & 2 & 1 & 1 & 0 & & & D & 58 \\
\hline 110 & 31 & 1 & 1 & 1 & \multirow[t]{2}{*}{5} & \multirow[t]{2}{*}{0} & $00000000 D$ & 59 \\
\hline 110 & 2 & 1 & 1 & 0 & & & D & 60 \\
\hline 406 & 32 & 1 & 1 & 1 & \multirow[t]{2}{*}{5} & \multirow[t]{2}{*}{0} & $01000000 \mathrm{D}$ & 61 \\
\hline 406 & 2 & 1 & 1 & 13 & & & D & 62 \\
\hline 406 & 33 & 1 & 1 & 2 & \multirow[t]{2}{*}{5} & \multirow[t]{2}{*}{0} & $01000000 \mathrm{D}$ & 63 \\
\hline 406 & 2 & 1 & 1 & 13 & & & D & 64 \\
\hline 406 & $3 \overline{4}$ & 1 & 1 & 1 & \multirow[t]{2}{*}{5} & \multirow[t]{2}{*}{0} & $01000000 \mathrm{D}$ & 65 \\
\hline 406 & 2 & 1 & 1 & 13 & & & D & 66 \\
\hline 406 & $3 \overline{5}$ & 1 & 1 & 1 & \multirow{2}{*}{5} & 0 & $01000000 \mathrm{D}$ & 67 \\
\hline 406 & 2 & 1 & 1 & 9 & & & D & 68 \\
\hline 416 & $3 \overline{6}$ & 1 & 1 & 1 & 5 & 0 & $01000000 D$ & 69 \\
\hline 416 & 2 & 1 & 1 & 2 & & & D & 70 \\
\hline 402 & 37 & 1 & 1 & 2 & 5 & 0 & $01000300 \mathrm{D}$ & 71 \\
\hline 402 & 2 & 1 & 1 & 18 & & & D & 72 \\
\hline 402 & 39 & 1 & 1 & 2 & 5 & 0 & $01000300 \mathrm{D}$ & 73 \\
\hline 402 & 2 & 1 & 1 & 18 & & & D & 74 \\
\hline 312 & 40 & 1 & 1 & 1 & 5 & 0 & $00010201 \mathrm{D}$ & 75 \\
\hline 312 & 2 & 1 & 1 & 0 & & & D & 76 \\
\hline 312 & 41 & 1 & 1 & 2 & 5 & 0 & $00010201 \mathrm{D}$ & 77 \\
\hline 312 & 2 & 1 & 1 & 0 & & & D & 78 \\
\hline 312 & 42 & 1 & 1 & 1 & 5 & 0 & $00010201 \mathrm{D}$ & 79 \\
\hline 312 & 2 & 1 & 1 & 0 & & & D & 80 \\
\hline 110 & 43 & 1 & 1 & 2 & 5 & 0 & $00000000 \mathrm{D}$ & 81 \\
\hline 110 & 2 & 1 & 1 & 0 & & & D & 82 \\
\hline 110 & $4 \overline{4}$ & 1 & 1 & 2 & 5 & 0 & $00000000 \mathrm{D}$ & 83 \\
\hline 110 & 2 & 1 & 1 & 0 & & & D & 84 \\
\hline 110 & 45 & 1 & 1 & 1 & 5 & 0 & $00000000 \mathrm{D}$ & 85 \\
\hline 110 & 2 & 1 & 1 & 0 & & & D & 86 \\
\hline 110 & 46 & 1 & 1 & 2 & 5 & 0 & $00000000 D$ & 87 \\
\hline 110 & 2 & 1 & 1 & 0 & & & D & 88 \\
\hline 110 & 47 & 1 & 1 & 1 & 5 & 0 & $00000000 \mathrm{D}$ & 89 \\
\hline 110 & 2 & 1 & 1 & 0 & & & D & 90 \\
\hline 406 & 48 & 1 & 1 & 1 & 5 & 0 & $01000000 \mathrm{D}$ & 91 \\
\hline 406 & 2 & 1 & 1 & 14 & & & D & 92 \\
\hline 406 & $4 \overline{9}$ & 1 & 1 & 2 & 5 & 0 & $01000000 \mathrm{D}$ & 93 \\
\hline 406 & 2 & 1 & 1 & 12 & & & D & 94 \\
\hline 402 & 50 & 1 & 1 & 1 & 5 & 0 & $01000000 \mathrm{D}$ & 95 \\
\hline 402 & 2 & 1 & 1 & 12 & & & D & 96 \\
\hline $0,4 \mathrm{H}$ & 4,5 & 1 & & & & & 1P & 1 \\
\hline & & & : & 1 & , 15; & & $3 P$ & 2 \\
\hline-0 & & & 0 & & & & $5 P$ & \\
\hline 0.0 &, 0 & & .5 & & & & $7 \mathrm{P}$ & 4 \\
\hline $\begin{array}{l}0.5 \\
0.0\end{array}$ & 0.0 & & 0 & & & & $9 P$ & $\begin{array}{l}5 \\
6\end{array}$ \\
\hline $\begin{array}{l}0 \\
-2\end{array}$ &, 2 & 5 & 0 & & & & $13 P$ & $\begin{array}{l}0 \\
7\end{array}$ \\
\hline & & & & & & & $15 \mathrm{P}$ & \\
\hline 2.0 & & & & & & & $17 \mathrm{P}$ & \\
\hline
\end{tabular}




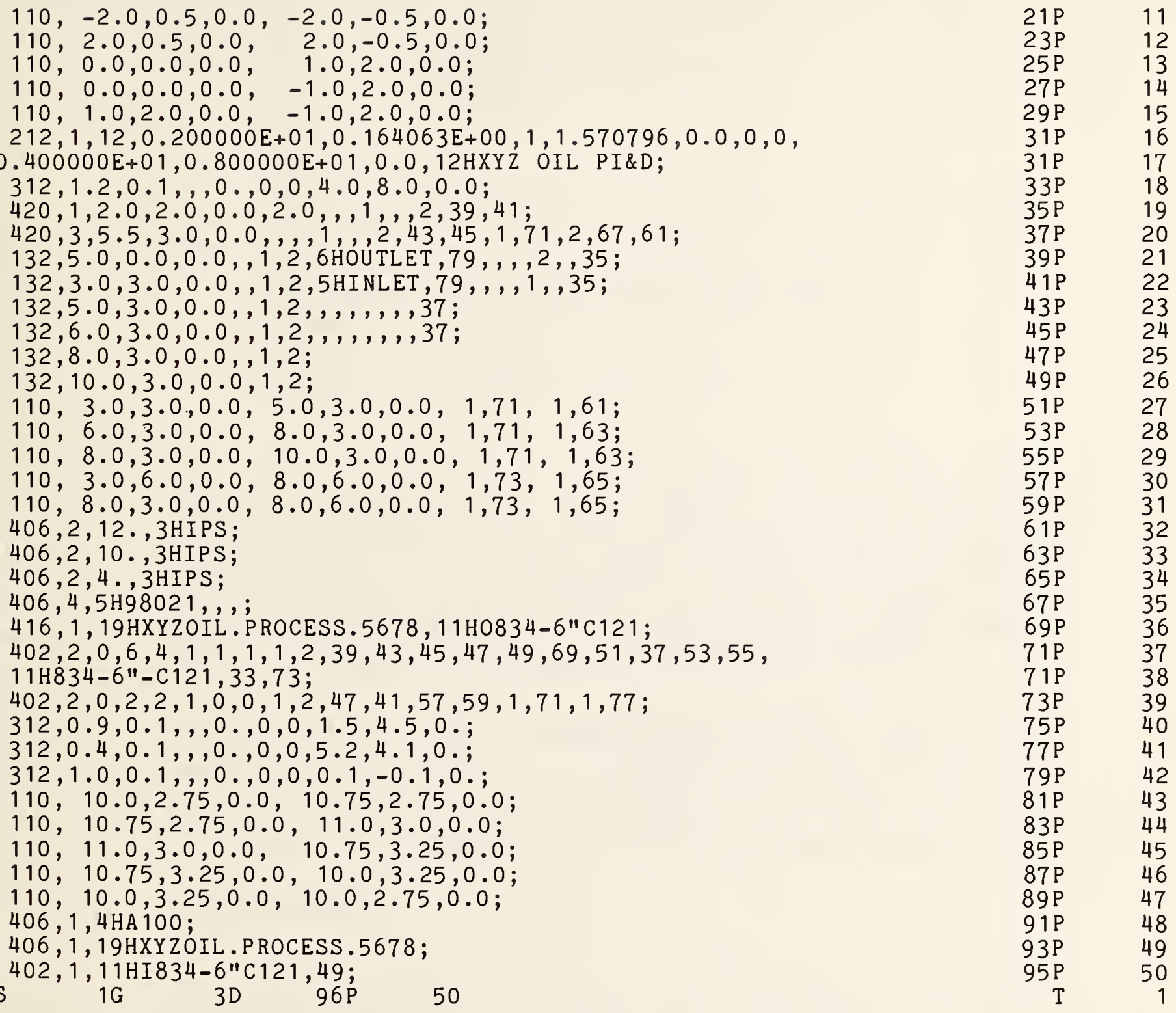


THIS PAGE LEFT BLANK 


\section{APPENDIX D \\ SPLINE REPRESENTATIONS}

\section{INTRODUCTION}

Section 3 of the Specification includes four different types of spline representations:
a. A Parametric Piecewise Cubic Polynomial (for curves)
b. A Rational B-Spline Curve
c. A Grid of General Bicubic Patches (for surfaces).
d. A Rational B-Spline Surface

Most of the spline types used in CAD/CAM systems can be mapped into these representations without change in shape. Spline types supported in Section 3 of this Specification include parametric cubics, piecewise linear, Wilson-Fowler, modified Wilson-Fowler, rational and non-rational B-splines, rational and nonrational cartesian product B-spline surfaces, and Coons patches. Spline types not supported include splines under tension and extended Coons patches.

Software to convert between parametric spline curves or surfaces and the corresponding rational B-spline curves or surfaces is available from the IGES office at the National Bureau of Standards. Materials provided include a magnetic tape of Pascal source code, a listing of the code, and accompanying documentation.

\section{D2 SPLINE FUNCTIONS}

In Section 3.8 of this Specification, spline curves are represented by a number of cubic spline functions, one for each of the $X, Y, Z$ coordinates. Each cubic spline function $\mathrm{S}(\mathrm{u})$ is defined by

a. N: The number of segments,

b. $T(1), \ldots, T(N+1)$ : The endpoints and the breakpoints separating the cubic polynomial segments,

c. $A(i), B(i), C(i), D(i), i=1, \ldots, N$ : The coefficients of the polynomials representing the spline in each of the $\mathrm{N}$ segments, 
d. CTYPE: The spline type (1=linear, 2=quadratic, 3=cubic, 4=Wilson-Fowler, $5=$ Modified Wilson-Fowler, 6=B-spline) of the originating system.

See section 3.8

e. $\quad H:$ Degree of continuity. See section 3.8.3.

To evaluate the spline at a point " $u$ ", first determine the segment containing " $u$ ", i.e., the segment " $i$ " such that $T(i) \leqslant u \leqslant T(i+1)$, then evaluate the cubic polynomial in that segment, i.e., compute

$S(u)=A(i)+B(i) *(u u)+C(i) *(u u) * * 2+D(i) *(u u) * * 3$

where $u u=u-T(i)$.

The polynomial is written in terms of the relative displacement uu (rather than $u$ ) so that the values of the spline at the breakpoints can be read directly out of the representation (i.e., $S(T(i))=A(i), i=1, \ldots, N$, and $S(T(N+1))=T P 0)$. Computations using the relative displacement also have less floating-point roundoff error.

This particular "piecewise polynomial" form is only one of many used to represent the spline segments in CAD/CAM systems. Other representations employed include:

a. End points E1,E2 and end slopes S1,S2: The spline can be evaluated using the "Hermite" basis (DEBO78, p. 59).

b. Values at four points: The spline value can be computed from the Lagrange or Newton interpolation formulae (DEBO78).

c. End points and "control" points: There are a number of schemes for computing splines from control points which will not be described here.

DeBoor (DEBO78) gives techniques for conversion between these representations. 
Splines can also be represented as a linear combination of the B-spline basis functions. In CAD/CAM systems, B-splines have been used directly in curve fitting (e.g., the B-spline Bezier polygon (GORD74)) and indirectly in various spline calculations (e.g., computing a cubic spline interpolate). For every set of breakpoints $\mathrm{T}(1), \ldots, \mathrm{T}(\mathrm{N}+1)$ and degree of continuity $\mathrm{H}$, a set of B-spline functions $\mathrm{B}(1, \mathrm{u}), \mathrm{B}(2, \mathrm{u}), \ldots, \mathrm{B}\left(\mathrm{n}^{\prime}, \mathrm{u}\right)$ can be constructed (DEBO78). Then, for any piecewise polynomial $S(u)$ with these breakpoints and continuity there is a set of B-spline coefficients $a(1), \ldots, a(n ')$ such that $S(u)$ can be represented as a linear combination of these B-splines

$S(u)=a(1) * B(1, u)+a(2) * B(2, u)+\ldots+a\left(n^{\prime}\right) * B\left(n^{\prime}, u\right)$

where $n^{\prime}=(\mathrm{N}-1) *(3-\mathrm{H})+4$.

B-splines can be computed from piecewise polynomials and vice versa (DEBO78, p. 116 and Subroutine BSPLPP).

Several other types of spline representations (e.g., cardinal bases) have been employed, but they are much less common and do not appear to present a problem for this Specification.

\section{SPLINE CURVES}

The comments in this section pertain primarily to section 3.8 .

Since curves in CAD/CAM problems are frequently many-valued, spline functions cannot represent such curves adequately. The most common approach to curve fitting is to parameterize the curves, i.e., to represent each curve as either two or three spline functions (one for each coordinate)

$$
\begin{aligned}
& X(u)=S x(u), \\
& Y(u)=S y(u), \text { and } \\
& Z(u)=S z(u)
\end{aligned}
$$

which sketch out the curve as the parameter $u$ varies from $T(1)$ to $T(N+1)$. 
All of the spline function representations of the previous section can be generalized to parametric curves and the algorithms for converting spline curves from one representation to the other follow easily from multiple applications of the corresponding function conversion algorithms.

Wilson-Fowler Curves: In the early sixties, the Wilson-Fowler spline (a special case of parametric cubics) was developed for curve fitting (IITR68). It is still used in many turnkey drafting systems. In the Wilson-Fowler representation, each spline segment is defined in a separate coordinate system whose $\mathrm{X}$-axis begins at one endpoint of the segment and passes through the other. Each spline segment is then defined by a cubic spline function $S w f(x)$ and the coordinates of the two endpoints. These WilsonFowler splines can be converted to splines defined in Section 3.8 by rotating the parametric spline $(\mathrm{u}, \mathrm{Swf}(\mathrm{u}))$ back into the current coordinate system; however, most types of splines defined in Section 3.8 cannot be conver ted to Wilson-Fowler splines.

The comments in this section pertain primarily to section 3.16 .

A rational B-spline curve is expressed parametrically in the form

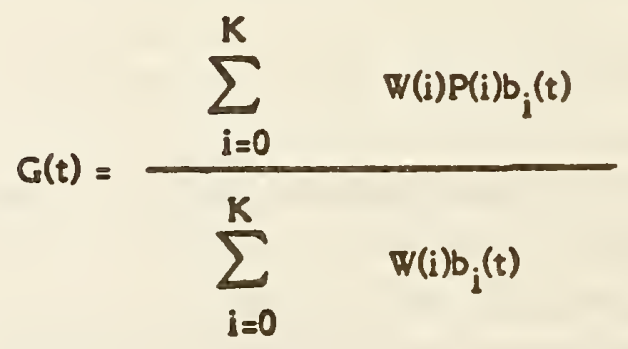

where the notation is interpreted as follows.

The W(i) are the weights (non-zero real numbers).

The $\mathrm{P}(\mathrm{i})$ are the control points (points in $\mathrm{R}^{3}$ ). 
The $b_{i}$ are the $B$-spline basis functions. These are defined as soon as their degree, $M$, and underlying knot sequence, $T$, are specified.

This is done as follows:

Let $N=K-M+1$. Then, the knot sequence consists of the non-decreasing set of real numbers; $T(-M), \ldots, T(0), \ldots, T(N), . ., T(N+M)$

The curve itself is parametrized for $V(0) \leqslant t \leqslant V(1)$ where $T(0) \leqslant V(0)<V(1) \leqslant T(N)$.

The $\mathrm{B}$-spline basis functions $b_{\mathrm{i}}$ are each non-negative piecewise polynomials of degree $M$. The function $b_{i}$ is supported by the interval $[T(i-M), T(i+1)]$. Between any two adjacent knot values $T(j), T(j+1)$ the function can be expressed as a single polynomial of degree $\mathrm{M}$.

For any parameter value $t$ between $T(0)$ and $T(N)$ the basis functions satisfy the identity

$$
\sum_{i=0}^{k} b_{i}(t)=1
$$

If the weights are all positive, the curve $G(t)$ is contained within the convex hull of its control points.

There are a number of ways to precisely define the B-spline basis functions. A recursive approach proceeds as follows.

Let $N\left(t \mid t_{i-m}, \ldots, t_{i+1}\right)$ denote the B-spline basis function of degree $m$ supported by the interval $\left[t_{i-m}, t_{i+1}\right]_{1}$.

With this notation, the degree 0 functions are simply characteristic functions of a half-open interval.

$$
N(t \mid a, b)=\quad \begin{aligned}
& 1 \text { if } a \leqslant t<b \\
& 0 \text { otherwise }
\end{aligned}
$$


The degree $\mathrm{k}$ functions are defined in terms of those of degree $\mathrm{k}-1$.

$$
\begin{aligned}
& N\left(t \mid s_{0}, \ldots, s_{k}\right)= \\
& \frac{\left(t-s_{0}\right) N\left(t \mid s_{0}, \ldots, s_{k-1}\right)}{s_{k-1}-s_{0}}+\frac{\left(s_{k}-t\right) N\left(t \mid s_{1}, \ldots, s_{k}\right)}{s_{k}-s_{1}}
\end{aligned}
$$

Since some of the denominators will be 0 in the case of multiple knots, the convention $0 / 0=0$ is adopted in the above definition.

Rational Bezier curves (and surfaces) can be expressed exactly as rational Bspline curves (and surfaces). (BLOM82).

Software to convert between parametric spline curves or surfaces and the corresponding rational B-spline curves or surfaces is available from the IGES office at the National Bureau of Standards. Materials provided include a magnetic tape of Pascal source code, a listing of the code, and accompanying documentation.

\section{SPLINE SURFACES}

The spline surface defined in Section 3.9 is the analog of the spline curve defined in section 3.8, i.e., it is also pieced together out of other primitive functions. The surface is a grid of parametric bicubic patches defined by:

a. M: The number of grid lines in $u$,

b. TU(1),..,TU(M+1): The breakpoints in $u$ (u values of grid lines),

c. N: The number of grid lines in $v$,

d. TV(1),...,TV $(\mathrm{N}+1)$ : The breakpoints in $v$ ( $v$ values of grid lines),

e. $A x(i, j), B x(i, j), \ldots, A y(i, j), \ldots, A z(i, j), \ldots$, for $i=1, \ldots, M ; j=1, \ldots, N$ :

The $M^{*} N$ sets of $3 * 16$ coefficients defining the bicubic polynomial for each of the three coordinates of the patch, 
f. CTYPE: The spline type. (1=linear, 2=quadratic, 3=cubic, 4=WilsonFowler, 5=Modified Wilson-Fowler, 6 = B-spline), and

g. PTYPE: The patch type. (1=Cartesian product, $0=$ unspecified).

To evaluate the spline at a point " $u, v$ ", first determine the patch containing the point " $u, v$ " in the parameter grid, i.e., the patch " $i, j$ " such that $T U(i) \leqslant u \leqslant T U(i+1)$ and $T V(j) \leqslant v \leqslant T V(j+1)$, then evaluate the bicubic polynomial in that patch, i.e., compute

$$
\begin{aligned}
& x(u, v)=A x(i, j) * v v * * 0 * u u * * 0+B x(i, j) * v v * * 0 * u u * 1 \\
& +C x(i, j) * v v * * 0 * u u * 2+D x(i, j) * v v * * 0 * u u * 3 \\
& +E x(i, j) * v v^{*} 1 * u u * 0+F x(i, j) * v v * * 1 * u u * 1 \\
& +G x(i, j) * v v * * 1 * u u * 2+H x(i, j) * v v * 1 * u u * 3 \\
& +\mathrm{Kx}(\mathrm{i}, \mathrm{j}) * v \mathrm{v} * * 2 * \mathrm{uu} * 0+\mathrm{Lx}(\mathrm{i}, \mathrm{j}) * v \mathrm{v} * * 2 * u u * 1 \\
& +M x(i, j) * v v * 2 * u u * 2+N x(i, j) * v v * * 2 * u u * * 3 \\
& +P x(i, j) * v v * 3 * u u * 0+Q x(i, j) * v v * 3 * u u * 1 \\
& +\mathrm{Rx}(\mathrm{i}, \mathrm{j}) * v v * * 3 * u u^{*} * 2+5 x(i, j) * v v * * 3 * u u * 3 \\
& Y(u, v)=A y(i, j) \ldots \\
& \mathrm{z}(\mathrm{u}, \mathrm{v})=\mathrm{Az}(\mathrm{i}, \mathrm{j}) \ldots \\
& \text { where } u u=u-T U(i) \text { and } v v=v-T V(j)
\end{aligned}
$$

The patches in the spline surface are equivalent to the bicubic surface patch (or the Coons patch, see (ROGE76, p. 170) for the conversion details). The parameters of the Coons patch are given as the corner points, corner slopes, and twist vectors (similar in spirit to the point/slope representation for curves).

However, because the Specification spline is more general than splines found in many CAD/CAM systems (e.g., the APT Wilson-Fowler spline), shapepreserving transformations out of the Specification spline format may not be possible. Difficulties encountered include restrictions such as uniform 
breakpoint spacing and smooth second derivatives. In these cases, the conversion must be accomplished by an interpolation or smoothing process.

The comments in this section pertain primarily to section 3.17 .

A rational B-spline surface is expressed parametrically in the form

$$
G(s, t)=\frac{\sum_{i=0}^{K 1} \sum_{j=0}^{K 2} w(i, j) P(i, j) b_{i}(s) b_{j}(t)}{\sum_{l=0}^{K 1} \sum_{j=0}^{K 2} w(i, j) b_{i}(s) b_{j}(t)}
$$

where the notation is analogous to that used for rational B-spline curves.

The $W(i, j)$ are the weights (non-zero real numbers).

The $\mathrm{P}(\mathrm{i}, \mathrm{j})$ are the control points (points in $\mathrm{R}^{3}$ ).

The $b_{i}$ are the B-spline basis functions of degree $M l$ determined by the knot sequence $\mathrm{S}(-\mathrm{M} 1), \ldots, \mathrm{S}(\mathrm{N} 1+\mathrm{M} 1)$. The $\mathrm{b}_{\mathrm{j}}$ are the $\mathrm{B}$-spline basis functions of degree $M 2$ determined by the knot sequence $T(-M 2), \ldots, T(N 2+M 2)$. Here, $\mathrm{N} 1=\mathrm{K} 1-\mathrm{M} 1+1$ and $\mathrm{N} 2=\mathrm{K} 2-\mathrm{M} 2+1$.

The surface itself is parameterized for $U(0) \leqslant s \leqslant U(1)$ and for $V(0) \leqslant t \leqslant V(1)$ where $S(0) \leqslant U(0)<U(1) \leqslant S(N 1)$ and $T(0) \leqslant V(0)<V(1) \leqslant T(N 2)$. Rational Bezier surfaces (and curves) can be expressed exactly as rational B-spline surfaces (and curves). (BLOM82).

If the surface is periodic with respect to the first parametric variable, set PROP4 to 1 ; otherwise set PROP4 to 0 . If the surface is periodic with respect to the second parametric variable, set PROP5 to 1; otherwise set PROP5 to 0 . 
Software to convert between parametric spline curves or surfaces and the corresponding rational B-spline curves or surfaces is available from the IGES office at the National Bureau of Standards. Materials provided include a magnetic tape of Pascal source code, a listing of the code, and accompanying documentation. 
THIS PAGE LEFT BLANK 


\section{APPENDIX E}

CONIC ARCS

Conic arcs as specified by the IGES standard are extremely sensitive to the data in two distinct ways:

\section{(a) Accuracy}

It is numerically sensitive; small changes in the coefficients can cause large changes in the locations of the points satisfying the conic equation.

\section{(b) Stability}

The determination of the conic type depends upon whether certain invariants are positive, zero or negative. Working in floating point arithmetic, a machine value of 0.0 is unlikely to be encountered. Furthermore, small changes in coefficient values can easily result in positive values when negative ones are intended and conversely.

It is assumed that data is put into a conic arc entity with the intent of preserving the geometric properties of the data (major and minor semi-axes, asymptotes, directrices, etc.) in addition to describing the points on the curve.

If the geometric properties are desired, the 104 entity should be used as described below.

This method primarily addresses the stability problem, though the accuracy of the conic should improve because the range of coefficient values will decrease. While the geometric properties are not explicitly defined in this representation, they can be obtained from it in a direct and arithmetically stable manner.

If both the sending and intended receiving system are known to use the A-F form of the 104 entity (Conic Arc) in their own databases the preprocessor may put the data into in the unchanged form. This minimizes the loss of information caused by truncation and roundoff errors as no changes are made to the data. The stability problem is presumably not of concern in this case. 
Here is one suggested set of values:

(1) Ellipse
$\mathrm{A}:=\overline{\mathrm{AXISY}}{ }^{2}$
$B:=0$
$\mathrm{C}:=\mathrm{AXISX}^{2}$
$\mathrm{D}:=0$
$E:=0$
$F:=-A * C$

where AXISY and AXISX are the lengths of the major and minor semiaxes (not necessarily in order).

(2) Hyperbola
$A:=-A_{X I S Y} 2 \quad\left(o r+A X I S Y^{2}\right)$
$\mathrm{B}:=0$
$C:=+\operatorname{AXISX} 2$ (or $-\operatorname{AXISX}^{2}$, if $\left.A \quad 0\right)$
$\mathrm{D}:=0$
$E:=0$
$F:=-A * C$.

where AXISY and AXISX are the lengths of the major and minor semiaxes (not necessarily in order).

(3) Parabola
$A:=0$
(or 1 )
$\mathrm{B}:=0$
$C:=1$
(or 0 , if $A=1$ )
$\mathrm{D}:=4 * \mathrm{DIST}$
(or 0 , if $A=1$ )
$E:=0$
(or $4 *$ DIST, if $A=1$ )
$F:=0$

where DIST is the distance of the vertex from the focus.

\section{Preprocessor Conic Handling}

The conic arc must be put into standard form, parallel to the $X$ and/or $Y$ axis(axes) and centered about the origin. An 124 transformation matrix must be used to move the conic arc into its desired position in space. In this form the coefficients in the format that should be 0.0 will be exactly so. In particular, for the ellipse and hyperbola $B, D$, and $E$ must be 0.0 , and for the parabola $B$ and $F$ and either $A$ and $E$ or $\mathrm{C}$ and $\mathrm{D}$ must be 0.0 .

Determination of the conic type from the equations becomes straight forward for the postprocessor.

For further mathematical details, see (THOM60). 


\section{APPENDIX F \\ COLOR SPACE VARIATIONS}

It is often more convenient to operate in some color space other than RGB. The relationship between RGB and CMY is given by:

$$
\begin{array}{lll} 
& \text { where: } & \\
R=100.0-C & R=\text { red } & C=\text { cyan } \\
G=100.0-M & G=\text { green } & M=\text { magenta } \\
B=100.0-Y & B=\text { blue } & Y=\text { yellow }
\end{array}
$$

The HSL color space can be defined in terms of RGB in several ways with subtle variations. A typical approach is given by the following transformation:

$$
\begin{array}{ll}
H=\frac{1}{2 \pi} \arctan \left[\frac{2 R-G-B}{\sqrt{3(G-B)}}\right] & \text { where: } \\
S=\left(R^{2}+G^{2}+B^{2}-R G-R B-B G\right)^{\frac{1}{2}} & H=\text { Hue } \\
L=\frac{1}{3}(R+G+B) & S=\text { Saturation }
\end{array}
$$

Variations on this transformation are given in (JOBL78) and SMIT78). 
THIS PAGE LEFT BLANK 
FILE CONVERSION UTILITIES

APPENDIX G

This Appendix gives details of utility programs to convert a file from the ASCII Form to the Compressed ASCII Form and back again. These utilities are written in Fortran 77 code, have been tested at several sites and are available from the IGES office at The National Bureau of Standards. 
APPENDIX G FILE CONVERSION

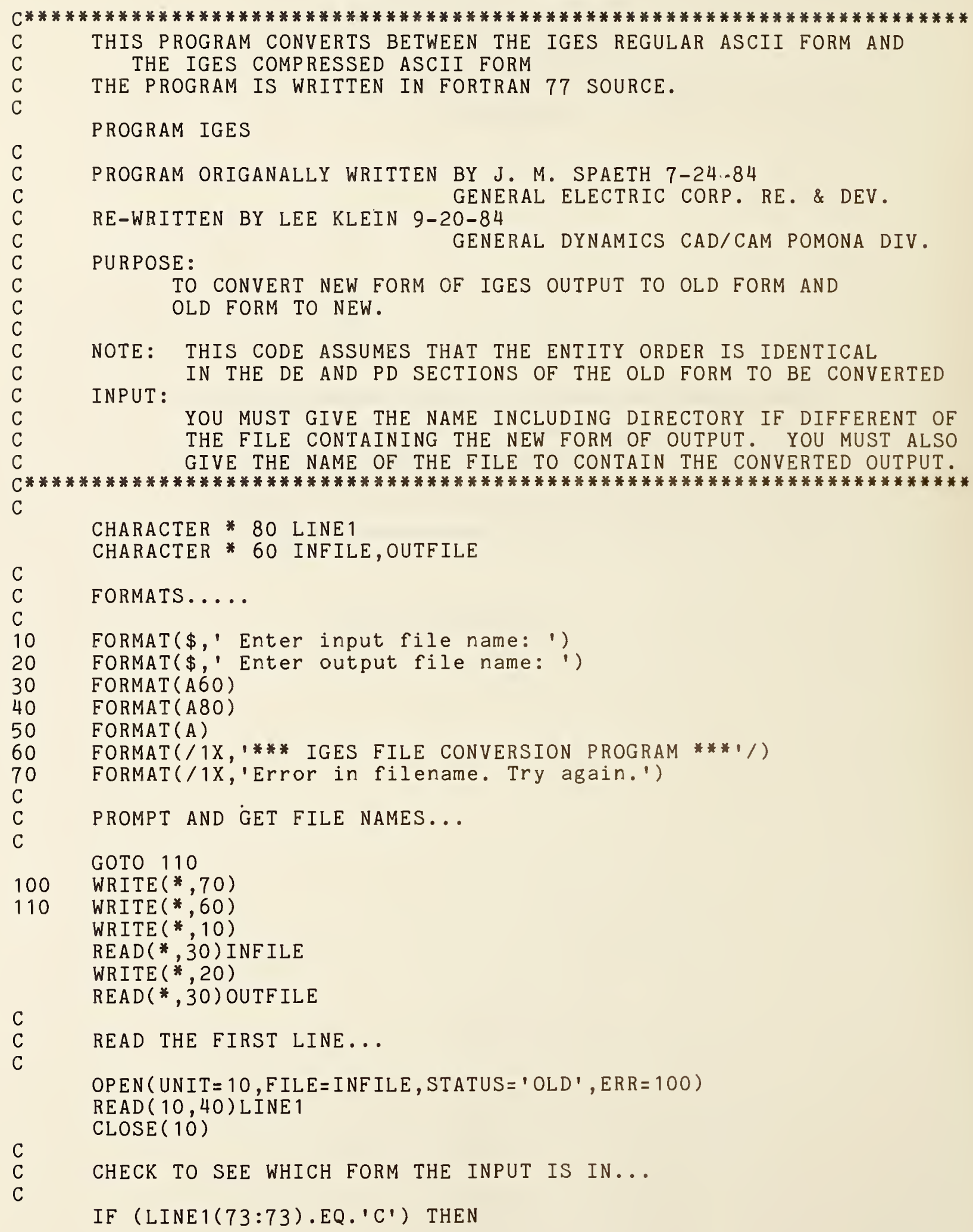

THIS PROGRAM CONVERTS BETWEEN THE IGES REGULAR ASCII FORM AND THE IGES COMPRESSED ASCII FORM

THE PROGRAM IS WRITTEN IN FORTRAN 77 SOURCE.

PROGRAM IGES

$\mathrm{C}$

C

PROGRAM ORIGANALLY WRITTEN BY J. M. SPAETH 7-24..84

RE-WRITTEN BY LEE KLEIN 9-20-84

GENERAL ELECTRIC CORP. RE. \& DEV.

C

C

C

PURPOSE :

GENERAL DYNAMICS CAD/CAM POMONA DIV.

TO CONVERT NEW FORM OF IGES OUTPUT TO OLD FORM AND OLD FORM TO NEW.

NOTE: THIS CODE ASSUMES THAT THE ENTITY ORDER IS IDENTICAL

INPUT: IN THE DE AND PD SECTIONS OF THE OLD FORM TO BE CONVERTED

YOU MUST GIVE THE NAME INCLUDING DIRECTORY IF DIFFERENT OF THE FILE CONTAINING THE NEW FORM OF OUTPUT. YOU MUST ALSO GIVE THE NAME OF THE FILE TO CONTAIN THE CONVERTED OUTPUT.

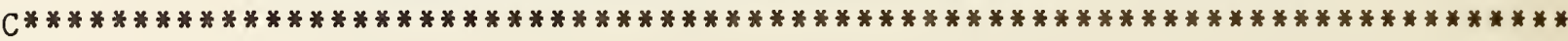

$\mathrm{C}$

CHARACTER * 80 LINE 1

CHARACTER * 60 INFILE, OUTF ILE

$\mathrm{C}$

C FORMATS....

10 FORMAT( $\$$, Enter input file name: ')

20 FORMAT( $\$$, 'Enter output file name: ')

30 FORMAT (A60)

40 FORMAT (A80)

50 FORMAT (A)

60 FORMAT $(/ 1 \mathrm{X}, 1 * * *$ IGES FILE CONVERSION PROGRAM *** $1 /$ )

70 FORMAT(/1X,'Error in filename. Try again.')

C

$\mathrm{C}$

PROMPT AND GET FILE NAMES...

C

100

GOTO 110

110 WRITE $(*, 60)$

WRITE $(*, 10)$

READ (*,30) INF ILE

WRITE $(*, 20)$

READ(*,30) OUTF ILE

C

READ THE FIRST LINE...

OPEN (UNIT $=10, F I L E=$ INF ILE, STATUS $=$ ' OLD', ERR $=100$ )

$\operatorname{READ}(10,40)$ LINE1

CLOSE (10)

$\mathrm{C}$
$\mathrm{C}$

CHECK TO SEE WHICH FORM THE INPUT IS IN...

IF (LINE1(73:73).EQ.'C') THEN 


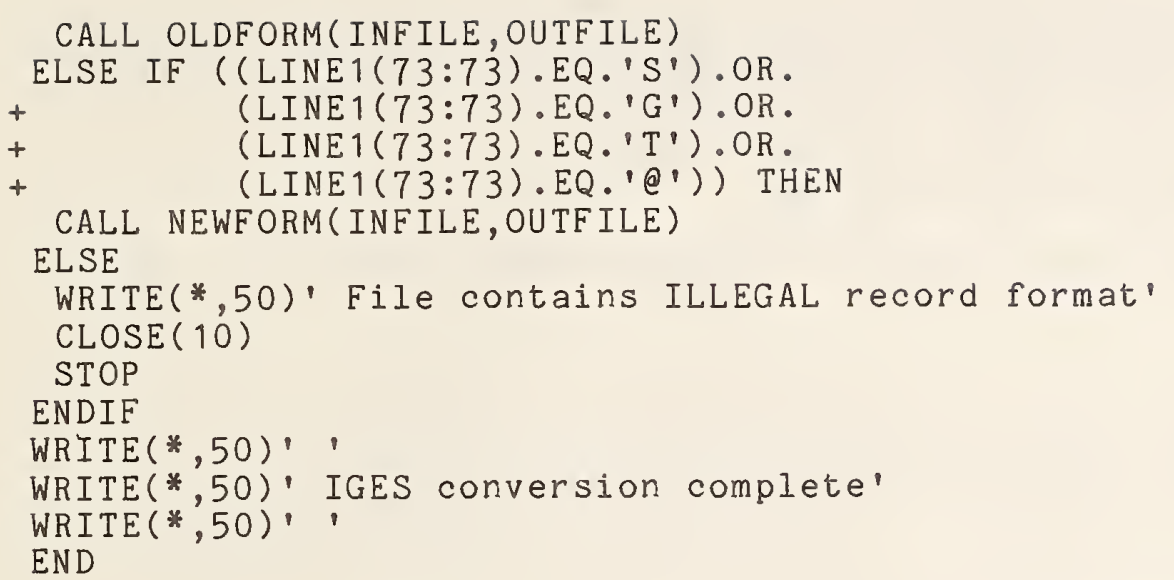

OLD FORM CONVERSION

SUBROUTINE OLDFORM(INFILE, OUTFILE)

PROGRAM ORIGANALLY WRITTEN BY J. M. SPAETH 7-24-84

GENERAL ELECTRIC CORP. RE. \& DEV . RE-WRITTEN BY LEE KLEIN 9-20-84

PURPOSE :

GENERAL DYNAMICS CAD/CAM POMONA DIV. TO CONVERT NEW FORM OF IGES OUTPUT TO OLD FORM...

VARIABLE DECLARATIONS...

CHARACTER * 60 INF ILE, OUTF ILE

CHARACTER * 8 BLNK, INARR(20), NEWARR(20)

CHARACTER 
CALL OLDFORM (INFILE, OUTFILE)

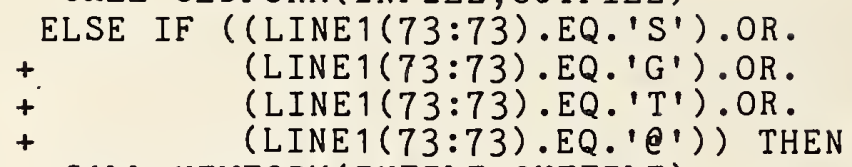

CALL NEWFORM(INFILE, OUTFILE)

ELSE

WRITE(*,50)' File contains ILLEGAL record format' CLOSE (10)

STOP

ENDIF

WRITE $(*, 50)$ ' '

WRITE $(*, 50)$ ' IGES conversion complete'

WRITE $(*, 50)$ ' '

END

C

\section{OLD FORM COMVERSION}

SUBROUTINE OLDFORM(INFILE, OUTFILE)

C

PROGRAM ORIGANALLY WRITTEN BY J. M. SPAETH 7-24-84

RE-WRITTEN BY LEE KLEIN 9-20-84

GENERAL ELECTRIC CORP. RE. \& DEV.

GENERAL DYNAMICS CAD/CAM POMONA DIV.

PURPOSE :

TO CONVERT NEW FORM OF IGES OUTPUT TO OLD FORM...

VARIABLE DECLARATIONS...

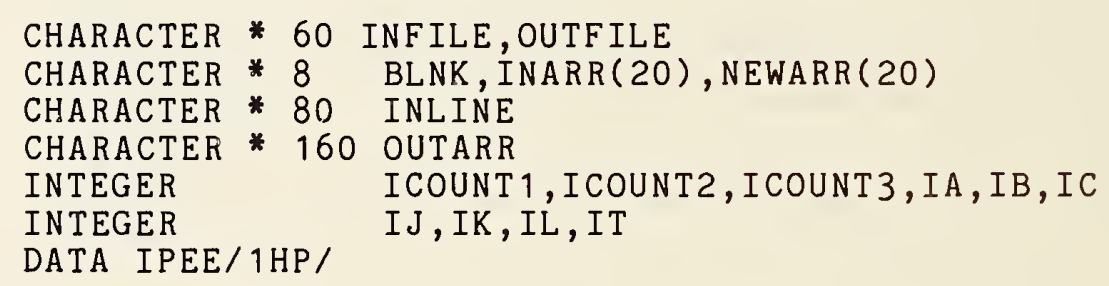

$\mathrm{C}$
$\mathrm{C}$
$\mathrm{C}$
1
2
3
5
6
$\mathrm{C}$
$\mathrm{C}$
$\mathrm{C}$
$\mathrm{C}$
$\mathrm{C}$
$\mathrm{C}$

FORMATS...

FORMAT (A80)

FORMAT ( A64, 1 I8, 1A 1, 1I7)

FORMAT ( 10A8)

FORMAT ( 9 A8, A, I7)

FORMAT ( A8, I8, A64)

INITIALIZE OUTARR TO BLANKS...

$\operatorname{OUTARR}(1: 160)=1 \quad$ '

OPEN INPUT AND TEMP FILES...

OPEN (UNIT=1,FILE= 'FILE1.TMP', STATUS= ' NEW' , CARRIAGECONTROL= 'LIST') OPEN (UNIT $=2$, FILE = 'FILE2. TMP', STATUS = ' NEW' ',CARRIAGECONTROL = 'LIST' $)$ OPEN (UNIT $=3$, FILE = ' FILE3. TMP' , STATUS = ' NEW' ', CARRIAGECONTROL= 'LIST' $)$ 
OPEN (UNIT $=4$, FILE= 'FILE4. TMP', STATUS = 'NEW', CARRIAGECONTROL= 'LIST')

OPEN (UNIT $=9$, F ILE = OUTFILE, STATUS = 'NEW' , CARRIAGECONTROL= 'LIST')

OPEN (UNIT $=10$, FILE $=$ INFILE, STATUS $=$ ' OLD')

INITIALIZE COUNTERS...

$I \operatorname{COUNT} 1=-1$

ICOUNT2 $=1$

ICOUNT3 $=0$

C

READ THE FILE AND SEPARATE INTO PARTS...

C

$100 \operatorname{READ}(10,1, \mathrm{END}=600)$ INLINE

IF ((INLINE(73:73).EQ.'S') .OR. (INLINE(73:73).EQ.'G')) GOTO 101

IF (INLINE( $73: 73)$.EQ.'T') GOTO 102

IF ((INLINE( $\left.1: 1) \cdot E Q \cdot ' \varrho ') . O R .\left(\operatorname{INLINE}(1: 1) \cdot E Q \cdot D^{\prime}\right)\right)$ GOTO 400

C

C

IF IT IS AN C THAN DELETE IT OFF BE READING THE NEXT LINE

IF (INLINE( $\left.73: 73) . E Q \cdot{ }^{\prime} C^{\prime}\right)$ GOTO 100

C

PUT THE PARAMETER DATA INTO FILE4.TMP...

$\operatorname{WRITE}(4,2)$ (INLINE $(1: 64), \operatorname{ICOUNT} 1, \operatorname{IPEE}, \operatorname{ICOUNT2})$

ICOUNT2 = ICOUNT2 + 1

GOTO 100

WRITE HEADER LINES INTO FILE1.TMP...

101 WRITE $(1,1)$ INLINE

GOTO 100

C

C

WRITE TERMINATION LINES INTO FILE2.TMP...

102 WRITE $(2,1)$ INLINE

GOTO 100

$103 \operatorname{WrITE}(3,6)$ (OUTARR $(1: 8), \operatorname{ICOUNT2,\operatorname {OUTARR}(17:80))}$

$\operatorname{WRITE}(3,1)$ OUTARR $(81: 160)$

ICOUNT $1=$ ICOUNT $1+2$

GOTO 100

C

REWRITE TO DE LINES IN THE NEW FORM...

GO THRU INLINE ONE CHAR. AT A TIME LOOKING FOR THE DELIMETER (e, ,_)...

400

C

DO $I L=1,80$

IF (INLINE(IL:IL).EQ.';') GOTO 103

IF (INLINE(IL:IL).EQ.'', ) GOTO 100

IF (INLINE(IL:IL).NE.'@') GOTO 501

C

DETERMINE IF THE FIELD IS ONE OR TWO CHARACTERS... 
C

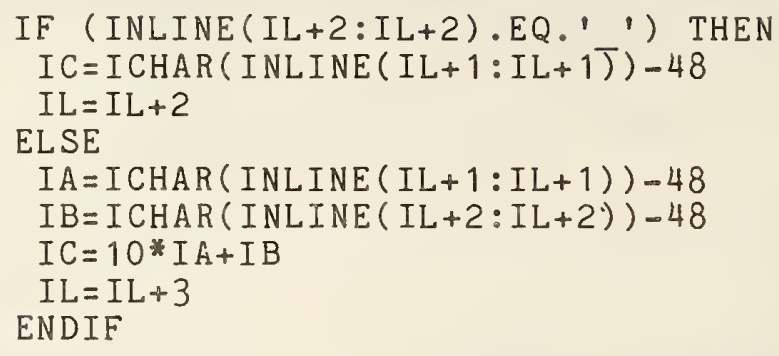

C

AT THIS POINT IC IS THE NUMBER OF THE RECORD FIELD BEING PROCESSED, AND INLINE(IL) =USCORE

C

I $\mathrm{T}=0$

$I K=0$

$I J=(I C-1) * 8+1$

C
C
C
C
C
C
C
C

RESET THE FIELD TO BE CHANGED TO ALL BLANKS IN ORDER TO CREATE A COMPLETELY NEW FILED...

$\operatorname{OUTARR}(I J: I J+7)=$ '

WE WILL NOW CONTINUE THRU THE LINE PICKING OFF THE CHAR.

OF THE RECORD FIELD ONE AT A TIME UNTIL A DELIMETER IS HIT...

$450 \quad I K=I K+1$

IF (INLINE(IL+IK:IL+IK).EQ.'C') GOTO 500

IF (INLINE(IL+IK:IL+IK).EQ.' ') THEN

IF (INLINE (IL+IK+1:IL+IK+1).EQ.' ') GOTO 500 ENDIF

IF (INLINE(IL+IK:IL+IK).EQ.';') GOTO 500

$I \mathrm{~T}=\mathrm{IT}+1$

$I J=(I C-1) * 8+I T$

OUTARR $(I J: I J)=I N L I N E(I L+I K: I L+I K)$

IF (IC.EQ.1) THEN

OUTARR $(I J+80: I J+80)=\operatorname{INLINE}(I L+I K: I L+I K)$

ENDIF

GOTO 450

$500 \quad I L=I L+I T$

501 END DO

GOTO 100

$\mathrm{C}$

REWIND ALL FILES BEFORE WE WRITE THEM TO OUTPUT...

C

600

REWIND 1

REWIND 2

REWIND 3

REWIND 4

C WRITE START AND GLOBAL RECORDS TO OUTPUT FILE...

$\mathrm{C}$

$601 \operatorname{READ}(1,1, \mathrm{END}=602)$ INLINE

WRITE $(9,1)$ INLINE 


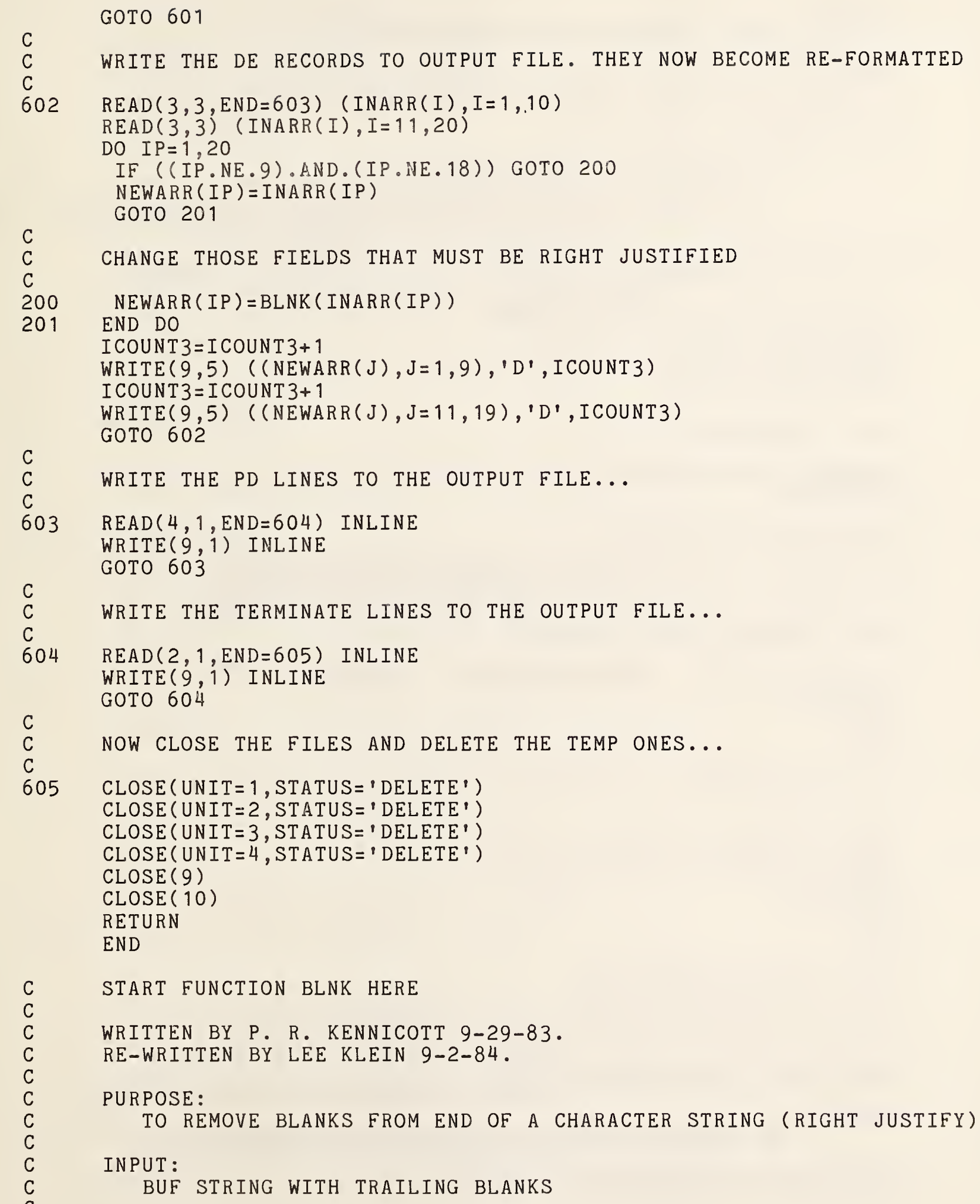


C OUTPUT:

C BLNK STRING WITH TRAILING BLANKS REMOVED

C

C

METHOD:

FIND FIRST BLANK, THEN TRANSLATE OUTPUT STRING RESTRICTIONS:

1. BUF $<=512$ CHARACTERS.

2. LENGTHS OF BUF \& BLNK MUST BE = .

3. FIRST CHARACTER MUST NOT BE BLANK OR NO CONVERSION.

VARIABLE DECLARATIONS...

CHARACTER* (*) FUNCTION BLNK (BUF)

CHARACTER* * * BUF

INTEGER I

$\mathrm{C}$

CHARACTER*512 IBUF

C

C

C

SET UP COUNTERS...

$N=\operatorname{INDEX}(\operatorname{BUF}(1:), 1,1)-1$

$M=\operatorname{LEN}(B U F)$

C

$\mathrm{C}$

C

C

C

C

CHECK FOR SIZE TOO BIG...

IF (M.GT.512) STOP 'Buffer too big at function BLNK'

CHECK FOR FIRST CHAR A BLANK...

IF (BUF( $1: 1) . E Q . '$ ' OR.BUF $(M: M) \cdot N E . '$ ') THEN

$B L N K=B U F$

RETURN

ENDIF

C

OK PROCESS STRING...

DO $10 \quad I=1, N$

10

$\operatorname{IBUF}(M-I+1: M-I+1)=B U F(N-I+1: N-I+1)$

$\operatorname{IBUF}(1: M-N)=1$,

$B L N K=I B U F$

RETURN

END

C START NEW SUBROUTINE HERE

$\mathrm{C}$

$\mathrm{C}$

C

C

C

C

C

C

C

PROGRAM ORIGANALLY WRITTEN BY J. M. SPAETH 7-24-84

GENERAL ELECTRIC CORP. RE. \& DEV.

RE-WRITTEN BY LEE KLEIN 9-20-84

GENERAL DYNAMICS CAD/CAM POMONA DIV.

PURPOSE:

TO CONVERT OLD FORM OF IGES OUTPUT TO NEW FORM...

SUBROUTINE NEWFORM(INFILE, OUTFILE) 
C

VARIABLE DECLARATIONS...

DIMENSION INLINE( 80$)$

CHARACTER * 60 INFILE, OUTFILE

DATA IPEE/1HP/

DATA IDEE/1HD/

DATA ITEE/1HT/

$\mathrm{C}$

C

OPEN THE INPUT AND TEMP FILES...

OPEN (UNIT $=10$, FILE = INFILE, STATUS $=$ ' OLD' )

OPEN (UNIT $=1$, FILE = 'TEST. TMP' , STATUS = 'NEW' , CARRIAGECONTROL= 'LIST')

OPEN (UNIT $=2$, FILE = 'FILE2. TMP' ', STATUS = 'NEW' $)$

OPEN (UNIT $=3$, FILE = 'FILE3. TMP', STATUS = 'NEW' )

OPEN (UNIT $=7$, , FILE = ' FILE5. TMP' ', STATUS= 'NEW')

C

WRITE THE HEADER WITH A "C" TO SHOW COMPRESSED ASCII FORM...

C

C

WRITE $(1,99)$ ' $C$ '

$99 \operatorname{FORMAT}(72 \mathrm{X}, \mathrm{A})$

C

SEPERATE THE PD AND DE RECORDS, WHILE WRITING G,S, \&T LINES TO THE OUTPUT FILE...

$\mathrm{C}$

C

102 FORMAT (80A1)

$200 \operatorname{READ}(10,102, \operatorname{END}=204)(\operatorname{INLINE}(I), I=1,80)$

IF (INLINE(73).EQ.IDEE) GOTO 201

IF (INLINE( 73).EQ.IPEE) GOTO 202

C

IF (INLINE(73).EQ.ITEE) GOTO 203

C

WRITE HEADER LINES INTO A TEST.TMP...

WRITE $(1,102) \quad(\operatorname{INLinE}(I), I=1,80)$

GOTO 200

$\mathrm{C}$

C

C

201 WRITE $(3,102)(\operatorname{INLINE}(I), I=1,80)$

GOTO 200

$\mathrm{C}$

C

$\mathrm{C}$

202 WRITE $(2,102)(\operatorname{InLinE}(J), J=1,80)$

GOTO 200

203 WRITE $(7,102)(\operatorname{InLinE}(J), J=1,80)$

GOTO 200

204 CALL XPD

REWIND 7

$205 \operatorname{READ}(7,102, \operatorname{END}=206) \quad(\operatorname{INLINE}(I), I=1,80)$

WRITE $(1,102)$ (INLINE $(J), J=1,80)$

GOTO 205 
206

C

C

C

C

C

C

C

C

C

C

1

C

C

C

C

C

C

C

C

C

10

$\mathrm{C}$

C

C

c

C

C

20

CALL COMPRESS (OUTFILE)

CLOSE FILES AND DELETE TEMP ONES...

CLOSE (UNIT $=1$, STATUS = 'DELETE')

CLOSE (UNIT $=2$, STATUS $=$ 'DELETE')

CLOSE (UNIT $=3$, STATUS= 'DELETE')

CLOSE (UNIT $=4$ )

CLOSE (UNIT $=7$, STATUS= 'DELETE')

CLOSE (UNIT $=10$ )

RETURN

END

SUBROUTINE XPD

PURPOSE :

TO TRANSFER ALL PD \& DE RECORDS FORM TEMPORY FILES TO OUTPUT FILE IN MERGED FORM...

VARIABLE DECLARATIONS... 
$\operatorname{READ}(3,1, \operatorname{END}=120)$ DELIN 1

$\operatorname{READ}(3,1)$ DELIN2

$\operatorname{DELIN} 1(73: 73)={ }^{\prime}$,

$\operatorname{DELIN} 2(73: 73)=0$

C

GET THE DATA FROM EACH FIELD OF THE DE RECORD SET ...

FLDBEG $=-7$

FLDEND $=1$

DO FLDNUM $=1,10$

$F L D B E G=F L D B E G+8$

FLDEND $=$ FLDEND + 8

READ (DELIN 1 (FLDBEG:FLDEND), 30) NEWDAT (FLDNUM)

READ (DELIN2(FLDBEG:FLDEND), 30) NEWDAT (FLDNUM+10)

30 FORMAT (A8)

END DO

$\mathrm{C}$

FIELD 18 MUST BE RIGHT JUSTIFIED...

$\operatorname{NEWDAT}(18)=\operatorname{BLNK}(\operatorname{NEWDAT}(18))$

DETERMINE THE LENGTH OF THE DATA WITHIN EACH FIELD

DO FLDNUM $=1,20$

DO $I=1,8$

IF (NEWDAT(FLDNUM) ( I:I).NE.' ') THEN

$\operatorname{LFLD}($ FLDNUM $)=9-I$

GOTO 40

ENDIF

END DO

40 END DO

C

C

WRITE THE DE SEQUENCE NUMBER AT THE BEGINNING OF THE OUTPUT DE RECORD

$\operatorname{ENCODE}(\operatorname{LFLD}(10)+1,60, \operatorname{NEWDE}(1: \operatorname{LFLD}(10)+1)) \operatorname{NEWDAT}(10)(9-\operatorname{LFLD}(10): 8)$

60 FORMAT ('D',A)

CHRPTR $=\operatorname{LFLD}(10)+2$

C

C

SEARCH NEW DE RECORD SET FOR CHANGED DATA; WHEN FOUNT WRITE CHANGED

DATA TO OUTPUT DE RECORD...

DO FLDNUM $=1,20$

$\mathrm{C}$

C

C

SKIP FIELDS THAT NO LONGER NEED PROCESSING...

IF ((FLDNUM.EQ. 2).OR .

(FLDNUM.EQ.10).OR .

(FLDNUM.EQ.11).OR .

(FLDNUM.EQ.20)) GOTO 100

IF (NEWDAT(FLDNUM).NE.LSTDAT(FLDNUM)) THEN

IF (FLDNUM.GT.9) THEN

CHRPTR $=$ CHRPTR $+4^{-}$ 
ELSE

80

ENCODE ( 3,80, NEWDE ( CHRPTR: CHRPTR+2)) FLDNUM

$$
\text { FORMAT ( 'e', I1,', ') }
$$

CHRPTR $=$ CHRPTR+3

$$
\text { ENDIF }
$$

IF (LFLD(FLDNUM).NE.0) THEN

IF (FLDNUM.NE.9) THEN

READ (NEWDAT (FLDNUM) (9-LFLD (FLDNUM):8), 90)

90

FORMAT (A)

CHRPTR $=$ CHRPTR+LFLD (FLDNUM)

ELSE

$\mathrm{C}$

C

C

FIELD 9 IS A SPECIAL CASE...

$\operatorname{READ}(\operatorname{NEWDAT}(9)(1: 8), 90)$ NEWDE (CHRPTR: CHRPTR+7) CHRPTR $=$ CHRPTR +8

ENDIF

ENDIF

ENDIF

C
C STORE DATA FORM CURRENT DE RECORD SET TO COMPARE WITH NEXT SET

100 END DO

LSTDAT $($ FLDNUM $)=$ NEWDAT $($ FLDNUM $)$

NEWDE (CHRPTR:CHRPTR) = SCOLN

C

$\mathrm{C}$

IF OUTPUT DE RECORD > 80 CHAR'S, WRITE 2 LINES...

IF (CHRPTR.GT.80) THEN

DO $I=1,11$

IF (NEWDE (82-I:82-I).EQ.' '') GOTO 110

END DO

110 WRITE $(1,111) \operatorname{NEWDE}(1: 81-\mathrm{I})$

111 FORMAT $(A<81-\mathrm{I}>)$

$\operatorname{NEWDE}(1: 80)=\mathrm{N} \operatorname{EWDE}(82-\mathrm{I}: 161-\mathrm{I})$

ENDIF

C

$\operatorname{WRITE}(1,1) \quad \operatorname{NEWDE}(1: 80)$

C ERASE UNNECESSARY DATA FORM PD RECORD AND WRITE TO OUTPUT FILE;

C THEN PURGE

C

$115 \operatorname{PDLINE}(65: 80)=$ ',

WRITE $(1,1)$ PDLINE

C $\operatorname{PDLINE}(1: 80)=$

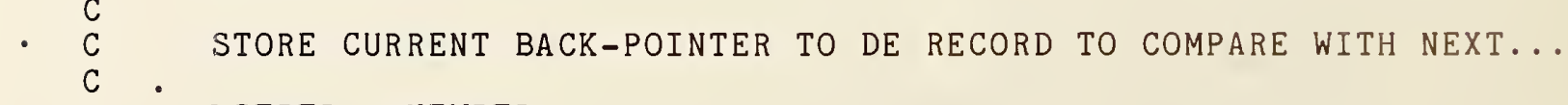

C L LSTPTR $=$ NEWPTR

C
C

GOTO 10

120 CONTINUE

999 RETURN

END 


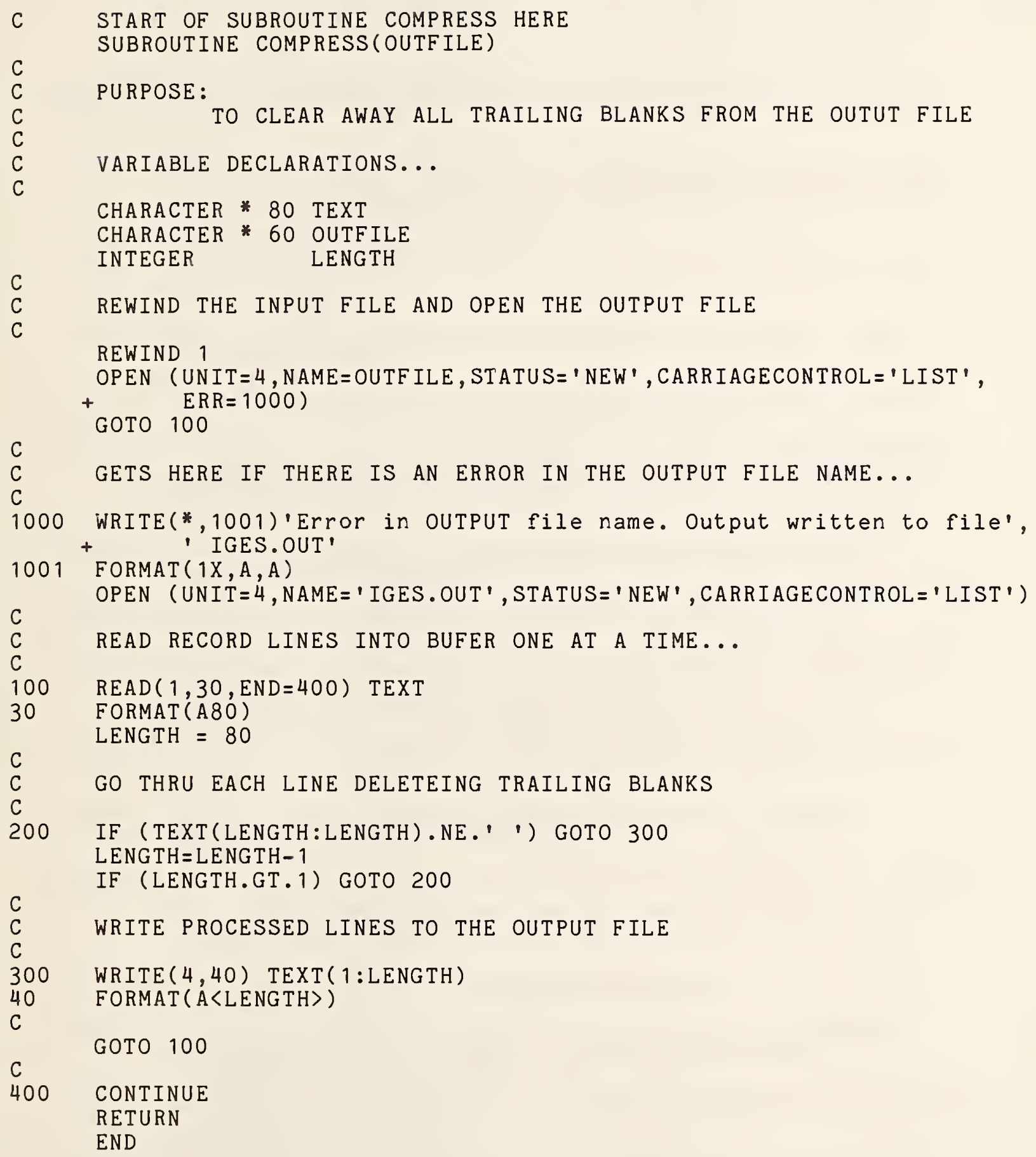





\section{LIST OF REFERENCES}

ANS168 American National Standards Institute, Code for Information Interchange (X3.4-1968), ANSI, 1968.

ANS174 American National Standards Institute, Code Extension Techniques for Use with the 7-Bit Coded Character Set of American National Standard Code for Information Interchange (ASCII), X3.4-1968 (X3.41-1974), ANSI, 1974.

ANSI77 American National Standards Institute, Code for Information Interchange (X3.4-1977), ANSI, 1977.

ANSI78 American National Standards Institute, Line Conventions and Let tering (Y14.2-1979), ANSI, 1978.

ANSI79 American National Standards Institute, FORTRAN (X3.9-1978), ANSI, 1978.

BLOM82 Blomgren, R. and R. Fuhr, Algorithms to Convert Between Rational B-spline and Rational Bezier Representations of Curves and Surfaces, National Bureau of Standards, 1982.

COON67 Coons, S. A., Surfaces for Computer Aided Design of Space Forms, MIT Project MAC TR-41, June 1967.

DEBO78 deBoor, C., A Practical Guide to Splines, Springer-Verlag, 1978.

DOD12D Department of Defense, Abbreviations for Use on Drawing, Specifications, Standards, and in Technical Documents (MIL-STD-12D).

DoCA76 DoCarmo, M. P., Differential Geometry of Curves and Surfaces, Prentice Hall, 1976.

FAUX79 Faux, I., and M. J. Pratt, Computational Geometry for Design and Manufacture, John Wiley and Sons, 1979.

GORD 74 Gordon, W. J. and R. F. Riesenfeld, "B-Spline Curves and Surfaces", published in Barnhill, R. E. and R. F. Riesenfeld, ed., Computer Aided Geometric Design, Academic Press, 1974.

HILD76 Hildebrand, F., Advanced Calculus for Applications, Prentice Hall, 1976.

IEEE260 Institute of Electrical and Electronics Engineers, IEEE Standard Letter Symbols for Units of Measurement (ANSI/IEEE Std 260), IEEE.

IEEE85 Institute of Electrical and Electronics Engineers, Standard for Binary FloatingPoint Arithmetic (ANSI/IEEE Std 754-1985), IEEE, 1985.

IITR68 Illinois Institute of Technology Research Institute, APT Computer System Manual: Volume 2 - Subroutine Library, IITRI, 1968.

JOBL78 Joblove, G. H. and D. Greenberg, Color Spaces for Computer Graphics, SIGGRAPH Proceedings, 1978. 
LIST OF REFERENCES

KAPL52 Kaplan, W., Advanced Calculus, Addison-Wesley, 1952.

ROGE76 Rogers, D. F. and J. A. Adams, Mathematical Elements for Computer Graphics, McGraw-Hill, 1976.

SMIT78 Smith, A. R., Color Gamut Transformation Pairs, Computer Graphics, 1978.

THOM60 Thomas, G., Calculus and Analytic Geometry, Addison-Wesley, 1960. 


\section{IGES GLOSSARY}

The spirit of this Glossary is to provide general, sometimes intuitive information pertaining to certain phrases and concepts either appearing in or alluded to by this document. The spirit is not to provide detailed mathematical definitions such as may be found within the document itself.

\section{ANGULAR DIMENSION ENTITY}

An annotation entity designating the measurement of the angle between two geometric lines.

\section{ANNOTATION}

Text or symbols, not part of the geometric model, which provide information.

\section{ASSEMBLY (IEEE 200-1975)}

A number of basic parts or subassemblies, or any combination thereof, joined together to perform a specific function.

\section{ASSOCIA TIVITY}

A structure entity, which defines a logical link or relationship between different entities.

\section{ASSOCIATIVITY DEFINITION ENTITY}

A structure entity which designates the type (link structure) and generic meaning of a relationship. (See PRE-DEFINED ASSOCIATIVITIES)

\section{ASSOCIATIVITY INSTANCE ENTITY}

A structure entity formed by assigning specific values to the data items defining an associativity.

\section{ATTRIBUTE}

Information, provided in specific fields within the directory entry of an entity, which serves to qualify the entity definition.

\section{AXONOMETRIC PROJECTION}

A projection in which only one plane is used, the object being turned so that three faces show. The main axonometric positions are isometric, dimetric, and trimetric.

\section{BACK POINTER}

A pointer in the parameter data section of an entity pointing to an associativity instance of which it is a member.

BASIC PART (IEEE 200-1975)

One piece, or two or more pieces joined together, which are not normally subject to disassembly without destruction of designed use. 


\section{BLANK STATUS FLAG}

A portion of the status number field of the directory entry of an entity designating whether a data item is to be displayed on the output device.

\section{BOUNDED PLANE}

A finite region defined in a plane.

\section{BREAKPOINT}

A member of an increasing sequence of real numbers which is a subsequence of the knot sequence used to specify parametric spline curves.

\section{B-SPLINE BASIS}

A set of functions which form a basis for the set of splines of specified degree on a specified knot sequence. B-spline basis functions are characterized by being splines of minimal support. See appendix A4 for more details.

\section{CENTERLINE ENTITY}

An annotation entity for representing the axis of symmetry for all symmetric views or portions of views, such as the axis of a cylinder or a cone.

\section{CIRCULAR ARC ENTITY}

A geometric entity which is a connected portion of a circle or the entire circle.

\section{CLASS}

A group of data items pertinent to a common logical relationship in an associativity definition.

\section{CLIP}

To abbreviate or terminate the intended display of an entity along an intersecting curve or surface.

\section{CLIPPING BOX}

A bounding set of surfaces which abbreviate the intended display of data to that portion which lies within the box.

\section{CLIPPING PLANE}

A bounding plane surface which abbreviates the intended display of data to that portion which lies on one or the other side of the plane.

\section{CLOSED CUR VE}

A curve with coincident start and terminate points. 


\section{COMPLEMENTARY ARC}

Either of the two connected components of a closed connected curve which has been divided by two distinct points lying on the curve.

\section{COMPONENT}

Typically a synonym for part (e.g., resistor, capacitor, microcircuit, etc.), but also may refer to a subassembly being treated as a part. The IGES representation of a component may be a collection of entities, associativities, and properties.

\section{COMPOSITE CUR VE}

A connected curve which is formed by concatenating two or more curve segments.

\section{CONIC ARC ENTITY}

A geometric entity which is a finite connected portion of an ellipse, a parabola, or a hyperbola.

\section{CONNECTED CURVE}

A curve such that for any two points $\mathrm{P} 1$ and $\mathrm{P} 2$, one can travel from $\mathrm{P} 1$ to $\mathrm{P} 2$ without leaving the curve.

\section{CONNECTION POINT ENTITY}

A geometric entity giving the $X Y Z$ location and other information (e.g., text labels) of a point of connection. May be independent or subordinate to a Network subfigure definition and/or instance. Used for netlist information.

\section{CONSTITUENT}

A member of a set.

\section{CONTROL POINT}

A point in definition space which appears in the numerator of the expression for a rational B-spline curve or surface. If the weights are all positive, the resulting curve or surface lies within the convex hull of the control points. Its shape resembles that of the polygon or polyhedron whose vertices are the control points. A control point is sometimes referred to as a B-spline coefficient. See appendices $A 4$ and $A 6$ for more details.

\section{COONS PATCH}

A three dimensional surface.

\section{COPIOUS DATA ENTITY}

A geometric entity sometimes used as an annotation entity, containing arrays of types of real numbers to which a specific meaning has been assigned. One form number corresponds to one special meaning. 
DEFINITION LEVEL (or DISPLAY LEVEL)

The graphics display level ior layer) on which one or more entities have been defined.

\section{DEFINITION MATRIX}

The matrix which transforms the coordinates represented in the definition space into the coordinates represented in the model space.

\section{DEFINITION SPACE}

A local Cartesian coordinate system chosen to represent a geometric entity for the purpose of mathematical simplicity.

\section{DEFINITION SPACE SCALE}

A scale factor applied within an entity definition space.

\section{DEVELOPABLE SURFACE}

A surface which can be unrolled onto a plane.

\section{DIAMETER DIMENSION ENTITY}

An annotation entity designating the measurement of a diameter of a circular arc. DIRECTED CUR VE

A curve with an associated direction derived from the start and terminate points.

\section{DIRECTORY ENTRY SECTION}

The section of an IGES file, consisting of fixed field data items for an index and attribute list of all entities in the file.

\section{DIRECTRIX}

The curve entity used in the definition of a tabulated cylinder entity.

\section{DISPLAY SYMBOL}

A method for graphically representing certain entities (plane, point, section) for identification purposes.

\section{DRAWING ENTITY}

A structure entity which specifies the projection(s) of a model onto a plane, with any required annotation and/or dimension.

\section{DRILLED HOLE PROPERTY}

A predefined property that assigns the physical attribute of a hole that can be made 
by a drill. May be used in electrical applications to 1) define a via from one printed circuit board, PCB, layer to another, 2) define a plated via hole, and 3) give the first physical drill diameter and/or the finished hole diameters. It is usually attached to a point, circle, subfigure definition or instance.

\section{EDGE VERTEX}

A method of geometric modeling in which a two- or three-dimensional object is represented by curve segments (edges of the object) connected to points or vertices of the object. A higher level of topological information can be contained in such a model than is implied by a 'wire-frame' terminology, but in the context of this specification the terms are used interchangeably.

\section{ENTITY}

The basic unit of information in a file. The term applies to single items which may be individual elements of geometry, collections of annotation to form dimensions, or collections of entities to form structured entities.

\section{ENTITY LABEL}

A one to eight character identifier for an entity. This term may implicitly include the entity subscript, providing for additional characters.

\section{ENTITY SUBSCRIPT}

A one to eight digit unsigned integer associated with the entity label. The label and subscript specify a unique instance of an entity within an array of entities.

\section{ENTITY TYPE NUMBER}

An integer used to specify the kind of the entity. For example, the circular arc entity has an entity type number of 100 .

\section{ENTITY USE FLAG}

A portion of the status number field of the directory entry of an entity to designate whether the entity is used as geometry, annotation, structure, logical, or other. For example, a circle used as part of a point dimension would have an entity use flag which designates annotation.

\section{EXTERNAL REFERENCE ENTITY}

A mechanism for referencing definitions which do not reside in the same IGES file as the instances of those definitions.

\section{FINITE ELEMENT}

A small part of a structure defined by the connection of nodes, material, and physical properties.

\section{FLAG NOTE ENTITY}

An annotation entity which takes label information and formats it such that the text is circumscribed by a flag symbol. 
FLASH

The Flash entity may be used to represent a repetitive artwork feature which is usually produced by photo-optical means. Examples include PC pads, targets, clearances and IC features.

\section{FLASH ENTITY}

A geometric entity used for photo-plotting apertures and other filled areas. May be used for representing metallic conductive material on a printed circuit board such as pads and traces. Also, may be used in integrated circuit, IC, chip masks.

\section{FLEXIBLE PRINTED CIRCUIT}

An arrangement of printed circuit and components utilizing flexible base materials with or without flexible cover layers.

\section{FLOW ASSOCIATIVITY ENTITY}

A predefined associativity that represents a flow path. In electrical applications such as schematics and physical descriptions for Printed Wiring Boards, PWB, Printed Circuit Boards, PCB, PCB assemblies, ICs, etc., it presents a common electrical signal (e.g., voltage). In piping applications it represents a flow path between only one source and sink, but branching is allowed to other Flow Associativities. It provides netlist information for a single flow.

\section{FONT CHARACTERISTIC}

An integer which is used to identify a text font. Font characteristic numbers may be positive which indicate an IGES-defined text font or may be negative which is interpreted as a text font definition entity.

\section{FORM NUMBER}

An integer which is used when needed to further define a specific entity. This becomes necessary when there are several interpretations of an entity type. For example, the form number of the conic arc entity indicates whether the curve is an ellipse, hyperbola, parabola, or unspecified. The form number is also used when necessary to supply sufficient information in the directory entry of an entity to allow the structure of the parameters in the parameter data entry to be decoded.

\section{GENERAL LABEL ENTITY}

An annotation entity consisting of a general note with one or more associated leaders.

\section{GENERAL NOTE ENTITY}

An annotation which consists of text which is to be displayed in some specific size and at some specific location and orientation.

\section{GENERATRIX}

The defining curve which is to be swept to generate a tabulated cylinder, or revolved to generate a surface of revolution. 
GEOMETRIC

Having to do with the shape information (points, curves, surfaces, and volumes), necessary to represent some object.

\section{GLOBAL SECTION}

The section of an IGES file consisting of general information describing the file, the file generator (pre-processor), and information needed by the file reader (postprocessor).

GRID

The set of (ui, vj) where ui and vj are the breakpoints on the $u$ and $v$ coordinates respectively used to specify a parametric spline or rational B-spline surface. The term grid is also applied to the projected image on the spline surface.

\section{GROUND PLANE}

A conductor layer, or portion of a conductor layer (usually a continuous sheet of metal with suitable clearances), used as a common reference point for circuit returns, shieldings, or heat sinking.

\section{GROUP ASSOCIATIVITY}

A predefined associativity for forming any collection of entities.

\section{HIER ARCHY}

A tree structure consisting of a root and one or more dependents. In general, the root may have any number of dependents, each of which may have any number of lower-level dependents, and so on, to any number of levels.

\section{INSTANCE}

A particular occurrance of some item or relationship. Several instances may reference the same item.

\section{KNOT SEQUENCE}

A nondecreasing sequence of real numbers used to specify parametric spline curves.

\section{LABEL DISPLAY ASSOCIATIVITY}

A pre-defined associativity that is used by those entities that have one or more possible displays for their entity label. Entities requiring this associativity will have pointers in their directory entry to a label display associativity instance entit;

\section{LEADER ENTITY}

An annotation entity, also referred to as arrow, which consists of an arrowhead and one or more line segments. In the case of an angular dimension entity, the line segment is replaced by a circular arc segment. In general, these entities are used in connection with other annotation entities to link text with some location. 


\section{LEVEL}

An entity attribute which defines a graphic display level to be associated with the entity.

\section{LEVEL FUNCTION PROPERTY}

A predefined property that assigns an "application data base defined functionality" to a level. This property may stand alone (e.g., DE status is independent), that is no other entity points to it. Also, see the level field in directory entry.

\section{LINE ENTITY}

A geometric entity consisting of a straight segment connecting two points in space.

\section{LINE FONT}

A pattern for the appearance of a curve. The pattern is a repeating sequence of blanked and unblanked line segments, or of subfigure instances.

\section{LINE FONT DEFINITION ENTITY}

A structure entity which defines a line font.

\section{LINE WEIGHT}

An entity attribute which is used.to determine the line display thickness for that entity.

\section{LINE WIDENING PROPERTY}

A predefined property that overrides the line weight given in the directory entry of an entity by providing a physical value for the actual width. May be used in electrical applications to describe metallization on a printed circuit board such as traces and off board connections. Also, see the FLASH and SECTION entities and the Region Fill property.

\section{LINEAR DIMENSION ENTITY}

An annotation entity used to represent a distance between two locations.

\section{LINEAR PATH ENTITY}

A geometric entity that defines a collection of linear segments that form a path. Also, see Copious Data Entity Forms 11 and 12.

\section{MACRO BODY}

The portion of a macro definition containing statements which define the action of the macro.

\section{MACRO DEFINITION ENTITY}

The structure entity, containing the macro body within its parameter data section, used to define a specific macro. 


\section{MACRO INSTANCE ENTITY}

A structure entity which will invoke a macro which has been defined using a macro definition entity.

\section{MIRROR}

To reflect through an axis.

\section{MODEL}

A particular collection of data in an IGES file which describes a product.

\section{MODEL SPACE}

A right-handed three-dimensional Cartesian coordinate space in which the product is represented.

\section{NEGATIVE BOUNDED PLANAR PORTION}

A hole.

\section{NETWORK SUBFIGURE DEFINITION ENTITY}

A structure entity used to define a schematic symbol, component or pipe in electrical and piping applications. Shall be used whenever associated Connect Point Entities need to be instanced with the Network Subfigure Instance Entity. For physical components it may have subordinate entities (copious data, simple closed area, subfigure definition or instance, etc.) that have attached a Region Restriction Property giving design rules for auto routing a Printed Circuit Board, PCB. Also, 2D component outlines and 3-D phsical descriptions may be defined.

\section{NETWORK SUBFIGURE INSTANCE ENTITY}

A structure entity used to specify an occurrence of a schematic symbol, component or pipe in electrical and piping applications. It has associated "instanced" Connect Point Entities that give the $X Y Z$ model space point of connections. Used in netlist information and part lists.

\section{NODAL DISPLACEMENT and ROTATIONAL ENTITY}

This entity is used to communicate finite element post processing data. It contains the node identifier, original node coordinates, and incremental displacements and rotations for each node for each load case.

\section{NODAL LOAD/CONSTRAINT ENTITY}

An entity used in a finite element model to apply a force, moment, or other loading or constraints at a specific node.

NODE

A point in space used to define a finite element topology. 


\section{ORDINATE DIMENSION ENTITY}

An annotation entity used to indicate dimensions from a common reference line in the direction of the XT or YT axis.

\section{ORTHONORMAL}

A term describing two vectors which are or thogonal and of unit length.

\section{PARAMETER DATA SECTION}

A section of an IGES file consisting of specific geometric or annotative information about the entities or pointers to related entities.

\section{PARAMETRIC SPLINE CURVE ENTITY}

A geometric entity consisting of polynomial segments subject to certain continuity conditions.

\section{PARAMETRIC SPLINE SURFACE ENTITY}

A geometric entity which is a smooth surface made from a grid of patches. The patches are regions between the component parametric curves.

\section{PARENT CUR VE}

The full curve on which a segment curve lies.

\section{PART NUMBER PROPERTY}

A predefined property that provides one or more text strings giving one to four distinct part numbers (Generic, MIL-STD, Vendor, and/or Internal) to an entity representing a physical part. May be used in electrical, piping or other applications. Usually, it is attached to a subfigure definition and/or instance that represents the part. May be used for part lists.

\section{PATCH}

A surface represented by parametric functions of two parameters which blend four given boundary curves.

\section{PIN NUMBER PROPERTY}

A predefined property that provides a text string giving a component pin number value to an entity representing an electrical component. Also, see the CONNECT POINT Entity.

\section{PLANE ENTITY}

A geometric entity which is a surface with the property that the straight line passing through any two distinct points on the surface lies entirely on the surface.

\section{PLATED-THROUGH HOLE (ANSI/IPC-T-50B)}

A hole in which electrical connection is made between internal or external conductive patterns, or both, by the deposition of metal on the wall of the hole. 
POINT ENTITY

A geometric entity which has no size but possesses a location in space.

\section{POINT DIMENSION ENTITY}

An annotation entity consisting of a leader, text, and an optional circle or hexagon enclosing the text.

\section{POINTER}

A number that indicates the location of an entity within an IGES file.

\section{POSITIVE BOUNDED PLANAR PORTION}

The top of a peg.

\section{POST-PROCESSOR}

A program which translates a file of product definition data from the form of this standard into the data base form of a specific CAD/CAM system.

\section{PRE-DEFINED ASSOCIATIVITIES}

Associativities which are defined within this standard.

\section{PRE-PROCESSOR}

A program which translates a file of product defintiion data from the data base form of a specific CAD/CAM system into the form of this standard.

PRINTED BOARD (ANSI/IPC-T-50B)

The general term for completely processed printed circuit or printed wiring configurations. It includes rigid or flexible, single, double, or multilayer boards.

\section{PRINTED CIRCUIT (ANSI/IPC-T-50B)}

A conductive pattern comprised of printed components, printed wiring, or a combination thereof, all formed in a predetermined design and intended to be at tached to a common base. (In addition, this is a generic term used to describe a printed board produced by any of a number of techniques.)

PRINTED CIRCUIT BOARD (ANSI/IPC-T-50B)

A part manufactured from rigid base material upon which a completely processed printed circuit has been formed.

PRINTED WIRING (ANSI/IPC-T-50B)

The conductive pattern intended to be formed on a common base, to provide pointto-point connection of discrete components, but not to contain printed components. 


\section{PRODUCT DEFINITION}

Data required to describe and communicate the characteristics of physical objects as manufactured products.

\section{PROPERTY ENTITY}

A structure entity which allows numeric or text information to be related to other entities.

\section{RADIUS DIMENSION ENTITY}

An annotation entity which is a measurement of the radius of a circuiar arc.

\section{RATIONAL B-SPLINE CUR VE}

A parametric curve which is expressed as the ratio of two linear combinations of Bspline basis functions. Each basis function in the numerator is multiplied by a scalar weight and a vector B-spline coefficient. Each corresponding basis function in the denominator is just multiplied by the corresponding weight.

\section{RATIONAL B-SPLINE SURFACE}

A parametric surface which is expressed as the ratio of two linear combinations of products of pairs of B-spline basis functions. Each product of basis functions in the numerator is multiplied by a scalar weight and a vector B-spline coefficient. Each corresponding product of basis functions in the denominator is multiplied by the corresponding weight.

\section{REFERENCE DESIGNATOR PROPERTY}

A predefined property that provides a text string giving a component reference designator value to an entity representing an electrical component. Also, see the Network Subfigure entity.

\section{REGION}

The bounded area enclosed by a closed curve or a combination of curves.

\section{REGION FILL PROPERTY}

A predefined property that is used to solid fill or unfill (nested) a closed area. May be used for cross-section material representations (i.e., concrete, steel, etc.) and ar twork. Also, see the SECTION entity. Also, see the FLASH and SECTION entities and the Line Widening property.

\section{REGION RESTRICTION PROPERTY}

A predefined property that provides design rules in electrical applications. Especially, region restrictions regarding Printed Circuit Board, PCB routing rules for prohibiting or permitting vias and traces under component outlines and placement of components on the printed circuit board. 


\section{RELATION}

An aspect or quality that connects two or more things or parts as being or belonging or working together or as being of the same kind.

\section{REPEATING PATTERN}

An ordered sequence of items (elements) which, after a certain point, repeats itself.

\section{RIGHT-HANDED CARTESIAN COORDINATE SYSTEM}

A coordinate system in which the axes are mutually perpendicular and are positioned in such a way that, when viewed along the positive $Z$ axis toward the origin, the positive $\mathrm{X}$ axis can be made to coincide with the positive $\mathrm{Y}$ axis by rotating the $\mathrm{X}$ axis 90 degrees in the counterclockwise direction.

\section{RULED SURFACE ENTITY}

A surface generated by connecting corresponding points on two space curves by a set of lines.

\section{SECTION ENTITY}

A pattern used to distinguish a closed region in a diagram. It is represented as a form of the copious data entity.

\section{SECTION DISPLAY SYMBOL}

An arrangement of fonted straight lines in a repetitive planar pattern at a specified spacing and angle.

SET (IEEE 200-1975)

A unit or units and necessary assemblies, subassemblies, and basic parts connected or associated together to perform an operational function.

\section{SIMPLE CLOSED AREA ENTITY}

A geometric entity used to give a 2-D mathematically simple closed area (no holes or boundary intersections). See Copious Data entity Form 63. May be used for electrical design rules in conjunction with the Region Restriction property.

\section{SINGLE PARENT ASSOCIATIVITY ENTITY}

A predefined associativity that provides logical grouping of a single parent entity to its many children entities.

SPLINE

A piecewise continuous polynomial.

\section{START SECTION}

The section of an IGES file containing a man-readable file prolog. 
SUBASSEMBLY (IEEE 200-1975)

Two or more basic parts which form a portion of an assembly or a unit, replaceable as a whole, but having a part or parts which are individually replaceable.

\section{SUBFIGURE DEFINITION ENTITY}

A structure entity which permits a single definition of a detail to be utilized in multiple instances.

\section{SUBFIGURE INSTANCE ENTITY}

A structure entity which specifies an occurrence of the subfigure definition.

\section{SUBORDINATE ENTITY SWITCH}

A portion of the status number field of the directory entry of an entity. An entity is subordinate if it is an element of a geometric or annotative entity structure or is a member of a logical relationship structure. The terms subordinate and dependent are equivalent within this document.

\section{SURFACE OF REVOLUTION ENTITY}

A geometric entity which is a surface generated by rotating a curve, called the generatrix, about an axis, called the axis of rotation.

SYSTEM (IEEE 200-1975)

A combination of two or more sets, generally physically separated when in operation, and other such units, assemblies, and basic parts necessary to perform an operational function or functions.

\section{TABULAR DATA PROPERTY}

The tabular data property provides a structure to accommodate point form data. The basic structure is a two-dimensional array containing data list for depenent and independent variable.

\section{TABULATED CYLINDER ENTITY}

A geometric entity which is a surface generated by moving a line parallel to itself along a space curve called the generatrix.

\section{TERMINATE SECTION}

The final section of an IGES file, indicating the sizes of each of the preceding file sections.

\section{TEXT DISPLAY TEMPLATE ENTITY}

An annotation entity used to define the display location of a text string. In electrical applications it gives a "relative" mode of location dependent on a connect point for pin number and/or pin function of components (e.g., Integrated Circuit, IC, chip pins). For electrical schematic symbols and physical components it may give the display location of the reference designator text and/or part number. 


\section{TEXT FONT}

The specification of the appearance of the characters.

\section{TEXT FONT DEFINITION ENTITY}

The entity used to define the appearance of characters in a text font. A character is defined by pairing its character code with a sequence of display strokes and positional information.

\section{TRANSFORMATION MATRIX ENTITY}

An entity which allows translation and rotation to be applied to other entities. This is used to define alternate coordinate systems for definition and viewing

\section{TRANSLATION VECTOR}

A three element vector which specifies the offsets (along the coordinate axes) required to move an entity linearly in space.

UNIT (IEEE 200-1975)

A major building block for a set or system, consisting of a combination of basic parts, subassemblies, and assemblies packaged together as a physically independent entity.

\section{VERSION NUMBER}

A means for uniquely designating one specification definition or translator implementation from a preceding or subsequent one.

\section{VIA HOLE (ANSI/IPC-T-50B)}

A plated-through hole used as a through connection, but in which there is no intention to insert a component lead or other reinforcing material.

\section{VIEW ENTITY}

A structure entity used to provide the definition of a human-readable representation of a two-dimensional projection of a selected subset of the model and/or nongeometry information.

\section{VIEWING BOX}

The clipping box used to define a view.

\section{WEIGHT}

A non-zero real number which appears in the numerator and denominator of the expression for a rational B-spline curve or surface. Increasing the weight associated with a particular control point will tend to draw the resulting curve or surface toward that control point. See appendices A4 and A6 for details. 


\section{GLOSSARY}

\section{WIRE-FRAME}

A method of geometric modeling in which a two- or three-dimensional object is represented by curve segments which are edges of the object. In the context of this specification, 'wire-frame' and 'edge-ver tex' models are considered as the same technique and the terms are used interchangeably.

\section{WITNESS LINE}

An annotation entity consisting of line segments and used in engineering drawings to indicate the beginning or the end of a measurement. 
INDEX OF TOPICS

$\underline{\text { Page(s) }}$

ANGULAR DIMENSION ENTITY

209

ANNOTATION

ANNOTATION ENTITIES

1

ANGULAR DIMENSION

206

CENTERLINE

209

DIAMETER DIMENSION

213

215

FLAG NOTE

GENERAL LABEL

217

GENERAL NOTE

221

LEADER (ARROW)

223

LINEAR DIMENSION

238

ORDINATE DIMENSION

243

245

$\begin{array}{ll}\text { POINT DIMENSION } & 247\end{array}$

RADIUS DIMENSION 249

SECTION

251

GENERAL SYMBOL

254

SECTIONED AREA

122,256

WITNESS LINE

ARC CENTER POINT

259

215,249

ARC LENGTH

146

ARROWHEAD TYPE

238

ASCII

ASSOCIATIVITY

CONNECT NODE

ENTITY LABEL DISPLAY

EXTERNAL REFERENCE FILE INDEX

9

6,264

301

275

90,269

DIMENSIONED GEOMETRY

FLOW

282

289

GROUP

266

GROUP WITHOUT BACK POINTERS

278

285,286

PLANAR

287

PRE-DEFINED

266

SIGNAL STRING

297

$\begin{array}{ll}\text { SINGLE PARENT } & 279\end{array}$

$\begin{array}{ll}\text { TEXT NODE } & 299\end{array}$

VIEW LIST $\quad 296$

VIEWS VISIBLE

VIEWS VISIBLE, PEN, LINE WEIGHT

6,270

272

262

264

262

ASSOCIATIVITY SCHEMA

ATTRIBUTE

ATTRIBUTES, DIRECTORY ENTRY

4

BLANK STATUS

COLOR NUMBER

31

37

ENTITY LABEL

37

ENTITY SUBSCRIPT NUMBER

38

ENTITY TYPE NUMBER

ENTITY USE FLAG

34 
FORM NUMBER

HIERARCHY

LABEL DISPI_AY ASSOCIATIVITY POINTER

LEVEL NUMBER

LINE FONT PATTERN NUMBER

LINE WEIGHT

PARAMETER DATA POINTER

PAR AMETER LINE COUNT NUMBER

SEQUENCE NUMBER

STATUS NUMBER

STRUCTURE POINTER

SUBORDINATE ENTITY SWITCH

TRANSFORMATION MATRIX POINTER

VIEW POINTER

B-SPLINE

BACK POINTER

BICUBIC POLYNOMIAL

BINARY REPRESENTATION

DIRECTORY ENTRY SECTION

GLOBAL SECTION

PARAMETER SECTION

START SECTION

TERMINATE SECTION

BLANK STATUS

BOUNDED PLANE

BREAKPOINTS

CENTERLINE ENTITY

CHARACTER APPEAR ANCE

CIRCULAR ARC ENTITY

CIRCULAR ARRAY SUBFIGURE INSTANCE ENTITY

401

CLASSES

CLIPPING

CLIPPING BOX

COLOR NUMBER

COMPOSITE CURVE ENTITY

108

305,413

414

37

111

CONE

CONIC ARC ENTITY

CONNECT NODE ASSOCIATIVITY

CONNECTIVITY

CONSTANTS

INTEGER

LANGUAGE STATEMENT

POINTER

REAL

STRING 
CONTROL POINTS FOR B-SPLINE

COONS' PATCH

COORDINATE SYSTEM

COPIOUS DATA ENTITY

475

$104,158,411$

122

CUBIC SPLINE

CUR VE ON A PARAMETRIC SURFACE ENTITY

132,470

CYLINDER

198

173

DATA FORM

DEFINITION

DEFINITION LEVEL

9

104

DEFINITION SPACE

6,345

DEGREE OF CONTINUITY

DEPTH

DEVELOPABLE SURFACE

$104,158,207$

132,472

207

147

DIAMETER DIMENSION ENTITY

215

DIMENSIONED GEOMETRY ASSOCIATIVITY

282

DIMENSIONS

ANGULAR DIMENSION ENTITY

DIAMETER DIMENSION ENTITY

LINEAR DIMENSION ENTITY

ORDINATE DIMENSION ENTITY

POINT DIMENSION ENTITY

RADIUS DIMENSION ENTITY

DIRECTED CUR VE

DIRECTRIX

209

215

243

245

247

249

106

155

25

126

DISPLAY SYMBOL

6,303

DRAWING ENTITY

DRAWING SIZE PROPERTY

DRAWING UNITS PROPERTY

392

393

351

DRILLED HOLE PROPERTY

133

EDGE-VERTEX

ELECTRICAL EXAMPLE

1

425

186

ELEMENT TOPOLOGY

ELLIPSE

ENCODED FILES (EXAMPLES)

ENTITY

ANGULAR DIMENSION

425,465

ASSOCIA TIVITY DEFINITION

209

262

ASSOCIATIVITY INST ANCE

264

CENTERLINE

CIRCULAR ARC

122,213

108

401

CIRCULAR ARRAY SUBFIGURE INST ANCE

111

116

CONIC ARC

122

198

CUR VE ON A PARAMETRIC SURFACE

215

DIAMETER DIMENSION

DRAWING

6,303

90,417

EXTERNAL REFERENCE

95,184

FINITE ELEMENT ENTITY

217 
$\begin{array}{ll}\text { FLASH } & 167\end{array}$

GENERAL LABEL 221

GENERAL NOTE $\quad 223$

LEADER (ARROW) 238

LINE $\quad 130$

LINE FONT DEFINITION $\quad 308$

LINEAR DIMENSION $\quad 243$

MACRO DEFINITION $\quad 321$

MACRO INSTANCE 334

NETWORK SUBFIGURE INSTANCE $\quad 403$

NODE

NODAL DISPLACEMENT AND ROTATION 192

NODAL LOAD/CONSTRAINT $\quad 419$

OFFSET CURVE 176

OFFSET SURFACE 195

ORDINATE DIMENSION 245

PARAMETRIC SPLINE CUR VE 132

PARAMETRIC SPLINE SURFACE 138

PLANE 126

$\begin{array}{ll}\text { POINT DIMENSION } & 247\end{array}$

$\begin{array}{ll}\text { POINT } & 144\end{array}$

PROPERTY $\quad 344$

RADIUS DIMENSION $\quad 249$

RATIONAL B-SPLINE CURVE 169

RATIONAL B-SPLINE SURFACE 172

RECTANGULAR ARRAY SUBFIGURE INSTANCE ENTITY 397

RULED SURFACE 146

SECTION 251

SECTIONED AREA 256

SUBFIGURE DEFINITION

SUBFIGURE INSTANCE $\quad 397$

SURFACE OF REVOLUTION

TABULATED CYLINDER 155

TRIMMED (PARAMETRIC) SURFACE 201

TEXT FONT DEFINITION 405

TRANSFORMATION MATRIX $\quad 7,98,158$

VIEW

WITNESS LINE

6,411

259

37

275

38

30,36

ENTITY TYPE NUMBER

ENTITY USE FLAG

EXTERNAL REFERENCE ENTITY

EXTERNAL REFERENCE FILE INDEX ASSOCIATIVITY

EXTERNAL REFERENCE FILE LIST PROPERTY 388

FILE STRUCTURE

DIRECTORY ENTRY SECTION

GLOBAL SECTION 18

PARAMETER SECTION

START SECTION 16

TERMINATE SECTION

FINITE ELEMENT ENTITIES

NODE ENTITY 95,181

ELEMENT ENTITY 99,184 
MATERIAL PROPERTIES (TABULAR DATA) 356

NODAL LOAD/CONSTRAINTS $\quad 419$

NODAL DISPLACEMENT AND ROTATIONS 192

$\begin{array}{lr}\text { FLAG NOTE ENTITY } & 217\end{array}$

$\begin{array}{ll}\text { FLASH ENTITY } & 167\end{array}$

FLOW ASSOCIATIVITY 289

FLOW LINE SPECIFICATION PROPERTY 390

FONT CHARACTERISTIC $\quad 223$

FONT NUMBER $\quad 223$

$\begin{array}{ll}\text { FORM NUMBER } & 37\end{array}$

$\begin{array}{ll}\text { FREE FORMAT } & 14\end{array}$

GENERAL LABEL ENTITY 221

GENERAL NOTE ENTITY 223

GENERATRIX

GEOMETRIC

GEOMETRY

CIRCULAR ARC

COMPOSITE CURVE $\quad 111$

CONIC ARC 116

COPIOUS DATA 122

LINE $\quad 130$

OFFSET CURVE

OFFSET SURFACE 195

PARAMETRIC SPLINE CURVE 132

PARAMETRIC SPLINE SURFACE 138

$\begin{array}{ll}\text { PLANE } & 126\end{array}$

POINT 144

RATIONAL B-SPLINE CURVE 169

RATIONAL B-SPLINE SURFACE 172

RULED SURFACE 146

SURFACE OF REVOLUTION 151

TABULATED CYLINDER 155

TRANSFORMATION MATRIX $\quad 7,98,158$

GLOBAL SECTION

GROUP ASSOCIATIVITY

18

$6,266,278,285,286$

HIER ARCHY

35

HIERARCHY PROPERTY

HYPERBOLA

$117,170,478$

$\begin{array}{ll}\text { INTEGER CONSTANT } & 11\end{array}$

KNOT SEQUENCE FOR B-SPLINE $\quad 469$

$\begin{array}{ll}\text { LABEL DISPLAY ASSOCIATIVITY } & 275\end{array}$

LABEL DISPLAY ASSOCIATIVITY POINTER 31

LANGUAGE STATEMENT CONSTANT 13

LEADER (ARROW) ENTITY 223

LEVEL

LEVEL FUNCTION PROPERTY

$30,345,347$

LEVEL NUMBER

LINE ENTITY 
LINE FONT DEFINITION ENTITY

170,308

LINE FONT PATTERN NUMBER

LINE REMOVAL

LINE WEIGHT NUMBER

LINE WIDENING PROPERTY

6

23,36

LINEAR DIMENSION ENTITY

349

243

LINEAR SPLINE

MACRO

ATTRIBUTES

CAPABILITY SECTION

314

314

DEFINITION ENTITY

EXAMPLES

INST ANCE ENTITY

SYNTAX

MACRO DEFINITION ENTITY

MACRO INSTANCE ENTITY

MATERIAL PROPERTIES

BEAM ELEMENT END RELEASES

BENDING COUPLING MATERIAL STIFFNESS MATRIX

BENDING MATERIAL STIFFNESS MATRIX

CONVECTIVE FILM COEFFICIENT

ELECTROMAGNETIC RADIATION PARAMETERS

ELEMENT THICKNESS

HEAT CAPACITY

LAMINATE MATERIAL STIFFNESS MATRIX

MATERIAL COORDINATE SYSTEM

MATERIAL MATRIX

MASS DENSITY

NODAL LOAD/CONSTRAINT DATA

NON-STRUCTURAL MASS

OFFSETS

POISSON'S RATIO

SECTION PROPERTIES FOR BEAM ELEMENTS

SHEAR MODULUS

STRESS RECOVERY INFORMATION

THERMAL CONDUCTIVITY

THERMAL EXPANSION COEFFICIENT

TRANSVERSE SHEAR MATERIAL STIFFNESS MATRIX

MODEL SPACE

MODIFIED WILSON-FOWLER SPLINE

NAME PROPERTY

$104,158,207$

133,470

NETWORK SUBFIGURE DEFINITION ENTITY

NETWORK SUBFIGURE INSTANCE ENTITY 
OBSOLETE ASSOCIATIVITY ENTITIES

OFFSET CUR VE ENTITY

OFFSET SURFACE ENTITY

ORDERED GROUP ASSOCIATIVITY

195

ORDINATE DIMENSION ENTITY

ORGANIZATION

ORIENTATION

PARABOLA

PARAMETER DATA POINTER

PARAMETER DATA SECTION

$117,170,478$

PARAMETER LINE COUNT NUMBER

30

55

37

PARAMETRIC PIECEWISE CUBIC POLYNOMIAL CUR VE

PARAMETRIC SPLINE CURVE ENTITY

132

PARAMETRIC SPLINE SURFACE ENTITY

138

PART NUMBER PROPERTY

354

PIN NUMBER PROPERTY

353

PLANAR ASSOCIATIVITY

287

PLANE ENTITY

POINT DIMENSION ENTITY

POINT ENTITY

POINTER CONSTANT

PRE-DEFINED ASSOCIATIVITIES

PRODUCT DEFINITION

PROPERTY

DEFINITION LEVELS

126,173

247

144

13

266

1,2

DRAWING SIZE

DRAWING UNITS

DRILLED HOLE

EXTERNAL REFERENCE FILE INDEX

FLOWLINE SPECIFICATION

390

HIER ARCHY

355

LEVEL FUNCTION

347

LINE WIDENING

349

NAME

391

NOMINAL SIZE

389

PART NUMBER

354

PIN NUMBER

REFERENCE DESIGNATOR

352

REGION FILL

348

REGION RESTRICTION

346

TABULAR DATA

PROPERTY ENTITY

QUADRATIC SPLINE

RADIUS DIMENSION ENTITY

RATIONAL B-SPLINE CURVE ENTITY

RATIONAL B-SPLINE SURFACE ENTITY

REAL CONSTANT 
REGION FILL PROPERTY

REGION RESTRICTION PROPERTY

ROTATION

ROTATION MATRIX

RULED SURFACE ENIITY

SECTION ENTITY

251

SECTIONED AREA ENTITY

122,256

SEQUENCE NUMBER

SEXTUPLES

SINGLE PARENT ASSOCIATIVITY

$9,36,38$

122

279

SLANT ANGLE

SPHERE

227

SPLINE CUR VE

SPLINE REPRESENTATION

SPLINE SURFACE

START ANGLE

START SECTION

STATUS NUMBER

STRING CONSTANT

STRUCTURE ENTITIES

ASSOCIATIVITY DEFINITION ENTITY

173

132,469

132,470

138,470

151

16

31

13

ASSOCIATIVITY INSTANCE ENTITY

EXTERNAL REFERENCE ENTITY

EXTERNAL REFERENCE FILE INDEX ASSOCIATIVITY

EXTERNAL REFERENCE FILE LIST PROPERTY

GROUP ASSOCIATIVITY ENTITY

LINE FONT DEFINITION

MACRO DEFINITION ENTITY

MACRO INSTANCE ENTITY

PRE-DEFINED ASSOCIATIVITIES

PROPERTY ENTITY

SUBFIGURE DEFINITION ENTITY

SUBFIGURE INSTANCE ENTITY

TEXT FONT DEFINITION ENTITY

VIEW ENTITY

VIEW LIST ASSOCIATIVITY

VIEWS VISIBLE

VIEWS VISIBLE, PEN, LINE WEIGHT ASSOCIATIVITY

STRUCTURE POINTER

STRUCTURES

SUBFIGURE DEFINITION ENTITY

SUBFIGURE INSTANCE ENTITY

SUBORDINATE ENTITY SWITCH

SURFACE OF REVOLUTION ENTITY

SURFACES

OFFSET SURFACE

262

264

90,417

90,269

388

$266,278,285,286$

308

321

334

266

344

394

397

405

6,411

296

270

272

30

85

394

397

31

151,173

PARAMETRIC SPLINE SURFACE ENTITY

195

138

172

RATIONAL B-SPLINE SURFACE ENTITY

146

RULED SURFACE ENTITY

151

SURFACE OF REVOLUTION ENTITY

155 
TABULAR DATA PROPERTY

TABULATED CYLINDER ENTITY

155,173

TERMINATE ANGLE

TERMINATE SECTION

58

TEXT BOX

227

TEXT FONT DEFINITION ENTITY

405

TORUS

173

TRANSFORMATION MATRIX POINTER

31

TRANSFORMATION MATRIX ENTITY

TRANSLATION

TRANSLATION VECTOR

TRIMMED (PARAMETRIC) SURFACE ENTITY

TRIPLES

$7,98,158$

7,103

104

201

122

UNBOUNDED PLANE

126

VERTEX POINT

209

VIEW ENTITY

6,411

VIEW LIST ASSOCIATIVITY

296

VIEW POINTER

30

VIEW POR T

VIEWING DIRECTION

303,411

411

270

272

VIEWS VISIBLE, PEN, LINE WEIGHT ASSOCIATIVITY

133,470

WILSON-FOWLER SPLINE

WIRE-FR AME

WITNESS LINE ENTITY

7

259

ZT DISPLACEMENT

207 

BIBLIOGRAPHIC DATA

SHEET (See instructions)

4. TITLE AND SUBTITLE

Init1al Graphics Exchange Specification (IGES) Version 3.0

5. SUTHCh(S)

Swith, B., Briggs, D., Colsher, R., Rennicott, P., Parks, C., Wellington, J. 8. IERFORMING ORGANIZATION (If joint or other thon NBS, see instructions)

nATIONAL DUREAU OF STAMDARDS

DEPARTMENT OF COMMERCE

7. ContracvGrant No.

WASHIM GTON, D.C. 20234

8. Type of Report 8. Period Covered Jan 1983 - Dec 1985

9. SPONSORING ORGANIZATION NAME AND COMPLETE ADDRESS (Streer, City. State, ZIP)

$\square$ Document describes a computer prozram: SF-185. FIPS Software Summary. is attached.

11. ABSTRACT (A 200-word or less foctuol summory of most significant information. If document includes a slgnificant bibliogrophy or literoture surver. mention (t here)

This document contains Version 3.0 of the Initial Graphics Exchange Specification, a defined format for the creation of a file which enables data found in today's commercially avallable CAD/CAM systems to be exchanged or archived.

IGES, Version 1.0, published as NBSIR 80-1978 (R) In January 1980, consisted of entity definitions for geometry, drafting and structural information. Definition entities were provided as a means of expanding the utility of IGES.

Version 3.0 further refines the concept and offers increased capability in both geometry and non-geometry data exchange. The applications of printed wiring boards and finite element models are well supported in addition to enhancements for mechanical products.

12. KEY WORDS (Six to twelve entries; alphabetical order; capitalize only proper names; and seporote key words by semicolons) Computer Alded Design (CAD), Computer Aided Manufacturing (CAM), design drawing, electrical information, exchange format, finite element modeling, geometrics, graphics.

X] Order From Superintendent of Documents, U.S. Govarnmant Printing Offica, Washington, D.C. 20402.

[D Order From National Technical Information Servica (NTIS), Springfield, VA. 22161

14. NO. OF PRINTED PAGES

$$
523
$$

15. Price

$$
\$ 40.95
$$


